\title{
Tracing fast roads towards bone regeneration
}

Citation for published version (APA):

Cámara Torres, M. (2021). Tracing fast roads towards bone regeneration: strategies to augment the bioactivity of additive manufactured scaffolds. [Doctoral Thesis, Maastricht University]. Gildeprint Drukkerijen. https://doi.org/10.26481/dis.20210913mt

Document status and date:

Published: 01/01/2021

DOI:

10.26481/dis.20210913mt

Document Version:

Publisher's PDF, also known as Version of record

\section{Please check the document version of this publication:}

- A submitted manuscript is the version of the article upon submission and before peer-review. There can be important differences between the submitted version and the official published version of record.

People interested in the research are advised to contact the author for the final version of the publication, or visit the DOI to the publisher's website.

- The final author version and the galley proof are versions of the publication after peer review.

- The final published version features the final layout of the paper including the volume, issue and page numbers.

Link to publication

\footnotetext{
General rights rights.

- You may freely distribute the URL identifying the publication in the public portal. please follow below link for the End User Agreement:

www.umlib.nl/taverne-license

Take down policy

If you believe that this document breaches copyright please contact us at:

repository@maastrichtuniversity.nl

providing details and we will investigate your claim.
}

Copyright and moral rights for the publications made accessible in the public portal are retained by the authors and/or other copyright owners and it is a condition of accessing publications that users recognise and abide by the legal requirements associated with these

- Users may download and print one copy of any publication from the public portal for the purpose of private study or research.

- You may not further distribute the material or use it for any profit-making activity or commercial gain

If the publication is distributed under the terms of Article $25 \mathrm{fa}$ of the Dutch Copyright Act, indicated by the "Taverne" license above, 


\section{Tracing fast roads towards bone regeneration: Strategies to augment the bioactivity of additive manufactured scaffolds}


Copyright 2021 @ María Cámara Torres, Maastricht. Neither this book nor its parts may be reproduced without written permission of the author.

Tracing fast roads towards bone regeneration: Strategies to augment the bioactivity of additive manufactured scaffolds

PhD Thesis, Maastricht University, Maastricht, The Netherlands

ISBN: 978-94-6423-383-4

Cover Art: Marco Antonio Cámara Palacios

Printed by: Gildeprint, Enschede 


\title{
Tracing fast roads towards bone regeneration: Strategies to augment the bioactivity of additive manufactured scaffolds
}

\author{
DISSERTATION
}

To obtain the degree of Doctor at the Maastricht University, on the authority of the Rector Magnificus, Prof.dr. Rianne M. Letschert in accordance with the decision of the Board of Deans, to be defended in public on Monday $13^{\text {th }}$ September 2021, at 13:00 hours.

\section{By}

María Cámara Torres

Born on the $16^{\text {th }}$ August 1991, in Madrid, Spain. 


\section{Promotor:}

Prof. Dr. Lorenzo Moroni

\section{Co-promotor:}

Dr. Carlos Mota

\section{Assessment committee:}

Prof. Dr. Pamela Habibovic (Chair)

Dr. Elizabeth Balmayor

Prof. Dr. ir. Sander Leeuwenburgh (Radboud University)

Dr. Ioannis Papantoniou (KU Leuven)

Prof. Dr. Martijn Poeze

The research described in this thesis was conducted at MELRN Institute for Technology Inspired Regenerative Medicine in Maastricht University. The work in this thesis was finantially supported by the European Commission H2020 Programm, call H2020-NMPPILOTS-2015, Project FAST- Functionally graded Additive Manufacturing scaffolds by hybrid manufacturing. 


\section{Table of Contents}

$\begin{array}{lll}\text { Chapter } 1 & \text { General introduction and aims } & 9\end{array}$

Chapter 2 Boosting bone regeneration using augmented melt extruded additive manufactured scaffolds

Chapter 3 Improving cell distribution on 3D additive manufactured scaffolds through engineered seeding media density and viscosity

Chapter 4 Tuning cell behavior on 3D scaffolds fabricated by atmospheric plasma assisted additive manufacturing

Chapter 5 Effect of highly loaded nanohydroxyapatite composite scaffolds prepared via melt extrusion additive manufacturing on the osteogenic differentiation of human mesenchymal stromal cells

Chapter 6 Effect of reduced graphene oxide (rGO) compaction degree and concentration on rGO-polymer composites printability and cell interactions

Chapter 7 3D Additive manufactured composite scaffolds with antibioticloaded lamellar fillers for bone infection prevention and tissue regeneration

Chapter 8 General discussion 289

$\begin{array}{lll}\text { Chapter } 9 & \text { Impact } & 315\end{array}$

$\begin{array}{ll}\text { Summary / Samenvatting } & 321\end{array}$

$\begin{array}{ll}\text { List of publications } & 327\end{array}$

$\begin{array}{ll}\text { Acknowledgement } & 331\end{array}$

$\begin{array}{ll}\text { Biography } & 335\end{array}$ 



\section{Chapter 1}

General introduction and aims 

As one of the most frequently damaged tissues in our body, bone has always been in the spotlight of clinicians and scientists. In principle, bone tissue can self-repair. However, there are some cases, in which the injury is too large and severe, or the local environment is subobtimal for this process to happen on its own demanding external help to manage the injury. Unlike the axolotl, the human body does not have the unique ability to perfectly self-regenerate after complex damage.

For decades, the use of autografts, allografts or methalic prosthesis have been considered as the gold standard clinical practices to reconstruct large bone defects or non-unions. However, these approaches have never been considered optimum solutions, due to donor morbidity, immunogenicity, unavailability and/or structural failure. With the birth of the tissue engineering field, which aims at combining biomaterials, cells and/or biophysical and chemical signals to promote endogenous tissue regeneration, a new research perspective appeared to confront non-union bone injuries. Since then, a wide variety of novel approaches have been suggested towards a more effective clinical solution. Most of these revolve around the design of an ideal scaffold, which upon implantation can serve as a temporary structure mimicking bone extracellular matrix (ECM) to promote cell adhesion, proliferation, differentiation and new tissue formation. Several biomaterials and biofabrication techniques have been investigated trying to create a scaffold fulfilling the required biodegradability, bioactivity and mechanical properties. In this regard, natural and synthetic polymers, ceramics, metals, and their combinations have been processed through a variety of fabrication methods, including particulate leaching, gas foaming, thermally induced phase separation, freeze-drying, electrospinning and additive manufacturing (AM). Among all, AM, and melt extrusion AM (ME-AM) in particular, has stood out over the other biofabrication techniques, as it allows the reproducible controll over scaffold size, shape, internal interconnected macroporosity, which are crucial factors for bone tissue engineered (BTE) scaffolds success. In spite of these benefits, ME-AM possess also its own drawbacks, associated with the inherent characteristics of synthetic polymers, which are the only processable materials through this technique and generally lack the required bioactivity to induce bone regeneration. In Chapter 2 we summarize the research that has been carried out over the last decades on strategies to augmenting the physicochemical properties of such ME-AM scaffolds, in order to enhance their interaction with cells. These include modifications of the scaffold internal architecture and surface properties, the fabrication of hybrid scaffolds, combining the ME-AM framework with fibrous or hydrogel networks, and the use of polymer composites containing inorganic, 
organic or metallic fillers. In spite of all these efforts, the search for optimal scaffolds that can boost bone regeneration continues, since an ideal ME-AM scaffold that shows an optimal in vivo tissue regeneration has not yet been found. To further contribute to the field, and by overcoming some of the identified challenges, this thesis aims at investigating different material-driven approaches to design and fabricate bioactive ME-AM scaffolds for BTE. These approaches are described in the different chapters of the thesis.

The first step towards the short and long-term functionality of a scaffold-supported engineered tissue is an efficient scaffold seeding. However, cell seeding on ME-AM scaffolds is challenging, due to the interplay of macroporosity, inert scaffold surface properties, and gravity. Chapter 3 and Chapter 4 propose two different alternatives to efficiently seed ME-AM scaffolds in terms of seeding efficiency and distribution. While some of the pre- or post-scaffold fabrication modification techniques presented in Chapter 2 have been used on an attempt to circumvent these intrinsic cell seeding difficulties, there is still the need of a simple and reliable method that does not depend on the scaffold architecture or its material physicochemical properties. In this regard, Chapter $\mathbf{3}$ describes a simple, unique and universal approach to statically seed ME-AM scaffolds in a precise and reproducible manner, regardless of the scaffold type. In this chapter, the independent addition to the cell seeding medium of two biocompatible macromolecules, dextran and Ficoll, is studied to adjust the medium viscosity and density, respectively, and tune cell settling velocity for better attachment during the seeding process. The influence of these seeding media on cell attachment and distribution along the scaffolds' cross section, as well as on the osteogenic differentiation capacity of human mesenchymal stromal cells (hMSCs), is evaluated.

In addition to the simple strategy presented in Chapter 3, the method described in Chapter 4 relies on the use of a high-end technology for attaining enhanced cell attachment on ME$A M$ via plasma modification. This technology, which combines a ME printhead and an atmospheric pressure plasma jet (APPJ) module, not only allows scaffold production and treatment in a single-step, but also ensures a controlled and homogeneous surface modification, in contrast to conventional post-fabrication plasma approaches. Here, MEAM polymeric scaffolds are plasma-polymerized with (3-Aminopropyl) trimethoxysilane and maleic anhydride-vinyltrimethoxysilane, which deposit rather stable amine- and carboxyl-based coatings on the scaffolds filaments. The effect of these uniquely performed 
treatments on scaffolds' hydrophilicity, protein adhesion, cell adhesion mechanism and hMSCs osteogenic differentiation is evaluated.

After ensuring an efficient cell attachment, composite ME-AM scaffolds comprising the copolymer PEOT/PBT and either nanohydroxyapatite $(\mathrm{nHA})$, reduced graphene oxide (rGO), or antibiotic-loaded lamellar fillers, are fabricated in Chapters 5, 6 and 7, respectively. Among all the strategies for augmenting the bioactivity of ME-AM polymeric scaffolds, the use of composites enable bulk scaffold properties modification (i.e. mechanical, physicochemical, electrical) without altering the scaffold architecture or the need of post-fabrication steps. To replicate the organic-inorganic composition of bone ECM, and taking advantage of the osteoinductive properties of calcium and phosphate, composite constructs comprising polymers and calcium phosphate materials, and in particular nHA, have been widely explored. Despite material advances and thorough studies on the printability of highly loaded nHA ME-AM composite scaffolds, the biological assessment of such scaffolds has been mostly overlooked and, in general, poorly characterized. In Chapter 5, we aimed to fill this important gap and provide an in depth in vitro biological characterization of highly loaded nHA scaffolds. Polymer-nHA composite scaffolds with up to $45 \mathrm{wt} \% \mathrm{nHA}$ were fabricated, and the effect of $\mathrm{nHA}$ content on the osteogenic differentiation of hMSCs was intensively evaluated using several biochemical and molecular assays, such as q-PCR, immunofluorescence, ELISA and calcium staining. In addition, a mechanical analysis was also performed.

Towards the fabrication of ME-AM scaffolds with outstanding physicochemical and electroactive properties for bone formation stimulation, graphene based materials can be used as fillers. In spite of its thermal stability, electroconductivity and easy production, compared to other graphene derivative, rGO has been mostly overlooked as filler within ME-AM composite scaffolds due to its low bulk density compromising its safe handling during melt compounding. In order to overcome this limitation, rGO was densified in Chapter 6, and the effect of densification on rGO compaction degree and on the resulting melt compounded composites physicochemical properties and printability via ME-AM was assessed. In addition, the effect of rGO concentration on the materials' physicochemical properties and cell-material interactions in 2D and 3D, including osteogenic differentiation, was also investigated.

Bone infection is a common complication after trauma or implant surgery. On an attempt to circumvent the use of high doses of systemic antibiotic therapy, local antibiotic delivery 
systems are desired. However, approaches to fabricate ME-AM scaffolds with antimicrobial properties are based either on post-fabrication and multi-step surface adsorption treatments, which lead to poor loading efficiencies and uncontrolled release kinetics, or on low temperature ME-AM $\left(<120^{\circ} \mathrm{C}\right)$ to preserve the bioactive molecules functionality, which limits the polymer choice. In Chapter 7, the possibility of incorporating antibiotics within the polymer matrix of scaffolds fabricated by high temperature ME-AM $\left(\sim 190^{\circ} \mathrm{C}\right)$, via antibiotic-loaded inorganic fillers, is discussed. In this chapter, two different antibiotic-nanofiller complexes are investigated, and the antibiotic release kinetics, drug stability after printing and scaffold antimicrobial properties against relevant orthopedic bacterial strains, evaluated. In addition, the osteogenic differentiation capability of hMSCs on the composite scaffolds is assessed to ensure their dual functionality.

In Chapter 8, the results described in the aforementioned chapters are discussed and place into the state of the art context, while providing future perspectives. To finalize, a reflection on the scientific and social impact of the research carried out in this thesis is presented in Chapter 9. 


\section{Chapter 2}

Boosting bone regeneration using augmented meltextruded additive manufactured scaffolds

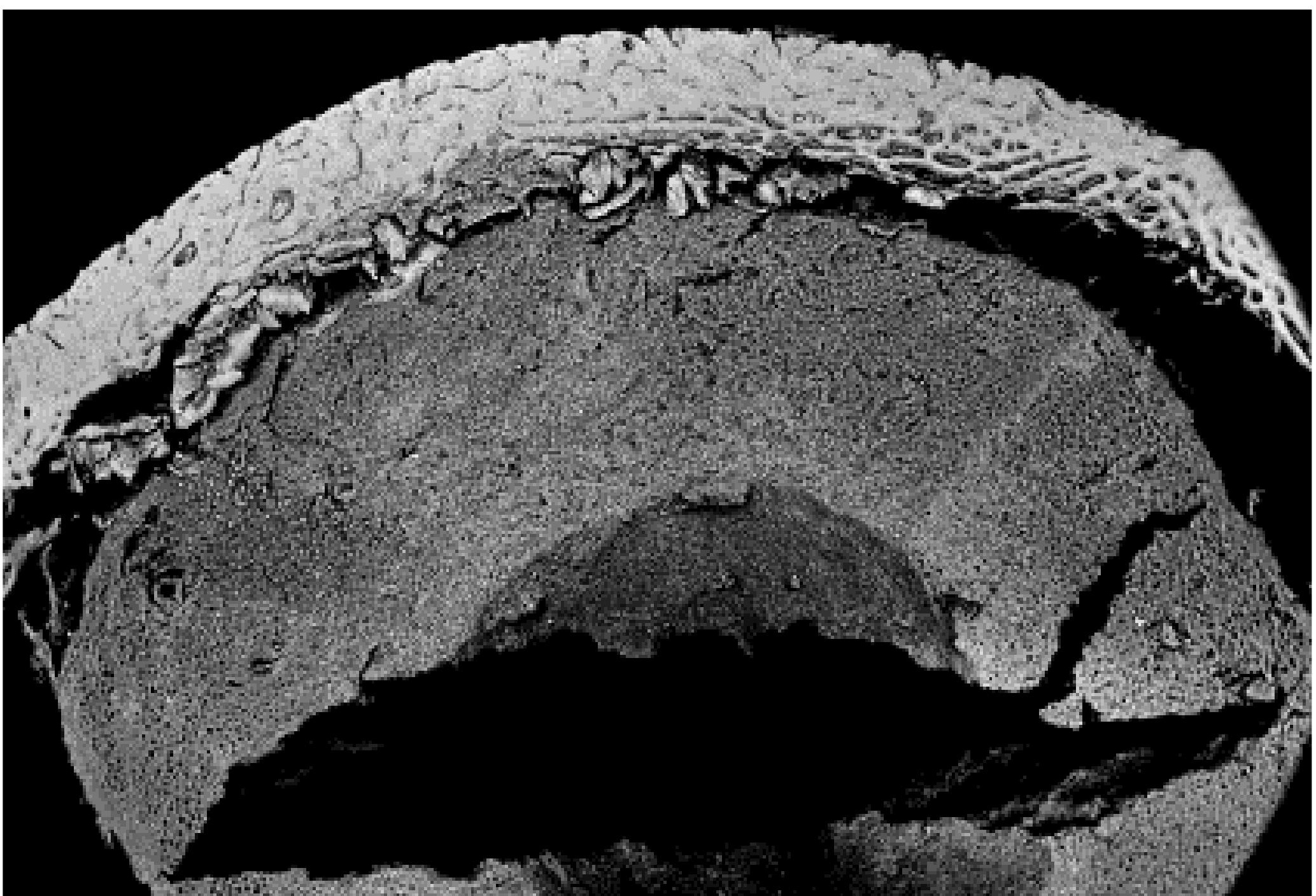





\begin{abstract}
The bone tissue engineering (BTE) field is in active search of the ideal scaffold to give a clinical solution for bone regeneration in non-union fracture scenarios. During the last decades, the use of additive manufacturing ( $\mathrm{AM}$ ) technologies and, in particular, melt extrusion $\mathrm{AM}$ (ME-AM), has been thoroughly investigated towards this aim. ME-AM enables the cost-effective fabrication of personalized 3D scaffolds, with a controlled and highly interconnected porosity, through the solvent-free processing of biodegradable and mechanically robust thermoplastic polymers. In addition to these properties matching the requirements for BTE scaffolds, the synthetic polymers used to fabricate these constructs are also more amenable for further functionalization than metals or ceramics, to influence cell behavior, making thermoplastic materials a preferred choice for BTE. This review provides a comprehensive analysis of various ME-AM scaffolds developed for BTE, along with the approaches used to augment their bioactivity, which include architectural modifications, surface physical and chemical modifications, the incorporation of secondary fibrous or hydrogel networks within the scaffolds pores, and the use of filler-polymer composites for ME-AM scaffold fabrication. A detailed examination on how each of these strategies affect the final scaffold physicochemical and mechanical properties is presented. Special emphasis is placed on discussing the effect of these modifications on promoting cell osteogenic differentiation in vitro and bone tissue development in vivo.
\end{abstract}




\section{Bone and its regenerative capacity}

Bone is a complex hierarchically structured tissue that plays a critical role in human physiology including locomotion, organ protection, blood production, mineral storage and homeostasis, and source of mesenchymal stem cells in the marrow compartment. ${ }^{1}$ The building blocks of the bone matrix consist of an inorganic phase of carbonated hydroxyapatite (HA), and an organic phase mainly composed of collagen type I (COL I), among other types of collagens, proteoglycans, fibronectin and specific bone proteins, such as osteopontin (OPN), bone sialoprotein (BSP) and osteocalcin (OCN), in minor percentages. ${ }^{2}$ In the cortical bone, which extends along the shaft or diaphysis of long bones and becomes thinner towards the distal and proximal ends or epiphysis, mineralized collagen nanofibers organize parallel into concentric lamellae and surround the Harversian channel constituting the osteons. ${ }^{3}$ On the other hand, cancellous bone, which is present in long bones mostly in their epiphysis, is organized into irregular and convoluted lamellae, which create an intricate porous trabecular framework as housing for the soft and highly cellularized bone marrow. ${ }^{3}$ Due to their differences in porosity and architecture, cortical and cancellous bone possess an elastic modulus at the macroscale of $15-20 \mathrm{GPa}$ and $0.1-2 \mathrm{GPa}$, and a compressive strength of $100-200 \mathrm{MPa}$ and 2-20 MPa, respectively. 4-5 These properties are highly dependent on age, health condition, anatomic site and loading direction. ${ }^{4,6-8}$ At the tissue level, cortical bone possess slightly higher density and mineral content compared to trabecular bone, as it presents a lower rate of turnover. ${ }^{9}$ Due to this, microtensile measurements have also revealed differences in the elastic modulus of both tissues at the microscale ( 18 GPa for cortical bone vs. $11 \mathrm{GPa}$ for cancellous bone). Yet, they are in the same order of magnitude, due to their similar lamellar nanostructure. 8 These impressively high mechanical properties are owed to the mineralization of the collagen fibers, which provides stiffness and structural reinforcement to the viscoelastic and tough collagen.

Bone is constantly undergoing remodelling to adapt to mechanical stresses, resulting from physical exercise or loading, and to maintain the integrity of the skeleton, by repairing micro fractures caused by the bending and torsion of bones during locomotion. ${ }^{10}$ This is a dynamic process orchestrated by a variety of biochemical and mechanical factors that requires the interplay between different cell phenotypes: the bone-forming osteoblasts, the bone-degrading osteoclasts, and the bone lining cells and the osteocytes. ${ }^{11}$ In line with its remodeling capacity, bone also has self-healing abilities after fracture. ${ }^{12}$ In bone fractures, 
not only the structural integrity of bone is disturbed, but also the vascular structures, limiting the nutrients flow at the injury site. Therefore, the healing process is much more complex than during spontaneous remodelling, and includes an inflammatory phase, a soft callous formation, and a remodelling phase, involving multiple cellular types, including mesenchymal stromal cells, and molecular activities that recapitulate the endochondral ossification happening during embryogenesis. ${ }^{13}$ However, improper stabilization, age and comorbidities of the patient, as well as large bone defects, caused by disease, traumatic injury, implant surgery, infection or tumor resection, can negatively influence the healing process, leading to non-union in up to $10 \%$ of the cases. ${ }^{14}$ Current strategies used in the clinics for the treatment of non-union or critical size bone defects mainly rely on: i) the distraction osteogenesis or llizarov method (Figure 1A), ${ }^{15}$ ii) the induced membrane or Masquelet technique, ${ }^{16-17}$ combined with autografts, such as intramedullary bone grafts (Figure 1B-C), iliac crest, or vascularized fibula graft (Figure 1D) ${ }^{18}$ iii) allografts, or iv) demineralized bone matrices. 19-20 Treatments based on the Illizarov or Masquelet methods are often complex, requiring external fixation and multiple surgeries. Similarly, natural bone grafts (autografts or allografts) possess several disadvantages, including donor site morbidity, restricted availability, high costs, pain, prolonged rehabilitation, lack of structural properties, and can trigger inflammation, immunological rejections in the case of allografts, or infections. ${ }^{1}$ Therefore, the management of non-union bone defects remains a major clinical challenge.

During the past decades, a large amount of efforts have been dedicated to investigate tissue-engineered strategies to regenerate bone in non-union scenarios, and avoid the aforementioned disadvantages associated with the currently used therapies. ${ }^{21}$ Particular attention has been given to additive manufacturing (AM) technologies, which enable to finely control the scaffold properties. In the following sections of this chapter, a brief overview on current AM techniques used to fabricate scaffolds for bone tissue engineering (BTE) applications will be provided, and special focus will be given to the advantages of melt extrusion AM (ME-AM) over other technologies. This includes the compatibility of the synthetic polymers used for ME-AM with various supplemental methodologies. While making this choice, we acknowledge the promising bone regeneration capacities of ceramic and biodegradable metal scaffolds, however they offer suboptimal biomechanical properties, being either too brittle or too stiff, respectively, compared to polymeric scaffolds. In this regard, current state-of-the-art strategies used to augment the bioactivity of polymeric ME-AM scaffolds towards an ideal BTE scaffold will be extensively discussed. 
The related in vitro cell responses and in vivo bone regeneration produced by these strategies will be highlighted.
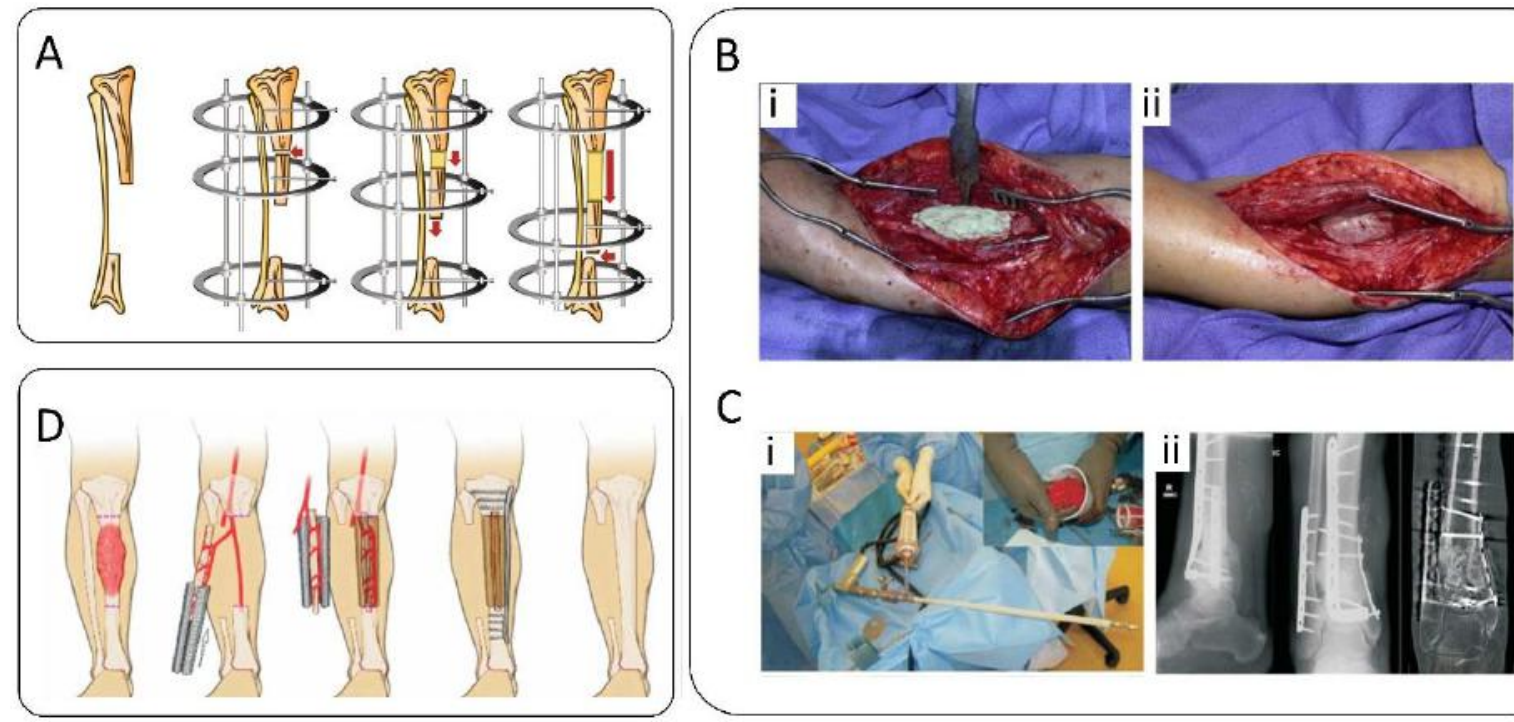

C
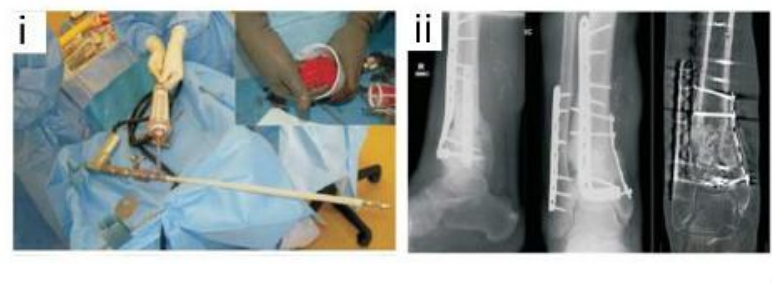

Figure 1 - Current gold standard techniques for the treatment of non-unions. (A) Schematic representation of the distraction osteogenesis technique. After the formation of a critical size defect, a bone segment is fractured and brought into close contact with the bone. The llizarov apparatus (external fixation) is used to gradually separate the bone segment, allowing new bone to form in the gap via conventional endochondral ossification during fracture healing. Adapted with permission from Ref. ${ }^{22}$ (B) Clinical case in which the Masquelet technique was applied to treat a humeral non-union. (i) After debridement of the bone ends, a cement spacer was inserted for 2 months. (ii) After this period, a pearly membrane was induced around the cement, which was filled with a bone graft after spacer removal. Adapted with permission from Ref. ${ }^{17}$ (C) Clinical case combining the Masquelet technique with an intramedullary bone graft to treat a tibial non-union. (i) After poly (methyl methacrylate) (PMMA) spacer removal and the induced membrane formation, the defect site was packed with autologous cancellous bone graft collected from the femur with the reamer-irrigator aspirator (RIA) technique. (ii) 7 months post-surgery X-rays. (D) Schematic representation of the vascularized fibula approach combined with a supporting construct comprising the following steps: resection of malignant tumor, construct placed around the vascularized fibular graft, insertion in the critical size defect and use of internal fixation. Adapted with permission from Ref. ${ }^{21}$

\section{Towards AM scaffolds for BTE applications}

One of the pillars of tissue engineering (TE) are scaffolds. An ideal scaffold should provide a physico-chemically biomimetic environment to support and boost bone tissue ingrowth and regeneration. ${ }^{23}$ In this regard, the use of biocompatible materials to avoid the risks of local toxicity and undesired immune/inflammatory responses is fundamental. Moreover, the scaffold degradation and the bone formation rates should match, ensuring the stability of the defect post implantation. At the same time, scaffolds should provide mechanical support and stability, ideally matching the mechanical properties of neighboring bone 
tissue to prevent stress shielding. ${ }^{24}$ Surface properties such as chemistry, surface charge and topography are also critical, as they will dictate cell-material interactions for bone tissue ingrowth. While the aforementioned properties are of utmost importance, the architectural parameters of the scaffold i.e. pore interconnectivity, porosity, and pore size and shape, will ultimately determine the success of the scaffold in promoting tissue ingrowth leading to the regeneration of a fully functional tissue. Interconnected pores are necessary for efficient management of nutrients, oxygen, metabolic waste and material biodegradation products, as well as for continuous vascularization and tissue ingrowth within the scaffold. Moreover, scaffolds with pore sizes of at least $300 \mu \mathrm{m}$ are needed to promote neovascularization and favor osteogenesis in vivo. ${ }^{25-27}$ Pore shape and curvature has also shown to influence bone formation by modulating cell response and rate of tissue regeneration. ${ }^{28-29}$ Since it is difficult to decouple porosity from mechanical properties and degradation rate, all these parameters need to be carefully balanced in order to optimize scaffolds for bone regeneration. ${ }^{25,27}$

It is already established that conventional scaffold fabrication techniques, such as particulate leaching, gas foaming, phase separation, solution casting or freeze drying, cannot ensure pore interconnectivity, nor the precise control over pore size, shape and spatial distribution. ${ }^{30-31}$ On the contrary, biofabrication technologies derived from AM techniques for rapid prototyping, have demonstrated their potential to generate reproducibly complex-shaped tissue engineered constructs with a high degree of control on architectural parameters. Depending on the material type and its processing method, the most commonly used AM technologies in biofabrication of BTE constructs can be classified into: ${ }^{32-33}$

i) powder based techniques, including 3D printing, selective laser sintering (SLS), selective laser melting (SLM) and electron beam melting (EBM),

ii) vat photopolymerization techniques, including stereolitography (SLA), digital light processing (DLP) and two-photon polymerization (2PP),

iii) ME techniques, including fused deposition modeling (FDM), 3D fiber deposition (3DF) and bioextrusion,

iv) solution extrusion, including direct ink writing, low temperature deposition manufacturing (LDM), pressure-assisted microsyringe (PAM), and wetspinning, and 
v) bioprinting, includding bioplotting, ink-jet bioprinting and laser-assisted bioprinting.

Among all the aforementioned, ME-AM techniques are the most used $\mathrm{AM}$ techniques in BTE. Here, a thermoplastic polymer or composite in the form of a filament (FDM) or pellets (3DF and bioextrusion) is hot-extruded in molten form through a nozzle upon application of pressure via rollers pushing the filament (FDM), pressurized gas/piston (3DF) or a screw (bioextrusion), and deposited as solidified strut in a layer-by-layer manner. ${ }^{34}$ Compared to other AM technologies, ME-AM does not require demanding hardware, its operation principle is straightforward, and the materials used do not require extensive development, in terms of chemistry. Moreover, ME-AM techniques offer one of the highest resolution-totime of manufacturing (RTM) ratio among all AM techniques ( $1 \times 10^{-3} \mathrm{~m}^{2} /$ minute). $\mathrm{RTM}$ is a parameter that defines the efficiency of the manufacturing process in terms of the ability to produce scaffolds with fine details in a short time, and is expressed as volume of material deposited per minute, divided by the minimum printed feature dimension. ${ }^{33}$ An RTM ratio of $\sim 1 \times 10^{-3} \mathrm{~m}^{2} /$ minute is also estimated for SLS, where a high power laser selectively sinters a powder bed layer-by-layer. In spite of producing scaffolds at a faster rate, SLS offers lower resolution than ME (400 $\mu \mathrm{m}$ vs. $200 \mu \mathrm{m}) .{ }^{33}$ Moreover, SLS and other powderbased methods such as 3D printing, which is based on the controlled deposition of a binder material on successive powder layers, require post-fabrication heat treatments to complete the binding reactions and increase the construct mechanical properties, as well as a depowdering step to remove excess material. ${ }^{35}$ Similarly, scaffolds fabricated by solution extrusion methods, in which the material in a solvent is extruded at low temperature, are followed by post-lyophilization (i.e. LDM) or drying steps (i.e. PAM and wet-spinning) to remove the organic solvent, which also leads to scaffolds shrinkage and loss of mechanical properties. ${ }^{36-37}$ Besides, the long time required to fabricate thick constructs by solvent extrusion, due to their low minimal feature size $(\sim 20 \mu \mathrm{m})$, contributes to a low RTM ratio ( $0.5 \times 10^{-3} \mathrm{~m}^{2} /$ minute). ${ }^{33}$ Generally, the use of binders and organic solvents is by itself a limitation of all the aforementioned processes. Such additives could potentially induce cytotoxic effects if not well removed prior to their contact with cells. Likewise, uncured resin and photoinitiator remnants in scaffolds produced by vat photopolymerization techniques, which work by selectively crosslinking layers of a photosensitive resin by laser (SLA and 2PP) or UV light (DLP), may be toxic. ${ }^{38}$ Despite their accuracy, ability to fabricate complex shapes without additional support, and high speed (especially DLP), ${ }^{33}$ the questionable biocompatibility of photopolymers and photoinitiators, together with the limited availability 
of such systems and their demanding rheological specifications, has delegated vat photopolymerization techniques to a secondary plane within the BTE field. ${ }^{38-39}$

Post-processing steps involving solvent and binder removal, or post-curing, are not necessary in ME-AM, as here the raw polymeric material is only subjected to melting and subsequent solidification. Moreover, most of the commonly used biodegradable thermoplastics for bone scaffolds fabrication, such as poly( $\varepsilon$-caprolactone) (PCL), poly(lactic acid) (PLA), and poly(lactic-co-glycolic) acid (PLGA), have already been approved for multiple applications by the Food and Drug Administration (FDA). These materials have been used for decades in the clinic, processed by melt-based techniques. Some examples include melt spun PCL or PLGA resorbable sutures, ${ }^{40-41}$ or injection molded PLA resorbable screws for anterior cruciate ligament reconstruction. ${ }^{42}$ The record of accomplishment of melt-based techniques in medical device manufacturing further emphasizes the enormous translational potential of ME-AM scaffolds to the clinic. Examples of this are the $3 \mathrm{D}$ implant Osteoplug ${ }^{\mathrm{TM}}$ and the thin interwoven mesh Osteomesh ${ }^{\mathrm{TM}}$, from Osteopore International, which are the first marketed 3D AM medical devices for bone regeneration applications. These implants are fabricated by FDM using PCL and used as Class II medical devices for craniofacial applications. These products have their origin in the research of Hutmacher et al., who first described the fabrication of FDM scaffolds for TE applications using PCL and PCL/tricalcium phosphate (TCP) in 2000. 23, 43 Promising in vitro research led to in vivo studies in large animal models and to firstin-human clinical studies to evaluate the feasibility of the PCL scaffolds for skull bone regeneration. ${ }^{44-47}$ Subsequent clinical studies were performed to study their safety and efficacy for alveolar ridge preservation after tooth extraction and orbital floor fractures reconstruction. ${ }^{48-49}$

PCL, together with other commonly used thermoplastics, such as PLA, PLGA, poly(ethylene oxide terephthalate)/poly(butylene terephthalate) (PEOT/PBT) and thermoplastic polyurethane (TPU), are biocompatible, biodegradable, possess relatively high mechanical properties, can be easily processed, and can support in vitro and in vivo tissue ingrowth which make them excellent candidates for bone scaffold materials. ${ }^{50-54}$ However, these synthetic polymers generally lack bioactivity to stimulate cell and protein adhesion, which are necessary for an efficient cell differentiation and tissue formation. This is due to such polymers being relatively hydrophobic, with static contact angles in the range of $75^{\circ}-90^{\circ}$, and lacking sufficient reactive side-chain groups for influencing cell 
behavior. ${ }^{55-56}$ For this reason, research has been focused over the last two decades on augmenting the bioactivity of ME-AM scaffolds for BTE made of synthetic polymers. These include i) modifications of the scaffold architecture, ii) modification of scaffolds filaments' surface, in terms of roughness, microporosity, chemistry or bioactive coatings, iii) the fabrication of hybrid scaffolds, combining the ME-AM framework with fibrous or hydrogel networks, and iv) the use of polymer composites containing inorganic, organic or metallic fillers. (note: while the terms 'hybrid' and 'composite' are broad, permitting overlaps in the above defined categories, here 'hybrid' will be used for scaffolds prepared using a combination of material classes or fabrication techniques, and 'composite' will be used for cases where a filler is loaded into the thermoplastic polymer).

\section{Scaffold architecture modification}

A major strategy in BTE is the fabrication of scaffolds mimicking the mechanical properties of human bone. ME-AM offers the possibility of modulating the porosity and, therefore, the mechanical properties of the scaffolds, by simply tuning layer-by-layer the fiber deposition pattern, in terms of fiber distance (FD) and deposition angle. ${ }^{57}$ Mechanical analysis of scaffolds fabricated from various polymers (PCL, poly(ester) based TPU (PEU) and PEOT/PBT) has proven that for a given deposition angle, increasing the FD in XY or the layer thickness in $Z$, leads to an increase in the scaffolds' porosity and a decrease in stiffness (Figure 2A). ${ }^{58-60}$ A larger amount of deposited polymer, and the subsequent decrease of porosity within the same scaffold volume, explained higher compression modulus upon FD reduction. Similarly, minimizing the angle of deposition from $90^{\circ}$ towards $15^{\circ}$ while keeping porosity, FD and layer thickness constant, has also shown to decrease the mechanical properties of scaffolds, due to reduced contact points between fibers increasing the local stress experienced by the structure (Figure 2B). ${ }^{61}$ In an attempt to maximize the scaffold stiffness while maintaining an optimal porosity ( 60\%), PCL scaffolds with triply periodic minimal surface (TPMS) geometries were recently manufactured by ME-AM (Figure 2C). ${ }^{62}$ Among other geometries, gyroid scaffolds had a compression modulus of 60-80 MPa, higher than previously reported PCL scaffolds with similar porosity but standard geometries. ${ }^{61,63}$ While the mechanical properties that can be attained with scaffolds fabricated with commonly used polymers, such as PCL, lies in the range of cartilage and cancellous bone mechanical properties (up to $120 \mathrm{MPa}$ ), other materials, such as PLA, can provide scaffolds with much higher mechanical properties 
(compression modulus up to $450 \mathrm{MPa}$ ), which are more suitable for load bearing applications. 64

Besides having an impact on the scaffold's mechanical properties, the scaffold pore architecture has also shown to influence cell seeding efficiency and distribution on scaffolds, which can directly affect the tissue formation rate and uniformity, and cell differentiation behavior. ${ }^{65-66}$ Generally, achieving a high seeding efficiency on ME-AM scaffolds is difficult, due to the open pore architectures and the poor cell-adhesion sites of the scaffold materials. In this regard, scaffolds displaying a gradient in pore size along the vertical direction have shown to improve cell seeding efficiency and distribution, without compromising porosity (Figure 2D). ${ }^{67}$ Cell differentiation has also been shown to be modulated by scaffold architecture. In this regard, Di Luca at al. showed that human bone marrow-derived mesenchymal stromal cells (hBM-MSCs) preferentially differentiate towards the osteogenic lineage, over chondrogenic differentiation, in PEOT/PBT scaffolds with high FD (900 - $1100 \mu \mathrm{m})$, compared to smaller pore size scaffolds (500 $\mu \mathrm{m})$, according to increased alkaline phosphatase (ALP) activity and ECM mineralization. ${ }^{68}$ This was in agreement with the study of Woodfield et al., where a higher glycosaminoglycan (GAG) and Collagen II production by chondrocytes was observed on small pore size PEOT/PBT scaffolds. 69 In addition, higher ALP activity and the upregulation of osteogenic related genes has also been shown to be better supported on PCL scaffolds with rhomboidal pore shapes, given by a $0 / 15^{\circ}$ lay down pattern, compared to other filament orientation angles. 70 In comparison, square shaped pores $\left(0 / 90^{\circ}\right)$ supported enhanced chondrogenic differentiation of hBM-MSCs. This pore shape limited the local oxygen availability, inducing the expression of hypoxia inducible factors and chondrogenesis, compared to rhomboidal pore scaffolds with same porosity. ${ }^{70}$ In contrast to this in vitro result, PCL/TCP scaffolds with a $0 / 90^{\circ}$ laydown pattern implanted on a rat calvarial critical bone defect for 12 weeks revealed the highest amount of bone formation compared to scaffolds with other angles of deposition. ${ }^{71}$ In vivo, the optimum porosity and FD have also not been clearly defined, as some studies have reported significantly higher amount of new bone formation on PCL scaffolds with low FD $(130 \mu \mathrm{m})$, compared to scaffolds with high FD (500 - $700 \mu \mathrm{m}),{ }^{72}$ while others have not observed differences in bone formation volume among PCL scaffolds with pore sizes ranging from $350 \mu \mathrm{m}$ to $800 \mu \mathrm{m}$ after 8 weeks of implantation. ${ }^{73}$ In short, pore sizes and shapes achievable using ME-AM provide a suitable way to tune tissue regeneration and implant mechanics, but attention needs to be paid to not modify 
another interdependent property and reverse the beneficial effects of tailored pores, as was possibly the case in the conflicting results cited above.
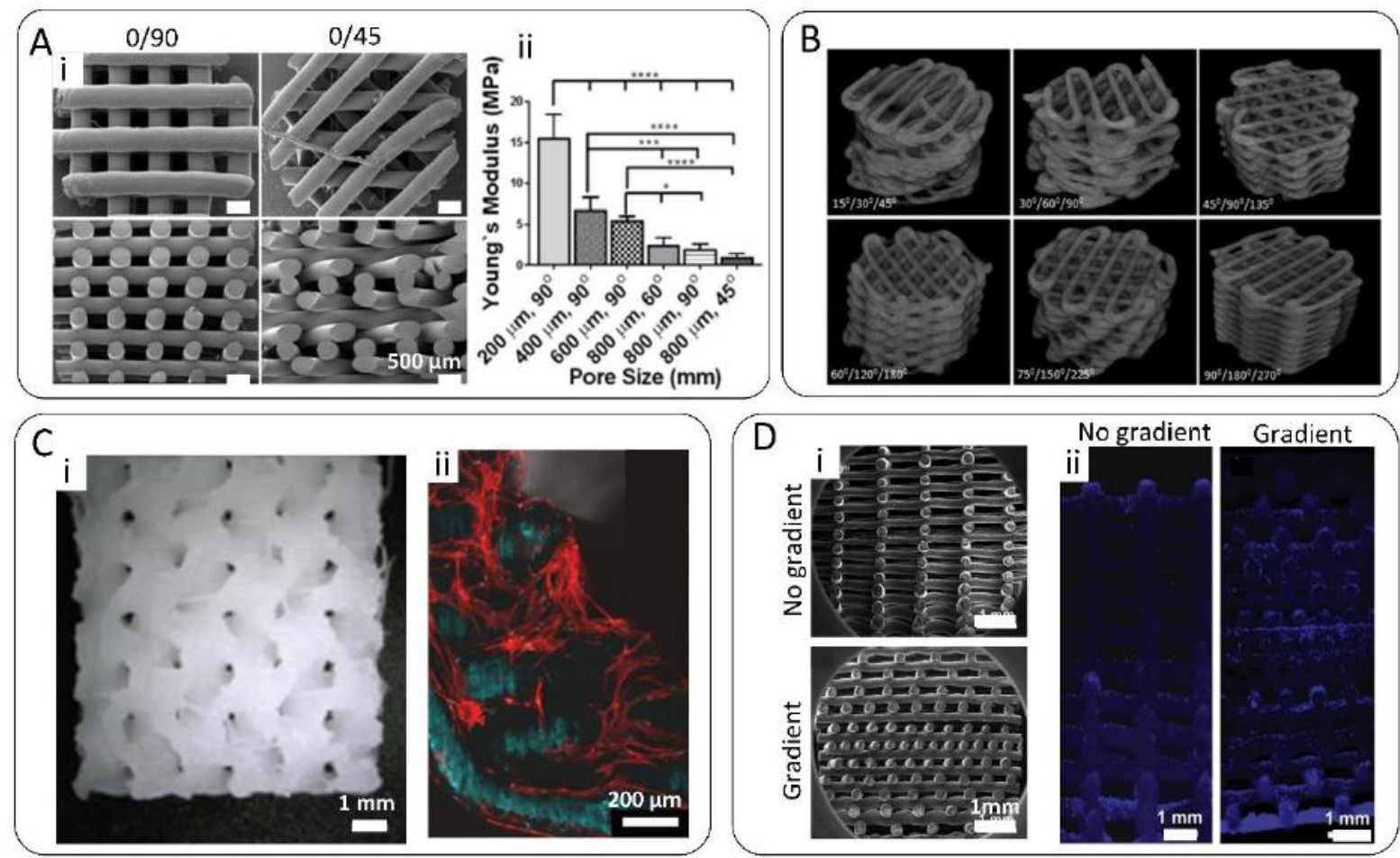

Figure 2 - Modification of the scaffold architecture. (A) (i) SEM images of the top view and cross section of PEU scaffolds with different angles of deposition. (ii) Compressive Young's moduli of PEU scaffolds with different FD and angles of deposition. Adapted with permission from Ref. 60 (B) $\mu C T$ images of PCL scaffolds with different lay-down patterns. Adapted with permission from Ref. ${ }^{61}$ (C) (i) PCL gyroid scaffold. (ii) Representative confocal microscopy images of human adipose derived MSCs (hAD-MSCs) cultured for 30 days on a PCL (calcium phosphate coated) gyroid scaffold. (F-actin, red). Adapted with permission from Ref. ${ }^{62}$ (D) (i) SEM images of the cross section of a homogeneous PCL scaffold (no gradient, pore size $750 \mu \mathrm{m}$ ), and a vertical pore size gradient PCL scaffold $(750 \mu \mathrm{m}->200 \mu \mathrm{m}->750 \mu \mathrm{m})$. (ii) Fluorescent microscopy images of the cross section of the aforementioned homogeneous and gradient scaffolds seeded with SaOs-2 cells. Adapted with permission from Ref. ${ }^{67}$

\section{Scaffold surface modification}

Since cell-scaffold interactions mainly occur at the interface, numerous techniques have been used to modify the surface of chemically inert ME-AM scaffolds, without altering the bulk scaffold material and architectural properties. These include wet chemical modifications, plasma treatment, and coatings with proteins, growth factors, calcium phosphate $(\mathrm{CaP})$ or other bioactive molecules. These can alter the scaffold surface roughness, surface topography, hydrophilicity, chemical composition and bioactivity, 
towards enhanced protein adsorption, and improved cell adhesion, proliferation and differentiation.

\section{Introduction of roughness and microporosity}

Surface roughness and nano/microtopography have been shown to enhance protein attachment and influence cell adhesion, proliferation and differentiation. ${ }^{74-76}$ However, the surface of the filaments of ME-AM polymeric scaffolds is rather smooth, due to rounding of free surfaces in the melt state before solidification, and surface roughness can be introduced via scaffold immersion in an etching solution. For instance, Kumar et al. etched PCL scaffolds by immersion in a dichloromethane-acetone solution post-fabrication (Figure 3A). 77 Scaffolds filaments showed a 5-fold increase in average surface roughness, which induced hBM-MSCs osteogenic differentiation and matrix mineralization after 62 days of culture under basic media conditions. This effect was attributed to a change of cell morphology, as cells in etched scaffolds presented a lower spread area and smaller size, similar to differentiated cells in previous studies, ${ }^{78}$ compared to the elongated and undifferentiated cells on non-etched scaffolds. Similarly, an alkaline treatment with $\mathrm{NaOH}$ has been shown to provide changes in the surface microtopography of PCL scaffolds, as well as to introduce functional carboxyl and hydroxyl groups to the filaments surface. ${ }^{79}$ In this case, a combination of physical and chemical changes led to an increase of ALP activity and calcium deposition by MC3T3-E1 pre-osteoblasts, compared to treated scaffolds. In spite of requiring post-etching solvent removal and washing, post-fabrication wet chemical surface modifications are considered greener compared to other techniques, such as solvent extrusion AM, in which the organic solvent is used during the scaffold manufacturing process and cytotoxic traces can remain within the material's core. Nevertheless, some studies have reported significant reduction of the filament diameter after solvent etching, which negatively affected the mechanical properties of the scaffold for the final application. ${ }^{80}$ This suggests that special care should be taken on the selection of the treatment parameters, in terms of solution concentration and immersion time.

Alternative to the introduction of surface topography by solvent etching, several organic solvent-free methodologies have been used to induce microporosity both in the surface and in the bulk of ME-AM scaffolds' filaments. Microporosity has previously been shown to increase the permeability of the scaffold and modify its degradation rate, as well as to enhance the integration between scaffold and surrounding tissue, essential for the 
mechanical stability of the implant. ${ }^{81-82}$ In this regard, ME-AM and porogen leaching have been combined to fabricate scaffolds with surface and intra-filament microporosity. For instance, elongated pores were formed along the long axis of the extruded filaments on TPU/Poly(vinyl alcohol) (PVA) scaffolds by composite printing followed by PVA dissolution in water. ${ }^{83}$ These scaffolds, in combination with a CaP coating enabled higher cell proliferation rates, as well as higher ALP activity and COL I deposition than smooth scaffolds. ${ }^{83}$ Similarly, a combination of ME-AM with salt leaching was used to fabricate dual porosity scaffolds for drug delivery applications (Figure 3B). ${ }^{84}$ Microporosity led to a significant increase in scaffold surface area, enabling a more efficient cefazolin (an antimicrobial molecule) loading upon soaking, and larger quantities released per timepoint. An additional Gelatin methacryloyl (GelMA) coating allowed to dampen the initial burst release and scaffolds showed antimicrobial activity over time. ${ }^{84}$ An alternative technique to create microporosity in ME-AM scaffolds is gas foaming. PCL/PVA composite scaffolds were subjected to a supercritical $\mathrm{CO}_{2}$ gas foaming step, followed by their immersion in water to leach the PVA and create open pores of around 2-10 $\mu \mathrm{m}$ within and on the surface of the filaments. ${ }^{85}$ Similarly, the combined extrusion of PLA with a foaming agent, which decomposed at the printing temperature for gas foaming, led to dual macro/microporous PLA scaffolds (micropore size 10-60 $\mu \mathrm{m}$ ) for significantly enhanced cell attachment and proliferation. ${ }^{86} \mathrm{~A}$ major limitation of the gas foaming step is the formation of a skin layer on the filaments surface, which hinders the exposure of the inner filament pores. Castano et al. showed that this layer can be removed and create surface porosity using the breath figure approach, which in turn requires the use of organic solvents. ${ }^{87}$ This technique consists on the short immersion of the scaffold in an organic solvent in a moist atmosphere. During the evaporation of the thin solvent layer deposited at the polymer surface, water vapor condensates as droplets on the surface of the scaffolds. After the complete evaporation of water and solvent, a honeycomb like porosity appears. Another drawback derived from the introduction of microporosity within the scaffolds filaments by all the aforementioned techniques is the loss of scaffold mechanical properties.

\section{Plasma treatment}

Plasma surface modification has the advantage of altering both the surface roughness and chemistry of ME-AM scaffolds, thus conferring hydrophilicity and positive or negative charge to the filaments, without the use of solvents and maintaining their bulk properties. 89 Surface charge does not only promote protein adsorption for cell attachment and to 
trigger proliferation and differentiation pathways via integrin binding, ${ }^{90-91}$ but it can also induce cell attachment through direct electrostatic interactions between the surface chemical groups and the cell surface. ${ }^{92-93}$ Oxygen, air, argon and helium non-thermal plasma (NTP) activation has been shown to increase the hydrophilicity of PCL or PLA MEAM scaffolds. ${ }^{94-97}$ This was achieved due to plasma etching, which increased the filaments surface roughness, and due to the introduction via post oxidation steps of oxygen containing functional groups (mostly peroxide and hydroperoxide groups). Similarly, ethylene/nitrogen and ammonia plasma activation have shown to directly deposit nitrogen containing groups (amines) on PCL ${ }^{98}$ and polystyrene (PS) scaffolds, ${ }^{99}$ respectively, also leading to a significant increase on the scaffold wettability. Compared to untreated scaffolds, plasma activated ones displayed enhanced cell attachment and better cell distribution along scaffold cross section, regardless of the plasma gas or cell type used (MG63 osteosarcoma cell line, 7F2 mouse osteoblast, human Saos-2 osteoblast or hBMMSCs). In addition, enhanced cell proliferation and early osteogenic differentiation in differentiation media cultures was also observed in some cases, when compared to untreated scaffolds. ${ }^{94-95,} 99$ Although in 3D scaffolds it has not been proven so far, cell differentiation on plasma modified 2D surfaces has been previously correlated to an enhanced integrin binding exposure of adhered proteins, as well as to high levels of recruitment of focal adhesion components and phosphorylation of focal adhesion kinases in the adhered cells, which are events required for osteoblast differentiation. ${ }^{90,} 100$

To obtain a more unique and stable coating with higher density of specific functional groups, the plasma discharge can be fed with monomers, such as alkyl amine or acrylic acid, to coat the surface with a thin amorphous polymeric film containing primarily amine or carboxyl groups, respectively. Cools et al. have reported the use of such a plasma process, referred to as plasma polymerization, to treat ME-AM scaffolds for cartilage TE. 101-102 Here, acrylic acid plasma polymerization successfully deposited carboxyl groups on the surface of PCL and PEOT/PBT scaffolds filaments, which enhanced cell adhesion of human adipose derived MSCs (hAD-MSCs), as well as proliferation and GAG production by chondrocytes, respectively, compared to untreated and helium activated scaffolds. ${ }^{101-}$ 102 

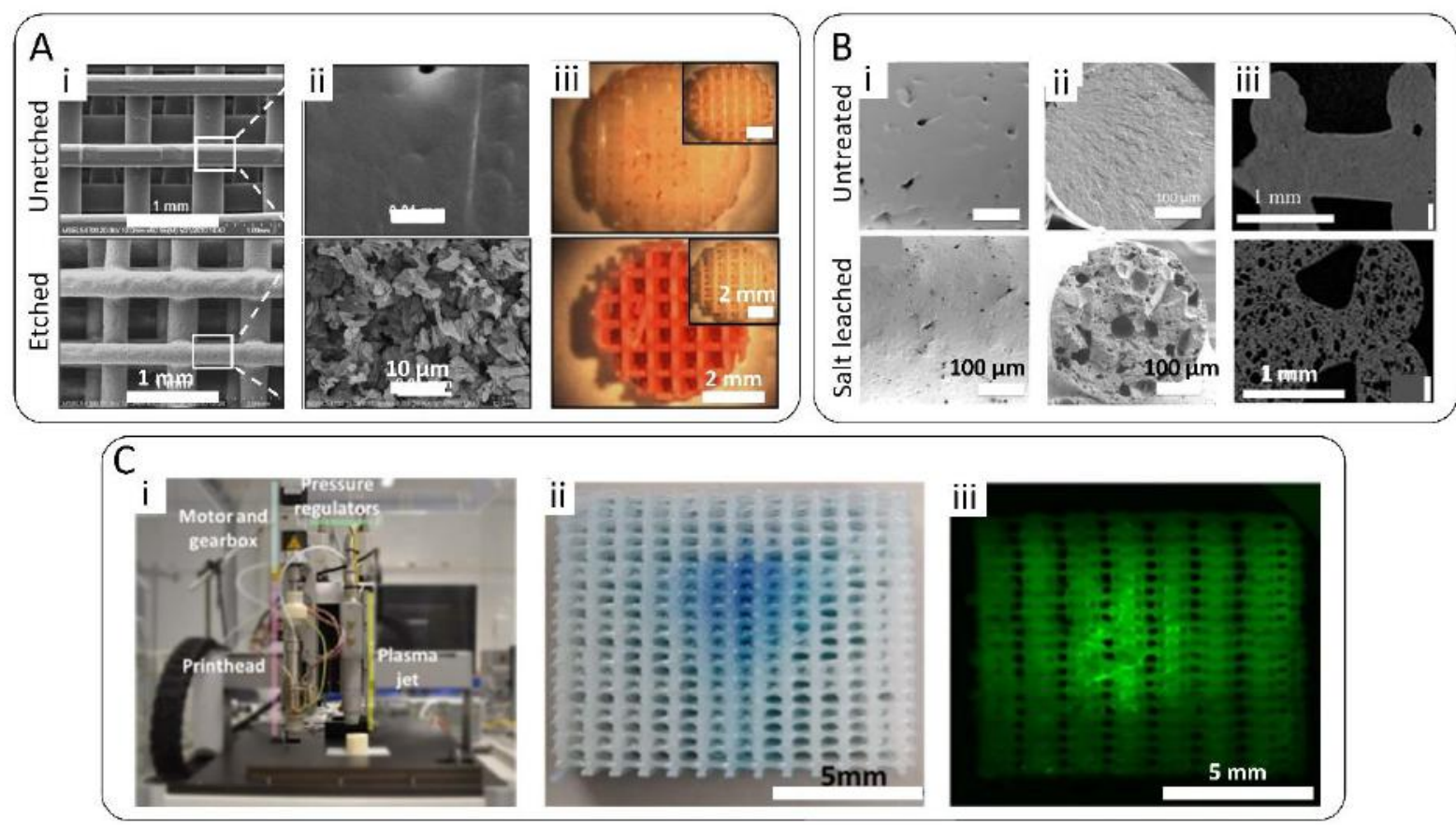

Figure 3 - Modification of the scaffold surface. (A) (i, ii) Introduction of surface roughness to PCL scaffolds filaments by post-fabrication solvent etching (dichloromethane:acetone). (iii) Alizarin red S (ARS) staining (calcium deposition) on the aforementioned unetched and etched scaffolds seeded with hBM-MSCs and cultured for 60 days in basic medium (without osteogenic factors). Insets correspond to the unseeded scaffolds cultured under the same media conditions. Adapted with permission from Ref. ${ }^{77}$ (B) (i, ii) Introduction of microporosity to the filaments of PCL scaffolds by combining ME-AM with salt leaching. SEM images depicting the scaffolds surface and cross section. (iii) $\mu \mathrm{CT}$ images of solid and microporous scaffolds at representative cross sectional planes. Adapted with permission from Ref. ${ }^{84}$ (C) (i) Hybrid AM platform consisting of a ME printhead and an atmospheric plasma jet for whole scaffold treatment from top, layer-by-layer, or zonally in specific regions of interest within the scaffold volume. (ii) Selective maleic anhydrideVinyltrimethoxysilane (MA-VTMOS) plasma polymerization treatment in a central spot of a PEOT/PBT scaffold using the hybrid AM platform. The patterned region was visualized using methylene blue staining. (iii) Specific hBM-MSCs attachment (F-actin, green) to an argon plasmapatterned scaffold. Adapted with permission from Ref. ${ }^{88}$

Despite promising results in enhancing ME-AM scaffolds surface bioactivity, NTP processes are usually carried out at sub-atmospheric pressures, and require costly vacuum and plasma chamber equipment. Most importantly, inhomogeneous functionalization along the scaffold volume, already observed for conventionally fabricated scaffolds, ${ }^{103-105}$ has been also pointed out in some reports, where ME-AM scaffolds were plasma treated. For instance, lower oxygen atomic concentration has been observed on the filaments within the core of PCL scaffolds, with respect to the edges, after helium plasma activation and acrylic acid plasma polymerization. 95, 101 In addition, the average oxygen content over the scaffold cross section decreased with decreasing scaffold pore size. ${ }^{101}$ Since plasma does not ignite inside the pores, the treatment of the core filaments 
is limited to diffusion, which drops while entering the scaffold and with decreasing pore size. This leads to a prolonged residence time of the monomer in the active plasma discharge, which in turn leads to a more extensive fragmentation of the precursor, and thus a lower oxygen incorporation. ${ }^{101}$ The use of atmospheric pressure plasma jets (APPJ) and their assembly within a ME-AM printing platform eliminates the need of a reactor chamber and vacuum equipment, avoids the formation of unwanted gradients, and enables plasma treatment during the manufacturing step. ${ }^{106}$ With such a hybrid setup, the flame exiting the jet can be directed into the scaffolds pores, and the scaffold can be treated as a whole from the top, layer-by-layer to reach deep pores in large anatomical scaffolds, or zonally in specific regions of interest within the scaffold volume. Two recent reports have explored these possibilities, employing oxygen plasma activation to treat PCL scaffolds, ${ }^{107}$ and plasma polymerization of maleic anhydride-Vinyltrimethoxysilane (MAVTMOS) on PEOT/PBT scaffolds, which deposits thin films containing carboxyl functional groups (Figure 3C). ${ }^{88}$ In the latter study, selective plasma treatment in a central spot within the scaffold core enabled specific cell attachment to the treated region. The subsequent dispersion of a second cell population throughout the scaffold volume showed the cell-patterning potential of this hybrid technique, with applications in cell co-culture experiments. 88

\section{Coatings with proteins, growth factors and other bioactive molecules}

The coating of the scaffold's filament surface with proteins, peptides, growth factors or antimicrobial molecules can stimulate cell attachment and differentiation, or provide the scaffold with antimicrobial properties. The simplest method for bioactive molecules incorporation is surface adsorption upon scaffold soaking into a solution. 108-110 For instance, the adsorption of the antimicrobial peptide polylysine onto PCL/HA scaffolds rendered their surface antibacterial. ${ }^{111}$ However, clear disadvantages of this technique are the requirement of potentially expensive highly concentrated solutions with the molecule of interest, the very low loading efficiency, and the loose binding to the polymer resulting in burst and uncontrolled desorption, which can lead to cytotoxicity effects or poor bioactivity in the long term. For instance, $50 \%$ of the total dexamethasone impregnated on PLA scaffolds by soaking was released within the first $6 \mathrm{~h}$ upon immersion in phosphate buffer saline (PBS). ${ }^{109}$ Similarly, complete VEGF released from PCL/HA scaffolds was observed after 2 days immersion in cell culture medium. ${ }^{110}$ Some studies have attempted to improve the binding efficiency of biomolecules by a prior protein coating. In this regard, 
Sawyer et al. pre-coated a PCL/TCP scaffold with COL I prior to bone morphogenetic protein- 2 (BMP-2) adsorption. ${ }^{112}$ BMPs play a crucial role on bone formation, are essential signaling molecules involved in the recovery of bone fractures, mediate cell proliferation and osteogenic differentiation. ${ }^{113}$ While BMP-2 was still completely released within the first $6 \mathrm{~h}$ of immersion in PBS at $37^{\circ} \mathrm{C}$, increased bone healing in a rat calvarial defect was observed compared to uncoated scaffolds. ${ }^{112}$ Similarly, Yan et al. performed a layer-bylayer assembly of positively charged chitosan and negatively charged deferoxamine (an iron chelator with potential to promote vascularization and bone regeneration) on the filaments of aminolysed PCL scaffolds to increase the deferoxamine loading. ${ }^{114}$

Alternative to an adsorption mechanism, bioactive molecules have been covalently bonded to the surface of ME-AM scaffolds. For instance, copper based click chemistry has been used to attach BMP-2 to amino acid based-PEU scaffolds. ${ }^{115}$ This is a highly efficient method, however it involves multiple reaction steps and cytotoxicity studies should be performed to ensure that the residual copper absorbed to the scaffold filaments does not reach cytotoxic concentrations. Alternatively, crosslinkers such as glutaraldehyde or 1ethyl-3-(3-dimethylaminopropyl)carbodiimide (EDC) have been used to mediate the conjugation of biomolecules to polymeric scaffolds. For instance, acetylated collagen was bonded to PLA scaffolds via glutaraldehyde and other linkers. 116 Similarly, hydroxypropyltrimethyl ammonium chloride chitosan (HACC) was covalently bound to the surface of PLGA/HA scaffolds via EDC/ N-hydroxysuccinimide (NHS) chemistry. 117-118 This latter functionalization provided antibacterial properties, as well as larger volumes of new bone formation in vivo, compared to control scaffolds. ${ }^{117-118}$ In spite of their versatility, crosslinker-based covalent immobilization methods require the introduction of amine or carboxyl functional groups to the scaffolds filaments surface prior to biomolecules binding, which is normally achieved by destructive aminolysis or hydrolysis steps. Avoiding these wet-chemical steps, reactive groups can be introduced through plasma activation. In most cases, plasma activation is followed by grafting of pre-synthesized polymer brushes, a process defined as plasma grafting. Polymer brushes are robust and versatile spacers, and offer a 3D distribution of functional groups, which can significantly increase the density of tethered biomolecules. ${ }^{119}$ Following this plasma grafting strategy, Declercq et al. activated PCL scaffolds with argon plasma, followed by 2-amino-ethylmethacrylate (AEMA) brushes UV grafting, which served for covalent attachment of gelatin, and subsequent physisorption of fibronectin. ${ }^{120}$ Histological analysis of the scaffolds cross section showed a higher MC3T3-E1 cells colonization in the center of these scaffold, which 
significantly helped osteogenic differentiation. In another study, Tardajos et al. coupled chitosan to the surface of PCL scaffolds through a similar surface functionalization procedure involving polymer brushes. First, scaffolds were plasma activated with argon, followed by methacrylic acid N-hydroxysuccinimide ester (NHSMA) brushes UV grafting, which enabled the coupling of chitosan. ${ }^{121}$ Such coating allowed cell growth while providing and antibacterial surface against $S$. aureus and $S$. epidermidis, which are bacterial strains commonly investigated when orthopedic implants are aimed. In addition to the aforementioned 'grafting to' approaches, 'grafting from' strategies, in which polymer brushes are grown into the surface via surface initiated polymerization, have also been used on ME-AM scaffolds for a better control of the functionality, density and thickness of the polymer brush. In this regard, poly[oligo (ethylene glycol) methacrylate] (POEGMA) brushes were uniformly grafted over the surface of PCL scaffolds, allowing the subsequent conjugation of fibronectin, for cell attachment, or of BMP-2 and transforming growth factor (TGF)- $\beta 3$, which triggered osteogenic and chondrogenic differentiation of hBM-MSCs, respectively (Figure 4A). ${ }^{122-123}$ Although immobilization of biomolecules through polymer brushes offers advantages in terms of high density of reactive functional groups, careful optimization of this technique is needed to avoid crowding of biomolecules, which can reduce their degree of conformational freedom and their resultant bioactivity. ${ }^{119}$

Polydopamine (PDA), which contains hydroxyl and amine functional groups, has also been investigated as primer coating for subsequent covalent conjugation of proteins onto $\mathrm{ME}$ AM scaffolds. The dopamine molecule possesses a similar chemical structure to that of combined lysine and 3,4-dihydroxyphenyl-L-alanine (DOPA) aminoacids, which confer the adhesive properties to the mussel adhesive proteins (MAPs) secreted by marine mussels. 124 Inspired by mussel adhesiveness, a PDA coating can be deposited onto any surface via the oxidative self-polymerization of dopamine at a slightly basic $\mathrm{pH}$. Following PDA coating, Teixeira et al. immobilized COL I on PLA scaffolds via EDC-NHS chemistry. ${ }^{125}$ Here, the combined coating enhanced hBM-MSCs adhesion and ALP activity compared to untreated scaffolds. Besides, various reports have immobilized BMPs on PDA coated scaffolds via Michael addition reaction. ${ }^{126-129}$ For instance, Lee et al. grafted recombinant BMP-2 to PCL-PDA scaffolds, which was sustain released over time and upregulated osteogenic gene expression on preosteoblasts (MC3T3-E1) cultured on the scaffolds. ${ }^{126}$ Similarly, bone forming peptide-1 (BFP-1, a short peptide derived from BMP-7) and SSVPT (an oligopeptide derived from BMP-2) were immobilized on PCL and PLA scaffolds, respectively, leading to enhanced cell adhesion and osteogenesis in vitro and increased 
bone growth in critical size calvarial defects in vivo (Figure 4B). ${ }^{128-129}$ Due to the high adsorption of serum proteins to PDA, which maintain an active conformation exposing integrin binding domains, ${ }^{130-131}$ a simple PDA coating onto ME-AM scaffolds has also been used to enhance cell adhesion and trigger differentiation. For instance, Kao et al. covered the filaments of a PLA scaffold with PDA. ${ }^{132}$ The PDA hydrophilic coating enhanced hADMSCs adhesion and proliferation, and supported osteogenesis by inducing the secretion of larger amounts of COL I and OCN, compared to bare PLA scaffolds. Interestingly, hADMSCs cultured on coated scaffolds also showed the upregulation of angiogenic genes. Due to its material-independent coating ability, simple deposition process, and high biocompatibility and stability in vitro, PDA constitutes a powerful tool for surface modification of ME-AM scaffolds. Moreover, preliminary in vivo studies have shown that PDA coatings can greatly reduce the inflammatory response of PLLA surfaces, evaluated during the first 14 days after implantation. ${ }^{133}$ Nevertheless, its long term in vivo stability, degradation mechanism and biocompatibility should be still evaluated.

An alternative method to improve the bioactivity of scaffold consists of the coating with cell-laid ECM, containing several bioactive factors that can stimulate osteogenic differentiation in vitro and in vivo. ${ }^{134}$ Compared to single protein coatings, which only provide fragmental components of the ECM that present specific functional receptors for cell attachment, such an approach better mimics the complexity of ECM. In this regard, Wu et al. cultured MG63 on PCL scaffolds for 5 days before a decellularization process with Triton, $\mathrm{NH}_{4} \mathrm{OH}$ and DNAse. ${ }^{135}$ Human Wharton's Jelly-derived MSCs (hWJ-MSCs) seeded on these ECM-coated scaffolds exhibited enhanced cell adhesion, proliferation and the upregulation of ALP, COL I, BSP and OCN genes, as well as increased calcium deposition, compared to uncoated scaffolds. Bone formation on a rat calvarial defect filled with these scaffolds was also higher than on uncoated scaffolds. ${ }^{135}$ In another study, the tissue formed by human nasal inferior turbinate tissue-derived MSCs (hNIT-MSCs) on PCL/PLGA/TCP scaffolds was decellularized through freeze-thaw cycles, which minimally affected the ECM bioactive components, compared to chemical methods. ${ }^{136}$ Subsequently seeded hNIT-MSCs also showed osteogenic genes upregulation, and coated scaffolds induced greater ectopic bone formation in vivo than bare scaffolds (Figure 4C). ${ }^{136}$ Similar to ECM, platelet rich plasma (PRP) can stimulate osteogenic differentiation and has been used to coat ME-AM scaffolds. ${ }^{137} \mathrm{PRP}$ is a source of growth factors that play an essential role in tissue healing, including platelet derived growth factor (PDGF), vascular endothelial growth factor (VEGF), TGF- $\beta 1$, basic fibroblast growth factor (bFGF), epidermal growth 
factor (EGF), and insulin-like growth factor. ${ }^{138-139}$ PCL scaffolds coated with freeze dried PRP, which had previously been proven to preserve growth factors at high levels, ${ }^{140}$ have shown to promote ALP activity and osteogenic gene upregulation on dental pulp stem cells, as well as higher levels of bone formation in a rat calvarial defect, compared to uncoated scaffolds. ${ }^{137}$ Overall, coatings comprising a cocktail of biomolecules can exert a more profound effect on the phenotypic behavior of cells, compared to single proteins, peptides or growth factors. Moreover, they avoid the lack of reproducibility, availability, and tunability of bioactive decellularized ECM scaffolds while embracing their advantages. Nevertheless, further investigation on variables such as cell source, culture duration and media type, is necessary to optimize these unique and complex biologically engineered systems.
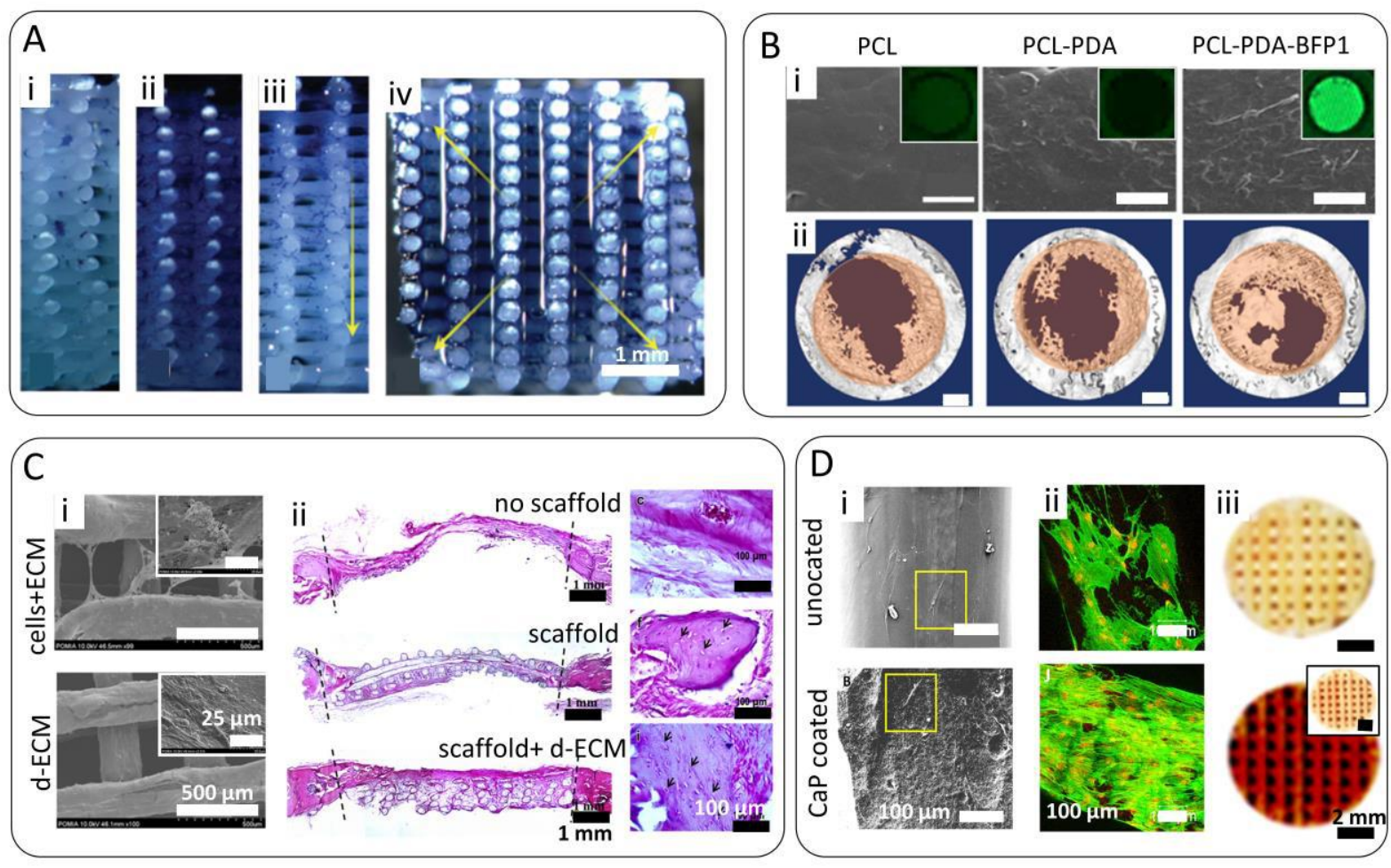

Figure 4 - Scaffolds surface modification via functional coatings. (A) (i) Methylene blue staining of hBM-MSCs adhered to PCL functionalized with POEGMA brushes. (ii) Homogeneous cell attachment on PCL-POEGMA scaffold grafted with fibronectin (FN). (iii) Vertical and (iv) radial gradient in coupled FN demonstrates gradients in cell attachment. The yellow arrows indicate the direction of decreasing FN concentration. Adapted with permission from Ref. ${ }^{122}$ (B) (i) SEM images of the surface morphology of PCL scaffolds coated with PDA and grafted with BFP1. Insets correspond to fluorescence images of BFP1 tethered on the scaffolds using FITC-labelled BFP1. (ii) Radiographic evaluation of bone formation around the scaffolds at 8 weeks post-implantation in a calvarial critical size defect in rabbits. Adapted with permission from Ref. ${ }^{129}$ (C) (i) SEM images of uncoated and d-ECM-coated PCL/PLGA/TCP. (ii) Histological sections stained with H\&E of critical size rat calvarial defects treated with the aforementioned scaffolds. Adapted with permission from Ref. ${ }^{136}$ (D) (i) SEM images of uncoated and CaP-coated PLA scaffolds. (ii) Confocal 
microscopy images showing cell attachment to CaP-coated scaffolds after 7 days of culture (Facting, green, nuclei, red). (iii) ARS staining on scaffold seeded with hBM-MSCs and cultured for 14 days in mineralization media. Insets depict stained scaffolds without cells but cultured under the same media conditions. Adapted with permission from Ref. ${ }^{141}$

\section{CaP coatings}

The presence of $\mathrm{CaP}$ deposits on the surface of ME-AM scaffolds can potentially stimulate cells' attachment and osteogenic differentiation through several cues, such as the release of calcium ions, the adsorption of serum proteins, or the nanoscale surface roughness. However, the direct growth of apatite layers on a polymeric surface is a challenging task due to the lack of nucleation sites. To overcome this, Jo et al. exploited the ability of PDA to nucleate apatite to create a bioinspired coating on PCL scaffolds, which allowed increased MC3T3-E1 preosteoblast infiltration and adherence within the scaffolds core. ${ }^{142}$ Here, the catecholamine moieties in PDA acted as nucleation sites of calcium and phosphate from simulated body fluid (SBF, 1.5x), in which the scaffolds were incubated for 14 days. ${ }^{143}$ Following a different approach, Murab et al. activated the surface of PCL scaffolds with oxygen plasma, leading to the introduction of hydroxyl and carboxyl groups, which nucleated biomimetic HA crystals upon immersion in cell culture medium. ${ }^{144}$ Alternatively, Holmes et al. conjugated nanao $\mathrm{HA}(\mathrm{nHA})$ particles to the surface of aminolysed PLA scaffolds through a glutaraldehyde crosslinker. ${ }^{145}$ This coating promoted osteogenic differentiation, observed in the form of $\mathrm{COL} I$ and calcium deposition, and vascularization upon co-culture of hBM-MSCs and human umbilical vein endothelial cells (HUVECs). Following a milder strategy, Jaidev et al. modified the surface of $\mathrm{NaOH}$ pretreated PLA scaffolds by grafting polyethyleneimine and subsequently immobilizing citric acid. ${ }^{141}$ Citric acid, which has been identified as a critical role player in precipitating the apatite phase during bone formation, ${ }^{146-147}$ mediated $\mathrm{CaP}$ precipitation from 1.5x SBF onto the scaffold after 7 days incubation. HBM-MSCs cultured on these scaffolds showed BMP2 gene upregulation and increased calcium deposition, compared to cells on bare PLA scaffolds (Figure 4D). ${ }^{141} \mathrm{In}$ an attempt to incorporate larger amounts of ceramics, Moroni and coworkers press fitted HA pillars into the pores of PEOT/PBT 3DF scaffolds. ${ }^{148-149}$ Such approach enabled larger amounts of calcium and phosphate ions release near cells cultured on the scaffolds, which influence stem cell osteogenic differentiation leading to upregulation of OPN gene expression, compared to bare and PEOT/PBT-HA composite scaffolds. 
In vivo studies assessing bone formation on $\mathrm{CaP}$-coated $\mathrm{ME}$-AM scaffolds have not always reported the beneficial effect of the CaP coating. For instance, Kim et al., who dip-coated PLGA/TCP scaffolds in a HA slurry, did not observe differences in new bone formation area among PLGA/TCP coated and uncoated scaffolds upon implantation in a rabbit femoral defect for 12 weeks. ${ }^{150}$ Similarly, Chim et al. observed less vasculogenesis and mineralization on precalcified PCL scaffolds implanted subcutaneously in mice for 14 weeks, compared to uncoated PCL scaffolds. ${ }^{151}$ Here, the coating was prepared by priming the PCL scaffolds with a silicate gel prior to 4 weeks immersion in SBF (1 week in 1x SBF, followed by 3 weeks in $1.5 x$ SBF). In both of the aforementioned studies it was hypothesized that flaking of the apatite coating might have impaired tissue ingrowth and osteogenesis, and that thinner layers might have yield more promising results. In accordance, Maia-Pinto, et al. reported increased bone formation on PLA scaffolds coated with a thinner CaP layer, only after 1 month of implantation in a rat calvarial defect, compared to bare PLA scaffolds. ${ }^{152}$ Here, the thin apatite layer was achieved through a shorter immersion time in SBF, 2 weeks (1 week in 1x SBF, followed by 1 week in $1.5 x$ SBF), compared to the 4 weeks reported in the study of Chim et al. Taking into consideration that the aforementioned results derive from different in vivo models, we can still suggest that the optimization of the apatite layer, in terms of thickness, is necessary in order to obtain a functional coating for the final in vivo application. In this regard, ensuring a good interfacial bonding by selecting an appropriate scaffold priming methodology seems also crucial for the success of the coating.

\section{Hybrid scaffolds}

\section{Fibrous networks}

While the large pore size of ME-AM scaffolds $(300-1000 \mu \mathrm{m})$ is advantageous for media permeation in vitro, as well as for tissue ingrowth and vascularization in vivo, such dimensions can turn out to be a limitation for cell seeding and scaffold coverage in an in vitro setting. Due to these large FDs, there is limited bridging between cells on different filaments, and pore closure happens slowly by cells forming concentric circles around the pore. ${ }^{29}$ This can significantly slow the process of tissue maturation, for which cell-cell contact is essential. ${ }^{153}$ Inspired by the fibrillary structure of ECM, some studies have embedded ME-AM scaffolds in a hydrogel solution and subsequent freeze-drying for the 
creation of a fibrous network within the pores of the scaffolds. This strategy can create an environment to enhance cell retention during the seeding process, as well as cell-cell interactions and mobility on a mechanically stable and highly porous printed structure. For instance, Jensen et al. dip-coated a PCL scaffold with a mixture of hyaluronic acid and TCP, followed by freeze-drying to create a fibrous coating containing TCP particles around the scaffolds filaments. ${ }^{154-155}$ Human dental pulp stem cells (hDPSCs) and MSC derived from human induced-pluripotent stem cells (hiPS-MSCs) proliferated at a higher rate on these scaffolds, deposited higher amounts of calcium and showed an upregulation of osteogenic genes, compared to uncoated scaffolds. Similarly, Chen et al. freeze-dried PCL scaffolds immersed in hyaluronic acid to create an interconnected fibrous network. HMSCs were seeded in a methylated collagen and terpolymer hydroxylethyl methacrylate- methyl methacrylate- methacrylic acid (HEMA-MMA-MAA) solution. ${ }^{156}$ This complex mimicked the constitution of the ECM, helped to achieve a more homogeneous cell distribution across the scaffold upon seeding and led to osteogenic genes upregulation, owed to the exposed integrin domains of the coated methylated collagen promoting focal adhesion assembly and activating osteogenesis pathways.

Inspired by the submicron key features in the ECM, some studies have reported the combination of ME-AM and electrospinning (ES), in order to introduce nanofibers to the microporous scaffolds. Some studies have reported the preparation of such hybrid scaffolds by alternating ES layers within ME layers, 157-159 while others have opted for the surface addition of the ES fibers once the 3D scaffold was completely printed. ${ }^{160-161}$ Interesting observations have been extracted from some of these studies. For instance, Park et al., who presented scaffolds consisting of $\mathrm{PCL} /$ collagen dense ES fibers intercalated within the layers of a PCL ME-AM scaffold, observed the proportional relation between number of intercalated ES layers and cell seeding efficiency and proliferation rate. ${ }^{157}$ Moreover, the reports of Mota et al. and Vyas et al. described the alignment of nanofibers perpendicular to the direction of the printed filaments, which acted as a parallel plate collector, when depositing low fiber density electrospun layers (Figure 5A). ${ }^{159-160}$ These crossing nanofibers acted as bridges for cells to easily reach parallel printed filaments, otherwise not possible on conventional ME-AM scaffolds. Interestingly, LaraPadilla et al. implemented a cooling system to allow the layer-by-layer intercalation of PCL nanofibers between PLA printed layers. ${ }^{158}$ This was to avoid the PCL fibers to partially melt and lose their integrity upon ME of subsequent PLA layers, due to the higher melting point of PLA. However, the resulting hybrid scaffolds presented lower mechanical 
properties, due to fast cooling reducing the bonding between printed layers. Going one step further, the combination of the ES and ME-AM technologies has given rise to gradient scaffolds for bone to ligament or bone to cartilage interfaces. ${ }^{162-163}$ In the latter case, the electrospun layer served as the tidemark of the osteochondral tissue and prevented blood vessel invasion towards the cartilage ME-AM region. In order to have a better control on the porosity of the secondary fibrillary network, Yang et al. used electrohydrodynamic direct writing (EHD-DW), instead of ES, in combination with ME-AM. ${ }^{164}$ Here, layers of ME PCL filaments were alternated with layers of EHD-DW PCL threads ( 100 $\mu \mathrm{m}$, composed of microfibers of $\sim 3 \mu \mathrm{m}$ ) precisely deposited with an offset with respect to the ME filaments. This created a secondary interwoven network with smaller dimensions and porosity, which allowed higher MG63 cell retention, ALP activity and calcium deposition than ME-AM or hybrid ES-ME-AM scaffolds (Figure 5B).

Overall, hierarchical scaffolds comprising ME filaments and a secondary fibrous network mimicking the micro/nanoscale details observed in tissues, such as bone, are desired to increase cell retention and promote tissue formation within the scaffold. On-going research on including such biomimetic features to ME-AM scaffolds has contributed to the development of combinatorial technologies, such as ES or EHD-DW, with ME-AM, which enable the automation and control over the whole scaffold fabrication process. While this requires currently complex experimental setups, more integrative technologies are expected to be designed in the future.

\section{Hydrogels}

Natural or synthetic hydrogels have also been used in combination with ME-AM scaffolds for cell encapsulation within the scaffold's pores. Such hybrid systems allow the retention of large cell concentrations compared to any other seeding methods, as the whole seeding volume containing the cells is maintained within the scaffolds pores after hydrogel crosslinking. For instance, sheep hBM-MSCs have been resuspended into a PRP solution and loaded on PCL/TCP scaffolds. The PRP solution formed a gel after crosslinking with thrombin, which enabled to retain the cells within the scaffold prior to their implantation on a sheep's tibial defect. ${ }^{168-169}$ With the same mechanism of action, fibrinogen has been used for seeding hBM-MSCs, which were embedded within the pores of a PCL scaffold after the addition of thrombin and fibrinogen polymerization into a fibrin gel. ${ }^{170}$ After 2-3 days of culture, and in parallel to hydrogel degradation, cells started migrating towards the 
PCL filaments. While in these systems the hydrogel was used as a degradable cell carrier, and the ME-AM scaffold was the main hybrid construct component for cells to grow on, other approaches have considered the ME-AM part solely as a structural support for the cell-laden hydrogel. For instance, Wang et al. took advantage of the round morphology of rabbit hBM-MSCs within PLGA-PEG-PLGA hydrogel carriers to stimulate chondrogenic differentiation, which was demonstrated to be better supported on the hybrid scaffolds, compared to bare PCL scaffolds. ${ }^{171}$ Moreover, Heo et al. used a PLA ME-AM scaffold to reinforce the surrounding hAD-MSCs-laden GelMA hydrogel, which in addition contained RGD conjugated gold nanoparticles for osteogenic gene upregulation and enhanced calcium deposition. ${ }^{172}$ Furthermore, hydrogels contained within ME-AM scaffolds have been used for the pre-vascularization of the constructs. For example, methacrylated hyaluronic acid/gelatin hydrogels carrying hAD-MSCs and HUVEC promoted the formation of capillary like networks within PCL/HA ME-AM scaffolds both in vitro and in vivo (Figure 5C). 165

Besides the incorporation of the cell-laden hydrogel component within ME-AM scaffolds by surface injection, the combination of bioprinting and ME-AM has been used to intercalate cell-laden hydrogel filaments within thermoplastic polymer filaments in a layerby-layer manner. This hybrid multi-printhead deposition process enables i) the automation of the tissue construct fabrication, as it does not require post-scaffold fabrication cell seeding, ii) the precise control over the deposition of the hydrogel bioink at specific locations within the construct, and iii) the possibility of multimaterial and multicellular bioprinting within the same hybrid construct. 166, 173-175 Yet, specific hydrogel rheological properties are required for bioprinting, and special attention should be taken to cell viability after shear during the extrusion process. Moreover, since the bioprinted filaments get in direct contact with the hot ME filament shortly after extrusion, low melting temperature thermoplastics, such as PCL, are normally used in these cases, to avoid heating of the cell-laden hydrogel by conduction. Working around these constraints, scaffolds with optimized microenvironment for the growth of 3D complex tissues have been fabricated. For instance, bioinks consisting of decellularized ECM of different tissues and hAD-MSCs have been bioprinted in combination with PCL ME-AM scaffolds to promote tissue specific differentiation. 176 Alternatively, Mekhileri et al. bioprinted human articular and nasal chondrocytes microtissues within the pores of PEOT/PBT scaffolds after the completion of the ME-AM scaffolds, in contrast to the aforementioned layer-by-layer process. The use of 
microtissues instead of single cells enabled building complex tissues, such as the osteochondral interface, of clinically relevant sizes (Figure 5D). ${ }^{166}$

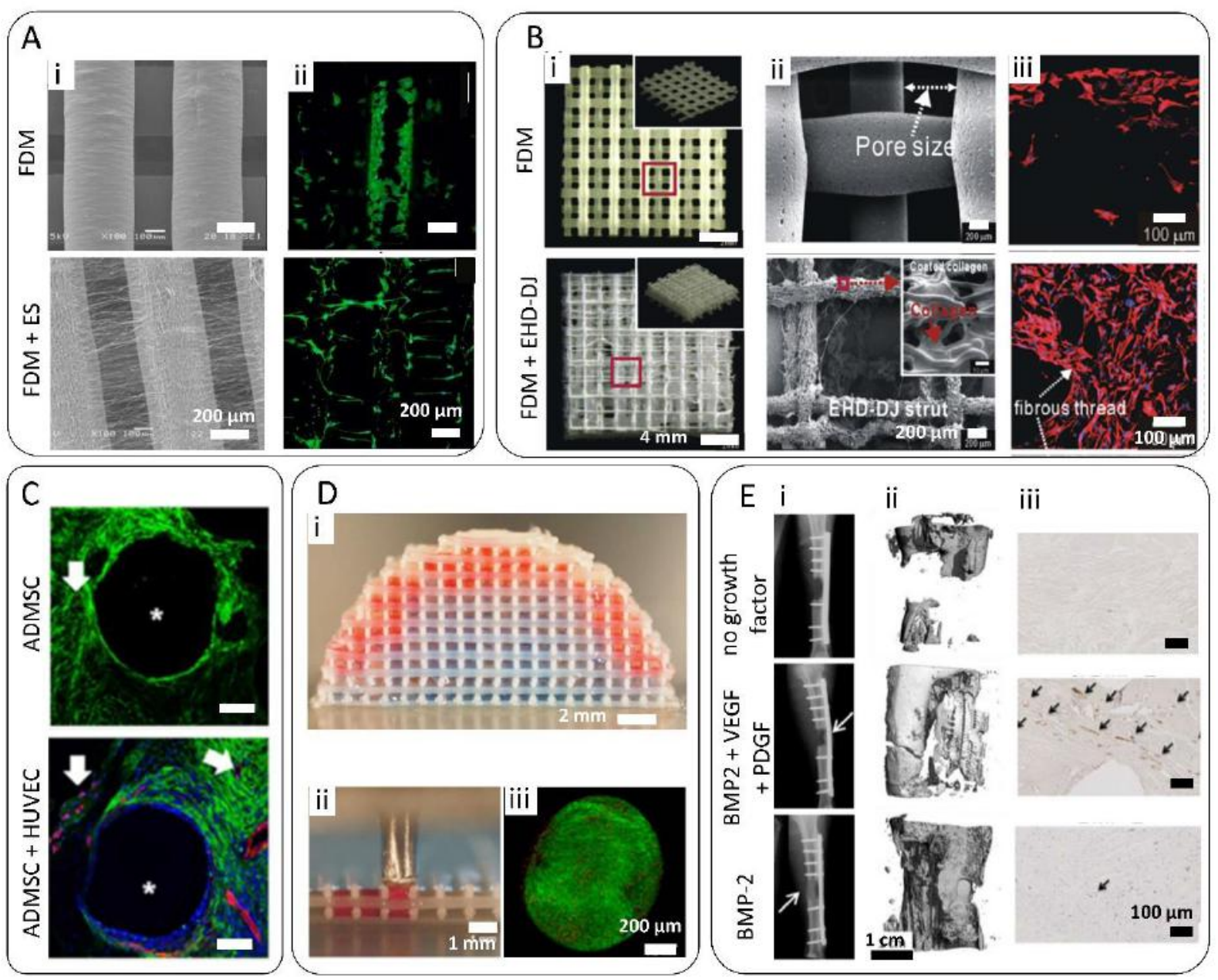

Figure 5 Combination of ME-AM scaffolds with fibrous and hydrogel networks. (A) (i) SEM images of bare PCL scaffolds, and scaffolds fabricated combining ME-AM of PCL and ES of PLGA nanofibers. (ii) Fluorescent microscopy images of MC3T3 (F-actin, green) cultured for 7 days on the aforementioned scaffolds. Adapted with permission from Ref. ${ }^{160}$ (B) (i) Optical and (ii) SEM images of bare PCL scaffolds, and hybrid scaffolds fabricated combining ME-AM of PCL and EHDDJ of collagen struts. (iii) Confocal fluorescent microscopy images of MG63 (F-actin, red; nuclei, blue) cultured for 7 days on the aforementioned scaffolds. Adapted with permission from Ref. ${ }^{164}$ (C) Immunohistochemistry fluorescent images of in vivo vascularization within PCL/HA scaffolds seeded with cell laden GelMA hydrogels containing either hAD-MSC, or hAD-MSC combined with HUVEC (CD31, red; a-smooth muscle actin, green; nuclei, blue). Scaffolds were implanted for 4 weeks subcutaneously. Asterisks indicate the PCL/HA scaffold filaments. Arrows indicate the stained cells/microvessels positive for human CD31. Adapted with permission from Ref. ${ }^{165}$ (D) (i) Biphasic hemispherical construct with stained GelMA hydrogel micro-spheres representing the chondrogenic (red) and osteogenic (blue) phase of an osteochondral construct. (ii) This construct was fabricated by applying a bottom-up automated tissue bioassembly strategy, consisting on the automated insertion of micro tissues within the ME-AM scaffold pores. (iii) Fluorescence microscopy images of injected human articular cartilage micro tissues stained with Calcein (live cells, green) and Propidium lodide (dead cells, red). Adapted with permission from Ref. ${ }^{166}$ (E) (i) Representative X-rays and (ii) $\mu \mathrm{CT}$ reconstructions of bone formation after hybrid scaffolds implantation on ovine tibial defects. Scaffolds consisted on a PCL ME-AM structure, loaded with a 
platelet rich plasma hydrogel containing microparticles for the delivery of BMP-2 or a combination of BMP-2, VEGF and PDGF. (iii) Representative images from defect mid sections showing immunohistochemical staining for the endothelial marker Von Willebrand Factor. Adapted with permission from Ref. ${ }^{167}$

Hydrogels have also been used as cell-free delivery systems of growth factors within MEAM scaffolds. They are an alternative strategy to surface adsorption, covalent immobilization or melt blending for the incorporation of bioactive molecules, which offer tunable and controlled release rates, as a function of hydrogel mesh size and degradation rate. PCL/PLGA and PCL/TCP scaffolds filled with a collagen hydrogel containing rhBMP2 enabled the controlled release of the growth factor, which improved and accelerated bone formation in vivo. 168, 177 Similarly, decellularized ECM hydrogel containing human recombinant BMP-2 was dispensed within the filaments of a PCL/TCP ME-AM scaffold enabling a sustained release, which resulted in a larger volume of new bone formation, compared to PCL/TCP and PCL/TCP/decellularized ECM scaffolds. ${ }^{175}$ In another study, the central region of a PCL scaffold was loaded with a PRP hydrogel containing degradable PLGA microparticles, which carried VEGF, PDGF and BMP-2, or BMP-2 only. 167 The polymeric microparticles were used to achieve a more sustained release, while the growth factors combination was chosen for obtaining a stable vasculature and induce bone formation in a sheep tibial segmental defect (Figure 5E).

Hydrogel laden ME-AM scaffolds bring together the ECM-like properties of hydrogels and the high structural support of ME-AM constructs, and the addition of cell, growth factors, drugs, or their combination to the equation adds up for extra functionality. The on-going research focused on the development of cell-laden hydrogels for bioprinting will allow in the future for further optimization of such hybrid systems towards advanced scaffolds for bone regeneration.

\section{Composites}

\section{CaP based materials}

In an attempt to mimic the organic-inorganic composition of bone ECM, CaP-polymer composites have been one of the most used combination of materials for the design of BTE scaffolds. CaP fillers can enhance the polymeric scaffolds mechanical properties, as well as offer osteoinductive and osteoconductive properties, which are dependent on their 
specific chemical phase, structural properties (crystallinity, surface roughness, and porosity), mechanical properties and associated degradation behavior. 178-180 Among all the CaP phases, HA and TCP, have been the most commonly used fillers for ME-AM scaffolds. Considering the average mineral content of bone tissue, which lies within the range of 50 to $74 \mathrm{wt} \%$, depending on the body location, ${ }^{181-182}$ tremendous efforts have been conveyed to increase the CaP content within polymeric scaffolds to optimize their mechanical properties while retaining their printability. For instance, Esposito-Corcione et al. reported the fabrication of PLA/HA 50 wt\% scaffolds, which presented a 2-fold increase in compression modulus, compared to bare PLA scaffolds. ${ }^{183}$ Similarly, Jiang et al. and Ding et al. reported the fabrication of PCL/HA scaffolds with up to $40 \mathrm{wt} \%$ mineral content. 184-186 Such scaffolds also presented a 2-fold increase in compression modulus and compressive strength compared to bare PCL scaffolds. Moreover, surface roughness and hydrophilicity were significantly increased, which enhanced cell attachment and proliferation. Interestingly, composites with higher HA concentration (60 wt\%) were not printable in a continuous manner, due to particle aggregation leading to nozzle clogging and filament discontinuity. ${ }^{186}$ In another report, Yu et al. fabricated PEU/HA scaffolds with up to $40 \mathrm{wt} \%$ mineral content, which were shown to support osteogenic differentiation in mineralization media cultures. ${ }^{187}$ Here, MC3T3-E1 cells seeded on the highly loaded composite scaffolds presented higher proliferation rates, as well as increased ALP activity and BSP and OCN gene upregulation, compared to cells on scaffolds with lower HA content and bare PEU scaffolds. Enhanced osteogenesis was attributed to HA increasing protein adsorption and activating focal adhesion kinase (FAK) and mitogen-activated protein kinase/ extracellular signal-regulated kinase (MAPK/ERK) signaling pathways. ${ }^{187}$ In order to further increase the osteogenic differentiation potential of polymer-HA composite scaffolds, Cho et al. eroded the surface of PCL/HA 40 wt\% scaffolds with a $\mathrm{NaOH}$ surface treatment. ${ }^{188-189}$ This was done to increase the exposure of HA particles on the filaments' surface, and thus the possibility of $\mathrm{Ca}$ and $\mathrm{P}$ ions exchange with the medium, since $\mathrm{Ca}$ and $\mathrm{P}$ (re)precipitation from CaP materials is known to affect osteogenic pathways. ${ }^{190-192}$ In this regard, $\mathrm{NaOH}$ erosion led to higher cell proliferation rates, calcium deposition and apatite layer formation in vitro, compared to other scaffold types. ${ }^{188}$ In vivo, HA based scaffolds allowed for significantly higher bone formation compared to bare PCL scaffolds, and eroded PCL/HA scaffolds showed the best bone regeneration ability (Figure 6A). 189 
While osteogenic differentiation has been supported in vitro on highly loaded HA scaffolds, enhanced osteogenesis has not been observed on scaffolds with low HA content, such as PEOT/PBT/HA or PCL/PLGA/HA with $15 \%$ and $10 \%$ inorganic content, respectively. ${ }^{193-}$ 194 However, in vivo experiments have revealed the osteoinductive/ osteoconductive properties of such low HA content scaffolds. For instance, new bone formation on the aforementioned PCL/PLGA/HA 10 wt\% HA scaffolds was $100 \%$ higher than in bare polymeric scaffolds in a rat calvarial defect. ${ }^{194}$ Besides, mechanical properties are still enhanced by low HA loadings, which makes these scaffolds still desirable for BTE applications. ${ }^{193-194} \mathrm{PCL} / \mathrm{HA}$ scaffolds with low HA content (10 wt\% or 15 wt\% HA) have also shown to promote bone formation in vivo. ${ }^{195-196}$

A couple of studies have assessed the effect of different $\mathrm{CaP}$ materials on the performance of composite ME-AM scaffolds. For instance, Nyberg et al. compared the in vitro osteoinductivity of PCL/HA, PCL/TCP, PCL/Bio-Oss ${ }^{\circledR}$ (bovine bone mineral matrix) and PCL/DBM (decellularized bone matrix) scaffolds with 30 wt\% of inorganic content, posttreated with $\mathrm{NaOH} .197$ Overall, greater osteoinductivity was observed on hAD-MSCs cultured on PCL/Bio-Oss and PCL/DBM compared to PCL/HA and PCL/TCP scaffolds, in terms of calcium deposition and COL I and osteonectin gene upregulation in basic and mineralization media. Nevertheless, Runt-related transcription factor 2 (RUNX2) and OPN were also upregulated on PCL/HA scaffolds in basic media, compared to other scaffolds types. Here, TCP showed lower bioactivity compared to other CaP fillers. Similarly, Huang et al. also showed that despite PCL/TCP 20 wt\% scaffolds displayed better compression modulus before implantation than PCL/HA 20 wt\% scaffolds, they presented lower bioactivity, in terms of cell attachment and proliferation. 198 In spite of this, a large amount of reports have explored the use of TCP as filler for ME-AM scaffolds. ${ }^{46}$ Several have attempted the addition of TCP up to $60 \mathrm{wt} \%$ to PCL composite scaffolds, which has shown to lead to an increase in compression modulus of up to $50 \%$, although these high wt\% diminished significantly the scaffolds compressive yield strength. ${ }^{199-201}$ For this reason, composite scaffolds with up to $20 \mathrm{wt} \%$ TCP have been mostly investigated, which have proven their bone regenerative capacity in vivo. 46, 168, 202 In addition, promising in vivo results have led to clinical trials investigations, where the applicability of PCL/TCP scaffolds has been tested for craniomaxilofacial bone regeneration (NCT01119144, NCT03735199). The fast degradation of TCP compared to HA can confer the scaffold with more optimum ion exchange dynamics to stimulate bone formation through calcium activated pathways. ${ }^{190-191,203}$ In order to accelerate the degradation dynamics of the 
scaffold and of TCP, and to enhance the replacement of the scaffold material by newly formed bone, $\mathrm{NaOH}$ treatment ${ }^{204-207}$ and PLGA $208-210$ have been further included into TCP based scaffolds. Yet, balancing the speed of degradation of TCP containing scaffolds is important, in order to keep the released calcium and phosphate concentration within the cell proliferation and differentiation stimulating range, without reaching apoptotic values. 192,211

In order to further mimic the in vivo tissue structure and stimulate bone formation, polymerinorganic composite scaffolds with gradients in HA or TCP concentration have been fabricated. For instance, Bittner et al. and Smith at al. have reported PCL/HA and PCL/TCP composite scaffolds, respectively, with vertical discrete gradients in inorganic content (from 30 or 20 to $0 \mathrm{wt} \% \mathrm{HA}$ or TCP) to mimic the bone to cartilage interface (Figure 6B). ${ }^{212-213}$ In contrast to these multi-printhead and discrete gradient fabrication approaches, Sinha et al. have recently reported the fabrication of continuously graded HA-based ME-AM scaffolds using a single printhead approach (Figure 6C). ${ }^{88}$ This printhead allowed the in situ mixing of two materials (PEOT/PBT only, and PEOT/PBT/HA 45 wt\%) to obtain scaffolds with continuous HA composition gradients (among other inorganic fillers), which better mimic in vivo tissue interfaces and possess better mechanical properties than discrete gradient scaffolds. ${ }^{214}$ Other studies have focused on the production of scaffolds with radial gradients in inorganic content and porosity have also been reported, to resemble the mechanical properties and bulk mineral content of the cortical to cancellous bone interface. ${ }^{215-216}$

CaP-polymer composite ME-AM scaffolds have extensively proven their mechanical and biological functionality for BTE, while benefiting from a single-step fabrication approach, compared to post-processing coating strategies. In spite of extensive material characterization and in vitro research, further in vivo investigations are still required to unravel the optimum scaffold composition, in terms of polymer type, CaP type and content, and degree of CaP exposure to the scaffold filament surface, which balance printability, mechanical properties and bone regeneration ability.

\section{Silicates}

Calcium silicate (CS) based materials, composed of $\mathrm{CaO}, \mathrm{SiO}_{2}$, and $\mathrm{MgO}$ or $\mathrm{AlO}$, have been used as fillers in ME-AM scaffolds, due to their bioactive properties and charged surfaces being highly compatible with polymeric matrices. ${ }^{217-218}$ An apatite layer is 
generally formed on CS when in contact with body fluid, which acts stronger interface and bonding with surrounding tissues upon implantation. Besides the known osteogenic capacity of $\mathrm{Ca}$, the released $\mathrm{Si}$ ions also have the ability of upregulating MAPK/ERK and p38 signaling pathways, which are known to be involved in osteogenesis, and stimulating angiogenesis as well. ${ }^{219}$ Lin et al. fabricated PCL/Mg-CS scaffolds, which released Mg and $\mathrm{Si}$, and reprecipitated $\mathrm{Ca}$ and $\mathrm{P}$ when immersed in SBF, forming an apatite layer (Figure 6D). 220 The highest hWJ-MSCs attachment, proliferation and osteogenic genes upregulation was observed for scaffolds with $50 \mathrm{wt} \% \mathrm{Mg}-\mathrm{CS}$, which released larger amounts of the aforementioned ions. These scaffolds also showed the highest degradation rate, due to lower polymer content. Zhang et al. also observed the good performance of $\mathrm{PCL} /$ gliadin scaffolds containing 30 wt\% Mg-CS. ${ }^{221}$ Here, both Mg-CS and the natural polymer gliadin were added to the scaffold to enhance its bioactivity and degradability. In a rabbit femoral defect, scaffolds with $30 \mathrm{wt} \% \mathrm{Mg}-\mathrm{CS}$ allowed for the fastest degradation rate and largest new bone volume formation, compared to lower Mg-CS percentages and bare PCL/gliadin scaffolds.

A very widely used type of $\mathrm{CS}$ is bioactive glass (BG), which in addition to $\mathrm{CaO}$ and $\mathrm{SiO}_{2}$, is composed of $\mathrm{Na}_{2} \mathrm{O}$ and $\mathrm{P}_{2} \mathrm{O}_{3}$. In vitro, $\mathrm{BG}$ has been shown to outperform other $\mathrm{CaP}$, like $\mathrm{HA}$, in terms of biological response and osteogenic commitment when incorporated within a PLA scaffold at the same concentration, potentially due to enhanced protein adsorption. 222 Yet, in vivo studies have not reported differences among PCL/BG and PCL/CaP scaffolds within 16 weeks implantation time, in terms of bone formation. ${ }^{223}$ Strontiumsubstituted BG ( $\mathrm{SrBG}$ ) has also been investigated as filler for ME-AM scaffolds, as $\mathrm{Sr}$ is an important trace element in bone and can stimulate bone formation and reduce bone resorption. ${ }^{224}$ In this regard, Poh et al. prepared PCL/SrBG 10 wt\%, which enhanced the formation of calcium deposits, compared to PCL/BG 10wt\% scaffolds. ${ }^{225}$

$\mathrm{CS}$ based materials appear to be valid substitutes of $\mathrm{CaP}$ as osteoinductive materials for bone regeneration applications, due to their richer bioactive ion composition. Although these established advantages, insufficient efforts have been dedicated so far to investigate their use as fillers within ME-AM scaffolds. A reason for that could rely on their fast degradation rates compromising the scaffold mechanical properties already at early stages of the regeneration process. ${ }^{226}$ Nevertheless, further research is required to better understand the performance of CS in comparison with CaP fillers. 


\section{Metallic particles}

Metals, such as titanium based alloys or cobalt chromium alloys, have been extensively used in the orthopedics field as permanent implants due to their good biocompatibility and high mechanical strength and wear properties. ${ }^{227-228}$ Others, such as iron- or magnesium $(\mathrm{Mg})$-based metals, are preferred for bone regeneration applications, since they are resorbable and can be degraded to be replaced by newly formed bone. In particular, Mgbased metals have been used as fillers for ME-AM scaffolds. Apart from improving the mechanical properties of composites, $\mathrm{Mg}$ ions are essential to bone metabolism, have been shown to be associated with the formation of biological apatite, and can stimulate osteogenic activity. ${ }^{229-231}$ Since bare $\mathrm{Mg}$ corrodes in aqueous environment leading to the formation of gas cavities, ${ }^{232}$ more stable compounds such as magnesium hydroxide $(\mathrm{MgOH})$, magnesium oxide $(\mathrm{MgO})$ or magnesium fluoride $(\mathrm{MgF})$ have been used as fillers within ME-AM scaffolds. For instance, Abdal-hay et al. prepared PCL/MgOH scaffolds with up to $20 \mathrm{wt} \%$ of $\mathrm{MgOH} .{ }^{233}$ Despite not enhancing the scaffolds' mechanical properties, potentially due to poor nanoparticle dispersion at high concentrations, $\mathrm{Mg}$ ions were released from the scaffolds, which led to increased cell proliferation and ALP activity. In another study, Shen et al. prepared PCL-PEG-PCL/MgO composite scaffolds. ${ }^{234}$ Here, silanization of the $\mathrm{MgO}$ particles allowed the addition of up to $4 \mathrm{wt} \% \mathrm{MgO}$ without the formation of agglomerates, which resulted in a 2-fold increase in compression modulus compared to bare PCL scaffolds. Yet, the most optimum magnesium release rate was achieved with 1 wt\% $\mathrm{MgO}$, which favored cell adhesion, ALP activity and calcium deposition, as well as bone formation in vivo, compared to other $\mathrm{MgO}$ concentrations (Figure 6E). ${ }^{234}$ Hansske et al. also pre-coated MgF nanoparticles, in this case with a poly (ethylene glycol) (PEG)-based biomimetic coupling agent to improve their stability and compatibility with the polymer matrix, prior to loading within a PCL scaffold. ${ }^{235}$ In a subsequent study, these compatibilized MgF nanoparticles were used as fillers in a ternary composite scaffold consisting of $\mathrm{PCL}, \mathrm{nHA}$ and $\mathrm{MgF}$. ${ }^{236}$ The sole addition of $1 \mathrm{wt} \% \mathrm{MgF}$ to the PCL/HA scaffolds enhanced the attachment and osteogenic differentiation of human fetal-derived MSCs (hF-MSCs), in terms of ALP activity, osteonectin gene expression and calcium deposition. In addition to the bioactivity of $\mathrm{Mg}, \mathrm{F}$ is also known to play a key role in the development of mineralized tissues. ${ }^{237}$

Silver has also been used as metallic filler within ME-AM scaffolds. Besides enhancing mechanical properties, silver has shown to provide antimicrobial properties to the 
scaffolds. For instance, Radhakrishnan et al. prepared PCL/silver 3 wt\% scaffolds, which showed antibacterial activity against $E$. coli while maintaining osteoblast cell viability. ${ }^{238}$

Overall, metallic particles are an attractive filler option for ME-AM composite scaffolds, especially due to their ability to significantly enhance the scaffold's mechanical properties at very low concentrations. Nevertheless, compatibility and dispersion within polymers are the major issue associated to these materials, and the main reason why they have not been widely explored so far.
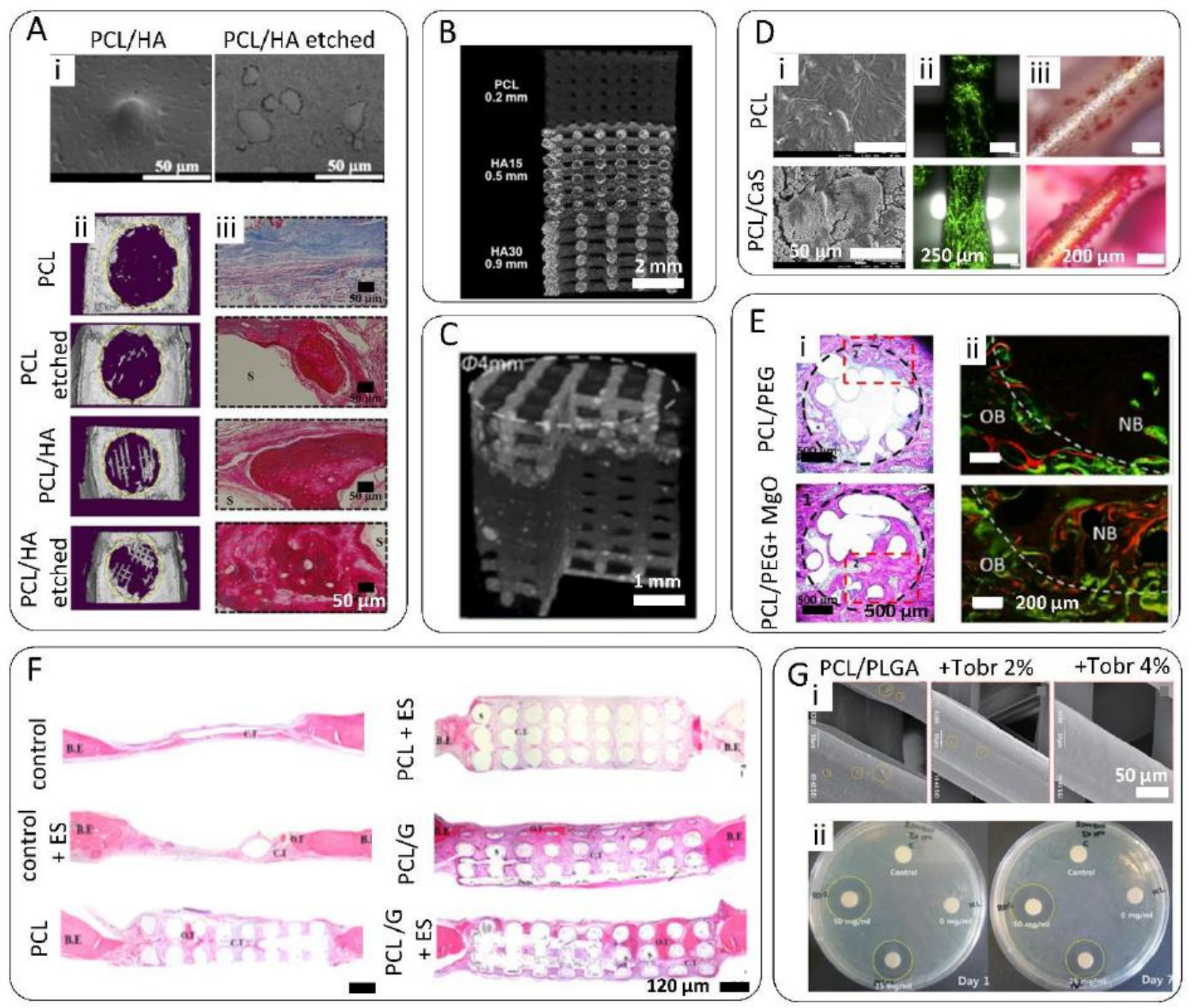

Figure 6 - Composite scaffolds. (A) (i) SEM images of the surface of PCL/HA 40 wt\% composite scaffolds before and after etching with $\mathrm{NaOH}$. Adapted with permission from Ref. ${ }^{188}$ (ii) $\mu$-CT images of critical size rat calvarial defects depicting new bone formation after 8 weeks implantation of the aforementioned scaffolds. Increased bone formation is observed in the composite scaffolds, compared to the PCL and PCL etched scaffolds controls. (iii) Representative Masson's trichrome stain images of the calvarial defects 8 weeks post-implantation, depicting mineralized areas (red), which were more abundant in the HA containing scaffolds. Adapted with permission from Ref. ${ }^{189}$ (B) $\mu C T$ images of PCL scaffolds with a discrete vertical gradient in HA concentration. Adapted with permission from Ref. ${ }^{212}$ (C) $\mu \mathrm{CT}$ images of PEOT/PBT scaffolds with a continuous vertical gradient in HA concentration. Adapted with permission from Ref. ${ }^{88}$ (D) (i) SEM images of the 
surface of PCL and PCL/CaS 50 wt\% scaffolds, after immersion in SBF for 7 days, depicting the ability of PCL/CaS of nucleating HA. (ii) Fluorescent microscopy images of hWJ-MSCs (F-actin, green) cultured on the aforementioned scaffolds for 7 days, showing enhanced cell coverage on the composite scaffolds. (iii) ARS staining of calcium nodules deposited on the PCL and PCL/CaS scaffolds cultured with hWJ-MSC for 3 weeks. Adapted with permission from Ref. ${ }^{220}$ (E) (i) Giemsa staining on histological sections of lateral epicondyle defects in rats 8 weeks post-implantation of PCL/PEG and PCL/PEG/MgO $1 \mathrm{wt} \%$ composite scaffolds (red, mineralized tissue). (ii) Fluorochrome-labeling analysis of new bone formation and mineralization 8 weeks postimplantation of the aforementioned scaffolds (calcein green, between 4 and 6 weeks; xylenol orange, red, between 6 and 8 weeks). 'OB' and 'NB' refer the location of original bone and newly formed bone, respectively. Adapted with permission from Ref. ${ }^{234}(\mathrm{~F}) \mathrm{H} \& \mathrm{E}$ stained sections of rat calvarial defects with implanted PCL and PCL/G (graphene) 0.78 wt\% scaffolds, with or without electrical stimulation. Adapted with permission from Ref. ${ }^{239}$ (G) (i) SEM images of the surface of PCL/PLGA and tobramycin-loaded PCL/PLGA scaffolds with various loading concentrations (2 $\mathrm{wt} \%$ and $4 \mathrm{wt} \%$ ). (ii) Results of disk diffusion test on day 1 (left) and day 7 (right), showing the aforementioned scaffolds' antimicrobial activity against with $\mathrm{S}$. aureus. Adapted with permission from Ref. ${ }^{240}$

\section{Carbon-based nanomaterials}

Polymer nanocomposites based on carbon-based materials have been used to improve the mechanical, electrical, chemical and osteogenic properties of polymers. The basic structure of carbon-based materials used for these applications is graphene, which is a one atom thick hexagonal lattice of sp2 hybridized carbon atoms, a structure that confers outstanding mechanical properties $(\mathrm{E}$ modulus $=1 \mathrm{TPa}$, ultimate strength $=130 \mathrm{GPa})$, thermal conductivity (5000 W/ mK), electrical conductivity (up to $6000 \mathrm{~S} / \mathrm{m}$ ) and chemical properties. ${ }^{241}$ Depending on its dimensional arrangement, graphene is referred as 0-D fullerene or wrapped graphene, 2D- carbon nanotube (CNT) or rolled graphene, and 3Dgraphite or stacked graphene. ${ }^{242}$ CNTs, generally prepared by chemical vapor deposition methods, can be single walled (SWCNTs), with a diameter of $1 \mathrm{~nm}$, or with several nanotubes arranged concentrically into a multi-walled structure (MWCNTs), with diameters generally between 10 and $100 \mathrm{~nm} .{ }^{243}$ Despite their narrow diameter, they possess very high aspect ratios, which makes them ideal reinforcing fibers in composites. Moreover, their geometrical structure can be tuned to adjust their electronic properties, from metallic to semiconducting materials. ${ }^{244}$ A few studies have reported the use of MWCNTs as fillers on ME-AM scaffolds for bone regeneration applications. For instance, Huang et al. loaded PCL scaffolds with up to 3 wt\% MWCNTs, which were shown to be aligned in the extrusion direction. ${ }^{245}$ This resulted in an increase in the compressive modulus from $\sim 50 \mathrm{MPa}$ to $~$ $88 \mathrm{MPa}$. Moreover, hAD-MSCs showed increased proliferation with increasing MWCNTs content. Taking advantage of the alignment of the MWCNTs along the extrusion direction, 
a subsequent study focused on the development of a ternary composite consisting of PCL, MWCNTs (0.75 wt\%) and $\mathrm{nHA}(20 \mathrm{wt} \%)$, which aimed at mimicking the bone tissue structure at the nanoscale. ${ }^{246}$ The aligned MWCNTs induced the PCL chains in their vicinity to recrystallize along their walls, which surrounded by $\mathrm{nHA}$ particles, mimicked the highly aligned and mineralized collagen fibrils in bone. Such ternary scaffolds also possessed higher compression moduli and showed enhanced upregulation of ALP, COL I and OCN gene expression of hAD-MSCs, compared to bare PCL and PCL/MWCNT scaffolds. ${ }^{246}$

Wang et al. compared the effect of MWCNTs and graphene sheets on PCL scaffolds mechanical properties and cell-affinity. 247 PCL/MWCNTs and PCL/graphene scaffolds with up to $3 \mathrm{wt} \%$ filler content were prepared. Interestingly, at a given filler concentration, $\mathrm{PCL} /$ graphene scaffolds presented higher compression modulus and strength, as well as higher protein adsorption and hAD-MSCs proliferation rates than PCL/MWCNT scaffolds. This was correlated to the better mechanical, physicochemical and conductive properties of graphene compared to MWCNTs. Indeed, graphene sheets prepared by liquid phase exfoliation of graphite, have been used as filler for PCL scaffolds in several studies from Bartolo's group. ${ }^{239,248-250}$ In all of them, a maximum of 0.78 wt $\%$ filler was used to avoid potential hAD-MSCs cytotoxicity effects of graphene upon complete scaffold degradation, which have been previously shown to be dose and size dependent. 251 These reports demonstrated that the sole addition of 0.78 wt $\%$ graphene improved the scaffolds compression modulus by $50 \%$. Moreover, PCL/graphene scaffolds showed enhanced hAD-MSCs proliferation compared to bare PCL scaffolds, ${ }^{248}$ which could be further improved by a chemical treatment with $\mathrm{NaOH},{ }^{249}$ or by the pre-adsorption of the P1-latex protein to the scaffolds filaments. ${ }^{250}$ Ultimately, untreated PCL/graphene scaffolds with $0.78 \mathrm{wt} \%$ filler were implanted in a rat calvarial defect, and electrical stimulation was applied, to assess their in vivo bone formation ability (Figure 6F). ${ }^{239}$ Histological analysis revealed that electrically stimulated PCL/graphene $0.78 \mathrm{wt} \%$ scaffolds led to more tissue infiltration and mineralization at early timepoints (60 days), compared to other conditions. Yet, values were leveled at later timepoints (120 days). ${ }^{239}$

Due to the high synthesis costs and low scale production of graphene through the aforementioned CVD or direct exfoliation of graphite methods, currently the most commonly used method for large-scale production of graphene-like materials relies on graphene oxide (GO), the oxidized version of graphite, as starting material. ${ }^{252} \mathrm{GO}$ can be 
easily mass produced by oxidation of graphite through well-established methods. Moreover, GO possesses oxygen-containing functional groups disrupting each carbon plane, including epoxy, carbonyl, ketone and hydroxyl groups, which offer the possibility of functionalization with biomolecules to tune its bioactivity. ${ }^{253}$ Unagolla et al. prepared PCL/GO $0.5 \mathrm{wt} \%$ and showed that while the addition of GO did not influence significantly the mechanical properties of the scaffolds, it led to a slight increase of matrix mineralization and osteogenic proteins expression at early timepoints, compared to bare PCL scaffolds. 254 In contrast, Belaid et al. reported that the addition of $0.3 \mathrm{wt} \% \mathrm{GO}$ to PLA scaffolds led to a $30 \%$ increase in the compression modulus. Moreover, MG63 proliferation was enhanced on these scaffolds, as well as calcium deposition when cultured in osteogenic medium. ${ }^{255}$ Chen et al. significantly augmented the filler loading, preparing TPU/PLA/GO scaffolds with up to $5 \mathrm{wt} \% \mathrm{GO}$, which led to a $125 \%$ increase in compression modulus, and good biocompatibility. ${ }^{256}$ Despite its facile production, GO is non-conductive and possesses poor thermal stability, which can lead to the formation of air pockets within the scaffold filaments when processed at high temperatures, like the ones required for PLA, PLGA or PEOT/PBT extrusion $\left(200^{\circ} \mathrm{C}\right) .{ }^{256}$ Electrical conductivity and thermal stability can be restored by chemical or thermal reduction of the GO, leading to exfoliated wrinkled reduced graphene oxide ( $\mathrm{rGO}$ ) sheets, similar to pristine graphene, but with some oxygen defects at the laminar edges, and holes on the carbon skeleton. ${ }^{257}$ Angulo-Pineda et al. fabricated PCL/rGO scaffolds with up to 10 wt\% filler, which showed to be not only conductive, but also antimicrobial against $S$. aureus. ${ }^{258}$ Electrical stimulation on such scaffolds further enhanced the antimicrobial effect, while supporting higher hBM-MSCs proliferation rates than on non-stimulated scaffolds. In spite of rGO-polymer composite scaffolds possessing interesting bioactive properties for bone regeneration, very few reports have studied them. This might very well be due to the very low bulk density of rGO upon thermal reduction, which can lead to particle inhalation during handling and lack of free-flowability into twin-screw compounders, which is the main cost-effective route for composites production at large scale. ${ }^{259}$

The aforementioned research examples demonstrate the great potential of graphenebased materials as fillers for ME-AM scaffolds for BTE, since they can act as reinforcement agents, boost cell attachment and osteogenic differentiation, and provide antimicrobial activity. However, there are still concerns on the in vivo toxicity of these materials and their degradation products. Studies have reported that the cytotoxicity of graphene and derivatives is dependent on their production method and purity, physicochemical 
properties (lateral dimensions, oxidation state, aggregation state, functionalization), concentration, exposure time and administration route. ${ }^{260}$ Yet, this information is scarce and contradictory results are often found, due to the various synthesis routes leading to diverse materials, which often are not properly characterized or described in literature. ${ }^{261}$ In addition, biodistribution studies have mostly been performed upon single intravenous injection of dispersed graphene materials, 262 and the effect of local, sustained and long term injections, simulating the release of graphene form a polymeric scaffold, have not yet been considered. Overall, more thorough in vitro and in vivo biocompatibility studies paired to the growing interest in graphene-based scaffolds development are necessary. This would help to determine the maximum graphene-based filler loading within ME-AM scaffold, to ensure their functionality while retaining their biocompatibility in an in vivo scenario.

\section{Bioactive molecules}

The direct incorporation of drugs or antibiotics within the scaffolds filaments is desired in order to simplify the fabrication process, as well as to improve the loading capability and release profile. However, the main limiting factor for ME polymer-drug composites is the thermal stability of the incorporated molecules. This limits the bioactive molecule and polymer choice, as the large majority of the thermoplastics used for biomedical applications require high processing temperatures $\left(\sim 175^{\circ} \mathrm{C}-220^{\circ} \mathrm{C}\right),{ }^{60,} 141$ which are above the thermal stability limits of most commonly used antibiotics for orthopedic applications ( $120{ }^{\circ} \mathrm{C}$, depending on exposure time and antibiotic type). ${ }^{263}$. As an exception, $\mathrm{PCL}$ has low melting $\left(\sim 60^{\circ} \mathrm{C}\right)$ and printing temperatures (up to $\left.100^{\circ} \mathrm{C}\right)$, thus several reports have investigated the incorporation of antibiotics or growth factors within PCL scaffolds. For instance, Lee et al. reported the fabrication of a PCL/rifampicin $2.5 \mathrm{wt} \%$ composite, which was melt blended and printed at $60^{\circ} \mathrm{C}$. ${ }^{264}$ During the 14 days evaluated, the antibiotic was sustain released and exerted antimicrobial activity against $E$. coli and $S$. aureus, while maintaining cell viability (Figure $6 \mathbf{G}$ ). Shim et al. reported the preparation of PCL/PLGA/tobramycin scaffolds at $110^{\circ} \mathrm{C}$. $240 \mathrm{Here}$, the drug was sustain released for a period of 50-60 days and when implanted on a rat femur model with osteomyelitis, was able to reduce inflammation and exert antimicrobial activity. In another study, Costa et al. printed PCL/poloxamine/dexamethasone scaffolds. ${ }^{265}$ The addition of the poloxamine copolymer in the blend led to filaments with lower surface porosity, which slowed down the release rate of dexamethasone. Similarly, the addition of HA to PLA/ nitrofurantoin 
scaffolds allowed to tune the antibiotic release, since HA facilitated the infiltration of water within the polymer matrix, leading to faster drug release rates. 266 The aforementioned studies also investigated the effect of the drug content (wt\%) on the release profile, leading to the general observation that higher loadings led to higher drug amounts released over time. ${ }^{265-266}$ On the other hand, Kempin et al. investigated the effect of polymer type on drug release, using quinine as a model, concluding that for a given drug, desired release profiles might be obtained by choosing the appropriate polymer. ${ }^{267}$ For a given polymer type and drug concentration, drug type will also define the release profile, as observed by the comparison among two independent studies: PCL/gentamicin $5 \mathrm{wt} \%$ scaffolds led to a burst release of gentamicin $(60 \%$ release in $24 \mathrm{~h})$, while quinine was released in a sustained manner from PCL/quinine $5 \mathrm{wt} \%$ scaffolds (60\% release in 7 days). ${ }^{267-268}$

As presented, loading bioactive molecules within the polymer matrix of ME-AM ensures higher incorporation efficiencies, allows for larger loading amounts and avoids fast release rates, compared to other strategies. However, this simple and cost-effective technique is at the moment limited to low melting temperature polymers, such as PCL.

\section{Other organic phase combinations}

The addition of organic fillers within ME-AM scaffolds has been investigated to improve their mechanical or physicochemical properties. For instance, cellulose nanomaterials have been used to reinforce PLA filaments for FDM, as well as PCL scaffolds, due to their crystalline fibrillar structure. ${ }^{269-270}$ Composite PCL/microcrystalline cellulose scaffolds showed an increase in compression and elastic modulus from $25 \mathrm{MPa}$ to $32 \mathrm{MPa}$ and from 60 to $72 \mathrm{MPa}$, respectively, with the sole addition of $2 \mathrm{wt} \%$ filler. ${ }^{270}$ Besides its reinforcing capabilities, this biopolymer possesses high specific surface area and surface reactivity, which allows for the introduction of well controlled chemical, structural, and topographical features at a cell relevant scale, which is of great interest for the TE field, but have not yet been widely explored for ME-AM scaffolds. ${ }^{271}$ Silk has also been used to improve the mechanical properties of ME-AM scaffolds. For instance, Aleman-Dominguez et al. demonstrated that the addition of up to $30 \mathrm{wt} \%$ silk microparticles within a PCL polymer matrix significantly increased the bulk and nano-mechanical properties of the scaffold, as measured by nanoindentation. 272 However, scaffold hydrophobicity was also significantly enhanced at this high concentration, which led to lower protein adsorption and subsequent poor cell affinity. This demonstrated that while a high filler content might be desired for 
significantly augmenting the scaffold's mechanical properties, cellular interactions, which are of outmost importance for the ultimate scaffold application, might be compromised at the same time. In this regard, the concentration of polyaniline (PANI) particles within a PCL scaffold was reduced to a minimum to ensure its biocompatibility. ${ }^{273}$ While the addition of this conductive polymer increased both the conductivity and compressive mechanical properties of the scaffold, concentrations above $0.1 \mathrm{wt} \%$ were shown to be cytotoxic. Despite these limitations, PCL/PANI $0.1 \mathrm{wt} \%$ scaffolds possessed a conductivity of $2.46 \pm$ $0.85 \times 10^{-4} \mathrm{~S} / \mathrm{cm}$, which enabled their use as electroactive structures to modulate cellular response and enhance tissue regeneration. ${ }^{273}$ Camarero-Espinosa et al. also reported the fabrication of scaffolds with the ability of providing cell stimulation, consisted of PCL/PLA Janus filaments that can be nano-deflected via external application of ultrasounds. ${ }^{274}$ Such Janus scaffolds were spontaneously formed by phase separation of PCL/PLA blends during $\mathrm{ME}$, and could perform as ultrasound transducers, enhancing cell proliferation, matrix deposition and osteogenic differentiation of hBM-MSCs, via the formation and activation of voltage-gated calcium ion channels.

\section{Conclusions and future perspective}

ME-AM enables the fabrication of complex-shaped personalized scaffolds for BTE applications, with an interconnected and highly controlled porosity and from a wide number of biodegradable and mechanically robust thermoplastic polymers. Compared to other AM technologies, ME-AM is a cost-effective and relatively simple technique, and does not require the use of potentially toxic binders, curing agents or solvents, which simplifies the clinical translation of the fabricated constructs. Furthermore, while synthetic thermoplastic polymers are often bioinert and lack cell-recognition sites to promote cell attachment, proliferation and osteogenic differentiation in vitro and in vivo, they are quite amenable to bioactive functionalization. A large amount of research has been carried out over the last decades on augmenting the bioactivity of ME-AM scaffolds. Modifying the scaffold architecture by adjusting the scaffold pore shape and size has been shown to influence cell attachment and differentiation. Similarly, the introduction of microporosity to the scaffold filaments' bulk and surface via ME-AM combined with salt leaching or gas foaming, has shown to increase the scaffold surface area for enhanced cell attachment. While these approaches have shown benefits, they can, in some cases, limit nutrient availability and diminish the scaffold's mechanical properties. On the contrary, surface 
modification strategies aiming at the introduction of roughness, specific chemical functional groups, or bioactive coatings to the filaments surface, do not compromise the scaffold structural integrity, and thus have been more extensively studied. In particular, plasma surface modification has stood out as a multi-approach technique for enhancing cell attachment and steering cell differentiation towards a specific lineage. In this regard, surface roughness can be introduced via plasma activation, specific polar functional groups can be deposited via plasma polymerization, and scaffold filaments can be primed for the subsequent covalent binding of biomolecules via plasma grafting. In addition to the conjugation of biomolecules, biomimetic PDA, CaP and full ECM coatings have also been investigated to enhance the surface functionality of scaffolds towards enhanced cell attachment and differentiation.

Although surface modifications can successfully augment the bioactivity of ME-AM scaffolds, we have seen that these approaches frequently involve the use of wet chemistry, and most importantly, they often require complex and multiple post-fabrication steps. Further incrementing the scaffold complexity, fibrous or hydrogel networks have been combined with the ME-AM framework towards hierarchical and hybrid scaffolds, which have been shown to increase cell retention and provide a biomimetic platform for tissue formation. Polymer-filler composite scaffolds have shown to induce specific cell behavior with the sole addition of fillers to the polymer matrix, avoiding the need of post fabrication steps. Moreover, composites can enhance not only the scaffold physicochemical properties, but also their mechanical and degradation properties, towards a better scaffold performance. In addition, composite production via melt blending by twin screw compounding ensures a complete solvent-free scaffold fabrication process. For all these reasons, a large body of literature has been focused on the fabrication of composite MEAM scaffolds using a variety of fillers, which include CaPs, silicates, metallic particles, carbon based materials, bioactive molecules and other organic materials, such as cellulose. Research has been focused on trying to identify the ideal filler type and concentration necessary to generate an optimum physicochemical and biological response, without compromising material printability, and while ensuring scaffold optimum mechanical properties and biocompatibility.

In spite of all these efforts, a ME-AM scaffold with optimum properties that can ensure optimum cell attachment and enhanced osteogenic differentiation towards a fully functional engineered in vitro and in vivo tissue is yet to be developed. There are still some 
challenges to overcome to achieve this aim, as well as aspects that would need attention in future research.

Currently, newly developed scaffolds can be easily characterized, in terms of printability, surface physicochemical analysis, filler dispersion in a composite, and mechanical properties thanks to the fast pace with which material science behind scaffold fabrication is progressing. Yet, the in vitro biological evaluation methodologies of such scaffolds are often insufficient to determine their in vivo efficacy. As a common strategy, a short in vitro evaluation of cell attachment, proliferation, differentiation, and early stage mineralization is often discussed. While these parameters are relevant for an initial estimation of the scaffolds functionality, long-term evaluations, coupled to the long process of bone regeneration, would be necessary to accurately determine the actual scaffold performance. In order to obtain more reliable in vitro short and long term information, as well as to reduce the number of animal experiments towards clinical translation, scaffolds could be cultured in bioreactors, where they could potentially be exposed to relevant cell populations and pro-osteogenic external stimulus (chemical, mechanical, electrical, ultrasonic). ${ }^{275-276}$ The flexible (sometimes elastomeric) synthetic thermoplastic polymers used in AM are best suited for mechanical stimulation. Such dynamic systems, in combination with computational models, could help to better understand and predict how specific scaffold architectures and the subsequent forces transmitted to cells (mechanical strain, fluid shear stress) can affect cell fate. ${ }^{277-278}$ This information could be used to improve the scaffold design towards enhanced tissue formation. In addition, bioreactor systems would also allow to analyze the long term stability of the scaffold modifications, such as plasma treatments, protein coatings, or bioactive CaP layers. Moreover, polymer degradation profiles and fillers release rates from composite scaffolds could be monitored to evaluate their in situ cytocompatibility. This could lead to more complete and focused studies and help finding the ideal scaffold for bone regeneration.

Another overlooked matter on ME-AM scaffolds for BTE is the formation of a vascular network. Due to their macroporous structure and their relatively small size for in vitro cultures, ME-AM scaffolds can support cell ingrowth and waste-nutrients exchange in vitro without the need of vascularization. Yet, scaffolds aimed to be used in large bone defects will likely require the integration of a vascular network to support tissue formation. Therefore, it is of great significance to implement cues to promote vascularization within ME-AM scaffolds, in addition to cues for cell attachment and osteogenic differentiation. In 
this regard, ME-AM scaffolds providing gradients in growth factors (i.e. VEGF) could induce blood vessels infiltration into the scaffold core. ${ }^{279}$ As presented in this review, current research is focused on the incorporation of bioactive cues to polymeric ME-AM scaffolds, while little efforts are being placed on developing new polymers, where the lack of functionality relies. Future research lines could relate to the design of new functional synthetic polymers, which can influence cellular behavior on their own, without the need of additional elements. Overall, this could lower the scaffold complexity, which would potentially simplify the production process, the in vitro and in vivo evaluation, the clinical translation path and, at the end of the chain, the final medical product price.

Despite current shortcomings, ME-AM scaffolds hold great promise in the future of bone regeneration. Appropriate scaffold design and architecture, specific bioactive cues for promoting cell attachment, differentiation and tissue formation, and thorough material and biological evaluations shall certainly be combined towards the construction of functional scaffolds for bone tissue regeneration. 


\section{References}

1. Porter, J. R.; Ruckh, T. T.; Popat, K. C., Bone Tissue Engineering: A Review in Bone Biomimetics and Drug Delivery Strategies. Biotechnology progress 2009, 25 (6), 1539-1560.

2. Rho, J.-Y.; Kuhn-Spearing, L.; Zioupos, P., Mechanical Properties and the Hierarchical Structure of Bone. Medical Engineering \& Physics 1998, 20 (2), 92-102.

3. Buckwalter, J.; Glimcher, M.; Cooper, R.; Recker, R., Bone Biology. J Bone Joint Surg Am 1995, 77 (8), 1256-1275.

4. Keaveny, T. M.; Morgan, E. F.; Yeh, O. C., Bone Mechanics. Standard handbook of biomedical engineering and design 2004, 1-24.

5. Reilly, D. T.; Burstein, A. H., The Elastic and Ultimate Properties of Compact Bone Tissue. J Biomech 1975, 8 (6), 393-405.

6. Kopperdahl, D. L.; Keaveny, T. M., Yield Strain Behavior of Trabecular Bone. J Biomech 1998, 31 (7), 601 608.

7. Morgan, E. F.; Keaveny, T. M., Dependence of Yield Strain of Human Trabecular Bone on Anatomic Site. J Biomech 2001, 34 (5), 569-577.

8. Rho, J. Y.; Ashman, R. B.; Turner, C. H., Young's Modulus of Trabecular and Cortical Bone Material: Ultrasonic and Microtensile Measurements. Journal of Biomechanics 1993, 26 (2), 111-119.

9. Gong, J. K.; Arnold, J. S.; Cohn, S. H., Composition of Trabecular and Cortical Bone. The Anatomical Record 1964, 149 (3), 325-331.

10. Burr, D. B., Targeted and Nontargeted Remodeling. Bone 2002, 30 (1), 2-4.

11. Crockett, J. C.; Rogers, M. J.; Coxon, F. P.; Hocking, L. J.; Helfrich, M. H., Bone Remodelling at a Glance. Journal of cell science 2011, 124 (Pt 7), 991-998.

12. Keating, J. F.; Simpson, A. H.; Robinson, C. M., The Management of Fractures with Bone Loss. The Journal of bone and joint surgery. British volume 2005, 87 (2), 142-150.

13. Marsell, R.; Einhorn, T. A., The Biology of Fracture Healing. Injury 2011, 42 (6), 551-555.

14. Green, E.; Lubahn, J. D.; Evans, J., Risk Factors, Treatment, and Outcomes Associated with Nonunion of the Midshaft Humerus Fracture. J Surg Orthop Adv 2005, 14 (2), 64-72.

15. Catagni, M. A.; Guerreschi, F.; Lovisetti, L., Distraction Osteogenesis for Bone Repair in the 21st Century: Lessons Learned. Injury 2011, 42 (6), 580-586.

16. Giannoudis, P. V.; Faour, O.; Goff, T.; Kanakaris, N.; Dimitriou, R., Masquelet Technique for the Treatment of Bone Defects: Tips-Tricks and Future Directions. Injury 2011, 42 (6), 591-598.

17. Karger, C.; Kishi, T.; Schneider, L.; Fitoussi, F.; Masquelet, A. C., Treatment of Posttraumatic Bone Defects by the Induced Membrane Technique. Orthopaedics \& Traumatology: Surgery \& Research 2012, 98 (1), 97-102.

18. Myeroff, C.; Archdeacon, M., Autogenous Bone Graft: Donor Sites and Techniques. JBJS 2011, 93 (23).

19. Spiegelberg, B.; Parratt, T.; Dheerendra, S. K.; Khan, W. S.; Jennings, R.; Marsh, D. R., llizarov Principles of Deformity Correction. Annals of the Royal College of Surgeons of England 2010, 92 (2), 101-105.

20. Gruskin, E.; Doll, B. A.; Futrell, F. W.; Schmitz, J. P.; Hollinger, J. O., Demineralized Bone Matrix in Bone Repair: History and Use. Advanced Drug Delivery Reviews 2012, 64 (12), 1063-1077.

21. Henkel, J.; Woodruff, M. A.; Epari, D. R.; Steck, R.; Glatt, V.; Dickinson, I. C.; Choong, P. F.; Schuetz, M. A.; Hutmacher, D. W., Bone Regeneration Based on Tissue Engineering Conceptions-a 21st Century Perspective. Bone research 2013, 1 (3), 216-248.

22. Iyer, K. M., The Principles of the Ilizarov Apparatus. In General Principles of Orthopedics and Trauma, lyer, K. M.; Khan, W. S., Eds. Springer International Publishing: Cham, 2019; pp 607-617.

23. Hutmacher, D. W., Scaffolds in Tissue Engineering Bone and Cartilage. Biomaterials 2000, 21 (24), 25292543.

24. Leong, K. F.; Chua, C. K.; Sudarmadji, N.; Yeong, W. Y., Engineering Functionally Graded Tissue Engineering Scaffolds. Journal of the Mechanical Behavior of Biomedical Materials 2008, 1 (2), 140-152. 
25. Karageorgiou, V.; Kaplan, D., Porosity of 3d Biomaterial Scaffolds and Osteogenesis. Biomaterials 2005, 26 (27), 5474-5491.

26. Van Bael, S.; Chai, Y. C.; Truscello, S.; Moesen, M.; Kerckhofs, G.; Van Oosterwyck, H.; Kruth, J. P.; Schrooten, J., The Effect of Pore Geometry on the in Vitro Biological Behavior of Human PeriosteumDerived Cells Seeded on Selective Laser-Melted Ti6al4v Bone Scaffolds. Acta Biomaterialia 2012, 8 (7), 2824-2834.

27. Zadpoor, A. A., Bone Tissue Regeneration: The Role of Scaffold Geometry. Biomaterials Science 2015, 3 (2), 231-245.

28. Rumpler, M.; Woesz, A.; Dunlop, J. W.; van Dongen, J. T.; Fratzl, P., The Effect of Geometry on ThreeDimensional Tissue Growth. Journal of the Royal Society Interface 2008, 5 (27), 1173-1180.

29. Bidan, C. M.; Kommareddy, K. P.; Rumpler, M.; Kollmannsberger, P.; Fratzl, P.; Dunlop, J. W. C., Geometry as a Factor for Tissue Growth: Towards Shape Optimization of Tissue Engineering Scaffolds. Advanced Healthcare Materials 2013, 2 (1), 186-194.

30. Sachlos, E.; Czernuszka, J. T., Making Tissue Engineering Scaffolds Work. Review: The Application of Solid Freeform Fabrication Technology to the Production of Tissue Engineering Scaffolds. Eur Cell Mater 2003, 5, 29-39; discussion 39-40.

31. Wubneh, A.; Tsekoura, E. K.; Ayranci, C.; Uludağ, H., Current State of Fabrication Technologies and Materials for Bone Tissue Engineering. Acta Biomaterialia 2018, 80, 1-30.

32. Mota, C.; Puppi, D.; Chiellini, F.; Chiellini, E., Additive Manufacturing Techniques for the Production of Tissue Engineering Constructs. Journal of tissue engineering and regenerative medicine 2015, 9 (3), 174190.

33. Moroni, L.; Boland, T.; Burdick, J. A.; De Maria, C.; Derby, B.; Forgacs, G.; Groll, J.; Li, Q.; Malda, J.; Mironov, V. A.; Mota, C.; Nakamura, M.; Shu, W.; Takeuchi, S.; Woodfield, T. B. F.; Xu, T.; Yoo, J. J.; Vozzi, G., Biofabrication: A Guide to Technology and Terminology. Trends in Biotechnology 2018, 36 (4), 384-402.

34. Calore, A. R.; Sinha, R.; Harings, J.; Bernaerts, K. V.; Mota, C.; Moroni, L., Additive Manufacturing Using Melt Extruded Thermoplastics for Tissue Engineering. Methods in molecular biology (Clifton, N.J.) 2021, 2147, 75-99.

35. Butscher, A.; Bohner, M.; Hofmann, S.; Gauckler, L.; Müller, R., Structural and Material Approaches to Bone Tissue Engineering in Powder-Based Three-Dimensional Printing. Acta Biomaterialia 2011, 7 (3), 907-920.

36. Liu, W.; Wang, D.; Huang, J.; Wei, Y.; Xiong, J.; Zhu, W.; Duan, L.; Chen, J.; Sun, R.; Wang, D., LowTemperature Deposition Manufacturing: A Novel and Promising Rapid Prototyping Technology for the Fabrication of Tissue-Engineered Scaffold. Materials Science and Engineering: C 2017, 70, 976-982.

37. Puppi, D.; Chiellini, F., Wet-Spinning of Biomedical Polymers: From Single-Fibre Production to Additive Manufacturing of Three-Dimensional Scaffolds. Polymer International 2017, 66 (12), 1690-1696.

38. Melchels, F. P.; Feijen, J.; Grijpma, D. W., A Review on Stereolithography and Its Applications in Biomedical Engineering. Biomaterials 2010, 31 (24), 6121-6130.

39. Hutmacher, D. W.; Sittinger, M.; Risbud, M. V., Scaffold-Based Tissue Engineering: Rationale for Computer-Aided Design and Solid Free-Form Fabrication Systems. TRENDS in Biotechnology 2004, 22 (7), 354-362.

40. Conn, J.; Oyasu, R.; Welsh, M.; Beal, J. M., Vicryl (Polyglactin 910) Synthetic Absorbable Sutures. The American Journal of Surgery 1974, 128 (1), 19-23.

41. Bezwada, R. S.; Jamiolkowski, D. D.; Lee, I.-Y.; Agarwal, V.; Persivale, J.; Trenka-Benthin, S.; Erneta, M.; Suryadevara, J.; Yang, A.; Liu, S., Monocry|® Suture, a New Ultra-Pliable Absorbable Monofilament Suture. Biomaterials 1995, 16 (15), 1141-1148.

42. Barber, F. A.; Dockery, W. D., Long-Term Absorption of Poly-L-Lactic Acid Interference Screws. Arthroscopy 2006, 22 (8), 820-826.

43. Hutmacher, D. W.; Schantz, T.; Zein, I.; Ng, K. W.; Teoh, S. H.; Tan, K. C., Mechanical Properties and Cell Cultural Response of Polycaprolactone Scaffolds Designed and Fabricated Via Fused Deposition Modeling. Journal of Biomedical Materials Research: An Official Journal of The Society for Biomaterials, The Japanese Society for Biomaterials, and The Australian Society for Biomaterials and the Korean Society for Biomaterials 2001, 55 (2), 203-216. 
44. Schantz, J.-T.; Lim, T.-C.; Ning, C.; Teoh, S. H.; Tan, K. C.; Wang, S. C.; Hutmacher, D. W., Cranioplasty after Trephination Using a Novel Biodegradable Burr Hole Cover: Technical Case Report. Operative Neurosurgery 2006, 58 (suppl_1), ONS-E176-ONS-E176.

45. Low, S. W.; Ng, Y. J.; Yeo, T. T.; Chou, N., Use of Osteoplug Polycaprolactone Implants as Novel BurrHole Covers. Singapore medical journal 2009, 50 (8), 777-780.

46. Youssef, A.; Hollister, S. J.; Dalton, P. D., Additive Manufacturing of Polymer Melts for Implantable Medical Devices and Scaffolds. Biofabrication 2017, 9 (1), 012002.

47. Ming; Hui, H. J.; W., V. D.; Chou, N.; Tsai, T., Cranial Reconstruction Using a Polycaprolactone Implant after Burr Hole Trephination. Journal of 3D Printing in Medicine 2020, 4 (1), 9-16.

48. Zhang, Z.; Teoh, S. H., Novel 3d Polycaprolactone Scaffold for Ridge Preservation-a Pilot Randomised Controlled Clinical Trial. 2014.

49. Teo, L.; Teoh, S. H.; Liu, Y.; Lim, L.; Tan, B.; Schantz, J.-T.; Seah, L. L., A Novel Bioresorbable Implant for Repair of Orbital Floor Fractures. Orbit 2015, 34 (4), 192-200.

50. Deschamps, A.; Claase, M.; Sleijster, W.; de Bruijn, J.; Grijpma, D.; Feijen, J., Design of Segmented Poly(Ether Ester) Materials and Structures for Tissue Engineering of Bone. Journal of controlled release : official journal of the Controlled Release Society 2002, 78, 175-186.

51. Huang, M.-N., Biodegradable and Bioactive Porous Polyurethanes Scaffolds for Bone Tissue Engineering. Journal of Biomedical Science and Engineering 2009, 02, 36-40.

52. Yu, N. Y.; Schindeler, A.; Little, D. G.; Ruys, A. J., Biodegradable Poly (A-Hydroxy Acid) Polymer Scaffolds for Bone Tissue Engineering. Journal of Biomedical Materials Research Part B: Applied Biomaterials: An Official Journal of The Society for Biomaterials, The Japanese Society for Biomaterials, and The Australian Society for Biomaterials and the Korean Society for Biomaterials 2010, 93 (1), 285-295.

53. Dwivedi, R.; Kumar, S.; Pandey, R.; Mahajan, A.; Nandana, D.; Katti, D. S.; Mehrotra, D., Polycaprolactone as Biomaterial for Bone Scaffolds: Review of Literature. Journal of Oral Biology and Craniofacial Research 2020, 10 (1), 381-388.

54. Griffin, M.; Castro, N.; Bas, O.; Saifzadeh, S.; Butler, P.; Hutmacher, D. W., The Current Versatility of Polyurethane Three-Dimensional Printing for Biomedical Applications. Tissue Engineering Part B: Reviews 2020, 26 (3), 272-283.

55. Chen, M.; Xu, L.; Zhou, Y.; Zhang, Y.; Lang, M.; Ye, Z.; Tan, W.-S., Poly(E-Caprolactone)-Based Substrates Bearing Pendant Small Chemical Groups as a Platform for Systemic Investigation of Chondrogenesis. Cell Proliferation 2016, 49 (4), 512-522.

56. Baran, E. H.; Erbil, H. Y., Surface Modification of 3d Printed Pla Objects by Fused Deposition Modeling: A Review. Colloids and interfaces 2019, 3 (2), 43.

57. Olubamiji, A. D.; Izadifar, Z.; Si, J. L.; Cooper, D. M.; Eames, B. F.; Chen, D. X., Modulating Mechanical Behaviour of 3d-Printed Cartilage-Mimetic Pcl Scaffolds: Influence of Molecular Weight and Pore Geometry. Biofabrication 2016, 8 (2), 025020.

58. Zein, I.; Hutmacher, D. W.; Tan, K. C.; Teoh, S. H., Fused Deposition Modeling of Novel Scaffold Architectures for Tissue Engineering Applications. Biomaterials 2002, 23 (4), 1169-1185.

59. Moroni, L.; De Wijn, J.; Van Blitterswijk, C., 3d Fiber-Deposited Scaffolds for Tissue Engineering: Influence of Pores Geometry and Architecture on Dynamic Mechanical Properties. Biomaterials 2006, 27 (7), 974 985.

60. Camarero-Espinosa, S.; Calore, A.; Wilbers, A.; Harings, J.; Moroni, L., Additive Manufacturing of an Elastic Poly(Ester)Urethane for Cartilage Tissue Engineering. Acta Biomaterialia 2020, 102, 192-204.

61. Ostrowska, B.; Di Luca, A.; Moroni, L.; Swieszkowski, W., Influence of Internal Pore Architecture on Biological and Mechanical Properties of Three-Dimensional Fiber Deposited Scaffolds for Bone Regeneration. Journal of Biomedical Materials Research Part A 2016.

62. Valainis, D.; Dondl, P.; Foehr, P.; Burgkart, R.; Kalkhof, S.; Duda, G. N.; van Griensven, M.; Poh, P. S. P., Integrated Additive Design and Manufacturing Approach for the Bioengineering of Bone Scaffolds for Favorable Mechanical and Biological Properties. Biomedical Materials 2019, 14 (6), 065002.

63. Bartnikowski, M.; Klein, T. J.; Melchels, F. P.; Woodruff, M. A., Effects of Scaffold Architecture on Mechanical Characteristics and Osteoblast Response to Static and Perfusion Bioreactor Cultures. Biotechnology and bioengineering 2014, 111 (7), 1440-1451. 
64. Liang, X.; Gao, J.; Xu, W.; Wang, X.; Shen, Y.; Tang, J.; Cui, S.; Yang, X.; Liu, Q.; Yu, L., Structural Mechanics of 3d-Printed Poly (Lactic Acid) Scaffolds with Tetragonal, Hexagonal and Wheel-Like Designs. Biofabrication 2019, 11 (3), 035009.

65. Holy, C. E.; Shoichet, M. S.; Davies, J. E., Engineering Three-Dimensional Bone Tissue in Vitro Using Biodegradable Scaffolds: Investigating Initial Cell-Seeding Density and Culture Period. Journal of Biomedical Materials Research: An Official Journal of The Society for Biomaterials, The Japanese Society for Biomaterials, and The Australian Society for Biomaterials and the Korean Society for Biomaterials 2000, 51 (3), 376-382.

66. Li, Y.; Ma, T.; Kniss, D. A.; Lasky, L. C.; Yang, S. T., Effects of Filtration Seeding on Cell Density, Spatial Distribution, and Proliferation in Nonwoven Fibrous Matrices. Biotechnology progress 2001, 17 (5), 935 944.

67. Sobral, J. M.; Caridade, S. G.; Sousa, R. A.; Mano, J. F.; Reis, R. L., Three-Dimensional Plotted Scaffolds with Controlled Pore Size Gradients: Effect of Scaffold Geometry on Mechanical Performance and Cell Seeding Efficiency. Acta Biomaterialia 2011, 7 (3), 1009-1018.

68. Di Luca, A.; Ostrowska, B.; Lorenzo-Moldero, I.; Lepedda, A.; Swieszkowski, W.; Van Blitterswijk, C.; Moroni, L., Gradients in Pore Size Enhance the Osteogenic Differentiation of Human Mesenchymal Stromal Cells in Three-Dimensional Scaffolds. Scientific reports 2016, 6.

69. Woodfield, T. B.; Moroni, L.; Malda, J., Combinatorial Approaches to Controlling Cell Behaviour and Tissue Formation in 3d Via Rapid-Prototyping and Smart Scaffold Design. Combinatorial chemistry \& high throughput screening 2009, 12 (6), 562-579.

70. Di Luca, A.; Lorenzo-Moldero, I.; Mota, C.; Lepedda, A.; Auhl, D.; Van Blitterswijk, C.; Moroni, L., Tuning Cell Differentiation into a 3d Scaffold Presenting a Pore Shape Gradient for Osteochondral Regeneration. Advanced Healthcare Materials 2016, 5 (14), 1753-1763.

71. Berner, A.; Woodruff, M.; Lam, C.; Arafat, M.; Saifzadeh, S.; Steck, R.; Ren, J.; Nerlich, M.; Ekaputra, A. K.; Gibson, I., Effects of Scaffold Architecture on Cranial Bone Healing. International journal of oral and maxillofacial surgery 2014, 43 (4), 506-513.

72. Shim, J.-H.; Jeong, J.-h.; Won, J.-Y.; Bae, J.-H.; Ahn, G.; Jeon, H.; Yun, W.-S.; Bae, E.-B.; Choi, J.-W.; Lee, S.-H., Porosity Effect of 3d-Printed Polycaprolactone Membranes on Calvarial Defect Model for Guided Bone Regeneration. Biomedical Materials 2017, 13 (1), 015014.

73. Roosa, S. M. M.; Kemppainen, J. M.; Moffitt, E. N.; Krebsbach, P. H.; Hollister, S. J., The Pore Size of Polycaprolactone Scaffolds Has Limited Influence on Bone Regeneration in an in Vivo Model. Journal of Biomedical Materials Research Part A: An Official Journal of The Society for Biomaterials, The Japanese Society for Biomaterials, and The Australian Society for Biomaterials and the Korean Society for Biomaterials 2010, 92 (1), 359-368.

74. Rechendorff, K.; Hovgaard, M. B.; Foss, M.; Zhdanov, V.; Besenbacher, F., Enhancement of Protein Adsorption Induced by Surface Roughness. Langmuir 2006, 22 (26), 10885-10888.

75. Dalby, M. J.; Gadegaard, N.; Tare, R.; Andar, A.; Riehle, M. O.; Herzyk, P.; Wilkinson, C. D.; Oreffo, R. O., The Control of Human Mesenchymal Cell Differentiation Using Nanoscale Symmetry and Disorder. Nature materials 2007, 6 (12), 997-1003.

76. Faia-Torres, A. B.; Guimond-Lischer, S.; Rottmar, M.; Charnley, M.; Goren, T.; Maniura-Weber, K.; Spencer, N. D.; Reis, R. L.; Textor, M.; Neves, N. M., Differential Regulation of Osteogenic Differentiation of Stem Cells on Surface Roughness Gradients. Biomaterials 2014, 35 (33), 9023-9032.

77. Kumar, G.; Waters, M. S.; Farooque, T. M.; Young, M. F.; Simon, C. G., Jr., Freeform Fabricated Scaffolds with Roughened Struts That Enhance Both Stem Cell Proliferation and Differentiation by Controlling Cell Shape. Biomaterials 2012, 33 (16), 4022-4030.

78. Kumar, G.; Tison, C. K.; Chatterjee, K.; Pine, P. S.; McDaniel, J. H.; Salit, M. L.; Young, M. F.; Simon, C. G., The Determination of Stem Cell Fate by 3d Scaffold Structures through the Control of Cell Shape. Biomaterials 2011, 32 (35), 9188-9196.

79. Zamani, Y.; Mohammadi, J.; Amoabediny, G.; Visscher, D. O.; Helder, M. N.; Zandieh-Doulabi, B.; KleinNulend, J., Enhanced Osteogenic Activity by Mc3t3-E1 Pre-Osteoblasts on Chemically Surface-Modified Poly(E-Caprolactone) 3d-Printed Scaffolds Compared to Rgd Immobilized Scaffolds. Biomedical materials (Bristol, England) 2018, 14 (1), 015008.

80. Kosik-Kozioł, A.; Graham, E.; Jaroszewicz, J.; Chlanda, A.; Kumar, P. T. S.; Ivanovski, S.; Święszkowski, W.; Vaquette, C., Surface Modification of 3d Printed Polycaprolactone Constructs Via a Solvent Treatment: Impact on Physical and Osteogenic Properties. ACS Biomaterials Science \& Engineering 2019, 5 (1), 318328. 
81. Davies, J. E., Bone Bonding at Natural and Biomaterial Surfaces. Biomaterials 2007, 28 (34), 5058-5067.

82. Hing, K. A.; Annaz, B.; Saeed, S.; Revell, P. A.; Buckland, T., Microporosity Enhances Bioactivity of Synthetic Bone Graft Substitutes. Journal of Materials Science: Materials in Medicine 2005, 16 (5), 467 475.

83. Castro, N. J.; Tan, W. N.; Shen, C.; Zhang, L. G., Simulated Body Fluid Nucleation of Three-Dimensional Printed Elastomeric Scaffolds for Enhanced Osteogenesis. Tissue engineering. Part A 2016, 22 (13-14), 940-948.

84. Visscher, L. E.; Dang, H. P.; Knackstedt, M. A.; Hutmacher, D. W.; Tran, P. A., 3d Printed Polycaprolactone Scaffolds with Dual Macro-Microporosity for Applications in Local Delivery of Antibiotics. Materials Science and Engineering: C 2018, 87, 78-89.

85. Song, P.; Zhou, C.; Fan, H.; Zhang, B.; Pei, X.; Fan, Y.; Jiang, Q.; Bao, R.; Yang, Q.; Dong, Z.; Zhang, X., Novel 3d Porous Biocomposite Scaffolds Fabricated by Fused Deposition Modeling and Gas Foaming Combined Technology. Composites Part B: Engineering 2018, 152, 151-159.

86. Choi, W. J.; Hwang, K. S.; Kwon, H. J.; Lee, C.; Kim, C. H.; Kim, T. H.; Heo, S. W.; Kim, J.-H.; Lee, J.-Y., Rapid Development of Dual Porous Poly(Lactic Acid) Foam Using Fused Deposition Modeling (Fdm) 3d Printing for Medical Scaffold Application. Materials Science and Engineering: C 2020, 110, 110693.

87. Castaño, M.; Martinez-Campos, E.; Pintado-Sierra, M.; García, C.; Reinecke, H.; Gallardo, A.; RodriguezHernandez, J.; Elvira, C., Combining Breath Figures and Supercritical Fluids to Obtain Porous Polymer Scaffolds. ACS Omega 2018, 3 (10), 12593-12599.

88. Sinha, R.; Cámara-Torres, M.; Scopece, P.; Verga Falzacappa, E.; Patelli, A.; Moroni, L.; Mota, C., A Hybrid Additive Manufacturing Platform to Create Bulk and Surface Composition Gradients on Scaffolds for Tissue Regeneration. Nature Communications 2021, 12 (1), 500.

89. De Geyter, N.; Morent, R., 7 - Cold Plasma Surface Modification of Biodegradable Polymer Biomaterials. In Biomaterials for Bone Regeneration, Dubruel, P.; Van Vlierberghe, S., Eds. Woodhead Publishing: 2014; pp 202-224.

90. Keselowsky, B. G.; Collard, D. M.; García, A. J., Surface Chemistry Modulates Focal Adhesion Composition and Signaling through Changes in Integrin Binding. Biomaterials 2004, 25 (28), 5947-5954.

91. Curran, J. M.; Chen, R.; Hunt, J. A., The Guidance of Human Mesenchymal Stem Cell Differentiation in Vitro by Controlled Modifications to the Cell Substrate. Biomaterials 2006, 27 (27), 4783-4793.

92. Massia, S. P.; Hubbell, J. A., Immobilized Amines and Basic Amino Acids as Mimetic Heparin-Binding Domains for Cell Surface Proteoglycan-Mediated Adhesion. The Journal of biological chemistry 1992, 267 (14), 10133-10141.

93. Webb, K.; Hlady, V.; Tresco, P. A., Relative Importance of Surface Wettability and Charged Functional Groups on Nih 3t3 Fibroblast Attachment, Spreading, and Cytoskeletal Organization. J Biomed Mater Res 1998, 41 (3), 422-430.

94. Yildirim, E. D.; Pappas, D.; Güçeri, S.; Sun, W., Enhanced Cellular Functions on Polycaprolactone Tissue Scaffolds by O2 Plasma Surface Modification. Plasma Processes and Polymers 2011, 8 (3), 256-267.

95. Jacobs, T.; Declercq, H.; De Geyter, N.; Cornelissen, R.; Dubruel, P.; Leys, C.; Morent, R., Improved Cell Adhesion to Flat and Porous Plasma-Treated Poly-E-Caprolactone Samples. Surface and Coatings Technology 2013, 232, 447-455.

96. Jeon, H.; Lee, H.; Kim, G., A Surface-Modified Poly(E-Caprolactone) Scaffold Comprising Variable Nanosized Surface-Roughness Using a Plasma Treatment. Tissue Engineering Part C: Methods 2014, 20 (12), 951-963.

97. Wang, M.; Favi, P.; Cheng, X.; Golshan, N. H.; Ziemer, K. S.; Keidar, M.; Webster, T. J., Cold Atmospheric Plasma (Cap) Surface Nanomodified 3d Printed Polylactic Acid (Pla) Scaffolds for Bone Regeneration. Acta Biomaterialia 2016, 46, 256-265.

98. Domingos, M.; Intranuovo, F.; Gloria, A.; Gristina, R.; Ambrosio, L.; Bártolo, P. J.; Favia, P., Improved Osteoblast Cell Affinity on Plasma-Modified 3-D Extruded Pcl Scaffolds. Acta Biomaterialia 2013, 9 (4), 5997-6005.

99. Lerman, M. J.; Smith, B. T.; Gerald, A. G.; Santoro, M.; Fookes, J. A.; Mikos, A. G.; Fisher, J. P., Aminated 3d Printed Polystyrene Maintains Stem Cell Proliferation and Osteogenic Differentiation. Tissue Engineering Part C: Methods 2019, 26 (2), 118-131. 
100. Griffin, M. F.; Ibrahim, A.; Seifalian, A. M.; Butler, P. E. M.; Kalaskar, D. M.; Ferretti, P., Chemical GroupDependent Plasma Polymerisation Preferentially Directs Adipose Stem Cell Differentiation Towards Osteogenic or Chondrogenic Lineages. Acta Biomater 2017, 50, 450-461.

101. Cools, P.; Declercq, H.; Ghobeira, R.; Morent, R.; De Geyter, N., Acrylic Acid Plasma Coatings for Enhanced Cell Migration in Pcl 3d Additive Manufactured Scaffolds. Surface and Coatings Technology 2018, 350, 925-935.

102. Cools, P.; Mota, C.; Lorenzo-Moldero, I.; Ghobeira, R.; De Geyter, N.; Moroni, L.; Morent, R., Acrylic Acid Plasma Coated 3d Scaffolds for Cartilage Tissue Engineering Applications. Sci Rep 2018, 8 (1), 3830.

103. Cools, P.; Sainz-García, E.; Geyter, N. D.; Nikiforov, A.; Blajan, M.; Shimizu, K.; Alba-Elías, F.; Leys, C.; Morent, R., Influence of Dbd Inlet Geometry on the Homogeneity of Plasma-Polymerized Acrylic Acid Films: The Use of a Microplasma-Electrode Inlet Configuration. Plasma Processes and Polymers 2015, 12 (10), 1153-1163.

104. De Geyter, N.; Morent, R.; Leys, C., Penetration of a Dielectric Barrier Discharge Plasma into Textile Structures at Medium Pressure. Plasma Sources Science and Technology 2006, 15 (1), 78.

105. Sardella, E.; Salama, R. A.; Waly, G. H.; Habib, A. N.; Favia, P.; Gristina, R., Improving Internal Cell Colonization of Porous Scaffolds with Chemical Gradients Produced by Plasma Assisted Approaches. ACS Applied Materials \& Interfaces 2017, 9 (5), 4966-4975.

106. Lu, X.; Laroussi, M.; Puech, V., On Atmospheric-Pressure Non-Equilibrium Plasma Jets and Plasma Bullets. Plasma Sources Science and Technology 2012, 21 (3), 034005.

107. Liu, F.; Wang, W.; Hinduja, S.; Bártolo, P. J., Hybrid Additive Manufacturing System for Zonal PlasmaTreated Scaffolds. 3D printing and additive manufacturing 2018, 5 (3), 205-213.

108. Liu, Y.; Wang, R.; Chen, S.; Xu, Z.; Wang, Q.; Yuan, P.; Zhou, Y.; Zhang, Y.; Chen, J., Heparan Sulfate Loaded Polycaprolactone-Hydroxyapatite Scaffolds with 3d Printing for Bone Defect Repair. International journal of biological macromolecules 2020, 148, 153-162.

109. Farto-Vaamonde, X.; Auriemma, G.; Aquino, R. P.; Concheiro, A.; Alvarez-Lorenzo, C., Post-Manufacture Loading of Filaments and 3d Printed Pla Scaffolds with Prednisolone and Dexamethasone for Tissue Regeneration Applications. European Journal of Pharmaceutics and Biopharmaceutics 2019, 141, 100110.

110. Chen, C.-Y.; Chen, C.-C.; Wang, C.-Y.; Lee, A. K.-X.; Yeh, C.-L.; Lin, C.-P., Assessment of the Release of Vascular Endothelial Growth Factor from 3d-Printed Poly-E-Caprolactone/Hydroxyapatite/Calcium Sulfate Scaffold with Enhanced Osteogenic Capacity. Polymers 2020, 12 (7), 1455.

111. Tian, L.; Zhang, Z.; Tian, B.; Zhang, X.; Wang, N., Study on Antibacterial Properties and Cytocompatibility of Epl Coated 3d Printed Pcl/Ha Composite Scaffolds. RSC Advances 2020, 10 (8), 4805-4816.

112. Sawyer, A. A.; Song, S. J.; Susanto, E.; Chuan, P.; Lam, C. X. F.; Woodruff, M. A.; Hutmacher, D. W.; Cool, S. M., The Stimulation of Healing within a Rat Calvarial Defect by Mpcl-Tcp/Collagen Scaffolds Loaded with Rhbmp-2. Biomaterials 2009, 30 (13), 2479-2488.

113. Reddi, A. H., Role of Morphogenetic Proteins in Skeletal Tissue Engineering and Regeneration. Nature biotechnology 1998, 16 (3), 247-252.

114. Yan, Y.; Chen, H.; Zhang, H.; Guo, C.; Yang, K.; Chen, K.; Cheng, R.; Qian, N.; Sandler, N.; Zhang, Y. S.; Shen, H.; Qi, J.; Cui, W.; Deng, L., Vascularized 3d Printed Scaffolds for Promoting Bone Regeneration. Biomaterials 2019, 190-191, 97-110.

115. Li, S.; Xu, Y.; Yu, J.; Becker, M. L., Enhanced Osteogenic Activity of Poly(Ester Urea) Scaffolds Using Facile Post-3d Printing Peptide Functionalization Strategies. Biomaterials 2017, 141, 176-187.

116. Holmes, B.; Zhu, W.; Li, J.; Lee, J. D.; Zhang, L. G., Development of Novel Three-Dimensional Printed Scaffolds for Osteochondral Regeneration. Tissue engineering. Part A 2015, 21 (1-2), 403-415.

117. Yang, Y.; Yang, S.; Wang, Y.; Yu, Z.; Ao, H.; Zhang, H.; Qin, L.; Guillaume, O.; Eglin, D.; Richards, R. G.; Tang, T., Anti-Infective Efficacy, Cytocompatibility and Biocompatibility of a 3d-Printed Osteoconductive Composite Scaffold Functionalized with Quaternized Chitosan. Acta Biomater 2016, 46, 112-128.

118. Yang, Y.; Chu, L.; Yang, S.; Zhang, H.; Qin, L.; Guillaume, O.; Eglin, D.; Richards, R. G.; Tang, T., DualFunctional 3d-Printed Composite Scaffold for Inhibiting Bacterial Infection and Promoting Bone Regeneration in Infected Bone Defect Models. Acta Biomaterialia 2018, 79, 265-275.

119. Jiang, H.; Xu, F.-J., Biomolecule-Functionalized Polymer Brushes. Chemical Society Reviews 2013,42 (8), 3394-3426. 
120. Declercq, H. A.; Desmet, T.; Berneel, E. E.; Dubruel, P.; Cornelissen, M. J., Synergistic Effect of Surface Modification and Scaffold Design of Bioplotted 3-D Poly-Epsilon-Caprolactone Scaffolds in Osteogenic Tissue Engineering. Acta Biomater 2013, 9 (8), 7699-7708.

121. Tardajos, M. G.; Cama, G.; Dash, M.; Misseeuw, L.; Gheysens, T.; Gorzelanny, C.; Coenye, T.; Dubruel, P., Chitosan Functionalized Poly-E-Caprolactone Electrospun Fibers and 3d Printed Scaffolds as Antibacterial Materials for Tissue Engineering Applications. Carbohydrate Polymers 2018, 191, 127-135.

122. Gunnewiek, M. K.; Di Luca, A.; Bollemaat, H. Z.; van Blitterswijk, C. A.; Vancso, G. J.; Moroni, L.; Benetti, E. M., Creeping Proteins in Microporous Structures: Polymer Brush-Assisted Fabrication of 3d Gradients for Tissue Engineering. Advanced healthcare materials 2015, 4 (8), 1169-1174.

123. Di Luca, A.; Klein-Gunnewiek, M.; Vancso, J. G.; van Blitterswijk, C. A.; Benetti, E. M.; Moroni, L., Covalent Binding of Bone Morphogenetic Protein-2 and Transforming Growth Factor-B3 to 3d Plotted Scaffolds for Osteochondral Tissue Regeneration. Biotechnology Journal 2017, 12 (12), 1700072.

124. Madhurakkat Perikamana, S. K.; Lee, J.; Lee, Y. B.; Shin, Y. M.; Lee, E. J.; Mikos, A. G.; Shin, H., Materials from Mussel-Inspired Chemistry for Cell and Tissue Engineering Applications. Biomacromolecules 2015, 16 (9), 2541-2555.

125. Teixeira, B. N.; Aprile, P.; Mendonça, R. H.; Kelly, D. J.; Thiré, R. M. d. S. M., Evaluation of Bone Marrow Stem Cell Response to Pla Scaffolds Manufactured by 3d Printing and Coated with Polydopamine and Type I Collagen. Journal of Biomedical Materials Research Part B: Applied Biomaterials 2019, 107 (1), 3749.

126. Lee, S. J.; Lee, D.; Yoon, T. R.; Kim, H. K.; Jo, H. H.; Park, J. S.; Lee, J. H.; Kim, W. D.; Kwon, I. K.; Park, S. A., Surface Modification of 3d-Printed Porous Scaffolds Via Mussel-Inspired Polydopamine and Effective Immobilization of Rhbmp-2 to Promote Osteogenic Differentiation for Bone Tissue Engineering. Acta Biomater 2016, 40, 182-191.

127. Cheng, C. H.; Chen, Y. W.; Kai-Xing Lee, A.; Yao, C. H.; Shie, M. Y., Development of Mussel-Inspired 3dPrinted Poly (Lactic Acid) Scaffold Grafted with Bone Morphogenetic Protein-2 for Stimulating Osteogenesis. Journal of materials science. Materials in medicine 2019, 30 (7), 78

128. Zhang, X.; Lou, Q.; Wang, L.; Min, S.; Zhao, M.; Quan, C., Immobilization of Bmp-2-Derived Peptides on 3d-Printed Porous Scaffolds for Enhanced Osteogenesis. Biomedical Materials 2019, 15 (1), 015002.

129. Lee, S. J.; Won, J. E.; Han, C.; Yin, X. Y.; Kim, H. K.; Nah, H.; Kwon, I. K.; Min, B. H.; Kim, C. H.; Shin, Y. S.; Park, S. A., Development of a Three-Dimensionally Printed Scaffold Grafted with Bone Forming Peptide-1 for Enhanced Bone Regeneration with in Vitro and in Vivo Evaluations. Journal of colloid and interface science 2019, 539, 468-480.

130. Ku, S. H.; Park, C. B., Human Endothelial Cell Growth on Mussel-Inspired Nanofiber Scaffold for Vascular Tissue Engineering. Biomaterials 2010, 31 (36), 9431-9437.

131. Shin, Y. M.; Lee, Y. B.; Shin, H., Time-Dependent Mussel-Inspired Functionalization of Poly(L-Lactide-Co$\varepsilon$-Caprolactone) Substrates for Tunable Cell Behaviors. Colloids and surfaces. B, Biointerfaces 2011, 87 (1), 79-87.

132. Kao, C. T.; Lin, C. C.; Chen, Y. W.; Yeh, C. H.; Fang, H. Y.; Shie, M. Y., Poly(Dopamine) Coating of 3d Printed Poly(Lactic Acid) Scaffolds for Bone Tissue Engineering. Materials science \& engineering. C, Materials for biological applications 2015, 56, 165-173.

133. Hong, S.; Kim, K. Y.; Wook, H. J.; Park, S. Y.; Lee, K. D.; Lee, D. Y.; Lee, H., Attenuation of the in Vivo Toxicity of Biomaterials by Polydopamine Surface Modification. Nanomedicine 2011, 6 (5), 793-801.

134. Decaris, M. L.; Leach, J. K., Design of Experiments Approach to Engineer Cell-Secreted Matrices for Directing Osteogenic Differentiation. Annals of Biomedical Engineering 2011, 39 (4), 1174-1185.

135. Wu, Y. A.; Chiu, Y. C.; Lin, Y. H.; Ho, C. C.; Shie, M. Y.; Chen, Y. W., 3d-Printed Bioactive Calcium Silicate/Poly-E-Caprolactone Bioscaffolds Modified with Biomimetic Extracellular Matrices for Bone Regeneration. International journal of molecular sciences 2019, 20 (4).

136. Pati, F.; Song, T. H.; Rijal, G.; Jang, J.; Kim, S. W.; Cho, D. W., Ornamenting 3d Printed Scaffolds with Cell-Laid Extracellular Matrix for Bone Tissue Regeneration. Biomaterials 2015, 37, $230-241$.

137. Li, J.; Chen, M.; Wei, X.; Hao, Y.; Wang, J., Evaluation of 3d-Printed Polycaprolactone Scaffolds Coated with Freeze-Dried Platelet-Rich Plasma for Bone Regeneration. Materials (Basel, Switzerland) 2017, 10 (7).

138. Eppley, B. L.; Woodell, J. E.; Higgins, J., Platelet Quantification and Growth Factor Analysis from PlateletRich Plasma: Implications for Wound Healing. Plastic and Reconstructive Surgery 2004, 114 (6). 
139. Alsousou, J.; Thompson, M.; Hulley, P.; Noble, A.; Willett, K., The Biology of Platelet-Rich Plasma and Its Application in Trauma and Orthopaedic Surgery. The Journal of bone and joint surgery. British volume 2009, 91-B (8), 987-996.

140. Nakajima, Y.; Kawase, T.; Kobayashi, M.; Okuda, K.; Wolff, L. F.; Yoshie, H., Bioactivity of Freeze-Dried Platelet-Rich Plasma in an Adsorbed Form on a Biodegradable Polymer Material. Platelets 2012, 23 (8), 594-603.

141. Jaidev, L. R.; Chatterjee, K., Surface Functionalization of 3d Printed Polymer Scaffolds to Augment Stem Cell Response. Materials \& Design 2019, 161, 44-54.

142. Jo, S.; Kang, S. M.; Park, S. A.; Kim, W. D.; Kwak, J.; Lee, H., Enhanced Adhesion of Preosteoblasts inside 3d Pcl Scaffolds by Polydopamine Coating and Mineralization. Macromolecular bioscience 2013, 13 (10), 1389-1395.

143. Ryu, J.; Ku, S. H.; Lee, H.; Park, C. B., Mussel-Inspired Polydopamine Coating as a Universal Route to Hydroxyapatite Crystallization. Advanced Functional Materials 2010, 20 (13), 2132-2139.

144. Murab, S.; Gruber, S. M. S.; Lin, C. J.; Whitlock, P., Elucidation of Bio-Inspired Hydroxyapatie Crystallization on Oxygen-Plasma Modified 3d Printed Poly-Caprolactone Scaffolds. Materials science \& engineering. C, Materials for biological applications 2020, 109, 110529.

145. Holmes, B.; Bulusu, K.; Plesniak, M.; Zhang, L. G., A Synergistic Approach to the Design, Fabrication and Evaluation of $3 d$ Printed Micro and Nano Featured Scaffolds for Vascularized Bone Tissue Repair. Nanotechnology 2016, 27 (6), 064001-064001.

146. Rhee, S.-H.; Tanaka, J., Effect of Citric Acid on the Nucleation of Hydroxyapatite in a Simulated Body Fluid. Biomaterials 1999, 20 (22), 2155-2160.

147. Hu, Y. Y.; Rawal, A.; Schmidt-Rohr, K., Strongly Bound Citrate Stabilizes the Apatite Nanocrystals in Bone. Proceedings of the National Academy of Sciences 2010, 107 (52), 22425.

148. Moroni, L.; Hamann, D.; Paoluzzi, L.; Pieper, J.; de Wijn, J. R.; van Blitterswijk, C. A., Regenerating Articular Tissue by Converging Technologies. PloS one 2008, 3 (8), e3032.

149. Nandakumar, A.; Cruz, C.; Mentink, A.; Tahmasebi Birgani, Z.; Moroni, L.; van Blitterswijk, C.; Habibovic, P., Monolithic and Assembled Polymer-Ceramic Composites for Bone Regeneration. Acta Biomaterialia 2013, 9 (3), 5708-5717.

150. Kim, J.; McBride, S.; Tellis, B.; Alvarez-Urena, P.; Song, Y.-H.; Dean, D. D.; Sylvia, V. L.; Elgendy, H.; Ong, J.; Hollinger, J. O., Rapid-Prototyped Plga/B-Tcp/Hydroxyapatite Nanocomposite Scaffolds in a Rabbit Femoral Defect Model. Biofabrication 2012, 4 (2), 025003.

151. Chim, H.; Hutmacher, D. W.; Chou, A. M.; Oliveira, A. L.; Reis, R. L.; Lim, T. C.; Schantz, J. T., A Comparative Analysis of Scaffold Material Modifications for Load-Bearing Applications in Bone Tissue Engineering. International Journal of Oral and Maxillofacial Surgery 2006, 35 (10), 928-934.

152. Maia-Pinto, M. O. C.; Brochado, A. C. B.; Teixeira, B. N.; Sartoretto, S. C.; Uzeda, M. J.; Alves, A.; Alves, G. G.; Calasans-Maia, M. D.; Thiré, R., Biomimetic Mineralization on 3d Printed Pla Scaffolds: On the Response of Human Primary Osteoblasts Spheroids and in Vivo Implantation. Polymers (Basel) 2020, 13 (1).

153. Abo-Aziza, F. A. M.; A A, Z., The Impact of Confluence on Bone Marrow Mesenchymal Stem (Bmmsc) Proliferation and Osteogenic Differentiation. Int J Hematol Oncol Stem Cell Res 2017, 11 (2), 121-132.

154. Zou, L.; Luo, Y.; Chen, M.; Wang, G.; Ding, M.; Petersen, C. C.; Kang, R.; Dagnaes-Hansen, F.; Zeng, Y.; Lv, N.; Ma, Q.; Le, D. Q.; Besenbacher, F.; Bolund, L.; Jensen, T. G.; Kjems, J.; Pu, W. T.; Bünger, C., A Simple Method for Deriving Functional Mscs and Applied for Osteogenesis in 3d Scaffolds. Sci Rep 2013, 3, 2243.

155. Jensen, J.; Kraft, D. C.; Lysdahl, H.; Foldager, C. B.; Chen, M.; Kristiansen, A. A.; Rölfing, J. H.; Bünger, C. E., Functionalization of Polycaprolactone Scaffolds with Hyaluronic Acid and B-Tcp Facilitates Migration and Osteogenic Differentiation of Human Dental Pulp Stem Cells in Vitro. Tissue engineering. Part A 2015, $21(3-4), 729-739$.

156. Chen, M.; Le, D. Q. S.; Baatrup, A.; Nygaard, J. V.; Hein, S.; Bjerre, L.; Kassem, M.; Zou, X.; Bünger, C., Self-Assembled Composite Matrix in a Hierarchical 3-D Scaffold for Bone Tissue Engineering. Acta Biomaterialia 2011, 7 (5), 2244-2255.

157. Park, S. H.; Kim, T. G.; Kim, H. C.; Yang, D.-Y.; Park, T. G., Development of Dual Scale Scaffolds Via Direct Polymer Melt Deposition and Electrospinning for Applications in Tissue Regeneration. Acta Biomaterialia 2008, 4 (5), 1198-1207. 
158. Lara-Padilla, H.; Mendoza-Buenrostro, C.; Cardenas, D.; Rodriguez-Garcia, A.; Rodriguez, C. A., Influence of Controlled Cooling in Bimodal Scaffold Fabrication Using Polymers with Different Melting Temperatures. Materials (Basel, Switzerland) 2017, 10 (6), 640.

159. Vyas, C.; Ates, G.; Aslan, E.; Hart, J.; Huang, B.; Bartolo, P., Three-Dimensional Printing and Electrospinning Dual-Scale Polycaprolactone Scaffolds with Low-Density and Oriented Fibers to Promote Cell Alignment. 3D printing and additive manufacturing 2020, 7 (3), 105-113.

160. Mota, C.; Puppi, D.; Dinucci, D.; Errico, C.; Bártolo, P.; Chiellini, F., Dual-Scale Polymeric Constructs as Scaffolds for Tissue Engineering. Materials (Basel, Switzerland) 2011, 4 (3), 527-542.

161. Nandakumar, A.; Barradas, A.; de Boer, J.; Moroni, L.; van Blitterswijk, C.; Habibovic, P., Combining Technologies to Create Bioactive Hybrid Scaffolds for Bone Tissue Engineering. Biomatter 2013, 3 (2), e23705.

162. Criscenti, G.; Longoni, A.; Di Luca, A.; De Maria, C.; van Blitterswijk, C. A.; Vozzi, G.; Moroni, L., Triphasic Scaffolds for the Regeneration of the Bone-Ligament Interface. Biofabrication 2016, 8 (1), 015009.

163. Mellor, L. F.; Nordberg, R. C.; Huebner, P.; Mohiti-Asli, M.; Taylor, M. A.; Efird, W.; Oxford, J. T.; Spang, J. T.; Shirwaiker, R. A.; Loboa, E. G., Investigation of Multiphasic 3d-Bioplotted Scaffolds for Site-Specific Chondrogenic and Osteogenic Differentiation of Human Adipose-Derived Stem Cells for Osteochondral Tissue Engineering Applications. Journal of biomedical materials research. Part B, Applied biomaterials 2020, 108 (5), 2017-2030.

164. Yang, G. H.; Kim, M.; Kim, G., A Hybrid Pcl/Collagen Scaffold Consisting of Solid Freeform-Fabricated Struts and Ehd-Direct-Jet-Processed Fibrous Threads for Tissue Regeneration. Journal of colloid and interface science 2015, 450, 159-167.

165. Kuss, M. A.; Wu, S.; Wang, Y.; Untrauer, J. B.; Li, W.; Lim, J. Y.; Duan, B., Prevascularization of 3d Printed Bone Scaffolds by Bioactive Hydrogels and Cell Co-Culture. Journal of biomedical materials research. Part B, Applied biomaterials 2018, 106 (5), 1788-1798.

166. Mekhileri, N. V.; Lim, K. S.; Brown, G. C. J.; Mutreja, I.; Schon, B. S.; Hooper, G. J.; Woodfield, T. B. F., Automated 3d Bioassembly of Micro-Tissues for Biofabrication of Hybrid Tissue Engineered Constructs. Biofabrication 2018, 10 (2), 024103.

167. Kirby, G. T. S.; White, L. J.; Steck, R.; Berner, A.; Bogoevski, K.; Qutachi, O.; Jones, B.; Saifzadeh, S.; Hutmacher, D. W.; Shakesheff, K. M.; Woodruff, M. A., Microparticles for Sustained Growth Factor Delivery in the Regeneration of Critically-Sized Segmental Tibial Bone Defects. Materials 2016, 9 (4).

168. Reichert, J. C.; Cipitria, A.; Epari, D. R.; Saifzadeh, S.; Krishnakanth, P.; Berner, A.; Woodruff, M. A.; Schell, H.; Mehta, M.; Schuetz, M. A.; Duda, G. N.; Hutmacher, D. W., A Tissue Engineering Solution for Segmental Defect Regeneration in Load-Bearing Long Bones. Science Translational Medicine 2012, 4 (141), 141ra193.

169. Berner, A.; Reichert, J. C.; Woodruff, M. A.; Saifzadeh, S.; Morris, A. J.; Epari, D. R.; Nerlich, M.; Schuetz, M. A.; Hutmacher, D. W., Autologous Vs. Allogenic Mesenchymal Progenitor Cells for the Reconstruction of Critical Sized Segmental Tibial Bone Defects in Aged Sheep. Acta Biomaterialia 2013, 9 (8), 7874-7884.

170. Schantz, J.-T.; Brandwood, A.; Hutmacher, D. W.; Khor, H. L.; Bittner, K., Osteogenic Differentiation of Mesenchymal Progenitor Cells in Computer Designed Fibrin-Polymer-Ceramic Scaffolds Manufactured by Fused Deposition Modeling. Journal of Materials Science: Materials in Medicine 2005, 16 (9), 807-819.

171. Wang, S.-J.; Zhang, Z.-Z.; Jiang, D.; Qi, Y.-S.; Wang, H.-J.; Zhang, J.-Y.; Ding, J.-X.; Yu, J.-K., ThermogelCoated Poly(E-Caprolactone) Composite Scaffold for Enhanced Cartilage Tissue Engineering. Polymers 2016, 8 (5), 200.

172. Heo, D. N.; Castro, N. J.; Lee, S.-J.; Noh, H.; Zhu, W.; Zhang, L. G., Enhanced Bone Tissue Regeneration Using a 3d Printed Microstructure Incorporated with a Hybrid Nano Hydrogel. Nanoscale 2017, 9 (16), 5055-5062.

173. Schuurman, W.; Khristov, V.; Pot, M. W.; van Weeren, P. R.; Dhert, W. J. A.; Malda, J., Bioprinting of Hybrid Tissue Constructs with Tailorable Mechanical Properties. Biofabrication 2011, 3 (2), 021001.

174. Pati, F.; Shim, J.-H.; Lee, J.-S.; Cho, D.-W., 3d Printing of Cell-Laden Constructs for Heterogeneous Tissue Regeneration. Manufacturing Letters 2013, 1 (1), 49-53.

175. Bae, E. B.; Park, K. H.; Shim, J. H.; Chung, H. Y.; Choi, J. W.; Lee, J. J.; Kim, C. H.; Jeon, H. J.; Kang, S. S.; Huh, J. B., Efficacy of Rhbmp-2 Loaded Pcl/B-Tcp/Bdecm Scaffold Fabricated by 3d Printing Technology on Bone Regeneration. BioMed research international 2018, 2018, 2876135. 
176. Pati, F.; Jang, J.; Ha, D.-H.; Won Kim, S.; Rhie, J.-W.; Shim, J.-H.; Kim, D.-H.; Cho, D.-W., Printing ThreeDimensional Tissue Analogues with Decellularized Extracellular Matrix Bioink. Nature communications 2014, 5, 3935-3935.

177. Shim, J. H.; Kim, S. E.; Park, J. Y.; Kundu, J.; Kim, S. W.; Kang, S. S.; Cho, D. W., Three-Dimensional Printing of Rhbmp-2-Loaded Scaffolds with Long-Term Delivery for Enhanced Bone Regeneration in a Rabbit Diaphyseal Defect. Tissue engineering. Part A 2014, 20 (13-14), 1980-1992.

178. Barradas, A.; Yuan, H.; van Blitterswijk, C. A.; Habibovic, P., Osteoinductive Biomaterials: Current Knowledge of Properties, Experimental Models and Biological Mechanisms. Eur Cell Mater 2011, 21 (407), 29.

179. Habraken, W.; Habibovic, P.; Epple, M.; Bohner, M., Calcium Phosphates in Biomedical Applications: Materials for the Future? Materials Today 2016, 19 (2), 69-87.

180. Galván-Chacón, V. P.; Habibovic, P., Deconvoluting the Bioactivity of Calcium Phosphate-Based Bone Graft Substitutes: Strategies to Understand the Role of Individual Material Properties. Advanced Healthcare Materials 2017, 6 (13), 1601478.

181. Roschger, P.; Fratzl, P.; Eschberger, J.; Klaushofer, K., Validation of Quantitative Backscattered Electron Imaging for the Measurement of Mineral Density Distribution in Human Bone Biopsies. Bone 1998, 23 (4), 319-326.

182. Bloebaum, R. D.; Skedros, J. G.; Vajda, E. G.; Bachus, K. N.; Constantz, B. R., Determining Mineral Content Variations in Bone Using Backscattered Electron Imaging. Bone 1997, 20 (5), 485-490.

183. Esposito Corcione, C.; Gervaso, F.; Scalera, F.; Padmanabhan, S. K.; Madaghiele, M.; Montagna, F.; Sannino, A.; Licciulli, A.; Maffezzoli, A., Highly Loaded Hydroxyapatite Microsphere/ Pla Porous Scaffolds Obtained by Fused Deposition Modelling. Ceramics International 2019, 45 (2, Part B), 2803-2810.

184. Jiang, W.; Shi, J.; Li, W.; Sun, K., Morphology, Wettability, and Mechanical Properties of Polycaprolactone/Hydroxyapatite Composite Scaffolds with Interconnected Pore Structures Fabricated by a Mini-Deposition System. Polymer Engineering \& Science 2012, 52 (11), 2396-2402.

185. Ding, C.; Qiao, Z.; Jiang, W.; Li, H.; Wei, J.; Zhou, G.; Dai, K., Regeneration of a Goat Femoral Head Using a Tissue-Specific, Biphasic Scaffold Fabricated with Cad/Cam Technology. Biomaterials 2013, 34 (28), 6706-6716.

186. Jiang, W.; Shi, J.; Li, W.; Sun, K., Three Dimensional Melt-Deposition of Polycaprolactone/Bio-Derived Hydroxyapatite Composite into Scaffold for Bone Repair. Journal of Biomaterials Science, Polymer Edition 2013, 24 (5), 539-550.

187. Yu, J.; Xu, Y.; Li, S.; Seifert, G. V.; Becker, M. L., Three-Dimensional Printing of Nano Hydroxyapatite/Poly(Ester Urea) Composite Scaffolds with Enhanced Bioactivity. Biomacromolecules 2017, 18 (12), 4171-4183.

188. Cho, Y. S.; Choi, S.; Lee, S.-H.; Kim, K. K.; Cho, Y.-S., Assessments of Polycaprolactone/Hydroxyapatite Composite Scaffold with Enhanced Biomimetic Mineralization by Exposure to Hydroxyapatite Via a 3dPrinting System and Alkaline Erosion. European Polymer Journal 2019, 113, 340-348.

189. Cho, Y. S.; Quan, M.; Lee, S.-H.; Hong, M. W.; Kim, Y. Y.; Cho, Y.-S., Assessment of Osteogenesis for 3d-Printed Polycaprolactone/Hydroxyapatite Composite Scaffold with Enhanced Exposure of Hydroxyapatite Using Rat Calvarial Defect Model. Composites Science and Technology 2019, 184, 107844.

190. Barradas, A. M.; Monticone, V.; Hulsman, M.; Danoux, C.; Fernandes, H.; Tahmasebi Birgani, Z.; Barrerede Groot, F.; Yuan, H.; Reinders, M.; Habibovic, P.; van Blitterswijk, C.; de Boer, J., Molecular Mechanisms of Biomaterial-Driven Osteogenic Differentiation in Human Mesenchymal Stromal Cells. Integrative biology : quantitative biosciences from nano to macro 2013, 5 (7), 920-931.

191. Habibovic, P.; Yuan, H.; van der Valk, C. M.; Meijer, G.; van Blitterswijk, C. A.; de Groot, K., 3d Microenvironment as Essential Element for Osteoinduction by Biomaterials. Biomaterials 2005, 26 (17), 3565-3575.

192. Danoux, C. B. S. S.; Bassett, D. C.; Othman, Z.; Rodrigues, A. I.; Reis, R. L.; Barralet, J. E.; van Blitterswijk, C. A.; Habibovic, P., Elucidating the Individual Effects of Calcium and Phosphate lons on Hmscs by Using Composite Materials. Acta Biomaterialia 2015, 17, 1-15.

193. Nandakumar, A.; Cruz, C.; Mentink, A.; Birgani, Z. T.; Moroni, L.; van Blitterswijk, C.; Habibovic, P., Monolithic and Assembled Polymer-Ceramic Composites for Bone Regeneration. Acta biomaterialia 2013, 9 (3), 5708-5717. 
194. Moncal, K. K.; Heo, D. N.; Godzik, K. P.; Sosnoski, D. M.; Mrowczynski, O. D.; Rizk, E.; Ozbolat, V.; Tucker, S. M.; Gerhard, E. M.; Dey, M., 3d Printing of Poly (E-Caprolactone)/Poly (D, L-Lactide-CoGlycolide)/Hydroxyapatite Composite Constructs for Bone Tissue Engineering. Journal of Materials Research 2018, 33 (14), 1972-1986.

195. Chen, X.; Gao, C.; Jiang, J.; Wu, Y.; Zhu, P.; Chen, G., 3d Printed Porous Pla/Nha Composite Scaffolds with Enhanced Osteogenesis and Osteoconductivity in Vivo for Bone Regeneration. Biomedical Materials 2019, 14 (6), 065003

196. Zhang, H.; Mao, X.; Du, Z.; Jiang, W.; Han, X.; Zhao, D.; Han, D.; Li, Q., Three Dimensional Printed Macroporous Polylactic Acid/Hydroxyapatite Composite Scaffolds for Promoting Bone Formation in a Critical-Size Rat Calvarial Defect Model. Science and technology of advanced materials 2016, 17 (1), 136 148.

197. Nyberg, E.; Rindone, A.; Dorafshar, A.; Grayson, W. L., Comparison of 3d-Printed Poly-E-Caprolactone Scaffolds Functionalized with Tricalcium Phosphate, Hydroxyapatite, Bio-Oss, or Decellularized Bone Matrix. Tissue Engineering Part A 2016, 23 (11-12), 503-514.

198. Huang, B.; Caetano, G.; Vyas, C.; Blaker, J. J.; Diver, C.; Bártolo, P., Polymer-Ceramic Composite Scaffolds: The Effect of Hydroxyapatite and B-Tri-Calcium Phosphate. Materials (Basel, Switzerland) 2018, 11 (1).

199. Dávila, J.; Freitas, M.; Inforçatti Neto, P.; Silveira, Z.; Silva, J.; d’Ávila, M., Fabrication of Pcl/B-Tcp Scaffolds by 3d Mini-Screw Extrusion Printing. Journal of Applied Polymer Science 2016, 133 (15).

200. Bruyas, A.; Lou, F.; Stahl, A. M.; Gardner, M.; Maloney, W.; Goodman, S.; Yang, Y. P., Systematic Characterization of 3d-Printed Pcl/B-Tcp Scaffolds for Biomedical Devices and Bone Tissue Engineering: Influence of Composition and Porosity. J Mater Res 2018, 33 (14), 1948-1959.

201. Park, J.; Lee, S. J.; Jo, H. H.; Lee, J. H.; Kim, W. D.; Lee, J. Y.; Park, S. A., Fabrication and Characterization of 3d-Printed Bone-Like B-Tricalcium Phosphate/Polycaprolactone Scaffolds for Dental Tissue Engineering. Journal of Industrial and Engineering Chemistry 2017, 46, 175-181.

202. Kim, J. Y.; Ahn, G.; Kim, C.; Lee, J. S.; Lee, I. G.; An, S. H.; Yun, W. S.; Kim, S. Y.; Shim, J. H., Synergistic Effects of Beta Tri-Calcium Phosphate and Porcine-Derived Decellularized Bone Extracellular Matrix in 3d-Printed Polycaprolactone Scaffold on Bone Regeneration. Macromolecular bioscience 2018, 18 (6), e1800025.

203. Yuan, H.; Fernandes, H.; Habibovic, P.; de Boer, J.; Barradas, A. M. C.; de Ruiter, A.; Walsh, W. R.; van Blitterswijk, C. A.; de Bruijn, J. D., Osteoinductive Ceramics as a Synthetic Alternative to Autologous Bone Grafting. Proc Natl Acad Sci U S A 2010, 107 (31), 13614-13619.

204. Yeo, A.; Sju, E.; Rai, B.; Teoh, S. H., Customizing the Degradation and Load-Bearing Profile of 3d Polycaprolactone-Tricalcium Phosphate Scaffolds under Enzymatic and Hydrolytic Conditions. Journal of Biomedical Materials Research Part B: Applied Biomaterials 2008, 87B (2), 562-569.

205. Lam, C. X. F.; Hutmacher, D. W.; Schantz, J.-T.; Woodruff, M. A.; Teoh, S. H., Evaluation of Polycaprolactone Scaffold Degradation for 6 Months in Vitro and in Vivo. Journal of Biomedical Materials Research Part A 2009, 90A (3), 906-919.

206. Yeo, A.; Wong, W. J.; Khoo, H. H.; Teoh, S. H., Surface Modification of Pcl-Tcp Scaffolds Improve Interfacial Mechanical Interlock and Enhance Early Bone Formation: An in Vitro and in Vivo Characterization. Journal of Biomedical Materials Research Part A 2010, 92A (1), 311-321.

207. Yeo, A.; Wong, W. J.; Teoh, S. H., Surface Modification of Pcl-Tcp Scaffolds in Rabbit Calvaria Defects: Evaluation of Scaffold Degradation Profile, Biomechanical Properties and Bone Healing Patterns. J Biomed Mater Res A 2010, 93 (4), 1358-1367.

208. Kim, J. Y.; Yoon, J. J.; Park, E. K.; Kim, D. S.; Kim, S.-Y.; Cho, D.-W., Cell Adhesion and Proliferation Evaluation of Sff-Based Biodegradable Scaffolds Fabricated Using a Multi-Head Deposition System. Biofabrication 2009, 1 (1), 015002.

209. Shim, J.-H.; Moon, T.-S.; Yun, M.-J.; Jeon, Y.-C.; Jeong, C.-M.; Cho, D.-W.; Huh, J.-B., Stimulation of Healing within a Rabbit Calvarial Defect by a Pcl/PIga Scaffold Blended with Tcp Using Solid Freeform Fabrication Technology. Journal of Materials Science: Materials in Medicine 2012, 23 (12), 2993-3002.

210. Idaszek, J.; Bruinink, A.; Święszkowski, W., Ternary Composite Scaffolds with Tailorable Degradation Rate and Highly Improved Colonization by Human Bone Marrow Stromal Cells. Journal of Biomedical Materials Research Part A 2015, 103 (7), 2394-2404.

211. Meleti, Z.; Shapiro, I.; Adams, C. S., Inorganic Phosphate Induces Apoptosis of Osteoblast-Like Cells in Culture. Bone 2000, 27 (3), 359-366. 
212. Bittner, S. M.; Smith, B. T.; Diaz-Gomez, L.; Hudgins, C. D.; Melchiorri, A. J.; Scott, D. W.; Fisher, J. P.; Mikos, A. G., Fabrication and Mechanical Characterization of 3d Printed Vertical Uniform and Gradient Scaffolds for Bone and Osteochondral Tissue Engineering. Acta biomaterialia 2019, 90, 37-48.

213. Smith, B. T.; Bittner, S. M.; Watson, E.; Smoak, M. M.; Diaz-Gomez, L.; Molina, E. R.; Kim, Y. S.; Hudgins, C. D.; Melchiorri, A. J.; Scott, D. W., Multimaterial Dual Gradient Three-Dimensional Printing for Osteogenic Differentiation and Spatial Segregation. Tissue Engineering Part A 2020, 26 (5-6), 239-252.

214. Sinha, R.; Cámara-Torres, M.; Scopece, P.; Falzacappa, E. V.; Patelli, A.; Moroni, L.; Mota, C., A Hybrid Additive Manufacturing Platform to Create Bulk and Surface Composition Gradients on Scaffolds for Tissue Regeneration. bioRxiv 2020, 2020.2006.2023.165605.

215. Diaz-Gomez, L.; Kontoyiannis, P. D.; Melchiorri, A. J.; Mikos, A. G., Three-Dimensional Printing of Tissue Engineering Scaffolds with Horizontal Pore and Composition Gradients. Tissue Eng Part C Methods 2019, 25 (7), 411-420.

216. Diaz-Gomez, L.; Smith, B. T.; Kontoyiannis, P. D.; Bittner, S. M.; Melchiorri, A. J.; Mikos, A. G., Multimaterial Segmented Fiber Printing for Gradient Tissue Engineering. Tissue Eng Part C Methods 2019, $25(1), 12-24$

217. Gaharwar, A. K.; Rivera, C. P.; Wu, C.-J.; Schmidt, G., Transparent, Elastomeric and Tough Hydrogels from Poly (Ethylene Glycol) and Silicate Nanoparticles. Acta biomaterialia 2011, 7 (12), 4139-4148.

218. Wang, C.; Wang, S.; Li, K.; Ju, Y.; Li, J.; Zhang, Y.; Li, J.; Liu, X.; Shi, X.; Zhao, Q., Preparation of Laponite Bioceramics for Potential Bone Tissue Engineering Applications. PloS one 2014, 9 (6), e99585.

219. Shie, M. Y.; Ding, S. J., Integrin Binding and Mapk Signal Pathways in Primary Cell Responses to Surface Chemistry of Calcium Silicate Cements. Biomaterials 2013, 34 (28), 6589-6606.

220. Lin, Y. H.; Chiu, Y. C.; Shen, Y. F.; Wu, Y. A.; Shie, M. Y., Bioactive Calcium Silicate/Poly-E-Caprolactone Composite Scaffolds 3d Printed under Mild Conditions for Bone Tissue Engineering. Journal of materials science. Materials in medicine 2017, $29(1), 11$.

221. Zhang, Y.; Yu, W.; Ba, Z.; Cui, S.; Wei, J.; Li, H., 3d-Printed Scaffolds of Mesoporous Bioglass/Gliadin/Polycaprolactone Ternary Composite for Enhancement of Compressive Strength, Degradability, Cell Responses and New Bone Tissue Ingrowth. International journal of nanomedicine 2018, 13, 5433-5447.

222. Alksne, M.; Kalvaityte, M.; Simoliunas, E.; Rinkunaite, I.; Gendviliene, I.; Locs, J.; Rutkunas, V.; Bukelskiene, V., In Vitro Comparison of 3d Printed Polylactic Acid/Hydroxyapatite and Polylactic Acid/Bioglass Composite Scaffolds: Insights into Materials for Bone Regeneration. Journal of the Mechanical Behavior of Biomedical Materials 2020, 104, 103641.

223. Poh, P. S. P.; Hutmacher, D. W.; Holzapfel, B. M.; Solanki, A. K.; Stevens, M. M.; Woodruff, M. A., In Vitro and in Vivo Bone Formation Potential of Surface Calcium Phosphate-Coated Polycaprolactone and Polycaprolactone/Bioactive Glass Composite Scaffolds. Acta Biomater 2016, 30, 319-333.

224. Marie, P. J.; Ammann, P.; Boivin, G.; Rey, C., Mechanisms of Action and Therapeuticpotential of Strontium in Bone. Calcified Tissue International 2001, 69 (3), 121-129.

225. Poh, P. S. P.; Hutmacher, D. W.; Stevens, M. M.; Woodruff, M. A., Fabrication and in Vitro Characterization of Bioactive Glass Composite Scaffolds for Bone Regeneration. Biofabrication 2013, 5 (4), 045005.

226. Zhou, X.; Zhang, N.; Mankoci, S.; Sahai, N., Silicates in Orthopedics and Bone Tissue Engineering Materials. Journal of Biomedical Materials Research Part A 2017, 105 (7), 2090-2102.

227. Sidambe, A. T., Biocompatibility of Advanced Manufactured Titanium Implants—a Review. Materials 2014, 7 (12), 8168-8188.

228. Schröder, C.; Steinbrück, A.; Müller, T.; Woiczinski, M.; Chevalier, Y.; Weber, P.; Müller, P. E.; Jansson, V., Rapid Prototyping for <l>in Vitro</l> Knee Rig Investigations of Prosthetized Knee Biomechanics: Comparison with Cobalt-Chromium Alloy Implant Material. BioMed research international 2015, 2015, 185142.

229. Li, L.; Gao, J.; Wang, Y., Evaluation of Cyto-Toxicity and Corrosion Behavior of Alkali-Heat-Treated Magnesium in Simulated Body Fluid. Surface and Coatings Technology 2004, 185 (1), 92-98.

230. Rude, R. K.; Gruber, H. E.; Norton, H. J.; Wei, L. Y.; Frausto, A.; Kilburn, J., Dietary Magnesium Reduction to $25 \%$ of Nutrient Requirement Disrupts Bone and Mineral Metabolism in the Rat. Bone 2005, 37 (2), 211 219. 
231. Wong, H. M.; Wu, S.; Chu, P. K.; Cheng, S. H.; Luk, K. D. K.; Cheung, K. M. C.; Yeung, K. W. K., LowModulus Mg/Pcl Hybrid Bone Substitute for Osteoporotic Fracture Fixation. Biomaterials 2013, 34 (29), 7016-7032.

232. Zberg, B.; Uggowitzer, P. J.; Löffler, J. F., Mgznca Glasses without Clinically Observable Hydrogen Evolution for Biodegradable Implants. Nature Materials 2009, 8 (11), 887-891.

233. Abdal-hay, A.; Raveendran, N. T.; Fournier, B.; Ivanovski, S., Fabrication of Biocompatible and Bioabsorbable Polycaprolactone/ Magnesium Hydroxide 3d Printed Scaffolds: Degradation and in Vitro Osteoblasts Interactions. Composites Part B: Engineering 2020, 197, 108158.

234. Shen, J.; Wang, W.; Zhai, X.; Chen, B.; Qiao, W.; Li, W.; Li, P.; Zhao, Y.; Meng, Y.; Qian, S.; Liu, X.; Chu, P. K.; Yeung, K. W. K., 3d-Printed Nanocomposite Scaffolds with Tunable Magnesium Ionic Microenvironment Induce in Situ Bone Tissue Regeneration. Applied Materials Today 2019, 16, 493-507.

235. Hanßke, F.; Bas, O.; Vaquette, C.; Hochleitner, G.; Groll, J.; Kemnitz, E.; Hutmacher, D. W.; Börner, H. G., Via Precise Interface Engineering Towards Bioinspired Composites with Improved 3d Printing Processability and Mechanical Properties. Journal of Materials Chemistry B 2017, 5 (25), 5037-5047.

236. Bas, O.; Hanßke, F.; Lim, J.; Ravichandran, A.; Kemnitz, E.; Teoh, S.-H.; Hutmacher, D. W.; Börner, H. G., Tuning Mechanical Reinforcement and Bioactivity of 3d Printed Ternary Nanocomposites by Interfacial Peptide-Polymer Conjugates. Biofabrication 2019, 11 (3), 035028.

237. Robinson, C.; Kirkham, J., The Effect of Fluoride on the Developing Mineralized Tissues. Journal of Dental Research 1990, 69 (2_suppl), 685-691.

238. Radhakrishnan, S.; Nagarajan, S.; Belaid, H.; Farha, C.; latsunskyi, I.; Coy, E.; Soussan, L.; Huon, V.; Bares, J.; Belkacemi, K.; Teyssier, C.; Balme, S.; Miele, P.; Cornu, D.; Kalkura, N.; Cavaillès, V.; Bechelany, M., Fabrication of 3d Printed Antimicrobial Polycaprolactone Scaffolds for Tissue Engineering Applications. Materials Science and Engineering: C 2021, 118, 111525.

239. Wang, W.; Junior, J. R. P.; Nalesso, P. R. L.; Musson, D.; Cornish, J.; Mendonça, F.; Caetano, G. F.; Bártolo, P., Engineered 3d Printed Poly $(\mathcal{E}$-Caprolactone)/Graphene Scaffolds for Bone Tissue Engineering. Materials Science and Engineering: C 2019, 100, 759-770.

240. Shim, J.-H.; Kim, M.-J.; Park, J. Y.; Pati, R. G.; Yun, Y.-P.; Kim, S. E.; Song, H.-R.; Cho, D.-W., ThreeDimensional Printing of Antibiotics-Loaded Poly-E-Caprolactone/Poly(Lactic-Co-Glycolic Acid) Scaffolds for Treatment of Chronic Osteomyelitis. Tissue Engineering and Regenerative Medicine 2015, 12 (5), 283293.

241. Gu, M.; Liu, Y.; Chen, T.; Du, F.; Zhao, X.; Xiong, C.; Zhou, Y., Is Graphene a Promising Nano-Material for Promoting Surface Modification of Implants or Scaffold Materials in Bone Tissue Engineering? Tissue Engineering. Part B, Reviews 2014, 20 (5), 477-491.

242. Eivazzadeh-Keihan, R.; Maleki, A.; de la Guardia, M.; Bani, M. S.; Chenab, K. K.; Pashazadeh-Panahi, P.; Baradaran, B.; Mokhtarzadeh, A.; Hamblin, M. R., Carbon Based Nanomaterials for Tissue Engineering of Bone: Building New Bone on Small Black Scaffolds: A Review. Journal of Advanced Research 2019, 18, 185-201.

243. Tasis, D.; Tagmatarchis, N.; Bianco, A.; Prato, M., Chemistry of Carbon Nanotubes. Chemical Reviews 2006, 106 (3), 1105-1136.

244. Li, Z.; Wang, L.; Li, Y.; Feng, Y.; Feng, W., Carbon-Based Functional Nanomaterials: Preparation, Properties and Applications. Composites Science and Technology 2019, 179, 10-40.

245. Huang, B.; Vyas, C.; Roberts, I.; Poutrel, Q. A.; Chiang, W. H.; Blaker, J. J.; Huang, Z.; Bártolo, P., Fabrication and Characterisation of 3d Printed Mwcnt Composite Porous Scaffolds for Bone Regeneration. Materials science \& engineering. C, Materials for biological applications 2019, 98, 266-278.

246. Huang, B.; Vyas, C.; Byun, J. J.; El-Newehy, M.; Huang, Z.; Bártolo, P., Aligned Multi-Walled Carbon Nanotubes with Nanohydroxyapatite in a 3d Printed Polycaprolactone Scaffold Stimulates Osteogenic Differentiation. Materials science \& engineering. C, Materials for biological applications 2020, 108, 110374.

247. Wang, W.; Huang, B.; Byun, J. J.; Bártolo, P., Assessment of Pcl/Carbon Material Scaffolds for Bone Regeneration. Journal of the mechanical behavior of biomedical materials 2019, 93, 52-60.

248. Wang, W.; Caetano, G. F.; Chiang, W.-H.; Braz, A. L.; Blaker, J. J.; Frade, M. A. C.; Bartolo, P. J. D. S., Morphological, Mechanical and Biological Assessment of Pcl/Pristine Graphene Scaffolds for Bone Regeneration. International Journal of Bioprinting 2016, 2 (2). 
249. Wang, W.; Caetano, G.; Ambler, W. S.; Blaker, J. J.; Frade, M. A.; Mandal, P.; Diver, C.; Bartolo, P., Enhancing the Hydrophilicity and Cell Attachment of 3d Printed Pcl/Graphene Scaffolds for Bone Tissue Engineering. Materials (Basel, Switzerland) 2016, 9 (12).

250. Caetano, G. F.; Wang, W.; Chiang, W.-H.; Cooper, G.; Diver, C.; Blaker, J. J.; Frade, M. A.; Bártolo, P., 3d-Printed Poly(E-Caprolactone)/Graphene Scaffolds Activated with P1-Latex Protein for Bone Regeneration. 3D printing and additive manufacturing 2018, 5 (2), 127-137.

251. Akhavan, O.; Ghaderi, E.; Akhavan, A., Size-Dependent Genotoxicity of Graphene Nanoplatelets in Human Stem Cells. Biomaterials 2012, 33 (32), 8017-8025.

252. Dreyer, D. R.; Park, S.; Bielawski, C. W.; Ruoff, R. S., The Chemistry of Graphene Oxide. Chemical Society Reviews 2010, 39 (1), 228-240.

253. Kuila, T.; Bose, S.; Mishra, A. K.; Khanra, P.; Kim, N. H.; Lee, J. H., Chemical Functionalization of Graphene and Its Applications. Progress in Materials Science 2012, 57 (7), 1061-1105.

254. Unagolla, J. M.; Jayasuriya, A. C., Enhanced Cell Functions on Graphene Oxide Incorporated 3d Printed Polycaprolactone Scaffolds. Materials Science and Engineering: C 2019, 102, 1-11.

255. Belaid, H.; Nagarajan, S.; Teyssier, C.; Barou, C.; Barés, J.; Balme, S.; Garay, H.; Huon, V.; Cornu, D.; Cavaillès, V.; Bechelany, M., Development of New Biocompatible 3d Printed Graphene Oxide-Based Scaffolds. Materials Science and Engineering: C 2020, 110, 110595.

256. Chen, Q.; Mangadlao, J. D.; Wallat, J.; De Leon, A.; Pokorski, J. K.; Advincula, R. C., 3d Printing Biocompatible Polyurethane/Poly(Lactic Acid)/Graphene Oxide Nanocomposites: Anisotropic Properties. ACS Applied Materials \& Interfaces 2017, 9 (4), 4015-4023.

257. Pei, S.; Cheng, H.-M., The Reduction of Graphene Oxide. Carbon 2012, 50 (9), 3210-3228.

258. Angulo-Pineda, C.; Srirussamee, K.; Palma, P.; Fuenzalida, V. M.; Cartmell, S. H.; Palza, H., Electroactive 3d Printed Scaffolds Based on Percolated Composites of Polycaprolactone with Thermally Reduced Graphene Oxide for Antibacterial and Tissue Engineering Applications. Nanomaterials 2020, 10 (3), 428.

259. McAllister, M. J.; Li, J.-L.; Adamson, D. H.; Schniepp, H. C.; Abdala, A. A.; Liu, J.; Herrera-Alonso, M.; Milius, D. L.; Car, R.; Prud'homme, R. K.; Aksay, I. A., Single Sheet Functionalized Graphene by Oxidation and Thermal Expansion of Graphite. Chemistry of Materials 2007, 19 (18), 4396-4404.

260. Pinto, A. M.; Goncalves, I. C.; Magalhaes, F. D., Graphene-Based Materials Biocompatibility: A Review. Colloids and Surfaces B: Biointerfaces 2013, 111, 188-202.

261. Reina, G.; González-Domínguez, J. M.; Criado, A.; Vázquez, E.; Bianco, A.; Prato, M., Promises, Facts and Challenges for Graphene in Biomedical Applications. Chemical Society Reviews 2017, 46 (15), 44004416.

262. Ma, Y.; Shen, H.; Tu, X.; Zhang, Z., Assessing in Vivo Toxicity of Graphene Materials: Current Methods and Future Outlook. Nanomedicine 2014, 9 (10), 1565-1580.

263. Traub, W. H.; Leonhard, B., Heat Stability of the Antimicrobial Activity of Sixty-Two Antibacterial Agents. Journal of Antimicrobial Chemotherapy 1995, 35 (1), 149-154.

264. Lee, J.-H.; Baik, J.-M.; Yu, Y.-S.; Kim, J. H.; Ahn, C. B.; Son, K. H.; Kim, J.-H.; Choi, E. S.; Lee, J. W., Development of a Heat Labile Antibiotic Eluting 3d Printed Scaffold for the Treatment of Osteomyelitis. Scientific Reports 2020, 10 (1), 7554.

265. Costa, P. F.; Puga, A. M.; Díaz-Gomez, L.; Concheiro, A.; Busch, D. H.; Alvarez-Lorenzo, C., Additive Manufacturing of Scaffolds with Dexamethasone Controlled Release for Enhanced Bone Regeneration. International journal of pharmaceutics 2015, 496 (2), 541-550.

266. Water, J. J.; Bohr, A.; Boetker, J.; Aho, J.; Sandler, N.; Nielsen, H. M.; Rantanen, J., Three-Dimensional Printing of Drug-Eluting Implants: Preparation of an Antimicrobial Polylactide Feedstock Material. Journal of pharmaceutical sciences 2015, 104 (3), 1099-1107.

267. Kempin, W.; Franz, C.; Koster, L.-C.; Schneider, F.; Bogdahn, M.; Weitschies, W.; Seidlitz, A., Assessment of Different Polymers and Drug Loads for Fused Deposition Modeling of Drug Loaded Implants. European Journal of Pharmaceutics and Biopharmaceutics 2017, 115, 84-93.

268. Teo, E. Y.; Ong, S.-Y.; Khoon Chong, M. S.; Zhang, Z.; Lu, J.; Moochhala, S.; Ho, B.; Teoh, S.-H., Polycaprolactone-Based Fused Deposition Modeled Mesh for Delivery of Antibacterial Agents to Infected Wounds. Biomaterials 2011, 32 (1), 279-287. 
269. Dong, J.; Li, M.; Zhou, L.; Lee, S.; Mei, C.; Xu, X.; Wu, Q., The Influence of Grafted Cellulose Nanofibers and Postextrusion Annealing Treatment on Selected Properties of Poly(Lactic Acid) Filaments for 3d Printing. Journal of Polymer Science Part B: Polymer Physics 2017, 55 (11), 847-855.

270. Alemán-Domínguez, M. E.; Giusto, E.; Ortega, Z.; Tamaddon, M.; Benítez, A. N.; Liu, C., ThreeDimensional Printed Polycaprolactone-Microcrystalline Cellulose Scaffolds. Journal of biomedical materials research. Part B, Applied biomaterials 2019, 107 (3), 521-528.

271. Kuhnt, T.; Camarero-Espinosa, S., Additive Manufacturing of Nanocellulose Based Scaffolds for Tissue Engineering: Beyond a Reinforcement Filler. Carbohydrate Polymers 2021, 252, 117159.

272. Vyas, C.; Zhang, J.; Øvrebø, Ø.; Huang, B.; Roberts, I.; Setty, M.; Allardyce, B.; Haugen, H.; Rajkhowa, R.; Bartolo, P., 3d Printing of Silk Microparticle Reinforced Polycaprolactone Scaffolds for Tissue Engineering Applications. Materials Science and Engineering: C 2021, 118, 111433.

273. Wibowo, A.; Vyas, C.; Cooper, G.; Qulub, F.; Suratman, R.; Mahyuddin, A. I.; Dirgantara, T.; Bartolo, P., 3d Printing of Polycaprolactone-Polyaniline Electroactive Scaffolds for Bone Tissue Engineering. Materials (Basel, Switzerland) 2020, 13 (3), 512.

274. Camarero-Espinosa, S.; Moroni, L., Janus 3d Printed Dynamic Scaffolds for Nanodeflection-Driven Bone Regeneration. 2020.

275. McDermott, A. M.; Herberg, S.; Mason, D. E.; Collins, J. M.; Pearson, H. B.; Dawahare, J. H.; Tang, R.; Patwa, A. N.; Grinstaff, M. W.; Kelly, D. J.; Alsberg, E.; Boerckel, J. D., Recapitulating Bone Development through Engineered Mesenchymal Condensations and Mechanical Cues for Tissue Regeneration. Science Translational Medicine 2019, 11 (495), eaav7756.

276. Ciombor, D. M.; Aaron, R. K., The Role of Electrical Stimulation in Bone Repair. Foot and ankle clinics 2005, 10 (4), 579-593, vii.

277. Hendrikson, W. J.; Deegan, A. J.; Yang, Y.; van Blitterswijk, C. A.; Verdonschot, N.; Moroni, L.; Rouwkema, J., Influence of Additive Manufactured Scaffold Architecture on the Distribution of Surface Strains and Fluid Flow Shear Stresses and Expected Osteochondral Cell Differentiation. Frontiers in Bioengineering and Biotechnology 2017, 5 (6).

278. Hendrikson, W. J.; van Blitterswijk, C. A.; Rouwkema, J.; Moroni, L., The Use of Finite Element Analyses to Design and Fabricate Three-Dimensional Scaffolds for Skeletal Tissue Engineering. Front Bioeng Biotechnol 2017, 5, 30.

279. Freeman, F. E.; Pitacco, P.; van Dommelen, L. H. A.; Nulty, J.; Browe, D. C.; Shin, J.-Y.; Alsberg, E.; Kelly, D. J., 3d Bioprinting Spatiotemporally Defined Patterns of Growth Factors to Tightly Control Tissue Regeneration. Science Advances 2020, 6 (33), eabb5093. 


\section{Chapter 3}

Improving cell distribution on 3D additive manufactured scaffolds through engineered seeding media density and viscosity 



\title{
Improving cell distribution on 3D additive manufactured scaffolds through engineered seeding media density and viscosity
}

\author{
Maria Cámara-Torres ${ }^{1}$, Ravi Sinha ${ }^{1}$, Carlos Mota $^{1}$, Lorenzo Moroni $^{1}$ \\ Maastricht University, MERLN Institute for Technology-Inspired Regenerative Medicine, \\ Complex Tissue Regeneration Department, Maastricht, The Netherlands.
}

\begin{abstract}
In order to ensure the long-term in vitro and in vivo functionality of cell-seeded 3D scaffolds, an effective and reliable method to control cell seeding efficiency and distribution is crucial. Static seeding on 3D additive manufactured (AM) scaffolds made of synthetic polymers still remains challenging, as it often results in poor cell attachment, high cell sedimentation and non-uniform cell distribution, due to gravity and to the intrinsic macroporosity and surface chemical properties of the scaffolds. In this study, the biocompatible macromolecules dextran and Ficoll (Ficoll Paque) were used for the first time as temporary supplements to alter the viscosity and density of the seeding media, respectively, and improve the static seeding output. The addition of these macromolecules drastically reduced the cell sedimentation velocities, allowing for homogeneous cell attachment to the scaffold filaments. Both dextran- and Ficoll -based seeding methods supported human mesenchymal stromal cells viability and osteogenic differentiation post-seeding. Interestingly, the improved cell distribution led to increased matrix production and mineralization compared to scaffolds seeded by conventional static method. These results suggest a simple and universal method for an efficient seeding of 3D AM scaffolds, independent of their material and geometrical properties, and applicable for bone and various other tissue regeneration.
\end{abstract}




\section{INTRODUCTION}

In vivo, cells are surrounded by a complex 3D microenvironment that supports their growth and assembly into functional tissues. Upon tissue damage caused by trauma or disease, the structural integrity of this extracellular matrix (ECM) can be compromised leading to lack of essential cues for functional tissue performance, and the requirement of suitable replacements. By using scaffolds, tissue engineering and regenerative medicine (TERM) aim to provide artificial ECM-like structures, which try to mimic the native tissue in terms of architecture, chemistry or mechanical properties to guide cells into the first steps of the regeneration process. ${ }^{1-2}$ To allow cell infiltration, tissue ingrowth and vascularization, 3D scaffolds with a highly interconnected porous structure are required. ${ }^{3-4}$ In the past decade, additive manufacturing ( $A M$ ) and, more specifically, melt extrusion (ME)-based techniques, have emerged as one of the most appealing technologies to produce scaffolds for TERM. ${ }^{5-6}$ Compared to other conventional methods such as gas foaming/particulate leaching, ${ }^{7-8}$ freeze-drying ${ }^{9-10}$ or phase separation, ${ }^{11-12}$ AM allows the reproducible fabrication of complex 3D structures with interconnected, high porosity, from various types of biocompatible and biodegradable polymers with optimum mechanical properties desired for tissue regeneration applications.

Efficient cell seeding of 3D scaffolds is a key step towards the development of in vitro tissues for TERM applications. Cell density and their spatial distribution in macroporous scaffolds are critical to ensure the functionality of the engineered tissue, as these parameters will dictate intercellular and cell-material interactions, thereby affecting different cellular behaviors such as migration, proliferation, differentiation and matrix production. ${ }^{13-14}$ To improve cell distribution within 3D scaffolds, different dynamic seeding methods have been applied. Compared to static seeding, dynamic seeding methods, which apply an external force to facilitate cell penetration, such as flow perfusion, centrifugation, orbital shaking, and spinner flask, have shown to result in better cell distribution. 15-19 Moreover, culture of 3D scaffolds, fabricated with conventional techniques, in perfusion bioreactors after static seeding has also demonstrated to enhance cell distribution and tissue formation. ${ }^{20}$ However the use of bioreactors require specific lab equipment and expertise and perfusion flow right after seeding results in significant decrease in cell number in 3D AM scaffolds. ${ }^{21}$ Overall, due to the intrinsic scaffold characteristics, i.e. large and interconnected pore architectures, most of the dynamic techniques show limited applicability for seeding or culture AM scaffolds. Furthermore, the 
lack of specific cell adhesion sites in the majority of the polymers suitable for AM leads to poor cell-material interactions, which along with the gravitational force results in cell sedimentation during static seeding and, therefore, non-uniform cell distribution within the scaffold. Nevertheless, the simplicity of the static seeding over dynamic seeding has led to further developments of this method for seeding 3D AM scaffolds, starting by the modification of basic seeding parameters such as cell number, seeding volume, seeding time and seeding vessel optimization. For instance, seeding times up to $4 \mathrm{~h}$, seeding volumes comparable to the scaffold's free volume and not very large cell numbers have shown to give the highest cell seeding efficiency upon static seeding. ${ }^{22-24}$ On the other hand, different surface modification techniques capable of improving the chemical and biological performance of synthetic polymers have also shown to increase cell adhesion in 3D AM scaffolds. For example, plasma modification using oxygen ${ }^{25}$ or nitrogen ${ }^{26-27}$ have shown to increase cell adhesion of osteoblastic cell lines in poly( $\varepsilon$-caprolactone) (PCL) scaffolds. Chondrocytes adhesion on poly(ethylene oxide terephthalate)/poly(butylene terephthalate) (PEOT/PBT) 3D AM scaffolds has also been improved by the addition of carboxylic groups to the polymer surface via acrylic acid plasma polymerization. ${ }^{28}$ Surface functionalization using adhesive polymers such as poly(dopamine) have also demonstrated to improve adhesion of different cell types on PCL and poly(lactic acid) (PLA) scaffolds. ${ }^{29-31}$ As an alternative to chemical modification of polymer surfaces, better cell attachment along the scaffold cross section has been attained via decreasing the flow rate of the seeding media through the scaffold's pores ${ }^{32-33}$ By varying the strand distance or the angle of deposition in each layer, 3D AM scaffolds. with pore size gradients along the vertical direction ${ }^{32}$ or with different lay-down patterns ${ }^{33}$ were fabricated and resulted in enhanced cell attachment and distribution. This was, however, in exchange of varying the scaffolds mechanical properties and, more importantly, reducing the pore size, which hinders cell migration, nutrients and oxygen availability, waste management, and angiogenesis for pore sizes smaller than $300 \mu \mathrm{m}$. 4, 34-36

A large improvement in cell seeding efficiency and distribution was recently obtained by seeding 3D AM scaffolds with cell-laden hydrogels, which showed to retain almost the complete cell number mixed within the hydrogel precursor. ${ }^{37-41}$ However, these hybrid systems are multi-step and require fast gelation times. In addition, cells are exposed to two different microenvironments: the hard scaffold filaments and the soft hydrogel carrier. As it is widely accepted that substrate stiffness can direct cell fate, ${ }^{42}$ having these types 
of dual systems might mask the effect of the scaffold material and lead to unexpected results.

Although previous strategies have shown some improvement on cell seeding efficiency and distribution, they require modification of the scaffold's surface chemistry or architecture, influencing the overall microenvironment. Therefore, there is still lack of methodology and knowledge of an elegant cell seeding method applicable to all 3D AM scaffolds regardless of their characteristics. Here, we introduce a novel seeding technique based on controlling cell settling velocity upon static seeding that can be applied to all types of 3D AM scaffolds without requiring any chemical or architectural modification. Two biocompatible macromolecules (macroMs) were employed for the first time as supplements to independently tune i) the viscosity or ii) the density of the cell seeding media. Cells seeded with this novel technique were morphologically characterized using immunofluorescence and scanning electron microscopy. Furthermore, the osteogenic differentiation of human mesenchymal stromal cells (hMSCs) seeded with the macroMbased solutions on AM 3D scaffolds was investigated using various biochemical and molecular assays to evaluate the effect of macroMs on a specific cell differentiation.

\section{MATERIALS AND METHODS}

\section{Scaffolds fabrication}

Scaffolds were fabricated via a ME based AM technique (Bioscaffolder 3.1, SysENG, Germany). The copolymer PEOT/PBT, with a PEO molecular weight of $300 \mathrm{Da}$ and PEOT/PBT blocks ratio 55/45 wt\% (300PEOT55PBT45, PolyVation, The Netherlands) was used. Briefly, the cartridge was filled with PEOT/PBT pellets, heated at $195^{\circ} \mathrm{C}$ and extruded by applying a pressure of 4 bar, an auger screw rotation of $40 \mathrm{rpm}$ and a translation speed of $10 \mathrm{~mm}^{*} \mathrm{~s}^{-1}$. The scaffold architecture consisted of a 0-90 pattern, with a $250 \mu \mathrm{m}$ fiber diameter, $200 \mu \mathrm{m}$ layer thickness and $750 \mu \mathrm{m}$ strand distance (center to center), giving an expected $x-y$ porosity of approximately $500 \mu \mathrm{m}$. Cylindrical scaffolds of $4 \mathrm{~mm}$ diameter and $4 \mathrm{~mm}$ height were punched out from a 20x20x4 mm manufactured block using a biopsy punch and used for further experiments. 


\section{Density and viscosity measurements of Dextran and Ficoll based solutions}

Ficoll-Paque $^{\text {TM }}$ Plus solution (GE Healthcare) (Ficoll-Pq) was diluted to 80, 60, 40 and 20 vol\% with cell culture medium (CM), consisting of aMEM with Glutamax and no nucleosides (Gibco) supplemented with 10 vol\% FBS (Sigma-Aldrich), penicillin (100 U/ml) and streptomycin $(100 \mu \mathrm{g} / \mathrm{ml})$ (Fisher-Scientific). Similarly, 10, 5, and $2.5 \mathrm{wt} \%$ dextran (500 $\mathrm{kDa}$, Pharmacosmos) solutions were prepared in $\mathrm{CM}$. Densities were calculated by weighing known volumes of each of the solutions (accuracy $\pm 0.1 \mathrm{ml}$ ) in a high precision balance (accuracy $\pm 0.1 \mathrm{mg}$ ) and applying the density formula.. The relative viscosity (with respect to water) of the solutions was empirically determined by timing the flow of water and the same volume of each solution through the same fluidic circuit at the same constant pressure.

\section{Determination of hMSCs density using density gradient separation}

Density of hMSCs was measured using Ficoll-Pq density gradient centrifugation. The Ficoll-Pq solution was diluted to $80,60,40$ and 20 vol\% with appropriate volumes of CM containing phenol red, which helped to visualize the layers of the gradient. Starting from 100 vol\% Ficoll-Pq solution, $2 \mathrm{ml}$ of each solution were carefully layered on top of each other in decreasing order in a $15 \mathrm{ml}$ conical tube. One million cells in $\mathrm{CM}$ were layered on top of the $20 \%$ Ficoll-Pq layer. The tube was then centrifuged at $500 \mathrm{rcf}$ for 20 min at room temperature (RT). Subsequently, the layers were carefully separated by pipetting and the cells in each layer were imaged via brightfield microscopy and quantified using a Neubauer chamber. As a comparative method, cell density was additionally determined through buoyancy force-driven separation, which does not require the application of mechanical forces. For the buoyancy separation, the various concentrations of Ficoll-Pq media were similarly layered in a $15 \mathrm{ml}$ conical tube starting from 100 vol\% Ficoll-Pq solution. Here, 1 million cells in medium were carefully pipetted at the bottom of the tube under the $100 \%$ Ficoll-Pq layer using a long syringe needle, without disturbing the layers. The tube was then incubated at RT for $3 \mathrm{~h}$ to let cells be pushed by buoyancy forces to layers of similar density. After separation, the layers were collected separately and cells present in each solution were imaged and quantified using a Neubauer chamber. 


\section{Cell seeding and culture}

Cell expansion

HMSCs isolated from bone marrow were purchased from Texas A\&M Health Science Center, College of Medicine, Institute for Regenerative Medicine (Donor d8011L, female, age 22). Cryopreserved vials at passage 3 were plated at a density of $1,000 \mathrm{cells}^{*} \mathrm{~cm}^{-2}$ in tissue culture flasks and expanded until approximately $80 \%$ confluency in $\mathrm{CM}$ without Pen/Strep at $37^{\circ} \mathrm{C} / 5 \% \mathrm{CO}_{2}$.

\section{Cell seeding and culture in 3D scaffolds}

Scaffolds were disinfected in 70\% ethanol for 20 min, washed 3 times with phosphate buffered saline (PBS) and incubated overnight in CM for protein attachment. Before seeding, scaffolds were dried on top of a sterile filter paper and placed in the wells of a non-treated hydrophobic tissue culture well plate. Trypsinized passage 4 hMSCs suspension was centrifuged for $5 \mathrm{~min}$ at $500 \mathrm{rcf}$ and then resuspended in CM, Dextranbased CM, or Ficoll-Fq -based CM at a density of 200,000 cells per $37 \mu$ lapproximate scaffold free volume). Dextran-based CM consisted of 10 wt\% dextran in CM and Ficoll$\mathrm{Pq}$ based $\mathrm{CM}$ consisted of 60 vol\% Ficoll-Pq in a FBS adjusted cell culture medium ( $\alpha$ MEM supplemented with 25 vol\% FBS and 1 vol\% Penstrep, to obtain 10 vol\% FBS in the final medium). A $37 \mu \mathrm{l}$ droplet of each cell suspension each was placed on top of each scaffold, drawn into the scaffold's free volume by capillary forces and held inside the pores by the hydrophobic well under it. Scaffolds seeded with $\mathrm{CM}$ are referred to as conventional seeded (CS) scaffolds, while macroM seeded (MS) scaffolds are referred to as MS-Ficoll$\mathrm{Pq}$ and MS-Dextran, when seeded with Ficoll-Pq -based $\mathrm{CM}$ and dextran-based CM, respectively. Seeded scaffolds were incubated for $4 \mathrm{~h}$ at $37{ }^{\circ} \mathrm{C} / 5 \% \mathrm{CO}_{2}$ to allow cell attachment. After this time, scaffolds were transferred to new wells containing $1.5 \mathrm{ml}$ of basic media (BM) (CM supplemented with $200 \mu \mathrm{M}$ L-Ascorbic acid 2-phosphate (SigmaAldrich)). BM was replaced after 24h and every two or three days from then on. To evaluate hMSCs osteogenic differentiation, scaffolds were cultured for another 21 days in BM or mineralization media (MM) after 7 days in BM. MM consisted of BM supplemented with 10 nм dexamethasone (Sigma-Aldrich) and $10 \mathrm{~mm} \beta$-glycerophosphate (Sigma-Aldrich).

To visualize the potential entrapment of macroMs within the scaffold after seeding, cells were resuspended in $10 \mathrm{wt} \%$ dextran-FITC (Fluorescein Isothiocyanate-Dextran $500 \mathrm{kDA}$, Sigma-Aldrich) or 60 vol\% Ficoll-Pq-FITC at the same cell density $(200,000$ cells in $37 \mu \mathrm{l})$. 
The Ficoll-Pq-FITC based CM was prepared by first dissolving 5.7\% (wt/vol) polysucroseFITC (400 kDa, Sigma-Aldrich) and 9\% (wt/vol) sodium diatrizoate hydrate (Sigma-Aldrich) in sterile water, to match Ficoll-Pq composition. 60 vol\% of this solution was prepared in FBS adjusted cell CM as previously described. A $37 \mu \mathrm{l}$ droplet of each cell suspension was placed on top of each scaffold and incubated for $4 \mathrm{~h}$ at $37{ }^{\circ} \mathrm{C} / 5 \% \mathrm{CO}_{2}$ to allow cells attachment. After this time, scaffolds were transferred to new wells containing $1.5 \mathrm{ml}$ of $\mathrm{BM}$ and cultured for $24 \mathrm{~h}$.

To rule out macroM adhesion, as well as macroM-driven enhancement of serum proteins adsorption to the scaffold filaments, as the causes of improved cell seeding, scaffolds were pre-incubated for $4 \mathrm{~h}$ with dextran- and Ficoll-Pq - based CM. Non-adsorbed macroMs were removed by a single scaffold wash with PBS and the scaffolds were then dried on top of a sterile filter paper. Subsequently, scaffolds were seeded with $37 \mu \mathrm{l}$ of $\mathrm{CM}$ containing 200,000 cells and incubated for $4 \mathrm{~h}$ at $37^{\circ} \mathrm{C} / 5 \% \mathrm{CO}_{2}$ to allow cell attachment, after which scaffolds were analyzed.

\section{Cell seeding in 2D for viability and osteogenic differentiation evaluation}

Preliminary experiments in 2D were performed in order to asses the viability and osteogenic potential (mineralization) of cells pre-incubated in macroMs solutions. This was done in order to rule out the direct effect of macroM in cell death or in enhanced mineralization in a 3D setting. To assess the viability of cells at different timepoints when in a macroM solution, trypsinized cells were centrifuged and resuspended in $\mathrm{CM}$, dextranbased CM or Ficoll-Pq -based CM at a density of 200,000 cells per $37 \mu \mathrm{l}$. NucBlue ${ }^{\circledR}$ Live and NucGreen $\AA$ Dead reagents (ReadyProbes ${ }^{\circledR}$ Cell Viability Imaging Kit, Invitrogen) were added as well, at concentrations defined by the manufacturer protocol. Cell suspensions were incubated at $37^{\circ} \mathrm{C} / 5 \% \mathrm{CO}_{2}$ in plates mimicking the $4 \mathrm{~h}$ seeding in $3 \mathrm{D}$ scaffolds and imaged at $\mathrm{t}=0 \mathrm{~h}$ and $\mathrm{t}=4 \mathrm{~h}$ after seeding using an inverted fluorescent microscope (Eclipse, Ti2-e, NIKON). For optimal visualization in the reported images, live cells were false colored in green while dead cells were given a red color. In order to assess the osteogenic differentiation potential (mineralization) of cells pre-incubated with macroMs, cells pre-incubated for $4 \mathrm{~h}$ in macroM based solutions were centrifuged and resuspended in fresh $\mathrm{CM}$ to mimic the post-seeding culture condition of cells in scaffolds. Cells were counted and seeded at a density of 5,000 cells ${ }^{*} \mathrm{~cm}^{-2}$ in tissue culture polystyrene well plates. Media was refreshed every two or three days. Scaffolds were cultured for 21 days in BM or MM after 7 days in BM. 


\section{Imaging and quantification of cell distribution within scaffold cross section}

After seeding and $4 \mathrm{~h}$ incubation for attachment, CS and MS scaffolds were washed with PBS and fixed with $4 \mathrm{wt} \%$ paraformaldehyde for $30 \mathrm{~min}$, followed by washing steps with PBS. Cells were permeabilized using 0.1 vol\% Triton- $X$ for $30 \mathrm{~min}$, washed twice with PBS and incubated with phalloidin (Alexa Fluor 568, 1:75 dilution in PBS) for $1 \mathrm{~h}$ at RT. Finally, samples were washed with PBS. The bottom and cross section of scaffolds were imaged using an inverted fluorescent microscope. Cross section images were analyzed using ImageJ software. Briefly, images were converted to 8-bits format and the local contrast was enhanced (blocksize 50, maximum slope 3, no mask). Next, the background was subtracted and the images were converted to binary (rendering regions with cells in white and the rest in black). Each image was divided into four regions corresponding to heights of $0-1 \mathrm{~mm}, 1-2 \mathrm{~mm}, 2-3 \mathrm{~mm}, 3-4 \mathrm{~mm}$ starting from the bottom of the scaffolds and the total amount of white pixels in each region was quantified and normalized to the total number of pixels in the scaffold cross section. Values are reported as normalized cell coverage area in percentage.

\section{Imaging of cell viability and macroMs entrapment in 3D scaffold}

After $4 \mathrm{~h}$ seeding and 1 and 7 days of culture, dead cells were stained for 20 minutes prior to fixation using the LIVE/DEAD ${ }^{\mathrm{TM}}$ Fixable Dead Cell Stain Kit (Thermo Fisher Scientific) at a concentration of $0.5 \mu \mathrm{l}$ stain in $500 \mu \mathrm{l}$ Hank's Balanced Salt Solution per scaffold. Subsequently, samples were washed and fixed with $4 \mathrm{wt} \%$ paraformaldehyde for $30 \mathrm{~min}$, followed by three washing steps with PBS. Cells were stained with phalloidin as stated in section 2.5. Live/dead images of scaffolds at day 1 and 7 were acquired using an inverted fluorescent microscopy. Confocal laser scanning microscopy of macroMs entrapment/dead cells at $4 \mathrm{~h}$ and day 1 was performed with a Tandem confocal system (Leica TCS SP8 STED), equipped with a white light laser (WLL). Samples were excited with the dye specific wavelengths using the WLL or a photodiode 405 in the case of DAPI. Emission was detected with PMT detectors (DAPI) or HyD detectors (phalloidin, dextranFITC, Ficoll-FITC, dead cells).

\section{Scanning electron microscopy (SEM) imaging of cells in scaffolds}

After $4 \mathrm{~h}$ seeding, 1 day of culture in BM and 28 days of culture in MM, scaffolds were washed with PBS and fixed with $4 \mathrm{wt} \%$ paraformaldehyde for $30 \mathrm{~min}$, followed by washing 
steps with PBS. Sample water content was removed with a graded ethanol series $(30,50$, $70,80,90,96,100$ vol\%), each 15 min at RT. Drying of samples was achieved by 15 min incubation in $100 \%$ ethanol- hexamethyldisilazane (HMDS) at a $1: 1$ ratio for $15 \mathrm{~min}$, followed by 15 min incubation in HMDS. HMDS was removed and scaffolds allowed overnight drying at RT. Samples were gold sputter coated and examined using SEM (Phillips XL-30), with the detector operating at $5 \mathrm{kV}$.

\section{Biochemical assays}

Alkaline phosphatase (ALP) assay

ALP activity was evaluated after 7 and 21 days of culture in MM (time points day 14 and 28, respectively). 3D scaffolds were collected at every time point and washed with PBS, cut in half, stored at $-80^{\circ} \mathrm{C}$ and freeze-thawed 3 times. Samples were incubated for $1 \mathrm{~h}$ at $\mathrm{RT}$ in a cell lysis buffer composed of $0.1 \mathrm{M} \mathrm{KH}_{2} \mathrm{PO}_{4}, 0.1 \mathrm{M} \mathrm{K}_{2} \mathrm{HPO}_{4}$, and 0.1 vol\% Triton $\mathrm{X}-100$, at $\mathrm{pH}$ 7.8. $40 \mu \mathrm{l}$ of the chemiluminescent substrate for ALP CPD star- ready to use reagent was added to $10 \mu \mathrm{l}$ of cell lysate. After $15 \mathrm{~min}$ incubation, luminescence (emission $=470 \mathrm{~nm}$ ) was measured using a spectrophotometer (Biodrop). Remaining cell lysates were used for DNA quantification. Values are reported normalized to DNA content.

\section{DNA assay}

DNA assay was performed on cells cultured on 3D scaffolds after $4 \mathrm{~h}$ of seeding (time point 4h), after 7 days in BM (time point day 7), and after an extra 7 and 21 days of culture in BM or MM (time point day 14 and 28, respectively). In addition, DNA quantification was also performed on 2D samples using CyQUANT Cell Proliferation Assay Kit (Thermo Fisher Scientific). Samples (lysed samples from ALP activity assay or frozen samples after time point collection) were incubated overnight at $56^{\circ} \mathrm{C}$ in Proteinase $\mathrm{K}$ solution $\left(1 \mathrm{mg}^{*} \mathrm{ml}^{-}\right.$ 1 Proteinase K (Sigma-Aldrich) in Tris/EDTA) for matrix degradation and cell lysis. Subsequently, samples were freeze-thawed three times and incubated $1 \mathrm{~h}$ at RT with lysis buffer (cell lysis buffer from the kit diluted 20x in $\mathrm{dH}_{2} \mathrm{O}$ ) containing RNase $A(1: 500)$ to degrade cellular RNA. Lysed samples were incubated with the fluorescent dye provided by the Cyquant kit (1:1) for $15 \mathrm{~min}$ and fluorescence was measured (emission/excitation = $520 / 480 \mathrm{~nm}$ ) with a spectrophotometer. DNA concentrations were calculated from a DNA standard curve. 


\section{Alizarin red S (ARS) staining and quantification}

Calcium mineralization was qualitatively determined by ARS staining after 7 and 21 days of culture in BM or MM (time point day 14 and 28, respectively). 3D scaffolds were washed with PBS and fixed with $4 \mathrm{wt} \%$ paraformaldehyde for $30 \mathrm{~min}$, followed by three washing steps in distilled water. Subsequently, scaffolds were cut in half and each section was stained with ARS solution (60 mm, pH 4.1-4.3) for 20 min at RT, and washed several times with distilled water until no more stain was leaching out. Images were taken using a stereomicroscope (Nikon SMZ25).

After imaging, a protocol adapted from Gregory et al. ${ }^{43}$ was used to quantify calcium deposition. Briefly, stained samples were incubated in Eppendorf's for $1 \mathrm{~h}$ at RT with 30 vol\% acetic acid while shaking, followed by $10 \mathrm{~min}$ incubation at $85{ }^{\circ} \mathrm{C}$. Afterwards, scaffolds were removed and solutions were centrifuged at 20,000 rcf for $10 \mathrm{~min}$. An appropriate volume of $5 \mathrm{M}$ ammonium hydroxide was added to the supernatants to readjust the $\mathrm{pH}$ to $4.1-4.3$. The absorbance was measured at $405 \mathrm{~nm}$ using a spectrophotometer. Concentration of ARS was calculated from an ARS standard curve and the values were normalized to DNA content.

\section{Gene expression}

Gene expression analysis was performed at day 14 (7 days in MM) and day 28 (21 days in MM). RNA was extracted from cells in scaffolds using a Trizol isolation method at the selected time points. Briefly, samples were transferred to Eppendorf tubes and Trizol was added. ECM was precipitated at the bottom of the tube through a centrifugation step at 12,000 rcf for $5 \mathrm{~min}$. The supernatant was transferred to a new tube and chloroform was added to isolate the RNA, present in the aqueous phase after phase separation via centrifugation at 12,000 rcf for 5 min. RNA was further purified using RNeasy mini kit column (Qiagen), according to the manufacturer's protocol. The purity and quantity of total RNA was evaluated using a spectrophotometer. Reverse transcription was performed using iScript ${ }^{\mathrm{TM}}$ (Bio-Rad) following suppliers' protocol. The obtained cDNA was used in combination with SYBRGreen master mix (Qiagen) and the selected primers (Table S1, Supplementary Information) to perform qPCR using CFX Connect ${ }^{\mathrm{TM}}$ Real-Time System (Bio-Rad) under the following conditions: cDNA was denaturated for 3 min at $95{ }^{\circ} \mathrm{C}$, followed by 40 cycles consisting of $15 \mathrm{~s}$ at $95^{\circ} \mathrm{C}$ and $30 \mathrm{~s}$ at $65^{\circ} \mathrm{C}$. Additionally, a melting curve was generated for each reaction in order to test primer dimer formation and non- 
specific amplification. Gene transcription was normalized to the transcription of the housekeeping human B2M gene. The $2^{-\Delta \Delta \mathrm{Ct}}$ method was used to calculate relative gene expression for each target gene. Normalization was done with respect to relative gene expression of cells in CS scaffolds at day 14.

\section{Statistical analysis}

All data is shown as average with error bars indicating the standard deviation of at least three replicates. Analysis of statistics was conducted with GraphPad Prism (version 8.0.1). A one-way or two-way analysis of variance (ANOVA) was performed followed by a Tukey's or Sidak's post-hoc multiple comparison test (as recommended by the software) to evaluate statistical significance and correct for multiple comparisons.

\section{RESULTS}

\section{Optimization of the macroM seeding methods}

If cells in suspension are considered as rigid spheres, upon which frictional (viscosity related), buoyant (density related) and gravitational forces are exerted, their settling velocity in medium can be derived from the Stokes law. ${ }^{44}$ When the sum of the frictional and buoyancy forces exerted on the cell, due to the surrounding medium, balance the gravitational force, the constant settling velocity $(v)$ can be defined by:

$\mathrm{v}=\frac{2}{9} \frac{\left(\rho_{\text {cell }}-\rho_{\text {medium }}\right)}{\mu_{\text {medium }}} \mathrm{gR}^{2}$

where $\rho_{\text {cell }}$ refers to cell density, $\rho_{\text {medium }}$ refers to density of the surrounding cell culture medium, $\mu_{\text {medium }}$ refers to the viscosity of the surrounding cell culture medium, $g$ is the acceleration due to gravity, and $\mathrm{R}$ the cell hydrodynamic radius. According to this equation, to maintain cells in suspension the settling velocity can be decreased by increasing the media viscosity or reduced to zero by matching the density of the media and the cells. In this study, we investigated both approaches independently (Figure 1). The media viscosity was changed with the addition of dextran. As shown in Figure S1A, by the addition of dextran up to $10 \mathrm{wt} \%$, the viscosity of CM increases exponentially up to around 25 times. As our second approach, the density of the seeding medium was increased by the addition of Ficoll-Paque ${ }^{\mathrm{TM}}$ Plus (Ficoll-Pq) to match the cell density and therefore obtain a theoretical settling velocity equal to zero $(\mathrm{v}=0)$. Ficoll-Pq is an aqueous solution that also 
contains sodium diatrizoate, a compound that allows to have a high density solution while maintaining its viscosity relatively low (Figure S1B, Supplementary Information). Notably, the viscosity of this solution is only $\sim 3$ times higher than $\mathrm{CM}$ viscosity. A density gradient of Ficoll-Pq (Figure S1D) allowed for the experimental determination of the density of the hMSCs, which is known to be lower than $1.073 \mathrm{~g}^{*} \mathrm{ml}^{-1} 45-46$. Both separation by gradient centrifugation and by buoyancy forces determined that the density of the hMSCs population lied in between 1.034 and $1.052 \mathrm{~g} \mathrm{ml}^{-1}$ corresponding to $40 \mathrm{vol} \%$ and $60 \mathrm{vol} \%$ Ficoll-Pq in CM (Figure S2). In order to encompass the majority of the hMSCs population (i.e. to maintain most of the cells in suspension) 60 vol\% Ficoll-Pq solution (Ficoll-Pq based CM) was chosen as the density-based media for seeding the MS-Ficoll-Pq, with a relatively ignorable influence on the viscosity of $\mathrm{CM}$ (only 1.65 fold increase) (Figure S1B). On the other hand, the viscosity is considered the only effective parameter on the cell settling velocity in a $10 \mathrm{wt} \%$ Dextran solution (Dextran-based CM), which was chosen as the viscosity-based media for seeding the MS-Dextran scaffolds.The density of $10 \mathrm{wt} \%$ dextran $\left(1.024 \mathrm{~g} \mathrm{ml}^{*}\right)$ is significantly lower than 60 vol\% Ficoll-Pq density $\left(1.052 \mathrm{~g}^{*} \mathrm{ml}^{-1}\right)$ as well as 40 vol\% Ficoll-Pq density $\left(1.034 \mathrm{~g}^{*} \mathrm{ml}^{-1}\right)$, and therefore, lower than hMSCs density. Specifically, its density is comparable to that of a 20 vol\% Ficoll-Pq solution (1.027 $\mathrm{g}^{\star} \mathrm{ml}^{-1}$ ) (Figure S1C, E), where a minimum amount of cells were found during separation by Ficoll-Pq gradients (Figure S2).

\section{Cell distribution using MS methods}

To assess the cell distribution across highly porous PEOT/PBT scaffolds seeded with each of the seeding methods, cells in their cross sections and bottoms were imaged and the cross section images were divided into four horizontal regions of $1 \mathrm{~mm}$ each, from 0 to 4 $\mathrm{mm}$ height. Moreover, cell settling velocity and displacement towards the bottom of scaffolds during the seeding time was calculated and used to support the cell localization results. As demonstrated in Figure $\mathbf{2 A}, \mathbf{B}$, around $70 \%$ of the total cell number found in the scaffolds cross sections was concentrated in the lowest $1 \mathrm{~mm}$ region of the scaffold, as well as attached to the bottom when seeded with the CS method. Using this method, cells sedimented at a theoretical velocity of $1.8 \mu \mathrm{m}^{*} \mathrm{~s}^{-1}$ and displaced $2.6 \mathrm{~mm}$ towards the bottom of the scaffold ( $75 \%$ of the scaffold height) during the $4 \mathrm{~h}$ seeding (Figure S1F). In contrast, theoretical cell settling velocity decreased $\sim 50$-fold for MS-Dextran when compared to the CS method, or remained zero when seeded with the MS-Ficoll-Pq 

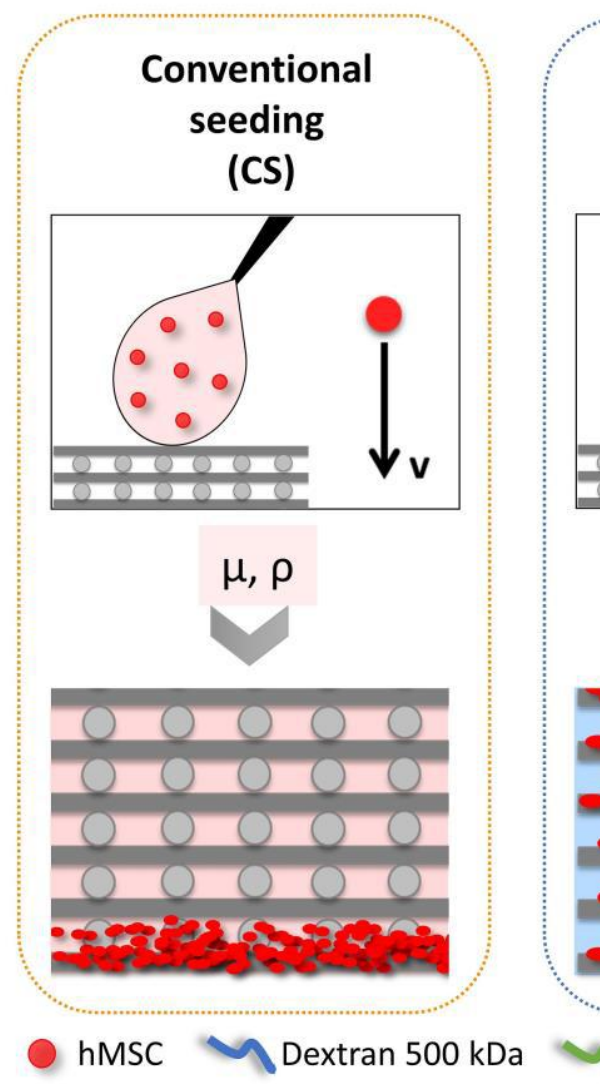

\section{MacroM seeding with 10 wt\% Dextran \\ (MS-Dextran)}

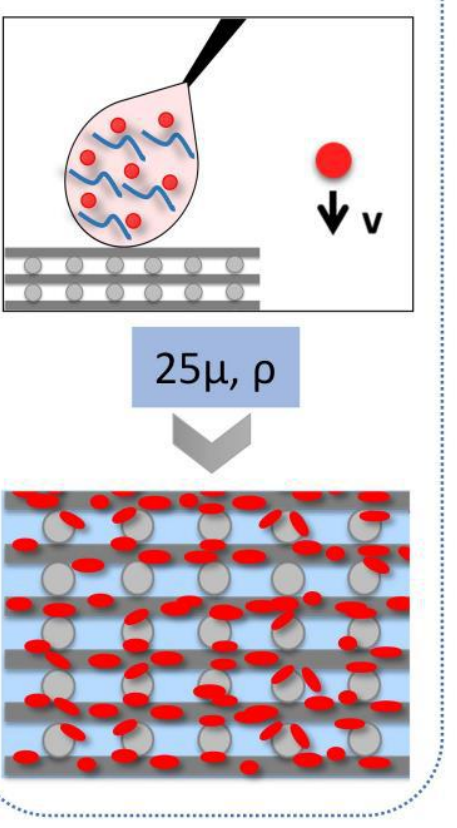

Ficoll (Ficoll-Pq)

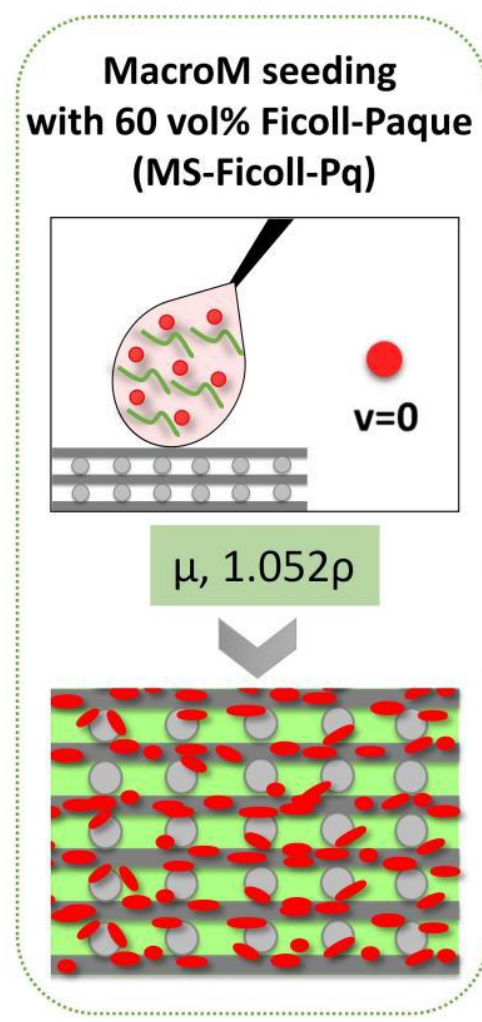

$\rho:$ medium density

Figure 1. Schematic representation of the static seeding approaches discussed in this study: Conventional seeding (CS), Macromolecular seeding with $10 \mathrm{wt} \%$ dextran (MS-Dextran) and macromolecular seeding with 60 vol\% Ficoll-Paque (MS-Ficoll-Pq). Influence of the properties of the cell seeding media (viscosity and density) on the settling velocity of cells and, therefore, on cell attachment along scaffold height is depicted

method, resulting in a theoretical displacement of $\sim 55 \mu \mathrm{m}$ or $0 \mu \mathrm{m}$ towards the bottom of the scaffold during the $4 \mathrm{~h}$ seeding, respectively (Figure S1F). This allowed the cells to remain in suspension and to attach to the filaments of the scaffolds in a more homogenous manner (Figure 2A). Around 20 to $30 \%$ of the total cell number in the cross section was found in each of the $1 \mathrm{~mm}$ subdivisions of the scaffold (Figure 2C, D), regardless of the use of the MS-Dextran or the MS-Ficoll-Pq method, suggesting that both approaches successfully improved cell distribution along scaffold's cross section. Furthermore, no cells were found at the bottom of scaffolds when seeded with the MS methods, contrary to what was observed in the case of CS scaffolds, which further demonstrates the enhanced cell attachment. Additionally, cells in suspension along the scaffold depth that did not attach to the filaments were washed away after immersing the scaffold in media. 
As observed in Figure 3A, cells preferentially attached to the top curved surface of the scaffold filaments during the seeding and, due to cell clustering and a larger number of cells attached, cell projections were not clear in the MS methods when compared to the spindle-like cells resulted from the CS method after $4 \mathrm{~h}$ seeding. Nevertheless, only after $24 \mathrm{~h}$ post-seeding cells in the MS scaffolds showed a clear elongated morphology, characteristic of migrating cells, and covered the whole surface of the filaments in contrast to the less crowded filaments on CS scaffolds (Figure 3B).
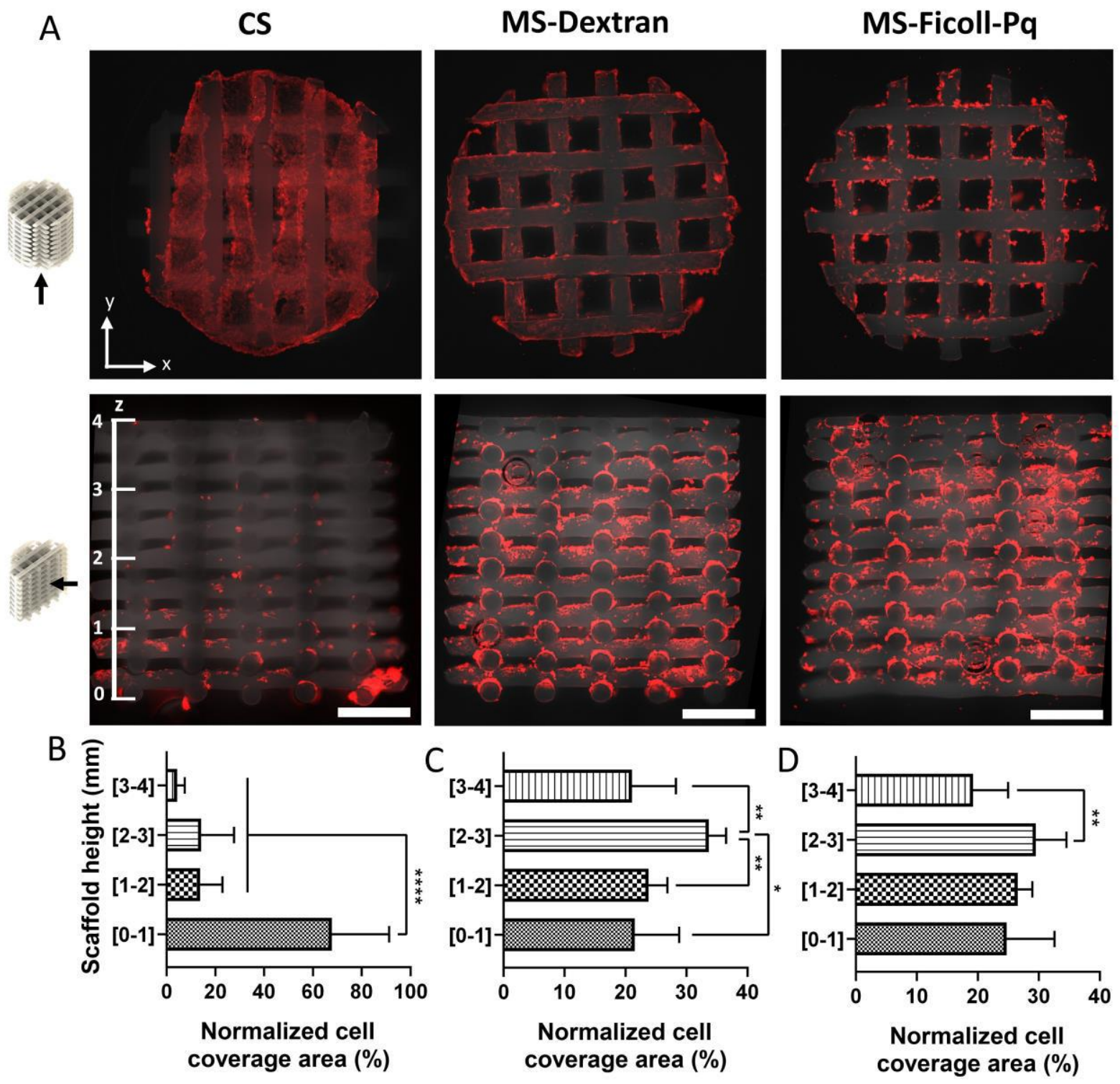

Figure 2. Fluorescent staining (F-actin, red) showing that MS methods improved cell distribution along scaffold cross section. (A) Fluorescence microscopy images of hMSCs in the bottom sides (top) and cross sections (bottom) of scaffolds seeded with the CS, MS-Dextran and MS-Ficoll-Pq methods and (B-D) respective quantification of cell distribution across the scaffold height. Statistical significance performed using one-way ANOVA with Tukey's multiple comparison test ${ }^{*} p<0.05$; $\left.{ }^{* *} p<0.01 ;{ }^{* * *} p<0.0001\right)$. Scale bars $1 \mathrm{~mm}$. 
CS

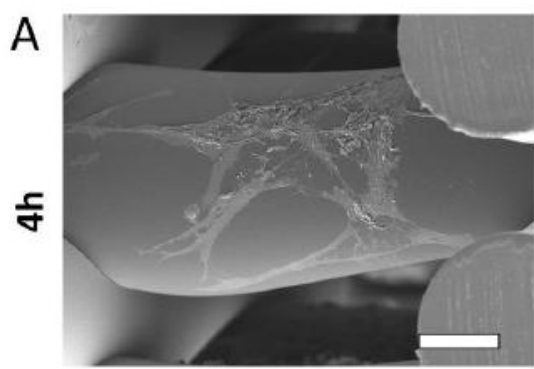

B
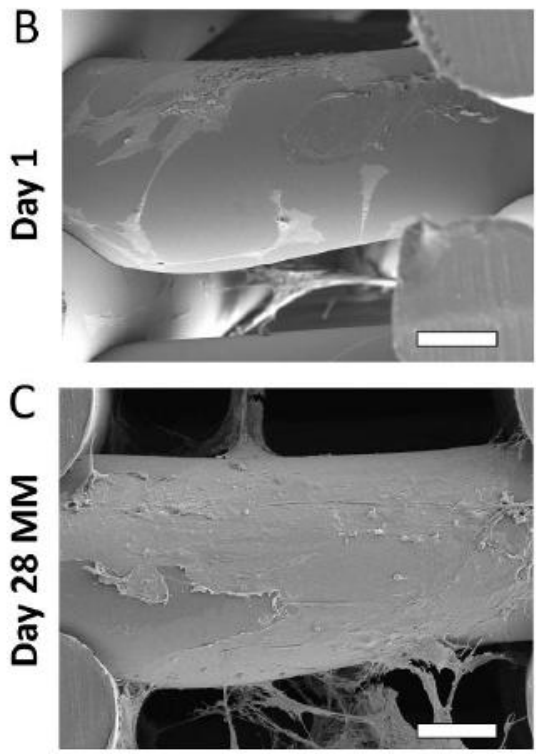

MS-Dextran
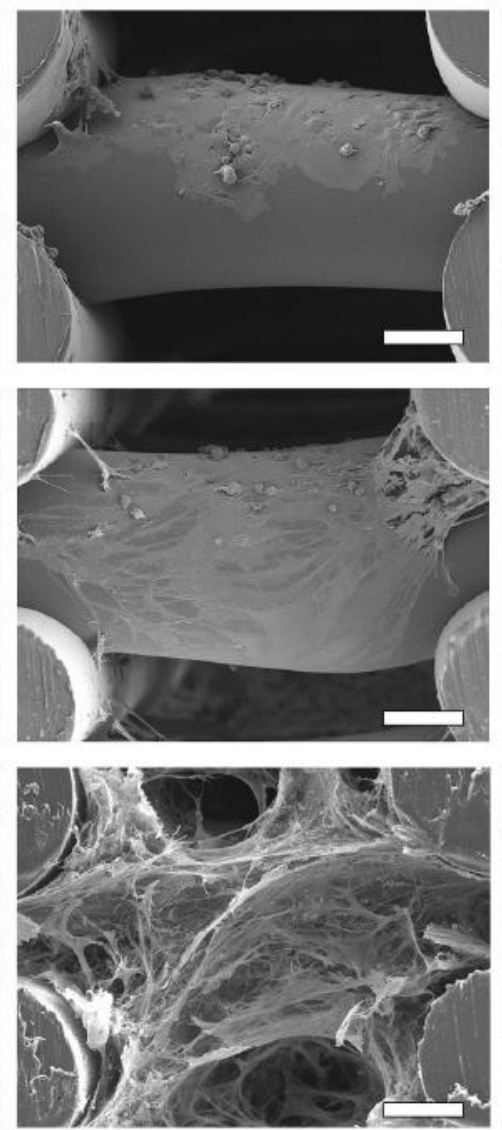

MS-Ficoll-Pq
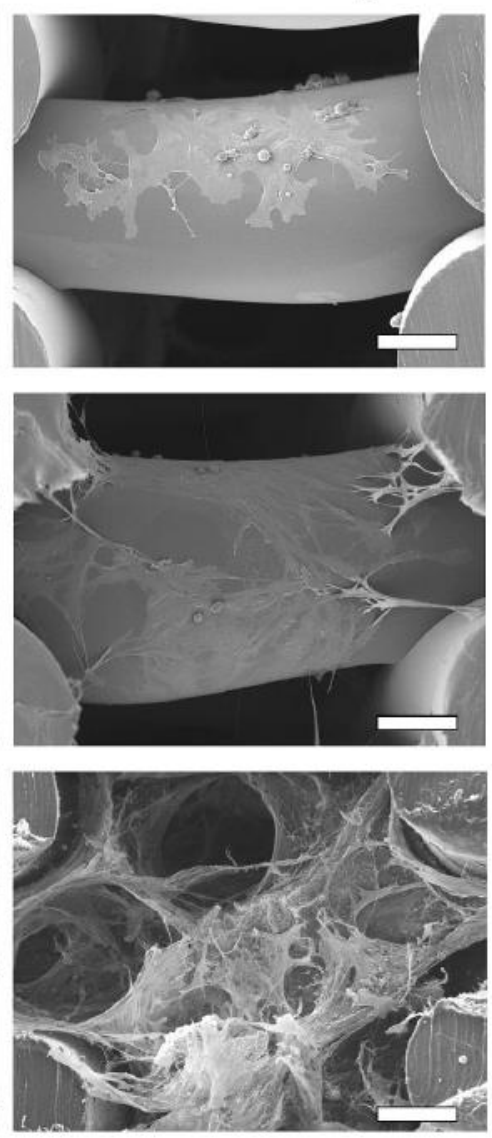

Figure 3. Higher cell coverage and matrix production on scaffolds seeded with the MS-methods. SEM micrographs of hMSCs on scaffolds seeded with the CS, MS-Dextran and MS-Ficoll-Pq methods after $4 \mathrm{~h}, 1$ day and 28 days of culture (21 days in MM) showing cell-scaffold interactions, cell coverage of scaffold filaments and matrix production. Scale bars $100 \mu \mathrm{m}$.

\section{MacroMs in scaffolds}

In order to evaluate the potential presence of macroMs in the scaffolds after seeding, scaffolds were seeded with solutions containing fluorescently labeled dextran or Ficoll. After $4 \mathrm{~h}$ seeding, macroMs were not found coating the scaffolds filaments. Besides, cells also sedimented in CS scaffolds, which were pre-incubated with dextran or Ficoll-Pq based CM (Figure S3). Collectively, both results suggest that macroMs did not adhere to the scaffold surface to act as cell adhesive molecules nor contributed to better cell adhesion by mediating adsorption of serum proteins to the scaffolds filaments. Interestingly, while for both MS-Dextran and MS-Ficoll-Pq, macroMs co-localized with the cells cytoplasm after $4 \mathrm{~h}$ seeding (Figure 4), their fluorescence signals were drastically reduced after $24 \mathrm{~h}$ and were found to co-localize only with dead cells, and (Figure 4 and Figure S4). Notably, cell death was visualized within the first minutes of hMSCs incubation with both macroMs 
based $\mathrm{CM}$ and $\mathrm{CM}$ (medium without macroMs) (Figure S5). Nevertheless, high cell viability was observed across the MS-Dextran and MS-Ficoll-Pq scaffolds over 7 days culture, as demonstrated in the Figure 5.

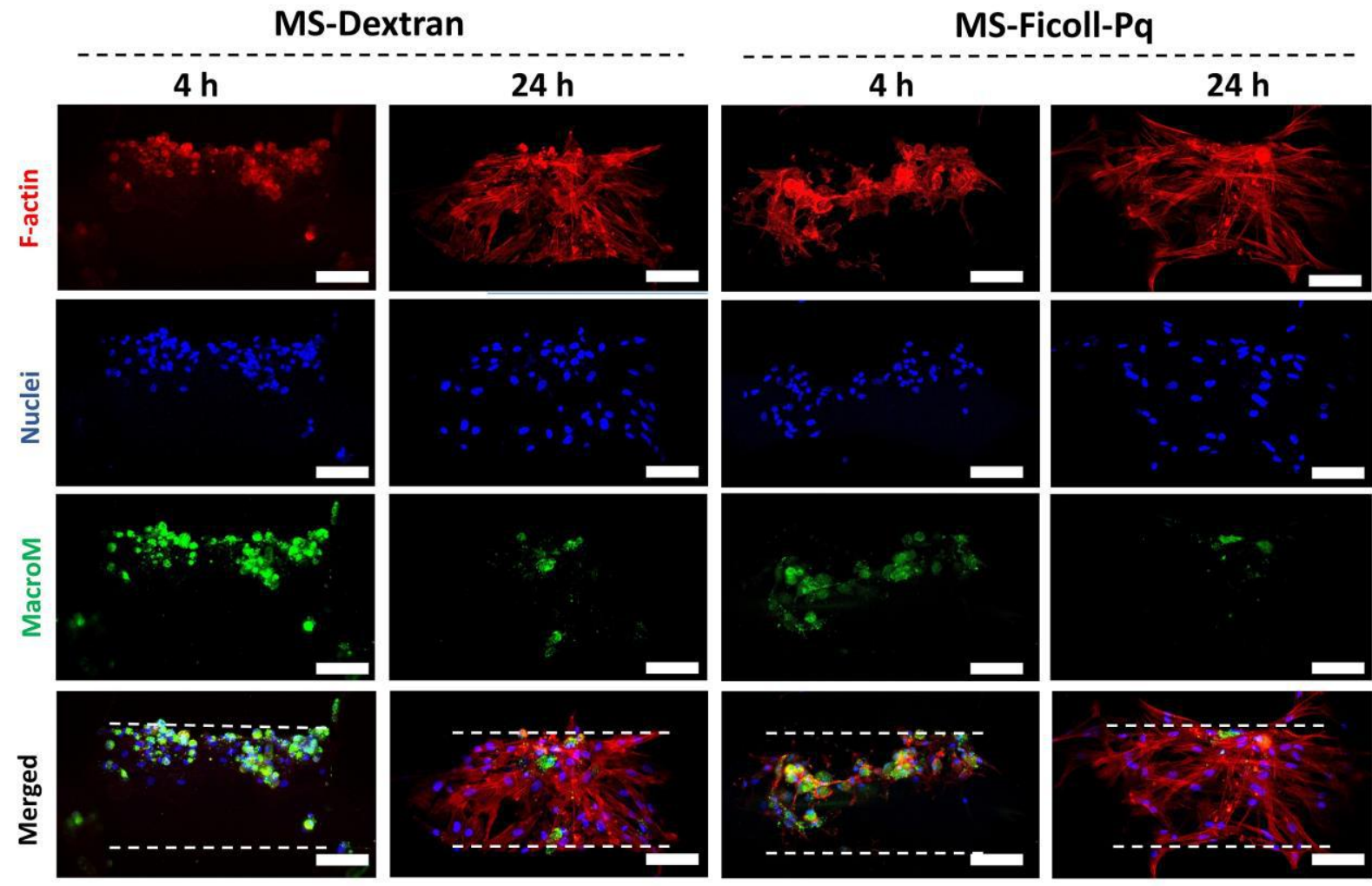

Figure 4. Initial retention (4h) and rapid elimination (24h) of macroMs in cells attached to the scaffolds. Representative confocal microscopy images of hMSCS (F-actin) and macroM (FITClabeled) on top of scaffold filament $4 \mathrm{~h}$ and $24 \mathrm{~h}$ post-seeding with the MS-Dextran and MS-Ficoll$\mathrm{Pq}$ methods. Dash lines delimitating the scaffold filament. Scale bars $100 \mu \mathrm{m}$.

\section{Osteogenic differentiation on MS scaffolds}

To fully assess the functionality of our novel MS methods to seed AM 3D scaffolds for bone regeneration, hMSCs potency and their ability to undergo osteogenic differentiation after seeding was evaluated. Preliminary 2D experiments confirmed that proliferation and mineralization were not affected when cells were pre-incubated in macroMs solutions and seeded in 2D (Figure S6). These initial positive results allowed us to further study hMSCs response to macroMs seeding in 3D. Despite the significant effect of MS methods in cell distribution (Figure 2), no statistical differences were found in the total number of cells attached to the scaffolds when compared to the CS method (Figure 6A). Similarly, looking at cell proliferation (Figure 6A), constant cell numbers were observed in scaffolds seeded with all the three methods over time, with the exception of day 28 in MM. At this timepoint, 
increased cell numbers were observed on MS scaffolds, compared with earlier time points and with CS scaffolds at day 28. SEM images in Figure 3C also demonstrated differences in ECM production at this timepoint among the seeding methods. In the case of MS scaffolds, cells were present both on the scaffold filaments and within pores' volume along with the formation of a dense ECM. On the contrary, ECM density was found to be much lower in CS scaffolds, which correlates to the low cell proliferation values over the whole culture period (Figure 6A).
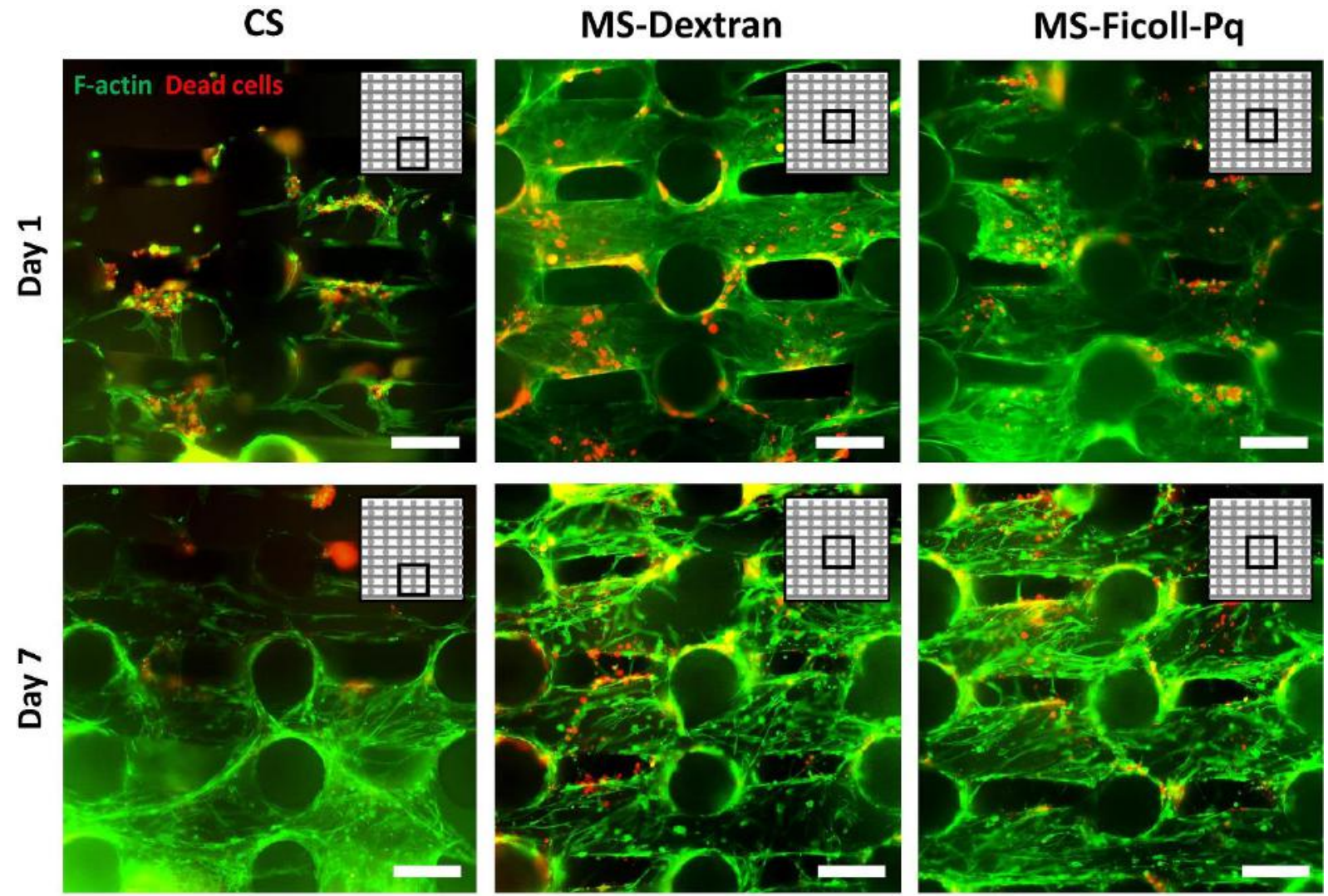

Figure 5. Cell viability is preserved after seeding with MS methods. Representative fluorescent images depicting cell viability ( $F$-actin, green; dead cells, red) 1 day and 7 days post-seeding with the CS, MS-Dextran and MS-Ficoll-Pq methods. Inserts represent the imaged area in scaffold cross-section. Scale bars $250 \mu \mathrm{m}$.

ALP activity was measured after 14 and 28 days of culture, corresponding to 7 and 21 days culture in MM (Figure 6B). No significant difference was observed in ALP activity at day 14 regardless of the seeding method. However, ALP activity was significantly lower at day 28 when comparing cells in scaffolds seeded with CS and with MS methods. Interestingly, ALP activity increased from day 14 to day 28 in cells seeded with CS method. 
In contrast, ALP decreased from day 14 to day 28 in cells seeded with MS methods despite the macroM type.
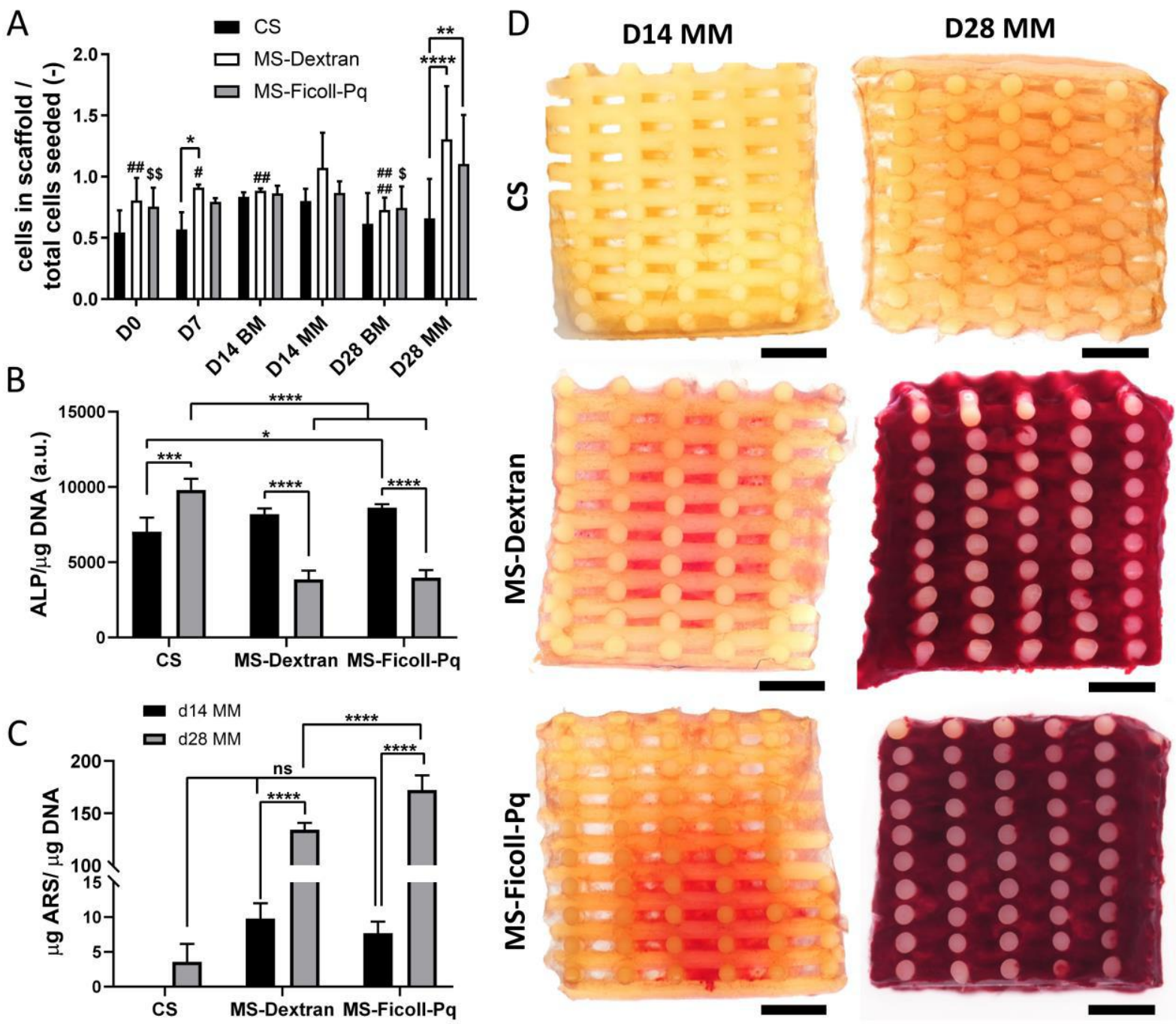

Figure 6. MS method does not hinder osteogenic differentiation of hMSCs: Evaluation of the osteogenic differentiation potential of hMSCS seeded with the MS-based methods compared to CS. (A) Cell seeding efficiency and cell number progression over 28 days of culture in BM and MM on scaffolds seeded with the CS, MS-Dextran and MS-Ficoll-Pq methods. Statistical significance performed using two-way ANOVA with Tukey's multiple comparison test ( $\# \$ p<0.05$; ${ }^{*} \# \# \$ \$ p<$ $0.01 ;{ }^{* * \star *} \# \# \# \$ \$ \$ p<0.0001 ;{ }^{*}$ for comparisons among seeding methods each time point; \# for comparisons with D28 MM among MS-Dextran; \$ for comparisons with D28 MM among MS-Ficoll$\mathrm{Pq}$ ). (B) Comparison of ALP activity at day 14 and day 28 of culture ( 7 and 21 days in MM, respectively) of hMSCs when seeded with the three different methods. (C) Quantification of the ARS staining extracted from scaffolds after 14 and 28 days of culture ( 7 and 21 days in MM, respectively), normalized to cell number. Statistical significance performed using two-way ANOVA with Sidak's multiple comparison test (n.s. $p>0.05$; ${ }^{*} p<0.05$; ${ }^{* * *} p<0.001$; ${ }^{* * \star *} p<0.0001$, for comparisons among seeding methods each time point or among time points each seeding method) (D) Stereomicroscopy images of scaffold cross sections stained with ARS after 14 and 28 days of culture (7 and 21 days in MM, respectively). Scale bars $1 \mathrm{~mm}$. 
To gain more insight into the potential of osteogenic differentiation of hMSCs in scaffolds seeded with our novel method, matrix mineralization was evaluated using ARS staining to visualize calcium deposits after 7 and 21 days in MM (day 14 and day 28, respectively). Scaffolds cultured in BM were used as controls to ensure the inert behavior of the macroMs in the mineralization process (Figure S7A). Intriguingly, as it is shown in Figure 6D, calcium deposition on the cross section of MS-scaffolds was observed from day 14 (7 days in $\mathrm{MM}$ ), and by day 28 these were distributed over the whole scaffold visualized by the dense red color homogeneously covering the cross section and outer surface of the scaffolds (Figure S7B). Quantification of the staining (Figure 6C) confirmed that calcium depositions increased $\sim 10$ to 15 -fold within these two time points. Moreover, statistical differences among the amount of calcium deposited by cells seeded by the MS-dextran and the MS-Ficoll-Pq methods were evident when quantified. No ARS staining was observed in CS scaffolds at day 14, and only a small amount of calcium deposits was quantified after 28 days culture, which was comparable to the mineralization in MS scaffolds at day 14.

Ultimately, the expression of relevant osteogenic genes on cells seeded with the MSmethods was evaluated and compared to the CS method. Notably, no significant differences were found in the expression of runt-related transcription factor (RUNX2), collagen type I (COL I), osteocalcin $(\mathrm{OCN})$ and osteonectin $(\mathrm{ON})$ among the different groups at day 14 (7 days in MM), suggesting that the seeding with macroMs did not alter the early stage of hMSCs differentiation (Figure 7). Importantly, the gene encoding RUNX2 was upregulated on cells seeded with the MS-Dextran and MS-Ficoll-Pq at day 28 (21 days in MM) when compared to CS method (Figure 7A). Similarly, COL I expression was higher in MS-Ficoll-Pq samples at this time point (Figure 7B). In addition, OCN was more expressed in MS-Dextran with respect to CS (Figure 7C). On the other hand, no significant differences were found in the ON encoding gene expression among time points or seeding methods (Figure 7D).

\section{DISCUSSION}

When cells are seeded on a 2D substrate, gravity acts as one of the main parameters favoring cell attachment. On the contrary, gravity acts as a counterforce to cell attachment during static seeding of 3D scaffolds fabricated from synthetic polymers and with large and interconnected pores, where cell attachment is already dramatically hindered because of 
lack of adhesion motifs, fast flow velocity and large scaffold's pore size. Rather than modifying the scaffold material properties or architecture, in this study we tackled the cell sedimentation problem from a different perspective, the cell seeding media physical properties (viscosity and density). This is particularly a beneficial approach as it can be applied to all AM 3D scaffolds independent of their material chemistry or geometries. In this study we tuned the viscosity and density of the seeding media by using two natural biocompatible macroMs, dextran and Ficoll. Dextran has previously shown to promote ECM deposition in vitro not only when used as macromolecular crowder mimicking the

A

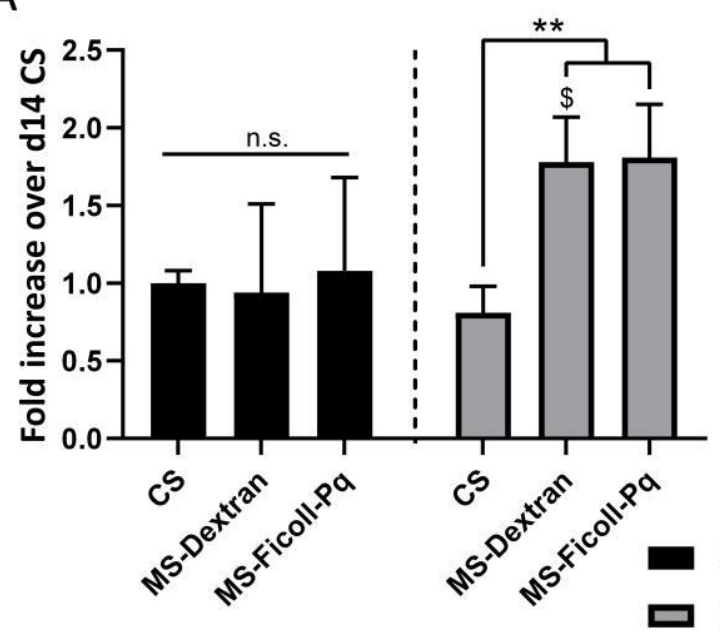

OCN

C

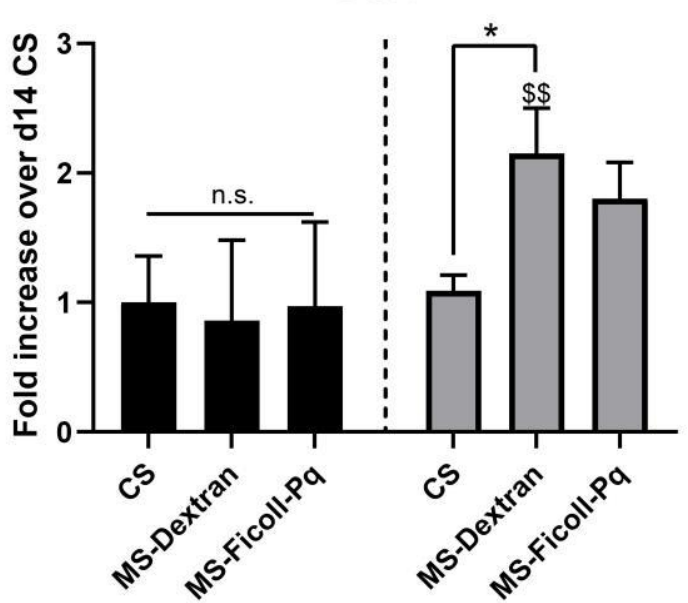

B

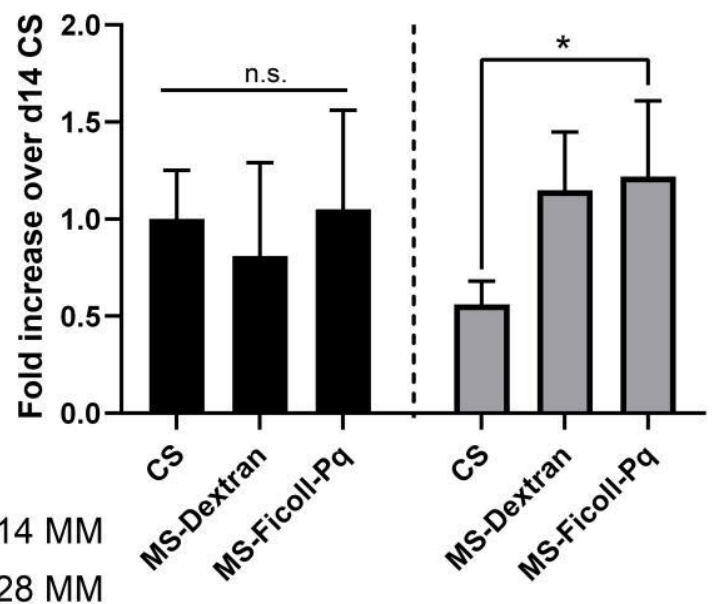

ON

D

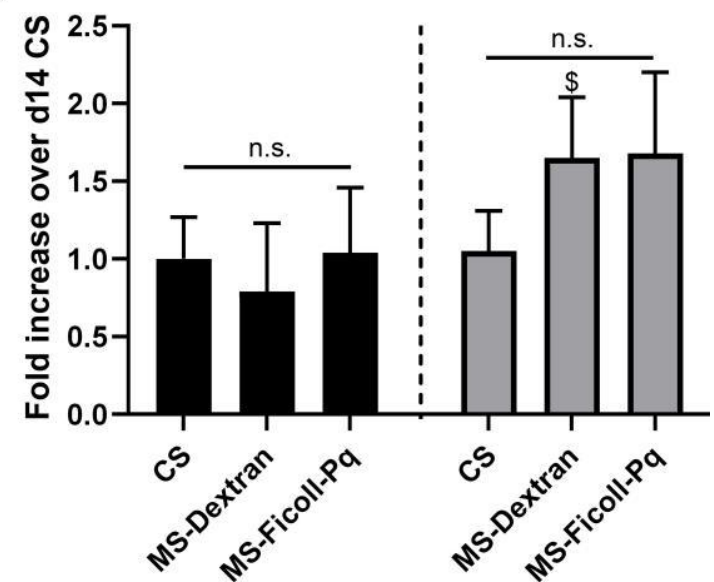

Figure 7. Gene expression of hMSCs after 14 and 28 days of culture (7 and 21 days in MM, respectively) comparing the three different seeding methods. (A) RUNX2, (B) COL I, (C) OCN and (D) ON fold change expression values are relative to CS d14 MM. Data presented as average \pm s.d. and statistical significance performed using two-way ANOVA with Sidak's multiple comparison test (n.s. $p>0.05 ;{ }^{*} \$ p<0.05 ;{ }^{* *} \$ p<0.01 ;{ }^{*}$ for comparisons among seeding methods each time point; $\$$ for comparisons among time points each seeding method). 
highly crowded/dense native ECM, ${ }^{47-49}$ but also when employed as media thickener to increase fluid shear forces in perfusion culture. ${ }^{50} \mathrm{It}$ has also been used to study erythrocyte aggregation and blood viscosity changes. ${ }^{51-53}$ On the other hand, Ficoll has been used for the isolation of cells from blood samples by centrifugation, ${ }^{54-55}$ as well as macromolecular crowding agent. 47-49, 56 Despite being widely used in the biomedical field, to the best of our knowledge, these macroMs were employed here for the first time as media supplements to enhance scaffolds static seeding. Moreover, we demonstrated that MS-Dextran and MS-Ficoll-Pq are two independent approaches, since viscosity and density are the effective parameters on cell settling velocity of the chosen dextran-based (10\% Dextran) and Ficoll-Pq -based (60 vol\% Ficoll-Pq) seeding solutions, and therefore the two methods can be studied and used separately. We showed that both increasing the seeding media viscosity or matching its density to that of hMSCs appropriately reduced cell settling velocity, allowing cells to float longer in the suspension, thereby giving them more time to attach and hence promoting homogeneous cell attachment on the PEOT/PBT scaffold filaments. On the other hand, cells poorly attached to the scaffold cross section when using the CS method. PEOT/PBT was chosen as model synthetic material to produce 3D AM scaffolds. These polyether-ester multiblock copolymers are thermoplastic elastomers, whose surface energies, swellability, degradation and mechanical properties can be tuned for specific applications by controlling the PEOT/PBT ratio ${ }^{2,57}$ and whose in vitro and in vivo applicability in the orthopedic field has been extensively studied. ${ }^{58-59}$ Due to their easy processability, they have been previously used to produce 3D AM scaffolds. ${ }^{60}$ However, they offer poor cell attachment upon conventional static seeding as shown here and in previous works. ${ }^{24,61}$

Direct comparison of our cell distribution results with previously published seeding techniques on 3D AM scaffolds is very challenging, as most of these studies have not shown the full characterization of cell distribution and sedimentation, but rather addressed the seeding efficiency and its direct effect on the tissue regeneration process. In one exception, the amount of DAPI nuclear staining was quantified in each of the slices subdividing the cross section of scaffolds with vertical pore size gradients. ${ }^{32}$ Despite the positive effect of gradients on cell attachment in each of the scaffold subdivisions, still large amounts of cells accumulated at the bottom of the scaffolds, leading to a non-uniform cell distribution over the scaffold cross section. Interestingly, the uniform cell distribution demonstrated by MS methods closely resembles a recently published report in which hMSCs were seeded in a polymeric scaffold by means of a cell-laden polyethylene glycol 
dithiothreitol hydrogel. 40 In this hybrid system, the cell carrier hydrogel was hydrolytically degraded after 7 days, time that cells used to populate the scaffold filaments attaining a good distribution. Importantly, when using our novel MS methods, cells attached directly to the filaments without the means of hydrogel carriers. Moreover, we proved that the macroMs did not act as a cell adhesive coating in the scaffolds, but viscosity or density of the media, depending on the macroM type, were the only factors leading to cell attachment. Previous reports demonstrating reduced protein adsorption and cell attachment in dextran coated surfaces support these results: dextran and Ficoll should be functionalized to render them cell adhesive. ${ }^{62,63}$

The fact that after the $4 \mathrm{~h}$ seeding the macroMs were found to colocalize with the cell cytoplasm and that the amount of macroMs was drastically reduced after $24 \mathrm{~h}$ culture in $\mathrm{CM}$, suggests that the macroMs were internalized by cells during the seeding period, followed by their exocytosis towards the cell culture media after $24 \mathrm{~h}$. Fluorescently labeled dextran and Ficoll have been shown to enter different cell types, including hMSCs, by macropinocytosis ${ }^{64-65}$ and micropinocytosis, ${ }^{66}$ respectively. Within the first $24 \mathrm{~h}$ after uptake, they have seen to accumulate in lysosomal compartments, were they degrade into glucose and fructose moieties. ${ }^{66-68}$ Moreover, since the cell culture media was renewed after $24 \mathrm{~h}$ culture and every 2 days from then on, the effect of the macroMs as macromolecular crowders influencing ECM deposition can be ruled out. The absence of macroM in the culture might be beneficial since no effect or even detrimental influence of macromolecular crowding has been shown on cartilaginous tissue formation in 3D. ${ }^{69}$ This was hypothesized to be mainly due to the fact that in a 3D cell confluent system cells already reside in the crowded microenvironment consisting of their secreted matrices. Moreover additional crowding could lead to inefficient oxygen and waste management and activation of degradation enzymes. However, there is lack of literature addressing the effect of macromolecular crowding in $3 \mathrm{D}$ cell systems and thus, no definitive conclusions can be drawn.

Regarding cell death, it is hypothesized that it occurred before their incubation with macroMs as i) it is known that internalized macroMs remain in the lysosomes with no effect on cellular function before their exocytosis to the extracellular space and ii) cell death was also visualized within the first minutes of hMSCs incubation even with medium without macroMs. Hence, the presence of dead cells in the scaffold might be attributed to the low media-to-cell ratio during the $4 \mathrm{~h}$ seeding and to the handling of cells prior to seeding 
(centrifugation and resuspension in a small volume of medium), which are unavoidable issues of the seeding process. In this regard, the visualization of macroMs in the scaffold even after $24 \mathrm{~h}$ culture is attributed to the ability of macroM to penetrate the permeable membrane of dead cells during the seeding and the subsequent inability of these cells to process the macroMs. ${ }^{70}$

Consistent with a previously published report, a cell monolayer was formed at the bottom of scaffolds seeded with CS method. ${ }^{24}$ Notably, this was not observed in the case of MS scaffolds. It is plausible that the high cell density at the bottom of CS scaffolds enabled cell-to-cell and cell-to-scaffold contact after their fast sedimentation, while the low cell number localized at the bottom filaments region of MS-scaffolds, due to no cell sedimentation during MS methods and unattached cells washing after seeding, prevented the formation of a cell monolayer. Importantly, the sedimented cells which attached to the bottom of CS scaffolds, might have significantly contributed to the relatively higher seeding efficiency values than expected ( $55 \%)$ for the high amount of cell settling observed, making it comparable to the MS methods seeding efficiency ( $80 \%$ and $\sim 75 \%$, for MSDextran and MS-Ficoll-Pq, respectively). In 2D, high cell seeding density (cells ${ }^{*} \mathrm{~cm}^{-2}$ ) has been demonstrated to direct and control early and late stage osteogenic markers and the temporal expression of MSCs genes towards osteogenesis. ${ }^{71}$ However, cell density coupled to an optimum distribution of cells occupying the whole scaffold surface area are the parameters that will ultimately favor osteogenesis in a 3D environment. Therefore, cell number alone is not a primary factor and "efficiency" is defined by both cell density and cell distribution when culturing 3D scaffolds. In our case, due to the higher chance of cell attachment when seeded with the MS methods, cells were confluently occupying the scaffold filaments from the beginning of the culture, resulting in surface saturation and contact inhibition between adjacent cells, ${ }^{72}$ which explains the lack of proliferation on these scaffolds over 28 days in BM. ${ }^{73}$ The increased matrix production in the MS scaffolds' pores volume when cultured in MM, might have offered the possibility to cells to grow not only on top of the scaffolds' filaments, but also on the ECM-filled scaffolds' pores. This increase in surface area available for cell proliferation between day 14 and day 28 was translated into an increase in cell number at late timepoints (day 28 in MM). Overall, cell confluency over the MS scaffolds, coupled to the osteogenic factors included in MM, showed to have positive effect on the osteogenic differentiation of the seeded hMSCs. This effect was initially demonstrated by the evaluation of ALP activity over the culture time, which is an early stage enzyme expressed in bone development and its upregulation 
precedes the upregulation of late osteogenic genes, such as $\mathrm{OCN}$, and matrix mineralization. ${ }^{74}$ Decreasing ALP activity over time, which enables the development of the bone matrix by preparing the extracellular matrix for the deposition of mineral, ${ }^{74}$ has previously been observed in scaffolds with high cell seeding numbers, and therefore possibly better cell distribution. ${ }^{75}$ Concordantly, a peak of ALP was seen at day 14 in MM, with values decreasing at day 28 in MM in cells seeded with MS methods despite the macroM type. On the contrary, ALP activity peaked after 28 days of culture in MM on scaffolds seeded with hMSCs via the CS method, as previously reported. ${ }^{61,76}$ These dissimilarities are likely due to the aforementioned differences in cell number coupled to their distribution profile within the scaffolds. Concordantly with the ALP activity progression, extensive ECM was produced over time on MS scaffolds and ARS staining results showed enhanced and progressively increased mineralization on MS scaffolds over the culture in MM. However, no clear hypothesis has been drawn to explain the increased calcium deposition on MS-Ficoll-Pq scaffolds at day 28 when compared to MSDextran, since cell viability was preserved in both cases and cell numbers and ALP activity values at the time point are comparable between both scaffold types.

Analyzing the gene expression, we observed that the upregulation of relevant protein encoding genes involved in osteogenic differentiation at day 28 in MM in cells seeded with MS-methods complements the abovementioned ALP activity and calcium deposition results. This further confirms that either there is a delay in the progression of osteogenesis in cells seeded with the CS method, or an acceleration in cells seeded with the MS methods, likely due to the differences in homogeneity in cell distribution. In particular, the gene encoding RUNX2, which is an essential transcription factor regulator of hMSCs differentiation into the osteogenic lineage, ${ }^{77-78}$ was upregulated for cells on MS scaffolds. It is known to target specific osteogenic genes, such as COL I, one of the major bone ECM components, or OCN, one of the major bone non-collagenous proteins with the ability to bind bone hydroxyapatite. ${ }^{79}$ Accordingly, COL I and OCN were upregulated in MS-Ficoll$\mathrm{Pq}$ and MS-dextran scaffolds, respectively, when compared to CS scaffolds, suggesting matrix maturation. Although $\mathrm{ON}$ did not differentially express in the MS scaffolds, this calcium binding non-collagenous protein has been found to be expressed in other nonmineralizing tissues, and therefore, lacks specificity. ${ }^{80}$ While longer culture periods of CS scaffolds in MM might possibly lead to comparable results among different seeding methods, ${ }^{81}$ the possibility of speeding the osteogenic process of hMSCs by MS-methods is desired for both the in vitro and in vivo applications of the scaffolds. 


\section{CONCLUSION}

Cell seeding on 3D AM scaffolds made of synthetic polymers with interconnected macroporosity still remains a challenge. The aim of this study was to develop a simple and reliable seeding technique to improve cell distribution upon static seeding valid for all 3D AM scaffolds regardless of their material properties and architecture. This was achieved by controlling the settling velocity of cells by altering the viscosity and density of the seeding media via the addition of two different macroMs, Dextran and Ficoll (Ficoll-Pq). Compared to scaffolds seeded with conventional static techniques, a homogeneous cell distribution was attained with direct cell attachment to the scaffold filaments. Importantly, macroMs did not affect viability or osteogenic differentiation of hMSCs and their complete removal was demonstrated after the seeding period. Intriguingly, the improved cell distribution from the macroMs based seeding led to enhanced mineralization and bone matrix maturation compared to conventional seeding. This newly proposed seeding method is simple, reproducible, and has the potential to improve the long-term functionality of in vitro and in vivo cell-seeded constructs for tissue engineering applications.

\section{Acknowledgement}

We are grateful to H2020-NMP-PILOTS-2015 (GA n. 685825) for financial support. Some of the materials used in this work were provided by the Texas A\&M Health Science Center College of Medicine Institute for Regenerative Medicine at Scott \& White through a grant from NCRR of the NIH (Grant \#P40RR017447). 


\section{References}

1. Place, E. S.; Evans, N. D.; Stevens, M. M., Complexity in Biomaterials for Tissue Engineering. Nature Materials 2009, 8, 457.

2. Deschamps, A. A.; Claase, M. B.; Sleijster, W. J.; de Bruijn, J. D.; Grijpma, D. W.; Feijen, J., Design of Segmented Poly(Ether Ester) Materials and Structures for the Tissue Engineering of Bone. Journal of Controlled Release 2002, 78 (1), 175-186.

3. Yang, S.; Leong, K.-F.; Du, Z.; Chua, C.-K., The Design of Scaffolds for Use in Tissue Engineering. Part I. Traditional Factors. Tissue engineering 2001, 7 (6), 679-689.

4. Karageorgiou, V.; Kaplan, D., Porosity of 3d Biomaterial Scaffolds and Osteogenesis. Biomaterials 2005, 26 (27), 5474-5491.

5. Mota, C.; Puppi, D.; Chiellini, F.; Chiellini, E., Additive Manufacturing Techniques for the Production of Tissue Engineering Constructs. J Tissue Eng Regen Med 2015, 9 (3), 174-190.

6. Guvendiren, M.; Molde, J.; Soares, R. M. D.; Kohn, J., Designing Biomaterials for 3d Printing. ACS Biomaterials Science \& Engineering 2016.

7. Nam, Y. S.; Yoon, J. J.; Park, T. G., A Novel Fabrication Method of Macroporous Biodegradable Polymer Scaffolds Using Gas Foaming Salt as a Porogen Additive. Journal of Biomedical Materials Research: An Official Journal of The Society for Biomaterials, The Japanese Society for Biomaterials, and The Australian Society for Biomaterials and the Korean Society for Biomaterials 2000, 53 (1), 1-7.

8. Kim, S.-S.; Park, M. S.; Jeon, O.; Choi, C. Y.; Kim, B.-S., Poly (Lactide-Co-Glycolide)/Hydroxyapatite Composite Scaffolds for Bone Tissue Engineering. Biomaterials 2006, 27 (8), 1399-1409.

9. Murphy, C. M.; Haugh, M. G.; O'Brien, F. J., The Effect of Mean Pore Size on Cell Attachment, Proliferation and Migration in Collagen-Glycosaminoglycan Scaffolds for Bone Tissue Engineering. Biomaterials 2010, 31 (3), 461-466.

10. Sultana, N.; Wang, M., Phbv/Plla-Based Composite Scaffolds Fabricated Using an Emulsion Freezing/Freeze-Drying Technique for Bone Tissue Engineering: Surface Modification and in Vitro Biological Evaluation. Biofabrication 2012, 4 (1), 015003.

11. Wei, G.; Ma, P. X., Structure and Properties of Nano-Hydroxyapatite/Polymer Composite Scaffolds for Bone Tissue Engineering. Biomaterials 2004, 25 (19), 4749-4757.

12. Akbarzadeh, R.; Yousefi, A. M., Effects of Processing Parameters in Thermally Induced Phase Separation Technique on Porous Architecture of Scaffolds for Bone Tissue Engineering. Journal of biomedical materials research. Part B, Applied biomaterials 2014, 102 (6), 1304-1315.

13. Luyten, F. P.; Dell'Accio, F.; De Bari, C., Skeletal Tissue Engineering: Opportunities and Challenges. Best Practice \& Research Clinical Rheumatology 2001, 15 (5), 759-769.

14. Hasegawa, T.; Miwa, M.; Sakai, Y.; Niikura, T.; Lee, S. Y.; Oe, K.; Iwakura, T.; Kurosaka, M.; Komori, T., Efficient Cell-Seeding into Scaffolds Improves Bone Formation. Journal of Dental Research 2010, 89 (8), 854-859.

15. Burg, K.; Holder Jr, W.; Culberson, C.; Beiler, R.; Greene, K.; Loebsack, A.; Roland, W.; Eiselt, P.; Mooney, D.; Halberstadt, C., Comparative Study of Seeding Methods for Three-Dimensional Polymeric Scaffolds. Journal of biomedical materials research 2000, 51 (4), 642-649.

16. Thevenot, P.; Nair, A.; Dey, J.; Yang, J.; Tang, L., Method to Analyze Three-Dimensional Cell Distribution and Infiltration in Degradable Scaffolds. Tissue Engineering Part C: Methods 2008, 14 (4), 319-331.

17. Weinand, C.; Xu, J. W.; Peretti, G. M.; Bonassar, L. J.; Gill, T. J., Conditions Affecting Cell Seeding onto Three-Dimensional Scaffolds for Cellular-Based Biodegradable Implants. Journal of Biomedical Materials Research Part B: Applied Biomaterials 2009, 91 (1), 80-87.

18. Alvarez-Barreto, J. F.; Linehan, S. M.; Shambaugh, R. L.; Sikavitsas, V. I., Flow Perfusion Improves Seeding of Tissue Engineering Scaffolds with Different Architectures. Annals of Biomedical Engineering 2007, 35 (3), 429-442.

19. Zhang, Z.-Z.; Jiang, D.; Wang, S.-J.; Qi, Y.-S.; Zhang, J.-Y.; Yu, J.-K., Potential of Centrifugal Seeding Method in Improving Cells Distribution and Proliferation on Demineralized Cancellous Bone Scaffolds for Tissue-Engineered Meniscus. ACS applied materials \& interfaces 2015, 7 (28), 15294-15302.

20. Magrofuoco, E.; Flaibani, M.; Giomo, M.; Elvassore, N., Cell Culture Distribution in a Three-Dimensional Porous Scaffold in Perfusion Bioreactor. Biochemical engineering journal 2019, 146, 10-19. 
21. Leferink, A. M.; Chng, Y.-C.; van Blitterswijk, C. A.; Moroni, L., Distribution and Viability of Fetal and Adult Human Bone Marrow Stromal Cells in a Biaxial Rotating Vessel Bioreactor after Seeding on Polymeric 3d Additive Manufactured Scaffolds. Frontiers in bioengineering and biotechnology 2015, 3, 169-169.

22. Impens, S.; Chen, Y.; Mullens, S.; Luyten, F.; Schrooten, J., Controlled Cell-Seeding Methodologies: A First Step toward Clinically Relevant Bone Tissue Engineering Strategies. Tissue Engineering Part C: Methods 2010, 16 (6), 1575-1583.

23. Chen, Y.; Bloemen, V.; Impens, S.; Moesen, M.; Luyten, F. P.; Schrooten, J., Characterization and Optimization of Cell Seeding in Scaffolds by Factorial Design: Quality by Design Approach for Skeletal Tissue Engineering. Tissue Engineering Part C: Methods 2011, 17 (12), 1211-1221.

24. Leferink, A. M.; Hendrikson, W.; Rouwkema, J.; Karperien, M.; van Blitterswijk, C.; Moroni, L., Increased Cell Seeding Efficiency in Bioplotted Three-Dimensional Peot/Pbt Scaffolds. Journal of tissue engineering and regenerative medicine 2016, 10 (8), 679-689.

25. Yildirim, E. D.; Pappas, D.; Güçeri, S.; Sun, W., Enhanced Cellular Functions on Polycaprolactone Tissue Scaffolds by O2 Plasma Surface Modification. Plasma Processes and Polymers 2011, 8 (3), 256-267.

26. Domingos, M.; Intranuovo, F.; Gloria, A.; Gristina, R.; Ambrosio, L.; Bártolo, P. J.; Favia, P., Improved Osteoblast Cell Affinity on Plasma-Modified 3-D Extruded Pcl Scaffolds. Acta Biomaterialia 2013, 9 (4), 5997-6005.

27. HoJun, J.; Hyeongjin, L.; GeunHyung, K., A Surface-Modified Poly(E-Caprolactone) Scaffold Comprising Variable Nanosized Surface-Roughness Using a Plasma Treatment. Tissue Engineering Part C: Methods 2014, 20 (12), 951-963

28. Cools, P.; Mota, C.; Lorenzo-Moldero, I.; Ghobeira, R.; De Geyter, N.; Moroni, L.; Morent, R., Acrylic Acid Plasma Coated 3d Scaffolds for Cartilage Tissue Engineering Applications. Sci Rep 2018, 8 (1), 3830.

29. Jo, S.; Kang, S. M.; Park, S. A.; Kim, W. D.; Kwak, J.; Lee, H., Enhanced Adhesion of Preosteoblasts inside $3 \mathrm{~d}$ Pcl Scaffolds by Polydopamine Coating and Mineralization. Macromolecular bioscience 2013, 13 (10), 1389-1395.

30. Lee, S. J.; Lee, D.; Yoon, T. R.; Kim, H. K.; Jo, H. H.; Park, J. S.; Lee, J. H.; Kim, W. D.; Kwon, I. K.; Park, S. A., Surface Modification of 3d-Printed Porous Scaffolds Via Mussel-Inspired Polydopamine and Effective Immobilization of Rhbmp-2 to Promote Osteogenic Differentiation for Bone Tissue Engineering. Acta Biomaterialia 2016, 40, 182-191.

31. Kao, C.-T.; Lin, C.-C.; Chen, Y.-W.; Yeh, C.-H.; Fang, H.-Y.; Shie, M.-Y., Poly(Dopamine) Coating of 3d Printed Poly(Lactic Acid) Scaffolds for Bone Tissue Engineering. Materials Science and Engineering: C 2015, 56, 165-173.

32. Sobral, J. M.; Caridade, S. G.; Sousa, R. A.; Mano, J. F.; Reis, R. L., Three-Dimensional Plotted Scaffolds with Controlled Pore Size Gradients: Effect of Scaffold Geometry on Mechanical Performance and Cell Seeding Efficiency. Acta Biomaterialia 2011, 7 (3), 1009-1018.

33. Ostrowska, B.; Di Luca, A.; Moroni, L.; Swieszkowski, W., Influence of Internal Pore Architecture on Biological and Mechanical Properties of Three-Dimensional Fiber Deposited Scaffolds for Bone Regeneration. Journal of Biomedical Materials Research Part A 2016.

34. Hutmacher, D. W., Scaffolds in Tissue Engineering Bone and Cartilage. Biomaterials 2000, 21 (24), 2529 2543.

35. Van Bael, S.; Chai, Y. C.; Truscello, S.; Moesen, M.; Kerckhofs, G.; Van Oosterwyck, H.; Kruth, J. P.; Schrooten, J., The Effect of Pore Geometry on the in Vitro Biological Behavior of Human PeriosteumDerived Cells Seeded on Selective Laser-Melted Ti6al4v Bone Scaffolds. Acta Biomaterialia 2012, 8 (7), 2824-2834.

36. Zadpoor, A. A., Bone Tissue Regeneration: The Role of Scaffold Geometry. Biomaterials Science 2015, 3 (2), 231-245.

37. Dong, L.; Wang, S.-J.; Zhao, X.-R.; Zhu, Y.-F.; Yu, J.-K., 3d- Printed Poly(E-Caprolactone) Scaffold Integrated with Cell-Laden Chitosan Hydrogels for Bone Tissue Engineering. Scientific reports 2017, 7 (1), $13412-13412$.

38. Papadimitropoulos, A.; Riboldi, S. A.; Tonnarelli, B.; Piccinini, E.; Woodruff, M. A.; Hutmacher, D. W.; Martin, I., A Collagen Network Phase Improves Cell Seeding of Open-Pore Structure Scaffolds under Perfusion. Journal of Tissue Engineering and Regenerative Medicine 2013, 7 (3), 183-191.

39. Markovic, M.; Van Hoorick, J.; Hölzl, K.; Tromayer, M.; Gruber, P.; Nürnberger, S.; Dubruel, P.; Van Vlierberghe, S.; Liska, R.; Ovsianikov, A., Hybrid Tissue Engineering Scaffolds by Combination of Three- 
Dimensional Printing and Cell Photoencapsulation. Journal of nanotechnology in engineering and medicine 2015, 6 (2), 021001.

40. Whitely, M.; Cereceres, S.; Dhavalikar, P.; Salhadar, K.; Wilems, T.; Smith, B.; Mikos, A.; CosgriffHernandez, E., Improved in Situ Seeding of 3d Printed Scaffolds Using Cell-Releasing Hydrogels. Biomaterials 2018, 185, 194-204.

41. Kim, W. J.; Yun, H.-S.; Kim, G. H., An Innovative Cell-Laden A-Tcp/Collagen Scaffold Fabricated Using a Two-Step Printing Process for Potential Application in Regenerating Hard Tissues. Scientific reports 2017, 7 (1), 3181-3181.

42. Engler, A. J.; Sen, S.; Sweeney, H. L.; Discher, D. E., Matrix Elasticity Directs Stem Cell Lineage Specification. Cell 2006, 126 (4), 677-689.

43. Gregory, C. A.; Gunn, W. G.; Peister, A.; Prockop, D. J., An Alizarin Red-Based Assay of Mineralization by Adherent Cells in Culture: Comparison with Cetylpyridinium Chloride Extraction. Analytical biochemistry 2004, 329 (1), 77-84.

44. Lamb, Hydrodynamics. 6th ed.; Cambridge University Press: Cambridge, 1994.

45. Chang, Y.; Hsieh, P. H.; Chao, C. C., The Efficiency of Percoll and Ficoll Density Gradient Media in the Isolation of Marrow Derived Human Mesenchymal Stem Cells with Osteogenic Potential. Chang Gung medical journal 2009, 32 (3), 264-275.

46. Insausti, C. L.; Blanquer, M. B.; Olmo, L. M.; López-Martínez, M. C.; Ruiz, X. F.; Lozano, F. J. R.; Perianes, V. C.; Funes, C.; Nicolás, F. J.; Majado, M. J.; Jiménez, J. M. M., Isolation and Characterization of Mesenchymal Stem Cells from the Fat Layer on the Density Gradient Separated Bone Marrow. Stem Cells and Development 2012, 21 (2), 260-272.

47. Chen, C.; Loe, F.; Blocki, A.; Peng, Y.; Raghunath, M., Applying Macromolecular Crowding to Enhance Extracellular Matrix Deposition and Its Remodeling in Vitro for Tissue Engineering and Cell-Based Therapies. Advanced Drug Delivery Reviews 2011, 63 (4), 277-290.

48. Satyam, A.; Kumar, P.; Fan, X.; Gorelov, A.; Rochev, Y.; Joshi, L.; Peinado, H.; Lyden, D.; Thomas, B.; Rodriguez, B.; Pandit, A.; Zeugolis, D., Macromolecular Crowding Meets Tissue Engineering by SelfAssembly: A Paradigm Shift in Regenerative Medicine. Advanced materials 2014, 26 (19), 3024-3034.

49. Lareu, R. R.; Subramhanya, K. H.; Peng, Y.; Benny, P.; Chen, C.; Wang, Z.; Rajagopalan, R.; Raghunath, M., Collagen Matrix Deposition Is Dramatically Enhanced in Vitro When Crowded with Charged Macromolecules: The Biological Relevance of the Excluded Volume Effect. FEBS letters 2007, 581 (14), 2709-2714.

50. Sikavitsas, V. I.; Bancroft, G. N.; Holtorf, H. L.; Jansen, J. A.; Mikos, A. G., Mineralized Matrix Deposition by Marrow Stromal Osteoblasts in 3d Perfusion Culture Increases with Increasing Fluid Shear Forces. Proceedings of the national academy of sciences 2003, 100 (25), 14683-14688.

51. Rosenblum, W. I., Effects of Dextran-40 on Blood Viscosity in Experimental Macroglobulinaemia. Nature 1968, 218 (5141), 591-593.

52. Pribush, A.; Zilberman-Kravits, D.; Meyerstein, N., The Mechanism of the Dextran-Induced Red Blood Cell Aggregation. European Biophysics Journal 2007, 36 (2), 85-94.

53. Neu, B.; Wenby, R.; Meiselman, H. J., Effects of Dextran Molecular Weight on Red Blood Cell Aggregation. Biophysical journal 2008, 95 (6), 3059-3065.

54. Bøyum, A., Separation of Blood Leucocytes, Granulocytes and Lymphocytes. Tissue antigens 1974, 4 (3), 269-274.

55. Fuss, I. J.; Kanof, M. E.; Smith, P. D.; Zola, H., Isolation of Whole Mononuclear Cells from Peripheral Blood and Cord Blood. Current protocols in immunology 2009, Chapter 7, Unit7.1.

56. Kumar, P.; Satyam, A.; Fan, X.; Collin, E.; Rochev, Y.; Rodriguez, B. J.; Gorelov, A.; Dillon, S.; Joshi, L.; Raghunath, M., Macromolecularly Crowded in Vitro Microenvironments Accelerate the Production of Extracellular Matrix-Rich Supramolecular Assemblies. Scientific reports 2015, 5, 8729.

57. Deschamps, A. A.; Grijpma, D. W.; Feijen, J., Poly(Ethylene Oxide)/Poly(Butylene Terephthalate) Segmented Block Copolymers: The Effect of Copolymer Composition on Physical Properties and Degradation Behavior. Polymer 2001, 42 (23), 9335-9345.

58. Van Blitterswijk, C.; Leenders, H.; Baaker, D., The Effect of Peo Ratio on Degradation, Calcification and Bone Bonding of Peo/Pbt Copolymer (Polyactive). Cells and Materials 1993, 3 (1), 2. 
59. Jansen, E. J. P.; Pieper, J.; Gijbels, M. J. J.; Guldemond, N. A.; Riesle, J.; Van Rhijn, L. W.; Bulstra, S. K.; Kuijer, R., Peot/Pbt Based Scaffolds with Low Mechanical Properties Improve Cartilage Repair Tissue Formation in Osteochondral Defects. Journal of Biomedical Materials Research Part A 2009, 89A (2), 444452.

60. Moroni, L.; De Wijn, J.; Van Blitterswijk, C., 3d Fiber-Deposited Scaffolds for Tissue Engineering: Influence of Pores Geometry and Architecture on Dynamic Mechanical Properties. Biomaterials 2006, 27 (7), 974 985.

61. Di Luca, A.; Ostrowska, B.; Lorenzo-Moldero, I.; Lepedda, A.; Swieszkowski, W.; Van Blitterswijk, C.; Moroni, L., Gradients in Pore Size Enhance the Osteogenic Differentiation of Human Mesenchymal Stromal Cells in Three-Dimensional Scaffolds. Scientific Reports 2016, 6, 22898.

62. Massia, S. P.; Stark, J.; Letbetter, D. S., Surface-Immobilized Dextran Limits Cell Adhesion and Spreading. Biomaterials 2000, 21 (22), 2253-2261.

63. Sun, G.; Mao, J. J., Engineering Dextran-Based Scaffolds for Drug Delivery and Tissue Repair. Nanomedicine 2012, 7 (11), 1771-1784.

64. Teo, B. K. K.; Goh, S.-H.; Kustandi, T. S.; Loh, W. W.; Low, H. Y.; Yim, E. K. F., The Effect of Micro and Nanotopography on Endocytosis in Drug and Gene Delivery Systems. Biomaterials 2011, 32 (36), 98669875.

65. Sánchez-Abarca, L. I.; Álvarez-Laderas, I.; Díez Campelo, M.; Caballero-Velázquez, T.; Herrero, C.; Muntión, S.; Calderón, C.; García-Guerrero, E.; Sánchez-Guijo, F.; del Cañizo, C.; San Miguel, J.; PérezSimón, J. A., Uptake and Delivery of Antigens by Mesenchymal Stromal Cells. Cytotherapy 2013, 15 (6), 673-678.

66. Rashid, R.; Beyer, S.; Blocki, A.; Le Visage, C.; Trau, D.; Wohland, T.; Raghunath, M., Mitochondrial Routing of Glucose and Sucrose Polymers after Pinocytotic Uptake: Avenues for Drug Delivery. Biomacromolecules 2014, 15 (6), 2119-2127.

67. Dragonetti, A.; Baldassarre, M.; Castino, R.; Demoz, M.; Luini, A.; Buccione, R.; Isidoro, C., The Lysosomal Protease Cathepsin D Is Efficiently Sorted to and Secreted from Regulated Secretory Compartments in the Rat Basophilic/Mast Cell Line Rbl. Journal of Cell Science 2000, 113 (18), 3289-3298.

68. Cohen, R.; Corwith, K.; Holowka, D.; Baird, B., Spatiotemporal Resolution of Mast Cell Granule Exocytosis Reveals Correlation with Ca2+ Wave Initiation. J Cell Sci 2012, 125 (12), 2986-2994.

69. Chen, B.; Wang, B.; Zhang, W. J.; Zhou, G.; Cao, Y.; Liu, W., Macromolecular Crowding Effect on Cartilaginous Matrix Production: A Comparison of Two-Dimensional and Three-Dimensional Models. Tissue Engineering Part C: Methods 2013, 19 (8), 586-595.

70. Moumaris, M.; Rajoelya, B.; Abuafa, N., Fluorescein Isothiocyanate-Dextran Can Track Apoptosis and Necrosis Induced by Heat Shock of Peripheral Blood Mononuclear Cells and Hela Cells. Open Biological Sciences Journal 2015, 1 (1).

71. Kim, K.; Dean, D.; Mikos, A. G.; Fisher, J. P., Effect of Initial Cell Seeding Density on Early Osteogenic Signal Expression of Rat Bone Marrow Stromal Cells Cultured on Cross-Linked Poly(Propylene Fumarate) Disks. Biomacromolecules 2009, 10 (7), 1810-1817.

72. Eagle, H.; Levine, E. M., Growth Regulatory Effects of Cellular Interaction. Nature 1967, 213 (5081), 11021106.

73. Lode, A.; Bernhardt, A.; Gelinsky, M., Cultivation of Human Bone Marrow Stromal Cells on ThreeDimensional Scaffolds of Mineralized Collagen: Influence of Seeding Density on Colonization, Proliferation and Osteogenic Differentiation. Journal of Tissue Engineering and Regenerative Medicine 2008, 2 (7), 400-407.

74. Owen, T. A.; Aronow, M.; Shalhoub, V.; Barone, L. M.; Wilming, L.; Tassinari, M. S.; Kennedy, M. B.; Pockwinse, S.; Lian, J. B.; Stein, G. S., Progressive Development of the Rat Osteoblast Phenotype in Vitro: Reciprocal Relationships in Expression of Genes Associated with Osteoblast Proliferation and Differentiation During Formation of the Bone Extracellular Matrix. Journal of cellular physiology 1990, 143 (3), 420-430.

75. Zhou, Y. F.; Sae-Lim, V.; Chou, A. M.; Hutmacher, D. W.; Lim, T. M., Does Seeding Density Affect in Vitro Mineral Nodules Formation in Novel Composite Scaffolds? Journal of Biomedical Materials Research Part A 2006, 78A (1), 183-193.

76. Di Luca, A.; Longoni, A.; Criscenti, G.; Mota, C.; van Blitterswijk, C.; Moroni, L., Toward Mimicking the Bone Structure: Design of Novel Hierarchical Scaffolds with a Tailored Radial Porosity Gradient. Biofabrication 2016, 8 (4), 045007. 
77. Komori, T.; Yagi, H.; Nomura, S.; Yamaguchi, A.; Sasaki, K.; Deguchi, K.; Shimizu, Y.; Bronson, R. T.; Gao, Y. H.; Inada, M.; Sato, M.; Okamoto, R.; Kitamura, Y.; Yoshiki, S.; Kishimoto, T., Targeted Disruption of Cbfa1 Results in a Complete Lack of Bone Formation Owing to Maturational Arrest of Osteoblasts. Cell 1997, 89 (5), 755-764.

78. Li, Y.; Ge, C.; Long, J. P.; Begun, D. L.; Rodriguez, J. A.; Goldstein, S. A.; Franceschi, R. T., Biomechanical Stimulation of Osteoblast Gene Expression Requires Phosphorylation of the Runx2 Transcription Factor. Journal of bone and mineral research : the official journal of the American Society for Bone and Mineral Research 2012, 27 (6), 1263-1274.

79. Ivaska, K. K.; Hentunen, T. A.; Vaaraniemi, J.; Ylipahkala, H.; Pettersson, K.; Vaananen, H. K., Release of Intact and Fragmented Osteocalcin Molecules from Bone Matrix During Bone Resorption in Vitro. The Journal of biological chemistry 2004, 279 (18), 18361-18369.

80. Rosset, E. M.; Bradshaw, A. D., Sparc/Osteonectin in Mineralized Tissue. Matrix biology : journal of the International Society for Matrix Biology 2016, 52-54, 78-87.

81. Holy, C. E.; Shoichet, M. S.; Davies, J. E., Engineering Three-Dimensional Bone Tissue in Vitro Using Biodegradable Scaffolds: Investigating Initial Cell-Seeding Density and Culture Period. Journal of Biomedical Materials Research: An Official Journal of The Society for Biomaterials, The Japanese Society for Biomaterials, and The Australian Society for Biomaterials and the Korean Society for Biomaterials 2000, 51 (3), 376-382. 


\section{SUPPLEMENTARY FIGURES}

Table S1. Primer sequences used for q-PCR. B2M: beta-2-microglobulin; RUNX2: runt-related transcription factor; COL1A1: collagen 1 alpha 1; OCN: osteocalcin: SPARC: osteonectin.

\begin{tabular}{c|c|c}
\hline Gene & Forward primer & Reverse primer \\
\hline B2M & ACAAAGTCACATGGTTCACA & GACTTGTCTTCAGCAAGGA \\
RUNX2 & TGGTIACTGTCATGGCGGGTA & TCTCAGATCGTTGAACCTTGCTA \\
COL1A1 & GAGGGCCAAGACGAAGACATC & CAGATCACGTCATCGCACAAC \\
OCN & TGAGAGCCCTCACACTCCTC & CGCCTGGGTCTCTTCACTAC \\
SPARC & AGCACCCCATTGACGGGTA & GGTCACAGGTCTCGAAAAAGC \\
\hline
\end{tabular}

A

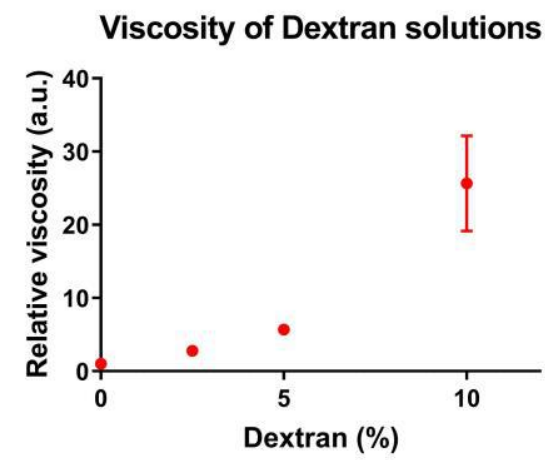

C

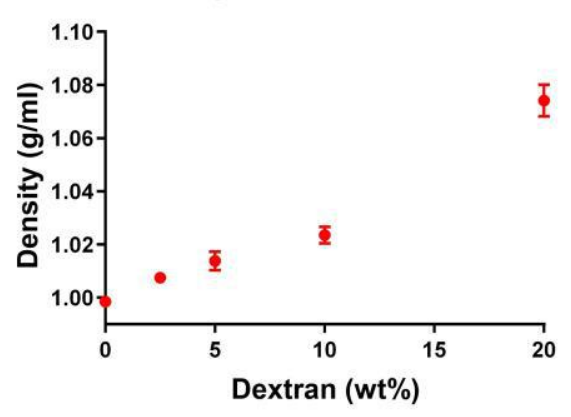

$\mathrm{E}$

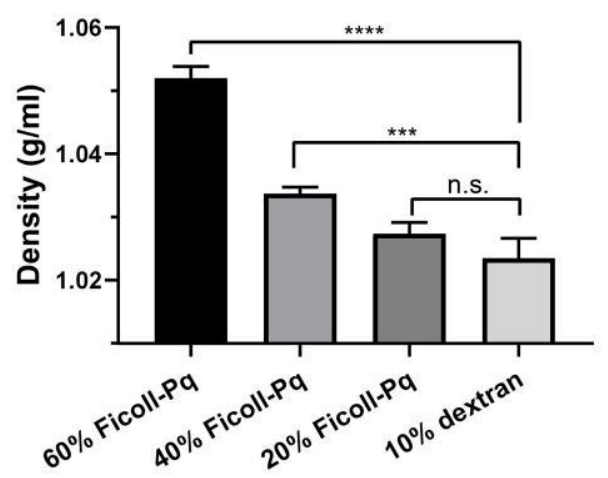

B

Viscosity of Ficoll-Pq solutions

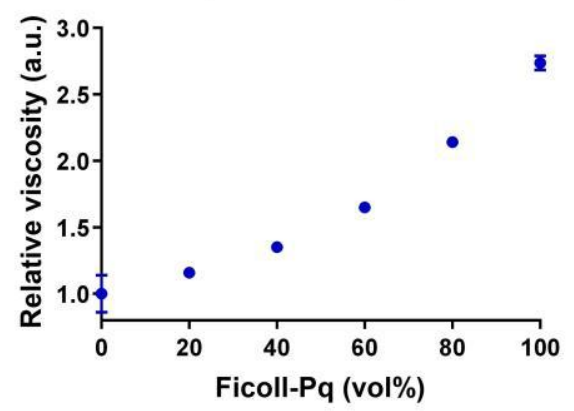

D

Density of Ficoll-Pq solutions

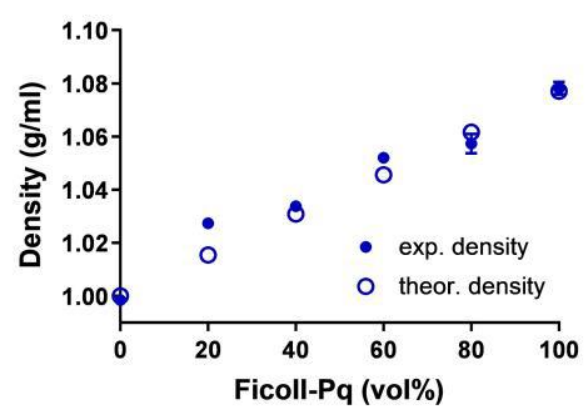

$\mathrm{F}$

Cell settling velocity calculation

\begin{tabular}{l|ccc}
\hline & CS & MS-Dextran & MS-Ficoll-Pq \\
\hline$R$ cell $(\mu \mathrm{m})$ & 5 & 5 & 5 \\
\hline$\rho$ cell $(\mathrm{g} / \mathrm{ml})$ & 1.052 & 1.052 & 1.052 \\
\hline$\rho$ medium $(\mathrm{g} / \mathrm{ml})$ & 1 & 1.024 & 1.052 \\
\hline$\mu$ medium $(\mathrm{kg} / \mathrm{m}-\mathrm{s})$ & 0.0010 & 0.0257 & 0.0016 \\
\hline $\mathbf{V}(\mu \mathrm{m} / \mathrm{s})$ & $\mathbf{1 . 8 1 3}$ & $\mathbf{0 . 0 3 8}$ & $\mathbf{0}$ \\
\hline $\begin{array}{l}\text { sedimentation in } \\
\text { 4h }(\mathrm{mm})\end{array}$ & 2.610 & 0.055 & 0 \\
\hline
\end{tabular}

Figure S1. (A, B) Relative viscosity and (C, D) density of cell culture media containing different concentrations of dextran and Ficoll. (E) Comparison of density values of media containing specific dextran and Ficoll concentrations. (F) Cell settling velocity in CS, MS-Dextran and MS-Ficoll methods. 


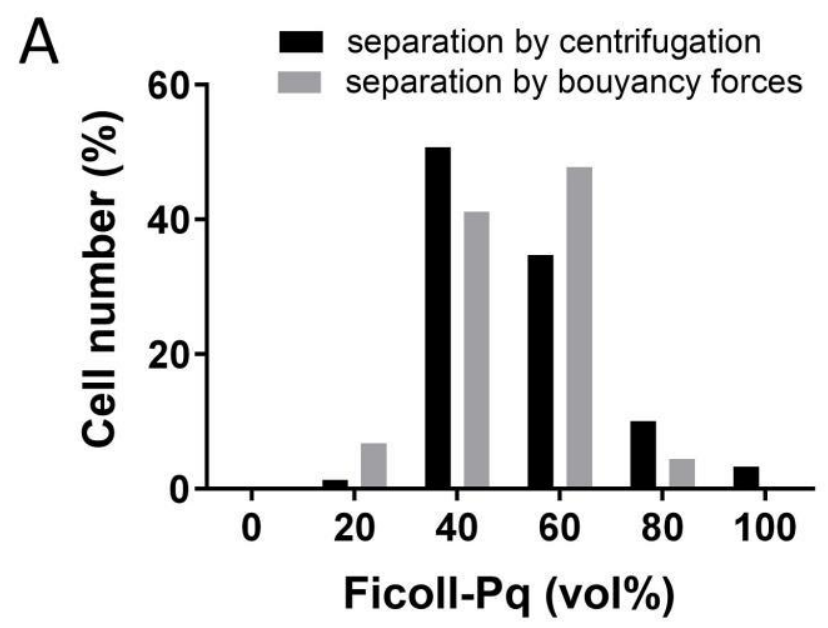

B Separation by centrifugation
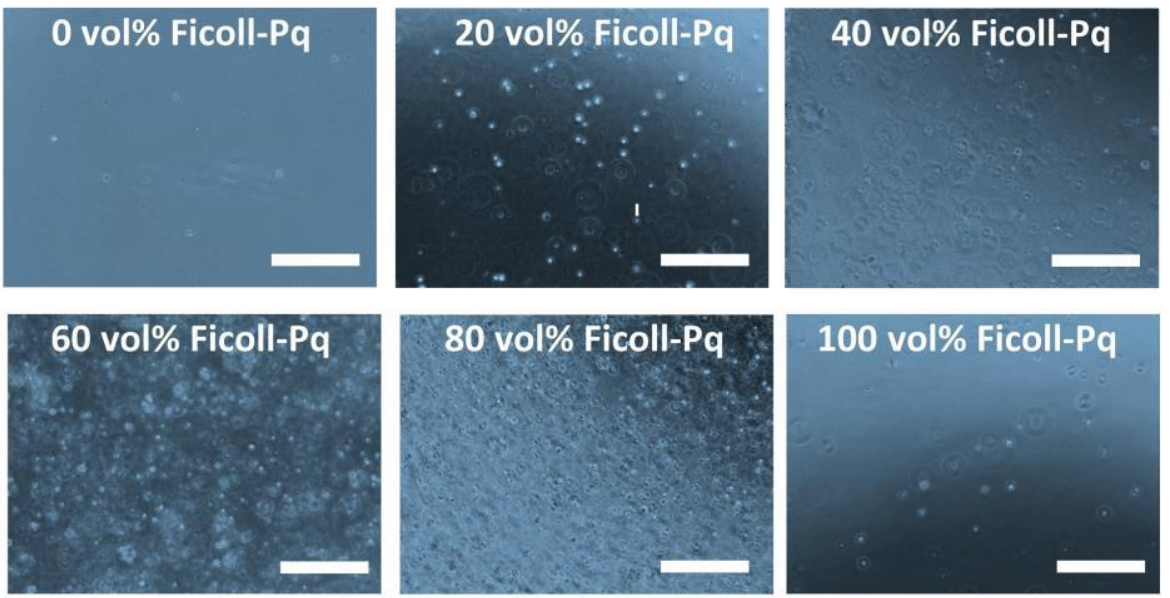

Separation by bouyancy forces
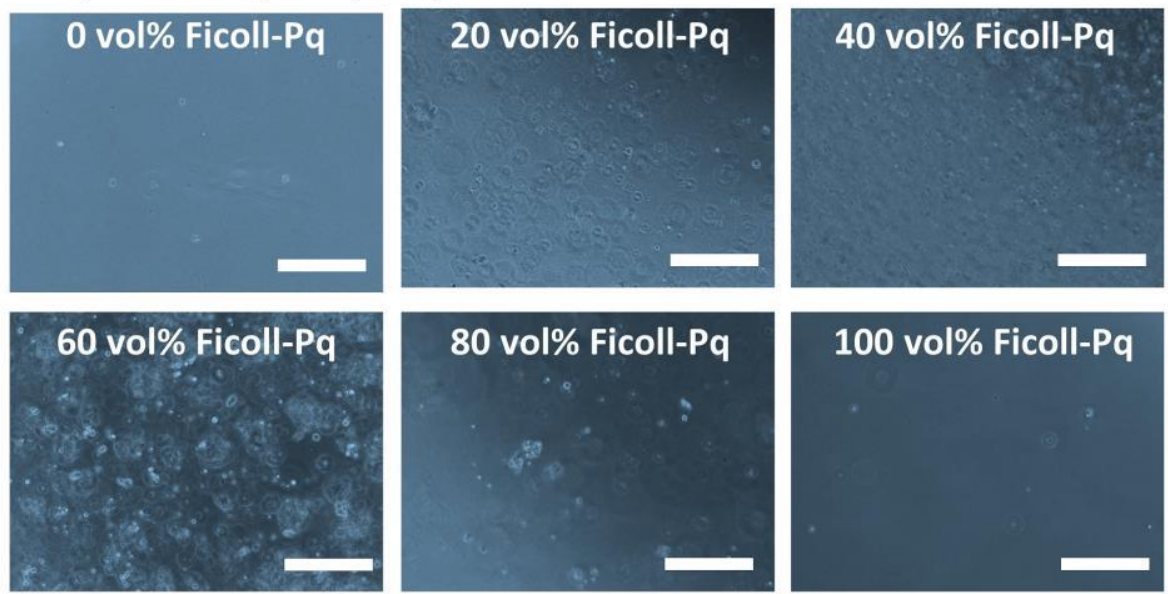

Figure S2. (A) Quantification of cell number in each layer of the Ficoll gradient used to determine hMSCs population density by separation by centrifugation or by separation by buoyancy forces. (B) Representative images of the cells found in each layer after each separation method. Scale bars $200 \mu \mathrm{m}$. 

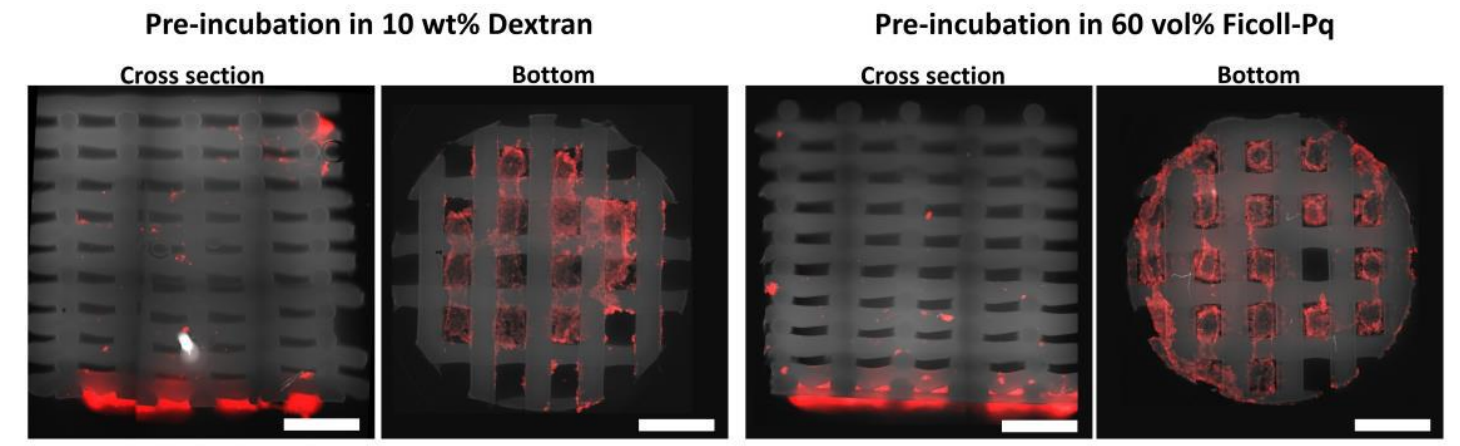

Figure S3. Fluorescence microscopy images of hMSCs in the cross sections and bottom lids of scaffolds pre-incubated with dextran and Ficoll based solutions and seeded with the CS method. Scale bar $1 \mathrm{~mm}$.
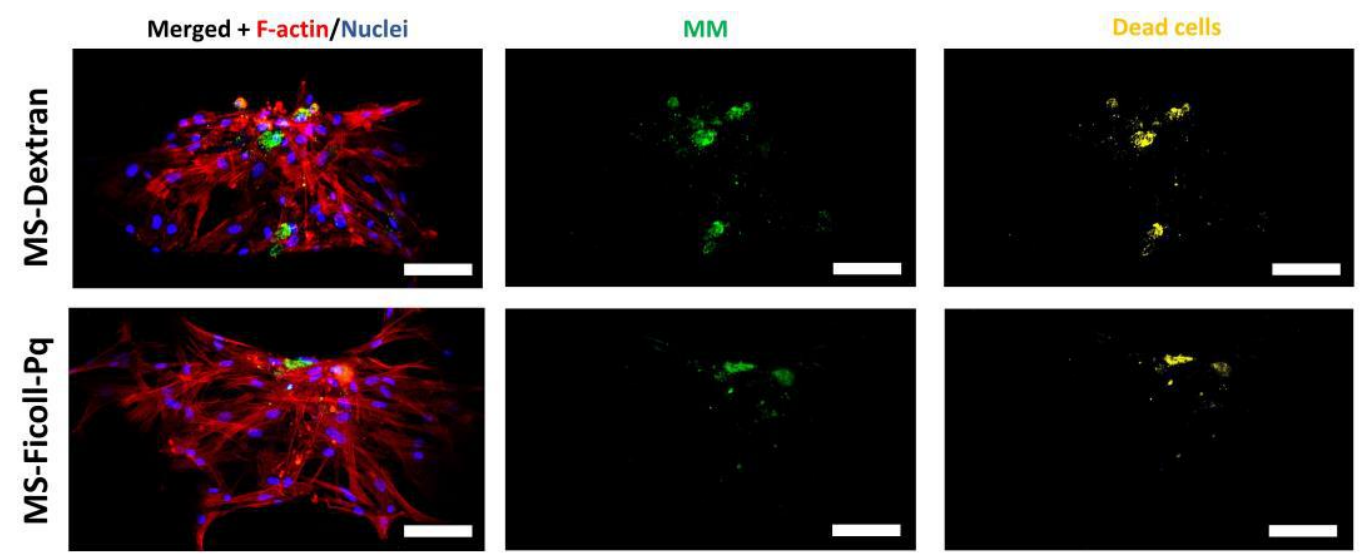

Figure S4. Representative confocal microscopy images of hMSCS (F-actin and nuclei), macroMs (FITC-labeled) and dead cells on top of scaffold fiber $24 \mathrm{~h}$ post-seeding with the MS-Dextran and MS-Ficoll methods. Scale bars $100 \mu \mathrm{m}$.
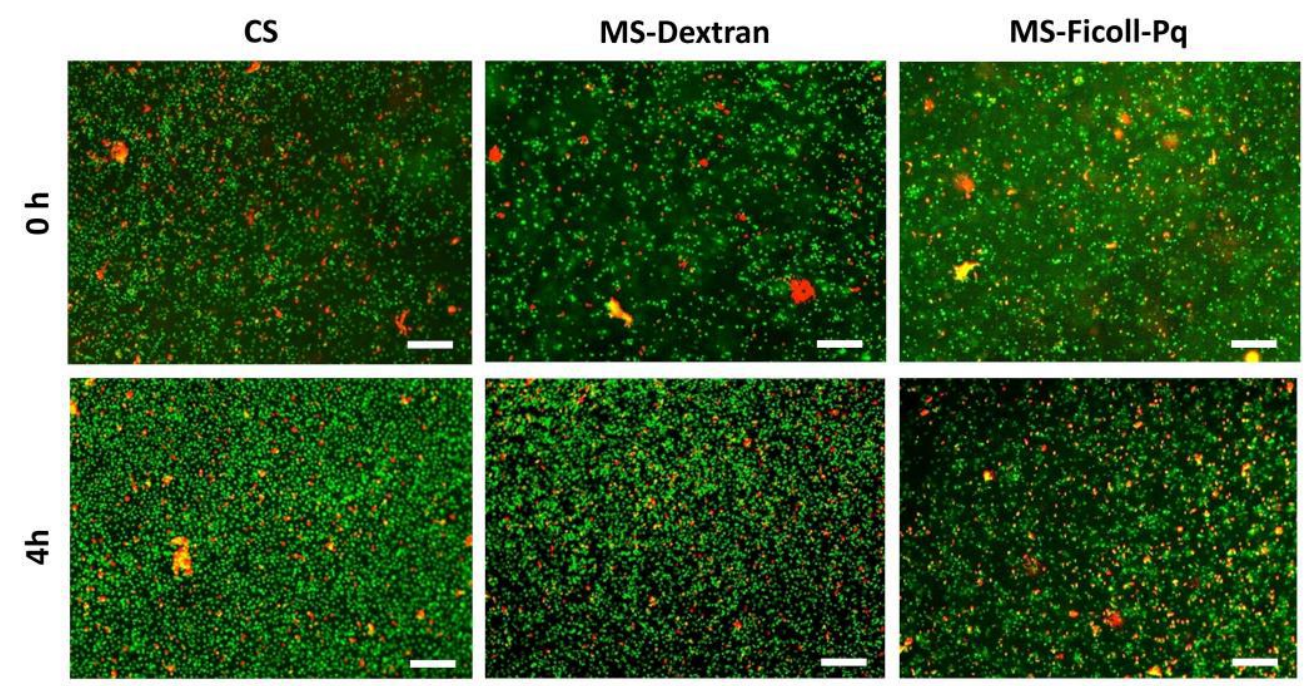

Figure S5. Representative fluorescent images depicting hMSCs viability (live, green; dead, red) right after their resuspension $(0 \mathrm{~h})$ and after $4 \mathrm{~h}$ incubation in macroM based solutions, as compared to control (CS). Scale bars $250 \mu \mathrm{m}$. 


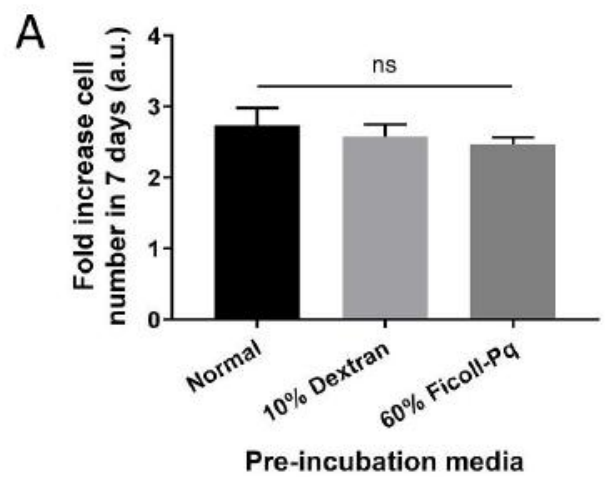

B

Pre-incubation media

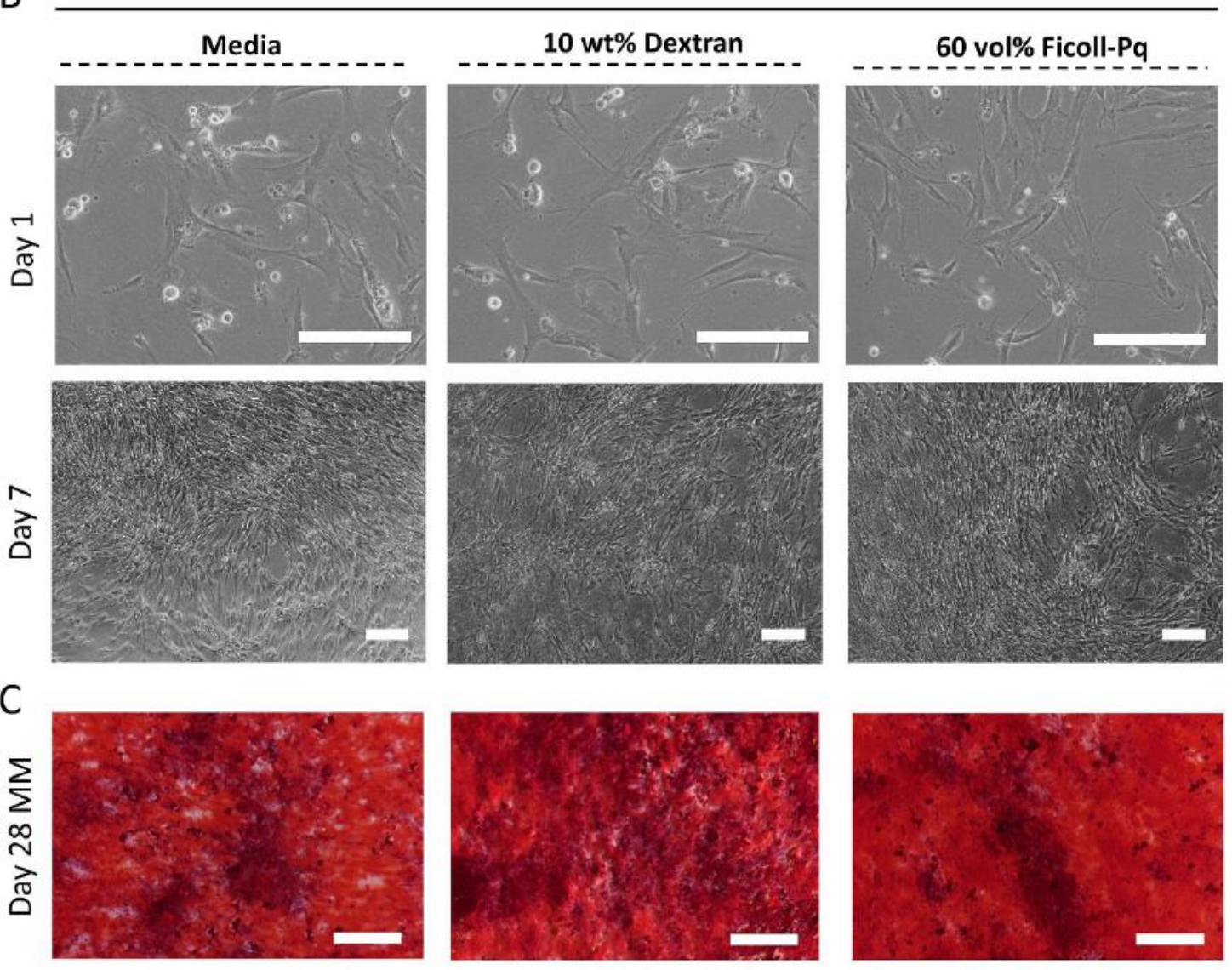

Figure S6. Cell culture and differentiation in 2D after hMSCs incubation in macroM based solutions during $4 \mathrm{~h}$ mimicking the scaffold seeding conditions. (A) Fold increase in cell number after 7 days of culture in BM and pre-incubation in normal, dextran or Ficoll based CM. (B) Representative brightfield images of cells pre-incubated in normal, dextran or Ficoll based CM after 1 and 7 days of culture in BM. (C) Stereomicroscopy images of cell monolayers stained with ARS after 28 days of culture (21 days in MM) and pre-incubated in normal, dextran or Ficoll based CM. Scale bars $250 \mu \mathrm{m}$. 


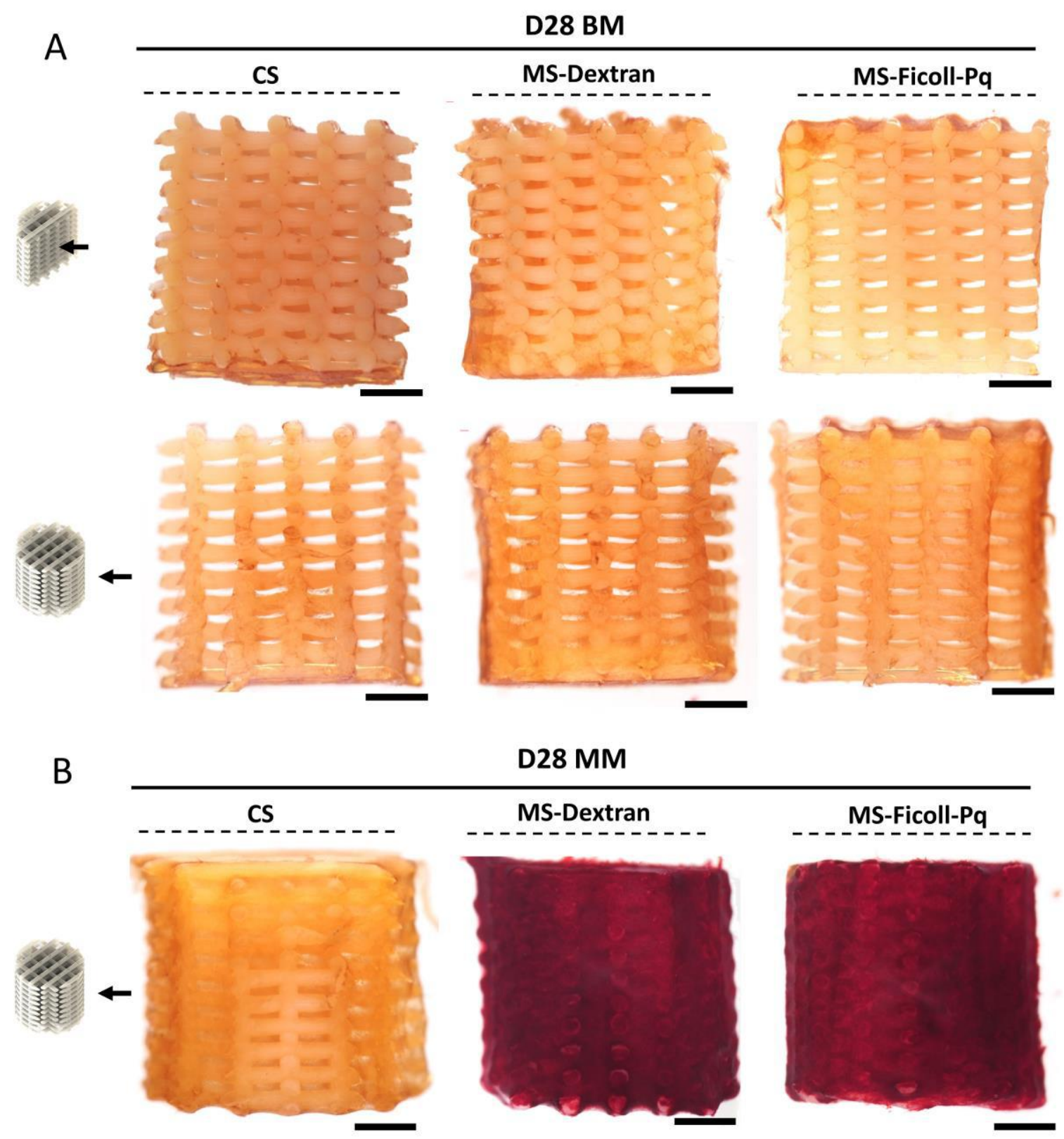

Figure S7. Stereomicroscopy images of ARS stained scaffolds. (A) Cross- and outer sections after 28 days of culture in BM and $(B)$ outer sections after 28 days of culture (21 days in MM). Scale bars $1 \mathrm{~mm}$. 


\section{Chapter 4}

Tuning cell behavior on 3D scaffolds fabricated by atmospheric plasma assisted additive manufacturing 



\title{
Tuning cell behavior on 3D scaffolds fabricated by atmospheric plasma assisted additive manufacturing
}

\begin{abstract}
Maria Cámara-Torres ${ }^{1}$, Ravi Sinha1 , Paolo Scopece², Thomas Neubert ${ }^{3}$, Kristina Lachmann ${ }^{3}$, Alessandro Patelli ${ }^{4}$, Carlos Mota ${ }^{1}$, Lorenzo Moroni ${ }^{1}$

${ }^{1}$ Maastricht University, MERLN Institute for Technology-Inspired Regenerative Medicine, CTR Department, Maastricht, The Netherlands, ${ }^{2}$ Nadir S.r.I., Venice, Italy, ${ }^{3}$ Fraunhofer Institute for Surface Engineering and Thin Films IST, Braunschweig, Germany, ${ }^{4}$ Department of Physics and Astronomy, Padova University, Padova, Italy.
\end{abstract}

\section{Abstract}

3D scaffolds with optimum physico-chemical properties are able to elicit specific cellular behaviors and guide tissue formation. However, cell-material interactions are limited in scaffolds fabricated by melt extrusion additive manufacturing (ME-AM) of synthetic polymers, and plasma treatment can be used to render the surface of the scaffolds more cell-adhesive. In this study, a hybrid AM technology, which combines a ME-AM technique with an atmospheric pressure plasma jet, was employed to fabricate and plasma treat scaffolds in a single process. The organosilane monomer (3-Aminopropyl) trimethoxysilane (APTMS) and a mixture of maleic anhydride and vinyltrimethoxysilane (MA-VTMOS) were used for the first time to plasma treat 3D scaffolds. APTMS treatment deposited plasma polymerized films containing positively charged amine functional groups, while MA-VTMOS introduced negatively charged carboxyl groups on the 3D scaffolds' surface. Argon plasma activation was used as control. All plasma treatments increased the surface wettability and protein adsorption to the surface of the scaffolds, and improved cell distribution and proliferation. Notably, APTMS treated scaffolds also allowed cell attachment by electrostatic interactions in the absence of serum. Interestingly, cell attachment and proliferation were not significantly affected by plasma treatment induced aging. Also, while no significant differences were observed between plasma treatments in terms of gene expression, human mesenchymal stromal cells (hMSCs) could undergo osteogenic differentiation on aged scaffolds. This is probably because osteogenic differentiation is rather dependent on initial cell confluency and surface chemistry might play a secondary role. 


\section{INTRODUCTION}

Over the past decades, additive manufacturing (AM), and in particular melt extrusion AM (ME-AM), has emerged as an advanced fabrication technique for the development of scaffolds for skeletal tissue engineering. This is due to the possibility of producing costeffective, customizable, biocompatible and biodegradable 3D constructs, with interconnected macro-pores facilitating tissue ingrowth, while maintaining sufficient mechanical properties for load bearing applications. ${ }^{1}$ In order to ensure tissue formation and to obtain reliable readouts when evaluating a scaffold functionality in vitro, an efficient cell attachment upon seeding, in terms of cell density and distribution, is particularly important. ${ }^{2-4}$ Moreover, cell colonization also plays a pivotal role to guarantee the in vivo success of both cell-laden and cell-free scaffolds. Despite their advantageous structural features, cell adhesion has shown to be challenging on ME-AM scaffolds, due to their large pores and lack of biological recognition sites to enable cell-material interactions, as they are mostly made of synthetic thermoplastic polymers, such as poly(E-caprolactone) (PCL), poly(lactic acid) (PLA), poly(lactic-co-glycolic acid) (PLGA), poly(urethane) (TPU), or poly(ethylene oxide terephthalate)/poly(butylene terephthalate) (PEOT/PBT). These factors, along with the gravity force, contribute to fast cell sedimentation towards the scaffold's bottom and mediocre attachment to the scaffold's filaments upon conventional static seeding, which is a simple and widely used seeding method that consists on placing a droplet of cell suspension on top of the scaffold surface, that gradually flows into the scaffold's pores. ${ }^{5-7}$

Different strategies have been considered to optimize cell attachment during static seeding of 3D ME-AM. For instance, loading cells into a natural or synthetic hydrogel solution, which crosslinks within the scaffolds pores, has shown to improve cell retention within the scaffold. ${ }^{8-9}$ Alternatively, we have recently proposed the use of macromolecules as temporary seeding media supplements to increase its viscosity or density, which has proven to reduce cell sedimentation velocity and result in homogeneous cell attachment along the scaffold's cross section. ${ }^{4}$ Besides the modification of the seeding solution, the alteration of the scaffold architecture, with regard to pore size, lay-down pattern, or the introduction of vertical gradients, has also been suggested to enhance cell attachment. ${ }^{6}$, 10 Furthermore, synthetic polymers have been blended with bioactive fillers, such as calcium phosphates, ${ }^{11}$ bioactive glass ${ }^{12}$ or graphene derivatives, ${ }^{13}$ to improve cell 
attachment and cell-material interactions by increasing scaffolds' hydrophilicity and /or surface roughness.

Surface coatings have also been considered as an alternative to increase polymeric scaffolds' bioactivity, as some of the methods described above involve the modification of scaffold geometry or the material bulk properties and these can negatively affect the scaffolds mechanical properties. For example, $\mathrm{NaOH}$ etching or other wet chemical surface modifications can increase the hydrophilicity and surface roughness, or deposit specific monomers with functional groups on 3D scaffolds. ${ }^{14}$ Yet, the use of harsh chemicals can potentially compromise the integrity of the scaffolds' structure. ${ }^{15}$ Other examples exploit scaffold's filaments coatings with extracellular matrix (ECM) proteins or cell adhesive peptides to improve cell adhesion. ${ }^{16}$ Nevertheless, this approach has not been extensively explored due to proteins' limited half-lives and high costs.

Non-thermal plasma (NTP) surface modification has the advantage of altering the polymer surface chemistry and/or topography without the use of solvents, while maintaining their bulk properties. ${ }^{17}$ Various types of gases, such as oxygen, helium, argon, nitrogen, or their combination can be used to generate the plasma discharge and activate polymer surfaces by the incorporation of hydroxyl, carboxyl, aldehyde or amine groups. To obtain more stable coatings with a higher density of functional groups, the plasma discharge can be fed with monomers, such as alkyl amine or ammonia, or acrylic acid, to coat the surface with a thin polymeric film containing amine or carboxyl groups, respectively, in a process known as plasma polymerization. NTP is an established technique to treat $2 \mathrm{D}$ polymeric surfaces, where extensive research has revealed enhanced protein adsorption and cell attachment due to the presence of functional groups compared to methyl only containing surfaces. 18-19 Moreover, carboxyl modified surfaces have shown to promote chondrogenesis, while amine groups have proved to direct cells towards the osteogenic pathway. ${ }^{20-22}$

Being an established technique to treat two-dimensional (2D) polymeric surfaces, NTP was used during the last decade to functionalize 3D ME-AM scaffolds as well. Oxygen plasma activation, as one of the primary focuses of reported studies, has shown to increase surface roughness, protein adsorption and cell attachment, as well as to promote alkaline phosphatase (ALP) expression and matrix mineralization on PCL scaffolds. ${ }^{23-24}$ Other studies have explored plasma polymerization techniques. Among those, nitrogen containing groups have been introduced on PCL or polystyrene scaffolds by allyl amine, 
ammonia or ethylene/ $\mathrm{N}_{2}$, promoting Runt-related transcription factor 2 (RUNX2) and ALP upregulation in human mesenchymal stromal cells (hMSCs) compared to untreated scaffolds. ${ }^{25-26}$ Alternatively, in a study using PEOT/PBT scaffolds treated with acrylic acid plasma polymerization, enhanced proliferation and glycosaminoglycan production by chondrocytes was shown. ${ }^{27}$ Despite promising results in enhancing ME-AM scaffolds surface bioactivity, NTP is still not a widely used technique for 3D scaffolds treatment. This is mainly due to the NTP process usually being carried out at sub-atmospheric pressures, where plasma has shown to not ignite inside the scaffold pores. ${ }^{28-29}$ This limits the treatment in the scaffold's core to diffusion, leading to inhomogeneous and uncontrolled gradient functionalization. Moreover, the process often requires costly vacuum equipment and its application demands multi-steps processes. Overcoming these limitations, atmospheric pressure plasma jets (APPJ) have been developed, ${ }^{30}$ where the need of a reactor chamber and vacuum equipment is eliminated and the NTP exiting the jet can be directed into the scaffolds pores, or a region of interest. Moreover, APPJ enables its assembly to an AM platform, where the printing and plasma treatment processes can take place in a single hybrid system. In addition, scaffolds can be either plasma treated as a whole from the top, or in a layer-by-layer manner to reach deep pores in large anatomical scaffolds, or treat specific scaffolds' zones. ${ }^{31-32}$

Taking these advantages, here we used a hybrid $\mathrm{AM}$ technique, consisting of a ME printhead and an APPJ module assembled on a 3-axis platform to fabricate plasma treated PEOT/PBT scaffolds in a single process. ${ }^{32}$ Despite its excellent processability and previous in vitro and in vivo applications for bone tissue engineering, PEOT/PBT demonstrates poor cell attachment upon cell seeding. 4,7 The organosilane monomers (3Aminopropyl) trimethoxysilane (APTMS) and maleic anhydride-Vinyltrimethoxysilane (MAVTMOS) were used to deposit polymer-like thin films containing amine and carboxyl functional groups, respectively, by plasma polymerization in argon on the PEOT/PBT scaffolds surface. Despite being already explored to modify 2D surfaces, ${ }^{33-34}$ these monomers were used here for the first time to treat 3D scaffolds. Compared to other precursors, organosilanes possess the advantage of providing a stable siloxane backbone to the coating, which is highly adherent and resistant to delamination in water conditions, ensuring good functional group retention under cell culture conditions. ${ }^{35-36}$ Pristine scaffolds, plasma polymerized scaffolds, and argon plasma activated scaffolds were seeded with hMSCs. Cell attachment and adhesion mechanism were evaluated. 
Moreover, proliferation and osteogenic differentiation were assessed and compared among the different plasma conditions.

\section{MATERIALS AND METHODS}

\section{Plasma treated scaffolds fabrication and characterization}

Scaffolds were fabricated and plasma treated with a hybrid AM platform, consisting of a 3D axis stage (Bioscaffolder 3.0, Gesim) equipped with a custom made ME printhead and an APPJ (Plasma Stylus Noble, Nadir srl), ${ }^{37}$ all enclosed in a polymethylmethacrylate box with fume extraction ventilation. In a first step, scaffolds were produced (Figure 1A). For that, PEOT/PBT pellets (300PEOT55PBT45, PEO molecular weight $=300 \mathrm{kDa}$, PEOT:PBT weight ratio = 55:45, intrinsic viscosity $0.51 \mathrm{dl} / \mathrm{g}$, Polyvation, The Netherlands) were heated up to $195^{\circ} \mathrm{C}$ in the cartridge of the printhead. Molten polymer was extruded through a $250 \mu \mathrm{m}$ internal diameter needle (pressure 7 bar, translation speed of $16 \mathrm{~mm} / \mathrm{s}$ ), depositing a 0-90 pattern block $\left(15 \times 15 \times 4 \mathrm{~mm}^{3}\right)$ with $200 \mu \mathrm{m}$ layer thickness and $750 \mu \mathrm{m}$ strand distance (center to center) (Figure 1A).

A

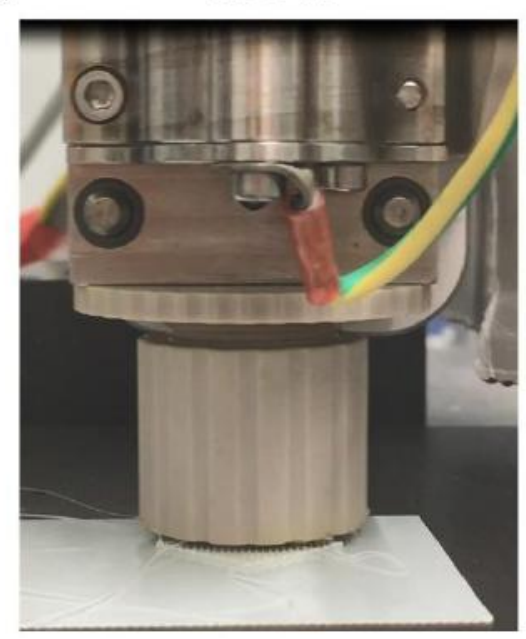

B

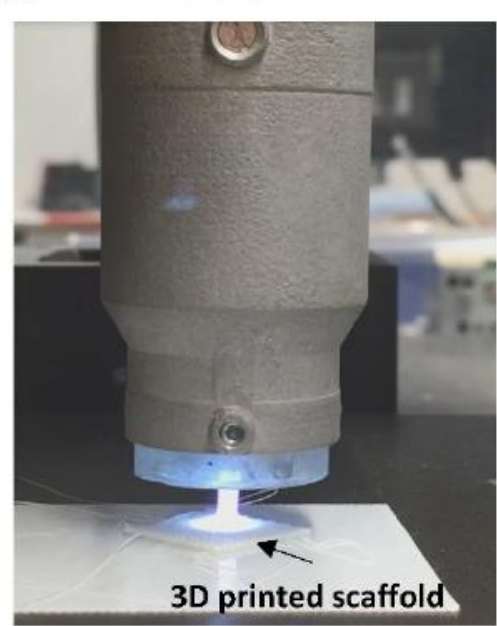

C

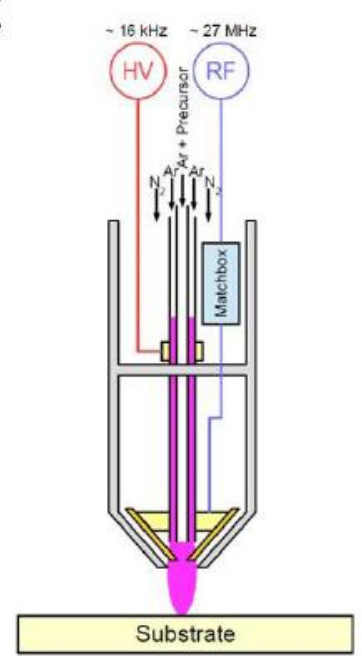

Figure 1. Hybrid $A M$ platform consisting of $(A)$ a $M E$ printhead and $(B)$ an atmospheric pressure plasma jet (APPJ), mounted on a 3D axis stage. ${ }^{1-2}(\mathrm{C})$ Schematics of the APPJ, which is based on a dielectric barrier discharge scheme simultaneously powered by a high-voltage (HV) generator and a radio-frequency (RF) generator. Electrodes are positioned externally to an alumina duct where argon is fluxed and plasma is ignited. Previously mixed precursors in vapor phase are carried by argon and mixed with the ignited plasma right before the RF generator through an inner coaxial tube, which is connected to external bubblers with precursors in liquid (APTMS, VTMOS) or solid (MA) phase. Nitrogen is carried through an outer coaxial duct for shielding and cooling at the exit of the torch. 
Right after fabrication, scaffolds were plasma treated with the APPJ (Figure 1B). The device is based on a dielectric barrier discharge scheme simultaneously powered by a high-voltage generator $(\mathrm{HV}, 10 \mathrm{kV}, 17 \mathrm{kHz})$ and a radio-frequency generator $(\mathrm{RF}, 27 \mathrm{MHz})$ (Figure 1C). Electrodes are positioned externally to an alumina duct where argon is fluxed (Ar 5.0 purity, $10 \mathrm{~L} / \mathrm{min}$ ) and plasma is ignited. An inner coaxial tube allows for the introduction of the precursors in vapor phase just before the RF electrodes. For APTMS plasma polymerization, APTMS (Sigma-Aldrich) was carried into the plasma zone by argon gas passing through a bubbler at room temperature (RT) (flow $=2 \mathrm{~L} / \mathrm{min}$ ). Similarly, for MAVTMOS plasma polymerization, MA (Sigma-Aldrich) and VTMOS (Sigma-Aldrich) in two independent bubblers were carried into the plasma zone by argon at $1.764 \mathrm{~L} / \mathrm{min}$ and $0.233 \mathrm{~L} / \mathrm{min}$, respectively. For argon activation, the RF generator was operated with a power output of $15 \mathrm{~W}$ in continuous mode. For APTMS and MA-VTMOS plasma polymerization, the RF generator was operated in pulsed mode, at a duty cycle of $5 \%$

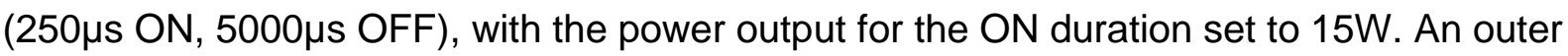
gas shell of nitrogen (15 L/min) was used during the process to prevent precursor oxidation by the environmental air during the deposition. The APPJ nozzle was positioned at $1 \mathrm{~mm}$ above the scaffold surface and moved in $X Y$ following the surface filaments' path at 1 $\mathrm{mm} / \mathrm{s}$. For further studies, plasma treated scaffolds were used within 2 days after plasma treatment ('fresh' samples) or after $\sim 10$ days being stored at RT in a sealed container ('aged' samples).

To assess the successful deposition of an APTMS plasma polymerized layer, fresh, aged and ethanol disinfected scaffolds, were incubated with the amine reactive fluorescent dye LIVE/DEAD ${ }^{\text {тм }}$ Fixable dead Cell Stain Kit (Thermo Fisher Scientific) for 45 min at a concentration of $0.5 \mu \mathrm{l} / 500 \mu \mathrm{l}$ PBS. Untreated, argon, and MA-VTMOS treated scaffolds were also stained as controls. After PBS washes, scaffolds were cut and the cross sections were imaged using a fluorescent microscope (Eclipse, Ti2-e, NIKON). In order to confirm the deposition of a MA-VTMOS layer, fresh, aged and ethanol disinfected scaffolds were incubated for $20 \mathrm{~s}$ in a methylene blue solution $(1 \mathrm{mg} / \mathrm{ml})$. Untreated, argon and APTMS treated scaffolds were also stained as controls. After PBS washes, scaffolds were cut and imaged with a stereomicroscope (Nikon SMZ25). In order to evaluate the dynamic wettability of the scaffolds, a $35 \mu$ water droplet was carefully deposited onto the surface of the scaffolds, and the wetting behavior was captured with a digital camera at 1 frame per second (Krüss DSA25S). 


\section{Plasma treated 2D films fabrication and characterization}

2D films were prepared from PEOT/PBT pellets. Briefly, 60 milligrams of pellets were molten at $190^{\circ} \mathrm{C}$ and pressed with a coverslip against a Teflon sheet to obtain films with $14 \mathrm{~mm}$ diameter and $\sim 300 \mu \mathrm{m}$ thickness. Subsequently, PEOT/PBT films were plasma treated with argon, APTMS or MA-VTMOS using the APPJ, according to the parameters in the section "Plasma treated scaffolds fabrication and characterization".

Static contact angle was measured in fresh and aged films using the sessile drop method. For that, a $4 \mu \mathrm{l}$ water droplet was placed on top of the substrates by an automatic syringe dispenser (Krüss DSA25S). 20 seconds after droplet formation, the contact angle was calculated automatically by the device's software using the Laplace-Young curve fitting.

Attenuated total reflectance-Fourier-transform-infrared spectroscopy (ATR-FTIR, Nicolet iS50, diamond ATR) was performed on untreated and fresh plasma treated films in order to further confirm the presence of the coating.

Zeta potential measurements were performed on fresh films with a size of $2 \times 4 \mathrm{~cm}^{2}$. Electrokinetic measurements were done at RT using a SurPASS ${ }^{\mathrm{TM}}$ system (Anton Paar $\mathrm{GmbH}$, Germany), with a $\mathrm{KCl}$ solution $(0.01 \mathrm{~m}, \mathrm{pH} 5-5.5)$ as electrolyte. The zeta potential was calculated from ten experimental points taken by the measurement of the streaming potential in a pressure range of 500 mbar (start) to $200 \mathrm{mbar}$ (end) in the flow cell.

\section{Cell seeding on 3D scaffolds}

HMSCs isolated from bone marrow were purchased from Texas A\&M Health Science Center, College of Medicine, Institute for Regenerative Medicine. Cryopreserved vials were plated at a density of $1,000 \mathrm{cells} \cdot \mathrm{cm}^{-2}$ in tissue culture flasks and expanded at $37^{\circ} \mathrm{C}$ / $5 \% \mathrm{CO}_{2}$ in cell culture media (CM) consisting of aMEM with Glutamax and no nucleosides (Gibco) supplemented with 10\% fetal bovine serum (FBS).

To investigate the cell attachment mechanism, fresh scaffolds were disinfected in $70 \%$ ethanol for 20 min and washed 3 times with Dulbecco's PBS (5 min each). Scaffolds were incubated overnight in CM with FBS ((+)FBS) or CM without FBS ((-)FBS). Before seeding, scaffolds were dried on top of a sterile filter paper and placed on non-treated well plates. HMSCs were trypsinized and resuspended in (+)FBS or (-)FBS CM. A droplet of cell suspension (37 $\mu$ l containing 200,000 cells) was placed on top of each scaffold, filling the pores within some seconds. Seeded scaffolds were incubated for 4 hours at $37{ }^{\circ} \mathrm{C} / 5 \%$ 
$\mathrm{CO}_{2}$ to allow for cell attachment. After this time, scaffolds were collected or transferred to new wells containing $1.5 \mathrm{ml}$ of $(+) F B S$ and cultured overnight before sample collection. Aged scaffolds incubated overnight in (+)FBS CM and seeded on (+)FBS CM were also cultured overnight before sample collection, to analyze the effect of aging on cell adhesion. To evaluate the effect of cell seeding density on scaffold coverage and proliferation, fresh and aged scaffolds were incubated overnight in CM and seeded with hMSCs in CM at a density of 200,000 (200k) or 400,000 (400k) cells/scaffold, concentrated in a $37 \mu$ droplet. After $4 \mathrm{~h}$ attachment, $200 \mathrm{k}$ and $400 \mathrm{k}$ scaffolds were transferred to new wells containing 1.5 or $3 \mathrm{ml} \mathrm{BM} \mathrm{(CM} \mathrm{supplemented} \mathrm{with} 200 \mu \mathrm{M}$ L-Ascorbic acid 2-phosphate), respectively, and cultured for 7 days.

To evaluate hMSCs osteogenic differentiation, aged plasma treated scaffolds were incubated overnight in (+)FBS. Scaffolds seeded with 200,000 cells were further cultured for 7 days in $\mathrm{BM}$ and for another 47 days in mineralization media (MM) consisting of $\mathrm{BM}$ supplemented with dexamethasone (10 nm) (Sigma-Aldrich) and $\beta$-glycerophosphate (10 mm) (Sigma-Aldrich). Media was replaced every two or three days. As osteogenic differentiation 2D controls, cells were seeded in tissue culture polystyrene wellplates at a density of 5,000 cells $\cdot \mathrm{cm}^{-2}$, and cultured in the same media conditions as 3D scaffolds.

\section{Imaging of cell attachment within scaffold cross sections}

Scaffolds were fixed with 4 wt\% paraformaldehyde for $30 \mathrm{~min}$, permeabilized using 0.1 vol\% Triton-X for $30 \mathrm{~min}$, and incubated with phalloidin (Alexa Fluor 488, 1:75 dilution in PBS) for $1 \mathrm{~h}$ at RT. The bottom and cross section of scaffolds were imaged using a fluorescent microscope. Background subtraction and contrast enhancement were performed on the images using the software Image $\mathrm{J}$, in order to clarify their visualization. To assess cell coverage, cross section images were converted to binary (rendering regions with cells in white and the rest in black), and the total amount of white pixels in the scaffold area was quantified and normalized to the total number of pixels.

\section{DNA quantification}

Scaffolds collected at the desired timepoints were freeze-thawed $3 x$ for cell lysis and incubated overnight at $56{ }^{\circ} \mathrm{C}$ in Proteinase $\mathrm{K}$ solution $(1 \mathrm{mg} / \mathrm{ml}$ Proteinase $\mathrm{K}$ (SigmaAldrich) in Tris/EDTA buffer) (1:1) for matrix degradation and cell lysis. Then, scaffolds were freeze-thawed $3 x$ and incubated $1 \mathrm{~h}$ at RT with a 20X diluted lysis buffer from the 
CyQUANT cell proliferation assay kit (Thermo Fisher Scientific) containing RNase A (1:500), to degrade cellular RNA. Lastly, samples were incubated with the fluorescent dye provided by the kit (1:1) for $15 \mathrm{~min}$ and fluorescence was measured with a spectrophotometer (emission/excitation $=520 / 480 \mathrm{~nm}$ ).

\section{Protein adsorption quantification}

Disinfected fresh and aged scaffolds were incubated overnight at $37^{\circ} \mathrm{C}$ in (+)FBS or ()FBS. After washing with PBS, scaffolds were blocked for $1 \mathrm{~h}$ at $37^{\circ} \mathrm{C}$ in $1 \mathrm{wt} / \mathrm{v} \% \mathrm{BSA} / \mathrm{PBS}$ and then incubated with specific bovine vitronectin (ab23444, Abcam) or fibronectin (ab2413, Abcam) primary antibodies, diluted 1:500 and 1:400, respectively, in $1 \mathrm{wt} / \mathrm{v} \%$ BSA/PBS. After washing three times in PBS, scaffolds were incubated for $1 \mathrm{~h}$ at $37^{\circ} \mathrm{C}$ in horseradish peroxidase (HRP)-conjugated anti-mouse secondary antibody (abcam, 1:100000 dilution 1\% BSA) for vitronectin detection, or HRP-conjugated anti-rabbit secondary antibody (abcam, 1:10000 dilution in 1\% BSA) for fibronectin detection. After three washes in $1 \% \mathrm{BSA}+0.05 \%$ tween 20 , scaffolds were blotted in an adsorbent paper and incubated in $150 \mu \mathrm{l}$ of 1-Step Ultra TMB ELISA substrate (Thermofisher Scientific). Color was allowed to develop for $10 \mathrm{~min}$ at RT and the reaction stopped with $50 \mu \mathrm{l}$ of sulfuric acid (2M). Supernatants absorbance was measured at $450 \mathrm{~nm}$ with a spectrophotometer (CLARIOstar®, BMG Labtech). Scaffolds incubated in (-)FBS and scaffolds incubated in (+)FBS and reacted with only secondary antibodies were used as controls.

In order to analyze albumin adsorption, disinfected fresh and aged scaffolds were incubated overnight at $37{ }^{\circ} \mathrm{C}$ with $1 \mathrm{mg} / \mathrm{ml}$ BSA-FITC (Sigma-Aldrich) solution. After washing three times with PBS, scaffolds were blotted in an adsorbent paper and incubated for $2 \mathrm{~h}$ at $\mathrm{RT}$ in a $1 \%$ SDS solution. Supernatants fluorescence was measured at excitation/emission $=495 \mathrm{~nm} / 519 \mathrm{~nm}$.

\section{Alizarin red S (ARS) staining}

Scaffolds were collected at day 35 and 54 of culture, fixed, washed with distilled water, cut in half and stained with ARS (60 mM, pH 4.1 - 4.3) for 20 min at RT. Samples were thoroughly washed to remove staining residues. Scaffolds' cross sections were imaged using a stereomicroscope to visualize calcium deposition. 


\section{Immunostaining}

After fixation, scaffolds were permeabilized for $30 \mathrm{~min}$ by incubating on Triton-X $100(0.1$ V\%). Subsequently, scaffolds were blocked by $1 \mathrm{~h}$ incubation in blocking buffer (BB, $3 \%$ $B S A+0.01 \%$ Triton- $X 100)$, cut in half, and incubated overnight at $4{ }^{\circ} \mathrm{C}$ with a primary antibody: either collagen I (COL I) rabbit polyclonal (ab34710, Abcam), or vinculin mouse monoclonal (sc73264, Santa Cruz Biotechnology), both diluted 1:200 in washing buffer (10x diluted blocking buffer). Washed samples were incubated for $1 \mathrm{~h}$ at RT with the secondary antibody (1:200 Alexa Fluor 568 goat derived anti rabbit antibody, Thermo Fisher Scientific). Then, scaffolds were washed and stained for F-actin (488 Alexa Fluor Phalloidin, Thermo Fisher Scientific) for $1 \mathrm{~h}$ at RT and imaged with a confocal laser scanning microscope (Leica TCS SP8 STED), equipped with a white light laser (WLL). Emission was detected with HyD detectors.

\section{Gene expression}

Gene expression was analyzed with qRT-PCR at 14 and 54 days of culture. RNA was extracted from cells by incubating the scaffolds in Trizol. Subsequently, samples were centrifuged at $12,000 \mathrm{rcf}$ for $5 \mathrm{~min}$ to precipitate the scaffold and ECM at the bottom. In a last step, chloroform was added to the supernatant and centrifuged at 12,000 ref for $5 \mathrm{~min}$ to isolate the RNA, present in the aqueous phase. RNA was further purified using RNeasy mini kit column (Qiagen), according to the manufacturer's protocol and quantified using a spectrophotometer. Reverse transcription was performed using iScript $^{\mathrm{TM}}$ (Bio-Rad) following suppliers' protocol. qPCR was performed on the mix composed of cDNA, SYBRGreen master mix (Qiagen) and the selected primers (Table S1) using a CFX Connect $^{\mathrm{TM}}$ Real-Time System (Bio-Rad) under the following conditions: cDNA denaturation for $3 \mathrm{~min}$ at $95^{\circ} \mathrm{C}, 40$ cycles of $15 \mathrm{~s}$ at $95^{\circ} \mathrm{C}$, and $30 \mathrm{~s}$ at $65^{\circ} \mathrm{C}$. Gene transcription was normalized to the transcription of the housekeeping gene B2M. The $2^{-}$ $\Delta \Delta \mathrm{Ct}$ method was used to calculate relative gene expression for each target gene.

\section{Statistical analysis}

All data is shown as average with error bars indicating the standard deviation of at least three replicates. Analysis of statistics was conducted with GraphPad Prism (version 8.0.1). A one-way or two-way analysis of variance (ANOVA) was performed followed by a Tukey's 
post-hoc multiple comparison test to evaluate statistical significance and correct for multiple comparisons.

\section{RESULTS AND DISCUSSION}

Compared to conventional manufacturing techniques, the possibility of fabricating customizable scaffolds for bone tissue engineering in a reproducible manner by AM brings the produced constructs one step closer to clinical translation. The assembly of a surface modification module, such as an APPJ, into the printing platform further increases the value of the technology and fabricated scaffolds for their research and clinical applications. With such a hybrid AM platform developed within our groups, ${ }^{32}$ we were able to manufacture surface modified 3D PEOT/PBT scaffolds in a continuous process, where scaffolds are fabricated by ME-AM and subsequently functionalized by the APPJ module (Figure 2). To do this, the nozzle tip of the APPJ module was adjusted at $1 \mathrm{~mm}$ over the scaffold surface, ensuring the penetration of the plasma flame over the whole scaffold depth (4 mm), while moved in XY over the scaffolds filaments at the transitional speed of $1 \mathrm{~mm} / \mathrm{s}$, covering the whole scaffold block area $(15 \times 15 \mathrm{~mm})$.

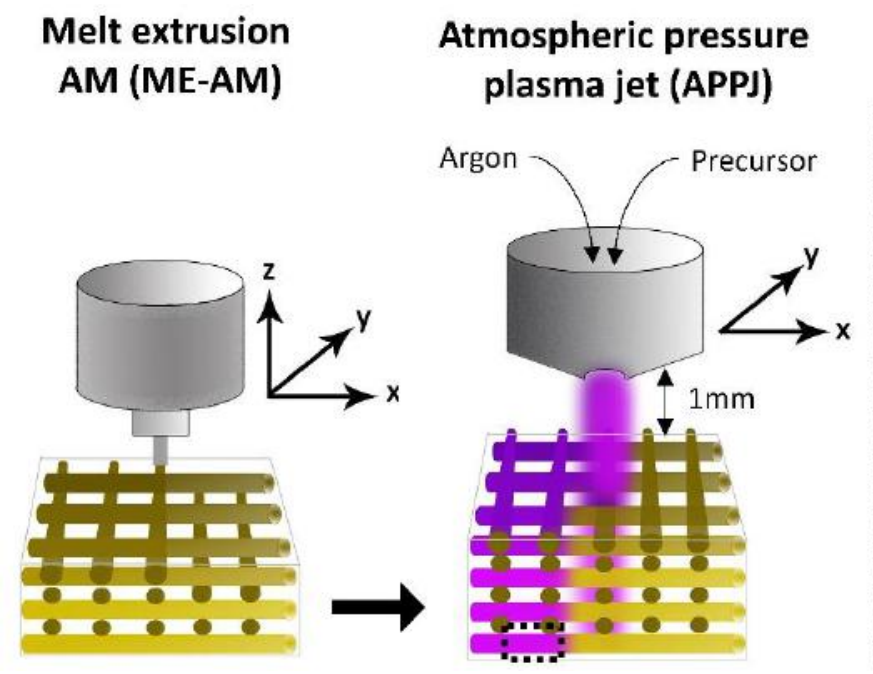

A.
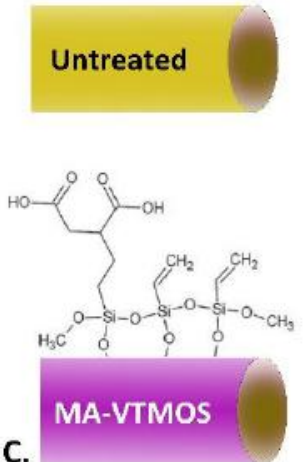

C.

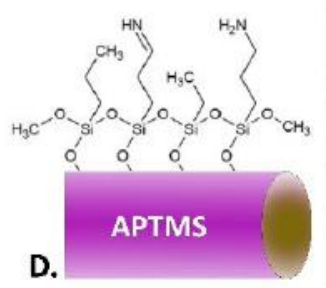

B.

\section{Argon}

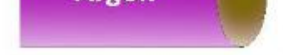

.

Figure 2. Schematic representation of the hybrid platform for scaffolds fabrication combined with plasma functionalization. 3D scaffolds are fabricated via ME-AM and consequently plasma treated from the top using an APPJ, which moves in XY following the scaffolds filaments, while the plasma penetrates the scaffold depth. As a result, (a) untreated, (b) argon activated or (c) MA-VTMOS and (d) APTMS plasma polymerized scaffolds can be obtained, each with a specific surface functionality. 


\section{Plasma scaffolds characterization}

Plasma polymerization of APTMS and MA-VTMOS on the surface of the scaffolds was carried out in a pulsing mode with $5 \%$ duty cycle, since compared to continuous plasma, pulsed plasma polymerization at low duty cycles has shown to allow for better preservation of the monomers' functional groups integrity during radical formation, as well as for the deposition of smoother surfaces. ${ }^{33,36,38}$ A homogeneous negatively charged coating covering the filaments all along the scaffolds volume after MA-VTMOS plasma polymerization was confirmed by staining with the cationic dye methylene blue, as previously described (Figure 3A, Figure S1), as well as with zeta potential measurements (Figure S2). ${ }^{39}$ FTIR analysis further identified the negatively charged functional groups as intact anhydride groups from the MA precursor $\left(-\mathrm{C}=\mathrm{O}\right.$ stretching at $\left.1750-1800 \mathrm{~cm}^{-1}\right)$, and carboxyl groups (-OH stretching at 3200-3650 $\mathrm{cm}^{-1}$ ) (Figure 3B, Figure S3A). ${ }^{38,40}$ In addition, vinyl groups from the VTMOS molecules ( $\mathrm{C}=\mathrm{C}$ stretching at $\left.1630-1660 \mathrm{~cm}^{-1}\right)$ were also identified. To visualize the formation of a homogeneous positively charged (Figure S2) thin polymer-like film containing amine groups $\left(-\mathrm{NH}_{2}\right)$ after APTMS plasma functionalization, an amine-reactive fluorescent dye was used to stain the scaffolds (Figure 3A, Figure S1). Primary amines vibrations were also observed in FTIR analysis $\left(\mathrm{N}-\mathrm{H}\right.$ bending at $1560-1630 \mathrm{~cm}^{-1}$ and $\mathrm{N}-\mathrm{H}$ stretching at $3000-3500 \mathrm{~cm}^{-1}$ ) (Figure 3C, Figure S3B). Stretching vibrations visible at $2980-2880 \mathrm{~cm}^{-1}$, confirmed the retention of some aminopropyl chains of the precursor. ${ }^{41}$ Nitrogen-containing groups were also visible as amides $\left(-\mathrm{C}=\mathrm{O}\right.$ stretching at $\left.1630-1695 \mathrm{~cm}^{-1}\right)$ and as oximes $(\mathrm{C}=\mathrm{N}$ stretching at 1650 $1680 \mathrm{~cm}^{-1}$ and $\mathrm{O}-\mathrm{H}$ stretching at $\left.3550-3600 \mathrm{~cm}^{-1}\right) .{ }^{42-43}$ The presence of oximes and amides was probably due to precursor oxidation during the deposition process, despite the nitrogen being flushed in the outer duct of the APPJ, probably due to the jet movement and the flow being disrupted by the scaffold structure. ${ }^{41}$ In addition, the APTMS and MAVTMOS plasma polymerized substrates spectra were dominated by absorption bands between 1000 and $1200 \mathrm{~cm}^{-1}$, confirming the presence of a siloxane network. In contrast to plasma polymerization, argon plasma activation is a less specific treatment, where a variety of polar groups can be introduced. According to previous reports on argon plasma on PEOT/PBT substrates, it is hypothesized that hydroperoxides $(-\mathrm{OOH})$ and peroxide (OO-) groups were incorporated, formed after post oxidation in air of the free radicals generated by plasma, along with aldehyde or ketone groups (-CHO, -CO-). ${ }^{44-46}$ The presence of these negatively charged groups exposed in the surface of the scaffolds was visualized by a low intensity methylene blue staining and confirmed by zeta potential 
measurements (Figure 3A, Figure S2). Yet, these changes were not detected by FTIR analysis, possibly due to the very subtle variation in surface chemistry (Figure 3D, Figure S3C). Moreover, it is plausible that argon plasma activation could have induced changes in the surface roughness of PEOT/PBT, as previously described. ${ }^{46}$ This effect has been attributed to plasma UV irradiation etching, mainly on the PEO amorphous regions of the copolymer, which are more mobile and susceptible to plasma than the PBT regions. However, SEM image analysis did not show any changes in the microscale surface roughness of argon scaffolds, compared to untreated ones (Figure S4). Similarly, no changes in microroughness were noted on the plasma polymerized scaffolds (Figure S4). This might be due to the low contact time of the plasma jet with the polymer, compared to the long exposure-driven changes reported in literature (from 5 to $30 \mathrm{~min}$ ), ${ }^{44-45}$ modifying only the roughness in the nanoscale, which was not appreciable with SEM.
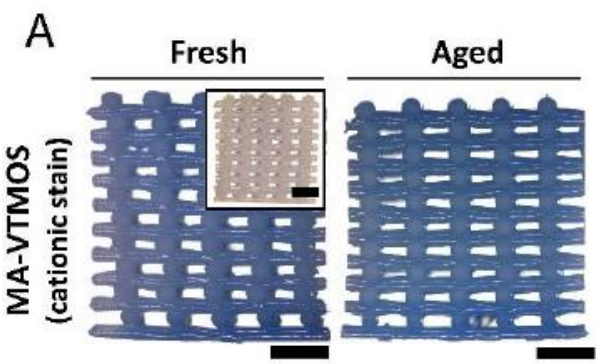

B
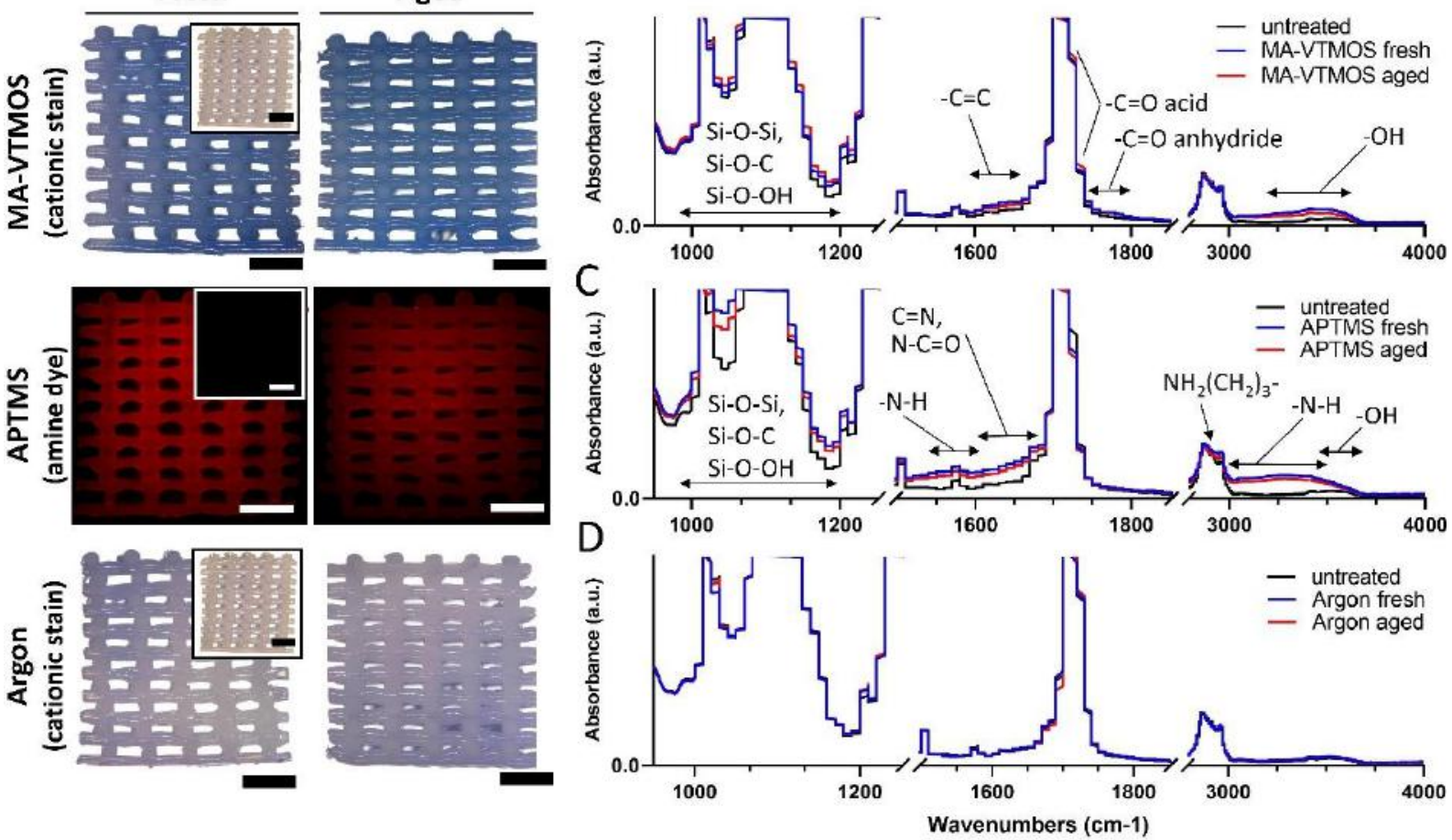

Figure 3. Characterization of plasma functionalized 3D scaffolds and comparison among fresh and aged conditions. (A) Verification of the treated scaffolds surface functionalization by specific staining. MA-VTMOS and Argon scaffolds were stained with the cationic dye methylene blue (blue staining in light microscopy) and APTMS scaffolds with an amine specific dye (red staining in fluorescence microscopy). Inserts represent stained control untreated PEOT/PBT scaffolds (white in light microscopy and grey in fluorescent microscopy). Scale bars $1 \mathrm{~mm}$. (B, C, D) FTIR spectra of MA-VTMOS, APTMS and Argon plasma treated 2D substrates (melt-pressed PEOT/PBT films). 
To evaluate the effect of plasma treatment on the polymer wettability, water contact angle (WCA) measurements were performed on melt pressed PEOT/PBT films, where enhanced hydrophilicity was observed due the introduction of polar groups by all the plasma conditions (Figure 4A). WCA decreased from $\sim 80^{\circ}$ to $\sim 55^{\circ}$ in argon and APTMS treated scaffolds, and down to $\sim 35^{\circ}$ in MA-VTMOS scaffolds. Interestingly, similar values were previously reported for self-assembled monolayers coated with these specific functional groups. ${ }^{47}$ In accordance to the WCA, the dynamic wettability of MA-VTMOS and argon treated scaffolds increased compared to untreated scaffolds. This was observed by a significant reduction in the time that a droplet of water placed on top of the scaffold took to fill the scaffolds pores (from 6 min on untreated scaffolds to $2 \mathrm{sec}$ on treated scaffolds), mimicking the static seeding process (Figure 4B). Surprisingly, despite the low contact angle of the APTMS coatings, the wettability of these scaffolds decreased compared to untreated scaffolds, potentially due to the lower density of functional groups and higher density of hydrophobic silane/siloxane in the scaffolds compared to the 2D films, where the WCA was measured. Yet, this did not affect cell culture experiments, since scaffolds were always pre-wetted before cell seeding.
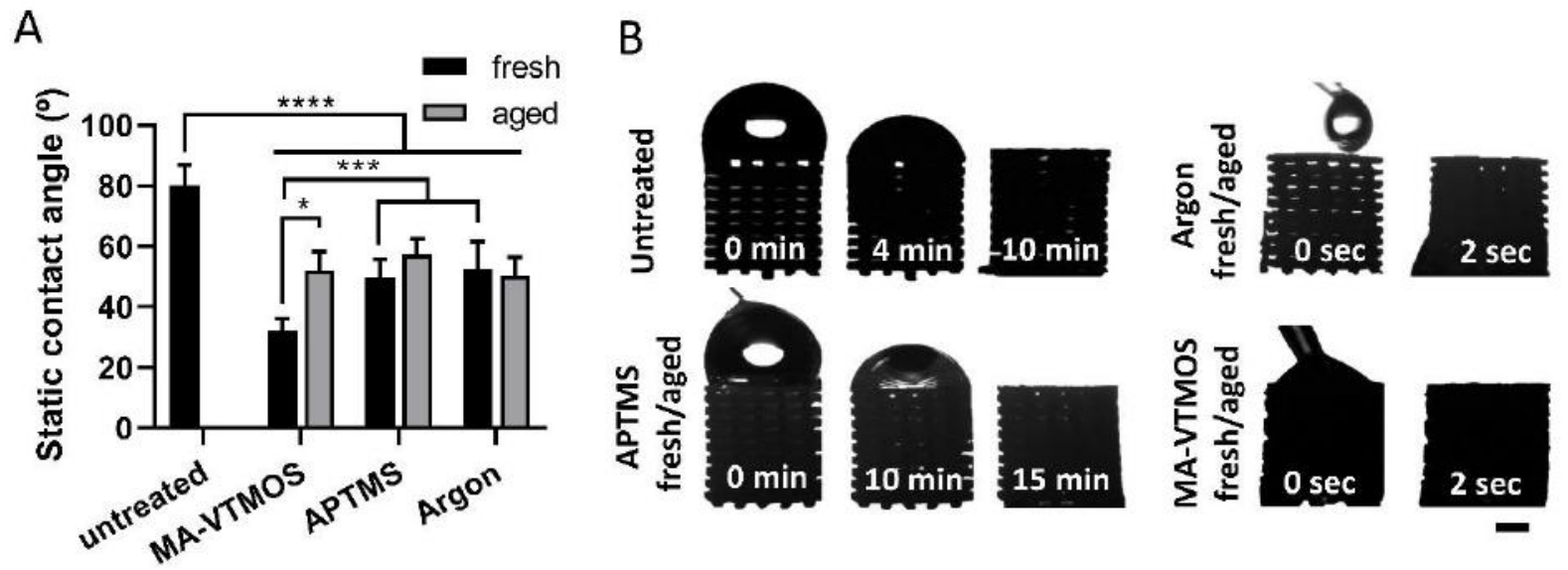

Figure 4. Hydrophilicity and wettability of plasma functionalized 3D scaffolds and comparison among fresh and aged conditions. A) Static contact angle measured on untreated and plasma treated 2D substrates. Data presented as average \pm s.d. and statistical significance performed using two-way ANOVA with Tukey's multiple comparison test $\left({ }^{*} p<0.05,{ }^{* * *} p<0.001,{ }^{* * * *} p<\right.$ 0.0001). (B) Dynamic wettability of plasma treated scaffolds. Scale bar $1 \mathrm{~mm}$.

The effect of storage ( 10 days at RT in a sealed container) on the preservation of the plasma activation or polymerized treatments was also evaluated. This is a relevant shelf life information due to the potential time-lapse between scaffold production and its use, both for in vitro and in vivo applications. It is well know that plasma treated polymeric 
surfaces can undergo ageing over time, which accounts for surface restructuring and hydrophobicity recovery towards a more energetically stable state. ${ }^{48}$ While bulk analysis in terms of staining intensity, contact angle values and wettability suggested stability of the coatings (Figure 3A, Figure 4A-B), FTIR analysis revealed slightly lower functional groups absorbance peaks on aged substrates compared to fresh ones (Figure 3B-D), as previously reported for such plasma treatments on 2D substrates. ${ }^{40,49}$ Moreover, it is worth noticing that the carbonyl stretching absorbance in MA-VTMOS spectra $(-\mathrm{C}=\mathrm{O}$ stretching at $1700-1725 \mathrm{~cm}^{-1}$ ) slightly increased in aged samples, which can be explained by the formation of carboxylic groups by the hydrolysis of the anhydrides through humidity. ${ }^{40}$ In general, these results suggest small changes in surface chemistry given by a reduction in the functional groups density on aged plasma polymerized substrates, which could potentially influence interactions such as protein adsorption or cell attachment. It is likely that the semi-crystalline property of PEOT/PBT and PBT the particularly efficient but cold and homogeneous APPJ configuration might have contributed to the limited aging effect, since it has previously been shown that the hydrophobic recovery decreases with increasing polymer crystallinity and with decreasing the degree of cross-linking due to plasma jet surface interaction. ${ }^{50-52}$

\section{Protein adsorption}

The effect of plasma treatment type and aging on protein adsorption after scaffolds incubation in cell culture media containing serum $((+) F B S)$ was analyzed (Figure 5). It was observed that fibronectin $(\mathrm{Fn})$ and vitronectin $(\mathrm{Vn})$, two relevant cell adhesive proteins present in serum, adsorbed in greater amounts to fresh plasma treated scaffolds than to untreated scaffolds (Figure 5A, B). Upon (+)FBS incubation, albumin, which is present at 100-1000 times higher concentration than adhesion-promoting proteins, adsorbs in the early phase of the protein layer formation. It is hypothesized that the hydrophobic interactions of albumin with the untreated PEOT/PBT are strong and resistant to displacement by $\mathrm{Fn}$ and $\mathrm{Vn}$, while these proteins can effectively displace the weakly adsorbed albumin on the hydrophilic and charged plasma treated surfaces. ${ }^{53-54}$ Notably, Fn and Vn adsorption was significantly higher on APTMS scaffolds, which can be attributed to the electrostatic interactions between the positively charged amine based scaffolds' surface and the negatively charged proteins in solution leading to high protein adsorption (Figure 5A, B). Yet, the theory of electrostatic interactions cannot explain the high adsorption of negatively charged $\mathrm{Fn}$ and $\mathrm{Vn}$ to the negative charge of the surface of MA- 
VTMOS and Argon scaffolds, compared to untreated scaffolds. Previous studies have also shown that Fn can be adsorbed at similar rates and amounts to both hydrophilic positively charged and negatively charged surfaces with zeta potential values similar to those measured within this study. ${ }^{55}$ This has been explained by charged micro domains of the proteins yielding short-range attractions, which can lead to hydrogen bonds formation once the Debye interactions overcome the macroscopic electrostatic repulsions. Alternatively, adsorption of $\mathrm{Fn}$ or $\mathrm{Vn}$ to negatively charged polymers has been explained by a first adsorbed layer of positively charged serum proteins, such as laminin, allowing subsequent electrostatic interactions with negatively charged proteins. ${ }^{56}$ Interestingly, the reduction in functional groups density and, potentially, surface charge, caused by aging led to a significant reduction in $\mathrm{Fn}$ and $\mathrm{Vn}$ adsorption, and a significant increase in albumin adsorption (Figure 5C), to MA-VTMOS and APTMS aged scaffolds. This resulted in comparable $\mathrm{Fn}$ and $\mathrm{Vn}$ adsorption to both scaffolds types, regardless their surface charge. Notably, argon scaffolds were not affected in this regard by aging and presented the largest relative cell-adhesive protein adsorption values among aged plasma treatment types. This observation further strengthens the hypothesis that argon plasma activation mostly led to a surface roughness change, rather than to a chemical change, and that is the nanoroughness, which remains unaltered overtime, the feature responsible of celladhesive protein adsorption. ${ }^{57}$
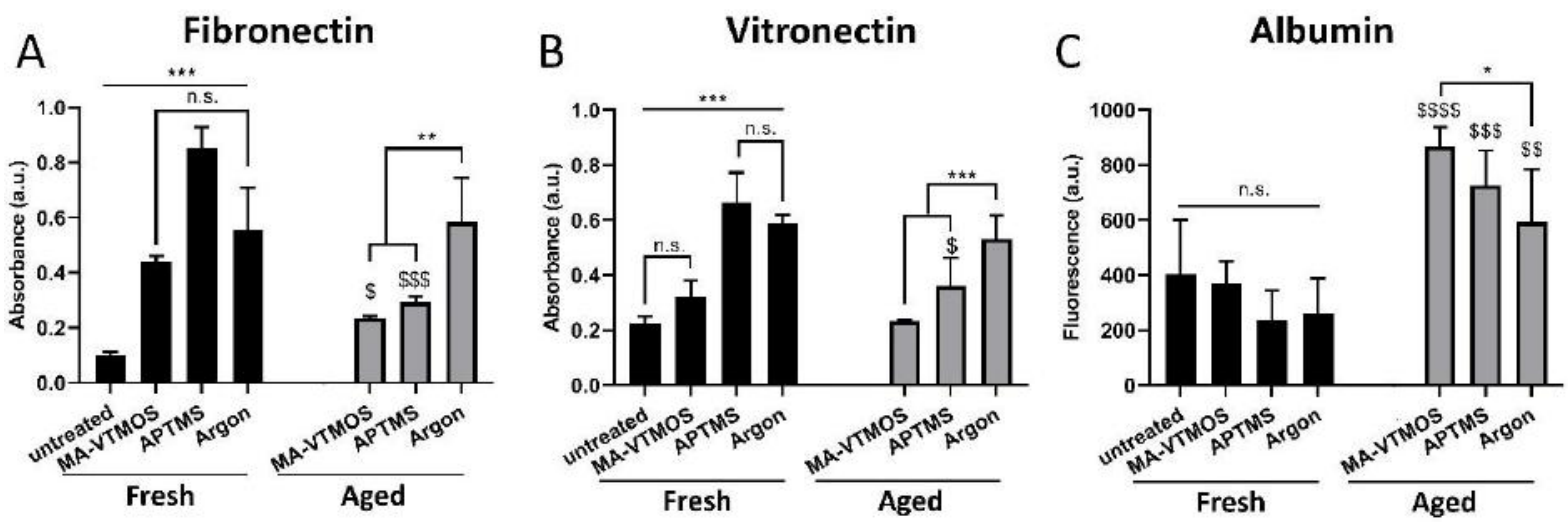

Figure 5. Protein adsorption to scaffolds upon incubation in serum containing medium $((+) F B S)$ : effect of plasma functionalization and aging conditions. Relative amounts of adsorbed $(A)$ fibronectin, (B) vitronectin, and (C) albumin to untreated scaffolds and fresh and aged MA-VTMOS, APTMS and Argon plasma treated scaffolds. Data presented as average \pm s.d. and statistical significance performed using two-way ANOVA with Tukey's multiple comparison test (n.s. $p>0.05$, ${ }^{*} \$ p<0.05,{ }^{* *} \$ p<0.01,{ }^{* * *} \$ \$ \$ p<0.0001 ;{ }^{*}$ for comparisons among treatments within aged or fresh scaffolds; $\$$ for comparisons among fresh and aged for each plasma treatment). 


\section{Cell adhesion mechanism to plasma treated scaffolds}

Enhanced cell attachment on 2D surfaces and 3D scaffolds containing polar functional groups has been attributed to surface wettability, ${ }^{58}$ surface charge potential, 55,59 or amount of protein adsorption. ${ }^{19}$ However, most of these studies have been performed in serum containing media, or in protein-coated surfaces, masking the potential effect of direct cell interactions with the functional groups, and therefore not fully characterizing the mechanism of cell adhesion to a specific surface chemistry. Thus, in order to discern among attachment mediated by electrostatic interactions with the charged surfaces, and attachment via integrin binding to proteins adsorbed from serum, fresh scaffolds were pre incubated for $24 \mathrm{~h}$ in (+)FBS or serum-free medium ((-)FBS), and subsequently seeded with hMSCs for 4h in (+)FBS or (-)FBS. Representative fluorescent images of hMSCs on scaffolds' cross section after overnight culture suggested an increase on cell attachment on plasma activated and polymerized scaffolds, compared to untreated scaffolds, when these were pre-incubated in (+)FBS, regardless of the presence or absence of serum during the $4 \mathrm{~h}$ seeding (Figure 6A). This can be attributed to the higher adsorption of cell adhesive proteins ( $F n$ and $V n$ ) to these scaffolds, as shown in Figure 5. Despite improving cell adhesion and distribution, cell sedimentation towards the bottom of the scaffold was observed both on untreated and treated scaffolds (Figure S5A). It is known that, regardless of the scaffolds surface properties, cells in the macropores, which are far from the scaffolds filaments during the seeding process, are unable to interact with the scaffold's surface, leading to sedimentation towards the bottom and a monolayer formation. Looking at the quantitative values on seeding efficiency presented in Figure $6 \mathrm{C}$, no differences in total cell attachment among scaffold types were observed, when pre-incubated and seeded in (+)FBS. In turn, slightly higher cell attachment was noted on MA-VTMOS and Argon scaffolds compared to APTMS and untreated scaffolds, when pre-incubated in (+)FBS but seeded in (-)FBS. In the case of APTMS, since cell distribution images show homogenous cell attachment in the cross section comparable to other plasma conditions and to its (+) FBS seeding counterpart, it is plausible that lower cell adhesion on the bottom of the scaffold might have contributed to lower overall cell adhesion values. Importantly, the sedimented cells that attached to the bottom of the untreated scaffolds, might have significantly contributed to the relatively higher seeding efficiency values than expected for the low cell coverage on their cross section. Interestingly, despite being pre-incubated in (-)FBS, homogeneous cell attachment was observed along the cross section of plasma 
A

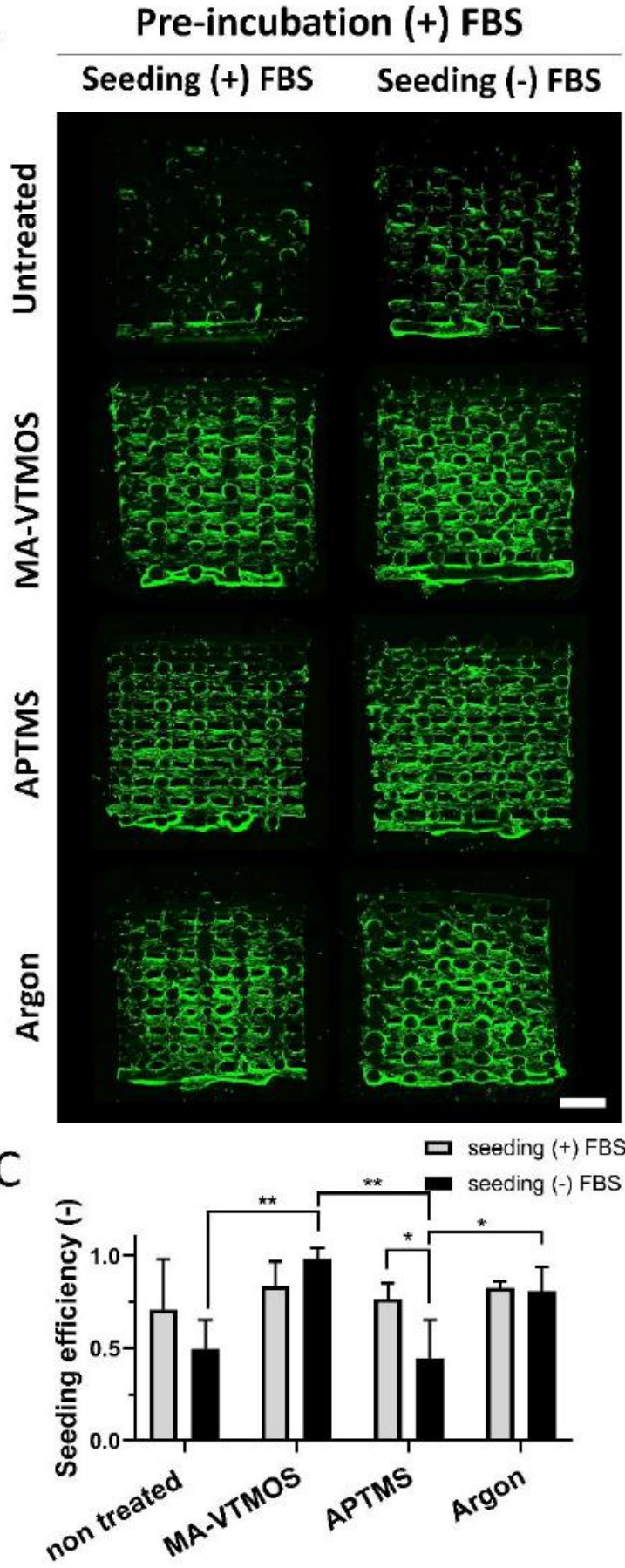

B

\begin{tabular}{c} 
Pre-incubation (-) FBS \\
\hline Seeding (+) FBS Seeding(-) FBS
\end{tabular}
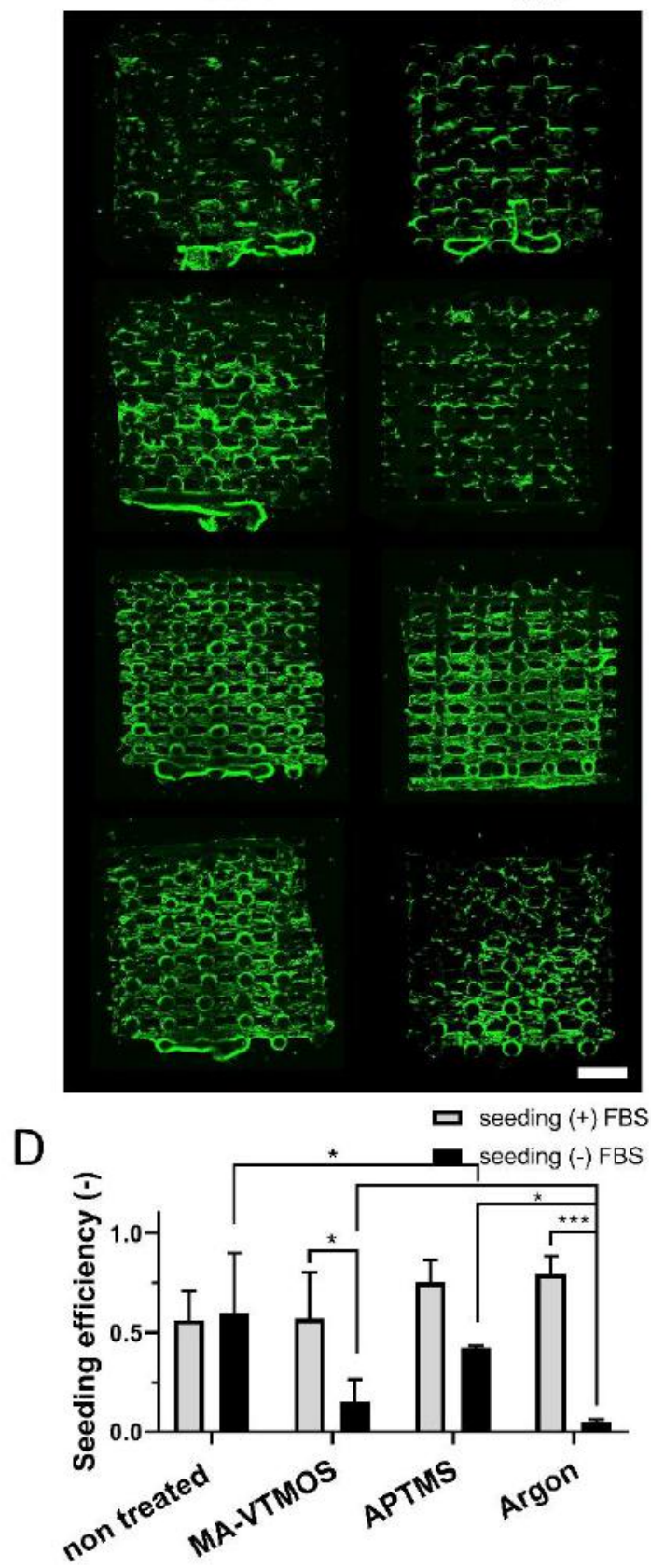

Figure 6. Cell coverage and cell seeding efficiency on fresh plasma treated scaffolds under different pre-incubation and seeding conditions. Fluorescence microscopy images ( $F$-actin, green) of hMSCs in the cross sections of scaffolds after $24 \mathrm{~h}$ of culture, and (A) pre-incubated with (+)FBS or (B) (-) FBS and seeded with (+)FBS or (-)FBS. Scale bars $1 \mathrm{~mm}$. Quantification of cell seeding efficiency on scaffolds (C) pre-incubated with (+)FBS and (D) pre-incubated with (-)FBS. Data presented as average \pm s.d. and statistical significance performed using two-way ANOVA with Tukey's multiple comparison test $\left({ }^{*} p<0.05 ;{ }^{* *} p<0.01 ;{ }^{* * *} p<0.001\right)$. 
treated scaffolds seeded in (+)FBS (Figure 6B), but not in untreated scaffolds. Furthermore, cell seeding efficiency values were comparable to those of scaffolds preincubated and seeded in (+)FBS (Figure S5C). These observations suggested that preincubation in (+)FBS was not necessary, when the seeding process was performed in $(+)$ FBS, regardless of the plasma treatment. This is likely due to protein adsorption reaching an equilibrium already in short incubation times. In fact, serum proteins have been shown to adsorb to surfaces containing polar functional groups within the first minutes of incubation. ${ }^{53,55}$ On the other hand, seeding with (+)FBS was necessary for optimum cell attachment on MA-VTMOS and argon scaffolds, when pre-incubated in ()FBS. In this case, seeding with (-)FBS on MA-VTMOS treated scaffolds led to poor, and comparable cell coverage in scaffold's cross section to untreated scaffolds (Figure 6B), which together with lack of sedimented cells layer formation on the bottom (cells remained on the well plate) contributed to significantly lower seeding efficiency (Figure 6D and Figure S5B). Similarly, lower cell coverage and absence of a cell monolayer at the bottom were observed in cross section of argon treated scaffolds pre-incubated and seeded in ()FBS, compared to other pre-incubation and seeding conditions. In both cases, due to the lack of protein attachment sites, the monolayer preferentially attached to the bottom of the seeding well plate rather than to the scaffold. Surprisingly, APTMS scaffolds pre-incubated and seeded in (-)FBS demonstrated a homogeneous and confluent cell coverage, as well as seeding efficiency values comparable to other pre-incubation and seeding conditions (Figure 6D and Figure S5D). Overall, these results suggest that i) MA-VTMOS plasma polymerization support cell attachment to scaffolds mostly through cell-protein interactions, and ii) cell attachment on APTMS plasma polymerized scaffolds is driven by cell-proteins interactions, in the presence of serum, and by electrostatic interactions between the cell and the amine groups coating the scaffolds filaments, in the absence of serum. The latter conclusion is in agreement with previously published reports studying cell adhesion on 2D surfaces suggesting that in protein-free conditions, direct interactions between negatively charged chondroitin sulfate proteoglycans in the cell membrane and the positively charged amine groups on a surface are responsible of promoting cell attachment. 58, 60 On the other hand, repulsive electrostatic interactions between the negatively charged groups on the MA-VTMOS scaffolds and the cells do not allow for cell attachment in serum free conditions. In the case of argon plasma treatment, we hypothesize that the nanoroughness itself 61 or combined with the lower presence of 
charged hydrophilic groups, might have favored a slightly higher cell attachment in these scaffolds in serum free conditions compared to MA-VTMOS treated scaffolds.

To further analyze cell attachment mechanism to the different plasma treated scaffolds, cell morphology and focal adhesion formation in the absence or presence of serum was studied (Figure 7). Pre-incubation and seeding in (+)FBS led to cell spreading and the formation of F-actin stress fibers on hMSCs adhered to both untreated and plasma treated scaffolds, only after the $4 \mathrm{~h}$ seeding. Moreover, while hMSCs did not show well defined vinculin structures on untreated scaffolds, established focal adhesions at the end of the stress fibers in the periphery of the cells were visualized on the plasma treated scaffolds, which was correlated to the higher amount of cell-adhesive proteins adsorbed on these surfaces allowing for integrin binding (Figure 7). Larger vinculin expression, as well as increased focal adhesion kinase expression, which localizes to focal adhesions to activate migration, proliferation and differentiation pathways, were also previously reported on amine, carboxyl, hydroxyl and argon functionalized surfaces pre-incubated in Fn ${ }^{20}$ or with (+)FBS medium, ${ }^{62-63}$ compared to untreated surfaces. On the contrary, the few attached hMSCs to the untreated, MA-VTMOS and argon scaffolds, showed a well-defined round morphology and lacked focal adhesion complexes when pre-incubated and seeded in ()FBS, due to the repulsive electrostatic forces and the lack of cell adhesive proteins hindering cell attachment spreading (Figure 7). The fact that, when looking at the scaffolds cross section, these cells were only found on the top surface of the filaments, further suggested that cells passively laid on top of the filament due to gravity during the seeding process, and that not active attachment occurred. On the contrary, hMSCs on APTMS scaffolds pre-incubated and seeded in (-)FBS were observed occupying the whole filament surface area. Interestingly, these cells were able to form multiple protrusions and F-actin fibers, which were not colocalized with focal adhesions, suggesting cell adhesion to the positively charged scaffold surface without mediation of integrin binding sites (Figure 7). Similar microfilament bundle formation and lack of focal contacts formation was previously reported on fibroblast adhesion to $2 \mathrm{D}$ amine coated surfaces. 60 

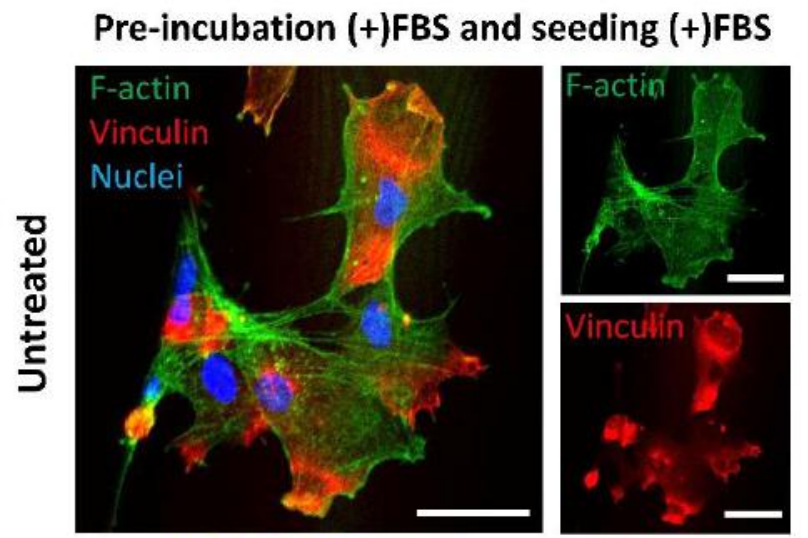

Pre-incubation (-)FBS and seeding (-)FBS
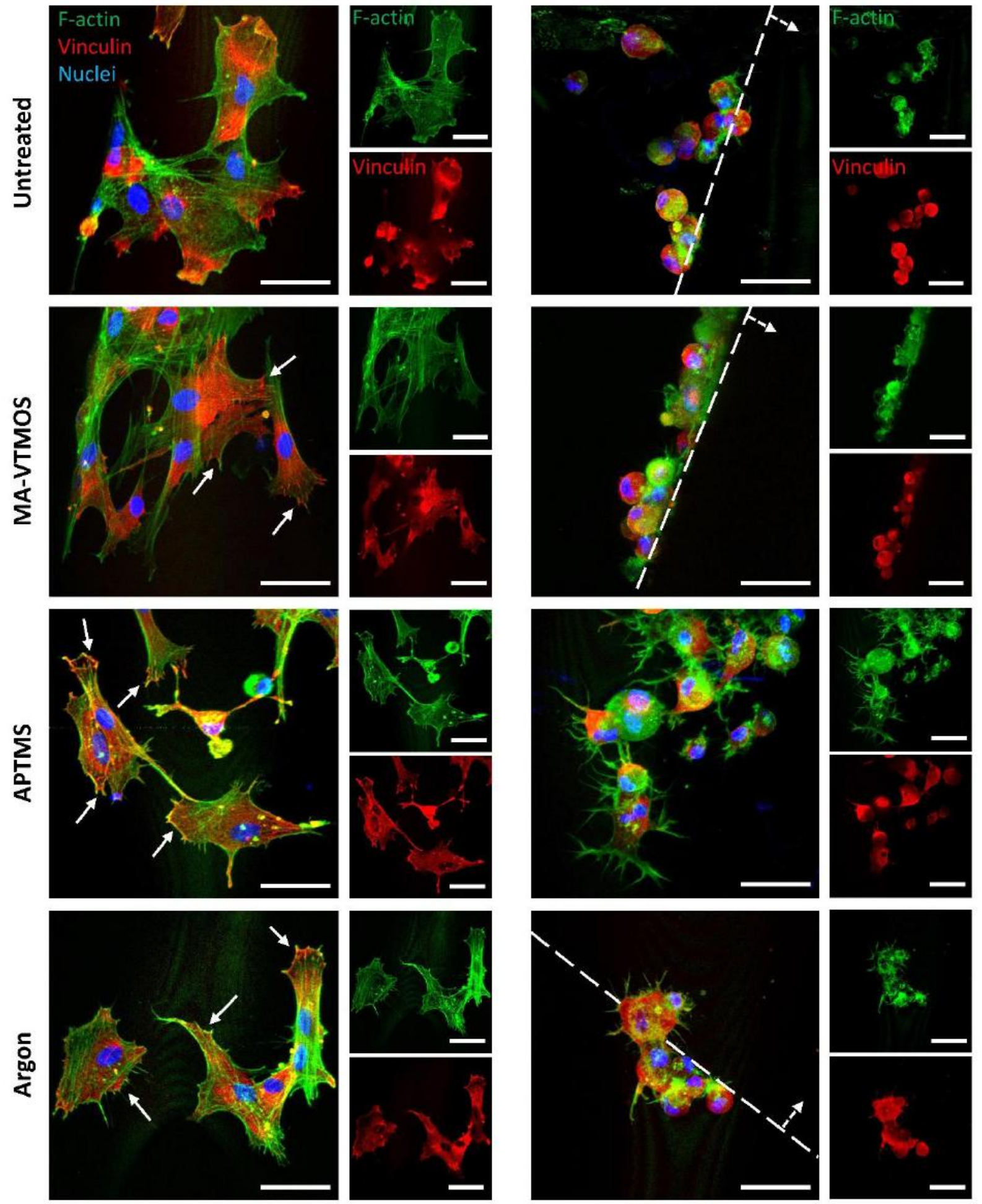

Figure 7. HMSCs morphology and focal adhesion formation after $4 \mathrm{~h}$ seeding on fresh plasma treated scaffolds pre-incubated and seeded in (+)FBS or (-)FBS. Representative confocal microscopy images of hMSCs (F-actin, green; nuclei, blue; vinculin, red) on top of scaffold filaments $4 \mathrm{~h}$ post-seeding. Continuous arrows indicating focal adhesions. Dashed lines delimitating the scaffold filament, with dashed arrows indicating the filament's surface. Scale bars $50 \mu \mathrm{m}$. 


\section{Cell proliferation}

Further cell culture studies aiming to assess cell behavior on the different plasma treated scaffolds were performed on scaffolds pre-incubated and seeded in (+)FBS, in order to have comparable cell number and distribution among scaffolds, as these parameters can influence cell behavior by their own. ${ }^{64}$ Initially, we confirmed that aging did not affect total cell attachment (Figure 8A) nor cell distribution (Figure S6), regardless of the plasma condition. Plasma effect on cell adhesion and distribution also showed not to be hMSCs donor dependent, suggesting the possibility of extrapolating our results to other hMSCs populations (Figure S6B, C). Interestingly, hMSCs did not proliferate significantly after 7 days of culture, neither on untreated nor on fresh or aged plasma scaffolds (Figure 8B). Despite this initial lack of proliferation, a significant increase in DNA content with respect to day 1 was observed on all fresh and aged plasma treated scaffolds after 14 days of culture ( 7 days in BM followed by 7 days in MM) (Figure $8 \mathrm{C}$ ). It is plausible that an increase in available surface area, given by ECM production in the scaffold pores volume when cultured in MM, offered cells the possibility to further proliferate on plasma treated scaffolds, as previously suggested. ${ }^{4}$ Previous research also showed significant differences in proliferation among surface functionalized and untreated 2D substrates only after long culture periods ( 10 days), ${ }^{25}$ whereas the use of dynamic culture systems or growth factors was found necessary to increase ECM production and boost cell proliferation on 3D plasma treated scaffolds. ${ }^{25,} 27$ Notably, no differences in hMSCs proliferation rate were found among fresh scaffolds, nor among aged scaffolds. Yet, while hMSCs in MA-VTMOS scaffolds proliferated at the same rate in fresh and aged scaffolds, higher levels in hMSCs proliferation were found on fresh APTMS and argon treated scaffolds, compared to their aged counterparts. In the case of APTMS, this might be due to the lower density of functional groups and adsorbed proteins on aged scaffolds.

\section{Osteogenic differentiation potential of hMSCs seeded on aged plasma treated scaffolds}

The analysis of osteogenic differentiation was performed on aged scaffolds, due to their higher applicability, as previously mentioned. Moreover, since comparable total cell numbers among conditions were observed at any timepoint evaluated during the culture on these scaffolds (Figure S7), it was then possible to focus solely on the influence of 

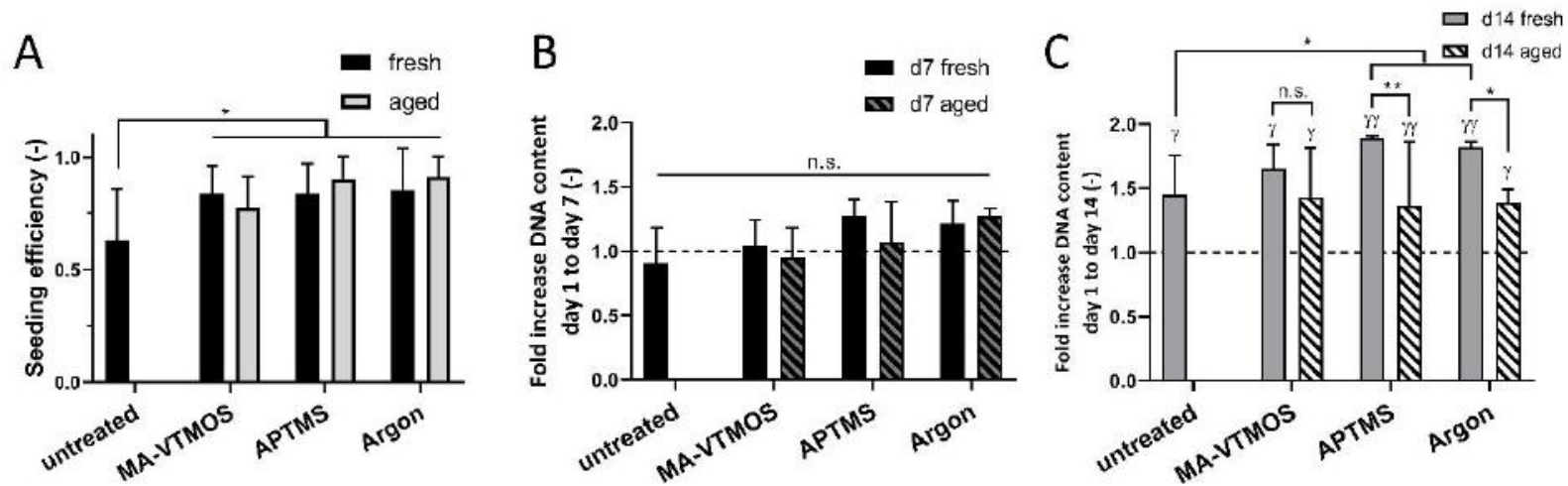

Figure 8. Effect of plasma treatment and aging conditions on cell seeding efficiency and proliferation. (A) Cell seeding efficiency, and fold increase in DNA content after (B) 7 days of culture (in $\mathrm{BM}$ ), and (C) 14 days of culture (7 days in BM and 7 days in $M M$ ) with respect to day 1 , which is marked with a dashed line. Scaffolds were pre-incubated and seeded with (+)FBS. Data presented as average \pm s.d. and statistical significance performed using two-way ANOVA with Tukey's multiple comparison test (n.s. $p>0.05,{ }^{*} \gamma p<0.05,{ }^{* *} \gamma \mathrm{p} p<0.01$; ${ }^{*}$ for comparisons among plasma treatments within aged or fresh scaffolds, and among fresh and aged scaffolds for each plasma treatment; $y$ for comparisons between the given timepoint and day 1 for each plasma treatment).

plasma activation and polymerization on differentiation, and exclude the cell number and distribution related effect on osteogenesis. Following this rationale, inefficiently seeded untreated control scaffolds were not included in the study. After seeding, scaffolds were cultured for a total of 54 days in MM ( 7 days in BM, followed by 47 days in $\mathrm{MM}$ ). Immunofluorescence was used to evaluate collagen I (COL I) deposition at early and late timepoints of the differentiation process (day 14 and day 54). Representative fluorescent microscopy images in Figure 9A revealed that hMSCs were able to produce COL I, one of the main bone ECM proteins, from early timepoints in all plasma treated scaffold conditions. These images also revealed that ECM became denser and pores more filled in the course of the culture. Furthermore, the expression of relevant osteogenic genes was screened though PCR (Figure 9B) at day 14 and 54 of culture. ALP, an early osteogenic marker, indicated no significant differences among plasma conditions or timepoints. Similarly, no statistical differences in OCN expression, a protein that bounds to hydroxyapatite, were found among plasma conditions and timepoints. RUNX2, a transcription factor modulating the expression of osteogenic proteins, was upregulated at day 54 in Argon treated scaffolds compared to other conditions. When analyzing osterix (OSX), bone sialoprotein (BSP) and bone morphogenetic protein-2 (BMP2) expression, proteins present in a maturated bone ECM, no upregulation was observed unless in argon treated scaffolds at day 54 compared to day 14 . Yet, no significant differences among 
plasma conditions were found at this time point. Overall, no clear trends were observed within this study and no plasma treatment showed to provide a distinct osteogenic stimulation to hMSCs over the others. In 2D functionalized surfaces, an enhanced osteogenic effect of amine groups compared to other functional groups has been correlated to the enhanced exposure of the integrin domain $\alpha 5 \beta 1$ in adsorbed $F n$, and to high levels of recruitment of focal adhesion components and phosphorylation of focal adhesion kinases in the adhered cells, which are events required for osteoblast differentiation. 20-22, 62, 65 In this regard, it is plausible that aging masked the osteogenic stimulation potential of the APTMS surfaces, and that some differences would have been evidenced under fresh conditions, since APTMS fresh scaffolds displayed the highest levels of protein adsorption and, potentially, of integrin domain $\alpha 5 \beta 1$. It is also plausible that the effect of surface chemistry is only pronounced at an early stage, when cells are in direct contact with the scaffolds surface, and that upon ECM production by cells during longer-term culture, cell-substrate contact becomes limited and ineffective. 66 Nevertheless, due to the lack of published reports simultaneously comparing the effect of different surface chemistries on osteogenic differentiation in 3D scaffolds, comparing our results with previous literature becomes challenging. To the best of our knowledge, a single study as such has demonstrated that salt leached poly(carbonate-urea) urethane scaffold modified by allylamine plasma polymerization, significantly enhanced osteogenic differentiation of adipose derived stem cells, compared to a carboxylic acid modified scaffold. ${ }^{67}$ Yet, no clear explanation on how the amine group affected the osteogenesis pathway was reported. Moreover, since those scaffolds were fabricated by a conventional manufacturing method, i.e. porogen leaching, the poor interconnected porosity might have induced a different cell behavior compared to what we observed in ME-AM scaffolds.

Besides, it is worth noticing that ECM mineralization was not observed in any scaffold type during the culture period evaluated (Figure S8A), despite the proven mineralization potential of these cells in 2D (Figure S8B). Also, no matrix mineralization was attained on fresh scaffolds (Figure S9C). Thus, it is believed that cells on the scaffolds did not reach yet a matured level of differentiation, and that the homogenous cell distribution after seeding given by a bioactive surface chemistry is not sufficient for a successful osteogenic differentiation and mineralization outcome. It is hypothesized that larger cell seeding numbers could have led to larger cell attachment, and therefore higher cell density and cell-cell contact for accelerated differentiation. ${ }^{68-70}$ In order to investigate this, preliminary experiments were conducted, in which scaffolds were seeded with a larger seeding density 
(400,000 cells per scaffold (400k), instead of 200,000 (200k)). Despite higher cell seeding efficiency, as per DNA quantification (Figure S9A), cells in scaffolds seeded with 400k tended to form a denser monolayer in the scaffolds' bottom, while maintaining the scaffolds' filaments as populated as when seeded with 200k (Figure S9B, C, day 1 of culture). Yet, some pores were already filled on the plasma treated scaffolds seeded with 400k at day 7 (Figure S9D), a cell confluency level that was not observed with lower cell seeding density, and that could potentially derive in earlier osteogenesis. Future work will be aimed at understanding if an enhancement of cell confluency at earlier timepoints, at the expense of needing a larger cell stock, might lead to mineralization and tissue maturation. This will help us to unravel if a specific plasma induced surface chemistry can offer enhanced osteogenic differentiation or if, on the contrary, cell confluency regardless of the surface chemistry is the primary parameter effectively playing a role on osteogenic differentiation on 3D AM scaffolds.

A
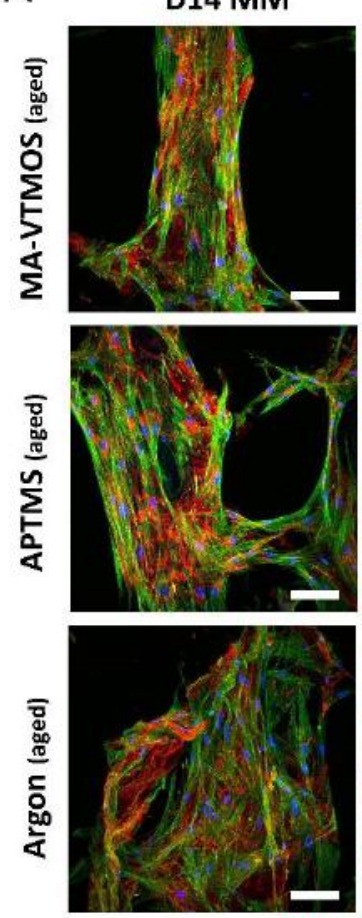

D54 MM
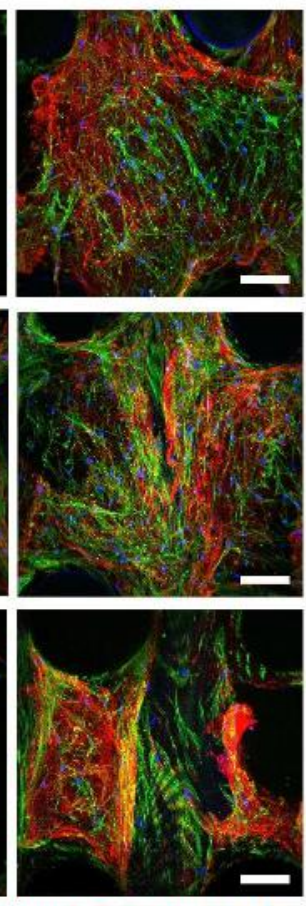

B

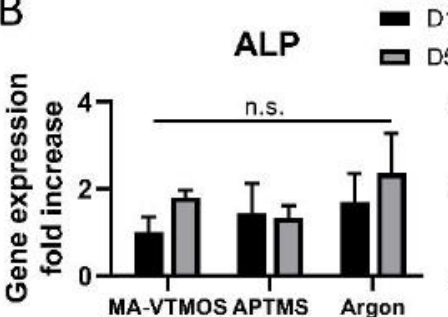

OCN

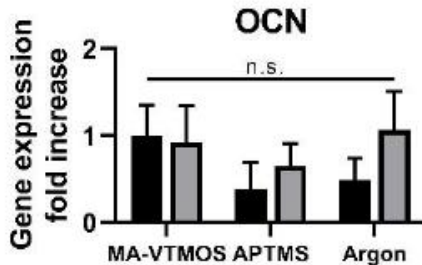

BSP

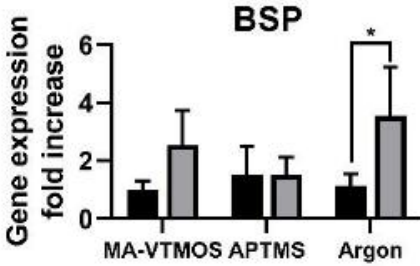

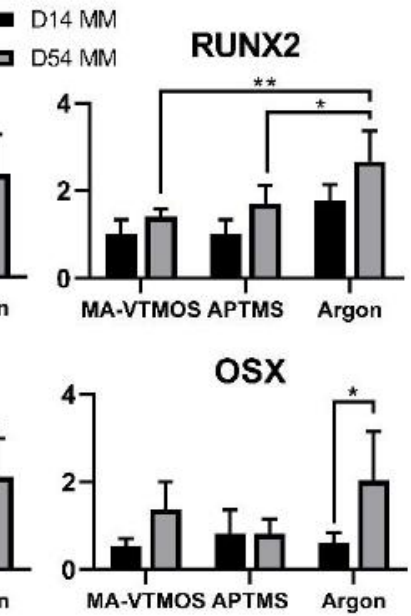

BMP2

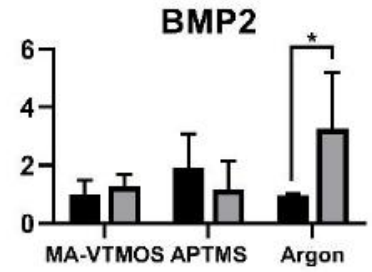

Figure 9. Evaluation of the osteogenic differentiation potential of hMSCs seeded on aged plasma treated scaffolds. (A) Representative confocal microscope images of hMSCs (F-actin, green) on top of scaffolds filaments after 14 and 54 days of culture (7 and 47 days in MM, respectively) and stained for the relevant osteogenic marker COL1 (red). Scale bars $100 \mu \mathrm{m}$. (B) Gene expression of hMSCs after 14 and 54 days of culture ( 7 and 47 days in MM, respectively) comparing the three different plasma treatments. ALP, RUNX2, OCN, OSX, BSP and BMP2 fold change expression values relative to MA-VTMOS day 14 . Data presented as average \pm s.d. and statistical significance performed using two-way ANOVA with Tukey's multiple comparison test (n.s. $p>0.05$; ${ }^{*} p<0.05$; $\left.{ }^{* *} p<0.01\right)$. 


\section{CONCLUSION}

ME-AM enables the reproducible fabrication of highly porous polymeric scaffolds, ideal for tissue regeneration. However, the lack of cell adhesion sites on synthetic polymers hinders an efficient cell adhesion to the scaffolds, the first step towards the development of a functional construct. The aim of this study was to fabricate 3D scaffolds using a hybrid AM technique that enabled scaffold fabrication by ME-AM and their subsequent atmospheric pressure plasma treatment, to enhance their bioactivity, within the same platform. While argon activation resulted in an unspecific surface plasma treatment, APTMS plasma polymerization enabled the deposition of a positively charged coating containing amine functional groups, and MA-VTMOS rendered the surface of the scaffold negatively charged, by depositing carboxyl and anhydride functional groups. All plasma treatments increased the surface wettability of the scaffolds, enhanced cell-adhesive proteins adsorption to their surface, and allowed for homogeneous cell attachment along the scaffold cross section. Interestingly, cell attachment was found to be driven by cell protein interactions in the presence of serum and by electrostatic interactions between the cell and the charged scaffold surface in serum free conditions. This latter feature allowed for cell attachment and scaffold population on APTMS treated scaffolds in the absence of serum, which possess relevant clinical applications. Scaffolds storage led to the aging of the surface treatment, in terms of a slight reduction of exposed functional groups. Yet, cell attachment and proliferation were not significantly affected. Notably, none of the plasma treatments stimulated osteogenic differentiation of hMSCs significantly more than the others, and it is hypothesized that initial cell confluency might play a major role, overruling effects of a specific surface chemistry on the osteogenic differentiation of hMSCs and ECM mineralization on 3D ME-AM scaffolds. Overall, this newly proposed method enabled an efficient workflow of scaffold production and surface treatment, and opens the door to future research on the effect of different plasma treatments on cell behavior on 3D ME-AM scaffolds.

\section{Acknowledgement}

We are grateful to the FAST project funded under the H2020-NMP-PILOTS-2015 scheme (GA n. 685825) for financial support. Some of the materials used in this work were provided by the Texas A\&M Health Science Center College of Medicine Institute for Regenerative Medicine at Scott \& White through a grant from NCRR of the NIH (Grant \#P40RR017447). 


\section{References}

1. Mota, C.; Puppi, D.; Chiellini, F.; Chiellini, E., Additive Manufacturing Techniques for the Production of Tissue Engineering Constructs. J Tissue Eng Regen Med 2015, 9 (3), 174-190.

2. Kim, K.; Dean, D.; Mikos, A. G.; Fisher, J. P., Effect of Initial Cell Seeding Density on Early Osteogenic Signal Expression of Rat Bone Marrow Stromal Cells Cultured on Cross-Linked Poly(Propylene Fumarate) Disks. Biomacromolecules 2009, 10 (7), 1810-1817.

3. Hasegawa, T.; Miwa, M.; Sakai, Y.; Niikura, T.; Lee, S. Y.; Oe, K.; Iwakura, T.; Kurosaka, M.; Komori, T., Efficient Cell-Seeding into Scaffolds Improves Bone Formation. Journal of Dental Research 2010, 89 (8), 854-859.

4. Cámara-Torres, M.; Sinha, R.; Mota, C.; Moroni, L., Improving Cell Distribution on 3d Additive Manufactured Scaffolds through Engineered Seeding Media Density and Viscosity. Acta Biomaterialia 2020, 101, 183-195.

5. Chen, Y.; Bloemen, V.; Impens, S.; Moesen, M.; Luyten, F. P.; Schrooten, J., Characterization and Optimization of Cell Seeding in Scaffolds by Factorial Design: Quality by Design Approach for Skeletal Tissue Engineering. Tissue Engineering Part C: Methods 2011, 17 (12), 1211-1221.

6. Sobral, J. M.; Caridade, S. G.; Sousa, R. A.; Mano, J. F.; Reis, R. L., Three-Dimensional Plotted Scaffolds with Controlled Pore Size Gradients: Effect of Scaffold Geometry on Mechanical Performance and Cell Seeding Efficiency. Acta Biomaterialia 2011, 7 (3), 1009-1018.

7. Leferink, A. M.; Hendrikson, W.; Rouwkema, J.; Karperien, M.; van Blitterswijk, C.; Moroni, L., Increased Cell Seeding Efficiency in Bioplotted Three-Dimensional Peot/Pbt Scaffolds. Journal of tissue engineering and regenerative medicine 2016, 10 (8), 679-689.

8. Dong, L.; Wang, S.-J.; Zhao, X.-R.; Zhu, Y.-F.; Yu, J.-K., 3d- Printed Poly(E-Caprolactone) Scaffold Integrated with Cell-Laden Chitosan Hydrogels for Bone Tissue Engineering. Scientific Reports 2017, 7 (1), 13412.

9. Whitely, M.; Cereceres, S.; Dhavalikar, P.; Salhadar, K.; Wilems, T.; Smith, B.; Mikos, A.; CosgriffHernandez, E., Improved in Situ Seeding of 3d Printed Scaffolds Using Cell-Releasing Hydrogels. Biomaterials 2018, 185, 194-204.

10. Ostrowska, B.; Di Luca, A.; Szlazak, K.; Moroni, L.; Swieszkowski, W., Influence of Internal Pore Architecture on Biological and Mechanical Properties of Three-Dimensional Fiber Deposited Scaffolds for Bone Regeneration. Journal of biomedical materials research. Part A 2016, 104 (4), 991-1001.

11. Yu, J.; Xu, Y.; Li, S.; Seifert, G. V.; Becker, M. L., Three-Dimensional Printing of Nano Hydroxyapatite/Poly(Ester Urea) Composite Scaffolds with Enhanced Bioactivity. Biomacromolecules 2017, 18 (12), 4171-4183.

12. Qi, X.; Pei, P.; Zhu, M.; Du, X.; Xin, C.; Zhao, S.; Li, X.; Zhu, Y., Three Dimensional Printing of Calcium Sulfate and Mesoporous Bioactive Glass Scaffolds for Improving Bone Regeneration in Vitro and in Vivo. Scientific Reports 2017, 7 (1), 42556.

13. Wang, W.; Caetano, G.; Ambler, W. S.; Blaker, J. J.; Frade, M. A.; Mandal, P.; Diver, C.; Bartolo, P., Enhancing the Hydrophilicity and Cell Attachment of 3d Printed Pcl/Graphene Scaffolds for Bone Tissue Engineering. Materials (Basel) 2016, 9 (12).

14. Zamani, Y.; Mohammadi, J.; Amoabediny, G.; Visscher, D. O.; Helder, M. N.; Zandieh-Doulabi, B.; KleinNulend, J., Enhanced Osteogenic Activity by Mc3t3-E1 Pre-Osteoblasts on Chemically Surface-Modified Poly(E-Caprolactone) 3d-Printed Scaffolds Compared to Rgd Immobilized Scaffolds. Biomedical materials (Bristol, England) 2018, 14 (1), 015008.

15. Kosik-Kozioł, A.; Graham, E.; Jaroszewicz, J.; Chlanda, A.; Kumar, P. T. S.; Ivanovski, S.; Święszkowski, W.; Vaquette, C., Surface Modification of 3d Printed Polycaprolactone Constructs Via a Solvent Treatment: Impact on Physical and Osteogenic Properties. ACS Biomaterials Science \& Engineering 2019, 5 (1), 318328.

16. Kang, S.-W.; Kim, J.-S.; Park, K.-S.; Cha, B.-H.; Shim, J.-H.; Kim, J. Y.; Cho, D.-W.; Rhie, J.-W.; Lee, S.$\mathrm{H}$., Surface Modification with Fibrin/Hyaluronic Acid Hydrogel on Solid-Free Form-Based Scaffolds Followed by Bmp-2 Loading to Enhance Bone Regeneration. Bone 2011, 48 (2), 298-306.

17. De Geyter, N.; Morent, R., 7 - Cold Plasma Surface Modification of Biodegradable Polymer Biomaterials. In Biomaterials for Bone Regeneration, Dubruel, P.; Van Vlierberghe, S., Eds. Woodhead Publishing: 2014; pp 202-224. 
18. Barrias, C. C.; Martins, M. C. L.; Almeida-Porada, G.; Barbosa, M. A.; Granja, P. L., The Correlation between the Adsorption of Adhesive Proteins and Cell Behaviour on Hydroxyl-Methyl Mixed SelfAssembled Monolayers. Biomaterials 2009, 30 (3), 307-316.

19. Arima, Y.; Iwata, H., Preferential Adsorption of Cell Adhesive Proteins from Complex Media on SelfAssembled Monolayers and Its Effect on Subsequent Cell Adhesion. Acta Biomaterialia 2015, 26, $72-81$.

20. Keselowsky, B. G.; Collard, D. M.; García, A. J., Surface Chemistry Modulates Focal Adhesion Composition and Signaling through Changes in Integrin Binding. Biomaterials 2004, 25 (28), 5947-5954.

21. Curran, J. M.; Chen, R.; Hunt, J. A., The Guidance of Human Mesenchymal Stem Cell Differentiation in Vitro by Controlled Modifications to the Cell Substrate. Biomaterials 2006, 27 (27), 4783-4793.

22. Chen, M.; Zhang, Y.; Zhou, Y.; Zhang, Y.; Lang, M.; Ye, Z.; Tan, W.-S., Pendant Small Functional Groups on Poly( $\epsilon$-Caprolactone) Substrate Modulate Adhesion, Proliferation and Differentiation of Human Mesenchymal Stem Cells. Colloids and Surfaces B: Biointerfaces 2015, 134, 322-331.

23. Yildirim, E. D.; Besunder, R.; Pappas, D.; Allen, F.; Guceri, S.; Sun, W., Accelerated Differentiation of Osteoblast Cells on Polycaprolactone Scaffolds Driven by a Combined Effect of Protein Coating and Plasma Modification. Biofabrication 2010, 2 (1), 014109.

24. Jeon, H.; Lee, H.; Kim, G., A Surface-Modified Poly(E-Caprolactone) Scaffold Comprising Variable Nanosized Surface-Roughness Using a Plasma Treatment. Tissue Engineering Part C: Methods 2014, 20 (12), 951-963.

25. Lerman, M. J.; Smith, B. T.; Gerald, A. G.; Santoro, M.; Fookes, J. A.; Mikos, A. G.; Fisher, J. P., Aminated 3d Printed Polystyrene Maintains Stem Cell Proliferation and Osteogenic Differentiation. Tissue Engineering Part C: Methods 2019, 26 (2), 118-131.

26. Domingos, M.; Intranuovo, F.; Gloria, A.; Gristina, R.; Ambrosio, L.; Bártolo, P. J.; Favia, P., Improved Osteoblast Cell Affinity on Plasma-Modified 3-D Extruded Pcl Scaffolds. Acta Biomaterialia 2013, 9 (4), 5997-6005.

27. Cools, P.; Mota, C.; Lorenzo-Moldero, I.; Ghobeira, R.; De Geyter, N.; Moroni, L.; Morent, R., Acrylic Acid Plasma Coated 3d Scaffolds for Cartilage Tissue Engineering Applications. Sci Rep 2018, 8 (1), 3830.

28. Cools, P.; Sainz-García, E.; Geyter, N. D.; Nikiforov, A.; Blajan, M.; Shimizu, K.; Alba-Elías, F.; Leys, C.; Morent, R., Influence of Dbd Inlet Geometry on the Homogeneity of Plasma-Polymerized Acrylic Acid Films: The Use of a Microplasma-Electrode Inlet Configuration. Plasma Processes and Polymers 2015, $12(10), 1153-1163$.

29. De Geyter, N.; Morent, R.; Leys, C., Penetration of a Dielectric Barrier Discharge Plasma into Textile Structures at Medium Pressure. Plasma Sources Science and Technology 2006, 15 (1), 78.

30. Lu, X.; Laroussi, M.; Puech, V., On Atmospheric-Pressure Non-Equilibrium Plasma Jets and Plasma Bullets. Plasma Sources Science and Technology 2012, 21 (3), 034005.

31. Liu, F.; Wang, W.; Hinduja, S.; Bártolo, P. J., Hybrid Additive Manufacturing System for Zonal PlasmaTreated Scaffolds. 3D Printing and Additive Manufacturing 2018, 5 (3), 205-213.

32. Sinha, R.; Cámara-Torres, M.; Scopece, P.; Falzacappa, E. V.; Patelli, A.; Moroni, L.; Mota, C., A Hybrid Additive Manufacturing Platform to Create Bulk and Surface Composition Gradients on Scaffolds for Tissue Regeneration. bioRxiv 2020, 2020.2006.2023.165605.

33. Hody, H.; Choquet, P.; Moreno-Couranjou, M.; Maurau, R.; Pireaux, J.-J., Optimization of Carboxyl Surface Functionalization by Ma-Vtms Copolymerization Using Atmospheric Pressure Plasma Dbd: Influence of the Carrier Gas. Plasma Processes and Polymers 2010, 7 (5), 403-410.

34. Chen, Z.; Zhao, J.; Jin, C.; Yuan, Y.; Zhang, Y.; Tatoulian, M.; Rao, X., Plasma Deposited Aptes: A Potential Film for Biomedical Application. Materials Letters 2020, 264, 127350.

35. Bitar, R.; Cools, P.; De Geyter, N.; Morent, R., Acrylic Acid Plasma Polymerization for Biomedical Use. Applied Surface Science 2018, 448, 168-185.

36. Bulou, S.; Lecoq, E.; Loyer, F.; Frache, G.; Fouquet, T.; Gueye, M.; Belmonte, T.; Choquet, P., Study of a Pulsed Post-Discharge Plasma Deposition Process of Aptes: Synthesis of Highly Organic Pp-Aptes Thin Films with Nh2 Functionalized Polysilsesquioxane Evidences. Plasma Processes and Polymers 2019, 16 (4), 1800177.

37. A. Patelli, E. V. F., P. Scopece, R. Pierobon, S. Vezzù Method for Generating Atmospheric Plasma Jet and Atmospheric Plasma Mini-Torch Device. 2015. 
38. Manakhov, A.; Moreno-Couranjou, M.; Boscher, N. D.; Rogé, V.; Choquet, P.; Pireaux, J.-J., Atmospheric Pressure Pulsed Plasma Copolymerisation of Maleic Anhydride and Vinyltrimethoxysilane: Influence of Electrical Parameters on Chemistry, Morphology and Deposition Rate of the Coatings. Plasma Processes and Polymers 2012, 9 (4), 435-445.

39. Zanini, S.; Grimoldi, E.; Riccardi, C., Development of Controlled Releasing Surfaces by Plasma Deposited Multilayers. Materials Chemistry and Physics 2013, 138 (2), 850-855.

40. Thomas, M.; von Hausen, M.; Klages, C.-P.; Baumhof, P., Generation of Stable Coatings with Carboxylic Groups by Copolymerization of Maa and Vtms Using Dbd at Atmospheric Pressure. Plasma Processes and Polymers 2007, 4 (S1), S475-S481.

41. Patelli, A.; Mussano, F.; Brun, P.; Genova, T.; Ambrosi, E.; Michieli, N.; Mattei, G.; Scopece, P.; Moroni, L., Nanoroughness, Surface Chemistry, and Drug Delivery Control by Atmospheric Plasma Jet on Implantable Devices. ACS Applied Materials \& Interfaces 2018, 10 (46), 39512-39523.

42. Lecoq, E.; Duday, D.; Bulou, S.; Frache, G.; Hilt, F.; Maurau, R.; Choquet, P., Plasma Polymerization of Aptes to Elaborate Nitrogen Containing Organosilicon Thin Films: Influence of Process Parameters and Discussion About the Growing Mechanisms. Plasma Processes and Polymers 2013, 10 (3), 250-261.

43. Mostofi Sarkari, N.; Doğan, Ö.; Bat, E.; Mohseni, M.; Ebrahimi, M., Assessing Effects of (3Aminopropyl)Trimethoxysilane Self-Assembled Layers on Surface Characteristics of OrganosilaneGrafted Moisture-Crosslinked Polyethylene Substrate: A Comparative Study between Chemical Vapor Deposition and Plasma-Facilitated in Situ Grafting Methods. Applied Surface Science 2019, 497, 143751.

44. Damanik, F. F. R.; Rothuizen, T. C.; van Blitterswijk, C.; Rotmans, J. I.; Moroni, L., Towards an in Vitro Model Mimicking the Foreign Body Response: Tailoring the Surface Properties of Biomaterials to Modulate Extracellular Matrix. Scientific Reports 2014, 4 (1), 6325.

45. Cools, P.; Asadian, M.; Nicolaus, W.; Declercq, H.; Morent, R.; De Geyter, N., Surface Treatment of Peot/Pbt (55/45) with a Dielectric Barrier Discharge in Air, Helium, Argon and Nitrogen at Medium Pressure. Materials (Basel) 2018, 11 (3), 391.

46. Olde Riekerink, M. B.; Claase, M. B.; Engbers, G. H. M.; Grijpma, D. W.; Feijen, J., Gas Plasma Etching of Peo/Pbt Segmented Block Copolymer Films. Journal of Biomedical Materials Research Part A 2003, $65 A(4), 417-428$.

47. Cao, B.; Peng, Y.; Liu, X.; Ding, J., Effects of Functional Groups of Materials on Nonspecific Adhesion and Chondrogenic Induction of Mesenchymal Stem Cells on Free and Micropatterned Surfaces. ACS Applied Materials \& Interfaces 2017, 9 (28), 23574-23585.

48. Nakamatsu, J.; Delgado-Aparicio, L. F.; Da Silva, R.; Soberon, F., Ageing of Plasma-Treated Poly(Tetrafluoroethylene) Surfaces. Journal of Adhesion Science and Technology 1999, 13 (7), 753-761.

49. Borris, J.; Thomas, M.; Klages, C.-P.; Faupel, F.; Zaporojtchenko, V., Investigations into Composition and Structure of Dbd-Deposited Amino Group Containing Polymer Layers. Plasma Processes and Polymers 2007, 4 (S1), S482-486.

50. Banik, I.; Kim, K. S.; Yun, Y. I.; Kim, D. H.; Ryu, C. M.; Park, C. S.; Sur, G. S.; Park, C. E., A Closer Look into the Behavior of Oxygen Plasma-Treated High-Density Polyethylene. Polymer 2003, 44 (4), 1163-1170.

51. Kim, K. S.; Ryu, C. M.; Park, C. S.; Sur, G. S.; Park, C. E., Investigation of Crystallinity Effects on the Surface of Oxygen Plasma Treated Low Density Polyethylene Using X-Ray Photoelectron Spectroscopy. Polymer 2003, 44 (20), 6287-6295.

52. Morent, R.; De Geyter, N.; Leys, C.; Gengembre, L.; Payen, E., Study of the Ageing Behaviour of Polymer Films Treated with a Dielectric Barrier Discharge in Air, Helium and Argon at Medium Pressure. Surface and Coatings Technology 2007, 201 (18), 7847-7854.

53. Arima, Y.; Iwata, H., Effects of Surface Functional Groups on Protein Adsorption and Subsequent Cell Adhesion Using Self-Assembled Monolayers. Journal of Materials Chemistry 2007, 17 (38), 4079-4087.

54. Arima, Y.; Iwata, H., Effect of Wettability and Surface Functional Groups on Protein Adsorption and Cell Adhesion Using Well-Defined Mixed Self-Assembled Monolayers. Biomaterials 2007, 28 (20), 3074-3082.

55. Lin, J.-H.; Chang, H.-Y.; Kao, W.-L.; Lin, K.-Y.; Liao, H.-Y.; You, Y.-W.; Kuo, Y.-T.; Kuo, D.-Y.; Chu, K.-J.; Chu, Y.-H.; Shyue, J.-J., Effect of Surface Potential on Extracellular Matrix Protein Adsorption. Langmuir 2014, 30 (34), 10328-10335.

56. Hoshiba, T.; Yoshikawa, C.; Sakakibara, K., Characterization of Initial Cell Adhesion on Charged Polymer Substrates in Serum-Containing and Serum-Free Media. Langmuir 2018, 34 (13), 4043-4051. 
57. Khang, D.; Kim, S. Y.; Liu-Snyder, P.; Palmore, G. T. R.; Durbin, S. M.; Webster, T. J., Enhanced Fibronectin Adsorption on Carbon Nanotube/Poly(Carbonate) Urethane: Independent Role of Surface Nano-Roughness and Associated Surface Energy. Biomaterials 2007, 28 (32), 4756-4768.

58. Webb, K.; Hlady, V.; Tresco, P. A., Relative Importance of Surface Wettability and Charged Functional Groups on Nih 3t3 Fibroblast Attachment, Spreading, and Cytoskeletal Organization. J Biomed Mater Res 1998, 41 (3), 422-430.

59. Chang, H.-Y.; Kao, W.-L.; You, Y.-W.; Chu, Y.-H.; Chu, K.-J.; Chen, P.-J.; Wu, C.-Y.; Lee, Y.-H.; Shyue, J.-J., Effect of Surface Potential on Epithelial Cell Adhesion, Proliferation and Morphology. Colloids and Surfaces B: Biointerfaces 2016, 141, 179-186.

60. Massia, S. P.; Hubbell, J. A., Immobilized Amines and Basic Amino Acids as Mimetic Heparin-Binding Domains for Cell Surface Proteoglycan-Mediated Adhesion. The Journal of biological chemistry 1992, 267 (14), 10133-10141.

61. Wang, M.; Favi, P.; Cheng, X.; Golshan, N. H.; Ziemer, K. S.; Keidar, M.; Webster, T. J., Cold Atmospheric Plasma (Cap) Surface Nanomodified 3d Printed Polylactic Acid (Pla) Scaffolds for Bone Regeneration. Acta Biomaterialia 2016, 46, 256-265.

62. Griffin, M. F.; Ibrahim, A.; Seifalian, A. M.; Butler, P. E. M.; Kalaskar, D. M.; Ferretti, P., Chemical GroupDependent Plasma Polymerisation Preferentially Directs Adipose Stem Cell Differentiation Towards Osteogenic or Chondrogenic Lineages. Acta Biomater 2017, 50, 450-461.

63. Griffin, M.; Palgrave, R.; Baldovino-Medrano, V. G.; Butler, P. E.; Kalaskar, D. M., Argon Plasma Improves the Tissue Integration and Angiogenesis of Subcutaneous Implants by Modifying Surface Chemistry and Topography. Int J Nanomedicine 2018, 13, 6123.

64. Lode, A.; Bernhardt, A.; Gelinsky, M., Cultivation of Human Bone Marrow Stromal Cells on ThreeDimensional Scaffolds of Mineralized Collagen: Influence of Seeding Density on Colonization, Proliferation and Osteogenic Differentiation. Journal of Tissue Engineering and Regenerative Medicine 2008, 2 (7), 400-407.

65. Moursi, A. M.; Globus, R. K.; Damsky, C. H., Interactions between Integrin Receptors and Fibronectin Are Required for Calvarial Osteoblast Differentiation in Vitro. J Cell Sci 1997, 110 ( Pt 18), 2187-2196.

66. Wang, P.-Y.; Clements, L. R.; Thissen, H.; Tsai, W.-B.; Voelcker, N. H., Screening Rat Mesenchymal Stem Cell Attachment and Differentiation on Surface Chemistries Using Plasma Polymer Gradients. Acta Biomaterialia 2015, 11, 58-67.

67. Griffin, M. F.; Ibrahim, A.; Seifalian, A. M.; Butler, P. E. M.; Kalaskar, D. M.; Ferretti, P., Chemical GroupDependent Plasma Polymerisation Preferentially Directs Adipose Stem Cell Differentiation Towards Osteogenic or Chondrogenic Lineages. Acta Biomaterialia 2017, 50, 450-461.

68. Holy, C. E.; Shoichet, M. S.; Davies, J. E., Engineering Three-Dimensional Bone Tissue in Vitro Using Biodegradable Scaffolds: Investigating Initial Cell-Seeding Density and Culture Period. Journal of Biomedical Materials Research: An Official Journal of The Society for Biomaterials, The Japanese Society for Biomaterials, and The Australian Society for Biomaterials and the Korean Society for Biomaterials 2000, 51 (3), 376-382.

69. Noda, S.; Kawashima, N.; Yamamoto, M.; Hashimoto, K.; Nara, K.; Sekiya, I.; Okiji, T., Effect of Cell Culture Density on Dental Pulp-Derived Mesenchymal Stem Cells with Reference to Osteogenic Differentiation. Scientific Reports 2019, 9 (1), 5430.

70. Abo-Aziza, F. A. M.; A A, Z., The Impact of Confluence on Bone Marrow Mesenchymal Stem (Bmmsc) Proliferation and Osteogenic Differentiation. Int J Hematol Oncol Stem Cell Res 2017, 11 (2), 121-132. 


\section{SUPPLEMENTARY FIGURES}

Table S1. Primer sequences used for qRT-PCR. ${ }^{a}$

\begin{tabular}{c|c|c}
\hline gene & forward primer & reverse primer \\
B2M & ACAAAGTCACATGGTTCACA & GACTTGTCTTTCAGCAAGGA \\
ALP & ACAAGCACTCCCACTTCATC & TTCAGCTCGTACTGCATGTC \\
RUNX2 & TCAACGATCTGAGATTTGTGGG & GGGGAGGATTTGTGAAGACGG \\
OCN & TGAGAGCCCTCACACTCCTC & CGCCTGGGTCTCTTCACTAC \\
SP7 & CCTCTGCGGGACTCAACAAC & AGCCCATTAGTGCTTGTAAAGG \\
BSP & CCCCACCTTTTGGGAAAACCA & TCCCCGTTCTCACTTTCATAGAT \\
BMP2 & ACTACCAGAAACGAGTGGGAA & GCATCTGTTCTCGGAAAACCT \\
\hline
\end{tabular}

${ }^{a}$ B2M: $\beta$-2-microglobulin, ALP: alkaline phosphatase, RUNX2: Runt-related transcription factor 2, OCN: osteocalcin, SP7: osterix, BSP: bone sialoprotein, and BMP2: bone morphogenetic protein2

A

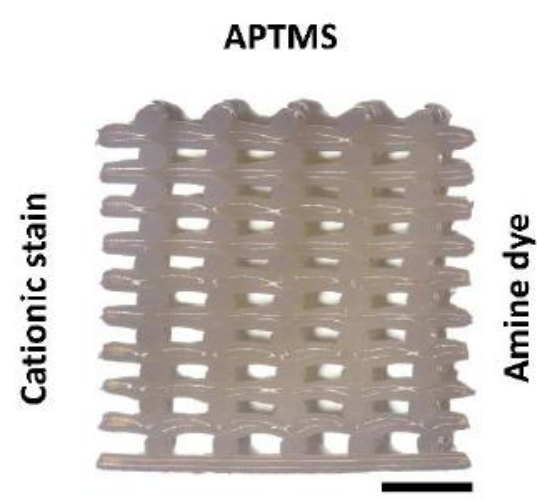

B
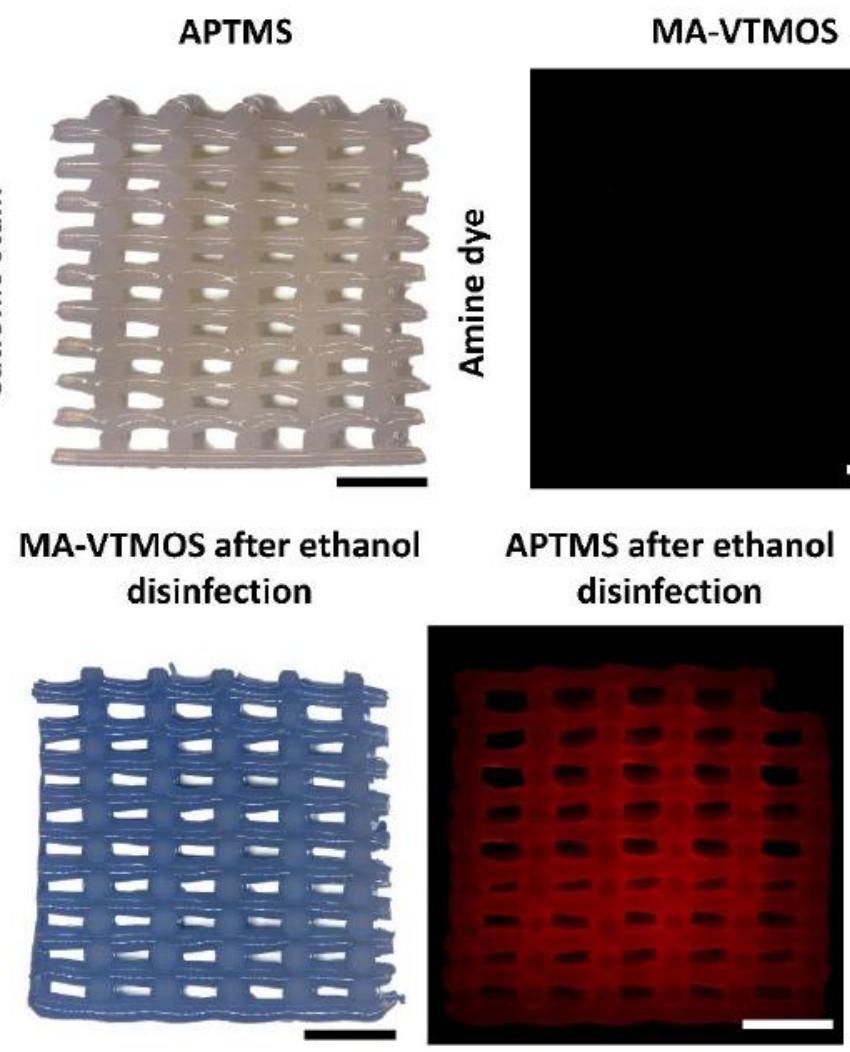

Figure S1. (A) Stereomicroscopy image of APTMS scaffold stained with the cationic dye methylene blue, and fluorescent microscopy images of MA-VTMOS and Argon scaffolds stained with amine specific dyes, to prove the specificity of the staining. (B) Stereomicroscopy image of MA-VTMOS scaffold and fluorescent microscopy image of APTMS scaffold stained with coating specific dyes after ethanol disinfection to verify the maintenance of the treatment before cell culture experiments. Scale bars $1 \mathrm{~mm}$. 


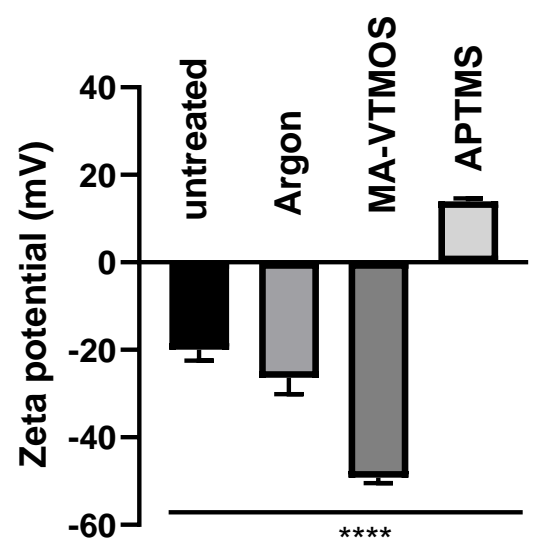

Figure S2. Zeta potential measurements of untreated, and Argon, MA-VTMOS and APTMS treated 2D substrates (melt-pressed films). Data presented as average \pm s.d. and statistical significance performed using one-way ANOVA with Tukey's multiple comparison test $\left({ }^{\star \star \star *} p<0.0001\right)$.

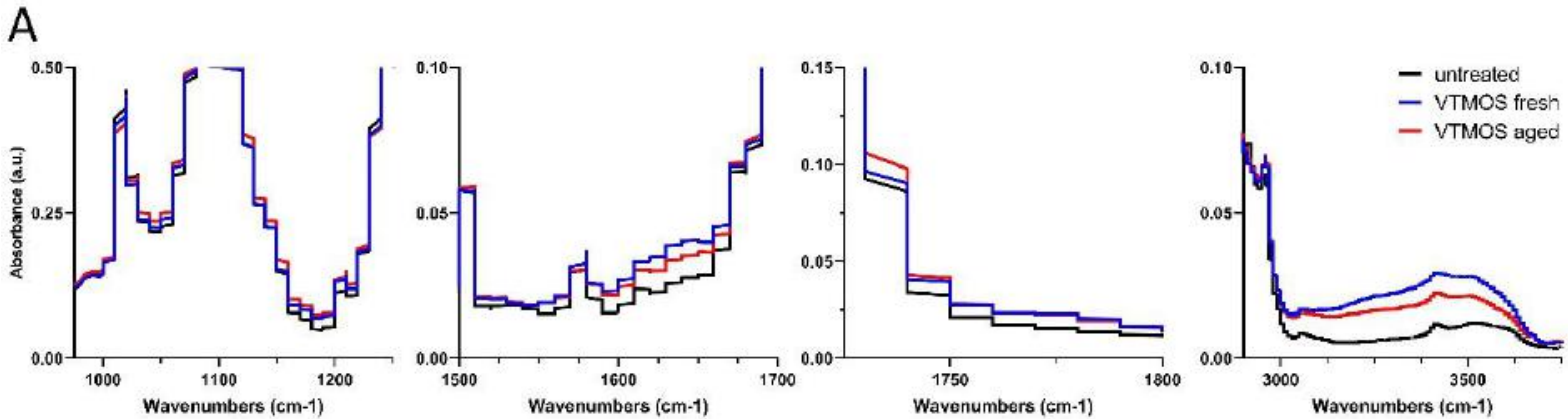

B
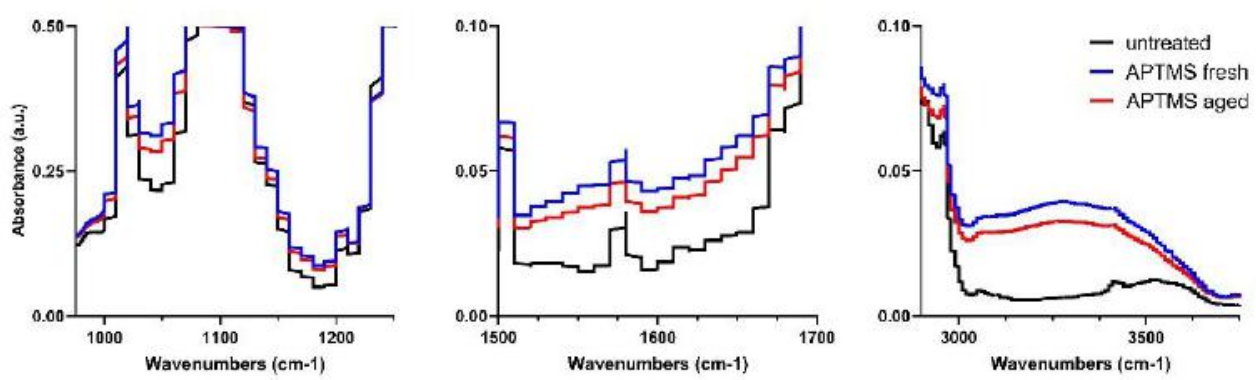

C
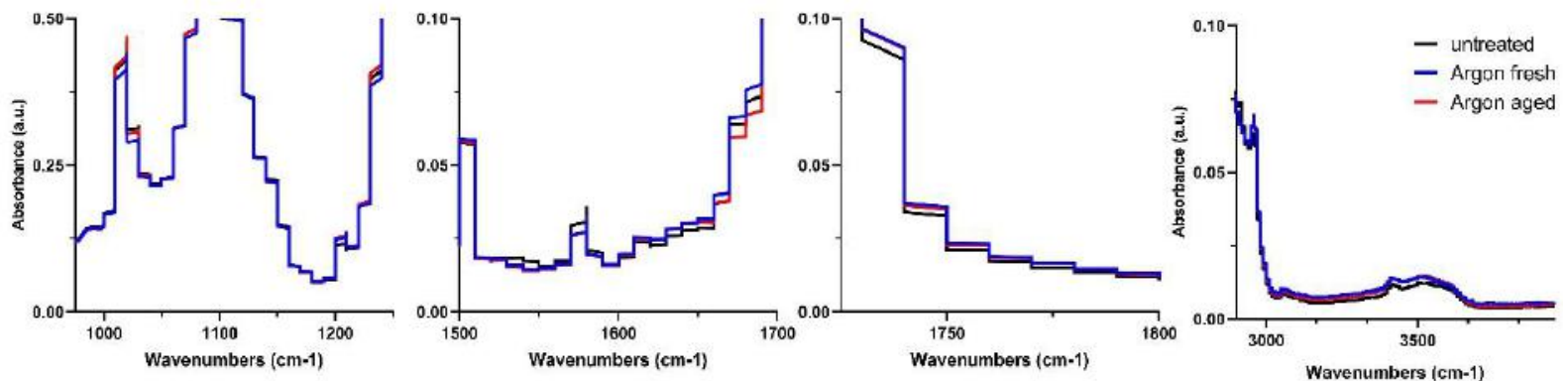

Figure S3. Regions of interest of the FTIR spectra of (A) MA-VTMOS, (B) APTMS and (C) Argon plasma treated 2D substrates (melt-pressed PEOT/PBT films). 


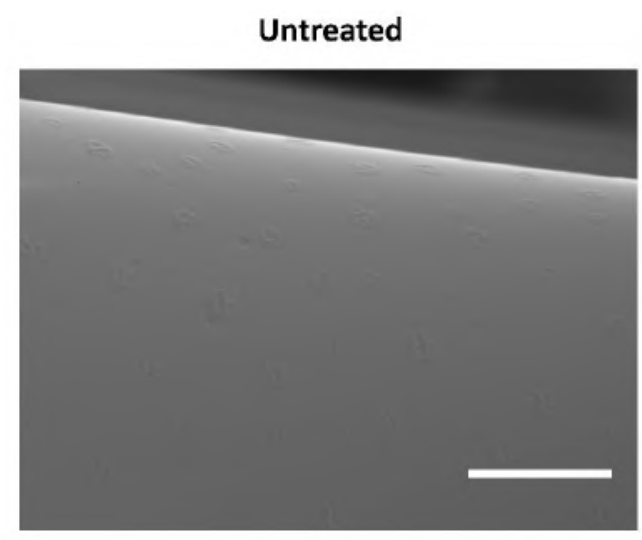

MA-VTMOS

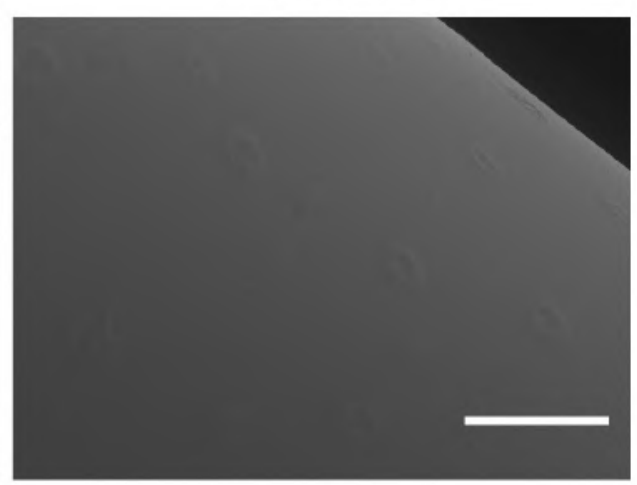

APTMS

Argon
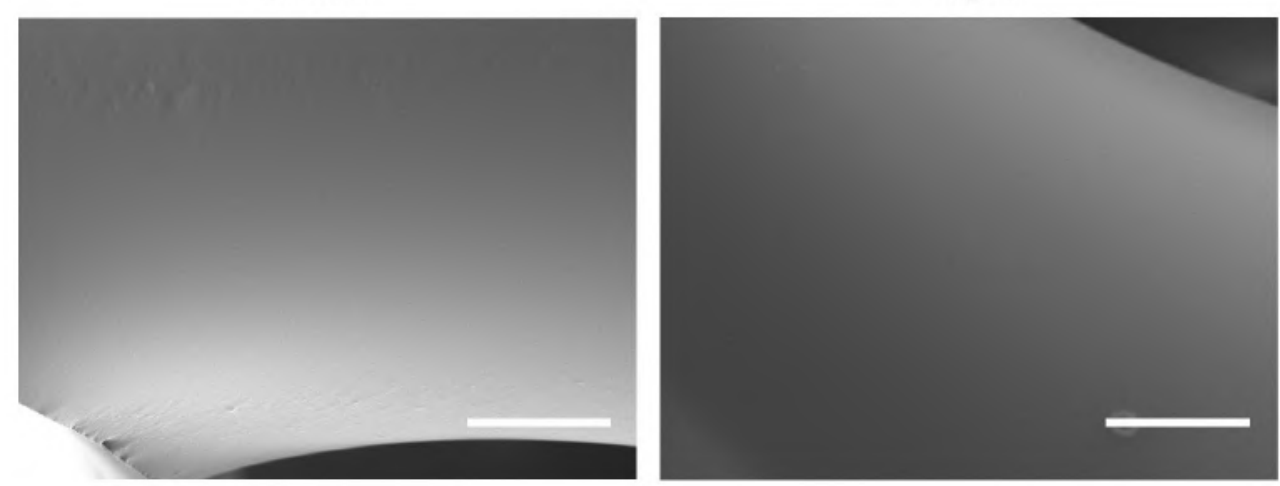

Figure S4. SEM micrographs the surface of untreated and MA-VTMOS, APTMS and Argon plasma treated scaffolds. Scale bars $50 \mu \mathrm{m}$. 
A

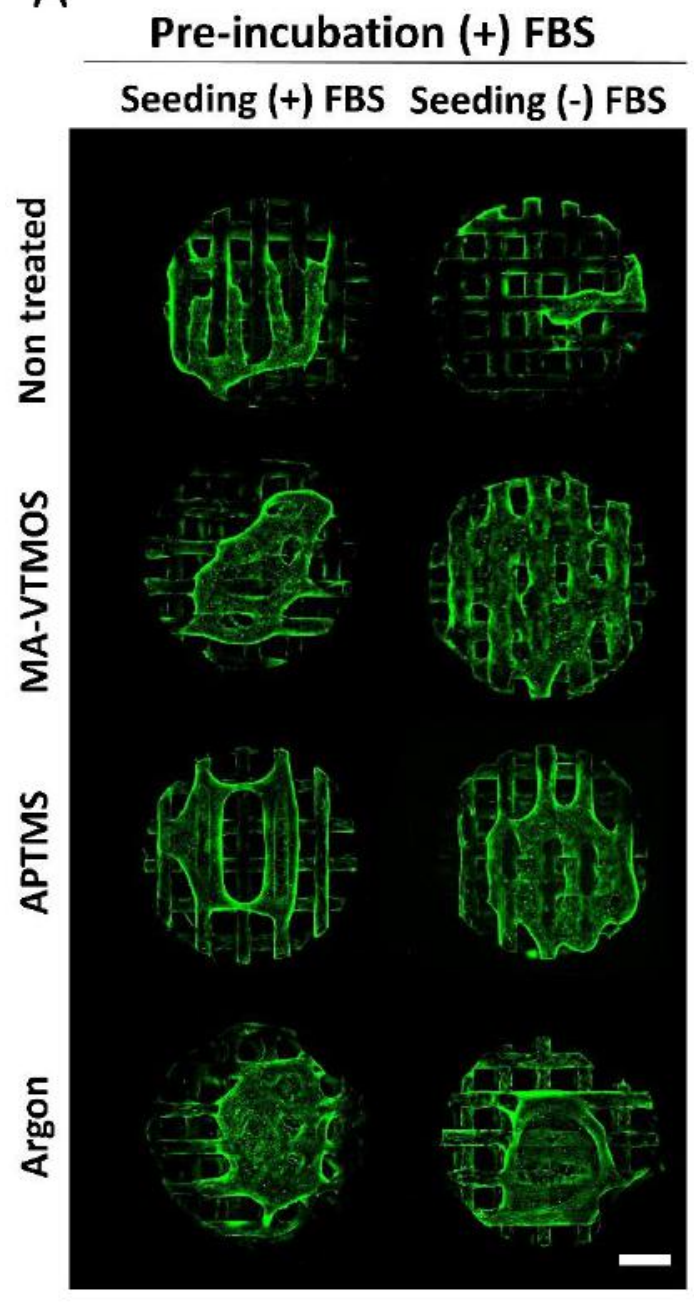

C Seeding (+) FBS

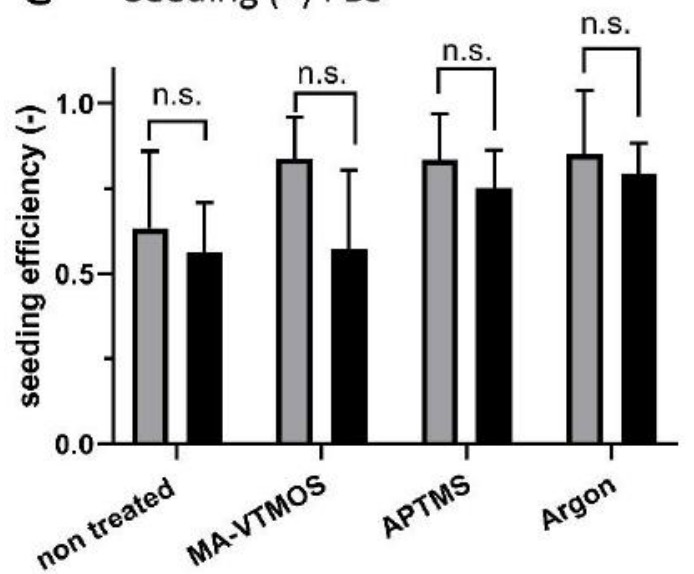

B

Pre-incubation (-) FBS

Seeding (+) FBS Seeding (-) FBS

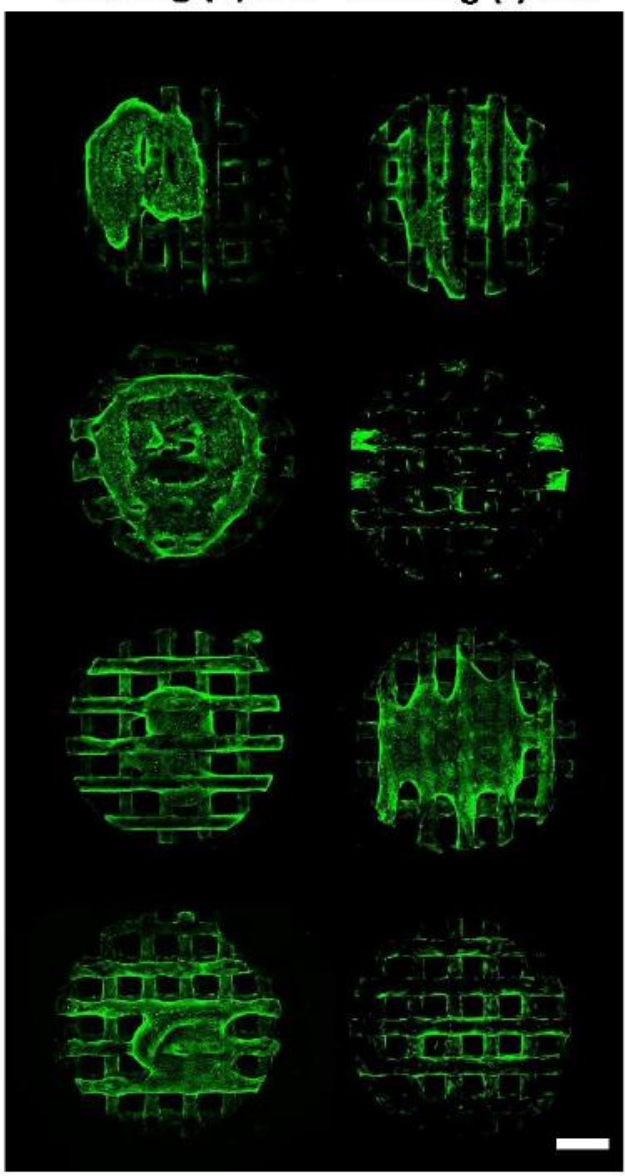

D Seeding (-)FBS $\square$ pre-incubation (+) FBS

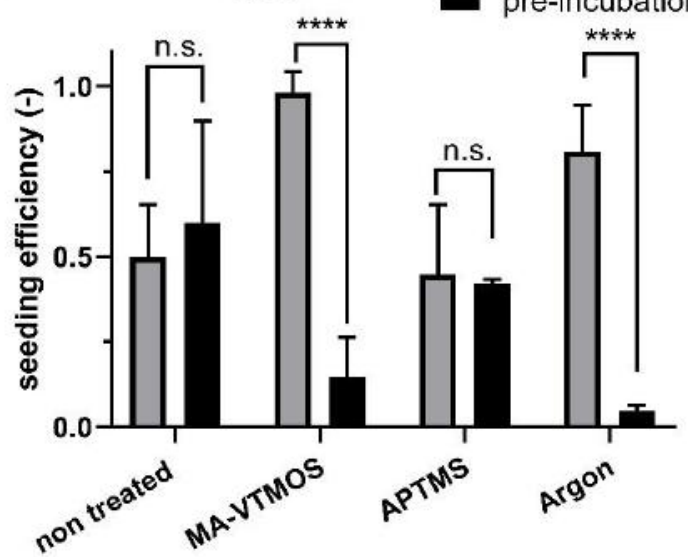

Figure S5. Fluorescence microscopy images (F-actin, green) of hMSCs in the bottom lids of scaffolds after $24 \mathrm{~h}$ of culture and pre-incubated with (A) (+)FBS or (B) (-) FBS and seeded with $(+)$ FBS or (-)FBS. Scale bars $1 \mathrm{~mm}$. Quantification of cell seeding efficiency on scaffolds (C) seeded with (+)FBS and (D) seeded (-)FBS. Data presented as average \pm s.d. and statistical significance performed using two-way ANOVA with Tukey's multiple comparison test (n.s. $p>0.05$; $\left.{ }^{* \star \star \star} p<0.0001\right)$. 


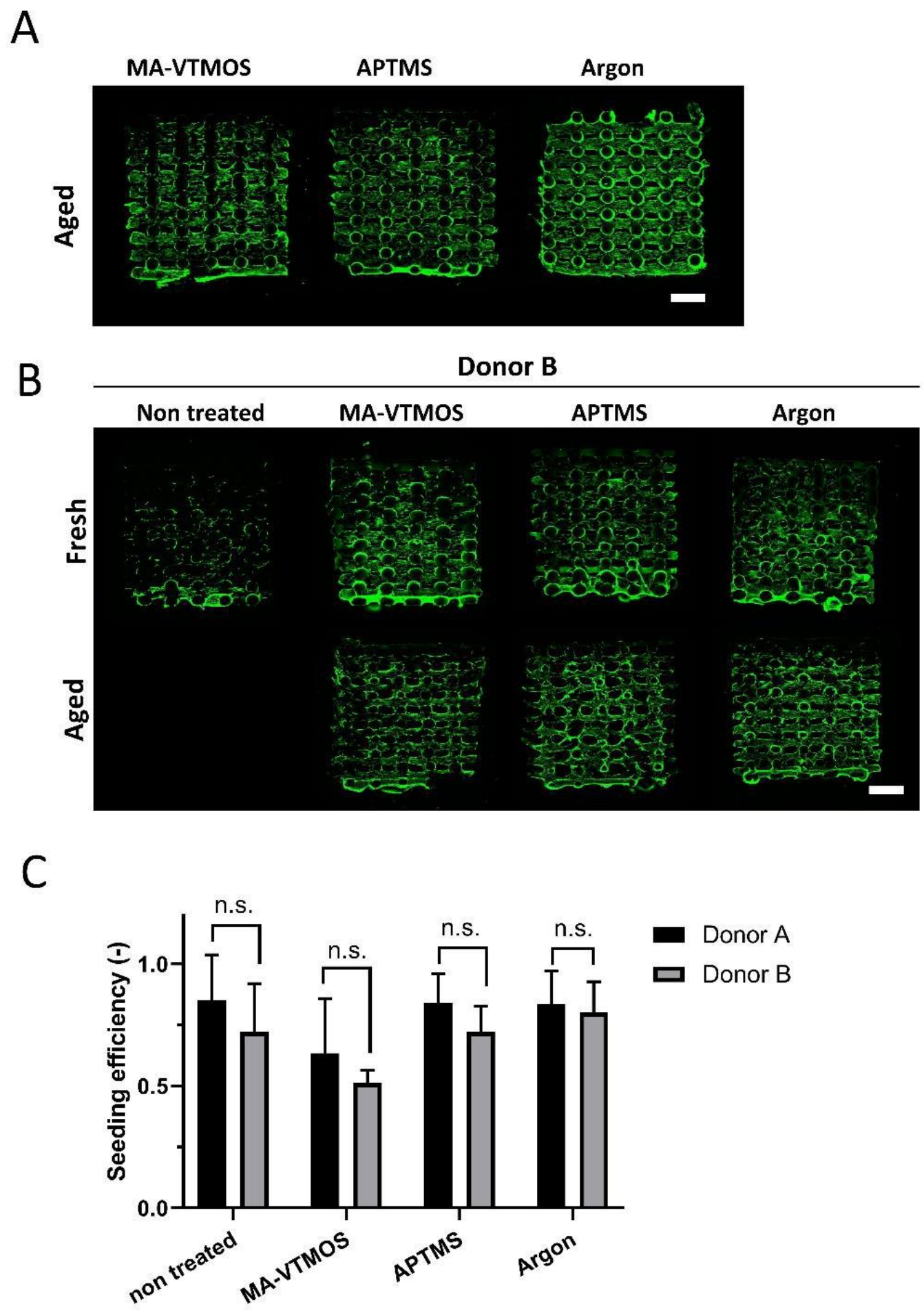

Figure S6. (A) Fluorescence microscopy images (F-actin, green) of hMSCs on the cross section of aged plasma treated scaffolds after $24 \mathrm{~h}$ of culture, and pre-incubated and seeded with (+)FBS. Scale bars $1 \mathrm{~mm}$. (B) Fluorescence microscopy images (F-actin, green) of hMSCs from a different donor (donor B) on the cross section of fresh and aged plasma treated scaffolds. Scaffolds were pre-incubated and seeded with (+)FBS. Scale bars $1 \mathrm{~mm}$. (C) Seeding efficiency on plasma treated scaffolds comparing hMSCs from 2 different donors. Data presented as average \pm s.d. and statistical significance performed using two-way ANOVA with Tukey's multiple comparison test (n.s. $p>0.05)$. 


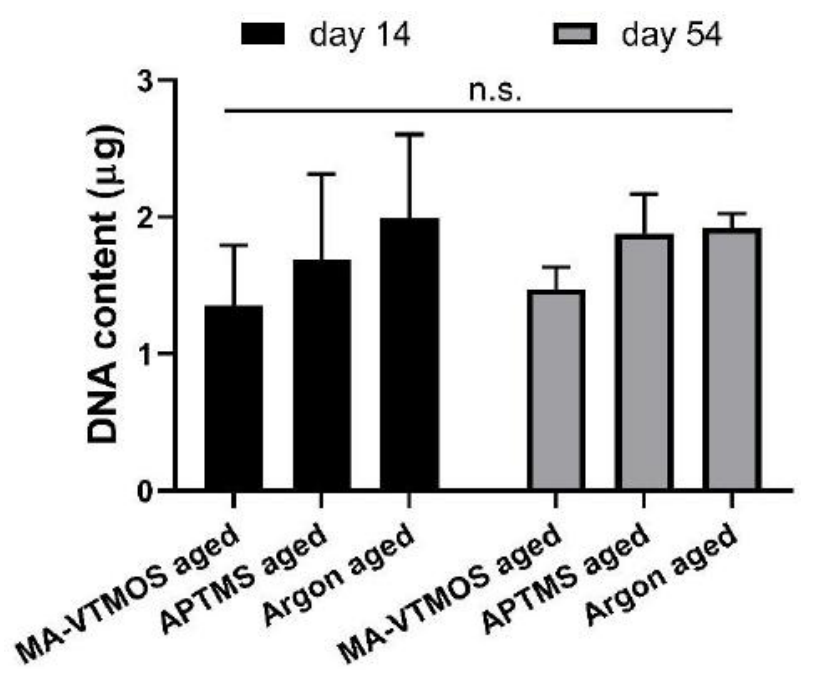

Figure S7. DNA content on aged plasma treated scaffolds after 14 or 54 days of culture ( 7 days or 47 days in $M M$, respectively). Data presented as average \pm s.d. and statistical significance performed using two-way ANOVA with Tukey's multiple comparison test (n.s. $p>0.05$ ). 
A

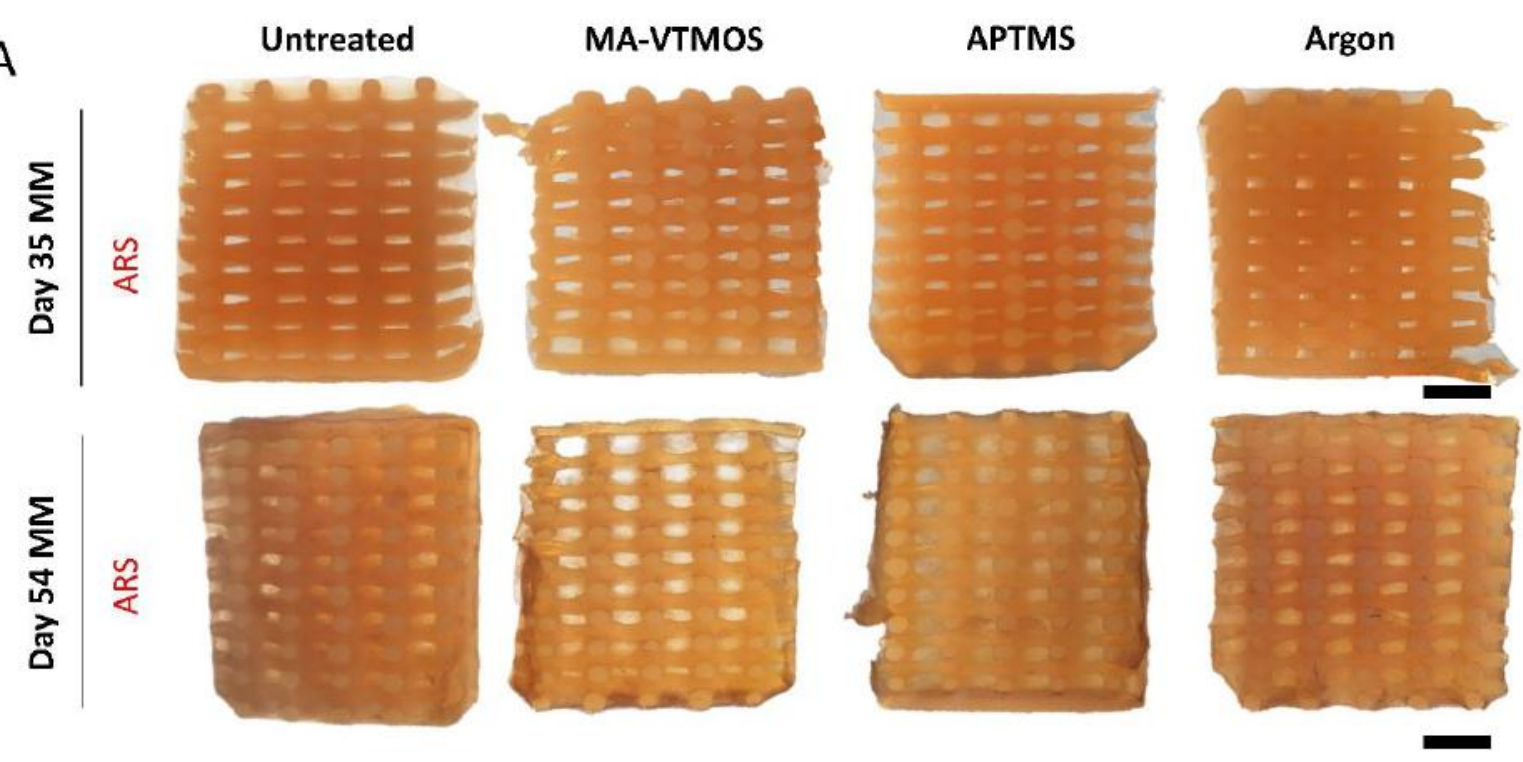

B

Basic media

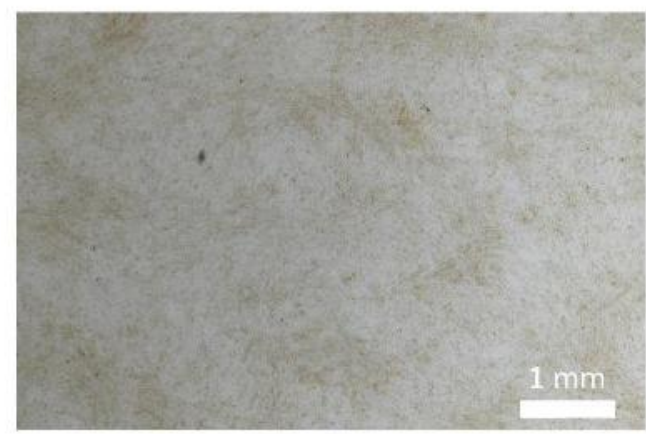

Mineralization media

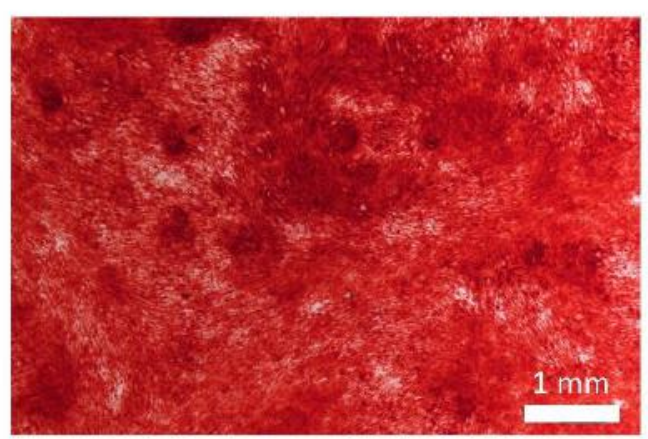

C
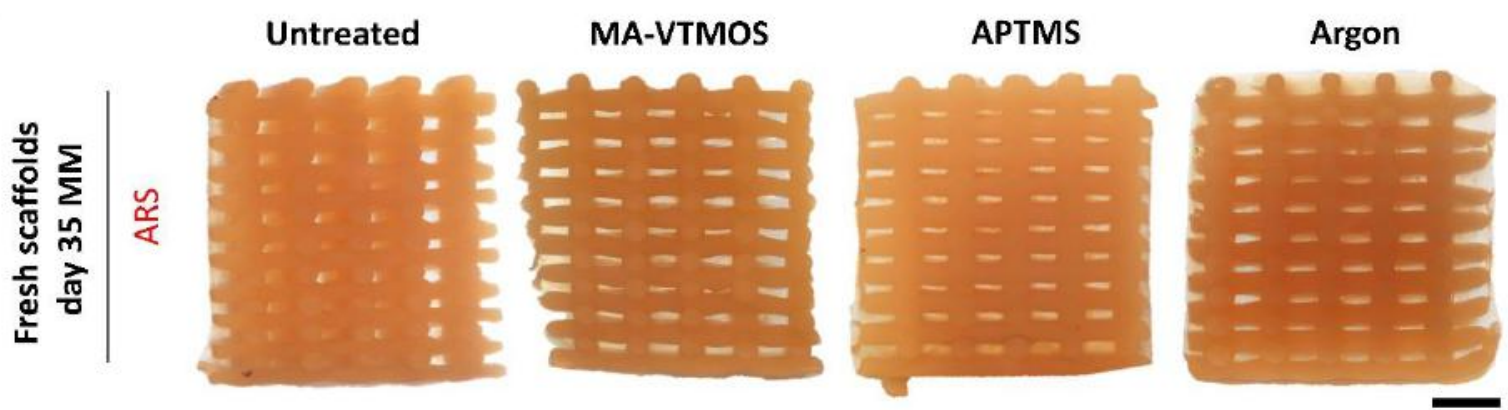

Figure S8. (A) Stereomicroscopy images of aged (plasma treatment) scaffold cross sections stained with ARS after 35 and 54 days of culture (28 and 47 days in MM, respectively) with hMSCs. (B) Stereomicroscopy images of hMSCs monolayers (osteogenic differentiation in 2D) stained with ARS after 35 days of culture (28 days in MM). (C) Stereomicroscopy images of fresh (plasma treatment) scaffold cross sections stained with ARS after 35 days of culture (28 days in MM) with hMSCs. Scale bars $1 \mathrm{~mm}$. 
A

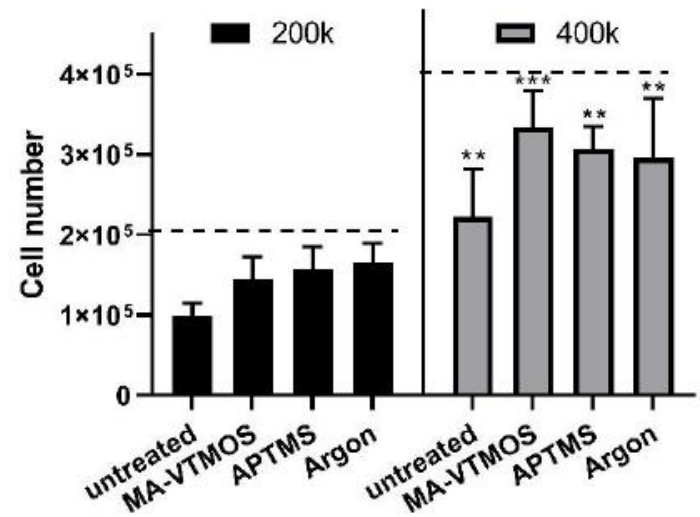

C

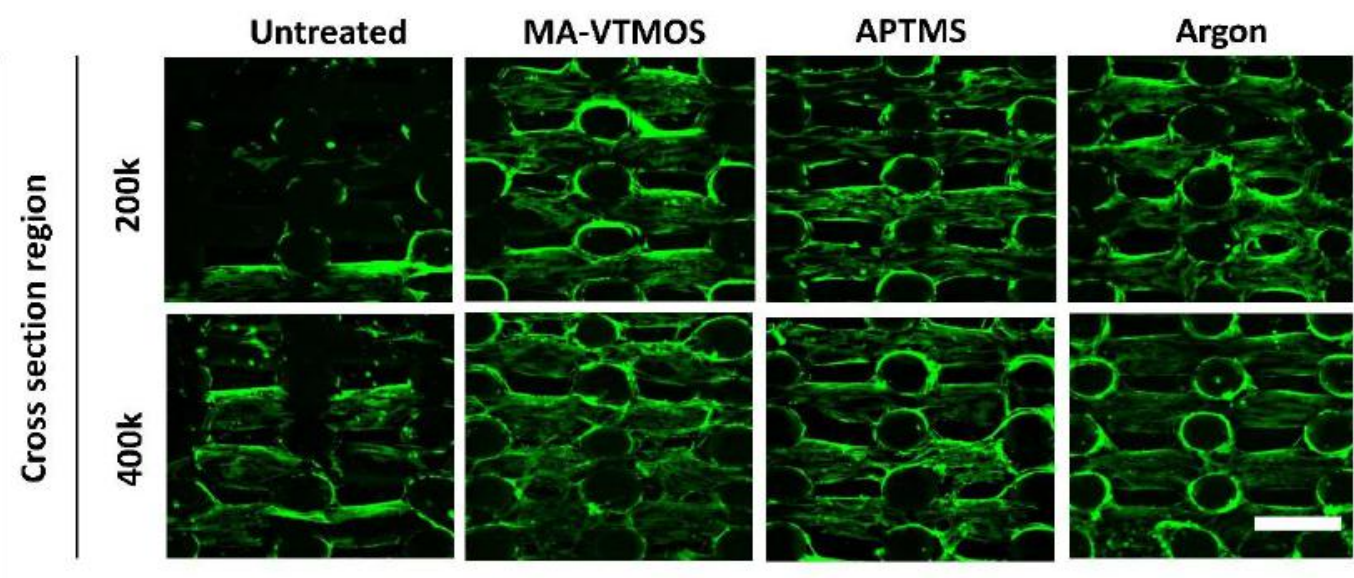

D
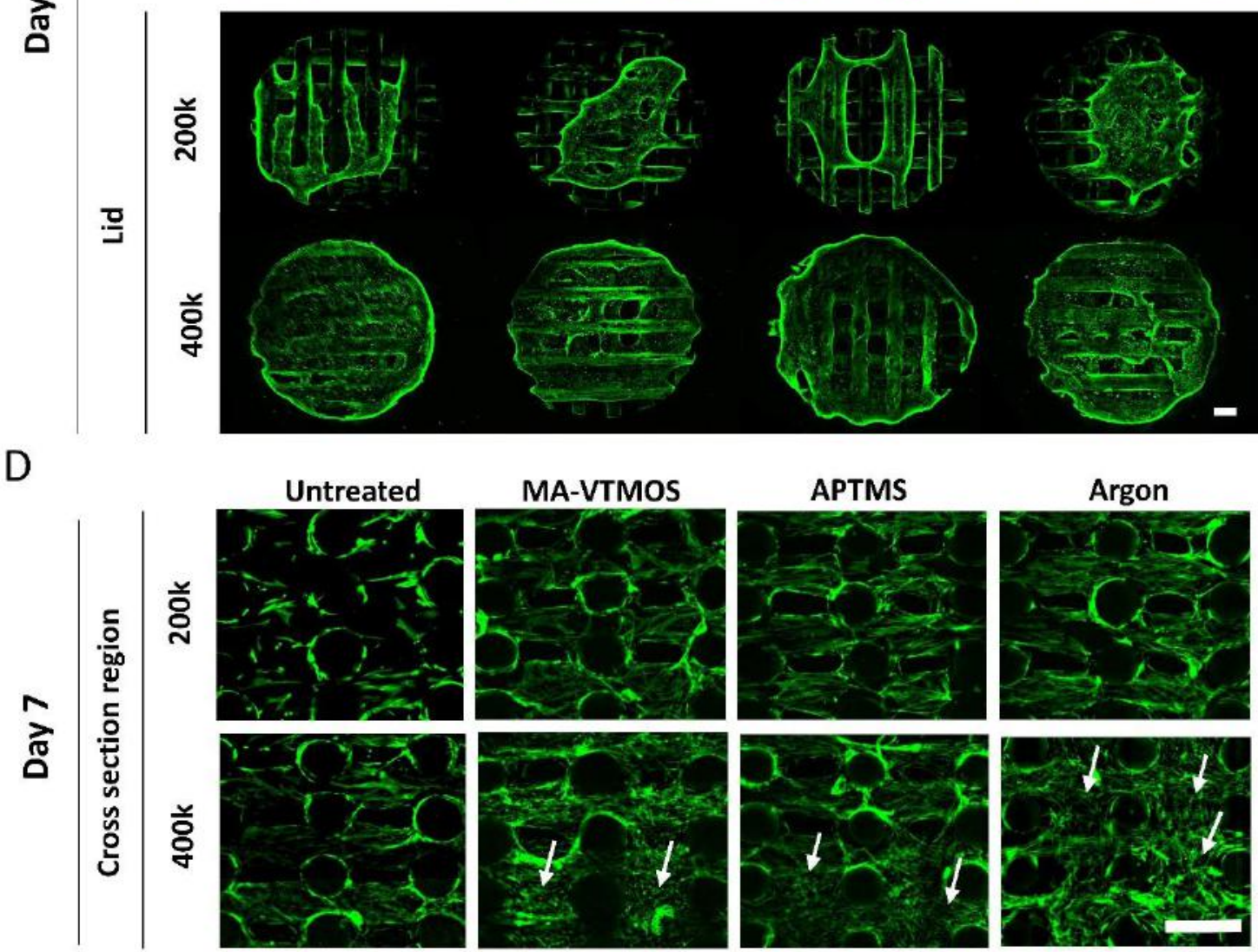

B

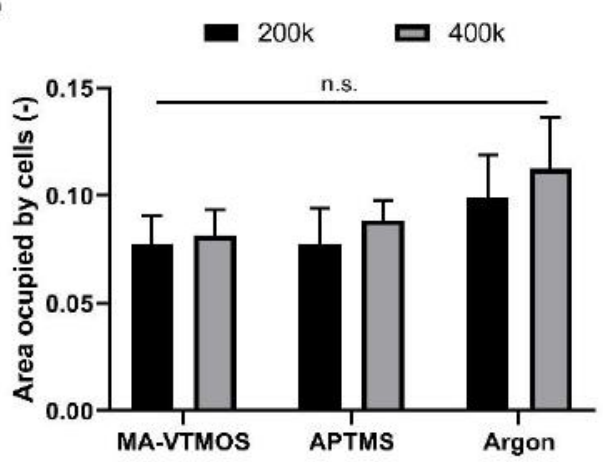

离

MA-VTMOS
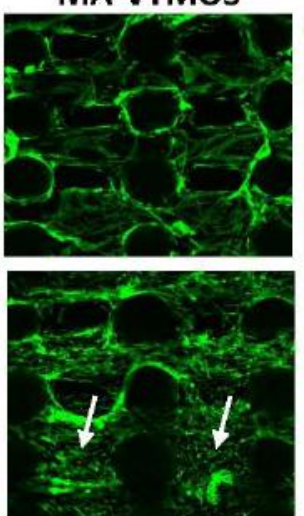

APTMS
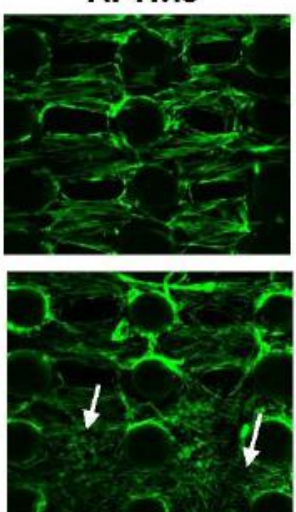

Argon

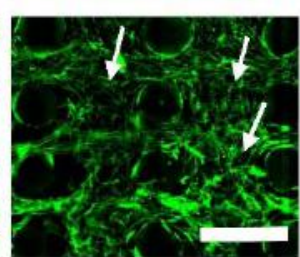

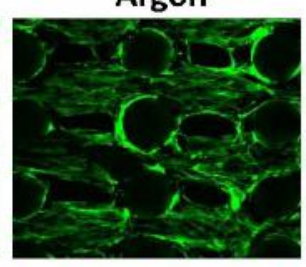


Figure 59. (A) DNA content on plasma treated scaffolds after seeding with two different cell densities: 200,000 cells (200k) or 400,000 cells (400k) per scaffold. Data presented as average \pm s.d. and statistical significance performed using two-way ANOVA with Tukey's multiple comparison test ${ }^{* *} p<0.01 ;{ }^{* * *} p<0.001$, for comparisons between $200 \mathrm{k}$ and $400 \mathrm{k}$ for each plasma treatment). (B) Quantification of cell coverage on plasma treated scaffolds seeded with $200 \mathrm{k}$ or $400 \mathrm{k}$ cells after 7 days of culture in BM. Data presented as average \pm s.d. and statistical significance performed using two-way ANOVA with Tukey's multiple comparison test (n.s. p > 0.05). (C) Comparison of cell coverage among plasma treated scaffolds seeded with 200k or 400k cells per scaffold: (C) Fluorescent microscopy images of hMSCs in a representative cross sectional area and in the bottom lids of scaffolds after 1 day of culture. (D) Fluorescent microscopy images of hMSCs in a representative cross sectional area of scaffolds after 7 day of culture in BM. White arrows signing scaffolds' pores coverage by cells Scale bars $500 \mu \mathrm{m}$.

\section{References}

1. Sinha, R.; Cámara-Torres, M.; Scopece, P.; Falzacappa, E. V.; Patelli, A.; Moroni, L.; Mota, C., A Hybrid Additive Manufacturing Platform to Create Bulk and Surface Composition Gradients on Scaffolds for Tissue Regeneration. bioRxiv 2020, 2020.06.23.165605.

2. Patelli, A.; FAlZACAPPA, E. V.; Scopece, P.; Pierobon, R.; Vezzu, S., Method for Generating an Atmospheric Plasma Jet and Atmospheric Plasma Minitorch Device. Google Patents: 2017. 
Chapter 4 


\section{Chapter 5}

Effect of Highly Loaded Nanohydroxyapatite Composite Scaffolds Prepared via Melt Extrusion Additive Manufacturing on the Osteogenic Differentiation of Human Mesenchymal Stromal Cells 



\title{
Effect of Highly Loaded Nanohydroxyapatite Composite Scaffolds Prepared via Melt Extrusion Additive Manufacturing on the Osteogenic Differentiation of Human Mesenchymal Stromal Cells
}

\author{
Maria Cámara-Torres ${ }^{1}$, Ravi Sinha ${ }^{1}$, Alberto Sanchez ${ }^{2}$, Pamela Habibovic ${ }^{3}$, Alessandro \\ Patelli ${ }^{4}$, Carlos Mota ${ }^{1}$, Lorenzo Moroni ${ }^{1}$ \\ ${ }^{1}$ Maastricht University, MERLN Institute for Technology-Inspired Regenerative Medicine, \\ Complex Tissue Regeneration Department, Maastricht, The Netherlands. ${ }^{2}$ TECNALIA, \\ Basque Research and Technology Alliance (BRTA), Donostia-San Sebastian, Spain. \\ Maastricht University, MERLN Institute for Technology-Inspired Regenerative Medicine, \\ Instructive Biomaterial Engineering Department, Maastricht, The Netherlands. \\ Department of Physics and Astronomy, Padova University, Padova, Italy.
}

\section{Abstract}

The field of bone tissue engineering seeks to mimic the bone extracellular matrix composition, balancing the organic and inorganic components. In this regard, additive manufacturing (AM) of highly loaded polymer-calcium phosphate (CaP) composites holds great promise towards the design of bioactive scaffolds. Yet, the biological performance of such scaffolds is still poorly characterized. In this study, melt extrusion AM (ME-AM) was used to fabricate poly(ethylene oxide terephthalate)/poly(butylene terephthalate) (PEOT/PBT)-nanohydroxyapatite (nHA) scaffolds with up to $45 \mathrm{wt} \% \mathrm{nHA}$, which presented significantly enhanced compressive mechanical properties, to evaluate their in vitro osteogenic potential as a function of $\mathrm{nHA}$ content. While osteogenic gene upregulation and matrix mineralization were observed on all scaffold types when cultured in osteogenic media, human mesenchymal stromal cells did not present an explicitly clear osteogenic phenotype, within the evaluated timeframe, in basic media cultures (i.e. without osteogenic factors). Yet, due to the adsorption of calcium and inorganic phosphate ions from cell culture media and simulated body fluid, the formation of a $\mathrm{CaP}$ layer was observed on PEOT/PBT-nHA 45 wt\% scaffolds, which is hypothesized to account for their osteoinductivity in the long term in vitro, and osteoconductivity in vivo. 


\section{INTRODUCTION}

Bone is one of the most recurrently damaged tissues in our body in form of fractures or defects caused by bone disease, traumatic injury, implant surgery, infection or tumor resection. ${ }^{1}$ In case of long bones, bone loss in length can lead to critical-size defects, which will not heal on their own. Current strategies used in the clinics for the treatment of critical size bone defects mainly rely on i) distraction osteogenesis and induced membrane techniques, ${ }^{2-3}$ or ii) on the use of graft materials, such as autografts, allografts, or demineralized bone matrices, that fill and / or help to regenerate the defect. ${ }^{4-5}$ Treatment methods based on the first option are often complex, demanding for the patient, and require external fixation and multiple surgeries. Similarly, natural bone grafts, despite being osteoinductive and / or osteoconductive, possess several disadvantages, including donor site morbidity, restricted availability, high costs, pain, prolonged rehabilitation, lack of structural properties, and can trigger inflammation, immunological rejections or infections. ${ }^{6}$ Therefore, the management of critical-size bone defects still remains a major clinical challenge, and consequently, a large amount of efforts are dedicated to investigate alternative strategies, such as tissue-engineered constructs to heal and regenerate bone in such scenarios. ${ }^{7}$

Mimicking the physical and chemical properties of bone has been the main approach towards the design of the optimal scaffold for bone regeneration. The bone matrix mainly consists of an organic phase of collagen type I (COL I), among other proteins, and an inorganic phase of carbonated hydroxyapatite. ${ }^{8}$ Accordingly, bioceramics, and in particular calcium phosphates (CaP), have been extensively applied for hard tissue repair. Widely applied CaP phases include hydroxyapatite (HA), tricalcium phosphate (TCP), biphasic and triphasic $\mathrm{CaP}$, and amorphous $\mathrm{CaP},{ }^{9}$ and their osteoinductive and osteconductive performance is determined by various properties, such as chemical phase, structural properties (crystallinity, surface roughness, macro-, micro- and nanoporosity), mechanical properties and associated degradation behavior. ${ }^{10}$ Yet, the role of the individual properties and their combination on the biological response to CaP-based bone graft substitutes has not yet been fully elucidated, with inconsistency in results found in the literature, which hampers the selection or design of the optimal $\mathrm{CaP}$ material for a specific application. 11-12 Several reasons for this are that studies lack sufficient material and/or biological characterization, or use different cell types in vitro, unavoidable inter- and intra-species variations in in vivo experiments, convoluted material properties that cannot 
be easily tuned independently, and different fabrication techniques used for scaffolds manufacturing. ${ }^{11-12}$

CaP processing into 3D macroporous scaffolds favors cell migration, tissue ingrowth, and vascularization. Moreover, the increase in surface area and body fluid accessibility provided by macroporosity can accelerate the degradation rate of the $\mathrm{CaP}$ material and boost the cell-material interactions towards an enhanced biological response, in terms of adhesion, proliferation and differentiation. ${ }^{13}$ Compared to conventional 3D scaffold fabrication techniques for polymers, such as gas foaming, particulate leaching, freezedrying and solvent casting, additive manufacturing (AM) has emerged as a promising macroporous scaffold fabrication technique. AM enables the fabrication of patient specific constructs with a fully interconnected network of pores, whose size and shape can be tuned to control cell response and the scaffold mechanical properties, of special importance for bone tissue engineering. ${ }^{14}$ Nevertheless, pure CaP AM scaffolds, processed by powder bed and binder jetting techniques, require post-sintering steps, the removal of binders, and present poor mechanical properties, due to the inherent material brittleness and low fracture toughness, which overall limit their in vivo applications. 15-16 Therefore, CaP have been processed by solution extrusion AM in combination with carrier liquids or solvents ${ }^{17-19}$, or by melt extrusion AM (ME-AM) in combination with synthetic biodegradable thermoplastic polymers. Among these, ME-AM of polymer-CaP composite materials has been widely explored, as it mimics the organic-inorganic composition of bone tissue, displays a high processability window, and allows the fabrication of scaffolds with tunable degradation rates and mechanical properties. ${ }^{20-21}$ In particular, the combination of polymers with HA has gained interest, as HA closely mimics the bone mineral phase. Considering the average mineral content of bone tissue, which lies within the range of 50 $w t \%$ to $74 w t \%$, depending on the body location, ${ }^{22-23}$ tremendous efforts have been conveyed to increase the HA content in the scaffold, while retaining their printability and optimizing the mechanical properties. A few studies demonstrated feasibility of production of highly HA-loaded scaffolds processed by ME-AM, including poly( $\varepsilon$-caprolactone) (PCL)HA composite with 40 wt\% ceramic phase, ${ }^{24-25}$ poly(lactic acid) (PLA)-HA composite with $50 \mathrm{wt} \%$ ceramic, ${ }^{26}$ and poly(ether urethane) (PEU)-HA composite with 40 wt\% ceramic. 27 Despite material related advances, most of previously published reports focus only on the material synthesis and printability, and the structural characterization of the scaffold, 24-26, 28-30 while their biological properties are poorly characterized. ${ }^{31-32}$ 
Here, we studied the in vitro osteogenic differentiation potential of highly loaded poly(ethylene oxide terephthalate)/poly(butylene terephthalate) (PEOT/PBT) - nano HA (nHA) composite scaffolds, fabricated by ME-AM. The biodegradable thermoplastic polyether-ester multiblock copolymer PEOT/PBT was selected due to its processability, mechanical properties and proven in vitro and in vivo applicability in the orthopedic field. 33-37 While in previous studies this polymer has been blended with HA to a maximum of 25 wt $\%$ micro-HA, ${ }^{38-39}$ for the first time, PEOT/PBT-HA composite scaffolds with up to 45 wt\% nHA were fabricated using ME-AM in the present report. To study their functionality in bone regeneration, human mesenchymal stromal cells (hMSCs) were chosen, due to the suitability of these stem cells over already committed cell lines to study the ability of a material to support osteogenic differentiation, ${ }^{40}$ as well as for future clinical translation. We evaluated the effect of the scaffolds' nHA content on the osteogenic differentiation of hMSCs by analyzing cell attachment, proliferation, matrix production and mineralization, and the expression of a relevant set of osteogenic genes and proteins.

\section{MATERIALS AND METHODS}

\section{Polymer-nHA composite production by solvent blending and characterization}

The production of PEOT/PBT-HA composites with 20 wt\% and 45 wt\% nHA (20-nHA and 45-nHA, respectively) was carried out by a solvent blending procedure. PEOT/PBT pellets (PEO molecular weight $=300 \mathrm{kDa}$, PEOT $:$ PBT weight ratio= 55:45, intrinsic viscosity 0.51 $\mathrm{dl} / \mathrm{g}$, Polyvation, The Netherlands) were dissolved in chloroform (Scharlab) under magnetic stirring for $45 \mathrm{~min}$. An amount of 20 or $45 \mathrm{wt} \% \mathrm{nHA}$ powder (particle size $<200 \mathrm{~nm}$, Sigma Aldrich) was added to the polymer solution and mechanically stirred at $750-1000 \mathrm{rpm}$ for 15 min (Dissolver Dispermat CV3). After stirring, the dispersion was kept for $2 \mathrm{~h}$ at 250 rpm, time during which part of the chloroform was evaporated and the viscosity of the blend increased. Subsequently, the polymer composite was precipitated in a mixture of diethylether and chloroform (Scharlab) (diethylether:chloroform $=10: 1$ ), and the supernatant removed. The composite was dried at RT, followed by a vacuum drying step at $90{ }^{\circ} \mathrm{C}$ for $2 \mathrm{~h}$ to ensure the removal of solvent traces (diethylether $<0.01 \mathrm{ppm}$, chloroform $<1 \mathrm{ppm}$,). The foam-like composite formed during the precipitation step was compression molded at $150-175^{\circ} \mathrm{C}$ and cut into pellets. The dispersion of $\mathrm{nHA}$ within the polymer was 
examined using backscattered scanning electron microscopy (BSEM, FEl's Quanta 200 environmental scanning microscope, beam voltage $30 \mathrm{kV}$, spot size 5).

\section{Scaffolds fabrication and characterization}

45-nHA, 20-nHA and PEOT/PBT (0-nHA) scaffolds were fabricated via ME-AM. A commercial platform (Bioscaffolder 3.0, Gesim) was equipped with a custom-made printhead with two separate heating sources for the cartridge and extrusion screw. ${ }^{41}$ The printing parameters applied to each material are briefly described in Table 1. A needle with $250 \mu \mathrm{m}$ internal diameter was used to print 0-90 layout scaffolds, with $200 \mu \mathrm{m}$ layer thickness and $750 \mu \mathrm{m}$ strand distance (center to center). For cell culture experiments and

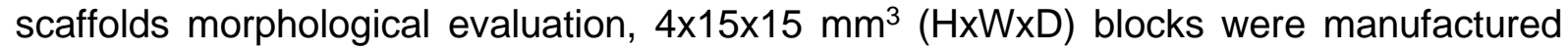
and cylindrical scaffolds of $4 \mathrm{~mm}$ diameter and $4 \mathrm{~mm}$ height were cored out using a biopsy punch. For mechanical testing, $4 \mathrm{~mm}$ diameter, $15 \mathrm{~mm}$ high cylindrical scaffolds were printed and directly used for analysis. These scaffold dimensions resemble the type of scaffold needed for a rabbit critical segmental bone defect study, as a real case example which will be performed in future experiments. The scaffolds' architecture consisted also on $250 \mu \mathrm{m}$ fiber diameter, $200 \mu \mathrm{m}$ layer height, and $750 \mu \mathrm{m}$ strand distance, and the scaffold layers had a 0-90 filament layout in the center, while at the edges, the filaments were deposited along the circular boundary. These scaffolds presented sufficient lateral porosity (Figure S4A-B).

Table 1. Printing parameters applied to obtained 3D scaffolds with each of the polymer composites

\begin{tabular}{|cccccc|} 
Scaffold & $\begin{array}{c}\text { T cartridge } \\
\left({ }^{\circ} \mathbf{C}\right)\end{array}$ & $\begin{array}{c}\text { T screw } \\
\left({ }^{\circ} \mathbf{C}\right)\end{array}$ & $\begin{array}{c}\text { Motor rotation } \\
(\mathbf{r p m})\end{array}$ & $\begin{array}{c}\text { Cartridge Pressure } \\
(\mathbf{b a r})\end{array}$ & $\begin{array}{c}\text { Printing speed } \\
(\mathbf{m m} / \mathbf{s})\end{array}$ \\
\hline 0-nHA & 195 & 195 & 60 & 7 & 16 \\
\hline 20-nHA & 205 & 210 & 45 & 8 & 18 \\
\hline 45-nHA & 210 & 220 & 30 & 8 & 15 \\
\hline
\end{tabular}

Scaffold morphology and porosity was assessed using a stereomicroscope (Nikon SMZ25) and scanning electron microscopy (SEM, XL-30, beam voltage $10 \mathrm{kV}$, spot size 3). Presence and distribution of fillers within gold sputter-coated scaffolds' filaments surface and cross-section was examined using BSEM (XL-30, beam voltage $20 \mathrm{kV}$, spot size 5). SEM operating at $25 \mathrm{kV}$ coupled with energy dispersive $X$-ray spectroscopy (EDS) was used to quantify the chemical composition of the samples. Particle size distribution on 
scaffolds was assessed on the acquired BSEM images and using the image analysis software tool XT Pro v3.2 (Soft Imaging System, GmbH).

Mechanical tests (compression at a rate of $0.1 \mathrm{~mm} / \mathrm{min}$., using a $100 \mathrm{~N}$ load cell) were carried out using an Instron Universal Mechanical test machine. Three scaffolds each, made from 0-nHA or 45-nHA were tested.

\section{Cell seeding and culture}

\section{Cell expansion}

HMSCs isolated from bone marrow were purchased from Texas A\&M Health Science Center, College of Medicine, Institute for Regenerative Medicine (Donor d8011L, female, age 22). Cryopreserved vials at passage 3 were plated at a density of 1,000 cells $\mathrm{cm}^{-2}$ in tissue culture flasks and expanded until approximately $80 \%$ confluency in culture media (CM), consisting of aMEM with Glutamax, no nucleosides (Gibco), supplemented with $10 \%$ fetal bovine serum (FBS), without penicillin-streptomycin (PenStrep) at $37^{\circ} \mathrm{C}$ and $5 \% \mathrm{CO}_{2}$.

\section{Cell seeding in $3 D$ scaffolds}

Scaffolds were disinfected in 70\% ethanol for 20 min, washed 3 times with Dulbecco's PBS and incubated overnight in CM for protein attachment. Before seeding, scaffolds were dried on top of a sterile filter paper and placed in the wells of non-treated plates. Passage $4 \mathrm{hMSCs}$ were trypsinized and resuspended in a dextran solution (10 wt/wt \% dextran in $\mathrm{CM}, 500 \mathrm{kDa}$, Farmacosmos) to slow down cell settling and guarantee homogeneous cell attachment throughout the scaffold. Dextran is a temporary supplement and will be washed away during the first $24 \mathrm{~h} .{ }^{42}$ A droplet of cell suspension (37 $\mu$ l containing 200,000 cells) was placed on top of each scaffold before slowly filling the scaffolds' pores. Seeded scaffolds were incubated for 4 hours at $37^{\circ} \mathrm{C}$ and $5 \% \mathrm{CO}_{2}$ to allow cells to attach. After this time, scaffolds were transferred to new wells containing $1.5 \mathrm{ml}$ of basic media (BM), consisting of CM supplemented with $200 \mu \mathrm{M}$ L-Ascorbic acid 2-phosphate and 1\% PenStrep. Scaffolds were cultured for 7 days in BM followed by another 28 days in BM or mineralization media (MM), consisting of BM supplemented with dexamethasone (10 nM) and $\beta$-glycerophosphate (10 mm) (Sigma-Aldrich). Media was replaced after $24 \mathrm{~h}$ and every two or three days during the culture period. 


\section{Biochemical assays}

Alkaline phosphatase (ALP) activity and DNA assay

ALP activity and DNA content were evaluated over the culture period. For that, scaffolds were collected at every timepoint and washed with PBS, stored at $-80{ }^{\circ} \mathrm{C}$ and freezethawed 3 times, to improve lysis efficiency. Samples were incubated for $1 \mathrm{~h}$ at RT in a cell lysis phosphate buffer containing Triton $X-100(0.1 \mathrm{v} \%)$, at pH 7.8. After the addition of the chemiluminescent substrate for ALP CDP star® ready to use reagent (Roche) at a 1:4 ratio, luminescence was measured using a spectrophotometer (CLARIOstar ${ }^{\circledR}$, BMG Labtech). Reported ALP values were normalized to DNA content, which was quantified using the CyQUANT cell proliferation assay kit (Thermo Fisher Scientific) on the remaining cell lysates. Samples for DNA quantification were incubated overnight at $56{ }^{\circ} \mathrm{C}$ in Proteinase K solution ( $1 \mathrm{mg} / \mathrm{ml}$ Proteinase K (Sigma-Aldrich) in Tris/EDTA buffer) (1:1) for further cell lysis and matrix degradation. Subsequently, samples were freeze-thawed 3 times and incubated $1 \mathrm{~h}$ at $\mathrm{RT}$ with a 20X diluted lysis buffer from the kit containing RNase A (1:500) to degrade cellular RNA. Samples were incubated with the fluorescent dye provided by the kit (1:1) for $15 \mathrm{~min}$ and fluorescence was measured (emission/excitation $=520 / 480 \mathrm{~nm}$ ) with a spectrophotometer.

\section{Extracellular matrix (ECM) mineralization assay}

Calcium ( $\mathrm{Ca}$ ) deposits were qualitatively determined by alizarin red $S$ (ARS) staining on composite scaffolds after 35 days of culture in BM or MM. Freshly fabricated scaffolds, and non-cell seeded scaffolds cultured in BM or MM and treated as cell seeded scaffolds in terms of media refreshment, were used as controls. Briefly, every timepoint scaffolds were fixed with $4 \%$ paraformaldehyde and washed with distilled water. Subsequently, scaffolds were stained with ARS (60 mm, pH 4.1 - 4.3) for 20 min at RT, and washed with distilled water. Scaffolds' cross sections were imaged using a stereomicroscope. After imaging, ARS was extracted from the scaffolds by $1 \mathrm{~h}$ incubation at RT with $1 \mathrm{ml} 30 \mathrm{v} \%$ acetic acid while shaking, followed by $10 \mathrm{~min}$ at $85^{\circ} \mathrm{C}$. Afterwards, scaffolds were removed and solutions were centrifuged at 20,000 rff for $10 \mathrm{~min}$. An appropriate volume of ammonium hydroxide $(5 \mathrm{~m})$ was added to the supernatants to bring the $\mathrm{pH}$ to 4.2. The absorbance was measured at $405 \mathrm{~nm}$ using a spectrophotometer. Concentration of ARS was calculated from an ARS standard curve and the values were normalized to DNA content. The signal from control scaffolds was subtracted. 


\section{Osteocalcin $(\mathrm{OCN})$ secretion}

OCN secretion in media was analyzed after 14, 21 and 35 days of culture in BM or MM. Scaffolds' culture media was refreshed on each timepoint by adding $1.5 \mathrm{ml}$ of the corresponding media without FBS, in order to avoid background OCN levels from the FBS. Upon collection (48 h later), media was centrifuged at 2,000 rcf for 10 minutes to remove debris and stored at $-80^{\circ} \mathrm{C}$ until analysis. OCN content in medium was detected using a quantitative sandwich ELISA kit (ab195214) according to the manufacturer's protocol.

\section{Gene expression}

Gene expression analysis was performed after 7, 14, 21 and 35 days of culture. RNA was extracted from cells in scaffolds using a TRIzol isolation method. Briefly, TRIzol was added to each sample in an Eppendorf tube and centrifuged at 12,000 rcf for $5 \mathrm{~min}$ to precipitate the scaffold and ECM at the bottom. Chloroform was added to the supernatant and centrifuged at 12,000 rcf for $5 \mathrm{~min}$ to isolate the RNA, present in the aqueous phase. RNA was further purified using RNeasy mini kit column (Qiagen), according to the manufacturer's protocol. The purity and quantity of total RNA was evaluated using a spectrophotometer. Reverse transcription was performed using iScript ${ }^{\mathrm{TM}}$ (Bio-Rad) following suppliers' protocol. qPCR was performed on the mix composed of cDNA, SYBRGreen master mix (Qiagen) and the selected primers (Table 2) using a CFX Connect $^{\mathrm{TM}}$ Real-Time System (Bio-Rad) under the following conditions: CDNA denaturation for $3 \mathrm{~min}$ at $95^{\circ} \mathrm{C}, 40$ cycles of $15 \mathrm{~s}$ at $95^{\circ} \mathrm{C}$, and $30 \mathrm{~s}$ at $65^{\circ} \mathrm{C}$. Additionally, a melting curve was generated for each reaction in order to test primer dimer formation and non-specific amplification. Gene transcription was normalized to the transcription of the housekeeping gene B2M. The $2^{-\Delta \Delta C t}$ method was used to calculate relative gene expression for each target gene. Normalization was done with respect to relative gene expression of cells in 0-nHA scaffolds at day 7 in BM.

Table 2. Primer sequences used for q-PCR. B2M: beta-2-microglobulin, RUNX2: runt-related transcription factor, COL I: collagen 1 alpha 1, OPN: osteopontin, OCN: osteocalcin, BSP: bone sialoprotein, SP7: osterix.

\begin{tabular}{l|l|l}
\hline Gene & Forward primer & Reverse primer \\
\hline B2M & ACAAAGTCACATGGTTCACA & GACTTGTCTTTCAGCAAGGA \\
RUNX2 & TCAACGATCTGAGATTTGTGGG & GGGGAGGATTTGTGAAGACGG \\
COL I & GAGGGCCAAGACGAAGACATC & CAGATCACGTCATCGCACAAC \\
OPN & CTCCATTGACTCGAACGACTC & CAGGTCTGCGAAACTTCTTAGAT
\end{tabular}




\begin{tabular}{l|l|l} 
OCN & TGAGAGCCCTCACACTCCTC & CGCCTGGGTCTCTTCACTAC \\
BSP & CCCCACCTTTTGGGAAAACCA & TCCCCGTTCTCACTTTCATAGAT \\
SP7 & CCTCTGCGGGACTCAACAAC & AGCCCATTAGTGCTTGTAAAGG \\
\hline
\end{tabular}

\section{Immunofluorescence}

Cells in scaffolds were fixed in $4 \%$ paraformaldehyde after 35 days of culture in BM or MM, and permeabilized by $30 \mathrm{~min}$ incubation in Triton-X 100 (0.1 v\%). Subsequently, the scaffolds were cut in half and washed 3 times with PBS. For assessing cell content and distribution on scaffolds, some scaffolds were directly stained for F-actin (488 Alexa Fluor Phalloidin, Thermo Fisher Scientific) for $1 \mathrm{~h}$. Scaffolds cross-sections were imaged with a fluorescent microscope (Eclipse, Ti2-e, NIKON). Background subtraction and brightness adjustments were performed on the images using the software Image $\mathrm{J}$, in order to improve their visualization. For antibody staining, scaffolds were further blocked for $1 \mathrm{~h}$ in blocking buffer (3 \% BSA + 0.01\% Triton-X 100). Afterwards, primary antibodies diluted 1:200 in washing buffer (10x diluted blocking buffer) were added (COL I rabbit polyclonal (ab34710, Abcam), osteopontin (OPN) rabbit polyclonal (ab8448, Abcam), OCN rabbit polyclonal (ab93876), each to a different sample half, and incubated overnight at $4{ }^{\circ} \mathrm{C}$. Washed samples were incubated for $1 \mathrm{~h}$ at RT with the secondary antibody (1:200 Alexa Fluor 647 goat derived anti-rabbit antibody, Thermo Fisher Scientific). Then, scaffolds were washed and stained for F-actin (488 Alexa Fluor Phalloidin, Thermo Fisher Scientific) for $1 \mathrm{~h}$, followed by three washing steps with PBS. Confocal laser scanning microscopy was performed with a tandem confocal system (Leica TCS SP8 STED), equipped with a white light laser (WLL). Samples were excited with the dye specific wavelengths and emission was detected with $\mathrm{HyD}$ detectors. For optimal visualization in the reported images, F-actin was colored in green, COL I in red, OPN in blue and OCN in magenta.

\section{Imaging of cultured scaffolds}

After 35 days of culture, cell seeded and non-cell seeded scaffolds in BM or MM were fixed with $4 \mathrm{wt} \%$ paraformaldehyde for $30 \mathrm{~min}$. Scaffolds were dehydrated by a graded ethanol series $(30,50,70,80,90,96,100 \mathrm{v} \%)$, during $15 \mathrm{~min}$ each at RT, followed by $15 \mathrm{~min}$ incubation in 100\% ethanol- hexamethyldisilazane (HMDS) at a 1:1 ratio, and $15 \mathrm{~min}$ incubation in HMDS. HMDS was removed and scaffolds were allowed further drying overnight at RT in the fume hood. Samples were gold sputter coated. Cells, ECM and scaffolds surface were visualized on scaffolds cross-section using scanning electron 
microscopy (SEM, beam voltage $10 \mathrm{kV}$, spot size 3). The presence of mineral deposits on cell seeded scaffolds was examined by backscattered SEM (BSEM, beam voltage $20 \mathrm{kV}$, spot size 5). SEM operating at $25 \mathrm{kV}$ coupled with Energy-dispersive X-ray spectroscopy (EDS) was used to identify $\mathrm{Ca}$, phosphorous $(\mathrm{P})$, and other elements on the ECM.

\section{Bioactivity test in Simulated Body Fluid (SBF)}

An SBF solution was prepared as previously described ${ }^{43}$. Briefly, chemicals were dissolved in distilled water in the following order: $\mathrm{NaCl}, \mathrm{NaHCO}_{3}, \mathrm{KCl}, \mathrm{K}_{2} \mathrm{HPO}_{4}$, $\mathrm{MgCl}_{2} \cdot 6 \mathrm{H} 2 \mathrm{O}, \mathrm{CaCl}_{2} \cdot 2 \mathrm{H}_{2} \mathrm{O}$ and $\mathrm{Na}_{2} \mathrm{SO}_{4}$. The solution was then buffered to $\mathrm{pH} 7.4$ at $36.5^{\circ}$ $\mathrm{C}$ using Tris and $\mathrm{HCl}(1 \mathrm{M})$. The final solution had an ion concentration ( $\mathrm{mM}$ ) as follows: $\mathrm{Na}^{+}, 142.0 ; \mathrm{K}^{+}, 5.0 ; \mathrm{Mg}^{+}, 1.5 ; \mathrm{Ca}^{+}, 2.5 ; \mathrm{Cl}^{-}, 147.8 ;(\mathrm{CO} 3)^{-}, 4.2 ;(\mathrm{PO} 4)^{2-}, 1.0 ;(\mathrm{SO} 4)^{2-}, 0.5$. Scaffolds were immersed in $1 \mathrm{ml} \mathrm{SBF}$ and incubated at $37^{\circ} \mathrm{C}$. SBF was refreshed every 34 days. After 4 and 14 days, scaffolds were carefully rinsed in distilled water, blotted on an adsorbent paper and dried at RT. Then they were cut in half, gold sputtered and observed with SEM at $15 \mathrm{kV}$, spot size 3 .

\section{Ion release analysis}

Before media refreshment at timepoints day 7, 14, 21 and 35 of the cell culture experiment, medium was collected from each scaffold and stored at $-30^{\circ} \mathrm{C}$ until analysis. In a separated experiment, scaffolds were incubated in $1 \mathrm{ml}$ simulated $(0.8 \% \mathrm{NaCl}, 50 \mathrm{mM}$ HEPES, pH 7.3) for 28 days at $37^{\circ} \mathrm{C}$. This was done to analyze $\mathrm{Ca}$ and $\mathrm{P}$ release in a non $\mathrm{Ca}$ and $\mathrm{P}$ saturated solution. Every 3-4 days, an aliquot of the supernatant was collected, stored at $-30^{\circ} \mathrm{C}$ until analysis, and the same volume of SPS was refreshed. Concentrations of $\mathrm{Ca}$ and $\mathrm{P}$ in cell culture medium and SPS were quantified by Inductively coupled plasma mass spectrometry (ICP-MS, iCaP Q, Thermo Scientific). Upon analysis, all samples, including fresh cell culture medium or fresh SPS, were thawed, vortexed for $30 \mathrm{~s}$, centrifuged at 20,000 rcf for $15 \mathrm{~min}$ to remove debris, and diluted $1: 50$ in $1 \mathrm{v} \% \mathrm{HNO}_{3}$ with the addition of $20 \mathrm{ppb}$ scandium as internal standard. A standard curve of 20, 10, 5, 2.5 and $1.25 \mathrm{ppm} \mathrm{Ca}$ and $\mathrm{P}$ in $1 \mathrm{v} \% \mathrm{HNO}_{3}$ and $20 \mathrm{ppb}$ scandium was prepared. Quantification was performed in standard mode with $\mathrm{He}$ as collision gas. 


\section{Statistical analysis}

All data is shown as average with error bars indicating the standard deviation of at least three replicates. Analysis of statistics was conducted with GraphPad Prism (version 8.0.1). A one-way or two-way analysis of variance (ANOVA) was performed followed by a Tukey's post-hoc multiple comparison test to evaluate statistical significance and correct for multiple comparisons.

\section{RESULTS}

\section{Scaffolds fabrication, nHA distribution and mechanical properties.}

Composite pellets prepared by solvent blending of PEOT/PBT and 20 or $45 \mathrm{wt} \% \mathrm{nHA}$ (Figure S1) were used to fabricate PEOT/PBT-nHA composite scaffolds via ME-AM (Figure 1). SEM micrographs in Figure 1A, B showed that both 20-nHAand 45-nHA scaffolds maintained an interconnected macroporosity, comparable to the control 0-nHA scaffolds. BSEM images in Figure 1C and EDS analysis in Figure S2A confirmed an increasing amount of $\mathrm{nHA}$, and therefore $\mathrm{Ca}$ and $\mathrm{P}$, on the scaffold filaments with increasing $\mathrm{nHA}$ content in the composite blend. The presence of $\mathrm{nHA}$ on the surface of the 20-nHA and 45-nHA scaffolds was further confirmed by ARS, which binds to the $\mathrm{Ca}$ of $\mathrm{nHA}$ (Figure S2B, C). An increase in the pink-red stain intensity on the surface of scaffolds was observed with increasing $\mathrm{nHA}$ content. Despite the nanometer size of the raw $\mathrm{nHA}$ $(<200 \mathrm{~nm})$, particle aggregation was already noticed in the solvent blending process prior to $\mathrm{ME}$, possibly occurring during the precipitation step of the polymer composite solution in the non-solvent (Figure S1B). In the 45-nHA scaffolds, nHA micro aggregates occupied around $24 \%$ of the filaments' surface area. Quantification on their size distribution revealed that $\sim 75 \%$ of the $\mathrm{nHA}$ micro aggregates possessed a diameter in the range of 2 $10 \mu \mathrm{m}$, while $\sim 5 \%$ of the particles diameter lied in the range of 30 to $90 \mu \mathrm{m}$ (Figure S3). The other $76 \%$ of the $45-\mathrm{nHA}$ scaffolds filaments surface area was occupied by the polymer and nanometer size $\mathrm{nHA}$ particles. The presence of these nHA micro aggregates provided a higher surface roughness to the composite scaffolds when compared to the smooth 0-nHA scaffolds (Figure 1B). The addition of nHA up to $45 \mathrm{wt} \%$ led to a 2 -fold increase in the scaffolds' compressive modulus, compared to copolymer only scaffolds (Figure S4C). Compressive strength at yield was also slightly increased on the 45-nHA 
scaffolds (Figure S4D). Yet, no significant differences were observed compared to 0-nHA scaffolds.
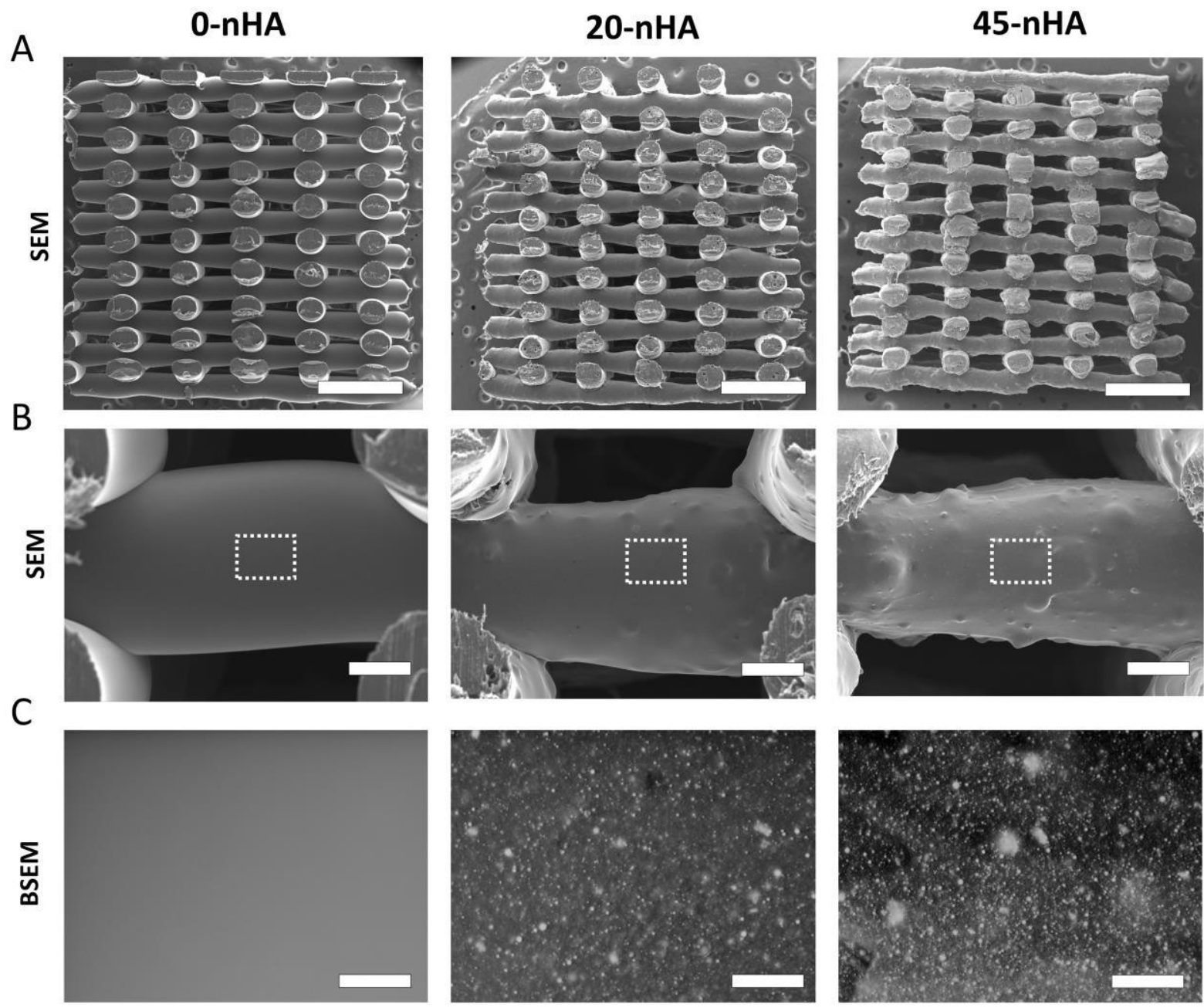

Figure 1. PEOT/PBT-nHA composite scaffolds fabrication and nHA distribution in scaffold filaments. (A) SEM micrographs of scaffolds cross-section showing their macroporosity. Scale bars $1 \mathrm{~mm}$. (B) Representative BSEM micrographs of scaffolds filaments depicting $\mathrm{nHA}$ distribution and increasing surface roughness with increasing $\mathrm{nHA}$ content. Scale bars $100 \mu \mathrm{m}$. (C) Magnified images of the regions marked with dashed lines in (B). Scale bars $20 \mu \mathrm{m}$.

\section{hMSCs seeding efficiency, proliferation and osteogenic differentiation on PEOT/PBT-nHA composite scaffolds}

Scaffolds were seeded using a viscous seeding solution to ensure homogeneous hMSCs attachment throughout the scaffold. Consequently, all scaffolds qualitatively showed uniform cell distributions from the earliest timepoints. Moreover, high seeding efficiency values in the order of $\sim 80 \%$ were maintained among different scaffold types without statistical differences in terms of cell attachment (Figure 2A). To understand the effect of nHA on the osteogenic differentiation of hMSCs, scaffolds were cultured up to 35 days in 
BM or MM. To support cell proliferation before the addition of osteogenic factors, scaffolds were cultured initially for 7 days in BM. Yet, DNA content analysis demonstrated no cell proliferation during this period (Figure 2A). DNA content was further monitored over the culture time (Figure 2B). Although no statistical differences were observed in terms of cell number among scaffold types and time points, a decrease in cell number from day 14 to day 35 of culture in BM was observed for all scaffold types. Overall, Figure 2B also demonstrated lower cell numbers in scaffolds cultured in BM when compared to $\mathrm{MM}$ at late timepoints (day 21 and day 35).

A

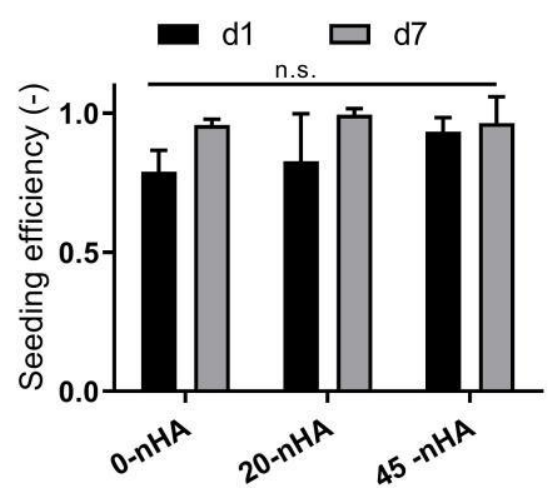

C

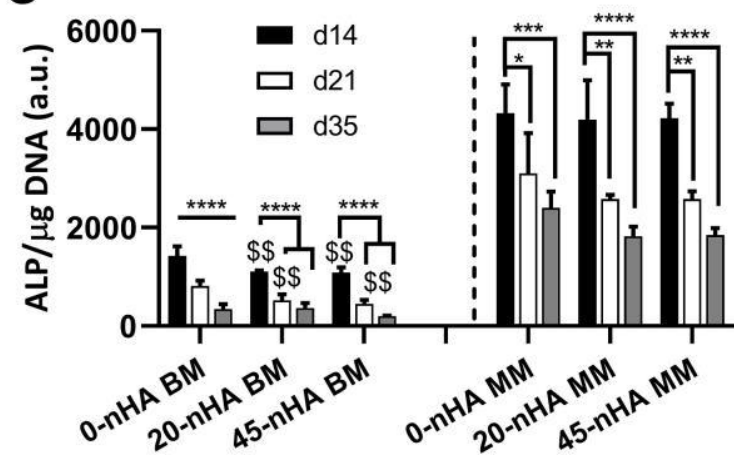

B
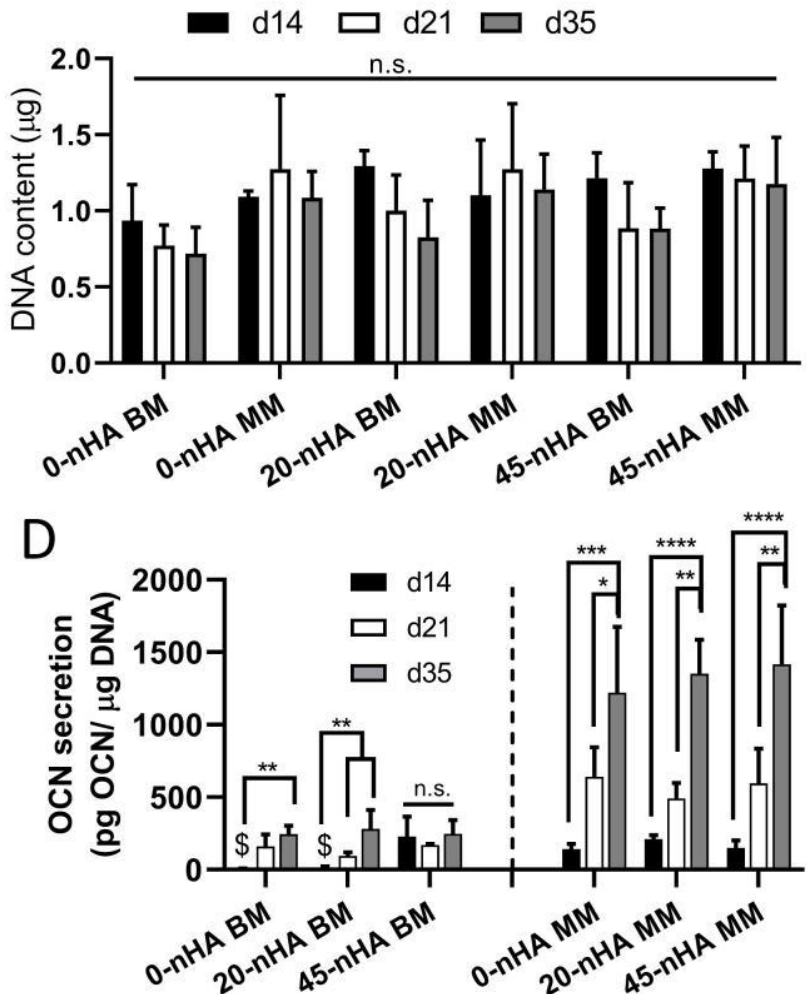

Figure 2. Cell seeding and osteogenic differentiation on PEOT/PBT-nHA composite scaffolds. (A) Seeding efficiency and proliferation 7 days after seeding. (B) DNA content progression on 0-nHA, 20-nHA, 45-nHA scaffolds over 35 days of culture in BM or MM. (D) ALP activity of hMSCs progression over 35 days when seeded on the different scaffold types in BM or MM. (E) OCN secretion by hMSCs in media progression over 35 days when seeded on the different scaffold types in $\mathrm{BM}$ or MM. Data presented as average \pm s.d. and statistical significance performed using twoway ANOVA with Tukey's multiple comparison test. For $(A, B)$ : n.s. $p>0.05$. For $(C)$ : ${ }^{*} \$ p<0.05$; ${ }^{* *} \$ \$ p<0.001 ;{ }^{* * *} \$ \$ \$ p<0.001 ;{ }^{* * * *} \$ \$ \$ p<0.0001 ;$; for comparisons among timepoints each scaffold type; $\$$ for comparisons to $0-\mathrm{nHA}$ each timepoint; comparisons among BM and MM samples performed separately. For (D): n.s. $p>0.05 ;{ }^{*} \$ p<0.05 ;{ }^{* *} \$ \$ p<0.001 ;{ }^{* *} \$ \$ \$ p<0.001$; ${ }^{* * * *} \$ \$ \$ \$ p<0.0001$; * for comparisons among timepoints each scaffold type; $\$$ for comparisons to 45-nHA each timepoint; comparisons among BM and MM samples performed separately. 
ALP activity, an early osteogenic marker, was evaluated at day 14, 21 and 35 (Figure 2C). ALP values were higher when cells were cultured in MM compared to BM. A peak in ALP activity was shown at day 14, with a progressive and significant decrease on day 21 and day 35, both in BM and MM. While in MM no statistical differences among scaffold types were observed at any time point, ALP values in BM were found to be significantly higher at day 14 and 21 in 0-nHA scaffolds compared to 20-nHA and 45-nHA scaffolds. When analyzing OCN secretion in media, higher values were observed in MM than in BM (Figure 2D). Since $\mathrm{OCN}$ is a late marker for osteogenic differentiation, a peak was observed at day 35, in contrast to ALP. As noted with ALP, no significant differences were observed in OCN secretion among scaffold types in MM. Nevertheless, a significantly higher OCN signal was recorded on 45-nHA scaffolds already at an early time point (day 14) when cultured in BM, compared to 20-nHA and 0-nHA scaffolds in BM. Notably, other scaffold types cultured in BM reached this levels of OCN secretion at day 21 or 35 at the earliest.

\section{Gene expression}

The expression of bone related transcription factors and protein encoding genes was assessed at day 14, 21 and 35, under BM or MM conditions. Figure 3 shows the relative gene expression change with respect to 0 -nHA day 7 BM condition (Figure S5). In general, RUNX2 gene expression was found to be higher in MM than in BM for all scaffold types over the whole culture period (Figure 3A). At day 14 in MM, significantly higher expression on 0-nHA scaffolds was observed. However, no significant differences among scaffold types were observed at later time points in MM. Similarly, despite higher COL I gene expression on 0-nHA scaffolds at day 14 in MM compared to the nHA composite scaffolds, comparable COL I gene expression levels were maintained at later culture time points among all scaffold types (Figure 3B). Interestingly, the culture media appeared not to have any effect on COL I gene expression and no significant differences were found between $\mathrm{BM}$ and $\mathrm{MM}$ at a given time point and scaffold type over the culture period. In the case of OPN gene expression, higher levels were noticed when cultured in BM compared to MM for all scaffold types at days 14 and 21 (Figure 3C). Yet, at day 35 the gene expression level of OPN was comparable among all scaffold types in both BM and MM media. Independent of the culture media, a low gene expression of OCN was maintained in all scaffold types until a significant upregulation, slightly higher in 0-nHA scaffolds, occurred at day 35 in MM (Figure 3D). A significant upregulation in the BSP and Osterix gene expression was observed for all scaffold types cultured in MM from day 14 to day 21, and 
to day 35 in the case of Osterix (Figure 3E, F). At day 35, gene expression levels in 0$\mathrm{nHA}$ and $45-\mathrm{nHA}$ remained comparable and significantly higher than in $\mathrm{BM}$.
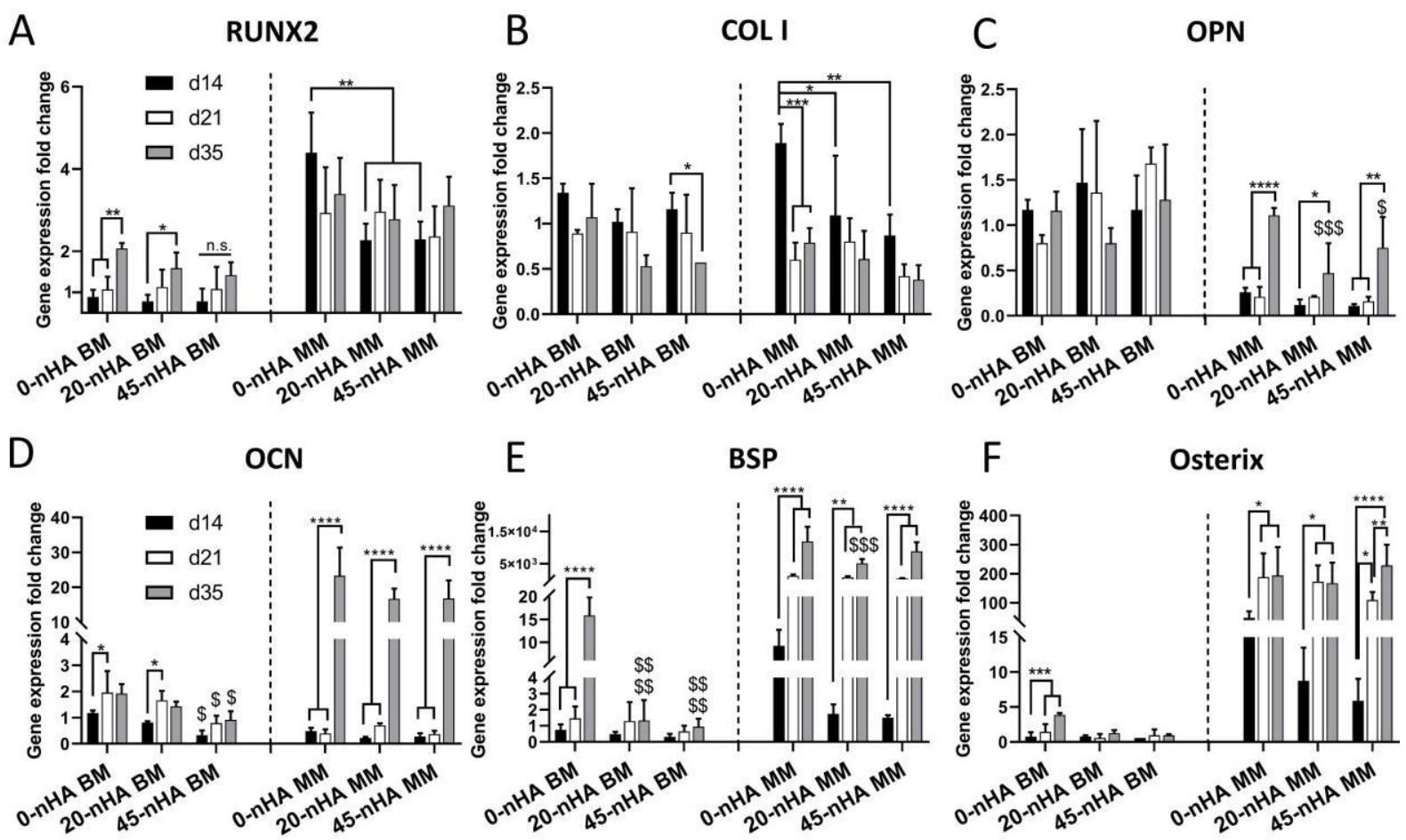

Figure 3. Gene expression of hMSCs cultured on scaffolds with different $\mathrm{nHA}$ concentrations for 14, 21 and 35 days in BM or MM. (A) RUNX2, (B) COL 1, (C) OPN, (D) OCN, (E) BSP, and (F) Osterix fold change expression values relative to 0 -nHA d7 BM. Data presented as average \pm s.d., and statistical significance performed using two-way ANOVA with Tukey's multiple comparison test $\left({ }^{*} \$ p<0.05 ;{ }^{* *} \$ p<0.01 ;{ }^{* * *} \$ \$ \$ p<0.001 ;{ }^{* * *} \$ \$ \$ \$ p<0.0001 ;{ }^{*}\right.$ for comparisons among timepoints each scaffold type; $\$$ for comparisons to 0-nHA (control) each timepoint; comparisons among BM and MM samples performed separately).

\section{Protein expression}

Complementary to gene expression, protein expression at day 35 was evaluated by immunofluorescence on all scaffold types and culture conditions. Extracellular COL I was found covering filaments on all scaffolds types and culture media conditions (Figure 4). Supporting the gene expression profiles, OPN protein expression was found slightly more abundant on scaffolds cultured in BM compared to MM, except in 45-nHA scaffolds in MM, where OPN protein expression was higher than in 20-nHA or 0-nHA scaffolds. Following an opposite trend, OCN protein expression was higher in scaffolds cultured in MM compared to BM (Figure 4). Interestingly, OCN protein expression was also recorded on nHA containing scaffolds (20-nHA and 45-nHA) cultured in BM. 

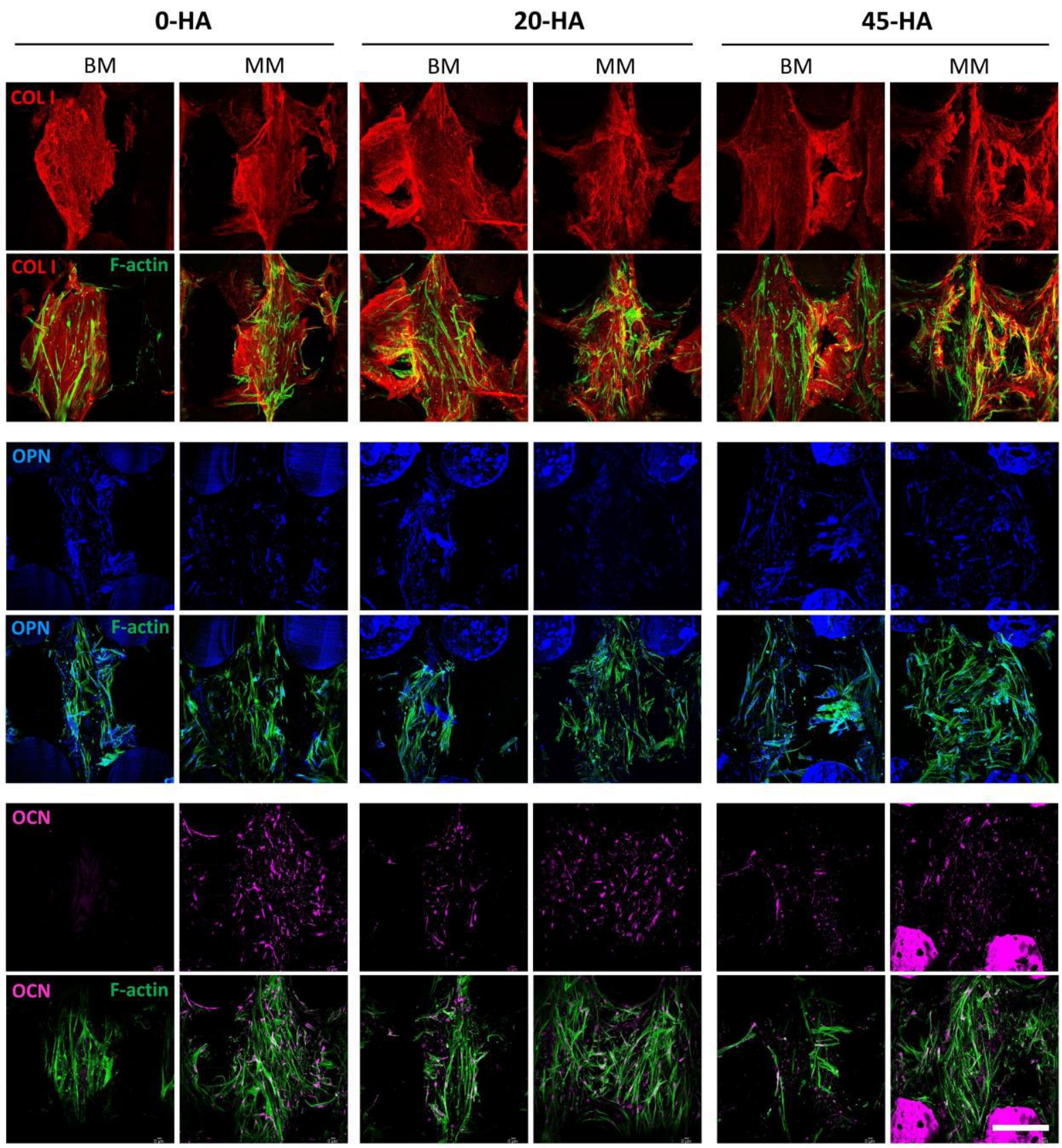

Figure 4. Influence of nHA content on the hMSCs expression of different osteogenic proteins. Representative confocal microscopy images of hMSCS (F-actin, green) on top of the filaments of $0-\mathrm{nHA}, 20-\mathrm{nHA}$ and $45-\mathrm{nHA}$ scaffolds after 35 days of culture in $\mathrm{BM}$ or $\mathrm{MM}$ and stained for the relevant osteogenic markers COL I (red), OPN (blue) and OCN (magenta). Scale bar for all images: $200 \mu \mathrm{m}$.

\section{Extracellular matrix (ECM) production and mineralization}

ECM mineralization was examined at day 35 of culture in BM and MM. An increasing amount of $\mathrm{Ca}$ deposition with increasing $\mathrm{nHA}$ content was observed, both quantitatively (Figure 5) and qualitatively, in scaffolds cultured in MM (Figure 6A, Figure S6). Although 
at lower levels than in MM, ARS quantification also revealed $\mathrm{Ca}$ deposits on 20-nHA and 45-nHA scaffolds when cultured in BM (Figure 5), after subtraction of the signal given by non-cell seeded stained scaffolds. In BM Ca deposition was mostly appreciable on 45-nHA scaffolds, where a more intense red color, compared to the non-seeded controls, was observed covering most of the surface area of the 45n-HA scaffolds filaments, as well as the scaffold filaments' exposed cross sections on the scaffolds outer surface (Figure 7A, Figure S6).

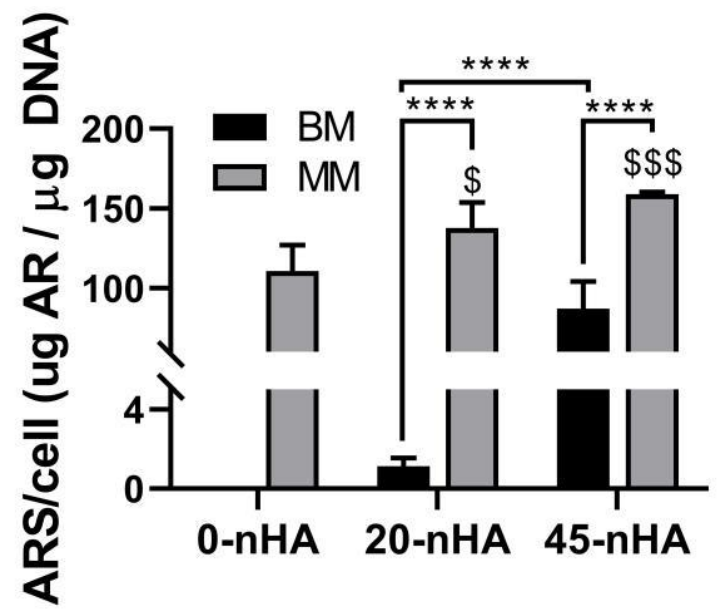

Figure 5. Quantification of the ARS staining extracted from scaffolds after 35 days of culture in BM or MM normalized to cell number. Data presented as average \pm s.d. and statistical significance performed using two-way ANOVA with Tukey's multiple comparison test. ${ }^{*} \$ p<0.05$; ${ }^{* * *} \$ \$ \$ p<$ $0.001 ;{ }^{* * *} \$ \$ \$ \$ p<0.0001 ; *$ for comparisons among BM and MM each scaffold type; $\$$ for comparisons among scaffold types each culture media type.

Next, cell and ECM coverage at day 35 of culture was investigated. When cultured in MM, cells in all scaffold types were confluent occupying both the scaffolds' filaments and pores along the whole scaffold cross-section (Figure 6B and Figure S7). Moreover, a dense and fibrillar ECM network produced by cells was observed for all scaffold types (Figure 6C and Figure S8). BSEM images in Figure 5D revealed bright points intercalated within the ECM in scaffolds pores, which were discriminated via EDS as $\mathrm{Ca}$ and $\mathrm{P}$ deposits, as well as a minimal amount of $\mathrm{Na}$ salts from the culture medium (Figure S9A). In addition, a peak of $\mathrm{N}$, corresponding to the ECM proteins, was also observed. In contrast to the dense ECM in scaffolds cultured in MM, a thin layer of cells and ECM was visualized covering the scaffolds' filaments when cultured in BM (Figure 7B-C, Figure S7, Figure S8). Notably, BSEM images of the ECM of cells cultured in BM did not depict bright points corresponding to mineral deposits (Figure 7D) and EDS analysis demonstrated significantly lower Ca and $\mathrm{P}$ signals compared to the analysis on scaffolds cultured on MM (Figure S9B). To be 
able to better discern among the EDS signal coming from the $\mathrm{Ca}$ and $\mathrm{P}$ already contained in the scaffold material, and the newly deposited minerals, not only the cell and ECM directly deposited on top of the scaffolds filaments was analyzed, but also the cell and ECM layer detached from the filament or present in the pores. $\mathrm{Ca}$ and $\mathrm{P}$ content in the ECM on the pores of MM scaffolds was found to be significantly higher than that on BM scaffolds (Figure S9).

MM

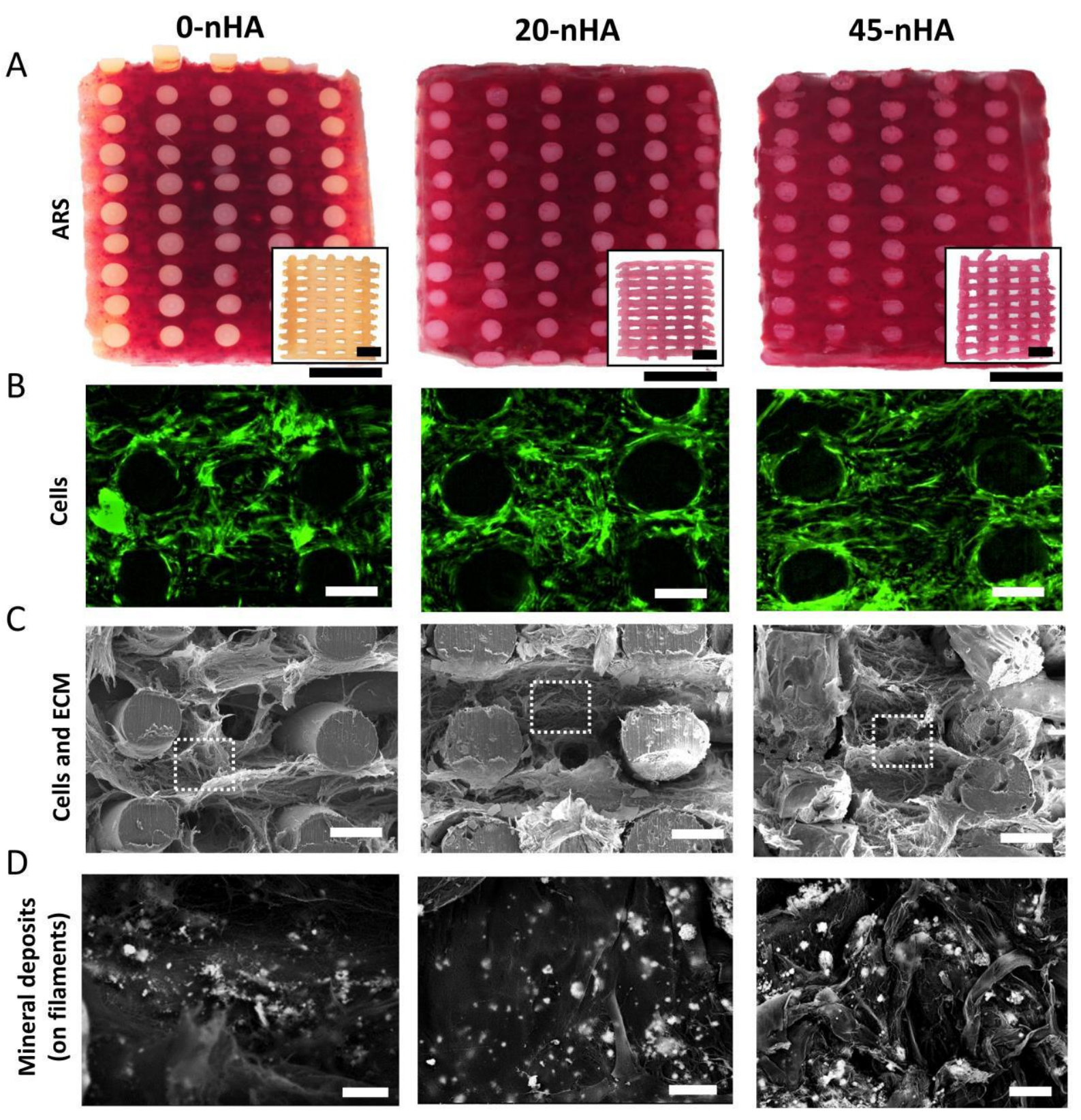

Figure 6. Influence of scaffolds' nHA content on matrix mineralization when cultured for 35 days in MM (7 days in BM followed by 28 days in MM). (A) Representative stereomicroscope images of scaffolds cross sections stained with ARS. Inserts represent the corresponding control scaffolds 
without cells incubated in MM and stained with ARS. Scale bars main images and insets $1 \mathrm{~mm}$. (B) Representative fluorescent microscopy images of hMSCs (F-actin, green) on scaffolds' cross sections. Scale bars $200 \mu \mathrm{m}$. (C) Representative SEM micrographs of scaffolds' cross sections depicting cell and ECM coverage. Scale bars $200 \mu \mathrm{m}$. (D) BSEM images of the regions marked with dash lines (ECM in scaffolds' pores) in (C) for examining the mineral deposits. Scale bars 20 $\mu \mathrm{m}$.

\section{BM}
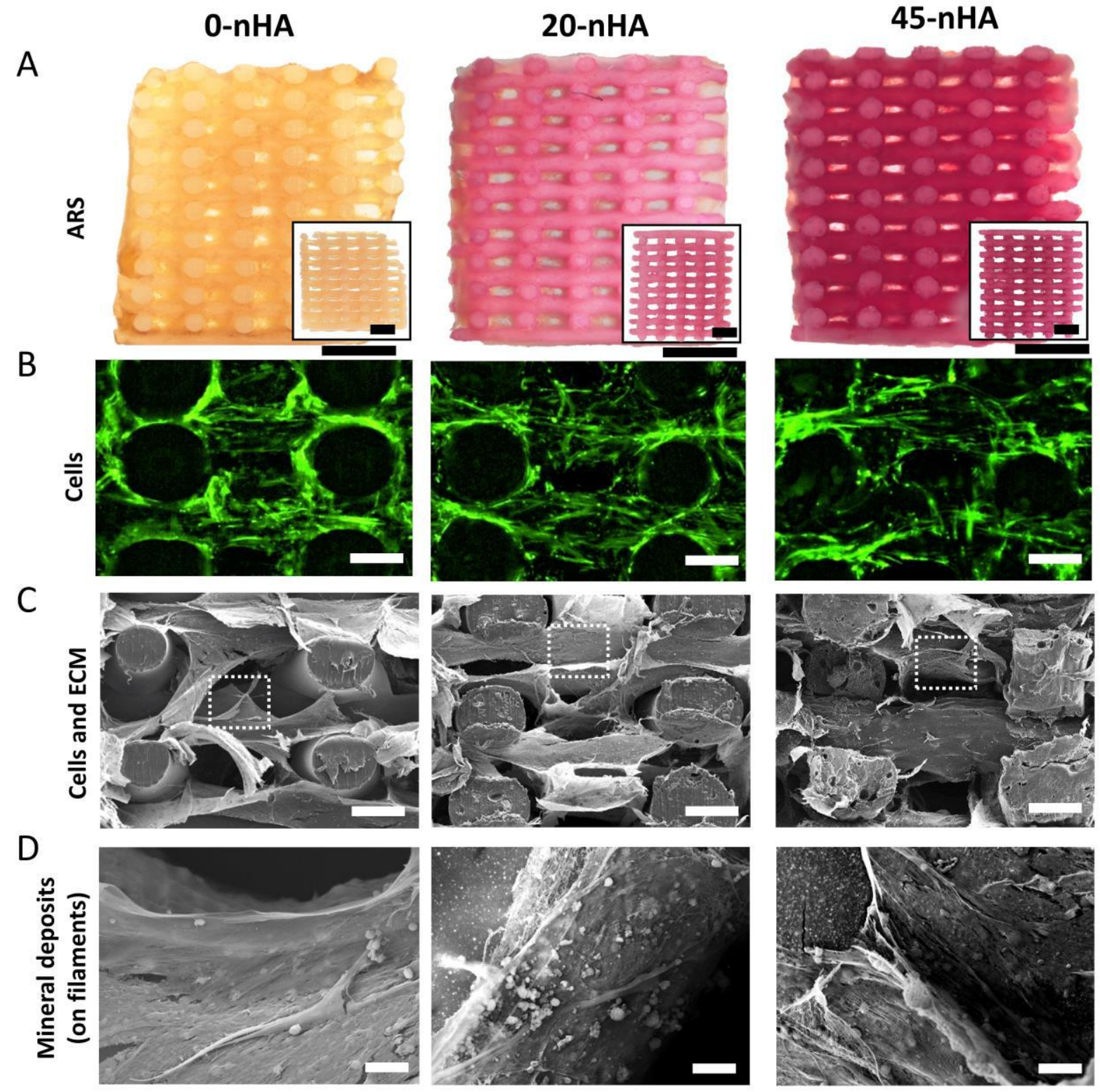

Figure 7. Influence of scaffolds' $\mathrm{nHA}$ content on matrix mineralization when cultured for 35 days in BM. (A) Representative stereomicroscope images of scaffolds cross sections stained with ARS. Inserts represent the corresponding control scaffolds without cells incubated in BM and stained with ARS. Scale bars main images and insets $1 \mathrm{~mm}$. (B) Representative fluorescent microscopy images of hMSCs (F-actin, green) on scaffolds' cross sections. Scale bars: $200 \mu \mathrm{m}$. (C) Representative SEM micrographs of scaffolds' cross sections depicting ECM coverage. Scale bars: $200 \mu \mathrm{m}$. (D) BSEM images of the regions marked with dash lines (ECM in scaffolds' pores) in (C) for examining mineral deposits. Scale bars $20 \mu \mathrm{m}$. 


\section{$C a$ and $P$ ion exchange dynamics with the medium}

To investigate the degradation of the $\mathrm{nHA}$ contained in the scaffolds, Ca release into SPS, which does not contain these ions, was quantified by ICP-MS. nHA-containing scaffolds released Ca into SPS continuously over the period evaluated (26 days) (Figure S10). In agreement with the nHA content on the scaffolds, 45-nHA scaffolds released larger amounts and at a higher rate (50 - $200 \mu \mathrm{m}$ every 3-4 days) than 20-nHA scaffolds (10 - 50 нм every 3-4 days).

To investigate the $\mathrm{Ca}$ and $\mathrm{P}$ content of the medium when scaffolds are in a cell culture setting, ICP-MS analysis was carried out on media collected at different time points during culture. This enabled to elucidate the ion exchange dynamics of $\mathrm{Ca}$ and $\mathrm{P}$ between the scaffolds and the media. The lower concentrations of $\mathrm{Ca}$ in media in contact with scaffolds compared to fresh media concentrations demonstrated that all scaffolds, cell seeded and non-cell seeded, depleted the media from $\mathrm{Ca}$, especially when cultured in MM (Figure S11). Similarly, scaffolds cultured in MM depleted the media from $P$, while $P$ levels in BM remained similar to fresh media ones. Interestingly, in general no significant differences in $\mathrm{Ca}$ and $\mathrm{P}$ depletion from media were found among cell seeded and non-cell seeded control scaffolds, nor among scaffolds with different nHA concentrations (Figure S11). To complement these results, the surface of the filaments of cell seeded (areas without cells) and non-cell seeded scaffolds cultured in BM and MM were analyzed by SEM and EDS (Figure S12 and Figure S13). Interestingly, while according to ICP-MS 0-nHA scaffolds depleted the media from $\mathrm{Ca}$ in $\mathrm{BM}$, and from $\mathrm{Ca}$ and $\mathrm{P}$ in $\mathrm{MM}$, no $\mathrm{Ca}$ and $\mathrm{P}$ was observed on any of the 0-nHA scaffolds surface. In contrast, the surface of 20-nHA and 45-nHA scaffolds in MM was found to be covered by CaP spheres in non-cell seeded scaffolds and by a compact $\mathrm{CaP}$ layer in cell seeded scaffolds. In $\mathrm{BM}$, these scaffolds showed a higher amount of white particles in their surface, compared to as-prepared scaffolds, potentially corresponding to the deposition of a Ca based mineral phase. While not easy to verify by either imaging or EDS, the surface of 45-nHA scaffolds in BM also looked more populated with bright spots, potentially corresponding to $\mathrm{Ca}$ deposits.

The scaffolds were also immersed in SBF, a solution that closely mimics human blood plasma and is used to test the ability of a material to deposit mineral on its surface. SEM showed a more pronounced deposition of mineral on 45-nHA scaffolds compared to 20nHA scaffolds (Figure 8). As expected, no mineral deposition occurred on the 0-nHA 
scaffolds even after 14 days of immersion, when a thick CaP layer was already covering the 45-nHA scaffolds.
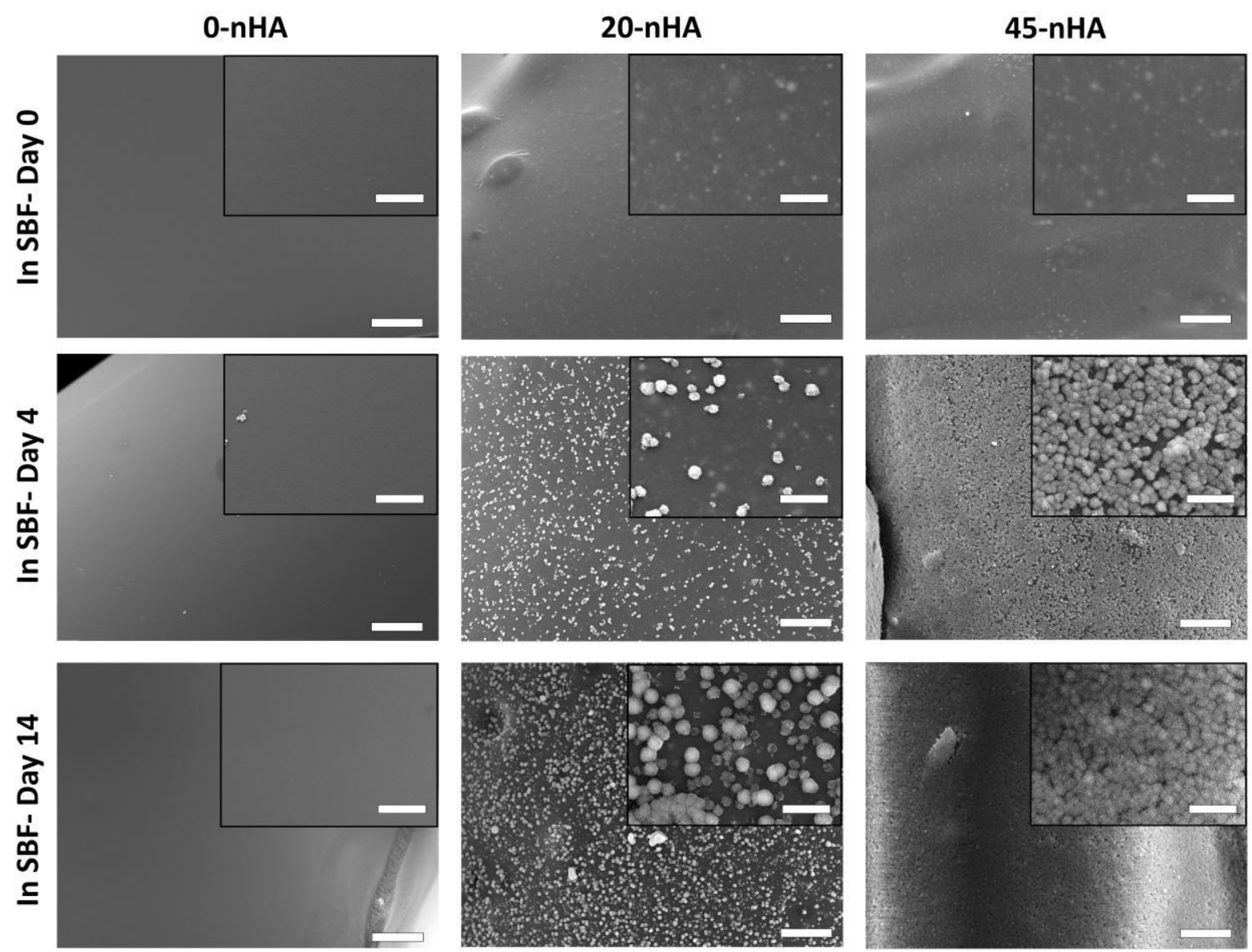

Figure 8. Representative SEM micrographs of scaffolds' filaments surface mineralization after immersion in SBF for 4 and 14 days. As-prepared scaffolds (day 0) are presented as controls. Scale bars: $20 \mu \mathrm{m}$. Inserts represent magnified regions. Scale bars $5 \mu \mathrm{m}$.

\section{DISCUSSION}

During the last decades, AM has rapidly grown into the field of tissue engineering offering patient specific 3D scaffolds from a wide range of biocompatible materials, including polymer-CaP composites. CaP fillers are highly desired when aiming to mimic the composition of bone and to provide scaffolds with high strength, tunable degradation and favorable bioactivity. While solvent extrusion has permitted the fabrication of AM scaffolds with up to $90 \mathrm{wt} \% \mathrm{HA},{ }^{44-45}$ the need of low volatility organic solvents, i.e. plasticizers, has shown to lead to solvent remnants within the scaffold, as well as to elastic constructs with poor applicability in load bearing applications. The advantage of thermoplastic ME-AM for bone tissue engineering over other AM techniques, such as stereolithography, mainly lies 
in the possibility of processing viscous composite materials including fillers, such as $\mathrm{CaP}$ particles, in a cost effective manner, and without requiring the use of organic solvents. Since the firstly reported PCL-CaP ME-AM scaffolds, containing up to 25 wt\% CaP, ${ }^{46-49}$ the field has largely evolved seeking to fabricate scaffolds with an inorganic content closer to native bone ECM, and with new polymeric materials. Here, we were able to fabricate composite 3D scaffolds based on PEOT/PBT, which have a great potential in the orthopedics field, and with up to $45 \mathrm{wt} \% \mathrm{nHA}$, closer to native bone mineral content than the majority of the until now reported scaffolds. Despite the high nHA loading, composites were printable and scaffolds' filaments maintained a good shape fidelity and macroporosity (pore size $500 \mu \mathrm{m}$ ), which is expected to favor tissue infiltration and vascularization in vivo. 50 When looking at the filaments' surface and cross-sections of the composite scaffolds, $\mathrm{nHA}$ aggregates with a size in the order of tens of microns were observed, in accordance with previous reports, where melt extruded filaments with HA concentrations above $10 \mathrm{wt} \%$ demonstrated HA agglomeration. ${ }^{51}$ In spite of these microaggregates, nanosized particles were still present in a well-dispersed manner within the polymer matrix of the scaffolds and on their surface, which were expected to elicit a higher degree of biological response compared to microsized counterparts, due to their high surface-to volume-ratio and to their small size, allowing uptake by cells. ${ }^{52-53}$ Most importantly, the high nHA content significantly enhanced the mechanical properties of the scaffolds, with 45-nHA scaffolds having a compressive modulus of $92 \pm 25 \mathrm{MPa}$ and compressive strength of $4.4 \pm 0.9$ $\mathrm{MPa}$, both in the range of cortical bone mechanical properties. ${ }^{54-55}$ Moreover, these scaffolds are stiffer than previously reported melt extruded AM scaffolds prepared with PCL-HA composites with similar inorganic loadings. This suggests the suitability of $45-$ $\mathrm{nHA}$ scaffolds for their application in bone regeneration scenarios, compared to bare PEOT/PBT scaffolds, as well as highly loaded PCL scaffolds, which is a commonly used polymer for bone scaffolds. ${ }^{24-25}$

In vivo, osteoinductive materials have the ability of recruiting stem cells and differentiating them towards an osteogenic phenotype in a heterotopic location. 12, 56 Due to the complexity of the in vivo microenvironment, in vitro models do not always reliably predict osteoinductivity. ${ }^{57}$ Yet, culturing stem cells on biomaterial scaffolds and analyzing cells' ability to differentiate can give some initial insights into a material osteogenic potential and aid to understand the osteoinduction mechanism. Thus, we cultured hMSCs on scaffolds in BM, without osteogenic factors, i.e. dexamethasone and $\beta$-glycerophosphate, and assessed their phenotype as a function of $\mathrm{nHA}$ content. In addition, we evaluated the 
synergistic effect of the material and these soluble factors that stimulate osteogenic differentiation/mineralization, by culturing in MM. Previously, it has been shown that cell distribution and density upon seeding can significantly influence hMSCs osteogenic differentiation in 3D scaffolds. ${ }^{42,58-59}$ Accordingly, poor cell attachment and lack of confluency within the scaffold cross-section have been shown to result in poor differentiation and lack of matrix mineralization in vitro. Besides, some studies reported enhanced cell attachment on polymer-CaP composites, likely due to the hydrophilicity and protein adsorption capacity of the CaP. ${ }^{60-61}$ In the present study, in order to decouple the osteogenic potential of the PEOT/PBT-nHA composite scaffolds from the effect of attachment efficiency, a viscous solution was used as seeding media, thereby ensuring comparable cell attachment and homogeneity among all scaffolds regardless of the $\mathrm{nHA}$ content, as previously reported. 42 Due to scaffold surface saturation with cells upon seeding, no cell proliferation was observed in the first 7 days of culture, nor during the subsequent 28 days in BM on any scaffold type, as ECM production was limited to the scaffolds filaments surface. On the other hand, ECM was also produced within the pores when cultured in MM, which increased the growth surface area enabling cell migration and a slight, yet not statistically significantly different, increase of cell number. An important general conclusion that can be drawn from the DNA quantification, and cell and ECM imaging on the scaffolds, is that 3D ME-AM PEOT/PBT scaffolds with up to $45 \mathrm{wt} \% \mathrm{nHA}$ are able to support cell growth, both in BM and MM.

Osteogenic differentiation was initially evaluated by ALP activity, an early marker for osteogenesis, ${ }^{62}$ and $\mathrm{OCN}$ secretion, one of the major bone non-collagenous proteins with the ability to bind to the bone HA. ${ }^{63}$ Moreover, the gene expression of RUNX2, an essential transcription factor to boost the expression of bone ECM proteins ${ }^{64-65}$, as well as other bone markers for osteogenesis at mRNA level (i.e. COL I, OPN, OCN, osterix and BSP), were analyzed during culture. According to the progression of bone markers during osteogenic differentiation suggested by literature, ALP activity was at its highest at early time points, and RUNX2 gene expression was upregulated from early time points, while OCN, osterix and BSP genes were highly expressed at later time points when cultured in MM. ${ }^{62,66-67} \mathrm{COL} I$ and OPN expression was maintained close to basal levels in BM, and slightly downregulated, with respect to day 7 , in MM. Interestingly, no significant effect of $\mathrm{nHA}$ was observed in MM, suggesting that the soluble osteogenic factors might play a more pronounced role than HA in the stimulation of osteogenesis, and the absence of a synergistic effect. In the case of culture in BM, all gene expression levels were much lower 
compared to MM cultures, maintaining gene expression at basal levels, with a slightly upregulation at day 35. Similar to MM cultures, no major differences in the gene expression levels were observed among scaffold types when cultured in BM. Yet, immunofluorescence revealed OCN and OPN protein expression at day 35 in BM only on 45-nHA scaffolds, while the ELISA analysis showed that OCN was also secreted in BM at early timepoints only in 45-nHA scaffolds. Despite these results suggesting the potential induction of osteogenesis by $45-\mathrm{nHA}$ scaffolds at late time points, this could not be concluded with a high degree of confidence, since the qualitative immunofluorescence observations were not supported by the aforementioned quantitative gene expression profiles. Moreover, OCN secretion in 45-nHA scaffolds measured by ELISA did not increase at later timepoints and levelled to other scaffold types. Previous studies have already suggested the lack of osteogenic response of cells to HA in composite scaffolds produced by ME-AM. This has been shown for polymer-HA composite scaffolds with different HA amounts. For example, PCL/PLGA-HA scaffolds with 10 wt $\%$ ceramic content in MM, as well as PEOT/PBT-HA scaffolds with 15wt\% HA in BM or MM, did not enhance osteogenic differentiation of rabbit MSCs and hMSCs, respectively, when compared to pure polymer scaffolds. ${ }^{39,60}$ Similarly, PLA-HA scaffolds with 50 wt\% HA seeded with hMSCs did not show any differences in gene expression compared to bare polymeric scaffolds when cultured in BM. 68 Consistently, our results also demonstrated that increasing the $\mathrm{nHA}$-to-copolymer ratio did not enhance the osteogenic potential of the scaffolds. We hypothesize that this is due to the suboptimal ion exchange dynamics between the scaffold and the medium. On one hand, we observed that upon incubation in SPS, scaffolds were able to release $\mathrm{Ca}$ and $\mathrm{P}$ from the $\mathrm{nHA}$ scaffolds, at the rate of 10 to $200 \mu \mathrm{M}$ every 3-4 days. On the other hand, ICP-MS measurements revealed overall $\mathrm{Ca}$ and $\mathrm{P}$ depletion from media, as previously reported for HA or HA-polymer composite scaffolds incubated in cell culture medium. ${ }^{68-70}$ Therefore, it is plausible that the slow release dynamics, compared to the fast (re)precipitation events, led to insufficient $\mathrm{Ca}$ concentration in the medium so to affect Ca signaling pathways involved in osteogenesis. Previous reports have shown that $\mathrm{Ca}$ and $\mathrm{P}$ salts added to culture media ${ }^{71-73}$ or released from polymeric scaffolds, ${ }^{74-75}$ at concentrations in the $\mathrm{mm}$ range, affect $\mathrm{Ca}$ and $\mathrm{P}$ signaling pathways involved in osteogenesis, leading to gene upregulation and matrix mineralization. Accordingly, scaffolds containing more soluble CaPs (e.g. biphasic $\mathrm{CaP}$ (BCP) or TCP) and, therefore, more optimum ion exchange dynamics, have shown to outperform HA based scaffolds, both in in vitro and in vivo scenarios. ${ }^{13,69,76-77}$ 
$\mathrm{Ca}$ and $\mathrm{P}$ depletion from media and SEM images of scaffolds surface after incubation in cell culture media and in SBF, confirmed $\mathrm{CaP}$ (re)precipitation on the scaffolds filaments surface. This was due to the presence of $\mathrm{nHA}$ particles on the filaments surface, negatively charged at physiological $\mathrm{pH}$, acting as nucleation sites and triggering electrostatic interactions with the $\mathrm{Ca}^{2+}$ cations in the media, which can accumulate on the surface of scaffold forming a positively charged Ca-rich layer and stimulate phosphate anions accumulation. ${ }^{78}$ This sequential process ultimately leads to the formation of a crystal phase of apatite, which in vivo has shown to promote the co-precipitation of endogenous proteins (e.g. BMPs), ultimately triggering undifferentiated cells to commit to the osteogenic lineage. 13, 40. This information, together with the lack of osteogenic differentiation in BM, suggests that the ARS staining in 20-nHA and $45 \mathrm{n}-\mathrm{HA}$ scaffolds after 35 days of culture in BM was not an hMSCs induced effect, but rather the consequence of a mineral phase (Ca carbonate or $\mathrm{CaP}$ ) precipitating from media onto the scaffolds' nHA. Notably, since both 45-nHA cell seeded and non-cell seeded scaffolds depleted BM equally of $\mathrm{Ca}$, and $\mathrm{MM}$ of $\mathrm{Ca}$ and $\mathrm{P}$, and yet non-cell seeded scaffolds presented lower ARS staining and thinner ions layer adsorption, it is hypothesized that the ECM produced by cells on the nHA scaffolds helped to further stabilize the mineral layers, respectively. While 0-nHA scaffolds demonstrated depletion of $\mathrm{Ca}$ from media over the culture period, they did not show any ARS staining in BM, or CaP layer formation in SBF. This is likely due to the lack of nucleation sites for $\mathrm{CaP}$ precipitation, and the weaker interaction of $\mathrm{Ca}$ with PEOT/PBT in 0-nHA scaffolds, leading to an unstable adsorption, in contrast to the strong Ca-nHA interactions on 45-nHA scaffolds. This hypothesis is supported by previous research on PEOT/PBT copolymers calcification, showing that efficient Ca-PEO complexations only occurred in copolymers with higher PEO molecular weight and higher PEOT-PBT ratios, compared to the one used in this study. ${ }^{33,} 79$ Consequently, it is plausible that CaP precipitated in the cell culture media.

To better elucidate the osteogenic property of the $\mathrm{nHA}$ containing scaffolds, longer in vitro culture times could be considered. Alternatively, enhancing the exposure of HA to the surface of the scaffold could potentially improve the ion exchange dynamics between scaffolds and medium and, therefore, improve the osteogenic potential of the $\mathrm{nHA}$ scaffolds. To do this, ME-AM scaffolds surface erosion using $\mathrm{NaOH},{ }^{77,80-81}$ as well as bare polymeric scaffolds coating with HA by ultrasonication, ${ }^{82}$ or pre-calcification by immersion in an SBF solution, ${ }^{83}$ have been proposed. While these studies commonly suggest the correlation of osteogenic genes upregulation or increased matrix mineralization to the 
enhanced HA exposure, we believe that careful investigations are still required, as using such surface optimization methods have also shown to dramatically change the surface roughness of the scaffolds, making it hard to decouple the effect of HA bioactivity and surface roughness, as the latter is known to affect osteogenic differentiation significantly. ${ }^{84}$

\section{CONCLUSION}

Due to a combination of interconnected macroporosity, tunable biodegradability, optimal mechanical properties and bioactivity, 3D polymer-HA composite scaffolds prepared by ME-AM are considered important candidates, when intending to regenerate critical sized bone defects. The aim of this study was the fabrication of highly loaded PEOT/PBT-nHA composite scaffolds with up to $45 \mathrm{wt} \% \mathrm{nHA}$ using ME-AM and the assessment of their mechanical and in depth biological performance as a function of the $\mathrm{nHA}$ content. 45-nHA scaffolds presented significantly enhanced compressive modulus, compared to bare PEOT/PBT copolymer scaffolds, which lied in the range of cancellous bone mechanical properties. In terms of cell behavior. hMSCs were able to differentiate into the osteogenic lineage in all scaffolds regardless of the HA content in MM. Although no differences were observed in osteogenic differentiation at the gene and protein level, increased matrix mineralization was observed on 45-nHA scaffolds compared to $0-\mathrm{nHA}$ and 20-nHA scaffolds. While no osteogenic differentiation of cells was observed in BM, the observed ARS signal in 45-nHA scaffolds in BM suggested the precipitation of a $\mathrm{CaP}$ layer from $\mathrm{Ca}$ and $\mathrm{P}$ ions present in the cell culture media. Since such a CaP layer was also formed upon immersion in SBF, 45-nHA scaffolds are thought to potentially serve as osteoconductive substrates in an in vivo situation, favoring osteoblasts adhesion and proliferation. Overall, our results suggest the enhanced mechanical properties of the presented highly loaded composite scaffolds (45-nHA) and their ability to reprecipitate a CaP layer, thus supporting their in vivo applicability. Yet, future research and further optimization of polymer-HA composite scaffolds prepared by ME-AM for stimulating bone regeneration, as well as on the validation of their performance through in vivo studies are needed.

\section{Acknowledgement}

We are grateful to H2020-NMP-PILOTS-2015 (GA n. 685825) for financial support. PH gratefully acknowledges the Gravitation Program 'Materials-Driven Regeneration', funded 
by the Netherlands Organization for Scientific Research (NWO) (024.003.013). Some of the materials used in this work were provided by the Texas A\&M Health Science Center College of Medicine Institute for Regenerative Medicine at Scott \& White through a grant from NCRR of the NIH (Grant \#P40RR017447). We would also like to thank Eva Gubbins from MERLN Institute for performing the ICP-MS measurements. 


\section{References}

1. Keating, J. F.; Simpson, A. H.; Robinson, C. M., The Management of Fractures with Bone Loss. The Journal of bone and joint surgery. British volume 2005, 87 (2), 142-150.

2. Karger, C.; Kishi, T.; Schneider, L.; Fitoussi, F.; Masquelet, A. C., Treatment of Posttraumatic Bone Defects by the Induced Membrane Technique. Orthopaedics \& Traumatology: Surgery \& Research 2012, 98 (1), 97-102.

3. Catagni, M. A.; Guerreschi, F.; Lovisetti, L., Distraction Osteogenesis for Bone Repair in the 21st Century: Lessons Learned. Injury 2011, 42 (6), 580-586.

4. Schmidmaier, G.; Capanna, R.; Wildemann, B.; Beque, T.; Lowenberg, D., Bone Morphogenetic Proteins in Critical-Size Bone Defects: What Are the Options? Injury 2009, 40, S39-S43.

5. Roddy, E.; DeBaun, M. R.; Daoud-Gray, A.; Yang, Y. P.; Gardner, M. J., Treatment of Critical-Sized Bone Defects: Clinical and Tissue Engineering Perspectives. European Journal of Orthopaedic Surgery \& Traumatology 2018, 28 (3), 351-362.

6. Porter, J. R.; Ruckh, T. T.; Popat, K. C., Bone Tissue Engineering: A Review in Bone Biomimetics and Drug Delivery Strategies. Biotechnology progress 2009, 25 (6), 1539-1560.

7. Henkel, J.; Woodruff, M. A.; Epari, D. R.; Steck, R.; Glatt, V.; Dickinson, I. C.; Choong, P. F.; Schuetz, M. A.; Hutmacher, D. W., Bone Regeneration Based on Tissue Engineering Conceptions-a 21st Century Perspective. Bone research 2013, 1 (3), 216-248.

8. Rho, J.-Y.; Kuhn-Spearing, L.; Zioupos, P., Mechanical Properties and the Hierarchical Structure of Bone. Medical Engineering \& Physics 1998, 20 (2), 92-102.

9. Habraken, W.; Habibovic, P.; Epple, M.; Bohner, M., Calcium Phosphates in Biomedical Applications: Materials for the Future? Materials Today 2016, 19 (2), 69-87.

10. Samavedi, S.; Whittington, A. R.; Goldstein, A. S., Calcium Phosphate Ceramics in Bone Tissue Engineering: A Review of Properties and Their Influence on Cell Behavior. Acta biomaterialia 2013, 9 (9), 8037-8045.

11. Galván-Chacón, V. P.; Habibovic, P., Deconvoluting the Bioactivity of Calcium Phosphate-Based Bone Graft Substitutes: Strategies to Understand the Role of Individual Material Properties. Advanced Healthcare Materials 2017, 6 (13), 1601478.

12. Barradas, A.; Yuan, H.; van Blitterswijk, C. A.; Habibovic, P., Osteoinductive Biomaterials: Current Knowledge of Properties, Experimental Models and Biological Mechanisms. Eur Cell Mater 2011, 21 (407), 29.

13. Habibovic, P.; Yuan, H.; van der Valk, C. M.; Meijer, G.; van Blitterswijk, C. A.; de Groot, K., 3d Microenvironment as Essential Element for Osteoinduction by Biomaterials. Biomaterials 2005, 26 (17), 3565-3575.

14. Hutmacher, D. W., Scaffolds in Tissue Engineering Bone and Cartilage. Biomaterials 2000, 21 (24), 2529 2543.

15. Trombetta, R.; Inzana, J. A.; Schwarz, E. M.; Kates, S. L.; Awad, H. A., 3d Printing of Calcium Phosphate Ceramics for Bone Tissue Engineering and Drug Delivery. Annals of Biomedical Engineering 2017, 45 (1), 23-44.

16. Moreno Madrid, A. P.; Vrech, S. M.; Sanchez, M. A.; Rodriguez, A. P., Advances in Additive Manufacturing for Bone Tissue Engineering Scaffolds. Materials Science and Engineering: C 2019, 100, 631-644.

17. Barba, A.; Diez-Escudero, A.; Maazouz, Y.; Rappe, K.; Espanol, M.; Montufar, E. B.; Bonany, M.; Sadowska, J. M.; Guillem-Marti, J.; Öhman-Mägi, C.; Persson, C.; Manzanares, M. C.; Franch, J.; Ginebra, M. P., Osteoinduction by Foamed and 3d-Printed Calcium Phosphate Scaffolds: Effect of Nanostructure and Pore Architecture. ACS Appl Mater Interfaces 2017, 9 (48), 41722-41736.

18. Raymond, S.; Maazouz, Y.; Montufar, E. B.; Perez, R. A.; González, B.; Konka, J.; Kaiser, J.; Ginebra, M.P., Accelerated Hardening of Nanotextured 3d-Plotted Self-Setting Calcium Phosphate Inks. Acta Biomaterialia 2018, 75, 451-462.

19. Jakus, A. E.; Rutz, A. L.; Jordan, S. W.; Kannan, A.; Mitchell, S. M.; Yun, C.; Koube, K. D.; Yoo, S. C.; Whiteley, H. E.; Richter, C.-P.; Galiano, R. D.; Hsu, W. K.; Stock, S. R.; Hsu, E. L.; Shah, R. N., 
Hyperelastic "Bone": A Highly Versatile, Growth Factor-Free, Osteoregenerative, Scalable, and Surgically Friendly Biomaterial. Science Translational Medicine 2016, 8 (358), 358ra127.

20. Zafar, M. J.; Zhu, D.; Zhang, Z., 3d Printing of Bioceramics for Bone Tissue Engineering. Materials (Basel, Switzerland) 2019, 12 (20).

21. Kumar, A.; Kargozar, S.; Baino, F.; Han, S. S., Additive Manufacturing Methods for Producing Hydroxyapatite and Hydroxyapatite-Based Composite Scaffolds: A Review. Frontiers in Materials 2019, 6 (313).

22. Bloebaum, R. D.; Skedros, J. G.; Vajda, E. G.; Bachus, K. N.; Constantz, B. R., Determining Mineral Content Variations in Bone Using Backscattered Electron Imaging. Bone 1997, 20 (5), 485-490.

23. Roschger, P.; Fratzl, P.; Eschberger, J.; Klaushofer, K., Validation of Quantitative Backscattered Electron Imaging for the Measurement of Mineral Density Distribution in Human Bone Biopsies. Bone 1998, 23 (4), 319-326.

24. Jiang, W.; Shi, J.; Li, W.; Sun, K., Morphology, Wettability, and Mechanical Properties of Polycaprolactone/Hydroxyapatite Composite Scaffolds with Interconnected Pore Structures Fabricated by a Mini-Deposition System. Polymer Engineering \& Science 2012, 52 (11), 2396-2402.

25. Jiang, W.; Shi, J.; Li, W.; Sun, K., Three Dimensional Melt-Deposition of Polycaprolactone/Bio-Derived Hydroxyapatite Composite into Scaffold for Bone Repair. Journal of Biomaterials Science, Polymer Edition 2013, 24 (5), 539-550.

26. Esposito Corcione, C.; Gervaso, F.; Scalera, F.; Padmanabhan, S. K.; Madaghiele, M.; Montagna, F.; Sannino, A.; Licciulli, A.; Maffezzoli, A., Highly Loaded Hydroxyapatite Microsphere/ Pla Porous Scaffolds Obtained by Fused Deposition Modelling. Ceramics International 2019, 45 (2, Part B), 2803-2810.

27. Yu, J.; Xu, Y.; Li, S.; Seifert, G. V.; Becker, M. L., Three-Dimensional Printing of Nano Hydroxyapatite/Poly(Ester Urea) Composite Scaffolds with Enhanced Bioactivity. Biomacromolecules 2017, 18 (12), 4171-4183.

28. Trachtenberg, J. E.; Placone, J. K.; Smith, B. T.; Fisher, J. P.; Mikos, A. G., Extrusion-Based 3d Printing of Poly(Propylene Fumarate) Scaffolds with Hydroxyapatite Gradients. J Biomater Sci Polym Ed 2017, 28 (6), 532-554.

29. Petrovskaya, T.; Toropkov, N.; Mironov, E.; Azarmi, F., 3d Printed Biocompatible PolylactideHydroxyapatite Based Material for Bone Implants. Materials and Manufacturing Processes 2018, 33 (16), 1899-1904.

30. Chen, S.; Shi, Y.; Zhang, X.; Ma, J., 3d Printed Hydroxyapatite Composite Scaffolds with Enhanced Mechanical Properties. Ceramics International 2019, 45 (8), 10991-10996.

31. Kim, M. H.; Yun, C.; Chalisserry, E. P.; Lee, Y. W.; Kang, H. W.; Park, S.-H.; Jung, W.-K.; Oh, J.; Nam, S. Y., Quantitative Analysis of the Role of Nanohydroxyapatite (Nha) on 3d-Printed Pcl/Nha Composite Scaffolds. Materials Letters 2018, 220, 112-115.

32. Serra, T.; Planell, J. A.; Navarro, M., High-Resolution Pla-Based Composite Scaffolds Via 3-D Printing Technology. Acta biomaterialia 2013, 9 (3), 5521-5530.

33. Van Blitterswijk, C.; Leenders, H.; Baaker, D., The Effect of Peo Ratio on Degradation, Calcification and Bone Bonding of Peo/Pbt Copolymer (Polyactive). Cells and Materials 1993, 3 (1), 2.

34. Kuijer, R.; Bouwmeester, S. M.; Drees, M. E.; Surtel, D. M.; Terwindt-Rouwenhorst, E. W.; Van Der Linden, A.; Van Blitterswijk, C.; Bulstra, S., The Polymer Polyactivetm as a Bone-Filling Substance: An Experimental Study in Rabbits. Journal of Materials Science: Materials in Medicine 1998, 9 (8), 449-455.

35. Deschamps, A. A.; Claase, M. B.; Sleijster, W. J.; de Bruijn, J. D.; Grijpma, D. W.; Feijen, J., Design of Segmented Poly(Ether Ester) Materials and Structures for the Tissue Engineering of Bone. Journal of Controlled Release 2002, 78 (1), 175-186.

36. Jansen, E. J. P.; Pieper, J.; Gijbels, M. J. J.; Guldemond, N. A.; Riesle, J.; Van Rhijn, L. W.; Bulstra, S. K.; Kuijer, R., Peot/Pbt Based Scaffolds with Low Mechanical Properties Improve Cartilage Repair Tissue Formation in Osteochondral Defects. Journal of Biomedical Materials Research Part A 2009, 89A (2), 444452.

37. Di Luca, A.; Ostrowska, B.; Lorenzo-Moldero, I.; Lepedda, A.; Swieszkowski, W.; Van Blitterswijk, C.; Moroni, L., Gradients in Pore Size Enhance the Osteogenic Differentiation of Human Mesenchymal Stromal Cells in Three-Dimensional Scaffolds. Scientific Reports 2016, 6 (1), 22898. 
38. Liu, Q.; De Wijn, J. R.; Bakker, D.; Van Blitterswijk, C. A., Surface Modification of Hydroxyapatite to Introduce Interfacial Bonding with Polyactivetm 70/30 in a Biodegradable Composite. Journal of Materials Science: Materials in Medicine 1996, 7 (9), 551-557.

39. Nandakumar, A.; Cruz, C.; Mentink, A.; Birgani, Z. T.; Moroni, L.; van Blitterswijk, C.; Habibovic, P., Monolithic and Assembled Polymer-Ceramic Composites for Bone Regeneration. Acta biomaterialia 2013, 9 (3), 5708-5717.

40. Lin, L.; Chow, K. L.; Leng, Y., Study of Hydroxyapatite Osteoinductivity with an Osteogenic Differentiation of Mesenchymal Stem Cells. Journal of Biomedical Materials Research Part A 2009, 89A (2), 326-335.

41. Sinha, R.; Cámara-Torres, M.; Scopece, P.; Falzacappa, E. V.; Patelli, A.; Moroni, L.; Mota, C., A Hybrid Additive Manufacturing Platform to Create Bulk and Surface Composition Gradients on Scaffolds for Tissue Regeneration. bioRxiv 2020, 2020.2006.2023.165605.

42. Cámara-Torres, M.; Sinha, R.; Mota, C.; Moroni, L., Improving Cell Distribution on 3d Additive Manufactured Scaffolds through Engineered Seeding Media Density and Viscosity. Acta Biomaterialia 2020, 101, 183-195.

43. Kokubo, T.; Takadama, H., How Useful Is Sbf in Predicting in Vivo Bone Bioactivity? Biomaterials 2006, 27 (15), 2907-2915.

44. Jakus, A. E.; Rutz, A. L.; Jordan, S. W.; Kannan, A.; Mitchell, S. M.; Yun, C.; Koube, K. D.; Yoo, S. C.; Whiteley, H. E.; Richter, C.-P., Hyperelastic "Bone": A Highly Versatile, Growth Factor-Free, Osteoregenerative, Scalable, and Surgically Friendly Biomaterial. Science translational medicine 2016, 8 (358), 358ra127-358ra127.

45. Huang, Y.-H.; Jakus, A. E.; Jordan, S. W.; Dumanian, Z.; Parker, K.; Zhao, L.; Patel, P. K.; Shah, R. N., Three-Dimensionally Printed Hyperelastic Bone Scaffolds Accelerate Bone Regeneration in Critical-Size Calvarial Bone Defects. Plastic and reconstructive surgery 2019, 143 (5), 1397-1407.

46. Schantz, J.-T.; Brandwood, A.; Hutmacher, D. W.; Khor, H. L.; Bittner, K., Osteogenic Differentiation of Mesenchymal Progenitor Cells in Computer Designed Fibrin-Polymer-Ceramic Scaffolds Manufactured by Fused Deposition Modeling. Journal of Materials Science: Materials in Medicine 2005, 16 (9), 807-819.

47. Zhou, Y. F.; Sae-Lim, V.; Chou, A. M.; Hutmacher, D. W.; Lim, T. M., Does Seeding Density Affect in Vitro Mineral Nodules Formation in Novel Composite Scaffolds? Journal of Biomedical Materials Research Part A 2006, 78A (1), 183-193.

48. Shor, L.; Güçeri, S.; Wen, X.; Gandhi, M.; Sun, W., Fabrication of Three-Dimensional Polycaprolactone/Hydroxyapatite Tissue Scaffolds and Osteoblast-Scaffold Interactions in Vitro. Biomaterials 2007, 28 (35), 5291-5297.

49. Rai, B.; Lin, J. L.; Lim, Z. X. H.; Guldberg, R. E.; Hutmacher, D. W.; Cool, S. M., Differences between in Vitro Viability and Differentiation and in Vivo Bone-Forming Efficacy of Human Mesenchymal Stem Cells Cultured on Pcl-Tcp Scaffolds. Biomaterials 2010, 31 (31), 7960-7970.

50. Karageorgiou, V.; Kaplan, D., Porosity of 3d Biomaterial Scaffolds and Osteogenesis. Biomaterials 2005, 26 (27), 5474-5491.

51. Backes, E. H.; Pires, L. D. N.; Beatrice, C. A. G.; Costa, L. C.; Passador, F. R.; Pessan, L. A., Fabrication of Biocompatible Composites of Poly(Lactic Acid)/Hydroxyapatite Envisioning Medical Applications. Polymer Engineering \& Science 2020, n/a (n/a).

52. Huang, Y.; Zhou, G.; Zheng, L.; Liu, H.; Niu, X.; Fan, Y., Micro-/Nano- Sized Hydroxyapatite Directs Differentiation of Rat Bone Marrow Derived Mesenchymal Stem Cells Towards an Osteoblast Lineage. Nanoscale 2012, 4 (7), 2484-2490.

53. Yang, X.; Li, Y.; Liu, X.; Zhang, R.; Feng, Q., In Vitro Uptake of Hydroxyapatite Nanoparticles and Their Effect on Osteogenic Differentiation of Human Mesenchymal Stem Cells. Stem cells international 2018, $2018,2036176$.

54. Keaveny, T. M.; Morgan, E. F.; Yeh, O. C., Bone Mechanics. Standard handbook of biomedical engineering and design 2004, 1-24.

55. Reilly, D. T.; Burstein, A. H., The Elastic and Ultimate Properties of Compact Bone Tissue. J Biomech 1975, 8 (6), 393-405

56. Albrektsson, T.; Johansson, C., Osteoinduction, Osteoconduction and Osseointegration. European spine journal 2001, 10 (2), S96-S101. 
57. Habibovic, P.; Woodfield, T.; de Groot, K.; van Blitterswijk, C., Predictive Value of in Vitro and in Vivo Assays in Bone and Cartilage Repair--What Do They Really Tell Us About the Clinical Performance? Advances in experimental medicine and biology 2006, 585, 327-360.

58. Noda, S.; Kawashima, N.; Yamamoto, M.; Hashimoto, K.; Nara, K.; Sekiya, I.; Okiji, T., Effect of Cell Culture Density on Dental Pulp-Derived Mesenchymal Stem Cells with Reference to Osteogenic Differentiation. Scientific Reports 2019, 9 (1), 5430.

59. Holy, C. E.; Shoichet, M. S.; Davies, J. E., Engineering Three-Dimensional Bone Tissue in Vitro Using Biodegradable Scaffolds: Investigating Initial Cell-Seeding Density and Culture Period. Journal of Biomedical Materials Research: An Official Journal of The Society for Biomaterials, The Japanese Society for Biomaterials, and The Australian Society for Biomaterials and the Korean Society for Biomaterials 2000, 51 (3), 376-382.

60. Moncal, K. K.; Heo, D. N.; Godzik, K. P.; Sosnoski, D. M.; Mrowczynski, O. D.; Rizk, E.; Ozbolat, V.; Tucker, S. M.; Gerhard, E. M.; Dey, M.; Lewis, G. S.; Yang, J.; Ozbolat, I. T., 3d Printing of Poly(ECaprolactone)/Poly(D,L-Lactide-Co-Glycolide)/Hydroxyapatite Composite Constructs for Bone Tissue Engineering. Journal of Materials Research 2018, 33 (14), 1972-1986.

61. Huang, B.; Caetano, G.; Vyas, C.; Blaker, J. J.; Diver, C.; Bártolo, P., Polymer-Ceramic Composite Scaffolds: The Effect of Hydroxyapatite and B-Tri-Calcium Phosphate. Materials 2018, 11 (1), 129.

62. Owen, T. A.; Aronow, M.; Shalhoub, V.; Barone, L. M.; Wilming, L.; Tassinari, M. S.; Kennedy, M. B.; Pockwinse, S.; Lian, J. B.; Stein, G. S., Progressive Development of the Rat Osteoblast Phenotype in Vitro: Reciprocal Relationships in Expression of Genes Associated with Osteoblast Proliferation and Differentiation During Formation of the Bone Extracellular Matrix. Journal of cellular physiology 1990, 143 (3), 420-430.

63. Ivaska, K. K.; Hentunen, T. A.; Vaaraniemi, J.; Ylipahkala, H.; Pettersson, K.; Vaananen, H. K., Release of Intact and Fragmented Osteocalcin Molecules from Bone Matrix During Bone Resorption in Vitro. The Journal of biological chemistry 2004, 279 (18), 18361-18369.

64. Komori, T.; Yagi, H.; Nomura, S.; Yamaguchi, A.; Sasaki, K.; Deguchi, K.; Shimizu, Y.; Bronson, R. T.; Gao, Y. H.; Inada, M.; Sato, M.; Okamoto, R.; Kitamura, Y.; Yoshiki, S.; Kishimoto, T., Targeted Disruption of Cbfa1 Results in a Complete Lack of Bone Formation Owing to Maturational Arrest of Osteoblasts. Cell 1997, 89 (5), 755-764.

65. Li, Y.; Ge, C.; Long, J. P.; Begun, D. L.; Rodriguez, J. A.; Goldstein, S. A.; Franceschi, R. T., Biomechanical Stimulation of Osteoblast Gene Expression Requires Phosphorylation of the Runx2 Transcription Factor. Journal of bone and mineral research : the official journal of the American Society for Bone and Mineral Research 2012, 27 (6), 1263-1274.

66. Roach, H. I., Why Does Bone Matrix Contain Non-Collagenous Proteins? The Possible Roles of Osteocalcin, Osteonectin, Osteopontin and Bone Sialoprotein in Bone Mineralisation and Resorption. Cell Biology International 1994, 18 (6), 617-628.

67. Ozawa, R.; Yamada, Y.; Nagasaka, T.; Ueda, M., A Comparison of Osteogenesis-Related Gene Expression of Mesenchymal Stem Cells During the Osteoblastic Differentiation Induced by Type-I Collagen and/or Fibronectin. International Journal of Oral-Medical Sciences 2003, 1 (2), 139-146.

68. Sun, L.; Danoux, C. B.; Wang, Q.; Pereira, D.; Barata, D.; Zhang, J.; LaPointe, V.; Truckenmüller, R.; Bao, C.; Xu, X.; Habibovic, P., Independent Effects of the Chemical and Microstructural Surface Properties of Polymer/Ceramic Composites on Proliferation and Osteogenic Differentiation of Human Mscs. Acta Biomaterialia 2016, 42, 364-377.

69. Barradas, A. M.; Monticone, V.; Hulsman, M.; Danoux, C.; Fernandes, H.; Tahmasebi Birgani, Z.; Barrerede Groot, F.; Yuan, H.; Reinders, M.; Habibovic, P.; van Blitterswijk, C.; de Boer, J., Molecular Mechanisms of Biomaterial-Driven Osteogenic Differentiation in Human Mesenchymal Stromal Cells. Integrative biology : quantitative biosciences from nano to macro 2013, 5 (7), 920-931.

70. Tahmasebi Birgani, Z.; van Blitterswijk, C. A.; Habibovic, P., Monolithic Calcium Phosphate/Poly(Lactic Acid) Composite Versus Calcium Phosphate-Coated Poly(Lactic Acid) for Support of Osteogenic Differentiation of Human Mesenchymal Stromal Cells. Journal of Materials Science: Materials in Medicine 2016, 27 (3), 54.

71. Barradas, A. M. C.; Fernandes, H. A. M.; Groen, N.; Chai, Y. C.; Schrooten, J.; van de Peppel, J.; van Leeuwen, J. P. T. M.; van Blitterswijk, C. A.; de Boer, J., A Calcium-Induced Signaling Cascade Leading to Osteogenic Differentiation of Human Bone Marrow-Derived Mesenchymal Stromal Cells. Biomaterials 2012, 33 (11), 3205-3215. 
72. Chai, Y. C.; Roberts, S. J.; Schrooten, J.; Luyten, F. P., Probing the Osteoinductive Effect of Calcium Phosphate by Using an in Vitro Biomimetic Model. Tissue Engineering Part A 2010, 17 (7-8), 1083-1097.

73. Beck, G. R.; Zerler, B.; Moran, E., Phosphate Is a Specific Signal for Induction of Osteopontin Gene Expression. Proceedings of the National Academy of Sciences 2000, 97 (15), 8352-8357.

74. Habibovic, P.; Bassett, D. C.; Doillon, C. J.; Gerard, C.; McKee, M. D.; Barralet, J. E., Collagen Biomineralization in Vivo by Sustained Release of Inorganic Phosphate Ions. Advanced Materials 2010, 22 (16), 1858-1862.

75. Danoux, C. B. S. S.; Bassett, D. C.; Othman, Z.; Rodrigues, A. I.; Reis, R. L.; Barralet, J. E.; van Blitterswijk, C. A.; Habibovic, P., Elucidating the Individual Effects of Calcium and Phosphate lons on Hmscs by Using Composite Materials. Acta Biomaterialia 2015, 17, 1-15.

76. Yuan, H.; Fernandes, H.; Habibovic, P.; de Boer, J.; Barradas, A. M. C.; de Ruiter, A.; Walsh, W. R.; van Blitterswijk, C. A.; de Bruijn, J. D., Osteoinductive Ceramics as a Synthetic Alternative to Autologous Bone Grafting. Proc Natl Acad Sci U S A 2010, 107 (31), 13614-13619.

77. Nyberg, E.; Rindone, A.; Dorafshar, A.; Grayson, W. L., Comparison of 3d-Printed Poly- $\varepsilon$-Caprolactone Scaffolds Functionalized with Tricalcium Phosphate, Hydroxyapatite, Bio-Oss, or Decellularized Bone Matrix. Tissue Engineering Part A 2016, 23 (11-12), 503-514.

78. Kim, H.-M.; Himeno, T.; Kawashita, M.; Kokubo, T.; Nakamura, T., The Mechanism of Biomineralization of Bone-Like Apatite on Synthetic Hydroxyapatite: An in Vitro Assessment. Journal of The Royal Society Interface 2004, 1 (1), 17-22.

79. Thoma, R.; Hung, T.; Nyilas, E.; Haubold, A.; Phillips, R., Metal Ion Complexation of Poly (Ether) Urethanes. In Advances in Biomedical Polymers, Springer: 1987; pp 131-145.

80. Cho, Y. S.; Choi, S.; Lee, S.-H.; Kim, K. K.; Cho, Y.-S., Assessments of Polycaprolactone/Hydroxyapatite Composite Scaffold with Enhanced Biomimetic Mineralization by Exposure to Hydroxyapatite Via a 3dPrinting System and Alkaline Erosion. European Polymer Journal 2019, 113, 340-348.

81. Cho, Y. S.; Quan, M.; Lee, S.-H.; Hong, M. W.; Kim, Y. Y.; Cho, Y.-S., Assessment of Osteogenesis for 3d-Printed Polycaprolactone/Hydroxyapatite Composite Scaffold with Enhanced Exposure of Hydroxyapatite Using Rat Calvarial Defect Model. Composites Science and Technology 2019, 184, 107844.

82. Si, J.; Lin, J.; Su, C.; Yu, S.; Cui, Z.; Wang, Q.; Chen, W.; Turng, L.-S., Ultrasonication-Induced Modification of Hydroxyapatite Nanoparticles onto a 3d Porous Poly(Lactic Acid) Scaffold with Improved Mechanical Properties and Biocompatibility. Macromolecular Materials and Engineering 2019, 304 (7), 1900081.

83. Nandakumar, A.; Barradas, A.; de Boer, J.; Moroni, L.; van Blitterswijk, C.; Habibovic, P., Combining Technologies to Create Bioactive Hybrid Scaffolds for Bone Tissue Engineering. Biomatter 2013, 3 (2), e23705.

84. Faia-Torres, A. B.; Guimond-Lischer, S.; Rottmar, M.; Charnley, M.; Goren, T.; Maniura-Weber, K.; Spencer, N. D.; Reis, R. L.; Textor, M.; Neves, N. M., Differential Regulation of Osteogenic Differentiation of Stem Cells on Surface Roughness Gradients. Biomaterials 2014, 35 (33), 9023-9032. 


\section{SUPPLEMENTARY FIGURES}

A

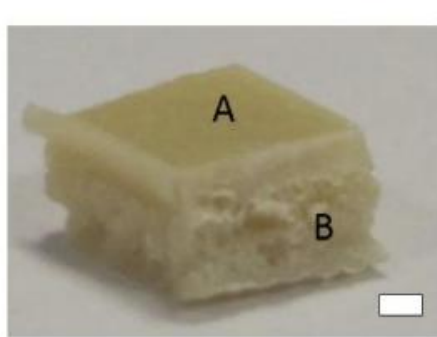

B

20-nHA (side B)
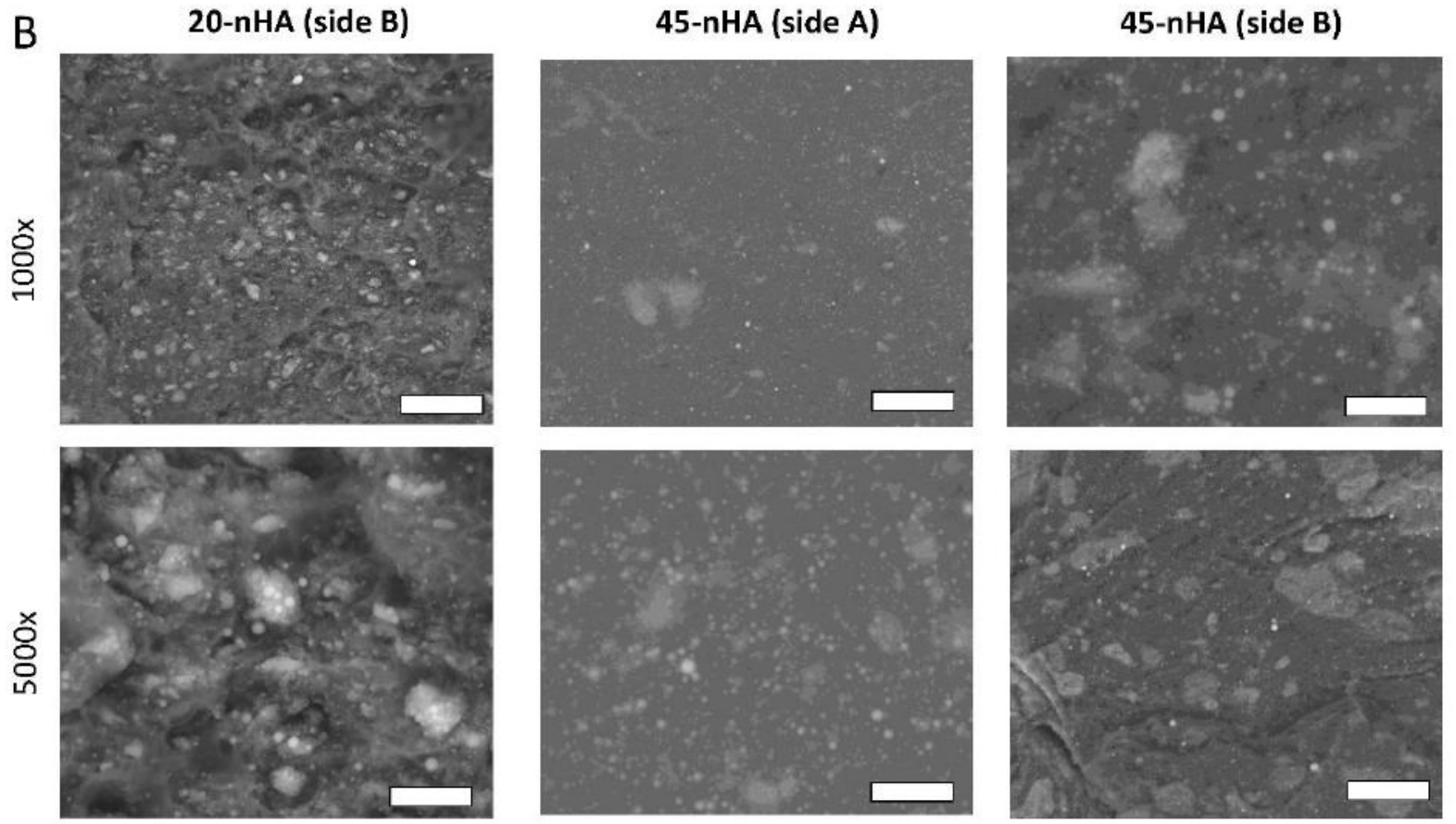

Figure S1. (A) Image of a 45-nHA pellet obtained after the solvent blending of $\mathrm{nHA}$ and PEOT/PBT process. The top side of the pellets is marked as side $A$ and the cross section of the pellet as side $B$. Scale bar: $1 \mathrm{~mm}$. (B) BSEM micrographs of the side $B$ of $20-n H A$ pellets, and the side $A$ and $B$ of 45-nHA pellets at two different magnifications (1000x and 5000x). Scale bars top row $50 \mu \mathrm{m}$, lower row $10 \mu \mathrm{m}$. 
A

0-nHA

20-nHA
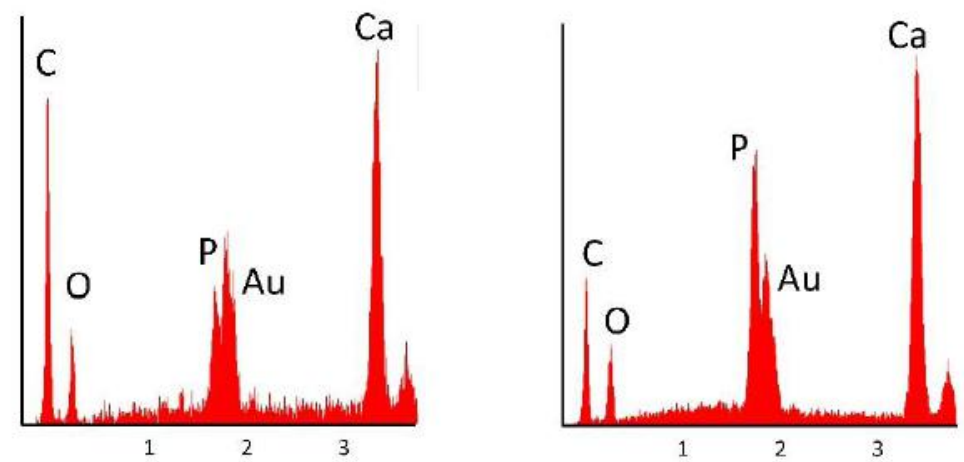

\begin{tabular}{|l|l|l|}
\hline Element & Wt\% & At \% \\
\hline $\mathrm{C}$ & 54.24 & 82,67 \\
\hline $\mathrm{O}$ & 12.44 & 14,24 \\
\hline $\mathrm{Au}$ & 33,32 & 3,1 \\
\hline
\end{tabular}

\begin{tabular}{|l|l|l|}
\hline Element & Wt \% & At \% \\
\hline $\mathrm{C}$ & 20.51 & 62.96 \\
\hline $\mathrm{O}$ & 6.59 & 15.2 \\
\hline $\mathrm{P}$ & 1.18 & 1.51 \\
\hline $\mathrm{Ca}$ & 9.57 & 8.8 \\
\hline $\mathrm{Au}$ & 62.16 & 11.64 \\
\hline
\end{tabular}

B

0-nHA

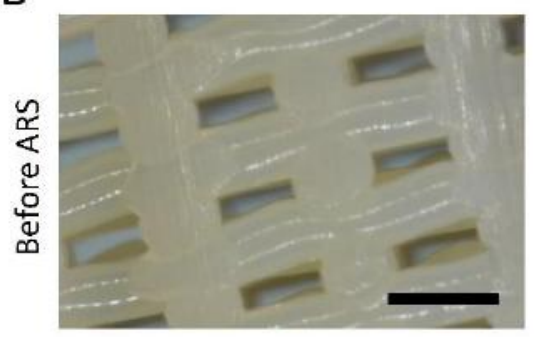

20-nHA

\begin{tabular}{|l|l|l|}
\hline Element & Wt \% & At \% \\
\hline $\mathrm{C}$ & 27.01 & 54.69 \\
\hline $\mathrm{O}$ & 15.66 & 23.8 \\
\hline $\mathrm{P}$ & 8 & 6.29 \\
\hline $\mathrm{Ca}$ & 18.87 & 11.45 \\
\hline $\mathrm{Au}$ & 30.46 & 3.76 \\
\hline
\end{tabular}

45-nHA
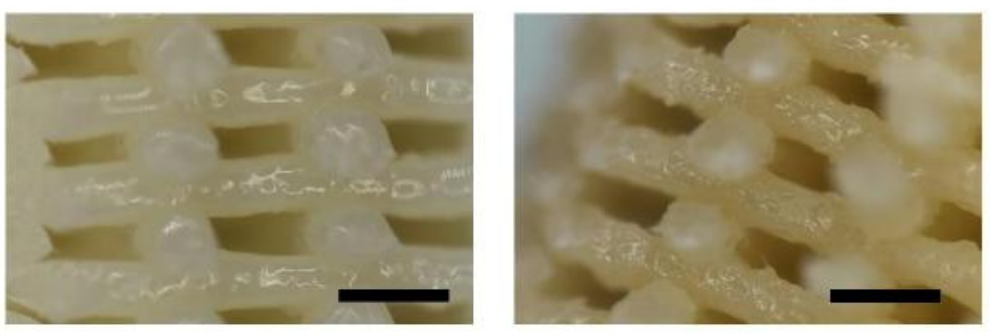

C
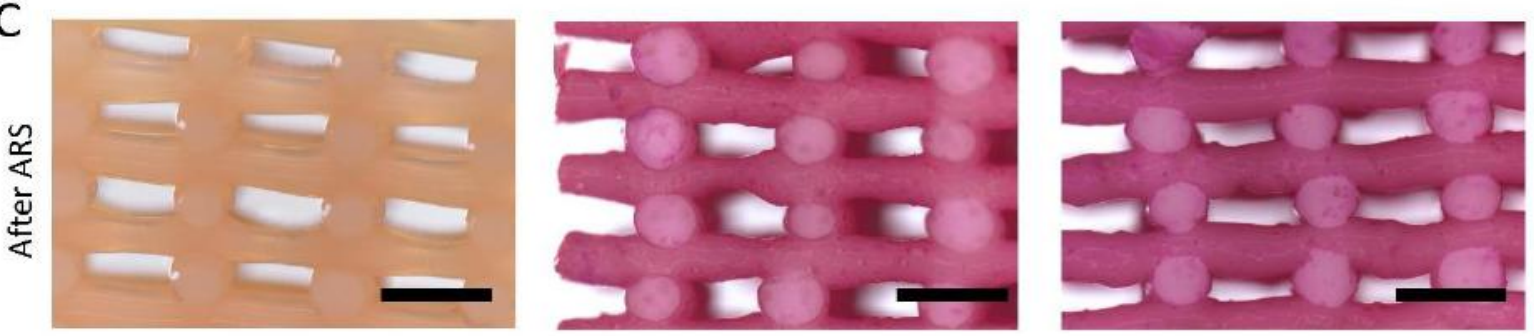

Figure S2. (A) Elemental analysis spectrum obtained with EDS and corresponding elements weight and atomic $\%$ of a $\sim 80 \times 80 \mu \mathrm{m}^{2}$ area from a 0 -nHA and 45-nHA surface filament and 45-nHA cross section. Stereomicroscopy images of a- prepared 0-nHA, 20-nHA and 45-nHA scaffolds (A) before ARS staining and $(B)$ after ARS staining. 
Effect of Highly Loaded Nanohydroxyapatite Composite Scaffolds Prepared via Melt Extrusion Additive Manufacturing on the Osteogenic Differentiation of Human Mesenchymal Stromal Cells

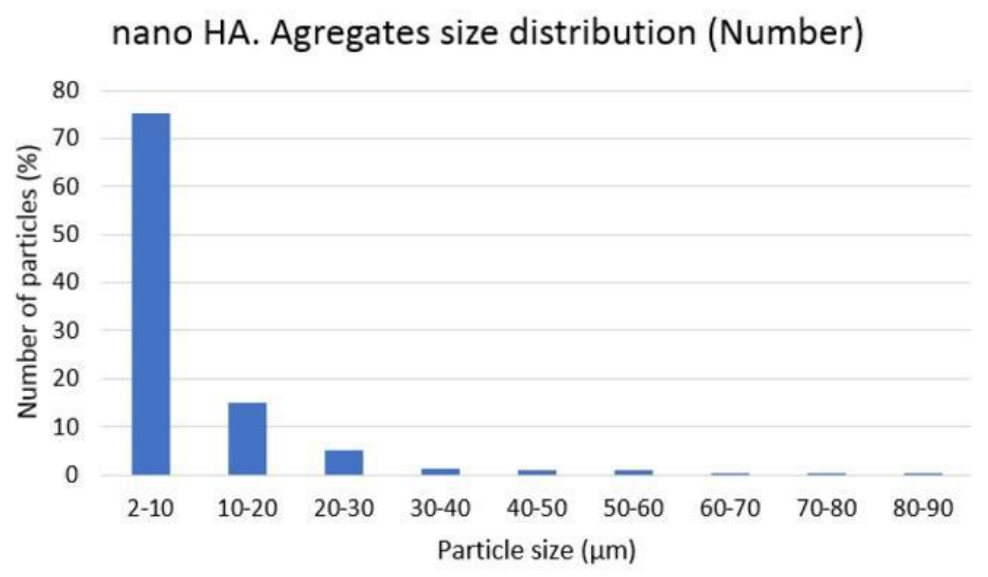

Figure S3. nHA aggregates size distribution on 45-nHA scaffolds.

A
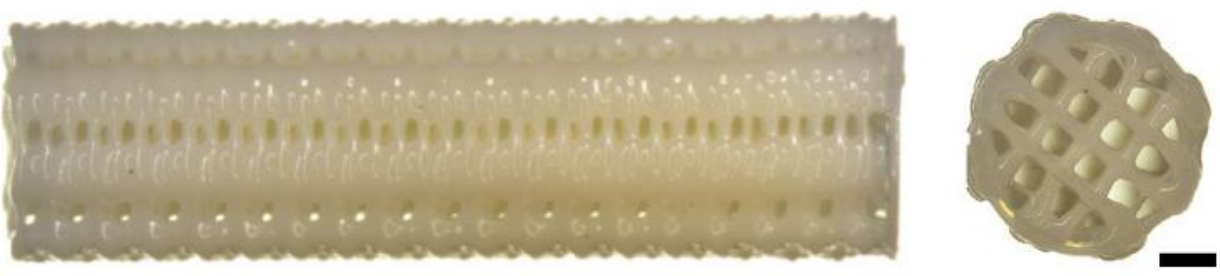

B
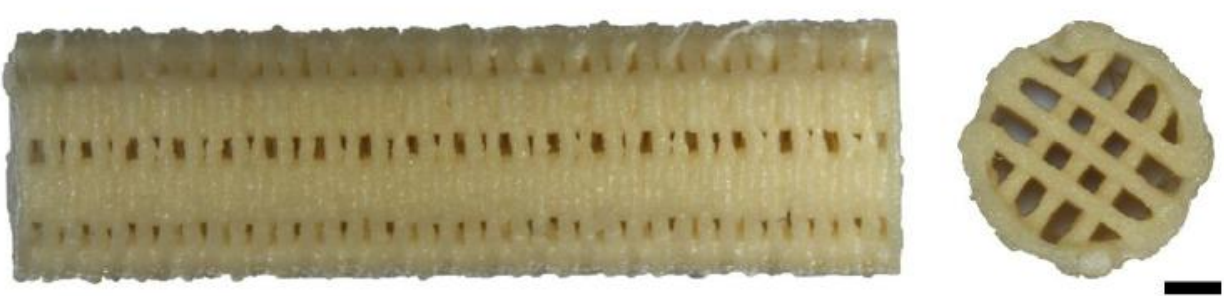

C

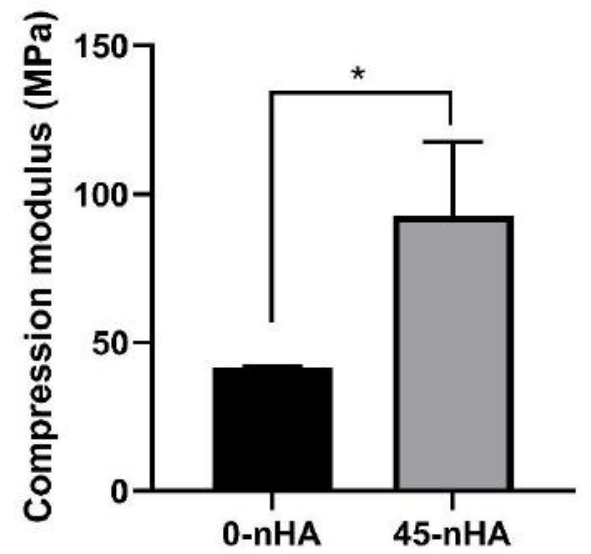

D

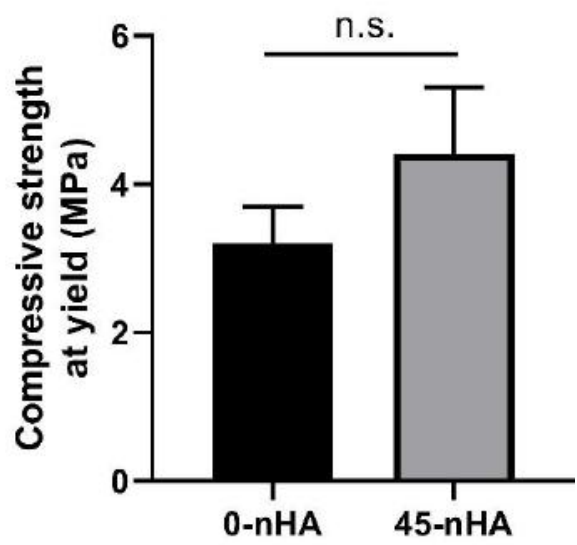

Figure S4. (A) Cylindrical 0 -nHA and (B) 45-nHA scaffolds with $15 \mathrm{~mm}$ height and $4 \mathrm{~mm}$ diameter. Scale bars $1 \mathrm{~mm}$. (C, D) Mechanical properties in compression of 0-nHA and 45-nHA scaffolds. 

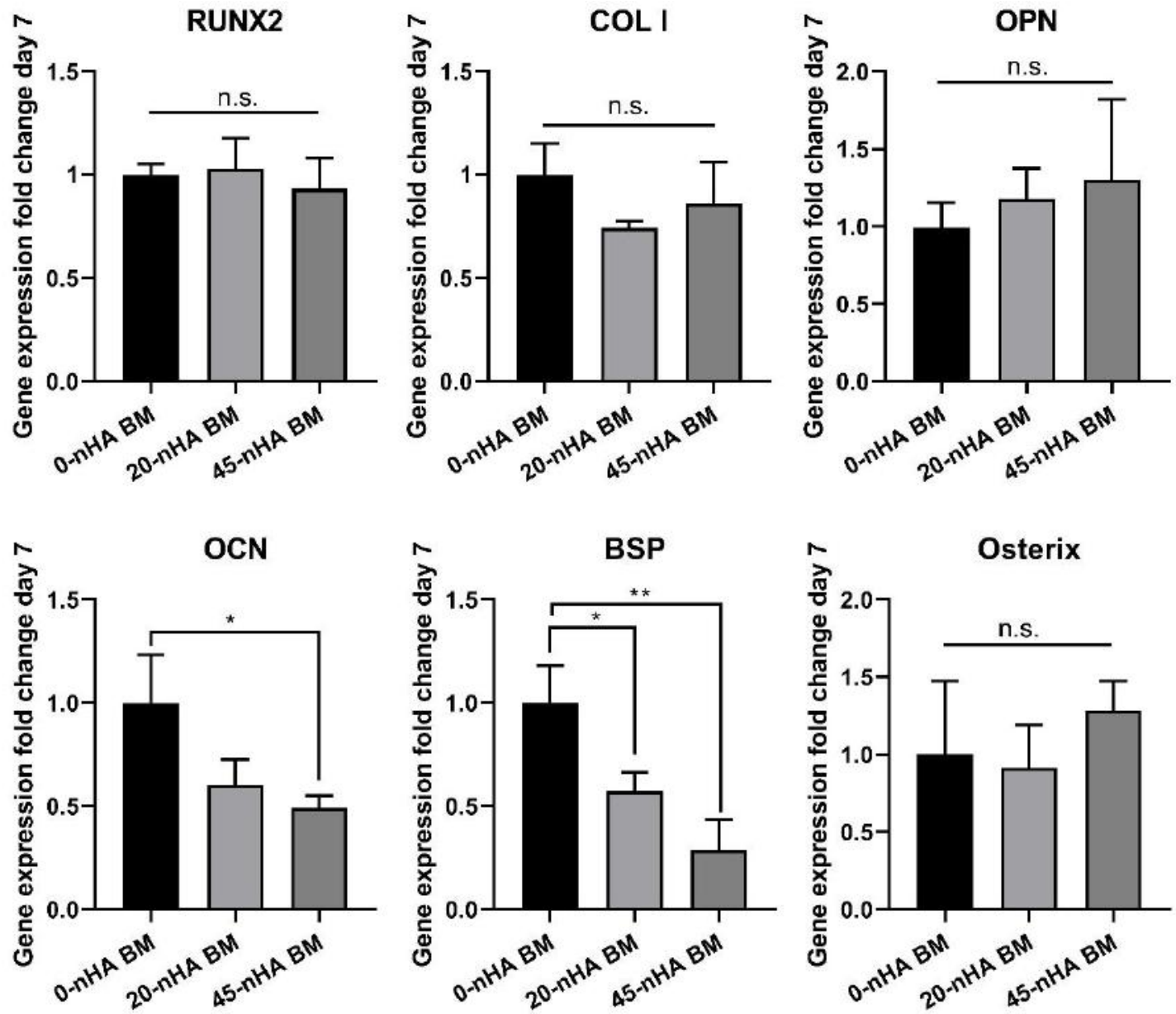

Figure S5. Gene expression fold change of hMSCs cultured on scaffolds with different $\mathrm{nHA}$ concentrations for 7 days in BM. Normalized to 0-nHA d7 BM. Data presented as average \pm s.d., and statistical significance performed using one-way ANOVA with Tukey's multiple comparison test (n.s. $p>0.05 ; * \$ p<0.05 ;{ }^{*} \$ \$ p<0.01$ ) 
A
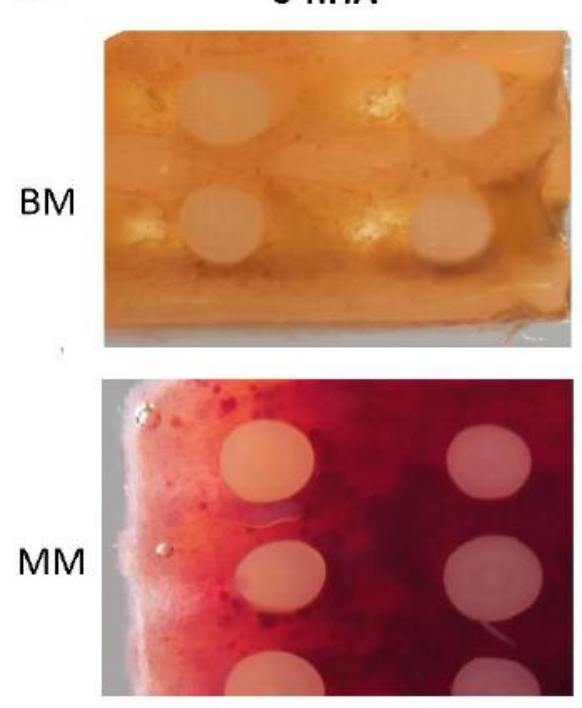

B
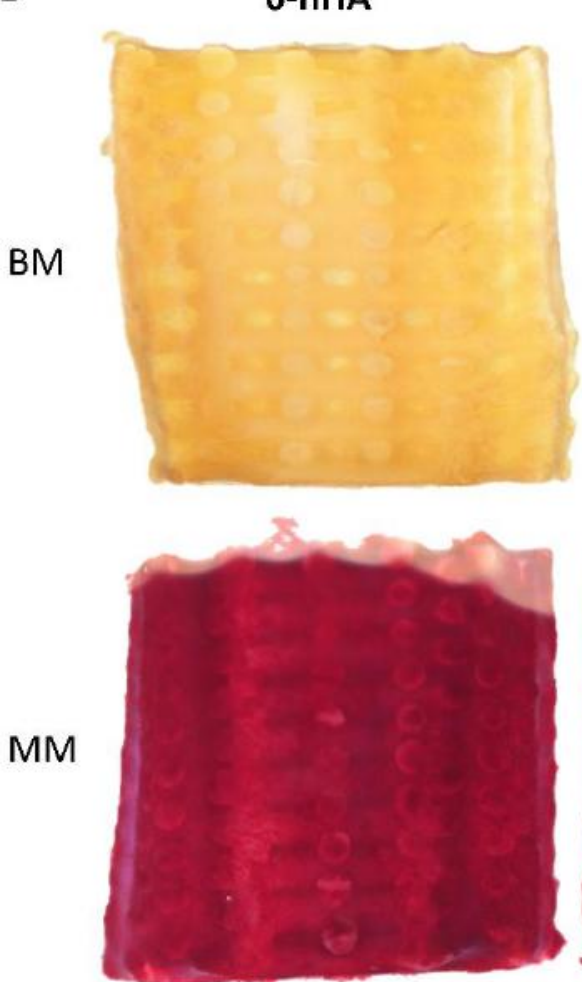

20-nHA
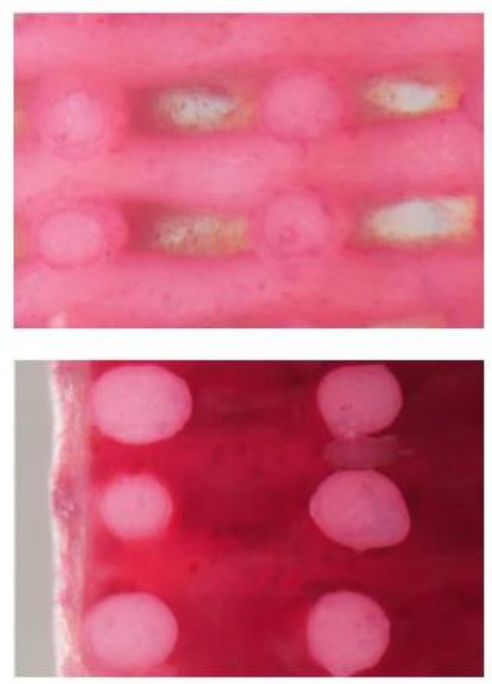

20-nHA
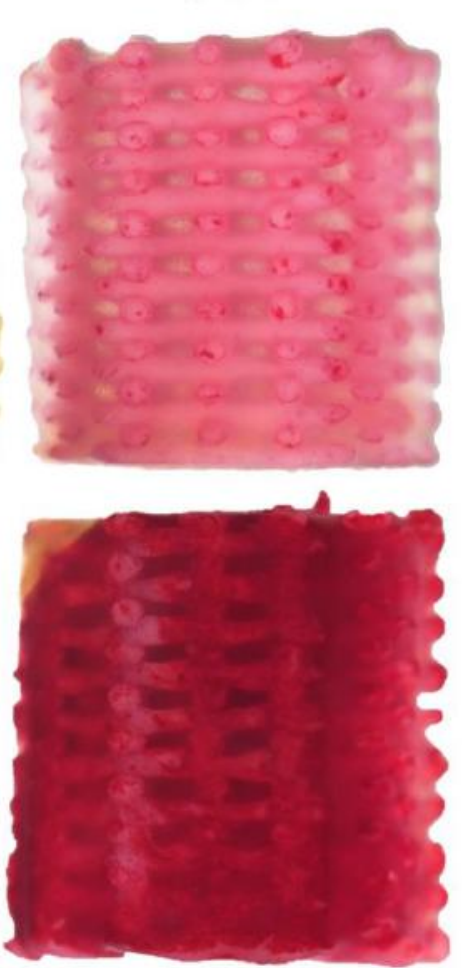

45-nHA
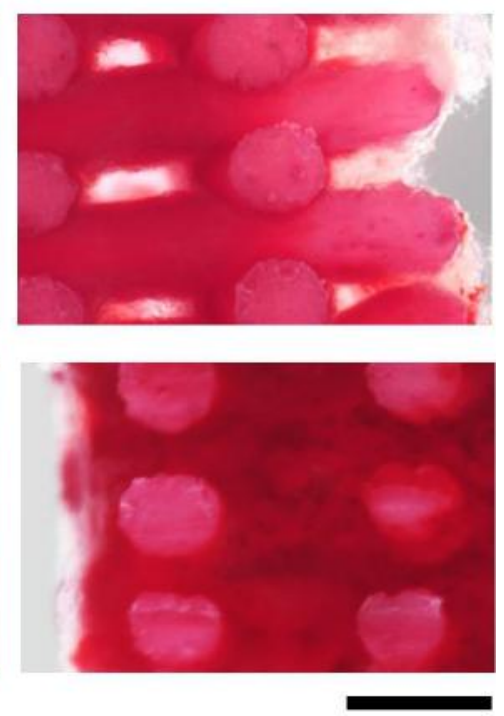

45-nHA
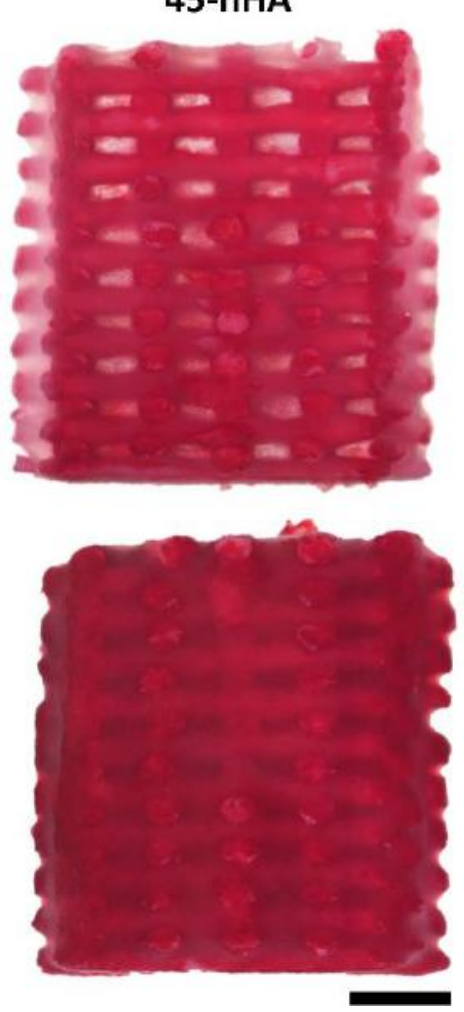

Figure S6. Stereomicroscopy images of scaffolds stained with ARS after 35 days of culture in BM or MM (7 days in BM followed by 28 days in MM). (A) High magnification images of scaffolds cross sections. Scale bar $500 \mu \mathrm{m}$. (B) Images of scaffolds outer surface of the scaffolds. Scale bar $1 \mathrm{~mm}$. 


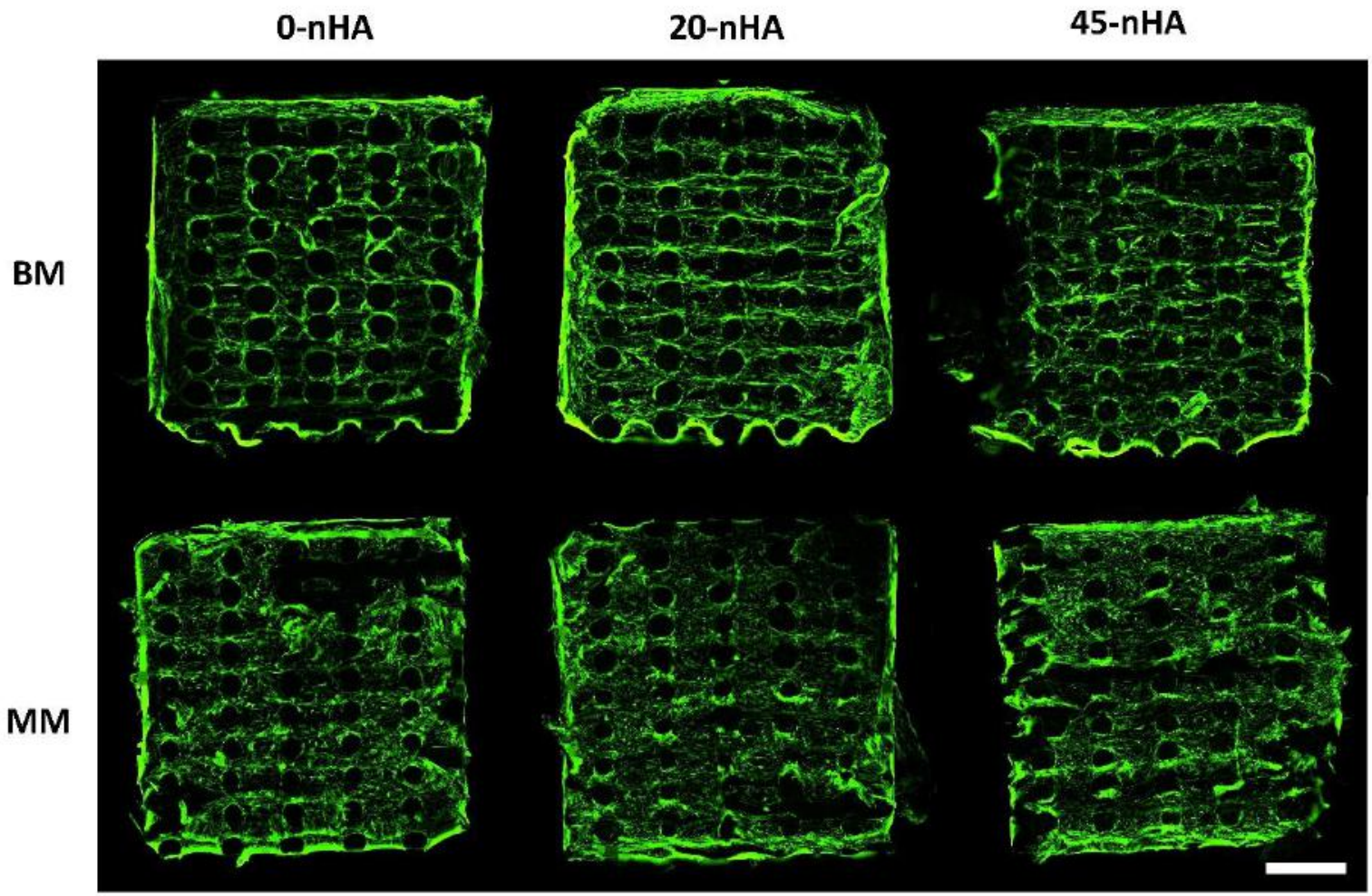

Figure S7. Fluorescent images of hMSCs (F-actin, green) on scaffolds cross sections after 35 days of culture in BM or MM. Scale bar $1 \mathrm{~mm}$.
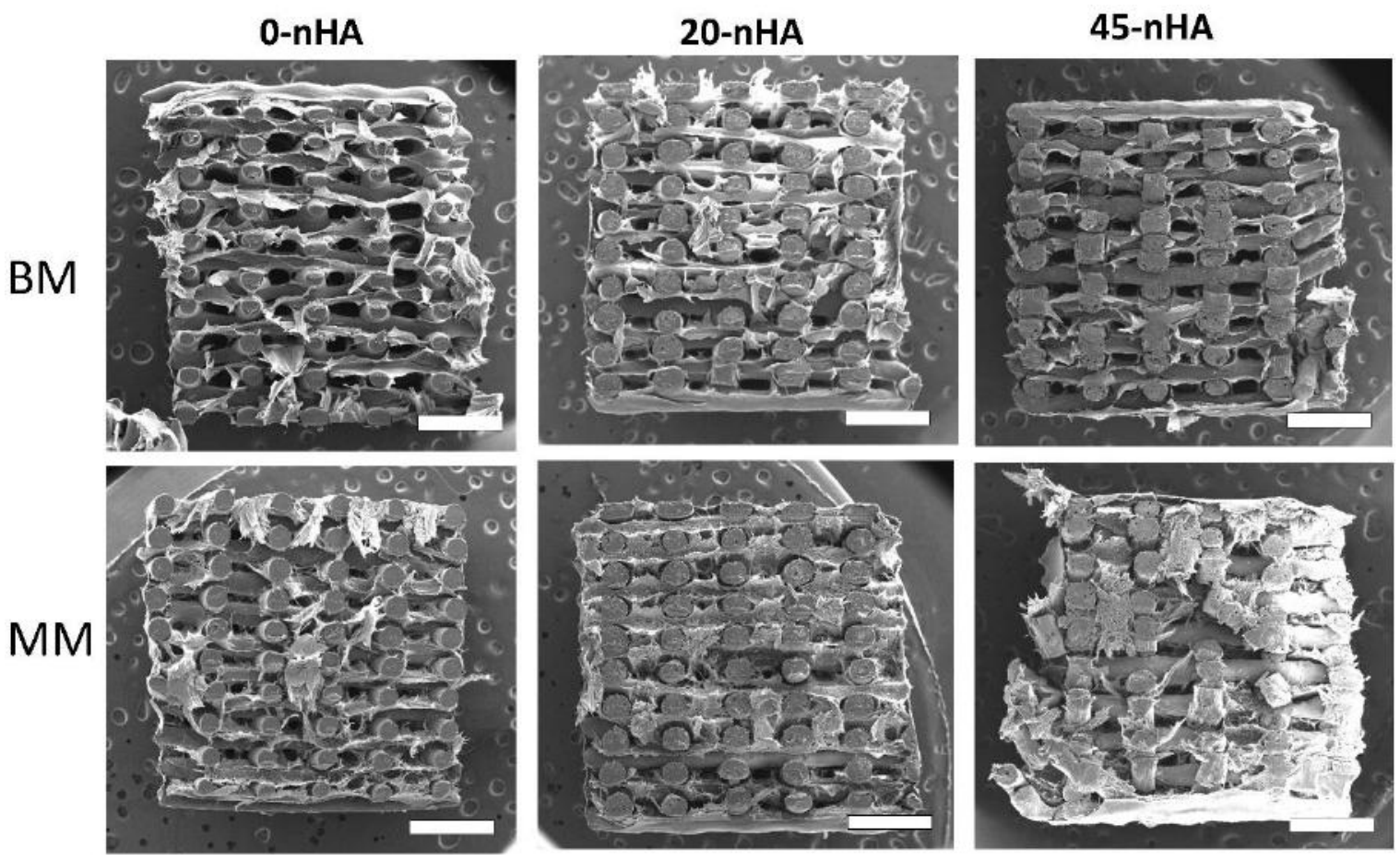

Figure S8. SEM micrographs of scaffolds cross sections after 35 days of culture in BM or MM. Scale bars $1 \mathrm{~mm}$. 
A
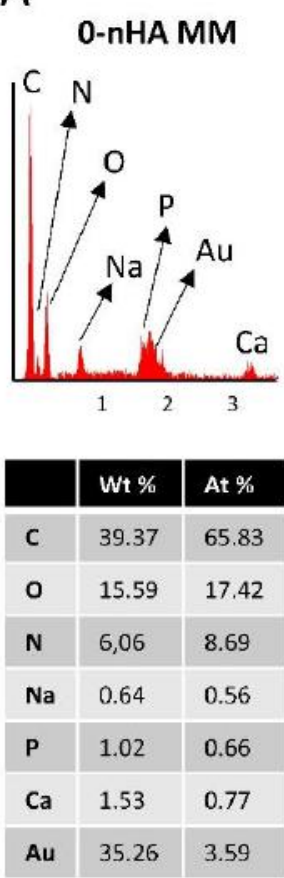

B

0-nHA BM

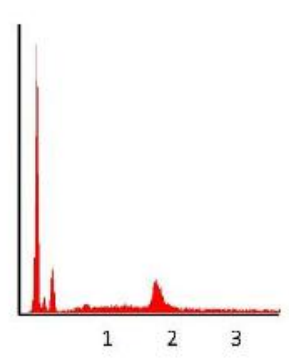

\begin{tabular}{|l|l|l|}
\hline & Wt \% & At \% \\
\hline $\mathbf{C}$ & 59.18 & 71.74 \\
\hline $\mathbf{O}$ & 17.82 & 16.22 \\
\hline $\mathbf{N}$ & 10.4 & 10.82 \\
\hline $\mathbf{N a}$ & 0.52 & 0.33 \\
\hline $\mathbf{A u}$ & 12.07 & 0.89 \\
\hline
\end{tabular}

\section{0-nHA MM}

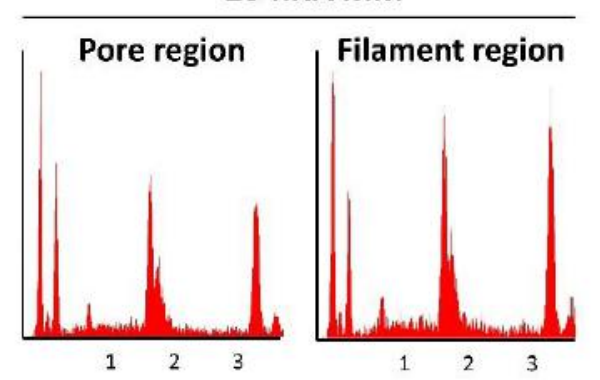

\begin{tabular}{|l|l|l|}
\hline & Wt \% & At \% \\
\hline C & 35.59 & 47.78 \\
\hline $\mathbf{O}$ & 29.65 & 29.79 \\
\hline $\mathbf{N}$ & 13.4 & 14.42 \\
\hline $\mathbf{N a}$ & 1.36 & 1.36 \\
\hline $\mathbf{P}$ & 4.68 & 4.68 \\
\hline $\mathbf{C a}$ & 6.67 & 6.67 \\
\hline Au & 33,32 & 3,1 \\
\hline
\end{tabular}

\begin{tabular}{|l|l|l|}
\hline & Wt \% & At \% \\
\hline C & 33.84 & 48.33 \\
\hline O & 26.03 & 27.92 \\
\hline $\mathbf{N}$ & 11.82 & 14.48 \\
\hline $\mathbf{N a}$ & 1.46 & 1.09 \\
\hline $\mathbf{P}$ & 5.4 & 2.99 \\
\hline $\mathrm{Ca}$ & 9.19 & 3.94 \\
\hline Au & 11.82 & 1.03 \\
\hline
\end{tabular}

20-nHA BM

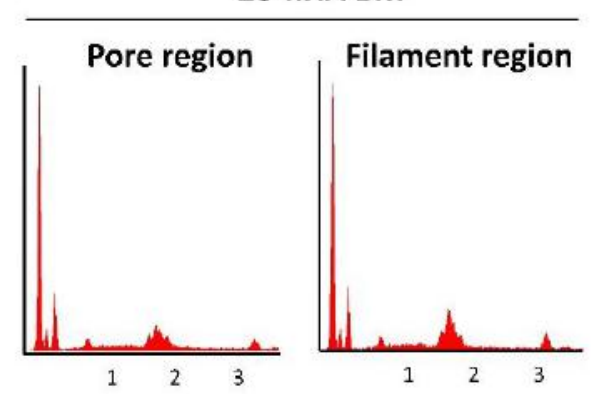

\begin{tabular}{|l|l|l|}
\hline & Wt \% & At \% \\
\hline $\mathbf{C}$ & 52.67 & 62.47 \\
\hline $\mathbf{O}$ & 20.96 & 18.66 \\
\hline $\mathbf{N}$ & 16.47 & 16.75 \\
\hline $\mathbf{N a}$ & 1.06 & 0.66 \\
\hline $\mathbf{P}$ & 0.71 & 0.33 \\
\hline $\mathbf{C a}$ & 1.07 & 0.38 \\
\hline Au & 6.43 & 0.46 \\
\hline
\end{tabular}

45-nHA MM

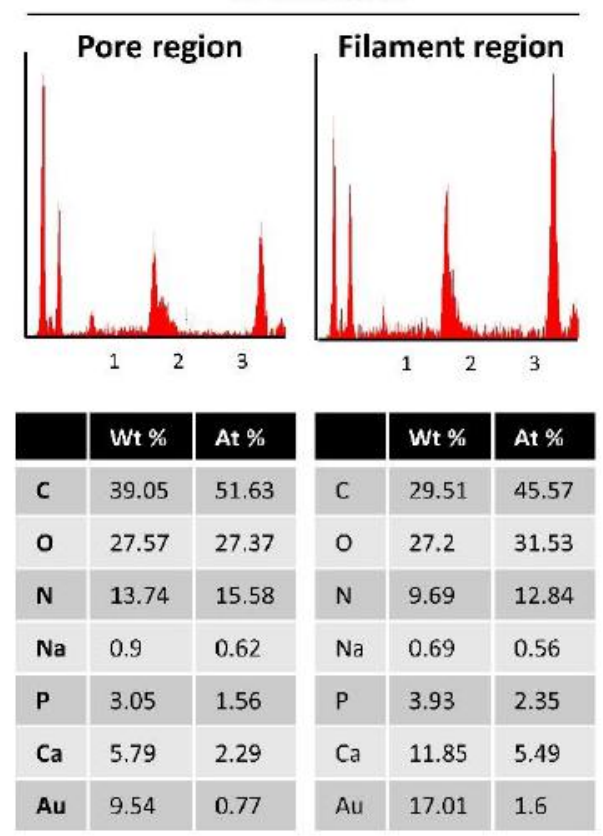

\section{5-nHA BM}

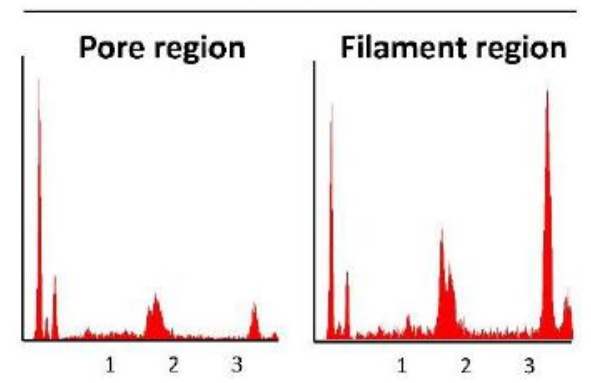

\begin{tabular}{|l|l|l|}
\hline & Wt \% & At \% \\
\hline $\mathbf{C}$ & 47.43 & 59.94 \\
\hline $\mathbf{O}$ & 20.09 & 19.91 \\
\hline $\mathbf{N}$ & 14.37 & 16.26 \\
\hline $\mathbf{N a}$ & 0.94 & 0.65 \\
\hline $\mathbf{P}$ & 1.4 & 0.72 \\
\hline $\mathbf{C a}$ & 2.93 & 1.16 \\
\hline Au & 14.42 & 1.16 \\
\hline
\end{tabular}

\begin{tabular}{|l|l|l|}
\hline & Wt \% & At \% \\
\hline $\mathbf{C}$ & 30.88 & 57.16 \\
\hline $\mathbf{O}$ & 12.26 & 17.18 \\
\hline $\mathbf{N}$ & 8.02 & 12.72 \\
\hline $\mathbf{N a}$ & 0.13 & 0.12 \\
\hline $\mathbf{P}$ & 2.65 & 1.9 \\
\hline $\mathbf{C a}$ & 12.47 & 6.92 \\
\hline $\mathbf{A u}$ & 33.11 & 3.74 \\
\hline
\end{tabular}

Figure S9. Elemental analysis spectrum obtained with EDS and corresponding elements weight and atomic \% of a $\sim 80 \times 80 \mu \mathrm{m} 2$ area of the ECM formed in 0-nHA and 45-nHA scaffolds after 35 days in culture in $\mathrm{BM}$ or $\mathrm{MM}$. 


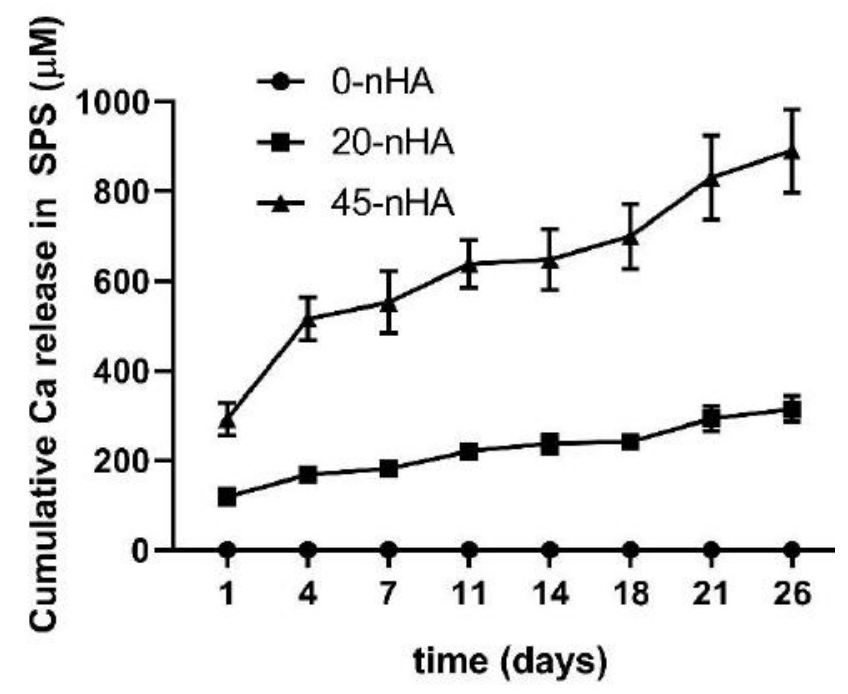

Figure S10. Ca ions release over time from nHA composite scaffolds upon immersion in SPS, measured by ICP-MS.

A

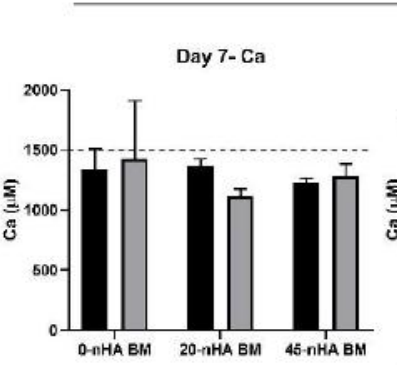

B

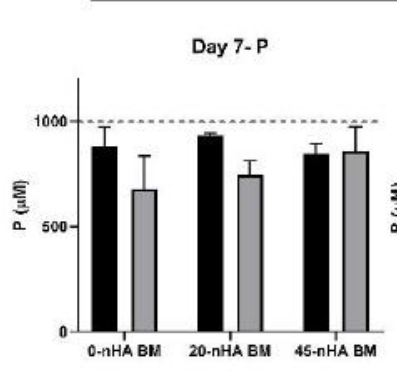

Ca in media

Day 14- Ca

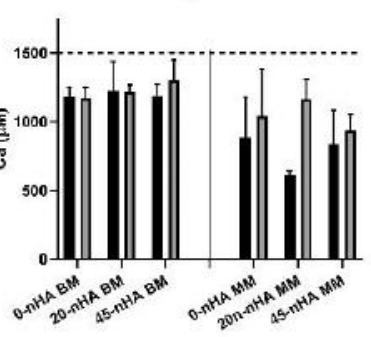

$P$ in media

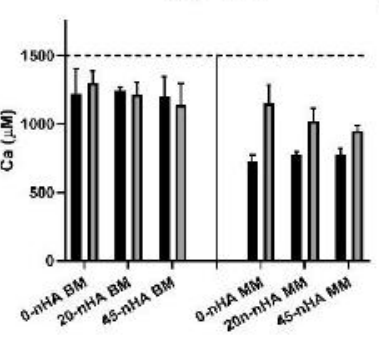

no cells Day 33- Ca

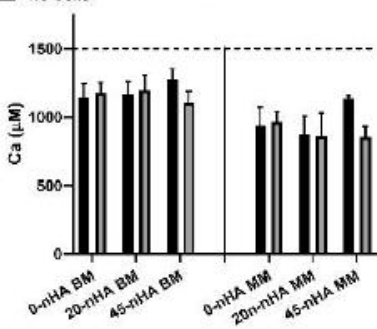

(a)
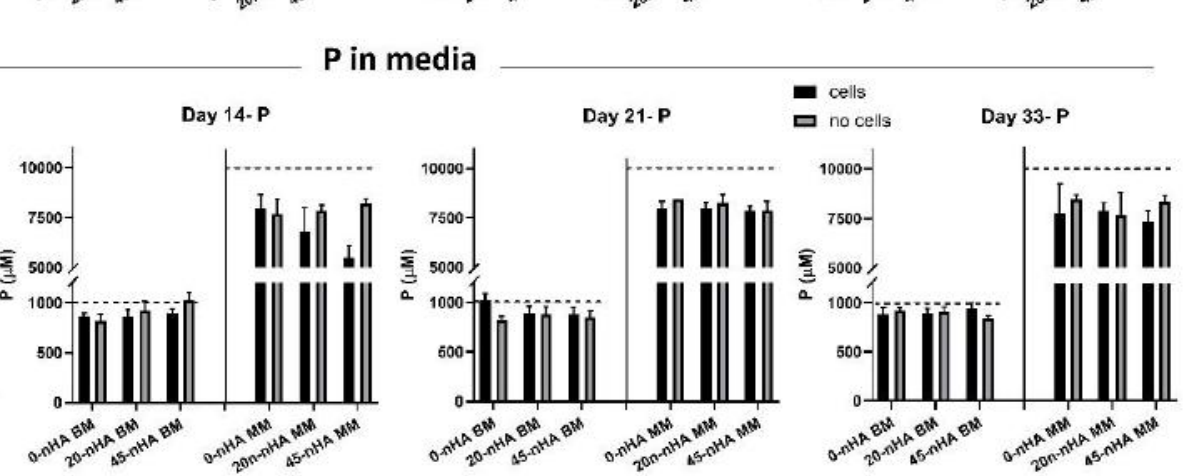

Figure S11. ICP-MS measurements of the calcium $(\mathrm{Ca})$ and phosphorous $(\mathrm{P})$ concentrations in cell culture medium after $7,14,21$, and 35 days of culture on 0 -nHA, 20-nHA and 45-nHA scaffolds with and without cells. The dash lines represent fresh medium concentrations. Data presented as average \pm s.d. 
Effect of Highly Loaded Nanohydroxyapatite Composite Scaffolds Prepared via Melt Extrusion Additive Manufacturing on the Osteogenic Differentiation of Human Mesenchymal Stromal Cells

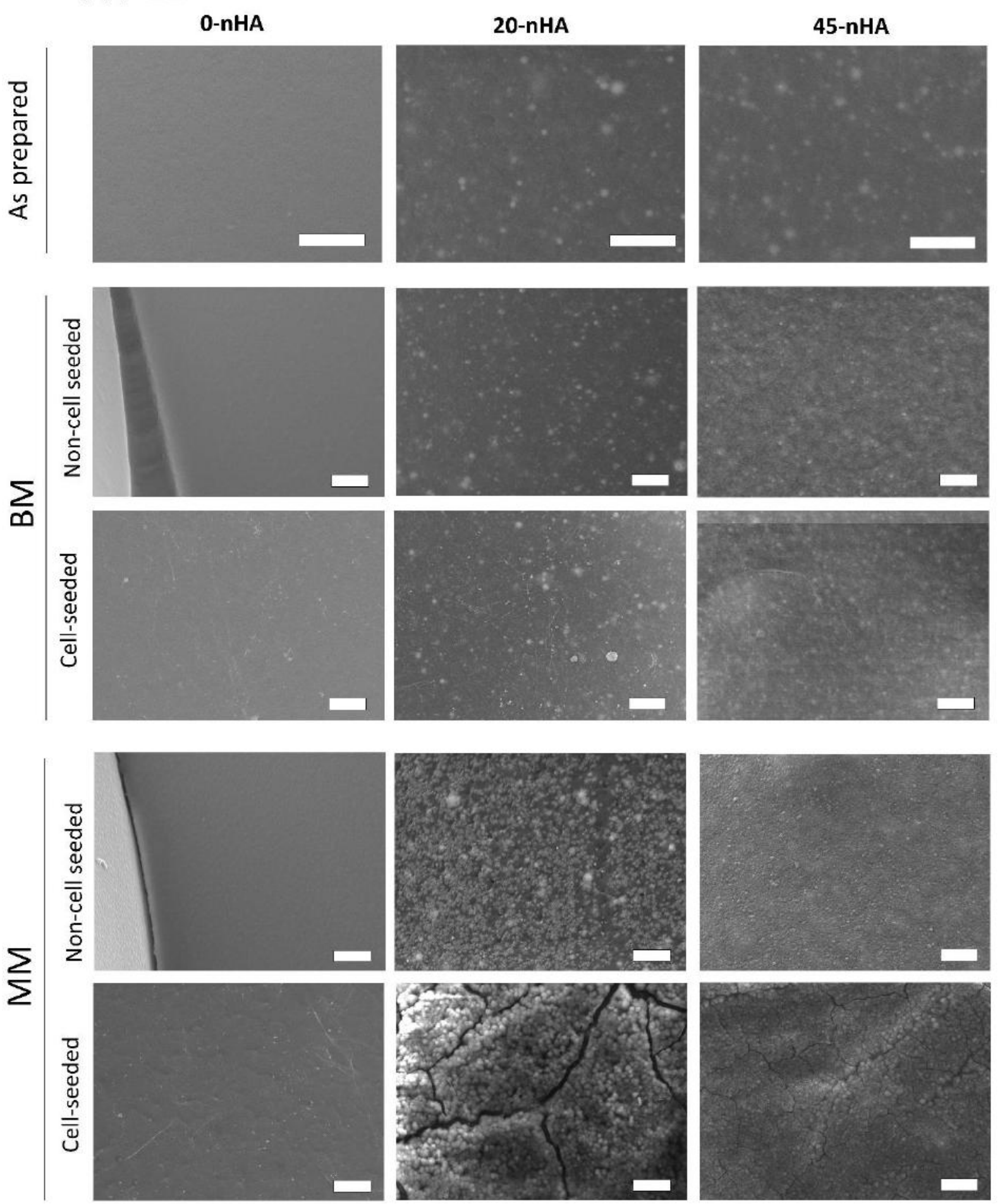

Figure S12. Representative SEM micrographs of 0-nHA, 20-nHA and 45-nHA non-cell seeded and cell seeded scaffolds incubated for 35 days in BM or MM. Scale bars $20 \mu \mathrm{m}$. 
0-nHA BM no cells

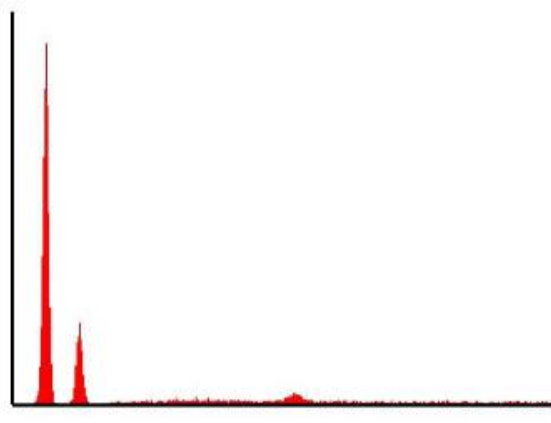

\section{Element}

\begin{tabular}{|l|l|l|}
\hline $\mathrm{C}$ & 66.3 & 76.7 \\
\hline $\mathrm{O}$ & 26.3 & 22.8 \\
\hline $\mathrm{Au}$ & 7.4 & 0.5 \\
\hline
\end{tabular}

0-nHA MM no cells

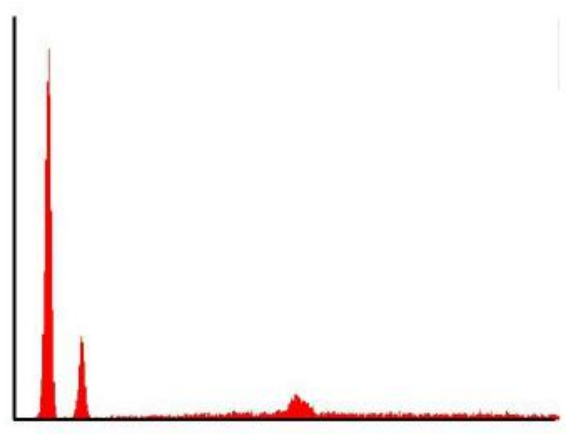

\section{Element \\ Weight \% \\ Atomic \%}

C

o

68.1

77

26.6

22.6

$\mathrm{Au}$

5.3

0.4

Figure S13. Elemental analysis spectrum obtained with EDS and corresponding elements weight and atomic \% of a $~ 80 \times 80 \mu \mathrm{m} 2$ area of the surface of filaments in 0-nHA non-cell seeded scaffolds after 35 days in culture in BM or MM. 


\section{$\underline{\text { Chapter } 6}$}

Effect of reduced graphene oxide ( $\mathrm{rGO}$ ) compaction degree and concentration on rGO-polymer composites printability and cell interactions 



\title{
Effect of reduced graphene oxide (rGO) compaction degree and concentration on rGO-polymer composites printability and cell interactions
}

\begin{abstract}
María Cámara-Torres ${ }^{1}$, Ravi Sinha ${ }^{1}$, Siamak Eqtesadi ${ }^{2}$, Rune Wendelbo ${ }^{2}$, Marco Scatto ${ }^{3}$, Paolo Scopece ${ }^{3}$, Alberto Sanchez ${ }^{4}$, Sara Villanueva ${ }^{4}$, Ainhoa Egizabal ${ }^{4}$, Noelia Álvarez ${ }^{4}$, Alessandro Patelli ${ }^{5}$, Carlos Mota ${ }^{1}$, Lorenzo Moroni ${ }^{1 *}$

${ }^{1}$ Maastricht University, MERLN Institute for Technology-Inspired Regenerative Medicine, Complex Tissue regeneration Department, Maastricht, The Netherlands. ${ }^{2}$ Abalonyx AS, Oslo, Norway. ${ }^{3}$ Nadir S.r.l., Venice, Italy. ${ }^{4}$ TECNALIA, Basque Research and Technology Alliance (BRTA), Donostia-San Sebastian, Spain. ${ }^{5}$ Department of Environmental Sciences, Informatics and Statistics, Ca' Foscari University of Venice, Venice, Italy. ${ }^{6}$ Department of Physics and Astronomy, Padova University, Padova, Italy.
\end{abstract}

\section{Abstract}

Graphene derivatives combined with polymers have attracted enormous attention for bone tissue engineering applications. Among others, reduced graphene oxide ( $\mathrm{rGO}$ ) is one of the preferred graphene-based fillers for the preparation of composites via melt compounding, and their further processing into 3D scaffolds, due to its established largescale production method, thermal stability, and electrical conductivity. In this study, rGO (low bulk density $10 \mathrm{~g} / \mathrm{L}$ ) was compacted by densification using a solvent (either acetone or water) prior to melt compounding, to simplify its handling and dosing into a twin-screw extrusion system. The effects of rGO bulk density (medium and high), densification solvent, and rGO concentration (3,10 and 15\% in weight) on rGO dispersion within the composite, electrical conductivity, printability and cell-material interactions were studied. High bulk density rGO (90 g/L) occupied a low volume fraction within polymer composites, offering poor electrical properties but a reproducible printability up to $15 \mathrm{wt} \% \mathrm{rGO}$. On the other hand, the volume fraction within the composites of medium bulk density rGO $(50 \mathrm{~g} / \mathrm{L})$ was higher for a given concentration, enhancing rGO particle interactions and leading to enhanced electrical conductivity, but compromising the printability window. For a given bulk density (50 g/L), rGO densified in water was more compacted and offered poorer dispersability within the polymer than rGO densified in acetone, and resulted in scaffolds 
with poor layer bonding or even lack of printability at high rGO percentages. A balance in printability and electrical properties was obtained for composites with medium bulk density rGO densified in acetone. Here, increasing rGO concentration led to more hydrophilic composites with a noticeable increase in protein adsorption. Moreover, scaffolds prepared with such composites presented antimicrobial properties even at low rGO contents (3 $w t \%)$. In addition, the viability and proliferation of human mesenchymal stromal cells (hMSCs) was maintained on scaffolds with up to 15\% rGO and with enhanced osteogenic differentiation on $3 \%$ rGO scaffolds. 


\section{INTRODUCTION}

Since the isolation of graphene, graphene based materials have been thoroughly exploited for various biomedical applications, and in particular for bone tissue engineering, due to their excellent mechanical, chemical and electrical properties, as well as to their unique ability of directing stem cell differentiation. ${ }^{1-3}$ Pristine graphene is a one atom thick, hexagonal lattice structure of sp2 hybridized carbon atoms, which confer all the aforementioned properties. ${ }^{4-6}$ Due to high synthesis costs and small scale production of graphene through bottom-up processes, such as chemical vapour deposition, ${ }^{7}$ or by direct exfoliation of graphite, such as micromechanical cleavage or direct sonication, ${ }^{8}$ currently the most promising method for large scale production of graphene-like materials relies on graphene oxide $(\mathrm{GO})$ as starting material. ${ }^{9} \mathrm{GO}$ can be easily mass-produced by oxidation of graphite through well-established methods, and used as-prepared or after exfoliation into graphene-like sheets. ${ }^{9}$ In addition, GO possesses oxygen containing functional groups disrupting each carbon plane, including epoxy, carbonyl, ketone and hydroxyl groups, which allows for good water and polymers dispersability and offers the possibility of functionalization with biomolecules to tune its bioactivity, unlike graphene sheets directly obtained from graphite. ${ }^{10}$ However, GO is electrically insulating and thermally unstable, requiring at least partial reduction to restore these properties when required for the final application. GO can be reduced by different chemical and thermal procedures, leading to exfoliated wrinkled reduced graphene oxide ( $\mathrm{rGO}$ ) sheets, similar to pristine graphene, but with some oxygen defects and holes on the carbon skeleton. ${ }^{11}$

In recent years, graphene, GO and rGO have been used as fillers in both natural and synthetic polymer nanocomposites to improve their physicochemical properties for bone tissue engineering applications. Among different types of such composites, including electrospun fibers, ${ }^{12-13}$ hydrogels, ${ }^{14-15}$ or additive manufactured (AM) scaffolds, ${ }^{16-19}$ the latter are superior when aiming towards load bearing applications, as they provide higher mechanical properties. Compared to other AM methods, melt extrusion AM (ME-AM) is considered a cost effective and established technique within the tissue engineering field, which enables the processing of a wide range of biocompatible and biodegradable thermoplastic materials, and provides full control on the internal pore architecture of the scaffolds. Accordingly, three main routes have been explored for the production of graphene derivatives-polymer composites to be used by ME-AM: solution blending, melt blending, and in situ polymerization. ${ }^{20}$ While solvent blending is a simple path to obtain a 
good filler dispersion, it generally requires the use of expensive and non-environmentally friendly organic solvents, which can potentially stay as residues within the composite matrix. ${ }^{21}$ In situ polymerization has shown to offer even a higher level of dispersion due to monomer intercalation between filler layers, followed by polymerization. However, this process can also require the use of solvents and is limited to specific polymer types. ${ }^{22}$ In spite of not providing the same level of filler dispersion as the aforementioned techniques, melt blending by twin screw compounding holds the most promise for large-scale composite fabrication, due to its lower cost, green production and industrial applicability. 23-24 However, some reports have described the formation of air pockets within extruded polymer composite filaments containing GO at high concentrations, due to its thermal instability and in situ thermal reduction at the extruding temperature. ${ }^{25} \mathrm{rGO}$ has also offered some challenges when used as a filler, despite its thermal stability. This is due to its very low bulk density and volatility after volume expansion upon thermal reduction, which impedes its free-flowability into melt compounders and can lead to nanoparticle intake by inhalation during handling. ${ }^{26}$ To overcome this, rGO compaction or densification by dispersion in a solvent and drying, ${ }^{24}$ pre-coating with polymer particles in solution, ${ }^{27}$ or the preparation of a highly concentrated masterbatch by solvent blending, prior to melt blending, have been considered. ${ }^{28}$ Yet, the attention from rGO as filler for ME-AM scaffolds for bone tissue engineering applications has been deviated and, to the best of our knowledge, the vast majority of previous studies have focused only on graphene and GO fillers for this application, despite pristine graphene's low yield production and GO's poor thermal stability and lack of electrical conductivity. ${ }^{17,29-31}$

Here, we prepared densified rGO and studied, for the first time, the effect of rGO densification on its dispersion within melt blended rGO- poly(ethylene oxide terephthalate)/poly(butylene terephthalate) (PEOT/PBT) composites, at various rGO concentrations. While most of previously reported graphene based ME-AM scaffolds contain only up to $3 \mathrm{wt} \%$ filler content, and very rarely up to $10 \mathrm{wt} \%$, in this study we investigated the feasibility of preparing composites with up to $15 \mathrm{wt} \% \mathrm{rGO}$, and evaluated their conductivity and printability as a function of rGO compaction and concentration. Moreover, the effect of rGO concentration on the material physicochemical properties, in terms of hydrophilicity, protein adsorption, and antimicrobial properties was assessed. To evaluate its application for bone tissue engineering, we further assessed human mesenchymal stromal cells (hMSCs) adhesion, proliferation and osteogenic differentiation on the prepared 3D ME-AM scaffolds. 


\section{MATERIALS AND METHODS}

\section{rGO synthesis and characterization}

GO was synthesized by Abalonix AS according to a proprietary in-house GO manufacturing method, and thermally reduced to obtain partially reduced rGO. During the reduction process, GO was introduced for few seconds into a tubular oven at $600{ }^{\circ} \mathrm{C}$. However, due to cold air flowing through the oven chamber, the real temperature that the material experiences was estimated to be $\sim 300{ }^{\circ} \mathrm{C}$. Three different $\mathrm{rGO}$ batches were produced following the same protocol. Densified / compacted (the two terms will be used interchangeably) forms of these rGOs (hereafter referred to as d-rGO) were produced by dispersion into a solvent and subsequent drying. rGO was dispersed in acetone at 50 $\mathrm{mg} / \mathrm{ml}$ and dried at room temperature to obtain d-rGO-B, with a bulk density of $\sim 50 \mathrm{~g} / \mathrm{L}$. To obtain d-rGO-A, rGO was dispersed in water at $50 \mathrm{mg} / \mathrm{ml}$ and dried at $100{ }^{\circ} \mathrm{C}$. The water dispersion and drying process was carried out two times to obtain d-rGO-C with a bulk density of $\sim 90 \mathrm{~g} / \mathrm{L}$. Overall, three different d-rGO materials were obtained: $d$-rGO-A (densified in water, bulk density $50 \mathrm{~g} / \mathrm{L}$ ), d-rGO-B (densified in acetone, bulk density 50 $\mathrm{g} / \mathrm{L}$ ) and d-rGO-C (densified in water, bulk density $90 \mathrm{~g} / \mathrm{L})$.

In order to evaluate the atomic composition of each d-rGO material, X-ray photoelectron spectroscopy (XPS, K-Alpha - Thermo Scientific, US) was used. Moreover, X-ray diffraction (XRD, D8 Advanced, Brukers, Germany) was employed to investigate the different rGO batches crystallinity and their layered structure. In addition, SEM was carried out to characterize the microstructure of the different $d$-rGO materials.

\section{Composite production and characterization}

d-rGO-polymer composites were obtained by compounding individually the various d-rGO materials (d-rGO-A, d-rGO-B and d-rGO-C) with PEOT/PBT (300PEOT55PBT45, pellets form, PEO Mw 300 kDa, PEOT:PBT weight ratio= 55:45, intrinsic viscosity $0.51 \mathrm{dl} / \mathrm{g}$, Polyvation, The Netherlands). Production was carried out in a lab scale co-rotating twinscrew extruder installed in Nadir S.r.l., consisting of a screw profile with 8 zones, 3 interposed kneading sections, and a screw dimensions of $11 \mathrm{~mm}$ diameter and 40 lengthto-diameter ratio. PEOT/PBT pellets and d-rGO powder were fed at different concentrations $(3,10,15 \mathrm{wt} \%)$ into the main hopper with a volumetric feeder. The screw rotation speed was fixed at $80 \mathrm{rpm}$, while the barrel temperature was set at $140^{\circ} \mathrm{C}$ for the 
first zone and $145-150^{\circ} \mathrm{C}$ for the following zones. Resulting composite wires were taken at the die exit, solidified in air and pelletized in a pelletizer machine. These will be referred to as " $X \%$ d-rGO-Y" pellets or composites, where " $X$ " refers to the rGO concentration in wt $\%$ $(3,10,15)$ and "Y" refers to the d-rGO batch $(A, B$, or $C)$.

TGA measurements of the d-rGO batches and composites were carried out in a vertical thermo-balance, TGA Discovery 55 analyser (Water-TA Instruments). About $11 \mathrm{mg}$ samples were conducted under nitrogen atmosphere $\left(99,999 \% \mathrm{~N}_{2}\right)$ with a flow rate of 90 $\mathrm{ml} / \mathrm{min}$ and using a temperature heating rate of $10^{\circ} \mathrm{C} / \mathrm{min}$ up to $900{ }^{\circ} \mathrm{C}$. Residual weight percentages at $550{ }^{\circ} \mathrm{C}$ extracted from the TGA curves were used to calculate the experimental $\mathrm{rGO}$ loading of each composite. The $550^{\circ} \mathrm{C}$ temperature was selected from the separate rGO and polymer only TGA's, as the temperature where most of the polymer was burnt off and most of the rGO was still left.

To analyze potential aggregation of d-rGO after the compounding process, pellets of each material were dissolved in chloroform. After mechanical stirring, a sample of each solution was dispensed on a scanning electron microscopy (SEM) sample holder, dried at room temperature, and imaged using SEM (XL-30) operating at a beam voltage of $15 \mathrm{kV}$ and a spot size of 3 . Particle size distribution was assessed using the image analysis software ImageJ.

\section{Scaffolds fabrication and characterization}

Scaffolds were fabricated via ME-AM. The platform consisted of a custom-made printhead, with separate heating sources for the cartridge (polymer reservoir) and extrusion screw, mounted on a three- axis stage (Bioscaffolder, Gesim). ${ }^{32}$ Briefly, the cartridge was filled with the pellets of the composite material to be printed, and scaffolds with a 0-90 architecture, $250 \mu \mathrm{m}$ fiber diameter, $200 \mu \mathrm{m}$ layer thickness and $750 \mu \mathrm{m}$ strand distance (center to center) were fabricated according to parameters in Table 1. Cylindrical scaffolds of $4 \mathrm{~mm}$ diameter and $4 \mathrm{~mm}$ height were punched out from $15 \times 15 \times 4 \mathrm{~mm}^{3}$ manufactured blocks using a biopsy punch and used for further experiments.

Scaffolds morphology and porosity were assessed using a stereomicroscope (Nikon SMZ25). Scaffolds surface roughness was examined using SEM, operating at $10 \mathrm{kV}$, and a spot size of 3 . The electrical conductivity of extruded filaments (length $6 \mathrm{~cm}$, diameter $340 \mu \mathrm{m}$ and $800 \mu \mathrm{m}$ ) of each material was measured using a digital multimeter (maximum measurable resistance: $2000 \mathrm{M} \Omega$ ). To do this, filaments with a known diameter and length 
were connected to the multimeter electrodes with conductive silver paste (Chemtronics Silver Conductive Adhesive Epoxy) to ensure good contact. The volume electrical conductivity was calculated by Equation 1.

Cell studies and antimicrobial analysis was performed only on the d-rGO-B scaffolds due to their superior combined properties for the final application, in terms of processability and electrical conductivity, compared to the d-rGO-A and d-rGO-C composites.

Table 1. Fabrication parameters of d-rGO-polymer composite scaffolds

\begin{tabular}{lccccc}
\hline Scaffold & $\begin{array}{c}\text { T reservoir } \\
\left({ }^{\circ} \mathbf{C}\right)\end{array}$ & $\begin{array}{c}\text { T screw } \\
\left({ }^{\circ} \mathbf{C}\right)\end{array}$ & $\begin{array}{c}\text { Pressure } \\
\text { cartridge } \\
(\text { bar })\end{array}$ & $\begin{array}{c}\text { Screw } \\
\text { rotation } \\
\text { speed }(\mathbf{r p m})\end{array}$ & $\begin{array}{c}\text { Translation } \\
\text { speed }(\mathbf{m m} / \mathbf{s})\end{array}$ \\
\hline 3\% d-rGO-A & 195 & 200 & 8 & 60 & 15 \\
$\mathbf{1 0 \%}$ d-rGO-A & 220 & 220 & 8 & 60 & $15-20$ \\
15\% d-rGO-A & not extrudable & & & & \\
3\% d-rGO-B & 195 & 200 & 8 & 60 & 15 \\
10\% d-rGO-B & 200 & 210 & 8 & 40 & $15-20$ \\
15\% d-rGO-B & 200 & 210 & 8 & 60 & $15-20$ \\
3\% d-rGO-C & 200 & 205 & 8 & 60 & 15 \\
10\% d-rGO-C & 200 & 205 & 8 & 60 & 15 \\
$\mathbf{1 5 \%}$ d-rGO-C & 200 & 210 & 8 & 60 & $15-20$ \\
\hline
\end{tabular}

Equation 1. Volume electrical conductivity $(\sigma)$ formula, where $I$ is the filament length $(6 \mathrm{~cm}), R$ is the electrical resistance and $A$ is the filament cross sectional area (diameter $340 \mu \mathrm{m}$ and $850 \mu \mathrm{m}$ ).

$$
\sigma=\frac{l}{R \cdot A}
$$

\section{Films preparation and characterization}

2D films were prepared from around 60 milligrams of $(3,10,15) \%$ rGO-B pellets and PEOT/PBT pellets, which were molten at $190-210^{\circ} \mathrm{C}$ and pressed with a coverslip against a Teflon sheet to obtain $14 \mathrm{~mm}$ diameter, $~ 300 \mu \mathrm{m}$ thickness films. Static contact angle was measured on these films using the sessile drop method. To do this, a $4 \mu$ water droplet was placed on top of the films by an automatic syringe dispenser (Krüss DSA25S). 20 seconds after droplet formation, the contact angle was calculated automatically by the device's software using the Laplace-Young curve fitting. 
Protein adsorption to the films was analysed by incubation in a bovine serum albuminFITC protein solution (1mg/ml, Sigma Aldrich) overnight at $37^{\circ} \mathrm{C}$. After washing in PBS, films were blotted in an adsorbent paper and incubated for $2 \mathrm{~h}$ at $\mathrm{RT}$ in a $1 \%$ SDS solution. Supernatants fluorescence was measured at excitation/emission $=495 \mathrm{~nm} / 519 \mathrm{~nm}$. Cell studies and antimicrobial analysis was performed only on the d-rGO-B films, due to their superior combined properties for the final application, in terms of processability and electrical conductivity, compared to the d-rGO-A and d-rGO-C composites.

\section{Antibacterial activity}

The antibacterial activity of $(3,10,15) \%$ d-rGO-B scaffolds and films against $P$. aeruginosa (CECT 116) and S. epidermidis (CECT 231) was evaluated by means of the flask shake test method. Briefly, scaffolds were immersed in concentrated bacterial suspensions $\left(10^{6}\right.$ $\left.\mathrm{CFU} \cdot \mathrm{L}^{-1}\right)$ in nutrient broth $(1: 500)$ for $24 \mathrm{~h}$ at $37^{\circ} \mathrm{C}$. After incubation, the number of viable bacteria present in the suspensions was measured by placing aliquots of the suspensions in sterile petri dishes with molten nutrient agar per triplicate and swirled gently. The petri dishes were incubated at $37^{\circ} \mathrm{C}$ for $24 \mathrm{~h}$, after which the colonies present on the plates were counted. Values are reported as the log base 10 reduction $(R)$, calculated as the difference in the log base 10 of the viable cell counts found on a suspension that has not been in contact with the scaffolds and the counts on a suspension that has been in contact with the scaffolds.

In addition, the antibacterial activity of $(3,10,15) \%$ d-rGO-B scaffolds was evaluated by means of the Agar disk-diffusion method. For that, scaffolds were incubated for 3 days in $1 \mathrm{ml}$ PBS. Mueller- Hinton agar plates were spread with a standardized inoculum of $P$. aeruginosa and $S$. epidermidis. At each timepoint (24h, 48h, $72 \mathrm{~h}$ ), $20 \mu \mathrm{l}$ of each of the scaffolds' supernatant was collected and used to impregnate commercial paper discs, which were subsequently placed on the agar surface. The agar plates were incubated under $37^{\circ} \mathrm{C}$ during 18-24 hours. After incubation, zones of growth inhibition (ZOI) around each of the discs (including disc diameter) were measured to the nearest millimeter. For reporting, the disc diameter was subtracted.

\section{Cell seeding and culture}

HMSCs isolated from bone marrow were purchased from Texas A\&M Health Science Center, College of Medicine, Institute for Regenerative Medicine (Donor d8011L, female, 
age 22). Cryopreserved vials at passage 3 or 4 were plated at a density of $1000 \mathrm{cell} s \times \mathrm{cm}^{-}$ 2 in tissue culture flasks and expanded until approximately $80 \%$ confluency in complete media (CM). CM was composed of aMEM with Glutamax and no nucleosides (Gibco) supplemented with $10 \%$ fetal bovine serum (FBS), without penicillin-streptomycin (PenStrep) at $37^{\circ} \mathrm{C}$ and $5 \% \mathrm{CO}_{2}$.

\section{Cell seeding on 2D films}

PEOT/PBT and $(3,10,15) \%$ d-rGO-B films were disinfected in 70\% ethanol for 20 min, washed 3 times with PBS, and fixed on the bottom of well-plates with the help of biocompatible O-rings (Eriks, 10023241). Prior to cell seeding, films were incubated in CM for $2 \mathrm{~h}$ at $37{ }^{\circ} \mathrm{C}$ and $5 \% \mathrm{CO}_{2}$ for protein adhesion. Trypsinized hMSCs were re-suspended in fresh media and seeded at 2,500 cells per film. HMSCs were cultured on films for 7 days, and media was replaced every 2 or 3 days.

\section{Cell seeding on scaffolds}

For cell attachment experiments, PEOT/PBT and (3, 10, 15)\% d-rGO-B scaffolds were disinfected in $70 \%$ ethanol and incubated in $\mathrm{CM}$ for $2 \mathrm{~h}$ at $37{ }^{\circ} \mathrm{C}$ and $5 \% \mathrm{CO}_{2}$ to allow protein attachment. Subsequently, scaffolds were blotted on top of a sterile filter paper and placed in the wells of a non-treated wellplate. Trypsinized hMSCs were resuspended in a dextran solution (500 kDa, Farmacosmos) (10 wt\% dextran in CM), to achieve uniform cell distribution, ${ }^{33}$ and were seeded at a density of $2 \times 10^{5}$ cells with $37 \mu \mathrm{l}$ of CM per scaffold. After $4 \mathrm{~h}$ incubation for cell attachment, scaffolds were transferred to new wells containing $1.5 \mathrm{ml}$ of basic media (BM) (CM supplemented with $200 \mu \mathrm{M} \mathrm{L-Ascorbic} \mathrm{acid} \mathrm{2-phosphate).}$ BM was replaced after $24 \mathrm{~h}$ and every 2 or 3 days from then on. Scaffolds were cultured for 7 days. For osteogenic differentiation experiments, PEOT/PBT and 3\% d-rGO-B scaffolds were seeded as mentioned before, and cultured for 7 days in BM, after which scaffolds were further cultured either in BM or in mineralization media (MM), consisting of BM supplemented with dexamethasone (10 nM) (Sigma-Aldrich) and $\beta$-glycerophosphate (10 mm) (Sigma-Aldrich) for another 28 days.

\section{Cells imaging on films and scaffolds}

Films and scaffolds seeded and cultured with cells were fixed in $4 \%$ paraformaldehyde and incubated for $30 \mathrm{~min}$ in Triton-X 100 (0.1 v\%). Then, cell cytoskeleton and nuclei were 
stained with 488 Alexa Fluor Phalloidin (Thermo Fisher Scientific, 1:75 dilution in PBS, 1h at RT) and DAPI $(0.1 \mu \mathrm{g} / \mathrm{ml}$ in PBS, $15 \mathrm{~min})$, respectively.

\section{Biochemical assays}

\section{Alkaline Phosphatase (ALP) activity}

To measure ALP activity, scaffolds were collected after 14 and 35 days of culture, freezethawed 3 times and incubated for $1 \mathrm{~h}$ at $\mathrm{RT}$ in a cell lysis buffer composed of $\mathrm{KH}_{2} \mathrm{PO}(0.1$ $\mathrm{m}), \mathrm{K}_{2} \mathrm{HPO}_{4}(0.1 \mathrm{~m})$, and Triton $\mathrm{X}-100(0.1 \mathrm{v} \%)$, at $\mathrm{pH}$ 7.8. The chemiluminescent substrate for ALP CDP star® ready to use reagent (Roche) was added to the cell lysate at a 1:4 ratio, and luminescence was measured using a spectrophotometer. Remaining cell lysates were kept for DNA quantification. ALP values were reported normalized to DNA content. DNA assay

DNA assay was performed on cells cultured on films and scaffolds using the CyQUANT cell proliferation assay kit (Thermo Fisher Scientific). Lysed scaffolds after ALP assay and frozen films after collection were incubated overnight at $56{ }^{\circ} \mathrm{C}$ in Proteinase $\mathrm{K}$ solution (1 $\mathrm{mg} \times \mathrm{ml}^{-1}$ Proteinase $\mathrm{K}$ (Sigma-Aldrich) in Tris/EDTA buffer) for matrix degradation and cell lysis. After three freeze-thawing cycles, samples were incubated for $1 \mathrm{~h}$ at RT with a 20X diluted lysis buffer from the kit containing RNase A (1:500) to degrade cellular RNA. Subsequently, the fluorescent dye provided by the kit (1:1) was added and fluorescence was measured after $15 \mathrm{~min}$ incubation (emission/excitation $=520 / 480 \mathrm{~nm}$ ). DNA concentrations were calculated from a DNA standard curve.

\section{Alizarin red $S(A R S)$ staining and quantification}

Calcium deposits on scaffolds cultured for 35 days in BM and MM were stained by ARS (60 mm, pH 4.1 - 4.3, 20 min incubation at RT) and visualized using a stereomicroscope (Nikon SMZ25). After imaging, ARS was extracted and quantified. To do this, scaffolds were incubated for $1 \mathrm{~h}$ at RT with $30 \mathrm{v} \%$ acetic acid, followed by $10 \mathrm{~min}$ incubation at 85 ${ }^{\circ} \mathrm{C}$. After a centrifugation step at $20,000 \mathrm{rcf}$ for $10 \mathrm{~min}$, ammonium hydroxide (5 M) was added to the supernatants to bring the $\mathrm{pH}$ to 4.2. The absorbance was measured at 405 $\mathrm{nm}$ using a spectrophotometer. Concentration of ARS was calculated from an ARS standard curve and the values were normalized to DNA content. 


\section{Statistical analysis}

All data is shown as average with error bars indicating the standard deviation of at least three replicates. Analysis of statistics was conducted with GraphPad Prism (version 8.0.1). A t-test analysis, or a one-way or two-way analysis of variance (ANOVA) followed by a Tukey's post-hoc multiple comparison test correcting for multiple comparisons, was performed to evaluate statistical significance.

\section{RESULTS AND DISCUSSION}

\section{Effect of densification on resulting d-rGO properties}

One of the most attractive methods for dispersing fillers into polymers, in terms of costs, scalability and green production, is melt compounding. ${ }^{34}$ Due to the low scale separation of exfoliated graphene sheets from bulk graphite, and the low thermal stability of GO, the use of rGO within melt blended polymer-graphene derivatives composites is preferred. ${ }^{35}$ However, the low bulk density of rGO makes its feeding into compounders very difficult. This low bulk density originates from the exfoliation and volume expansion of $\mathrm{GO}$ caused by the $\mathrm{CO}_{2}$ formed upon the decomposition of oxygen-containing functional groups during the high temperature thermal reduction process. ${ }^{24,26,28}$ For this reason, prior to compounding with PEOT/PBT, the as-produced very low bulk density rGO (10 g/L) was densified (d-rGO) to ease its handling and dosing into the twin-screw compounding system, as well as to reduce the nanoparticles' inhalation hazard. In order to investigate the effect of different densification parameters (i.e. densification solvent and final rGO bulk density) on the resulting d-rGO properties, as well as on the compounding and printing processes, two different densification solvents, acetone and water, were used. Previous literature suggests the applicability of these solvents as densification medium. ${ }^{24,36-37} \mathrm{Here}$, three d-rGO materials were obtained after dispersion in either of these two solvents (Figure 1): i) d-rGO-A: densified in water, bulk density $50 \mathrm{~g} / \mathrm{L}$ ( $\rho$ medium), ii) d-rGO-B: densified in acetone, bulk density $50 \mathrm{~g} / \mathrm{L}$ ( $\rho$ medium), and iii) $\mathrm{d}-\mathrm{rGO}-\mathrm{C}$ : densified in water, bulk density $90 \mathrm{~g} / \mathrm{L}$ ( $\rho$ high).

Since each of the d-rGO came from a different rGO source, their elemental composition was analysed to confirm equal $\mathrm{C} / \mathrm{O}$ ratios and to allow for subsequent comparisons. According to the XPS analysis in Table S1, all d-rGO materials were partially reduced, due to the presence of oxygen in their composition. The $\mathrm{C} / \mathrm{O}$ ratios were maintained between 


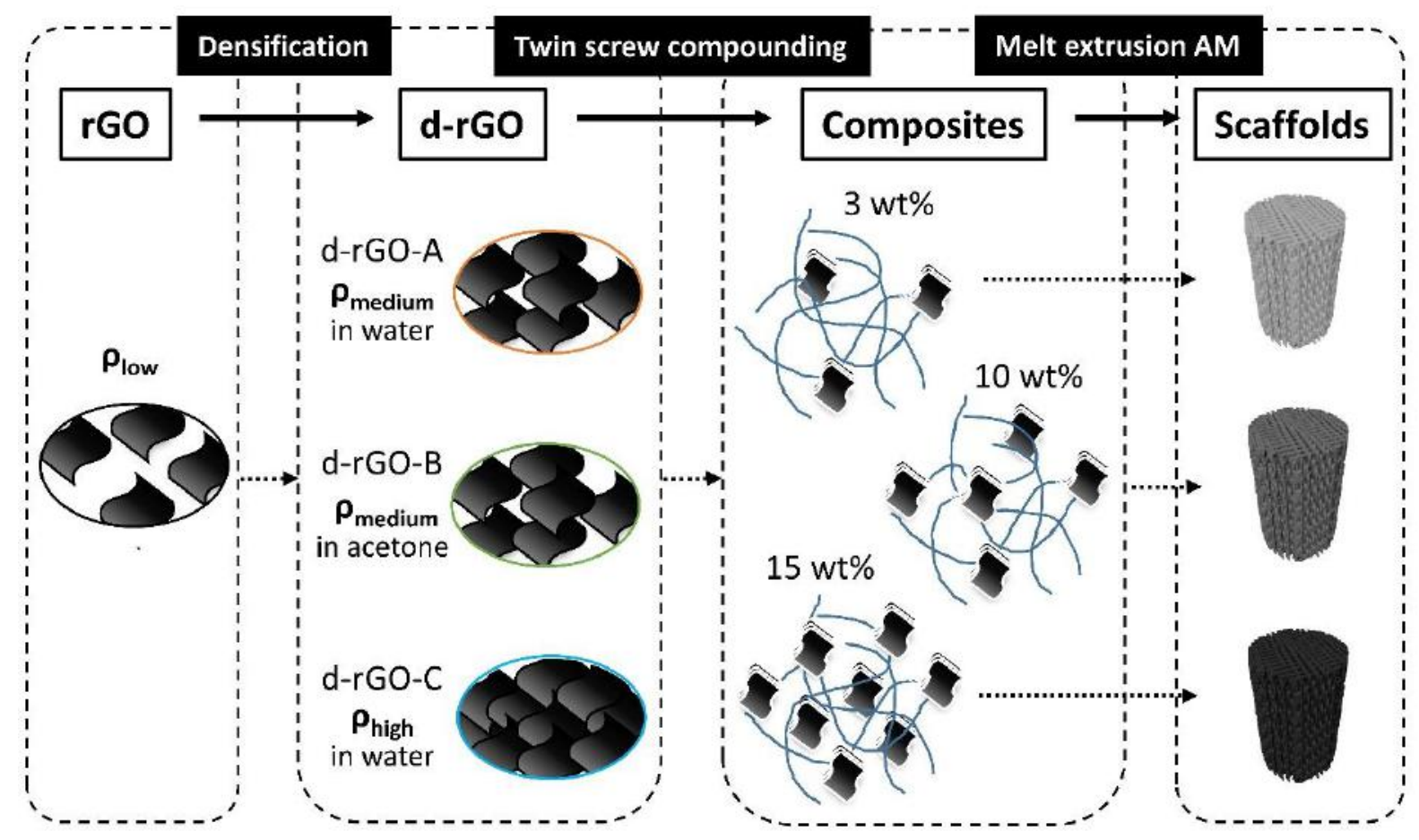

Figure 1. Schematic of the polymer-rGO composite scaffold fabrication route. The low bulk density as-prepared rGO was densified (d-rGO), and three different $d$-rGO types were obtained: $d$-rGO-A (densified in acetone, medium bulk density), d-rGO-B (densified in water, medium bulk density) and d-rGO-C (densified in water, high bulk density). Each of the d-rGO was melt blended via twin screw extrusion with PEOT/PBT to obtain composites at three different d-rGO concentrations (3, 10 and $15 \mathrm{wt} \%$ ). Each of the composites was used to fabricate scaffolds via melt extrusion AM.

5 and 6 in all rGO batches, verifying a consistent reduction degree among batches. Moreover, all batches presented traces of $\mathrm{N}, \mathrm{Al}, \mathrm{Si}, \mathrm{S}, \mathrm{Cl}$ or $\mathrm{Fe}$ lower than 1 atomic\%. Impurities of sulfur and nitrogen were likely to be due to the covalently bonded sulfates and nitrates during the Hummers method to produce GO. ${ }^{38-39}$ Metallic contaminations are thought to derive from the starting graphitic material itself or the synthesis process. ${ }^{40-41}$ The XRD patterns also showed no differences in the crystalline structure among the different d-rGO's (Figure S1). All diffractograms showed a (002) diffraction peak at 24 degrees, corresponding to rGO materials with an interlayer distance of $0.37 \mathrm{~nm}$, and a (100) peak at $\sim 42$ degrees. 38, 42 Upon calculation of the full width at half maximum (FWHM) of the (002) peaks, the same crystallite size among the different d-rGO's was confirmed (Figure S1B). The low intensity and broadness this peak revealed the amorphous structure and the short-range order of the d-rGO's. ${ }^{43-44}$ Other peaks at $~ 28$ 33 degrees, which correspond to crystalline mineral impurities, were also appreciated in some the diffractograms. ${ }^{45}$ In addition to these, some spectra showed low intensity sharp 
peaks at 26.5 degrees, which correspond to traces of unoxidized graphite. Overall, all drGO display similar XRD diffractrograms, suggesting that the densification step did not have an impact on the reduction degree, nanocrystalline structure or crystallite size of the samples. However, it is believed that the high porosity of as-prepared rGO created due to volume expansion and $\mathrm{CO}_{2}$ formation during the thermal reduction process, is reduced during densification, leading to a more compacted and aggregated material. rGO aggregation has been previously reported during the complete removal of water after an aqueous GO reduction process, or after drying $\mathrm{rGO}$ in an acetone solution. 24, 46 Furthermore, previous studies have revealed that re-dispersion of rGO in water or ethanol followed by a drying step, lead to agglomeration of the powder and a shrinkage of their macroporous and mesoporous structure. ${ }^{37}$ For these reasons, we hypothesize that d-rGO$\mathrm{C}$, with the highest bulk density, was the highest compacted material after solvent evaporation. This was confirmed by SEM, where both d-rGO-B and d-rGO-C displayed particles aggregating together, but d-rGO-C clearly revealed a less porous and more compacted structure at higher magnifications (Figure S2). Although possessing the same bulk density, differences among d-rGO-A and d-rGO-B were also expected, since they were densified in different solvents. Previous literature suggests that the shrinkage of rGO pore size after drying aqueous solutions is more pronounced compared to that of rGO dried in solvents with lower polarity, surface tension, and wettability, such as ethanol or, in our case, acetone. ${ }^{37}$ This is due to the better interaction of water with $\mathrm{rGO}$, which exerts a higher capillary force upon evaporation, causing a significant decrease in the macro- and mesopore volume. For this reason, d-rGO-A densified in water is hypothesized to be a more compacted material than d-rGO-B. Therefore, the three different $d-r G O$ could be classified according to their compaction degree, being d-rGO-C the most compacted (densified in water, bulk density $90 \mathrm{~g} / \mathrm{L}$ ), followed by the medium compacted d-rGO-A (densified in water, bulk density $50 \mathrm{~g} / \mathrm{L}$ ), and the low compacted d-rGO-B (densified in acetone, bulk density $50 \mathrm{~g} / \mathrm{L})$.

\section{Effect of d-rGO densification on melt compounding}

After densification, each of the d-rGO materials was melt compounded with PEOT/PBT to form PEOT/PBT-d-rGO composites (Figure 1). TGA measurements of the experimental d-rGO loading on each of the composites suggested the reproducibility of the melt compounding process regardless the d-rGO used, for all d-rGO concentrations (Table 2, Figure S3). Yet, it is worth noting that when d-rGO-B was blended at high concentrations 
(15 wt\%), the experimental values deviated slightly from the theoretical ones, and the composites contained lower d-rGO amounts than expected. This can be likely due to an inhomogeneous distribution of d-rGO within the pellets and the low amount of sample (a few pellets at most) used for TGA measurements.

Table 2. Experimental loadings (wt\%) of each of the composites calculated by TGA.

\begin{tabular}{cccc}
\hline & 3 wt $\%$ & 10 wt $\%$ & 15 wt $\%$ \\
\hline d-rGO-A & $2.91 \pm 0.34$ & $11.22 \pm 0.45$ & $17.73 \pm 0.28$ \\
d-rGO-B & $2.18 \pm 0.84$ & $11.68 \pm 1.03$ & $11.73 \pm 1.29$ \\
d-rGO-C & $4.64 \pm 0.28$ & $9.47 \pm 6.09$ & $15.01 \pm 1.03$ \\
\hline
\end{tabular}

The differences in compaction degree mentioned above correlated well with the filler dispersion within the PEOT/PBT-d-rGO composite after compounding. Filler aggregation as an indirect measure of d-rGO dispersion was evaluated by dissolving the composites in chloroform, and visualizing and quantifying the size of d-rGO particles. Figure 2A displays representative SEM images of d-rGO within each of the composites. Small particles up to $50 \mu \mathrm{m}$ were abundant for all conditions (relative frequency $\sim 80 \%$ ) (Figure 2A, B). In addition, big d-rGO aggregates were also observed mainly in d-rGO-A composites ( $\left.\rho_{\text {medium}}\right)$, at all d-rGO concentrations, and on d-rGO-B composites ( $\left.\rho_{\text {medium }}\right)$ at high concentrations (10\% and 15\%). Interestingly, d-rGO-C composites ( $\left.\rho_{\text {high}}\right)$ displayed much lower d-rGO aggregation, as revealed by the SEM images and the lower number of $>200 \mu \mathrm{m}$ aggregates compared to d-rGO-A and d-rGO-B composites (Figure 2B). Among all, the d-rGO-A composites displayed the largest number of $>200 \mu \mathrm{m}$ aggregates, especially at $3 \%$ and $15 \%$ d-rGO. It is believed that the high bulk density d-rGO-C occupied a smaller volume fraction within the composite at a given wt\% compared to d-rGO-A and d-rGO-B, even for high d-rGO concentrations. This avoided particle interaction within the composites and reduced the number of visible aggregates. On the other hand, due to the lower bulk density of d-rGO-A and d-rGO-B, these were able to reach a critical volume occupancy within the composites at much lower concentrations (wt\%). This increased the probability of d-rGO particles interaction, and the formation of particles overlaps into bigger agglomerates, by hydrophobic interactions. ${ }^{10,47-48}$ In addition, due to the lower porosity and higher compaction degree of d-rGO-A compared to d-rGO-B, filler exfoliation and dispersion within the d-rGO-A composites was more difficult, leading to the visualization 
of a larger number of aggregates. Compared to melt compounding, other composite production methods such as solvent blending or in situ polymerization, have shown to ensure more uniform d-rGO dispersions. ${ }^{24,49}$ Moreover, several reports have suggested the preparation by solvent blending of rGO-polymer masterbatches, to be used as starting material to prepare other rGO composites by melt compounding. ${ }^{28,50-51}$ This approach has shown to lead to better rGO dispersion, as it skips the densification or compaction step. However, it involves the use of organic solvents in the process, which is not desirable for biological applications, as the solvents can remain as residues within the composite matrix.

A
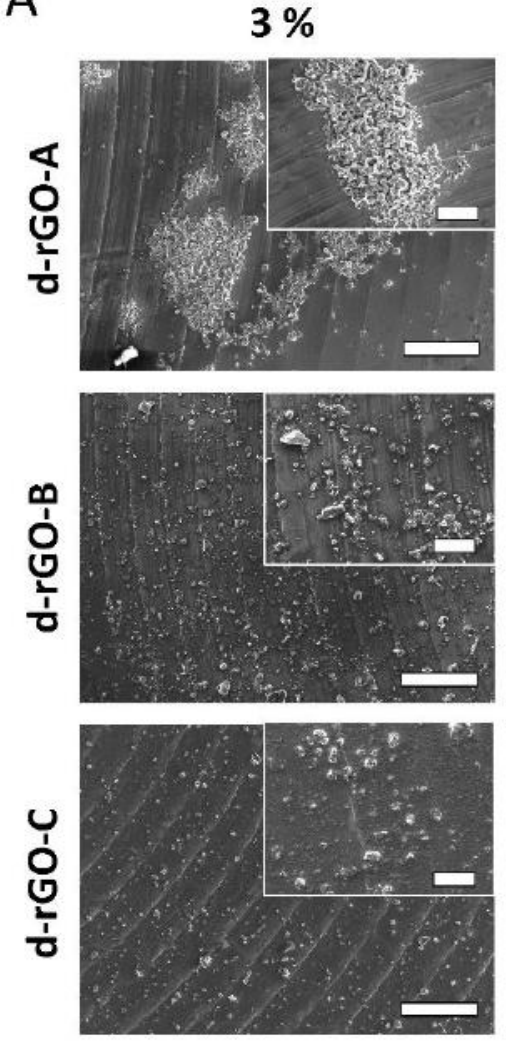

B

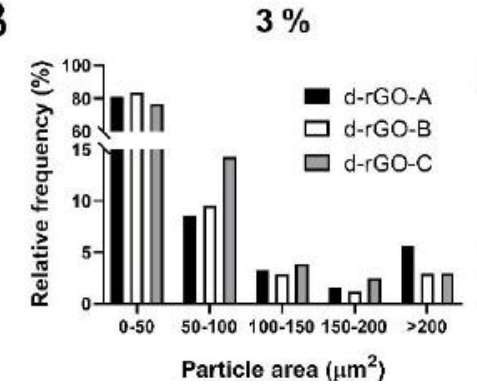

$10 \%$
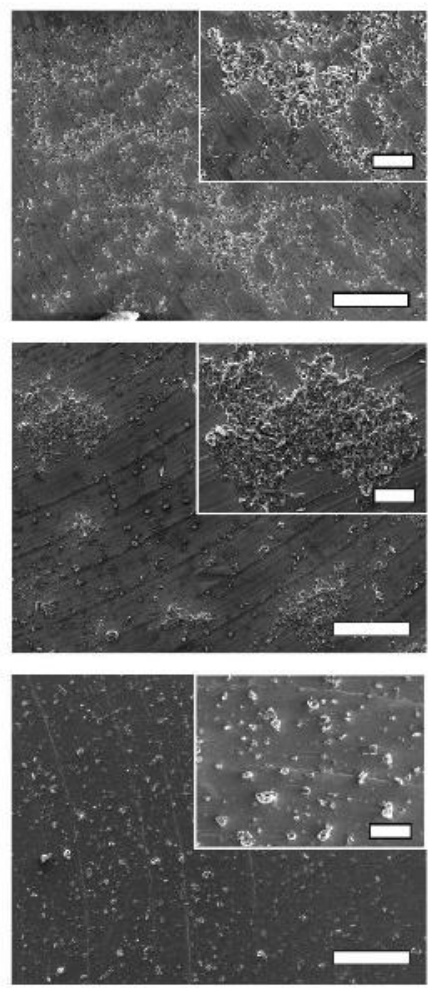

$10 \%$

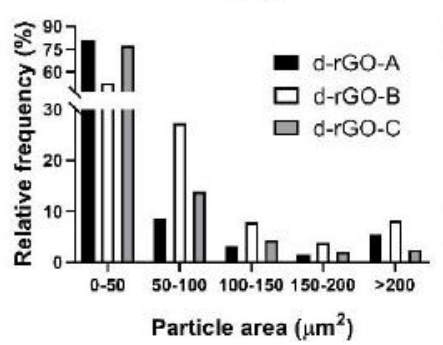

$15 \%$
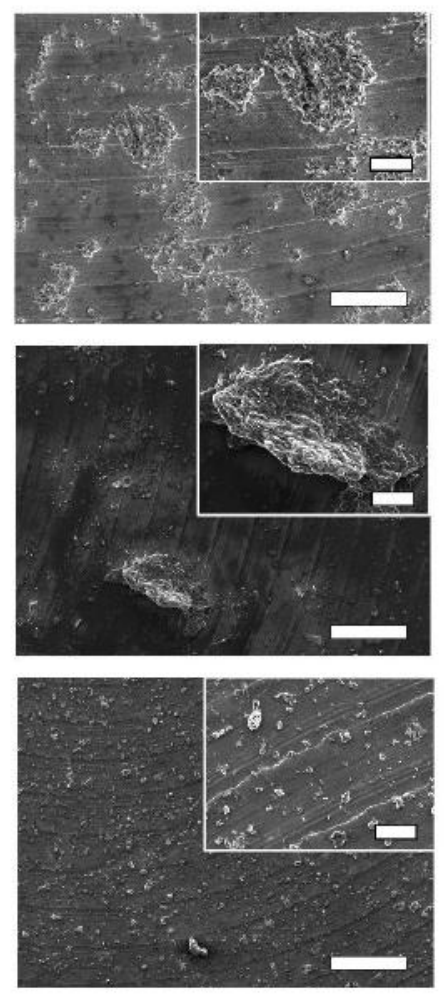

$15 \%$

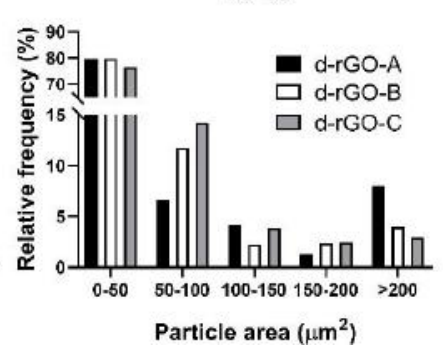

Figure 2. (A) Representative SEM micrographs of d-rGO particles and aggregates after dissolving each composite in chloroform. Scale bars $200 \mu \mathrm{m}$. Inserts correspond to high magnification images. Scale bars $50 \mu \mathrm{m}$. (B) Size distribution of d-rGO particles and aggregates measured from SEM micrographs in $(A)$. 
Notably, d-rGO volume occupancy and dispersion within the composite also correlated well with the electrical properties of extruded composite filaments, as presented in Table 3. d-rGO-C extruded filaments' high resistance (out of the device range) impeded the report of their conductivity values at any d-rGO concentration. In case of the d-rGO-A and d-rGO-B filaments, their electrical conductivity was measurable from $10 \% \mathrm{~d}$-rGO, and they were more conductive with increasing d-rGO content, as previously reported. 28 Interestingly, the conductivity of $10 \%$ d-rGO-A filaments was 35-fold and 3-fold higher compared to $10 \%$ d-rGO-B and $15 \%$ d-rGO-B, respectively, suggesting the lower percolation threshold of the d-rGO-A composites. As 15\% d-rGO-A was not extrudable, no filament was obtained for this measurement. It is evident that the high volume fraction and poor dispersability of d-rGO-A within the composites helped to form a more connected drGO network leading to conductive composites at lower loadings than the less compacted and better dispersed d-rGO-B. On the other hand, the lower volume fraction of d-rGO-C within the composites could not create such conductive pathways and electrical conductivity was not measurable. ${ }^{24,46,52-53}$

Table 3. Conductivity values of extruded filaments from each composite.

\begin{tabular}{cccc}
\hline & $\begin{array}{c}\text { Conductivity } \\
3 \%(\mathrm{mS} / \mathrm{m})\end{array}$ & $\begin{array}{c}\text { Conductivity } \\
10 \%(\mathrm{mS} / \mathrm{m})\end{array}$ & $\begin{array}{c}\text { Conductivity } \\
15 \%(\mathrm{mS} / \mathrm{m})\end{array}$ \\
\hline d-rGO-A & $<0.32$ & $13.8 \pm 1.1$ & n.a. \\
d-rGO-B & $<0.32$ & $0.44 \pm 0.1$ & $5.4 \pm 1.2$ \\
d-rGO-C & $<0.32$ & $<0.32$ & $<0.32$ \\
\hline
\end{tabular}

\section{Effect of d-rGO compaction on composite printability}

The d-rGO volume occupancy, compaction degree and dispersion also influenced composites printability by ME-AM (Figure 3). Interestingly, due to the large volume fraction and high frequency of large d-rGO aggregates in the 15\% d-rGO-A composites, as shown in Figure 2, this material remained a paste-like material rather than a melt, even at 220 ${ }^{\circ} \mathrm{C}$, and was not printable within the operational limits of the AM device (in terms of printing temperature and cartridge pressure). Reducing the filler loading down to $3 \mathrm{wt} \%$ made it possible to produce scaffolds with a fiber diameter of $250 \mu \mathrm{m}$ (Figure 3 and Figure S4). The fabrication of $10 \%$ d-rGO-A scaffolds was challenging, since lack of bonding between layers prevented their stability and handling, in spite of printing at the highest operational 
temperature $\left(220^{\circ} \mathrm{C}\right.$ ) and increasing the fiber diameter (up to $340 \mu \mathrm{m}$ ) for promoting a bigger area of contact in between layers (Figure 3 and Figure S5). On the other hand, scaffolds with a fiber diameter of $250 \mu \mathrm{m}$ were obtained with all d-rGO-B composites, including $10 \%$ and 15\% d-rGO-B (Figure 3, Figure S4). The printability and good interlayer bonding of these materials, compared to d-rGO-A, was possible due to the increased filler dispersion within the d-rGO-B composites and the lower number of aggregates compared to d-rGO-A (Figure 2). These parameters might have led to a lower viscosity of the $10 \%$ d-rGO-B melt compared to the $10 \%$ d-rGO-A melt at a given temperature, allowing an enhanced interlayer contact and bonding. ${ }^{54}$ Printing temperature was adjusted for each d-rGO-A composite, ranging between $195^{\circ} \mathrm{C}$ and $210^{\circ} \mathrm{C}$, since viscosity of the melt was found to increase with increasing d-rGO concentration for all composites. ${ }^{55}$ Notably, while 3 and $10 \%$ d-rGO-B scaffolds fabrication was reproducible in terms of resulting filament morphology and z-porosity, 15\% d-rGO-B scaffolds lateral porosity did not always remain constant across the scaffold height (Figure 3). This was possibly due to sudden viscosity changes during the extrusion process, probably caused by the discontinuous presence of $\mathrm{d}-\mathrm{rGO}-\mathrm{B}$ aggregates changing the flow of the molten composite and, therefore, causing filaments sagging at some points during the printing process. Interestingly, d-rGO-C scaffolds printing was very stable, and scaffolds with optimum lateral porosity were obtained for all d-rGO concentrations (Figure 3). Looking at d-rGO-C scaffolds filament cross section by SEM (Figure S6), it can be observed that PEOT/PBT (showing a flowy appearance) occupies much larger volume than d-rGO particles (with a grainy and spiky appearance), which are present as dispersed islands within a polymer matrix for all d-rGO concentrations, making these materials relatively easy to print. On the other hand, d-rGO dominantly occupied the d-rGO-A and d-rGO-B scaffolds filaments volume, especially on $10 \%$ and $15 \%$ d-rGO scaffolds, leading to difficulties when printing. Overall, these results suggest that d-rGO bulk density, and therefore volume fraction, as well as d-rGO densification solvent, which dictates compaction and dispersability degree, plays key roles in the maximum filler loading to process composites into ME-AM scaffolds. While previous studies have reported ME-AM scaffolds production only up to $10 \mathrm{wt} \%$ pristine graphene or $\mathrm{GGO}, 19,31$ we were able to produce scaffolds with up to $15 \mathrm{wt} \% \mathrm{~d}$-rGO. Despite the apparent lower rGO content in these studies, equivalent volume percentages or graphene densities would be required to make fair comparisons with the composites studied herein. Nevertheless, to the best of our knowledge, solvent blending and direct ink writing (solvent-based printing) are the only 
described manufacturing methods for scaffolds with higher graphene derivatives concentrations (50-75 wt\% graphene), which both suffer from requiring the application of organic solvents. ${ }^{18,53}$

$3 \%$
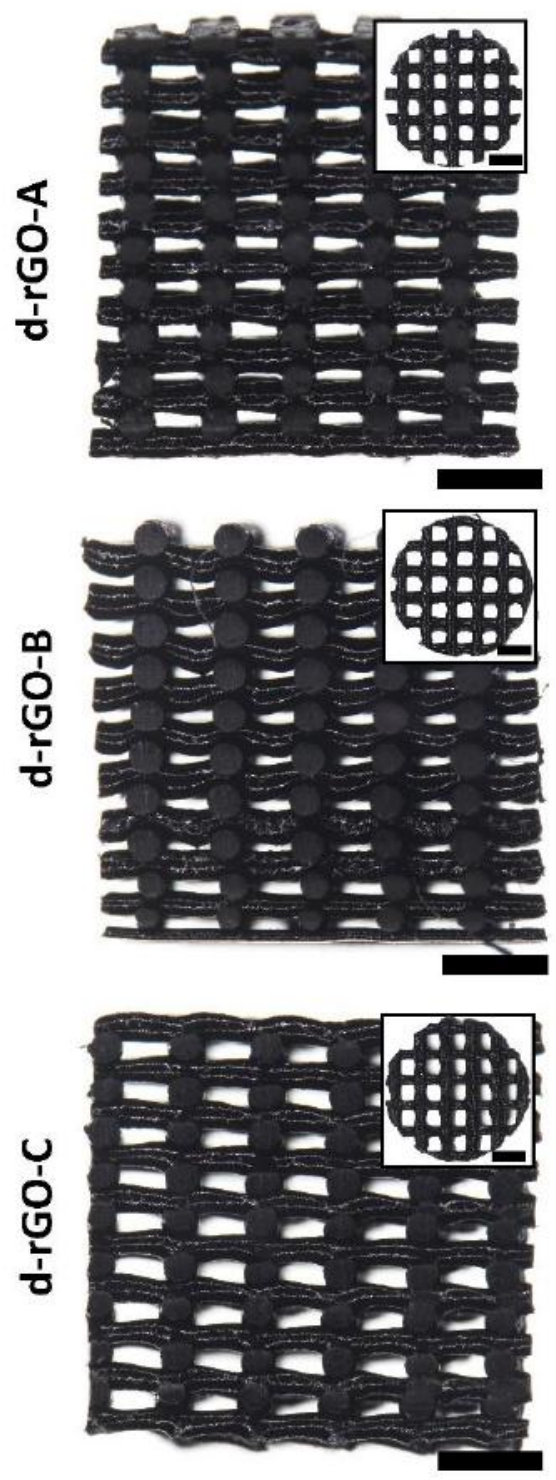

$10 \%$

inconsistent layer bonding
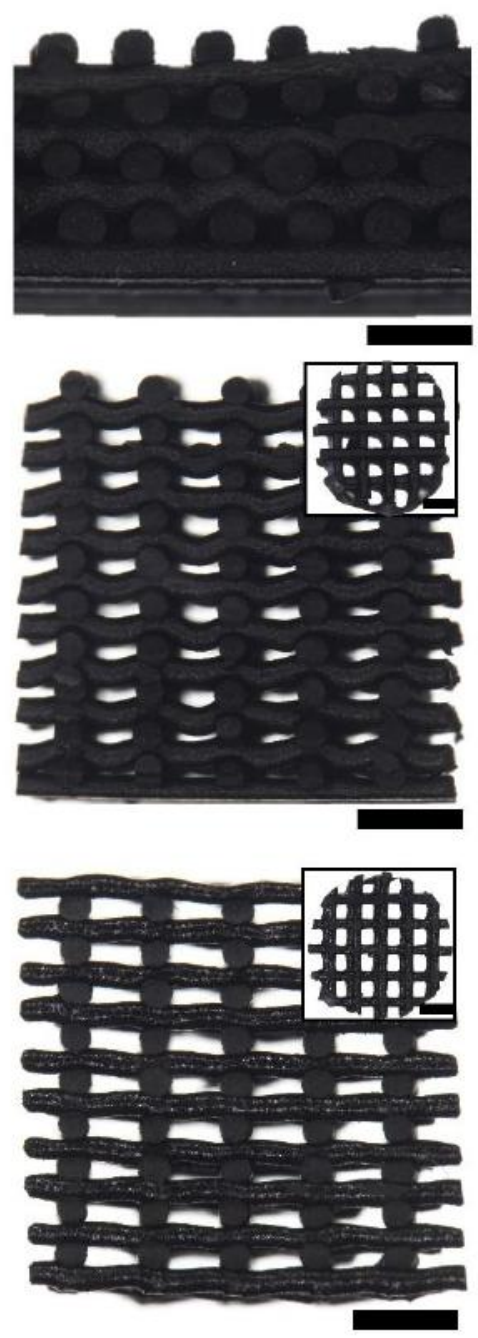

$15 \%$
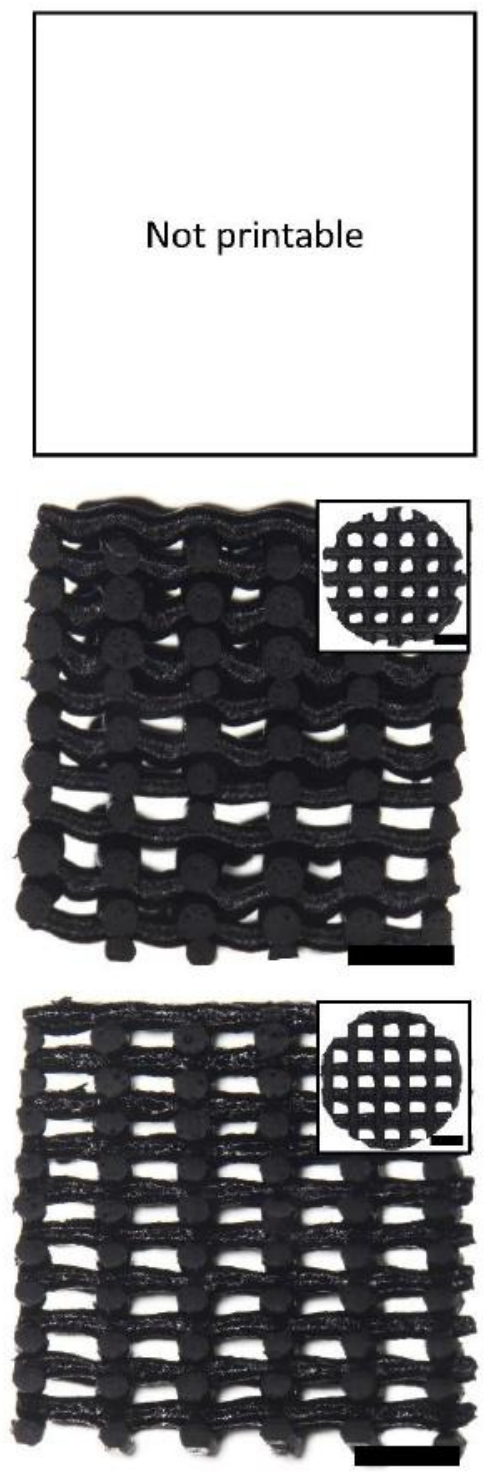

Figure 3. Stereomicroscopy images of 3D ME-AM scaffolds cross sections and top view (insets) obtained using each of the d-rGO composites, depicting scaffolds morphology and interconnected porosity. Scale bars $1 \mathrm{~mm}$.

The ME-AM scaffolds microscale surface roughness was analysed by SEM (Figure 4). As a general observation, the qualitative scaffold surface roughness increased with increasing $\mathrm{d}$-rGO content for all scaffolds types, given by the irregular protuberances formed by the underlying rGO particles. Interestingly, the surface of $3 \% \mathrm{~d}$-rGO scaffolds remained rather 
smooth, regardless of the d-rGO type (Figure 4). This is in agreement with previous reports, in which the surface of ME-AM scaffolds containing less than 5 wt\% graphene (or derivatives) did not present changes in their surface microroughness compared with bare polymeric scaffolds. ${ }^{56}$ In the case of $10 \%$ and 15\% d-rGO scaffolds, the degree of roughness was influenced by the type of d-rGO, i.e. its bulk density, compaction degree and volume fraction within the filament, for a given d-rGO concentration. In this regard, $10 \%$ d-rGO-A presented higher roughness than $10 \%$ d-rGO-B, and both higher than $10 \%$ d-rGO-C. Similarly, $15 \%$ d-rGO-B scaffold surface was much rougher than that of $15 \%$ drGO-C scaffolds, where only few wrinkles raised on the surface. This is likely due to the much lower volume fraction occupied by the highly compacted $d-r G O-C$ particles decreasing their probability of populating the filaments surface.
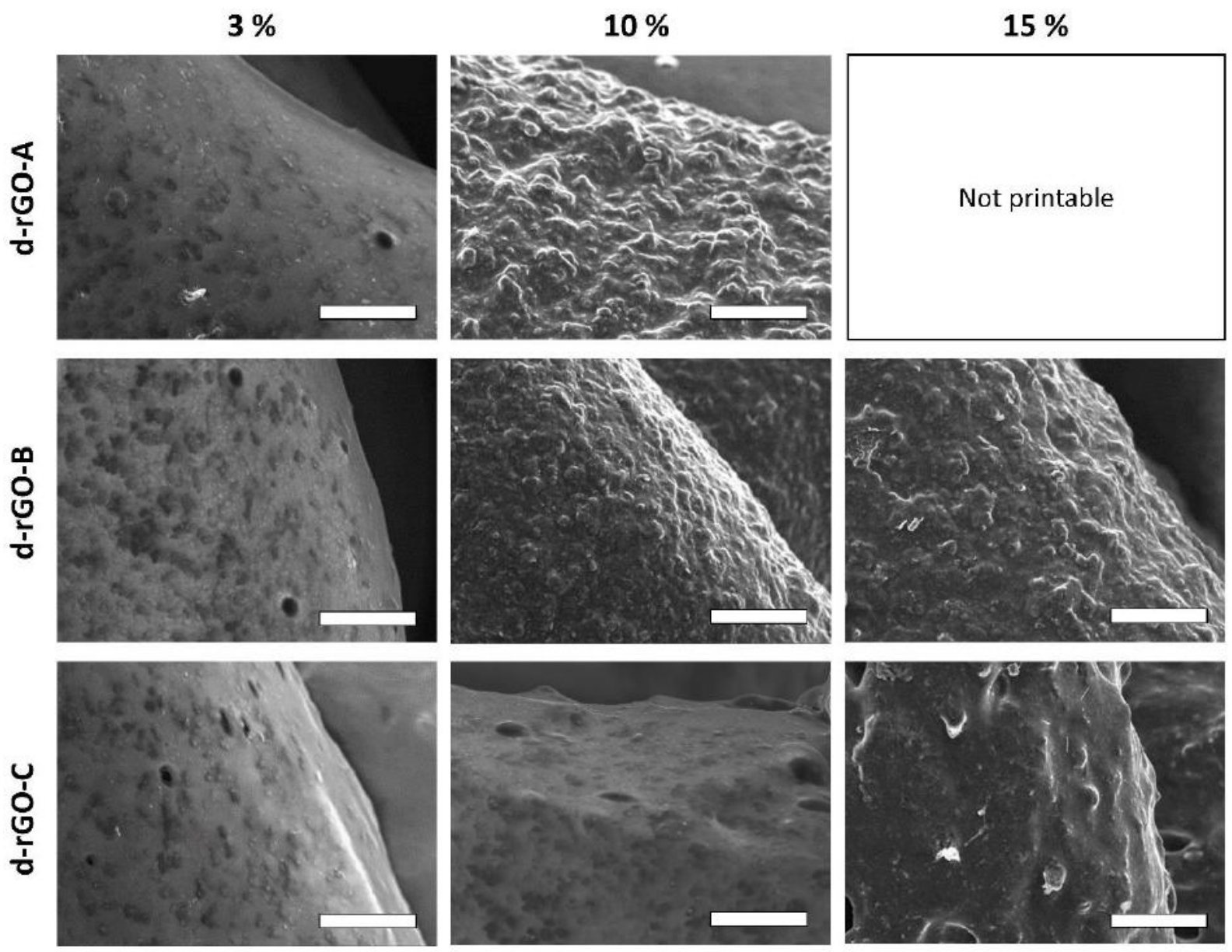

Figure 4. Representative SEM micrographs of composite scaffolds depicting the scaffold's surface roughness. Scale bars $50 \mu \mathrm{m}$. 


\section{Effect of $d$-rGO concentration on wettability and protein adsorption}

Prior to assessing the interaction of cells with the ME-AM scaffolds, different material properties, such as wettability and protein adsorption, as well as cell-material interactions in $2 \mathrm{D}$ were evaluated. For this and further studies, only the d-rGO-B composites were taken into consideration as they demonstrated a wider printability window (up to $15 \mathrm{wt} \%$ ) compared to d-rGO-A composites, and superior electrical conductivity compared to the $\mathrm{d}$ rGO-C composites, properties that are considered relevant for their application in bone tissue regeneration. The static contact angle measurements on composite films (Figure $5 \mathrm{~A}$ ), suggested that the material remained rather hydrophobic (contact angle $\sim 80^{\circ}$ ) up to $10 \%$ d-rGO-B. $15 \%$ d-rGO films contact angle was reduced down to $\sim 50^{\circ}$, denoting the composite hydrophilicity. While the increased hydrophilicity of polymers upon d-rGO-B addition is in agreement with some previous reports, ${ }^{19,57}$ others have suggested the opposite trend. ${ }^{58-59}$ The increase in hydophilicity of $15 \%$ d-rGO-B observed within our study might be explained by the d-rGO-B being partially reduced and containing remnant hydrophilic oxygen functional groups, as well as by the presence of larger amounts of $d$ $\mathrm{rGO}$ on the surface of the films with increasing $d-r G O-B$ concentrations. Protein adsorption was assessed by incubating the films in a BSA solution, as a model protein for studying protein-substrate affinity (Figure 5B). While 3\% d-rGO-B films adsorbed as much protein as PEOT/PBT films, d-rGO-B content over $3 \%$ led to a significant increase in protein adsorption to the films. It is believed that $\mathrm{rGO}$ interacts with proteins mainly through hydrophobic van der Waals and $\pi-\pi$ stacking interactions, due to the carbon structure. ${ }^{60-}$ ${ }^{61}$ Yet, electrostatic interactions and hydrogen bonding are also possible due to the partial presence of oxygen groups on rGO. ${ }^{60-61}$ As Figure 5B demonstrates, increasing the $\mathrm{d}$ rGO-B concentration up to $15 \%$ led to a 4 -fold increase in protein adsorption in $15 \% \mathrm{~d}$ rGO-B films, likely due to a larger amount of interaction points with proteins, compared to lower d-rGO-B content films.

Surface physicochemical properties, i.e. roughness, hydrophilicity and protein adsorption ability, can regulate cellular behaviour. To evaluate this effect, hMSCs were cultured on films pre-incubated in cell culture medium. Due to a potential partial loss of conformation (i.e. hiding of cell-binding sites) of the adsorbed proteins when adhered to the rGO-based surfaces, previously described in the literature, 62 as well as gravity acting as the main factor favouring cell attachment on 2D substrates, cell attachment to films did not significantly increase with increasing d-rGO-B content. This was observed upon DNA 
quantification at day 1 (Figure $5 \mathbf{C}$ ). Yet, compared to the spread morphology of cells on PEOT/PBT films, hMSCs cultured on d-rGO-B based films, especially at high d-rGO-B content (10 and $15 \mathrm{wt} \%$ ), showed a spindle-shape, elongated and branched morphology at both day 1 and 7 , suggesting higher affinity of hMSCs towards d-rGO-B containing materials, probably due to the aforementioned enhanced protein adsorption (Figure 5D). It is also plausible that the ridges and groves on the films rough surface, given by the underlying d-rGO-B, provided contact guidance cues for cells to acquire an elongated morphology. ${ }^{63-64}$

A

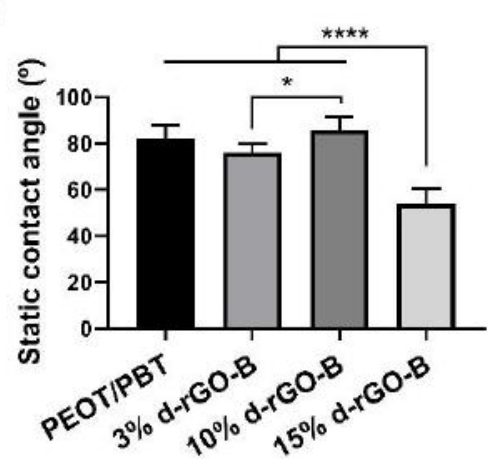

B

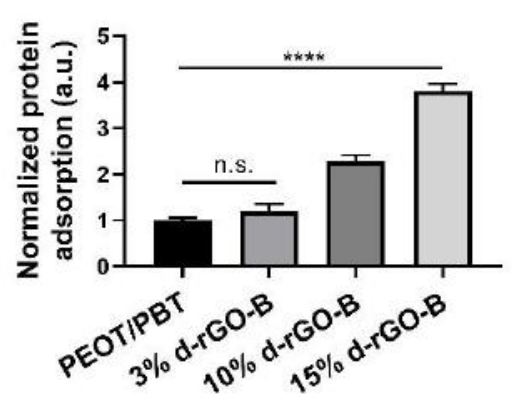

C

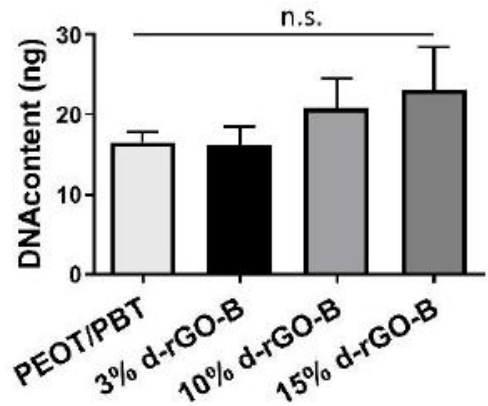

D

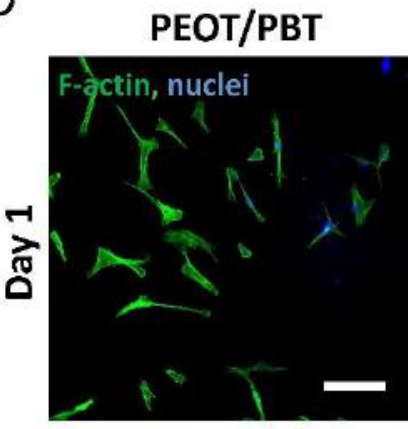

3\% d-rGO-B

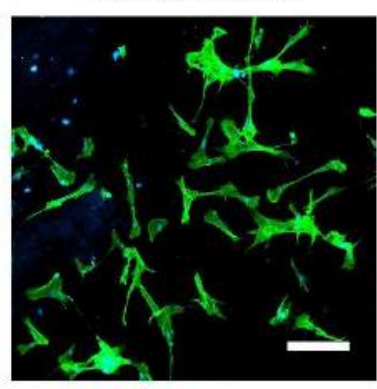

$10 \%$ d-rGO-B

$15 \%$ d-rGO-B
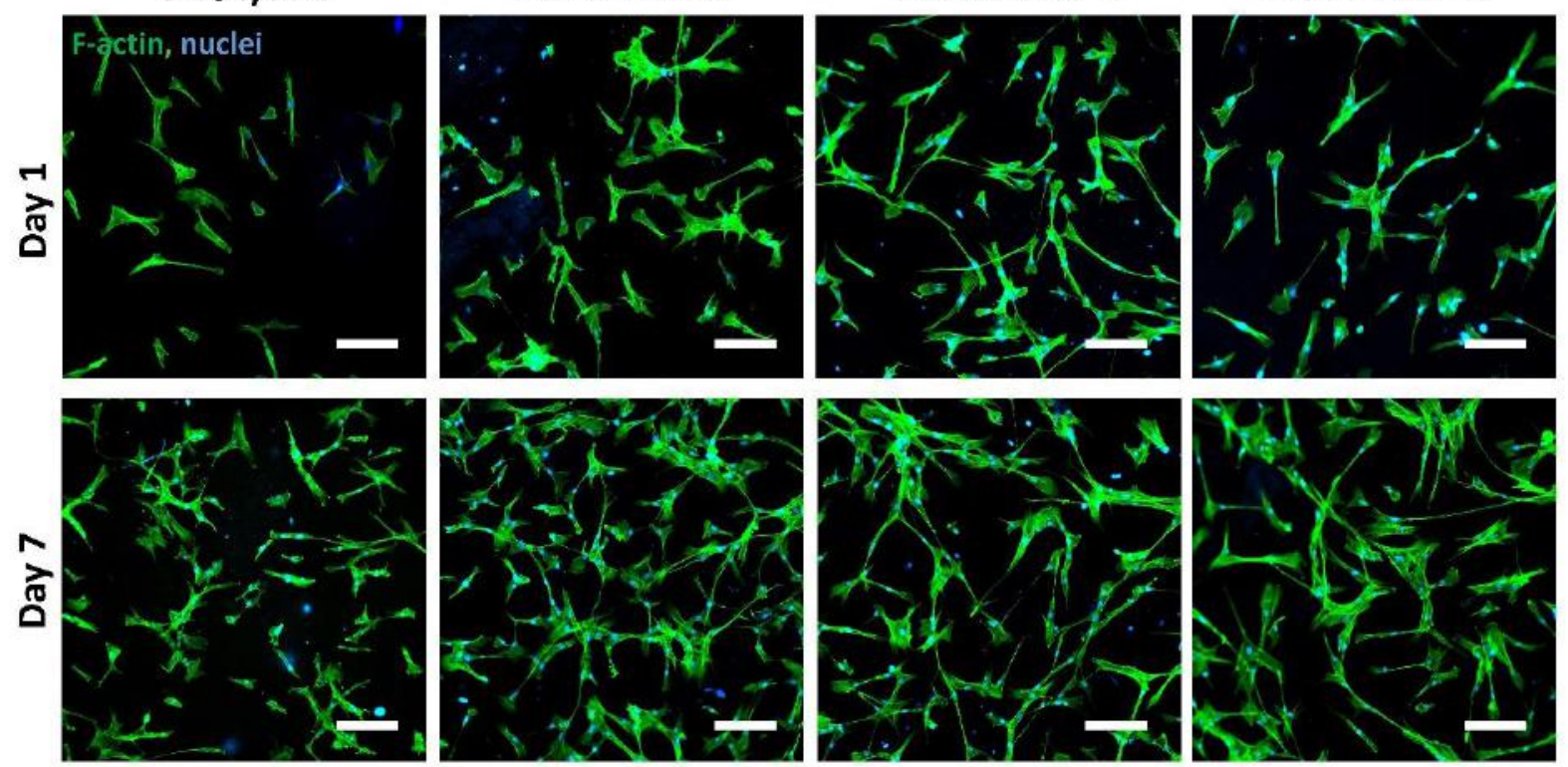

Figure 5. Effect of rGO concentration on physicochemical properties and cell-material interactions in 2D. (A) Static contact angle measured on PEOT/PBT and d-rGO-B composite films. (B) Protein adsorption to d-rGO-B films upon incubation in a BSA solution, normalized to protein adsorption to PEOT/PBT films. (C) Quantification of hMSCs attachment to d-rGO-B films after 1 day of culture. (D) Fluorescent microscopy images of hMSCs (F-actin in green, nuclei in blue) cultured on films for 1 and 7 days. Scale bars $100 \mu \mathrm{m}$. Data presented as average \pm s.d. and statistical significance performed using one-way ANOVA with Tukey's multiple comparison test (n.s. $p>0.05,{ }^{*} p<0.05$, $\left.{ }^{* * * *} p<0.0001\right)$. 


\section{Effect of d-rGO concentration on scaffolds antimicrobial activity}

The antimicrobial activity of scaffolds was evaluated against relevant Gram - and Gram + bacterial strains in the orthopedic field: $P$. aeruginosa and $S$. epidermidis respectively. Preliminary experiments demonstrated the biocidal activity of d-rGO-B at different concentrations and of composite films when in direct contact with bacteria (Table S2 and Table S3). Importantly, the d-rGO-B ME-AM scaffolds also demonstrated higher antibacterial activity with increasing d-rGO-B concentration within the scaffolds (Figure 6A). Interestingly, antibacterial effects were only evident when bacteria were placed in direct contact with the scaffolds, and no biocidal effect of the supernatant, in which scaffolds were incubated up to 3 days (Figure S7), was observed. This suggests that reactive oxygen species (ROS)-dependent oxidative stress might have not been the dominant antibacterial mechanism, and that membrane mechanical stress induced by sharp edges or corners of d-rGO-B exposed to the surface of the scaffolds, which act as nano-knives or nano-needles and disrupt bacteria membranes, could have been the prevailing mode of antibacterial action. ${ }^{19,59,65}$ Besides, rGO has been shown to produce lower oxidative stress compared to GO, because of the lower amount of oxygen functional groups. ${ }^{66}$ Mechanical stress causing bacterial damage would also explain the increasing antimicrobial activity with increasing $d-r G O-B$ concentration, potentially due to more $d$ rGO-B exposed to the filaments surface (Figure 6A). Furthermore, this mechanism would support scaffolds being more potent against $P$. aeruginosa (Gram -) than $S$. epidermidis $($ Gram +) (Figure 6A). As previously described, this could be attributed to the elongated and curved shape of $P$. aeruginosa being more vulnerable to rGO mechanical damage compared to $S$. epidermidis, with less surface area and a spherical shape. ${ }^{67}$

Due to the conductivity of rGO, charge-transfer oxidative stress can also be considered a main antimicrobial mechanism when bacteria get in contact with the surface of the scaffolds. Here, rGO can act as a conductive bridge over the bacteria lipid bilayer to mediate electron transfer from bacterial intracellular components to the external environment, interrupting the bacteria membrane respiratory chain, and leading to bacteria death. ${ }^{65-66}$ Overall, this and all the aforementioned antibacterial mechanisms will be a matter of investigation in future studies. 


\section{Cell seeding on scaffolds and osteogenic differentiation}

In spite of scaffolds displaying antibacterial activity, hMSCs viability was not compromised when seeded on the d-rGO-B composite scaffolds, at any d-rGO-B concentration. As fluorescent microscopy images of cells (F-actin) attached to the scaffolds' filaments in Figure $6 \mathrm{C}$ depict, cells populated the scaffolds from the beginning of the culture, and showed viable cells characteristics, i.e. a spread or elongated morphology. DNA analysis further confirmed the biocompatibility of the scaffolds up to $15 \%$ d-rGO-B, as cell number did not decrease after 7 days of culture, but was maintained rather constant (Figure 6B). While various reports have addressed the cyto- or genotoxicity of graphene and GO in contact with different cell lines, ${ }^{68-70}$ only a few have tried to understand the biocompatibility of $\mathrm{rGO}$, which greatly differs from the other derivatives in terms crucial factors for biointeractions, such as size (number of layers, available surface area), functional groups (reduction degree) and protein interactions. It has been demonstrated that the cytotoxic effect of GO and partially reduced rGO can be attenuated by proteins in serum binding to their surface, which can reduce the $\mathrm{GO} / \mathrm{rGO}$ ability to penetrate or physically damage the cell membrane, both in in vitro cell cultures and in vivo. ${ }^{71}$ In addition, $\mathrm{rGO}$ has been found to be, in general, more biocompatible than $\mathrm{GO}$ and to provide a reduced inflammatory response upon in vivo implantation, ${ }^{72}$ but still its dose- and size-dependent cytotoxicity towards hMSCs has been shown. ${ }^{73}$ Due to the d-rGO-B encapsulated within the polymer matrix and the polymer slow degradation rate such a cytotoxic effect was not evidenced during the culture period addressed here (35 days). ${ }^{74-75}$ Future studies on scaffolds degradation kinetics and d-rGO-B release rate will be essential, as polymer degradation will eventually occur over time in an in vivo situation, releasing potentially toxic amounts of d-rGO-B.

Considering the aforementioned potential long-term toxicity of highly loaded rGO scaffolds, preliminary osteogenic differentiation studies were performed with hMSCs seeded on $3 \%$ d-rGO-B scaffolds, a low concentration that has been commonly studied in previous reports. ${ }^{56,76-78} \mathrm{HMSC}$ were able to proliferate both in PEOT/PBT and $3 \% \mathrm{~d}$-rGO-B scaffolds over the culture period ( 35 days) especially in MM, likely due to more ECM formation within the scaffolds pores acting as cell growth support, compared to BM (Figure S8). As an early osteogenic marker, Figure 6D demonstrates the ALP activity of hMSCs scaffolds. For both bare PEOT/PBT and $3 \%$ d-rGO-B containing scaffolds, a peak in ALP activity was shown at day 14 , with a significant decrease on day 35 , both in BM and MM, 
which is in agreement with the osteogenic differentiation progression. ${ }^{79}$ However, no statistical differences on ALP activity were found among the d-rGO-B containing scaffolds and the PEOT/PBT control.

A

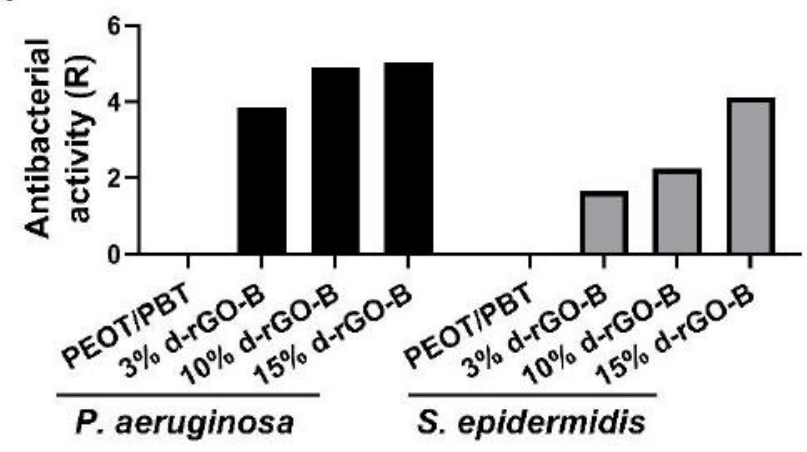

B

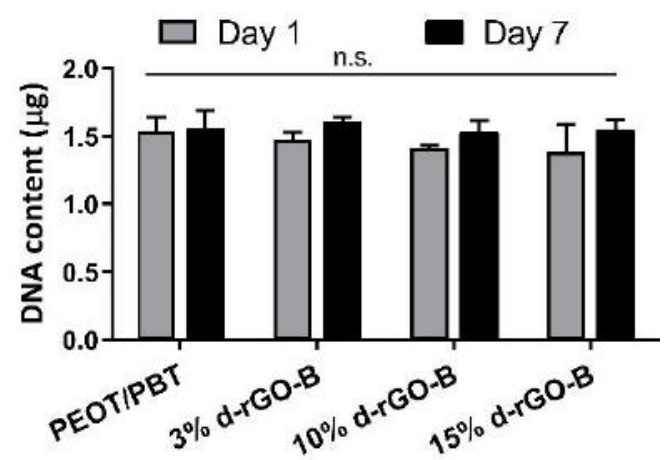

C

PEOT/PBT

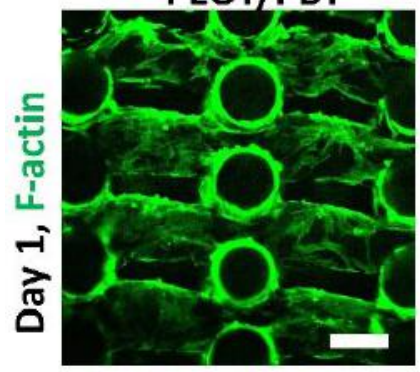

$3 \%$ d-rGO-B

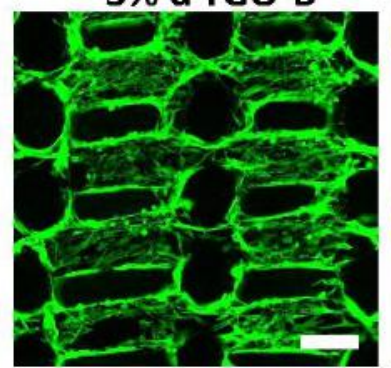

$10 \%$ d-rGO-B
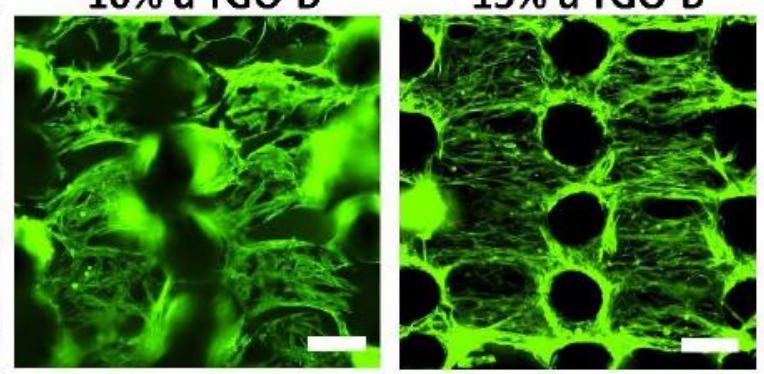
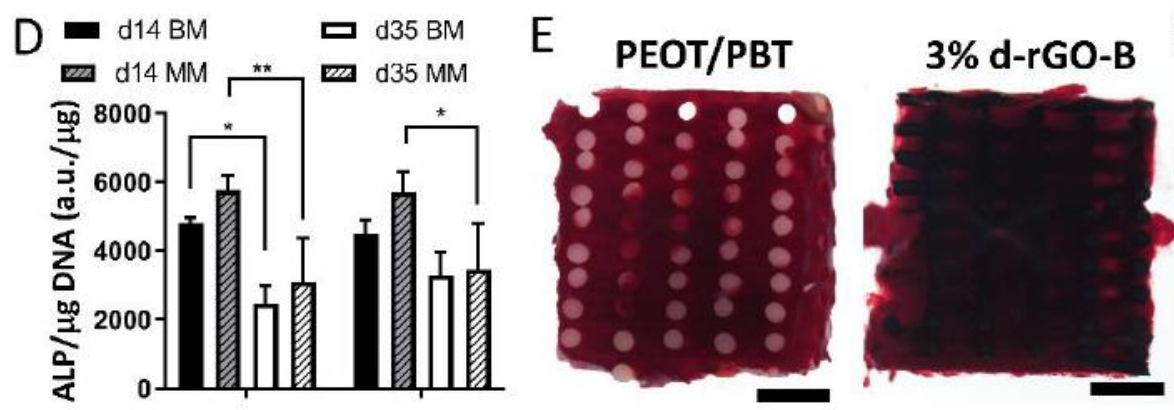

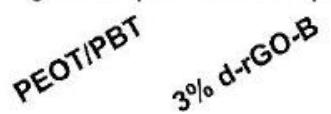
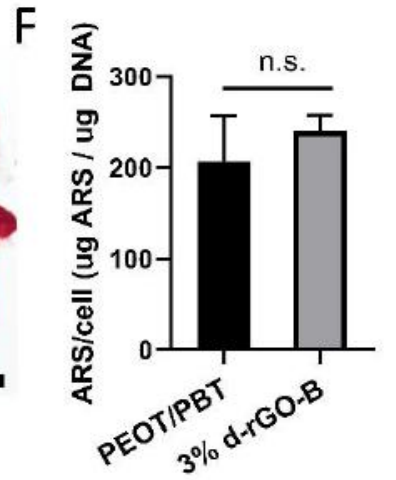

Figure 6. Cell-material interactions in d-rGO-B scaffolds: effect of d-rGO-B concentration. (A) Antibacterial activity of scaffolds against $P$. aeruginosa and $S$. epidermidis. (B) DNA content on scaffolds seeded with hMSCs after 1 and 7 days of culture. (C) Fluorescent microscopy images of hMSCs attachment (F-actin, green) to composite scaffolds cross section. Scale bars $250 \mu \mathrm{m}$. (D) ALP activity of hMSCs seeded on PEOT/PBT and 3\% d-rGO-B scaffolds after 14 and 35 days cultured in BM or MM. Data presented as average \pm s.d. and statistical significance performed using two-way ANOVA with Tukey's multiple comparison test $\left({ }^{*} p<0.05,{ }^{* *} p<0.01\right)$. (E) Stereomicroscopy images of PEOT/PBT and 3\% d-rGO-B scaffold cross sections stained with ARS after 35 days of culture in MM. Scale bars $1 \mathrm{~mm}$. (F) Quantification of the ARS staining extracted from scaffolds in $(E)$, normalized to cell number. Data presented as average \pm s.d. and statistical significance performed using T-test (n.s. $p>0.05$ ). 
ECM mineralization was examined at day 35 of culture in BM and MM with ARS, as a late osteogenic marker. Interestingly, both PEOT/PBT and 3\% d-rGO-B scaffolds revealed calcium deposition on the ECM matrix produced by cells inside the pores of scaffolds cultured in MM, as depicted by the ARS in Figure 6E. In spite of a slight increase in the calcium deposition on 3\% d-rGO-B scaffolds, no statistical differences were observed among these and the PEOT/PBT scaffolds (Figure 6F). These results imply that $3 \% \mathrm{~d}$ rGO-B scaffolds support osteogenic differentiation, observed by both early and late stage markers. Yet, these scaffolds seem to not enhance differentiation compared to bare polymeric scaffolds. While this is in disagreement with some previous reports, other studies only point out the enhanced cell adhesion and proliferation ability on composite scaffolds with such low graphene derivatives concentrations (up to $1 \mathrm{wt} \%$ ). ${ }^{16,29,76}$ As previously mentioned, this might very well be correlated to differences in rGO bulk density affecting the final volume fraction of rGO within the scaffolds. It is plausible that the low $\mathrm{d}$ rGO-B concentration used here (3 wt\%) did not allow for sufficient exposure of d-rGO-B to the scaffolds filament surface, preventing it to act as a pre-concentration platform for osteogenic inducers (e.g. dexamethasone and $\beta$-glycerolphosphate), which is believed to be the origin of the enhanced stem cell differentiation exerted by graphene derivatives. ${ }^{80-}$ 82 In this regard, we hypothesize that the increased hydrophilicity and protein adsorption on $10 \%$ and $15 \%$ d-rGO-B scaffolds, together with their surface microroughness, would induce accelerated osteogenic differentiation, compared to $3 \%$ d-rGO-B and PEOT/PBT scaffolds. Nevertheless, future studies will be dedicated to validate this hypothesis, as well as to strike a balance between in vivo biocompatibility and bone formation ability.

\section{CONCLUSION}

Due to its very low bulk density, rGO has to be densified prior to melt compounding with a polymer. The aim of this study was to understand the effect of rGO densification parameters (densification solvent and rGO bulk density after densification) on rGO compaction degree and on the resulting melt compounded composites physicochemical properties and printability via ME-AM. The effect of rGO concentration on the materials' physicochemical properties and cell-material interactions in $2 \mathrm{D}$ and $3 \mathrm{D}$ was also investigated. It was observed that high d-rGO bulk density $(90 \mathrm{~g} / \mathrm{L})$ correlated with higher d-rGO compaction, which was translated into smaller d-rGO volume fraction for a given drGO concentration within the polymer composite, and printability up to $15 \mathrm{wt} \% \mathrm{~d}$-rGO. On 
the other hand, medium bulk density d-rGO (50 g/L) occupied a greater volume within the melt compounded composites, which presented some challenges upon ME-AM at high drGO contents (10 and $15 \mathrm{wt} \%$ ), in terms of loss of filament fidelity, poor layer bonding, or even lack of printability. At a given bulk density (50 g/L), when comparing acetone and water as densification solvent, it was observed that densification in water led to a more compacted d-rGO than d-rGO densified in acetone, and d-rGO was poorly dispersed within the polymer matrix. This led to bigger aggregates formation within the composites, whose connections were able to create conductive pathways within the composites, making these materials the most conductive among all d-rGO types. Composites prepared medium bulk density d-rGO-B densified in acetone were chosen for further characterization, due to a balance of printability and electrical properties. It was observed that increasing $d-r G O$ content led to increasing material hydrophilicity and protein adsorption, as well as to increasing surface roughness due to higher rGO exposure to the surface. Scaffolds prepared with 3, 10 and 15\% d-rGO were found to possess increasing antibacterial properties with increased d-rGO content, without affecting hMSCs viability. Notably, $3 \% \mathrm{~d}$ rGO scaffolds were able to support hMSCs proliferation and osteogenic differentiation. Overall, this study demonstrated that rGO compaction degree and concentration greatly affects composites printability and scaffolds physicochemical and electrical properties. In this regard, a careful selection of the rGO densification parameters has to be made in order to ensure the most adequate properties of the final scaffolds required for bone tissue engineering applications.

\section{Acknowledgement}

We are grateful to the FAST project funded under the H2020-NMP-PILOTS-2015 scheme (GA n. 685825) for financial support. Some of the materials used in this work were provided by the Texas A\&M Health Science Center College of Medicine Institute for Regenerative Medicine at Scott \& White through a grant from NCRR of the NIH (Grant \#P40RR017447). 


\section{References}

1. Wright, Z. M.; Arnold, A. M.; Holt, B. D.; Eckhart, K. E.; Sydlik, S. A., Functional Graphenic Materials, Graphene Oxide, and Graphene as Scaffolds for Bone Regeneration. Regenerative Engineering and Translational Medicine 2019, 5 (2), 190-209.

2. Shin, S. R.; Li, Y.-C.; Jang, H. L.; Khoshakhlagh, P.; Akbari, M.; Nasajpour, A.; Zhang, Y. S.; Tamayol, A.; Khademhosseini, A., Graphene-Based Materials for Tissue Engineering. Advanced Drug Delivery Reviews.

3. Gu, M.; Liu, Y.; Chen, T.; Du, F.; Zhao, X.; Xiong, C.; Zhou, Y., Is Graphene a Promising Nano-Material for Promoting Surface Modification of Implants or Scaffold Materials in Bone Tissue Engineering? Tissue Engineering. Part B, Reviews 2014, 20 (5), 477-491.

4. Lee, C.; Wei, X.; Kysar, J. W.; Hone, J., Measurement of the Elastic Properties and Intrinsic Strength of Monolayer Graphene. science 2008, 321 (5887), 385-388.

5. Du, X.; Skachko, I.; Barker, A.; Andrei, E. Y., Approaching Ballistic Transport in Suspended Graphene. Nature Nanotechnology 2008, 3 (8), 491-495.

6. $\quad$ Loh, K. P.; Bao, Q.; Ang, P. K.; Yang, J., The Chemistry of Graphene. Journal of Materials Chemistry 2010, 20 (12), 2277-2289.

7. Wang, Y.; Chen, X.; Zhong, Y.; Zhu, F.; Loh, K. P., Large Area, Continuous, Few-Layered Graphene as Anodes in Organic Photovoltaic Devices. Applied Physics Letters 2009, 95 (6), 063302.

8. Novoselov, K. S.; Geim, A. K.; Morozov, S. V.; Jiang, D.; Zhang, Y.; Dubonos, S. V.; Grigorieva, I. V.; Firsov, A. A., Electric Field Effect in Atomically Thin Carbon Films. science 2004, 306 (5696), 666-669.

9. Dreyer, D. R.; Park, S.; Bielawski, C. W.; Ruoff, R. S., The Chemistry of Graphene Oxide. Chemical Society Reviews 2010, 39 (1), 228-240.

10. Kuila, T.; Bose, S.; Mishra, A. K.; Khanra, P.; Kim, N. H.; Lee, J. H., Chemical Functionalization of Graphene and Its Applications. Progress in Materials Science 2012, 57 (7), 1061-1105.

11. Pei, S.; Cheng, H.-M., The Reduction of Graphene Oxide. Carbon 2012, 50 (9), 3210-3228.

12. Rostami, F.; Tamjid, E.; Behmanesh, M., Drug-Eluting Pcl/Graphene Oxide Nanocomposite Scaffolds for Enhanced Osteogenic Differentiation of Mesenchymal Stem Cells. Materials Science and Engineering: $C$ 2020, 115, 111102.

13. Zhou, T.; Li, G.; Lin, S.; Tian, T.; Ma, Q.; Zhang, Q.; Shi, S.; Xue, C.; Ma, W.; Cai, X.; Lin, Y., Electrospun Poly(3-Hydroxybutyrate-Co-4-Hydroxybutyrate)/Graphene Oxide Scaffold: Enhanced Properties and Promoted in Vivo Bone Repair in Rats. ACS Applied Materials \& Interfaces 2017, 9 (49), 42589-42600.

14. Cha, C.; Shin, S. R.; Gao, X.; Annabi, N.; Dokmeci, M. R.; Tang, X. S.; Khademhosseini, A., Controlling Mechanical Properties of Cell-Laden Hydrogels by Covalent Incorporation of Graphene Oxide. Small 2014, $10(3), 514-523$.

15. Li, J.; Liu, X.; Crook, J. M.; Wallace, G. G., 3d Printing of Cytocompatible Graphene/Alginate Scaffolds for Mimetic Tissue Constructs. Frontiers in Bioengineering and Biotechnology 2020, 8 (824).

16. Wang, W.; Caetano, G.; Ambler, W. S.; Blaker, J. J.; Frade, M. A.; Mandal, P.; Diver, C.; Bartolo, P., Enhancing the Hydrophilicity and Cell Attachment of 3d Printed Pcl/Graphene Scaffolds for Bone Tissue Engineering. Materials (Basel, Switzerland) 2016, 9 (12).

17. Belaid, H.; Nagarajan, S.; Teyssier, C.; Barou, C.; Barés, J.; Balme, S.; Garay, H.; Huon, V.; Cornu, D.; Cavaillès, V.; Bechelany, M., Development of New Biocompatible 3d Printed Graphene Oxide-Based Scaffolds. Materials Science and Engineering: C 2020, 110, 110595.

18. Jakus, A. E.; Secor, E. B.; Rutz, A. L.; Jordan, S. W.; Hersam, M. C.; Shah, R. N., Three-Dimensional Printing of High-Content Graphene Scaffolds for Electronic and Biomedical Applications. ACS nano 2015, 9 (4), 4636-4648.

19. Angulo-Pineda, C.; Srirussamee, K.; Palma, P.; Fuenzalida, V. M.; Cartmell, S. H.; Palza, H., Electroactive 3d Printed Scaffolds Based on Percolated Composites of Polycaprolactone with Thermally Reduced Graphene Oxide for Antibacterial and Tissue Engineering Applications. Nanomaterials 2020, 10 (3), 428.

20. Potts, J. R.; Dreyer, D. R.; Bielawski, C. W.; Ruoff, R. S., Graphene-Based Polymer Nanocomposites. Polymer 2011, 52 (1), 5-25. 
21. Higginbotham, A. L.; Lomeda, J. R.; Morgan, A. B.; Tour, J. M., Graphite Oxide Flame-Retardant Polymer Nanocomposites. ACS Appl Mater Interfaces 2009, 1 (10), 2256-2261.

22. Fim, F. d. C.; Guterres, J. M.; Basso, N. R.; Galland, G. B., Polyethylene/Graphite Nanocomposites Obtained by in Situ Polymerization. Journal of Polymer Science Part A: Polymer Chemistry 2010, 48 (3), 692-698.

23. Paul, D. R.; Robeson, L. M., Polymer Nanotechnology: Nanocomposites. Polymer 2008, 49 (15), $3187-$ 3204.

24. Kim, H.; Kobayashi, S.; AbdurRahim, M. A.; Zhang, M. J.; Khusainova, A.; Hillmyer, M. A.; Abdala, A. A.; Macosko, C. W., Graphene/Polyethylene Nanocomposites: Effect of Polyethylene Functionalization and Blending Methods. Polymer 2011, 52 (8), 1837-1846.

25. Chen, Q.; Mangadlao, J. D.; Wallat, J.; De Leon, A.; Pokorski, J. K.; Advincula, R. C., 3d Printing Biocompatible Polyurethane/Poly(Lactic Acid)/Graphene Oxide Nanocomposites: Anisotropic Properties. ACS Applied Materials \& Interfaces 2017, 9 (4), 4015-4023.

26. McAllister, M. J.; Li, J.-L.; Adamson, D. H.; Schniepp, H. C.; Abdala, A. A.; Liu, J.; Herrera-Alonso, M.; Milius, D. L.; Car, R.; Prud'homme, R. K.; Aksay, I. A., Single Sheet Functionalized Graphene by Oxidation and Thermal Expansion of Graphite. Chemistry of Materials 2007, 19 (18), 4396-4404.

27. Kalaitzidou, K.; Fukushima, H.; Drzal, L. T., A New Compounding Method for Exfoliated GraphitePolypropylene Nanocomposites with Enhanced Flexural Properties and Lower Percolation Threshold. Composites Science and Technology 2007, 67 (10), 2045-2051.

28. Steurer, P.; Wissert, R.; Thomann, R.; Mülhaupt, R., Functionalized Graphenes and Thermoplastic Nanocomposites Based Upon Expanded Graphite Oxide. Macromolecular Rapid Communications 2009, 30 (4-5), 316-327.

29. Wang, W.; Caetano, G. F.; Chiang, W.-H.; Braz, A. L.; Blaker, J. J.; Frade, M. A. C.; Bartolo, P. J. D. S., Morphological, Mechanical and Biological Assessment of Pcl/Pristine Graphene Scaffolds for Bone Regeneration. International Journal of Bioprinting 2016, 2 (2).

30. Wang, W.; Junior, J. R. P.; Nalesso, P. R. L.; Musson, D.; Cornish, J.; Mendonça, F.; Caetano, G. F.; Bártolo, P., Engineered 3d Printed Poly( $\varepsilon$-Caprolactone)/Graphene Scaffolds for Bone Tissue Engineering. Materials Science and Engineering: C 2019, 100, 759-770.

31. Hou, Y.; Wang, W.; Bártolo, P., Novel Poly ( $\varepsilon$-Caprolactone)/Graphene Scaffolds for Bone Cancer Treatment and Bone Regeneration. 3D Printing and Additive Manufacturing 2020.

32. Sinha, R.; Cámara-Torres, M.; Scopece, P.; Falzacappa, E. V.; Patelli, A.; Moroni, L.; Mota, C., A Hybrid Additive Manufacturing Platform to Create Bulk and Surface Composition Gradients on Scaffolds for Tissue Regeneration. bioRxiv 2020, 2020.2006.2023.165605

33. Cámara-Torres, M.; Sinha, R.; Mota, C.; Moroni, L., Improving Cell Distribution on 3d Additive Manufactured Scaffolds through Engineered Seeding Media Density and Viscosity. Acta Biomaterialia 2020, 101, 183-195.

34. Rane, A. V.; Kanny, K.; Abitha, V. K.; Thomas, S., Chapter 5 - Methods for Synthesis of Nanoparticles and Fabrication of Nanocomposites. In Synthesis of Inorganic Nanomaterials, Mohan Bhagyaraj, S.; Oluwafemi, O. S.; Kalarikkal, N.; Thomas, S., Eds. Woodhead Publishing: 2018; pp 121-139.

35. Kim, H.; Abdala, A. A.; Macosko, C. W., Graphene/Polymer Nanocomposites. Macromolecules 2010, 43 (16), 6515-6530.

36. Araby, S.; Zaman, I.; Meng, Q.; Kawashima, N.; Michelmore, A.; Kuan, H.-C.; Majewski, P.; Ma, J.; Zhang, L., Melt Compounding with Graphene to Develop Functional, High-Performance Elastomers. Nanotechnology 2013, 24 (16), 165601.

37. Zhang, X.; Raj, D. V.; Zhou, X.; Liu, Z., Solvent Evaporation Induced Graphene Powder with High Volumetric Capacitance and Outstanding Rate Capability for Supercapacitors. Journal of Power Sources 2018, 382, 95-100.

38. Poh, H. L.; Šaněk, F.; Ambrosi, A.; Zhao, G.; Sofer, Z.; Pumera, M., Graphenes Prepared by Staudenmaier, Hofmann and Hummers Methods with Consequent Thermal Exfoliation Exhibit Very Different Electrochemical Properties. Nanoscale 2012, 4 (11), 3515-3522.

39. Dimiev, A.; Kosynkin, D. V.; Alemany, L. B.; Chaguine, P.; Tour, J. M., Pristine Graphite Oxide. Journal of the American Chemical Society 2012, 134 (5), 2815-2822. 
40. Guerrero-Contreras, J.; Caballero-Briones, F., Graphene Oxide Powders with Different Oxidation Degree, Prepared by Synthesis Variations of the Hummers Method. Materials Chemistry and Physics 2015, 153, 209-220.

41. Wong, C. H. A.; Sofer, Z.; Kubešová, M.; Kučera, J.; Matějková, S.; Pumera, M., Synthetic Routes Contaminate Graphene Materials with a Whole Spectrum of Unanticipated Metallic Elements. Proceedings of the National Academy of Sciences 2014, 111 (38), 13774.

42. Mohan, V. B.; Brown, R.; Jayaraman, K.; Bhattacharyya, D., Characterisation of Reduced Graphene Oxide: Effects of Reduction Variables on Electrical Conductivity. Materials Science and Engineering: $B$ 2015, 193, 49-60.

43. Díez, N.; Śliwak, A.; Gryglewicz, S.; Grzyb, B.; Gryglewicz, G., Enhanced Reduction of Graphene Oxide by High-Pressure Hydrothermal Treatment. Rsc Advances 2015, 5 (100), 81831-81837.

44. Joshi, S.; Siddiqui, R.; Sharma, P.; Kumar, R.; Verma, G.; Saini, A., Green Synthesis of Peptide Functionalized Reduced Graphene Oxide (Rgo) Nano Bioconjugate with Enhanced Antibacterial Activity. Scientific Reports 2020, 10 (1), 9441.

45. Simón, M.; Benítez, A.; Caballero, A.; Morales, J.; Vargas, O., Untreated Natural Graphite as a Graphene Source for High-Performance Li-lon Batteries. Batteries 2018, 4 (1).

46. Liao, K.-H.; Park, Y. T.; Abdala, A.; Macosko, C., Aqueous Reduced Graphene/Thermoplastic Polyurethane Nanocomposites. Polymer 2013, 54 (17), 4555-4559.

47. Zhao, X.; Zhang, Q.; Chen, D.; Lu, P., Enhanced Mechanical Properties of Graphene-Based Poly(Vinyl Alcohol) Composites. Macromolecules 2010, 43 (5), 2357-2363.

48. Shuai, C.; Feng, P.; Gao, C.; Shuai, X.; Xiao, T.; Peng, S., Graphene Oxide Reinforced Poly(Vinyl Alcohol): Nanocomposite Scaffolds for Tissue Engineering Applications. RSC Advances 2015, 5 (32), 25416-25423.

49. Mahmoud, W. E., Morphology and Physical Properties of Poly(Ethylene Oxide) Loaded Graphene Nanocomposites Prepared by Two Different Techniques. European Polymer Journal 2011, 47 (8), 1534 1540.

50. Shen, B.; Zhai, W.; Tao, M.; Lu, D.; Zheng, W., Enhanced Interfacial Interaction between Polycarbonate and Thermally Reduced Graphene Induced by Melt Blending. Composites Science and Technology 2013, $86,109-116$.

51. Shen, B.; Zhai, W.; Chen, C.; Lu, D.; Wang, J.; Zheng, W., Melt Blending in Situ Enhances the Interaction between Polystyrene and Graphene through П-П Stacking. ACS Applied Materials \& Interfaces 2011, 3 (8), 3103-3109.

52. Marsden, A. J.; Papageorgiou, D. G.; Vallés, C.; Liscio, A.; Palermo, V.; Bissett, M. A.; Young, R. J.; Kinloch, I. A., Electrical Percolation in Graphene-Polymer Composites. 2D Materials 2018, 5 (3), 032003.

53. Huang, K.; Yang, J.; Dong, S.; Feng, Q.; Zhang, X.; Ding, Y.; Hu, J., Anisotropy of Graphene Scaffolds Assembled by Three-Dimensional Printing. Carbon 2018, 130, 1-10.

54. Shanmugam, V.; Rajendran, D. J. J.; Babu, K.; Rajendran, S.; Veerasimman, A.; Marimuthu, U.; Singh, S.; Das, O.; Neisiany, R. E.; Hedenqvist, M. S.; Berto, F.; Ramakrishna, S., The Mechanical Testing and Performance Analysis of Polymer-Fibre Composites Prepared through the Additive Manufacturing. Polymer Testing 2021, 93, 106925.

55. Sinha, R.; Sanchez, A.; Camara-Torres, M.; Uriszar-Aldaca, I. C.; Calore, A. R.; Harings, J.; Gambardella, A.; Ciccarelli, L.; Vanzanella, V.; Sisani, M.; Scatto, M.; Wendelbo, R.; Perez, S.; Villanueva, S.; Matanza, A.; Patelli, A.; Grizzuti, N.; Mota, C.; Moroni, L., Additive Manufactured Scaffolds for Bone Tissue Engineering: Physical Characterization of Thermoplastic Composites with Functional Fillers. bioRxiv 2021, 2021.2003.2023.436548.

56. Wang, W.; Huang, B.; Byun, J. J.; Bártolo, P., Assessment of Pcl/Carbon Material Scaffolds for Bone Regeneration. Journal of the mechanical behavior of biomedical materials 2019, 93, 52-60.

57. Pei, S.; Ai, F.; Qu, S., Fabrication and Biocompatibility of Reduced Graphene Oxide/Poly(Vinylidene Fluoride) Composite Membranes. RSC Advances 2015, 5 (121), 99841-99847.

58. Arriagada, P.; Palza, H.; Palma, P.; Flores, M.; Caviedes, P., Poly(Lactic Acid) Composites Based on Graphene Oxide Particles with Antibacterial Behavior Enhanced by Electrical Stimulus and Biocompatibility. Journal of Biomedical Materials Research Part A 2018, 106 (4), 1051-1060.

59. Kumar, S.; Raj, S.; Kolanthai, E.; Sood, A. K.; Sampath, S.; Chatterjee, K., Chemical Functionalization of Graphene to Augment Stem Cell Osteogenesis and Inhibit Biofilm Formation on Polymer Composites for Orthopedic Applications. ACS Applied Materials \& Interfaces 2015, 7 (5), 3237-3252. 
60. Kumar, S.; Parekh, S. H., Linking Graphene-Based Material Physicochemical Properties with Molecular Adsorption, Structure and Cell Fate. Communications Chemistry 2020, 3 (1), 8.

61. Shi, X.; Chang, H.; Chen, S.; Lai, C.; Khademhosseini, A.; Wu, H., Regulating Cellular Behavior on FewLayer Reduced Graphene Oxide Films with Well-Controlled Reduction States. Advanced Functional Materials 2012, 22 (4), 751-759.

62. Hampitak, P.; Melendrez, D.; Iliut, M.; Fresquet, M.; Parsons, N.; Spencer, B.; Jowitt, T. A.; Vijayaraghavan, A., Protein Interactions and Conformations on Graphene-Based Materials Mapped Using a Quartz-Crystal Microbalance with Dissipation Monitoring (Qcm-D). Carbon 2020, 165, 317-327.

63. Lim, J. Y.; Hansen, J. C.; Siedlecki, C. A.; Runt, J.; Donahue, H. J., Human Foetal Osteoblastic Cell Response to Polymer-Demixed Nanotopographic Interfaces. Journal of The Royal Society Interface 2005, 2 (2), 97-108.

64. Patelli, A.; Mussano, F.; Brun, P.; Genova, T.; Ambrosi, E.; Michieli, N.; Mattei, G.; Scopece, P.; Moroni, L., Nanoroughness, Surface Chemistry, and Drug Delivery Control by Atmospheric Plasma Jet on Implantable Devices. ACS Applied Materials \& Interfaces 2018, 10 (46), 39512-39523.

65. Jia, Z.; Shi, Y.; Xiong, P.; Zhou, W.; Cheng, Y.; Zheng, Y.; Xi, T.; Wei, S., From Solution to Biointerface: Graphene Self-Assemblies of Varying Lateral Sizes and Surface Properties for Biofilm Control and Osteodifferentiation. ACS Applied Materials \& Interfaces 2016, 8 (27), 17151-17165.

66. Zou, X.; Zhang, L.; Wang, Z.; Luo, Y., Mechanisms of the Antimicrobial Activities of Graphene Materials. Journal of the American Chemical Society 2016, 138 (7), 2064-2077.

67. Sengupta, I.; Bhattacharya, P.; Talukdar, M.; Neogi, S.; Pal, S. K.; Chakraborty, S., Bactericidal Effect of Graphene Oxide and Reduced Graphene Oxide: Influence of Shape of Bacteria. Colloid and Interface Science Communications 2019, 28, 60-68.

68. Wang, K.; Ruan, J.; Song, H.; Zhang, J.; Wo, Y.; Guo, S.; Cui, D., Biocompatibility of Graphene Oxide. Nanoscale Res Lett 2010, 6 (1), 8.

69. Holt, B. D.; Arnold, A. M.; Sydlik, S. A., In It for the Long Haul: The Cytocompatibility of Aged Graphene Oxide and Its Degradation Products. Advanced Healthcare Materials 2016, 5 (23), 3056-3066.

70. Mukherjee, S. P.; Gliga, A. R.; Lazzaretto, B.; Brandner, B.; Fielden, M.; Vogt, C.; Newman, L.; Rodrigues, A. F.; Shao, W.; Fournier, P. M.; Toprak, M. S.; Star, A.; Kostarelos, K.; Bhattacharya, K.; Fadeel, B., Graphene Oxide Is Degraded by Neutrophils and the Degradation Products Are Non-Genotoxic. Nanoscale 2018, 10 (3), 1180-1188.

71. Hu, W.; Peng, C.; Lv, M.; Li, X.; Zhang, Y.; Chen, N.; Fan, C.; Huang, Q., Protein Corona-Mediated Mitigation of Cytotoxicity of Graphene Oxide. ACS Nano 2011, 5 (5), 3693-3700.

72. Sydlik, S. A.; Jhunjhunwala, S.; Webber, M. J.; Anderson, D. G.; Langer, R., In Vivo Compatibility of Graphene Oxide with Differing Oxidation States. ACS Nano 2015, 9 (4), 3866-3874.

73. Akhavan, O.; Ghaderi, E.; Akhavan, A., Size-Dependent Genotoxicity of Graphene Nanoplatelets in Human Stem Cells. Biomaterials 2012, 33 (32), 8017-8025.

74. Deschamps, A. A.; Grijpma, D. W.; Feijen, J., Poly (Ethylene Oxide)/Poly (Butylene Terephthalate) Segmented Block Copolymers: The Effect of Copolymer Composition on Physical Properties and Degradation Behavior. Polymer 2001, 42 (23), 9335-9345.

75. Deschamps, A.; van Apeldoorn, A. A.; Hayen, H.; de Bruijn, J. D.; Karst, U.; Grijpma, D. W.; Feijen, J., In Vivo and in Vitro Degradation of Poly (Ether Ester) Block Copolymers Based on Poly (Ethylene Glycol) and Poly (Butylene Terephthalate). Biomaterials 2004, 25 (2), 247-258.

76. Kumar, S.; Azam, D.; Raj, S.; Kolanthai, E.; Vasu, K. S.; Sood, A. K.; Chatterjee, K., 3d Scaffold Alters Cellular Response to Graphene in a Polymer Composite for Orthopedic Applications. Journal of Biomedical Materials Research Part B: Applied Biomaterials 2016, 104 (4), 732-749.

77. Unagolla, J. M.; Jayasuriya, A. C., Enhanced Cell Functions on Graphene Oxide Incorporated 3d Printed Polycaprolactone Scaffolds. Materials Science and Engineering: C 2019, 102, 1-11.

78. Krukiewicz, K.; Putzer, D.; Stuendl, N.; Lohberger, B.; Awaja, F., Enhanced Osteogenic Differentiation of Human Primary Mesenchymal Stem and Progenitor Cultures on Graphene Oxide/Poly(Methyl Methacrylate) Composite Scaffolds. Materials (Basel, Switzerland) 2020, 13 (13), 2991.

79. Owen, T. A.; Aronow, M.; Shalhoub, V.; Barone, L. M.; Wilming, L.; Tassinari, M. S.; Kennedy, M. B.; Pockwinse, S.; Lian, J. B.; Stein, G. S., Progressive Development of the Rat Osteoblast Phenotype in Vitro: Reciprocal Relationships in Expression of Genes Associated with Osteoblast Proliferation and 
Effect of reduced graphene oxide (rGO) compaction degree and concentration on rGO-polymer composites printability and cell interactions

Differentiation During Formation of the Bone Extracellular Matrix. Journal of cellular physiology 1990, 143 (3), 420-430.

80. Lee, W. C.; Lim, C. H. Y. X.; Shi, H.; Tang, L. A. L.; Wang, Y.; Lim, C. T.; Loh, K. P., Origin of Enhanced Stem Cell Growth and Differentiation on Graphene and Graphene Oxide. ACS Nano 2011, 5 (9), 73347341.

81. Luo, Y.; Shen, H.; Fang, Y.; Cao, Y.; Huang, J.; Zhang, M.; Dai, J.; Shi, X.; Zhang, Z., Enhanced Proliferation and Osteogenic Differentiation of Mesenchymal Stem Cells on Graphene Oxide-Incorporated Electrospun Poly(Lactic-Co-Glycolic Acid) Nanofibrous Mats. ACS Applied Materials \& Interfaces 2015, 7 (11), 6331-6339.

82. Nayak, T. R.; Andersen, H.; Makam, V. S.; Khaw, C.; Bae, S.; Xu, X.; Ee, P.-L. R.; Ahn, J.-H.; Hong, B. H.; Pastorin, G.; Özyilmaz, B., Graphene for Controlled and Accelerated Osteogenic Differentiation of Human Mesenchymal Stem Cells. ACS Nano 2011, 5 (6), 4670-4678. 


\section{SUPPLEMENTARY FIGURES}

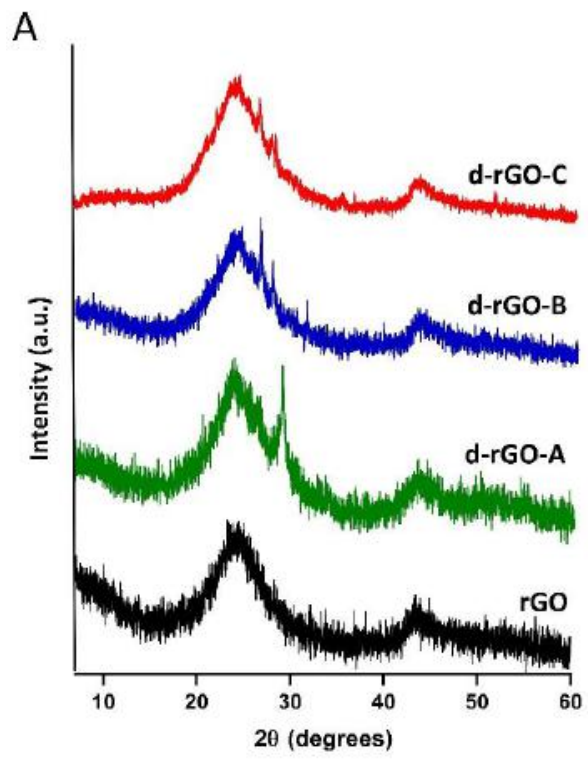

B

\begin{tabular}{cc}
\hline Sample & $\begin{array}{c}\text { FWHM peak (002) } \\
\text { (radians) }\end{array}$ \\
\hline rGO & 0.12 \\
d-rGO-A & 0.14 \\
d-rGO-B & 0.11 \\
d-rGO-C & 0.13 \\
\hline
\end{tabular}

Figure S1. (A) XRD diffraction patterns of the three different d-rGO used in this study, each coming from different $r G O$ batches. (B) FWHM of (002) diffraction peaks.
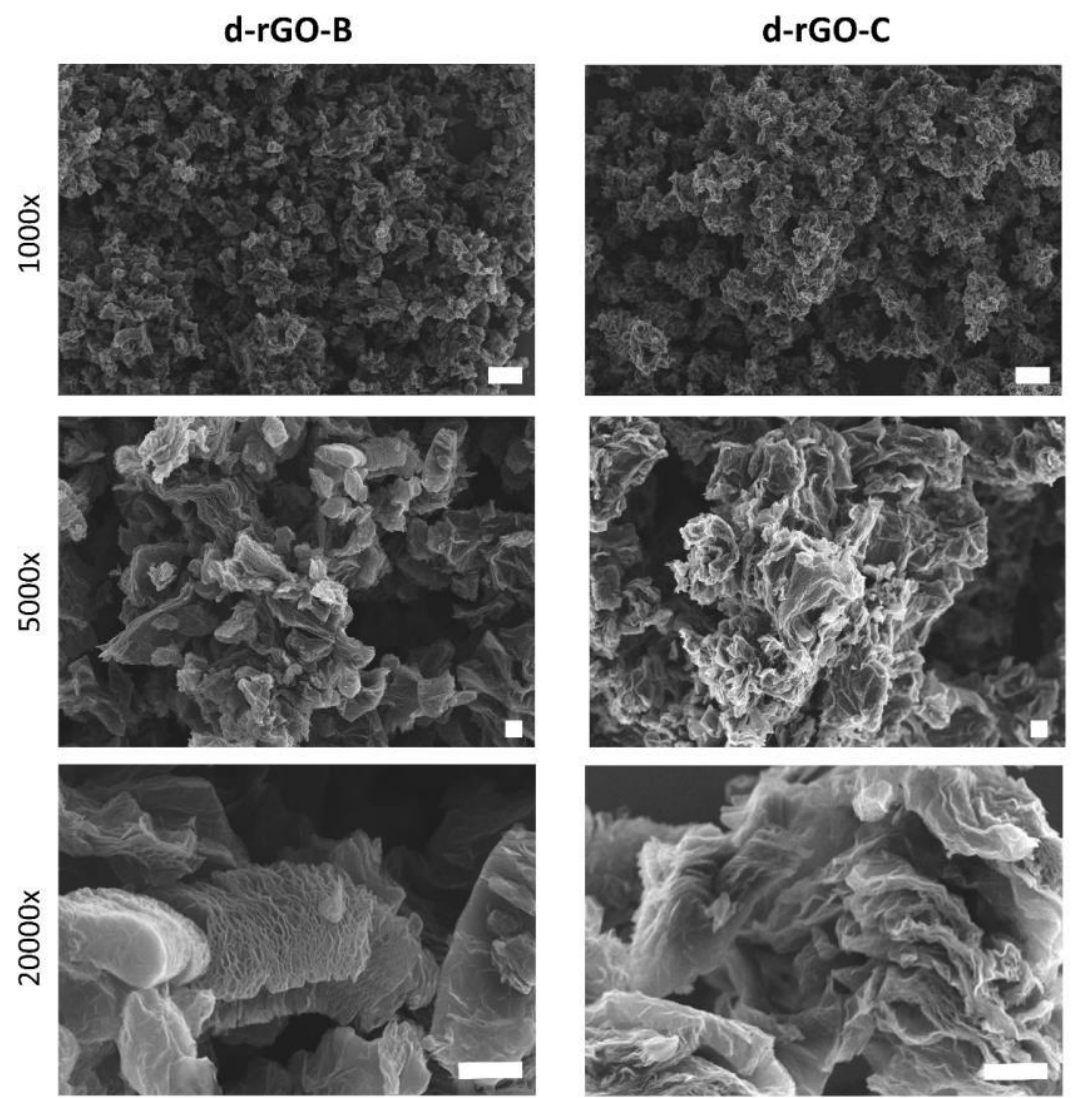

Figure S2. Representative SEM images of d-rGO-B and d-rGO-C at different magnifications, displaying different degrees of compaction. Scale bars $20 \mu \mathrm{m}$ (top row), and $2 \mu \mathrm{m}$ (middle and bottom row). 
A

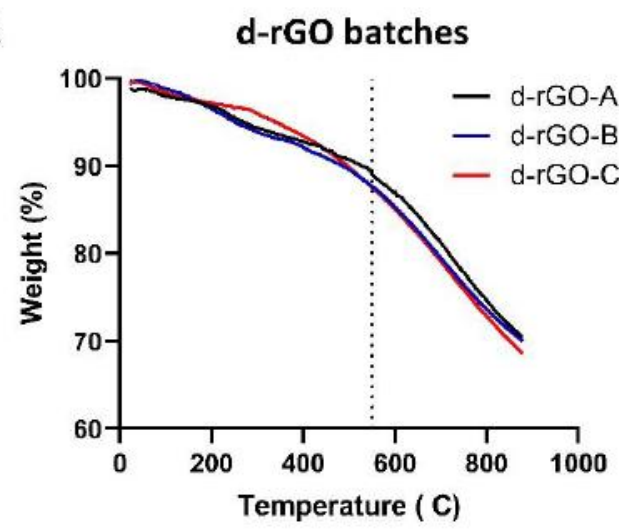

C

PEOT/PBT- d-rGO-B composites

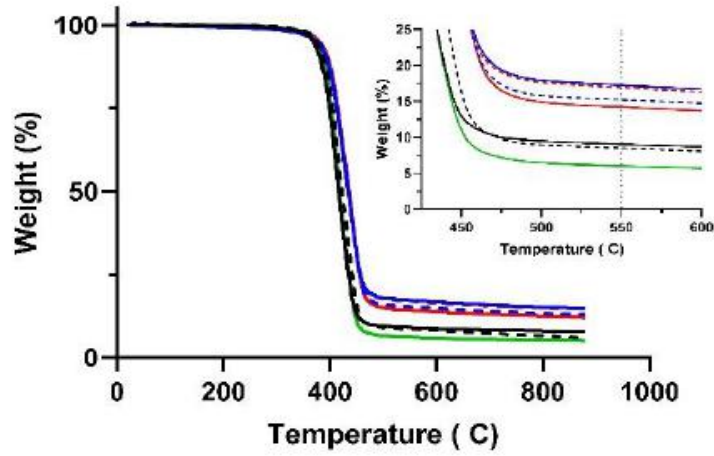

- $3 \%$ d-rGO-B $-10 \%$ d-rGO-B $-15 \%$ d-rGO-B

-.. $3 \%$ d-rGO-B - .. $10 \%$ d-rGO-B -.. $15 \%$ d-rGO-B

- PEOT/PBT
B PEOT/PBT- d-rGO-A composites

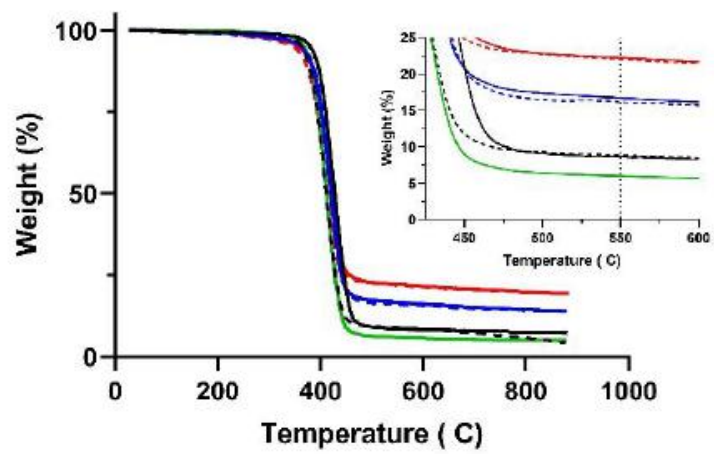

- $3 \%$ d-rGO-A - $10 \%$ d-rGO-A - $15 \%$ d-rGO-A

... $3 \%$ d-rGO-A -.. $10 \%$ d-rGO-A -.. $15 \%$ d-rGO-A

- PEOT/PBT

D PEOT/PBT- d-rGO-C composites

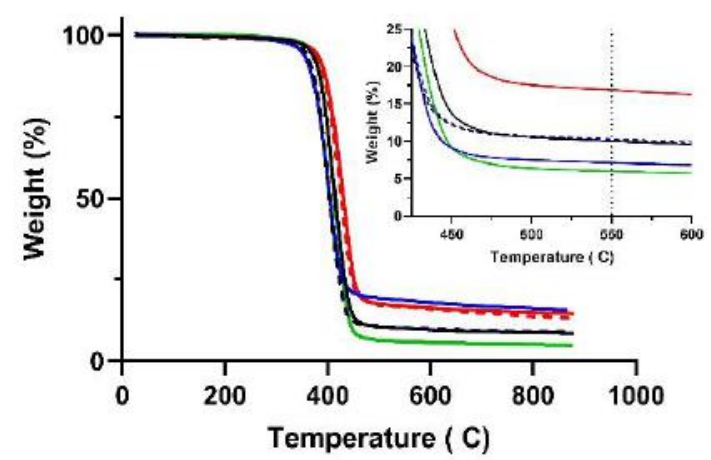

- 3\% d-rGO-C - $10 \%$ d-rGO-C $-15 \%$ d-rGO-C

... $3 \%$ d-rGO-C ... $10 \%$ d-rGO-C -.. $15 \%$ d-rGO-C

- PEOT/PBT

Figure S3. TGA curves of (A) d-rGO batches, (B) PEOT/PBT-d-rGO-A composites, (C) PEOT/PBTd-rGO-B composites and (D) PEOT/PBT-d-rGO-C composites. For each concentration, two curves, measured from two different samples are represented. 
$3 \%$
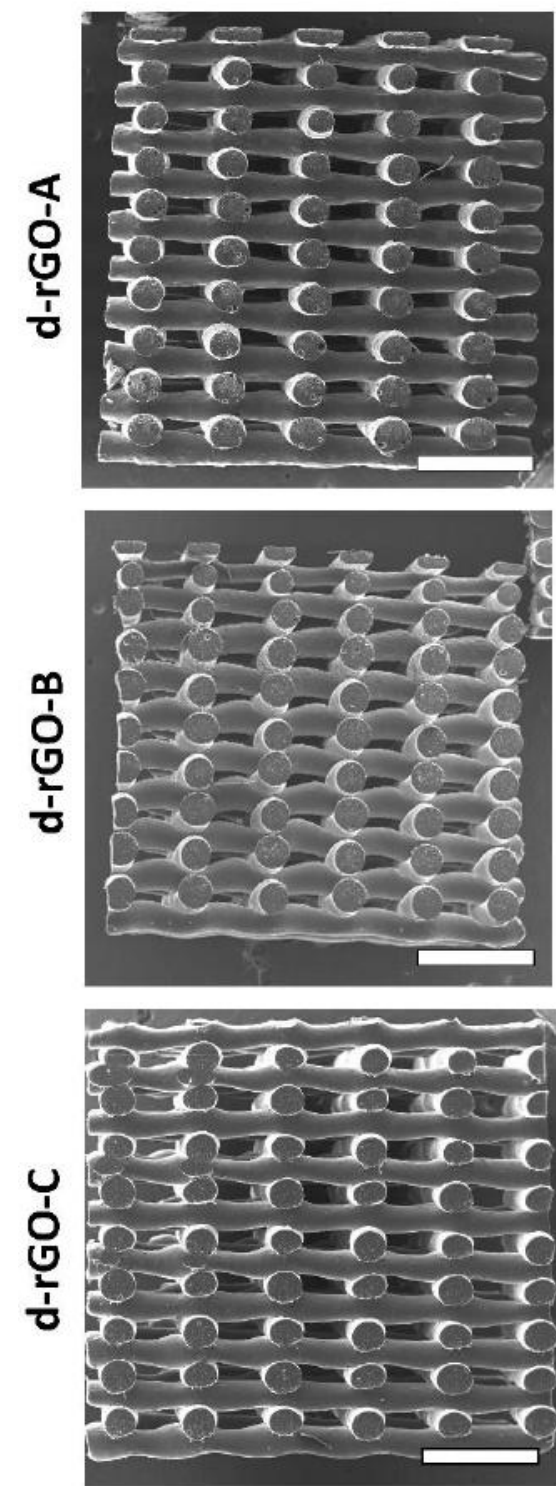

$10 \%$
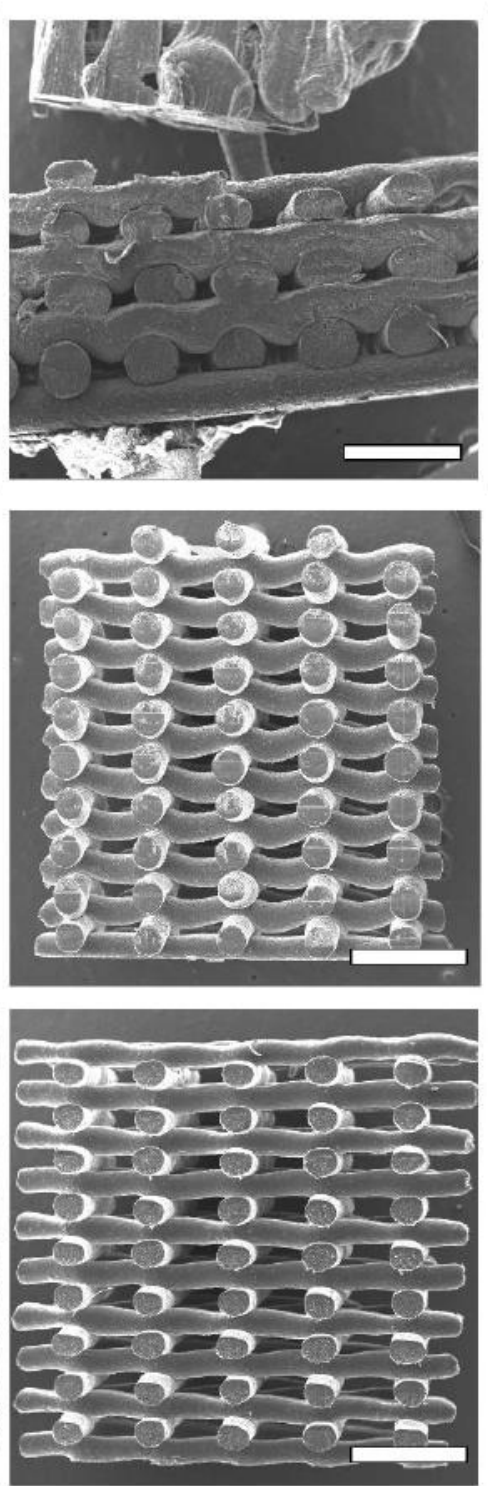

$15 \%$
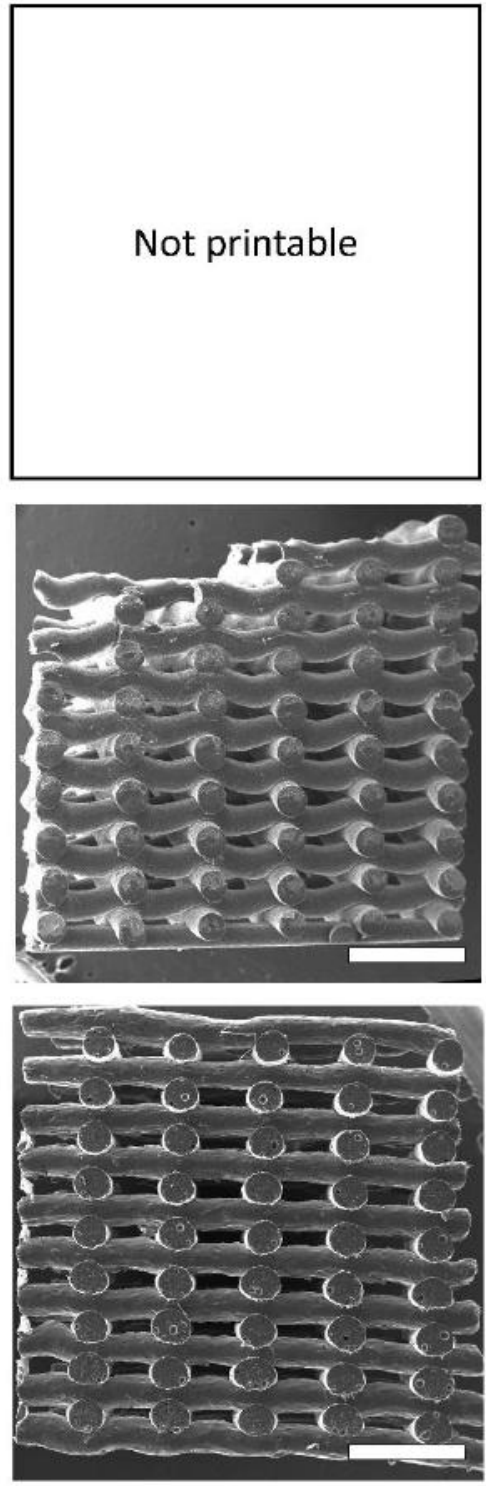

Figure S4. SEM micrographs of 3D ME-AM scaffolds cross sections obtained using each of the drGO composites, depicting scaffolds morphology and interconnected porosity. Scale bars $1 \mathrm{~mm}$. 
$10 \%$ d-rGO-A

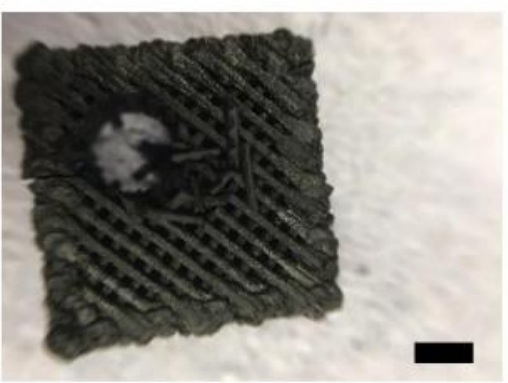

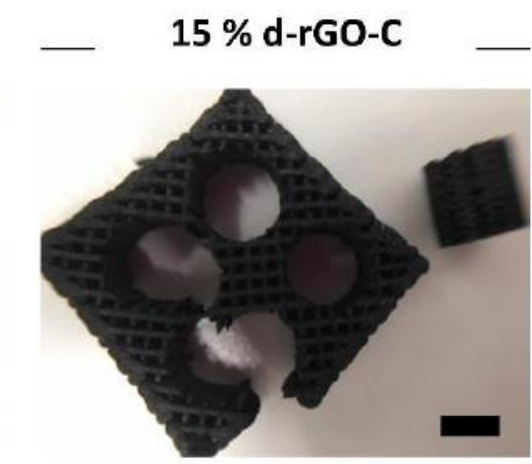

Figure S5. Demonstration of poor layer bonding of $10 \%$ d-rGO-A upon punching of scaffold using a biopsy puncher, compared to other scaffold types, such as the $15 \%$ d-rGO-C scaffold. Scale bars $2 \mathrm{~mm}$.
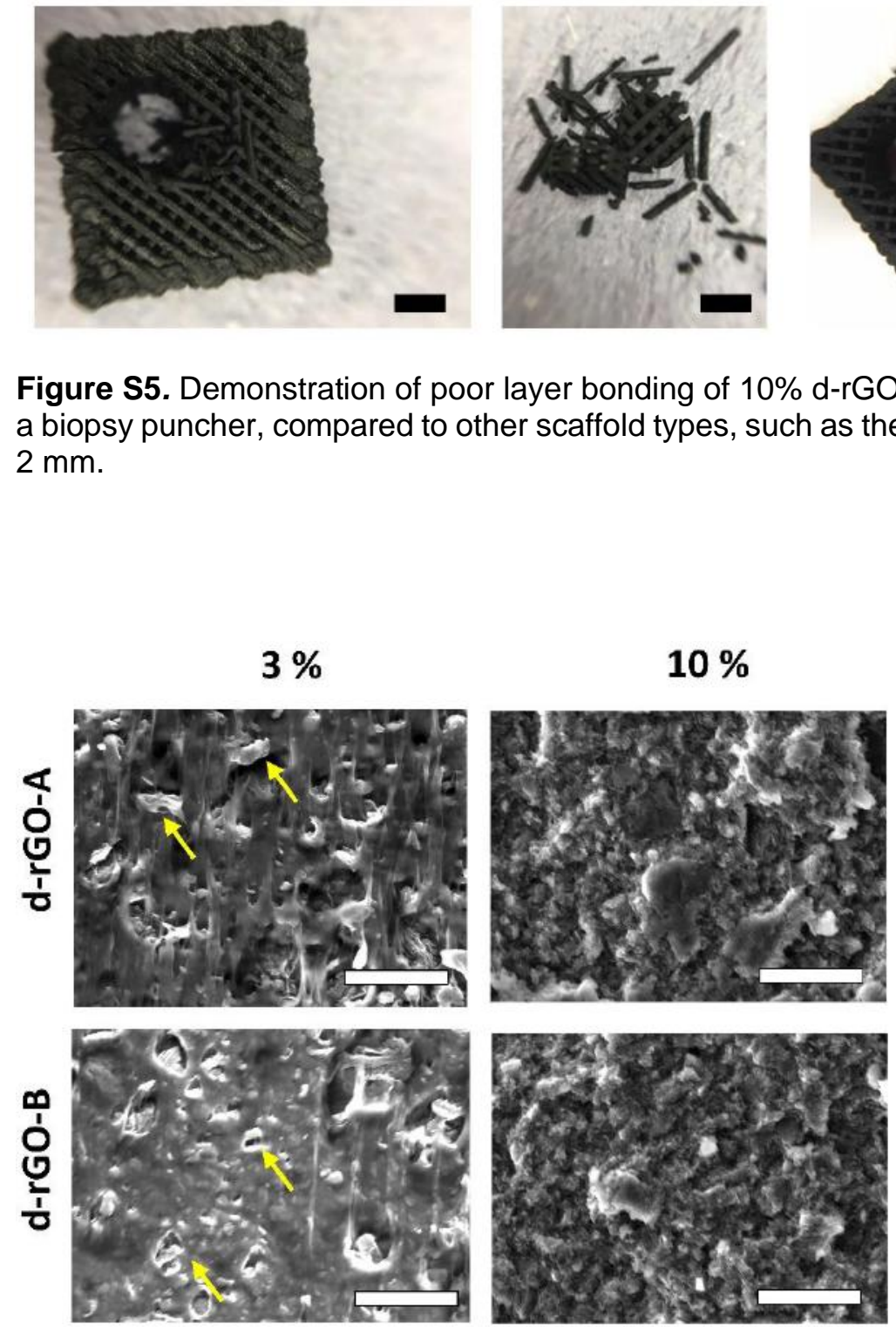

$15 \%$
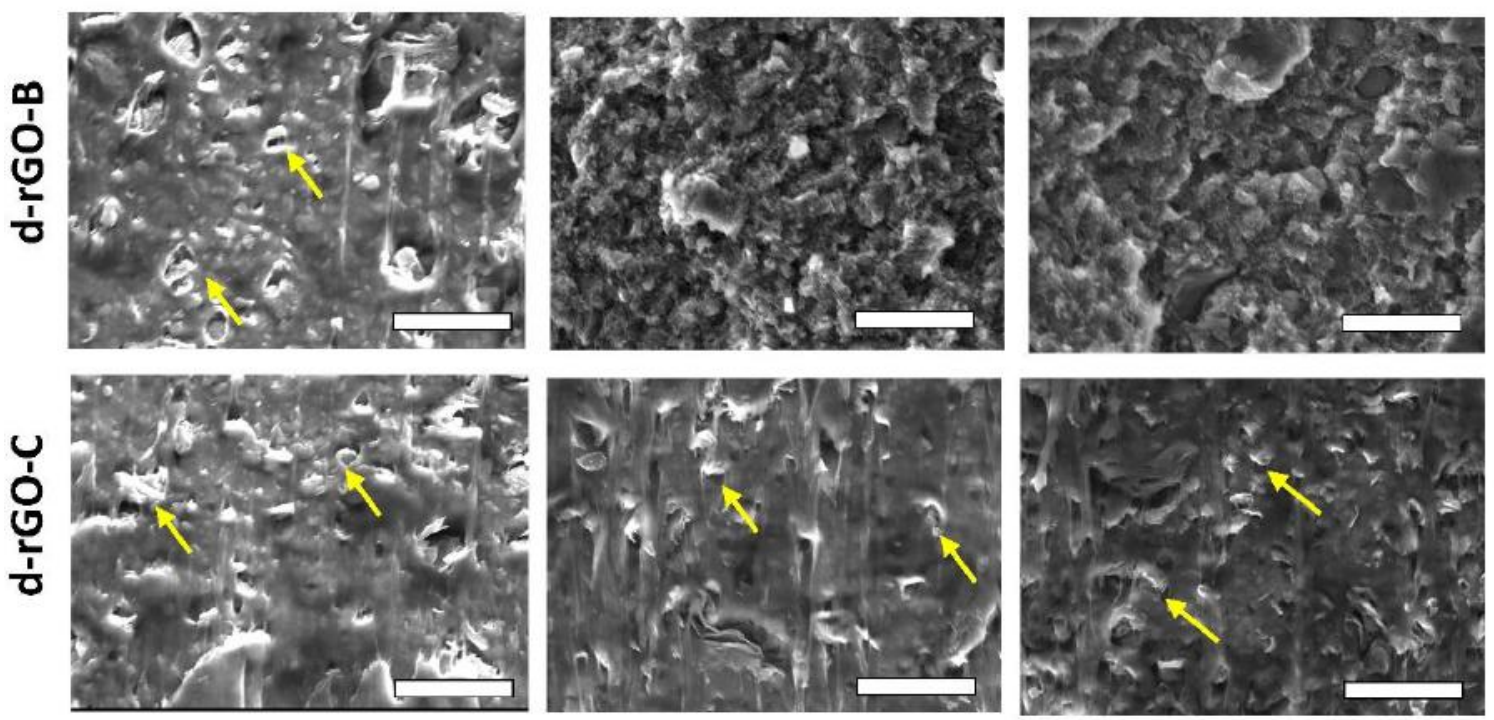

Figure S6. SEM micrographs of 3D ME-AM scaffolds filaments cross sections cut with a razor blade. Yellow arrows indicate rGO particles within the polymer matrix. Scale bars $50 \mu \mathrm{m}$. 
A Disk Diffusion Agar test

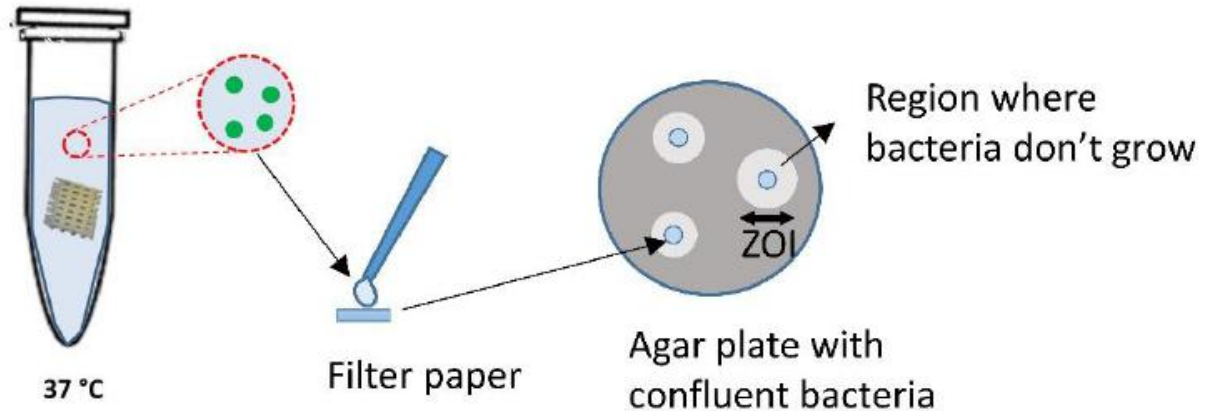

B

\begin{tabular}{c|ccc|ccc}
\hline & \multicolumn{3}{|c|}{$P$ aeruginosa $(\mathrm{ZOI})$} & \multicolumn{3}{c}{ S. epidermidis (ZOI) } \\
\cline { 2 - 7 } & $24 \mathrm{~h}$ & $48 \mathrm{~h}$ & $72 \mathrm{~h}$ & $24 \mathrm{~h}$ & $48 \mathrm{~h}$ & $72 \mathrm{~h}$ \\
\hline PEOT/PBT & 0 & 0 & 0 & 0 & 0 & 0 \\
$\mathbf{3 \%}$ d-rGO-B & 0 & 0 & 0 & 0 & 0 & 0 \\
$\mathbf{1 0 \%}$ d-rGO-B & 0 & 0 & 0 & 0 & 0 & 0 \\
$\mathbf{1 5 \%}$ d-rGO-B & 0 & 0 & 0 & 0 & 0 & 0 \\
\hline
\end{tabular}

C

$P$. aeruginosa (ZOI 24h)

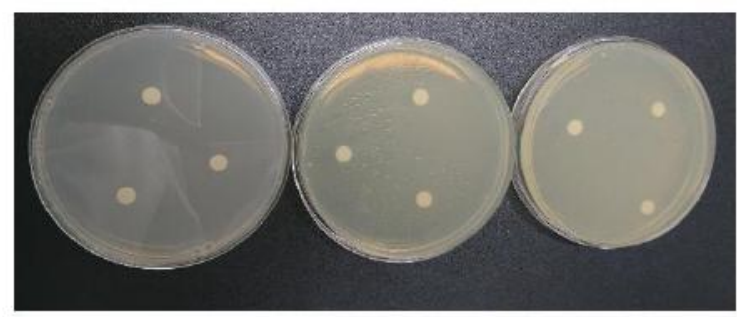

$3 \%(\mathrm{~d}-\mathrm{rGO}-\mathrm{B}) \quad 10 \%$

$15 \%$
S. epidermidis (ZOI 24h)

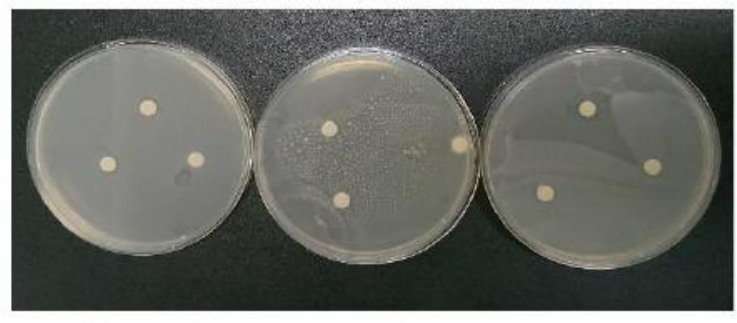

$3 \%(d-r G O-B) \quad 10 \%$

$15 \%$

Figure S7. (A) Disk diffusion agar test diagram. (B) Antimicrobial activity of d-rGO-B scaffolds against $P$. aeruginosa and $S$. epidermidis, measured through the disk diffusion agar test and reported as ZOI values. (C) Images of representative disk diffusion test plates depicting ZOls around a disk impregnated with an aliquot of the scaffold supernatant after the initial $24 \mathrm{~h}$ of incubation. 


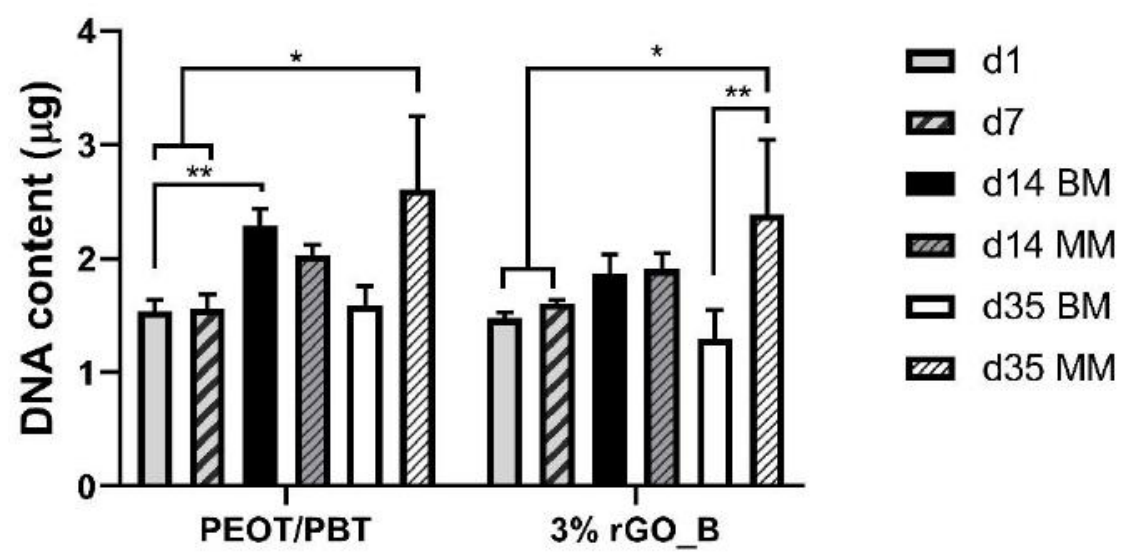

Figure S8. DNA content progression on PEOT/PBT and 3\% d-rGO-B scaffolds over 35 days of culture in BM or MM.

Table S1. Atomic compositions (\%) of each of the d-rGO measured by XPS.

\begin{tabular}{cccccccccc}
\hline & $\mathrm{C}$ & $\mathrm{O}$ & $\mathrm{C} / \mathrm{O}$ ratio & $\mathrm{N}$ & $\mathrm{Al}$ & $\mathrm{Si}$ & $\mathrm{S}$ & $\mathrm{Cl}$ & $\mathrm{Fe}$ \\
\hline d-rGO-A & 85.3 & 14.4 & 5.8 & 0.0 & 0.0 & 0.2 & 0.0 & 0.1 & 0.0 \\
d-rGO-B & 83 & 16 & 5.2 & 0.2 & 0.0 & 0.6 & 0.2 & 0.2 & 0.0 \\
d-rGO-C & 82 & 16.5 & 5.0 & 0.2 & 0.2 & 0.6 & 0.1 & 0.4 & 0.1 \\
\hline
\end{tabular}

Table S2. d-rGO-B antimicrobial activity at different concentrations in contact with $P$. aeruginosa and S. epidermidis.

\begin{tabular}{ccc}
\hline Samples & P. aeruginosa $(\mathrm{R})$ & S. epidermidis $(\mathrm{R})$ \\
\hline $\mathbf{3} \mathbf{m g} / \mathbf{m l ~ d - r G O - B}$ & $>5.8$ & $>4.8$ \\
$\mathbf{1 0} \mathbf{~} \mathbf{m} / \mathbf{m l ~ d - r G O - B}$ & $>5.8$ & $>4.8$ \\
$\mathbf{1 5} \mathbf{~} \mathbf{m} / \mathbf{m l ~ d - r G O - B}$ & $>5.8$ & $>4.8$ \\
\hline
\end{tabular}

Table S3. Antimicrobial activity against $P$. aeruginosa and $S$. epidermidis of PEOT/PBT- d-rGO-B films $(0.1 \mathrm{~g} / \mathrm{mL})$ containing different $\mathrm{d}-\mathrm{rGO}$ concentrations.

\begin{tabular}{ccc}
\hline Samples & P. aeruginosa $(\mathrm{R})$ & S. epidermidis $(\mathrm{R})$ \\
\hline 3\% d-rGO-B film & 1.3 & 2.0 \\
$\mathbf{1 0 \%}$ d-rGO-B film & 5.8 & 4.7 \\
$\mathbf{1 5 \%}$ d-rGO-B film & 6.2 & 4.7 \\
\hline
\end{tabular}





\section{Chapter 7}

3D Additive Manufactured Composite Scaffolds with Antibiotic-loaded Lamellar Fillers for Bone Infection Prevention and Tissue Regeneration 



\title{
3D Additive Manufactured Composite Scaffolds with Antibiotic- loaded Lamellar Fillers for Bone Infection Prevention and Tissue Regeneration
}

\begin{abstract}
María Cámara-Torres ${ }^{1}$, Stacy Duarte ${ }^{1}$, Ravi Sinha ${ }^{1}$, Ainhoa Egizabal ${ }^{2}$, Noelia Álvarez 2, Maria Bastianini ${ }^{3}$, Michele Sisani ${ }^{3}$, Paolo Scopece ${ }^{4}$, Marco Scatto ${ }^{4}$, Alessandro Bonetto ${ }^{5}$, Antonio Marcomini ${ }^{5}$, Alberto Sanchez ${ }^{2}$, Alessandro Patelli ${ }^{6}$, Carlos Mota ${ }^{1}$, Lorenzo Moroni ${ }^{1}$

${ }^{1}$ Maastricht University, MERLN Institute for Technology-Inspired Regenerative Medicine, Complex Tissue regeneration Dep., Maastricht, The Netherlands. ${ }^{2}$ TECNALIA, Basque Research and Technology Alliance (BRTA), San Sebastian, Spain. ${ }^{3}$ Prolabin \& Tefarm S.r.l., Perugia, Italy.

${ }^{4}$ Nadir S.r.l., Venice, Italy. ${ }^{5}$ Dep.of Environmental Sciences, Informatics and Statistics, Ca' Foscari University of Venice, Venice, Italy. ${ }^{6}$ Dep. of Physics and Astronomy, Padova University, Italy.
\end{abstract}

\section{Abstract}

Bone infections following open bone fracture or implant surgery remain a challenge in the orthopedics field. In order to avoid high doses of systemic drug administration, optimized local antibiotic release from scaffolds is required. 3D additive manufactured (AM) scaffolds made with biodegradable polymers are ideal to support bone healing in non-union scenarios and can be given antimicrobial properties by the incorporation of antibiotics. In this study, ciprofloxacin and gentamicin intercalated in the interlamellar spaces of magnesium aluminum layered double hydroxides (MgAl) and a-zirconium phosphates $(\mathrm{ZrP})$, respectively, are dispersed within a thermoplastic polymer by melt compounding and subsequently processed via high temperature melt extrusion $\mathrm{AM}\left(\sim 190{ }^{\circ} \mathrm{C}\right)$ into 3D scaffolds. The inorganic fillers enable a sustained antibiotics release through the polymer matrix, controlled by antibiotics counterions exchange or pH conditions. Importantly, both antibiotics retain their functionality after the manufacturing process at high temperatures, as verified by their activity against both Gram + and Gram - bacterial strains. Moreover, scaffolds loaded with filler-antibiotic do not impair human mesenchymal stromal cells osteogenic differentiation, allowing matrix mineralization and the expression of relevant osteogenic markers. Overall, these results suggest the possibility of fabricating dual functionality $3 \mathrm{D}$ scaffolds via high temperature melt extrusion for bone regeneration and infection prevention. 


\section{INTRODUCTION}

In the orthopedics field, surgical procedures involving fracture stabilizations or implants can develop infections in up to $5 \%$ of the cases, while this rate rises up to $50 \%$ in open bone fracture scenarios. ${ }^{1-2}$ Bacterial infections can trigger osteomyelitis, an inflammatory reaction in bone that can ultimately lead to bone destruction or osteolysis Moreover, bacteria can invade and survive within osteoblasts slowing down or preventing new bone formation, or and create biofilms, which present a challenge to the antibiotic therapy, due to reduced antimicrobial susceptibility leading to prolonged infection. ${ }^{3-5}$ The standard medical strategy to overcome osteomyelitis involves the debridement of infected bone and soft tissue and the removal of the implant or fixation device, accompanied by the systemic administration of antibiotics from 6 to 16 weeks depending on the severity of the infection. 6-7 Typically, high antibiotic concentrations are administered systemically to achieve effective concentrations at the infected site, which can lead to the development of resistant bacterial strains, as well as cell toxicity and adverse effects in the bone regeneration process. ${ }^{8}$ Since the removal of necrotic bone tissue commonly leaves a critical size defects, antibiotic-laden poly(methyl methacrylate) (PMMA) cements or beads were designed to act both as bone spacer and as local antibiotic delivery systems, maximizing target tissue concentrations and minimizing toxicity risks. ${ }^{9-10}$ Despite being one of the gold standard approaches, PMMA cements possess a series of disadvantages including lack of biodegradability, requirement of a second surgery to replace it by other material that allows bone regeneration. Furthermore, PMMA cements present a suboptimal drug elution kinetics, which is associated to a non-reproducible antibiotic-polymer mixing procedure, and to the exothermic polymerization of PMMA. Moreover, they are restricted to heatstable antibiotics. antibiotics can be incorporated. ${ }^{11-12}$ With the recent development in the field of biomaterials and bone tissue engineering, a number of alternative natural (i.e. collagen or calcium phosphates) and synthetic (i.e. poly(lactic acid) (PLA) and poly(lactides-co-glycolides) (PLGA)) carriers have been designed to allow biodegradability and the capability of delivering locally a wider variety of antimicrobial agents. ${ }^{13-14}$ These carriers are mainly collagen, chitosan, calcium sulfate, calcium phosphates, demineralized bone, as well as synthetic polymers, such as polyurethanes, polyanhydrides, PLA and PLGA. ${ }^{13-14}$ However, processing these materials into 3D scaffolds using conventional fabrication techniques, such as gas foaming/particulate leaching, freeze-drying or phase separation, can result in constructs that lack reproducibility or the structural integrity and 
mechanical properties needed to stabilize a non-union defect, making them also not fully functional candidates for facilitating bone healing following infection management.

In the past decade, additive manufacturing ( $\mathrm{AM}$ ) and, more specifically, melt extrusion (ME)-based techniques (ME-AM), have emerged as one of the most appealing technologies to produce scaffolds for bone tissue engineering. ${ }^{15}$ This method allows for the reproducible fabrication of patient-personalized 3D scaffolds, from a variety of biocompatible and biodegradable thermoplastic polymers, with an interconnected pore network, high porosity, and optimum mechanical properties for bone regeneration. Osteoinductive and antibacterial scaffolds have recently been developed by combining AM and drug delivery. Antimicrobial peptides, ${ }^{16-17}$ or other antimicrobial compounds, such as silver, ${ }^{18}$ bioactive glass, ${ }^{19}$ or quaternized chitosan, ${ }^{20-21}$ have been incorporated onto the surface of 3D AM scaffolds. These alternative compounds have been used to avoid the use of antibiotics due to their potential cell toxicity and bacterial resistance risks. Yet, the in vivo efficacy of these substitutes compared to conventional antibiotic therapies is still unproven. Therefore, as a common strategy, antibiotics have been adsorbed on the surface of AM scaffolds. However, surface adsorption via the immersion of the scaffold in an antibiotic solution has shown a limit retention of the loaded antibiotic, amount that correlates to the scaffold surface area, resulting in poor loading efficiency and leading to the burst release of the antibiotic. ${ }^{22}$ In order to increase the loading efficiency and allow for a more sustained drug release, different strategies have been explored, such as porogen leaching to create microporosity in the scaffold's filaments, hydrogel coatings, or loading of antibiotics within degradable polymeric microparticles. ${ }^{22-24}$ The combination of several of these loading methods in the same scaffold has also been considered to allow the incorporation of different antibiotics and to obtain a sequential release profile for a more potent therapy. ${ }^{25}$ However, in spite of enhancing the scaffold antimicrobial properties, these multi-step post-modification approaches are complex and laborious. Thus, the direct incorporation of the antibiotic within the scaffold material would be desired to simplify the fabrication process, as well as to improve the loading capability and release profile. With the exception of ME of polymers at low temperatures, such as polycaprolactone (PCL), ${ }^{26-}$ 27 the incorporation of antibiotics in the scaffold material is not frequently investigated due to the sensitivity of bioactive molecules to temperature. This in turn limits the polymer choice, as a large majority of the thermoplastic polymers for biomedical applications require long exposure to high processing temperatures $\left(\sim 175-220{ }^{\circ} \mathrm{C}\right),{ }^{28-29}$ which are above the thermal stability limits of most commonly used antibiotics $\left(\sim 120^{\circ} \mathrm{C}\right.$, depending 
on exposure time and antibiotic type). ${ }^{30}$ Moreover, most of current delivery systems lack the optimum release kinetics of the cargo. A desirable sustained drug release should ensure that an adequate antibiotic concentrations is delivered on site, above the minimal inhibitory concentration (MIC) values and over a sufficient time to cover the critical window period post-surgery, followed by a sustained release at an effective level for inhibiting the occurrence of a latent infection. ${ }^{31-32}$

Here, we introduce a novel approach that allows the direct fabrication of drug loaded scaffolds via high temperature ME-AM. The composite scaffolds consist of the biocompatible and biodegradable copolymer poly(ethyleneoxideterephthalate)/ poly(butyleneterephthalate) (PEOT/PBT) and the inorganic layered compounds magnesium aluminum layered double hydroxide (MgAl) or $\alpha$-Zirconium phosphate $(\mathrm{ZrP})$, which contain antibiotics intercalated within their lamellar structures. Ciprofloxacin (CFX) and gentamicin (GTM), two commonly used antibiotics to treat orthopedic infections, are used as model anionic and cationic molecules to be intercalated within the positively charged $\mathrm{MgAl}$ and negatively charged ZrP fillers, respectively. We evaluate the dual functionality of these scaffolds as i) local antibiotic delivery systems against Staphylococcus epidermidis (Gram +) and Pseudomonas aeruginosa (Gram -), identified as two of the major responsible for implant associated infections, ${ }^{33}$ and ii) their potential to support bone tissue formation.

\section{MATERIALS AND METHODS}

A list of materials described in this study and the abbreviations used to refer to them can be found in Table 1.

Table 1. List of materials

\begin{tabular}{|c|c|c|c|}
\hline Nomenclature & Description & Nomenclature & Description \\
\hline$C F X$ & Ciprofloxacin & $\begin{array}{l}5,10,20 \% \\
Z r P-G T M \_p\end{array}$ & $\begin{array}{l}\text { Pellets composed of } 5 \text {, } \\
10,20 \mathrm{wt} \% \text { ZrP-GTM } \\
\text { dispersed in PEOT/PBT }\end{array}$ \\
\hline GTM & Gentamicin & $\begin{array}{l}5,10,20 \% \\
\text { ZrP-GTM_s }\end{array}$ & $\begin{array}{l}\text { Scaffold composed of 5, } \\
10,20 \mathrm{wt} \% \text { ZrP-GTM } \\
\text { dispersed in PEOT/PBT }\end{array}$ \\
\hline
\end{tabular}




\begin{tabular}{|c|c|c|c|}
\hline$M g A l$ & $\begin{array}{l}\text { Magnesium aluminum } \\
\text { layer double hydroxide }\end{array}$ & $\begin{array}{l}\text { 5, 10, } 20 \% \\
\text { ZrP-GTM_f }\end{array}$ & $\begin{array}{l}\text { Film composed of } 5,10 \text {, } \\
20 \quad w t \% \text { ZrP-GTM } \\
\text { dispersed in PEOT/PBT }\end{array}$ \\
\hline$Z r P$ & $\begin{array}{l}\text { a-Zirconium phosphate } \\
\text { lamellar compound }\end{array}$ & $10 \%$ CFX_p & $\begin{array}{l}\text { Film composed of } 10 \\
\text { wt\% CFX dispersed in } \\
\text { PEOT/PBT }\end{array}$ \\
\hline$M g A l-C F X$ & $\begin{array}{l}\text { MgAl filler loaded with } \\
\text { CFX }\end{array}$ & $10 \%$ CFX_f & $\begin{array}{l}\text { Film composed of } 10 \\
\text { wt\% CFX dispersed in } \\
\text { PEOT/PBT }\end{array}$ \\
\hline ZrP-GTM & ZrP filler loaded with GTM & $10 \%$ GTM_p & $\begin{array}{l}\text { Film composed of } 10 \\
\text { wt\% GTM dispersed in } \\
\text { PEOT/PBT }\end{array}$ \\
\hline PEOT/PBT_p & PEOT/PBT pellets & $10 \%$ GTM_f & $\begin{array}{l}\text { Film composed of } 10 \\
\text { wt\% GTM dispersed in } \\
\text { PEOT/PBT }\end{array}$ \\
\hline PEOT/PBT_s & PEOT/PBT scaffolds & $10 \% M g A l \_p$ & $\begin{array}{l}\text { Film composed of } 10 \\
\text { wt\% } \\
\text { dispersed in PEOT/PBT }\end{array}$ \\
\hline $\begin{array}{l}\text { 5, 10, } 20 \% \\
M g A I-C F X \_p\end{array}$ & $\begin{array}{l}\text { Pellets composed of } 5 \text {, } \\
10,20 \text { wt } \% \text { MgAl-CFX } \\
\text { dispersed in PEOT/PBT }\end{array}$ & $10 \% M g A l s$ & $\begin{array}{l}\text { Scaffold composed of } \\
10 \mathrm{wt} \% \mathrm{MgAl}-\mathrm{CO}_{3} \\
\text { dispersed in PEOT/PBT }\end{array}$ \\
\hline $\begin{array}{l}\text { 5, 10, } 20 \% \\
M g A l-C F X \_s\end{array}$ & $\begin{array}{l}\text { Scaffold composed of } 5 \text {, } \\
10,20 \mathrm{wt} \% \mathrm{MgAl} \text {-CFX } \\
\text { dispersed in PEOT/PBT }\end{array}$ & $10 \% Z r P \_p$ & $\begin{array}{l}\text { Film composed of } 10 \\
\text { wt\% ZrP-H dispersed in } \\
\text { PEOT/PBT }\end{array}$ \\
\hline $\begin{array}{l}\text { 5, 10, } 20 \% \\
M g A I-C F X \_f\end{array}$ & $\begin{array}{l}\text { Film composed of } 5,10 \text {, } \\
20 \quad w t \% \quad \text { MgAl-CFX } \\
\text { dispersed in PEOT/PBT }\end{array}$ & $10 \% \mathrm{ZrP} \_s$ & $\begin{array}{l}\text { Scaffold composed of } \\
10 \mathrm{wt} \% \mathrm{ZrP}-\mathrm{H} \text { dispersed } \\
\text { in PEOT/PBT }\end{array}$ \\
\hline
\end{tabular}

\section{MgAl synthesis and CFX intercalation}

$\mathrm{MgAl}$ in nitrate form (MgAl) was obtained adapting the urea method from Costantino et al. ${ }^{34}$ Solid urea was added to $0.5 \mathrm{~m}$ magnesium nitrate hexahydrate (M II) - aluminum nitrate nonahydrate (M III), having molar fraction $\mathrm{M}(\mathrm{III}) /(\mathrm{M}$ (III) $+\mathrm{M}(\mathrm{II}))$ equal to 0.33 , until the molar ratio urea/M (III) reached the value of 6 . The mixture was heated at $100{ }^{\circ} \mathrm{C}$ for $48 \mathrm{~h}$. The final product was filtered, washed with water and dried in oven at $60{ }^{\circ} \mathrm{C}$. MgAl-CFX was prepared via anionic intercalation. A carbon dioxide free water solution of $\mathrm{NaOH} 1 \mathrm{~m}$ $(36 \mathrm{ml})$ was added to a suspension of CFX $(12.2 \mathrm{~g})$ in a hydroalcoholic solution $(370 \mathrm{ml}$, water: $\mathrm{EtOH} 1: 1)$ in order to obtain a sodium salt solution. Then MgAl (10 g) was added (MgAl:CFX 1:1 molar ratio). The suspension was kept under $\mathrm{N}_{2}$ and stirred for one day at 
RT. The reaction mixture was centrifuged, washed twice with a water-ethanol solution and dried in oven at $45^{\circ} \mathrm{C}$.

\section{ZrP-synthesis and GTM intercalation}

ZrP synthesis and GTM intercalation was carried out as previously described. ${ }^{35}$ Briefly, crystalline zirconium phosphate $\mathrm{Zr}\left(\mathrm{HPO}_{4}\right) 2 \cdot \mathrm{H}_{2} \mathrm{O}(\mathrm{ZrP})$ was obtained by refluxing zirconyl chloride $\left(\mathrm{ZrOCl}_{2} \cdot 8 \mathrm{H}_{2} \mathrm{O}\right)$ in a $10 \mathrm{~m}$ phosphoric acid solution for $48 \mathrm{~h}$. The residual solid was centrifuged, washed 3 times with water and dried in oven at $60{ }^{\circ} \mathrm{C}$. A pre-intercalated phase of ZrP with an expanded interlayer distance was obtained via ion exchange with propylamine, followed by $\mathrm{HCl}$ mixing to regenerate the acid form. ZrP-GTM was prepared by adding of GTM (18.9 g) to the gel. The mixture was stirred for $24 \mathrm{~h}$ at RT until complete exchange of the protons with GTM. The reaction mixture was centrifuged, washed twice with deionized water and dried in the oven at $40{ }^{\circ} \mathrm{C}$.

\section{Composite production}

The production of 5, 10, $20 \%$ MgAl-CFX_p, 5, 10, $20 \%$ ZrP-GTM_p, $10 \%$ MgAl_p and $10 \%$ ZrP_p composites was carried out in a lab scale co-rotating twin screw extruder installed in Nadir S.r.l., with a screw diameter of $11 \mathrm{~mm}$ and a length-to-diameter ratio (L/D) of 40 . The screw profile was composed of 8 zones with three interposed kneading sections. PEOT/PBT pellets (PEO molecular weight $=300 \mathrm{kDa}$, PEOT:PBT weight ratio $=55: 45$, intrinsic viscosity $0.51 \mathrm{dl} / \mathrm{g}$, Polyvation, The Netherlands, PEOT/PBT_p) were fed in the main hopper with a volumetric feeder. Using a double inlet, MgAl-CFX or ZrP-GTM powder were fed in main hopper at 5, 10, $20 \mathrm{wt} \%$. The screw rotation speed was fixed at $80 \mathrm{rpm}$ while the barrel temperature was set at $140^{\circ} \mathrm{C}$ for the first zone and temperatures from 145 to $150^{\circ} \mathrm{C}$ for following zones. The nanocomposite wire was taken at the die exit, solidified in air and pelletized in a pelletizer machine. The production of $10 \%$ CFX_p and $10 \%$ GTM_p was carried out in co-rotating twin screw extruder by pre-mixing grinded PEOT/PBT_p and CFX or GTM powder (9:1 by weight). The screw rotation speed was fixed at 80 while the barrel temperature was set at $150^{\circ} \mathrm{C}$. The nanocomposite wire was taken at the die exit, solidified in air and manually pelletized. 


\section{Composite materials characterization}

TGA measurements of CFX, GTM, MgAl-CFX, ZrP-GTM, MgAl-CFX_p and ZrP-GTM_p were carried out with an STD Q600 thermal analyser (TA Instruments, USA) in air flow with a heating rate of $10^{\circ} \mathrm{C} / \mathrm{min}$ up to $800^{\circ} \mathrm{C}$. Inorganic filler content within MgAl-CFX and ZrP-GTM was calculated to be $54 \mathrm{wt} \% \mathrm{MgAl}$ ( $46 \mathrm{wt} \% \mathrm{CFX}$ ) and $46 \mathrm{wt} \% \mathrm{ZrP}$ (54 wt\% GTM), respectively. These results in combination with the MgAl-CFX_p and ZrP-GTM_p TGA curves were used to calculate the experimental filler-antibiotic loading of the each polymer composite. Results are presented in Table S6.

Particle size distribution was assessed using the image analysis software tool XT Pro v3.2 (Soft Imaging System GmbH).

For high-performance liquid chromatography (HPLC) analysis, a precise amount of sample was weighted. When a polymer composite was analysed, sample was dissolved in chloroform by sonication for $20 \mathrm{~min}$ at RT, followed by 10 min centrifugation at $4600 \mathrm{rpm}$. The supernatant was aspirated and the residual chloroform was allowed to evaporate under $\mathrm{N}_{2}$. The pelleted MgAl-CFX or ZrP-GTM was treated with a suitable volume of $\mathrm{HCl}$ (1 $\mathrm{M})$ or in $\mathrm{HCl} / \mathrm{KCl}(3 \mathrm{M})$, respectively, for antibiotic extraction. Then, the solution was filtered (pore size $0,2 \mu \mathrm{m}$ ) or centrifuged for filler separation and appropriately diluted. The amount of CFX was assessed by means of a HPLC column (Zorbax SB C18 4,6 x 250 $\mathrm{mm}, 5 \mu \mathrm{m}$ ) with a flow rate of $0.8 \mathrm{ml} \mathrm{min}^{-1}$, and the eluent consisting of water and $2 \%$ acetic acid/acetonitrile at a ratio $84 / 16 \mathrm{v} / \mathrm{v} \%$. In order to assess the uncertainty involved in the samples dilutions, all samples were diluted and analyzed three times. A five point calibration curve was used to quantitatively determine the CFX content.

The amount of GTM was assessed by means of a LC-MS Bruker qTOF Compact. The chromatographic separation was conducted using an Agilent Zorbax SB-C18 column (4.6 $\mathrm{mm} \times 150 \mathrm{~mm}, 3.5 \mu \mathrm{m}$ ) with a flow rate of $0.4 \mathrm{ml} / \mathrm{min}$ and the eluent consisting of trifluoroacetic acid (TFA) water $(0.2 \mathrm{M})$ and methanol at a ratio $92 / 8 \mathrm{v} / \mathrm{v} \%$. The mass spectrometer was operated in full scan mode, and for the mass calibration, sodium formate clusters were used. According to literature, the four main isoform components of GTM were separated and quantified by extracting the high resolution mass chromatogram of the selected compounds with an uncertainty of 0.01 a.u.. For the quantification of the total amount of antibiotic loaded into the composites, the sum of all four species was taken into account. In order to assess the uncertainty involved in the samples dilutions, all samples were diluted and analyzed twice. 


\section{Scaffolds fabrication and characterization}

Scaffolds were fabricated via ME-AM. The platform (Bioscaffolder, Gesim) was equipped with a custom-made print head with two separate heating sources for the cartridge and screw. Briefly, the cartridge was filled with MgAl-CFX_p or ZrP-GTM_p, heated at $185^{\circ} \mathrm{C}$ and extruded at $190-195^{\circ} \mathrm{C}$ by applying a pressure of 8 bar, an auger screw rotation of 50-60 rpm and a translation speed of $15-20 \mathrm{~mm} / \mathrm{s}$. Parameters were adjusted slightly around these values for each material type and composition. 10\% MgAl_p and 10\% ZrP_p were also used to prepare only filler control scaffolds with the same aforementioned parameters. Similarly, PEOT/PBT_p were heated and extruded at $195^{\circ} \mathrm{C}$ by applying a pressure of $4 \mathrm{bar}$, an auger screw rotation of $30 \mathrm{rpm}$ and a translational speed of $15 \mathrm{~mm} / \mathrm{s}$. The scaffolds architecture consisted of a 0-90 pattern, with a $340 \mu \mathrm{m}$ fiber diameter, 250 $\mu \mathrm{m}$ layer thickness and $850 \mu \mathrm{m}$ strand distance (center to center), giving an expected $\mathrm{x}-\mathrm{y}$ porosity of $510 \mu \mathrm{m}$ and an expected z porosity of $90 \mu \mathrm{m}$. Cylindrical scaffolds of $4 \mathrm{~mm}$ diameter and $4 \mathrm{~mm}$ height were punched out from $15 \times 15 \times 4 \mathrm{~mm}^{3}$ manufactured blocks using a biopsy punch and used for further experiments.

Scaffold morphology and porosity was assessed using a stereomicroscope (Nikon SMZ25). Stereomicroscopy images were used to measure the experimental values of fiber diameter and $x-y-z$ porosity, and these values were inserted in the Landers equation (Equation 1) to calculate the average scaffold porosity. ${ }^{36}$ Presence and distribution of fillers within gold sputter-coated scaffolds' filaments surface and cross section was examined using backscattered scanning electron microscopy (BSEM, XL-30 beam voltage $20 \mathrm{kV}$, spot size 5). Scanning electron microscopy (SEM) operating at $25 \mathrm{kV}$ coupled with energy dispersive X-ray spectroscopy (EDS) was used to observe the chemical composition of the samples. Particle size distribution on scaffolds was assessed using the image analysis software tool XT Pro v3.2 (Soft Imaging System GmbH).

Equation 1. Landers formula for scaffold porosity calculation, where d1 is the experimental fiber diameter, $\mathrm{d} 2$ is the experimental strand distance and $\mathrm{d} 3$ is the experimental layer thickness.

$$
\operatorname{Porosity}(\%)=\left(1-\frac{\pi}{4} \cdot \frac{1}{\left(\frac{d 2}{d 1}\right)} \cdot \frac{1}{\left(\frac{d 3}{d 1}\right)}\right) \cdot 100
$$

\section{Antibiotic release kinetics from films and 3D scaffolds}

To assess the antibiotic release from each of the composite 3D scaffolds, 5, 10, $20 \%$ MgAl-CFX_s and 5, 10, 20\% ZrP-GTM_s were disinfected (20 min, 70\% ethanol), to mimic 
the disinfection performed to the samples used for in vitro culture, and subsequently incubated for 4 weeks in $1 \mathrm{ml}$ Dulbecco's phosphate buffered saline (dPBS) at $37^{\circ} \mathrm{C}$ at static conditions.

2D films were prepared from $10 \%$ CFX_p; $10 \%$ GTM_p; 5, 10 and $20 \%$ MgAl-CFX_p; and 5, 10, and $20 \%$ ZrP-GTM_p. Briefly, 60 milligrams of pellets were molten at $190{ }^{\circ} \mathrm{C}$ and pressed with a coverslip against a Teflon sheet to obtain $14 \mathrm{~mm}$ diameter, $\sim 300 \mu \mathrm{m}$ thickness films. To evaluate the effect of the filler on antibiotic release, films were incubated after disinfection (20 min, $70 \%$ ethanol) for 3 weeks in $1 \mathrm{ml} \mathrm{dPBS}$ at $37^{\circ} \mathrm{C}$ at static conditions, with both sides being exposed to the solution. To assess the effect of $\mathrm{pH}$ on antibiotic release, films were incubated in buffer solutions of acidic, neutral and basic $\mathrm{pH}$ : pH 4 (potassium hydrogen phthalate based buffer), $\mathrm{pH} 7.5$ (dPBS) and pH 10.5 (sodium tetraborate based buffer). To evaluate the effect of the eluent's ionic concentration on antibiotic release, $20 \% \mathrm{MgAl}$-CFX_f were incubated for 3 weeks in $1 \mathrm{ml}$ of dPBS (containing $10 \mathrm{~mm} \mathrm{Na}_{2} \mathrm{HPO}_{4}$, or $6.7 \mathrm{~mm} \mathrm{PO}_{4}{ }^{3-}$ ) or modified dPBS (with $2 \mathrm{~mm}$ or $50 \mathrm{~mm}$ $\mathrm{Na}_{2} \mathrm{HPO}_{4}$, equivalent to $1.3 \mathrm{~mm}$ or $33.5 \mathrm{~mm} \mathrm{PO}_{4}{ }^{3-}$, respectively). Similarly, $20 \%$ ZrP-GTM_f were incubated for 3 weeks in $1 \mathrm{ml}$ of dPBS (containing $135 \mathrm{~mm} \mathrm{NaCl}$, or $53 \mathrm{~mm} \mathrm{Na}$ ) or modified dPBS (with $27 \mathrm{~mm}$ or $685 \mathrm{~mm} \mathrm{NaCl}$, equivalent to $11 \mathrm{~mm}$ or $265 \mathrm{~mm} \mathrm{Na}^{+}$, respectively).

At each timepoint, the supernatant was collected and films or scaffolds placed on $1 \mathrm{ml}$ of fresh corresponding solution. CFX in the supernatants was detected by direct measurement of CFX absorbance at $275 \mathrm{~nm}$ using a nanodrop UV-Vis spectrophotometer (Biodrop). CFX concentrations were calculated from a CFX standard curve. GTM was detected by mixing equal volumes of supernatant, isopropanol and o-phthaldialdehyde reagent (Sigma-Aldrich) and adding $4 \mu \mathrm{ml}^{-1}$ 2-mecaptoethanol (Sigma Aldrich). ${ }^{37}$ After 30 min incubation at RT, the fluorescent complex formed was detected using a spectrophotometer (CLARIOstar®, BMG Labtech) at excitation/emission= 340/455 nm. GTM concentrations were calculated from a GTM standard curve.

To investigate the drug release mechanism, $20 \%$ MgAl-CFX_s and 20\% ZrP-GTM_s release curves ( $\%$ antibiotic release from the total loading vs. time) were fitted to 7 commonly used drug kinetic models: zero order, first order, Ritger-Peppas, Higuchi, Bhaskar, modified Freundlich and parabolic diffusion. 


\section{Antibacterial activity}

\section{Susceptibility of bacterial strains to CFX and GTM}

In order to analyze the susceptibility of bacterial strains to CFX and GTM the Agar diskdiffusion method was applied. Mueller- Hinton agar plates were spread with a standardized inoculum of $P$. aeruginosa (CECT 116) and $S$. epidermidis (CECT 231). Then, commercial filter paper discs impregnated with $20 \mu \mathrm{l}$ of antibiotics at different concentrations $(500,250$, $100,75,50,25,15,10,5$ and $0 \mu \mathrm{g} \mathrm{ml}^{-1}$ ) were placed on the agar surface. The Petri dishes were incubated under $37 \stackrel{\circ}{\circ} \mathrm{C}$ during 18-24 hours. After incubation, zones of growth inhibition (ZOI) around each of the discs (including disc diameter) were measured to the nearest millimeter.

\section{Antibacterial activity of filler-antibiotic complexes and films}

Antibacterial activity of fillers-antibiotic complexes (MgAl-CFX and ZrP-GTM) at different concentrations (5, 10 and $20 \mathrm{mg} / \mathrm{ml}$ ), and films (5, 10, 20\% MgAl-CFX_f and 5, 10, 20\% ZrP-GTM_f) was evaluated by shaking the samples in a concentrated bacterial suspension (106 CFU ml-1) in nutrient broth (1:500) for $24 \mathrm{~h}$ at $37^{\circ} \mathrm{C}$. After incubation, the number of viable bacteria present in the suspension was measured by placing aliquots of the suspensions and their dilutions (in triplicate) in sterile petri dishes with molten nutrient agar and swirled gently. The petri dishes were incubated at $37^{\circ} \mathrm{C}$ for $24 \mathrm{~h}$, after which the colonies present on the plates were counted. Values are reported as the $\log _{10}$ reduction (R), calculated as the difference in the log base 10 of the viable cell counts found on a suspension that has not been in contact with the sample and a suspension that has been in contact with the sample.

\section{Antibacterial activity of scaffolds}

The antibacterial activity of the antibiotics released from 5, 10, $20 \%$ MgAl-CFX_s and 5, 10, 20\% ZrP-GTM_s scaffolds incubated in dPBS solution was evaluated using the Agar disk-diffusion method, as described in previous section.

\section{Cell seeding and culture}

HMSCs)isolated from bone marrow were purchased from Texas A\&M Health Science Center, College of Medicine, Institute for Regenerative Medicine (Donor d8011L, female, age 22). Cryopreserved vials at passage 3 were plated at a density of $1000 \mathrm{cells} \mathrm{cm}^{-2}$ in tissue culture flasks and expanded until approximately $80 \%$ confluency in complete media 
(CM) consisting of aMEM with Glutamax and no nucleosides (Gibco) supplemented with $10 \%$ fetal bovine serum (FBS), without penicillin-streptomycin (PenStrep) at $37{ }^{\circ} \mathrm{C} / 5 \%$ $\mathrm{CO}_{2}$

\section{Metabolic activity assay to determine antibiotic toxicity}

The cytotoxicity of CFX and GTM was evaluated via PrestoBlue ${ }^{\text {TM }}$ assay. Initially, trypsinized hMSCs at passage 4 were seeded at a density of $5000 \mathrm{cell}^{-2}$ in the wells of a 48 wellplate, with $\mathrm{CM}$ without PenStrep, and allowed to attach for 24 hours at $37^{\circ} \mathrm{C} / 5 \%$ $\mathrm{CO}_{2}$. The medium was then replaced with a fresh medium containing CFX or GTM at different concentrations, starting from $1 \mathrm{mg} \mathrm{ml}^{-1}$ or $2.5 \mathrm{mg} \mathrm{ml}^{-1}$, respectively. CM without antibiotics was used as control. After $24 \mathrm{~h}$, cells were washed with PBS and $250 \mu \mathrm{l}$ of Presto Blue solution (10\% PrestoBlue in $\mathrm{CM}$ ) was added to each well and incubated for $1.5 \mathrm{~h}$ at $37{ }^{\circ} \mathrm{C} / 5 \% \mathrm{CO}_{2}$. After incubation, the supernatants were collected and the fluorescence was measured (emission/excitation $=590 / 560 \mathrm{~nm}$ ) using a spectrophotometer.

\section{Cell seeding on 3D scaffolds}

Scaffolds were disinfected in $70 \%$ ethanol for 20 min, washed 3 times with dPBS and incubated for $1.5 \mathrm{~h}$ in $\mathrm{CM}$ for initial burst release of the antibiotic. Scaffolds that were not pre-incubated in CM were used as controls. Before seeding, scaffolds were dried on top of a sterile filter paper and placed in the wells of a non-treated wellplate. Passage $4 \mathrm{hMSCs}$ were trypsinized, centrifuged $5 \mathrm{~min}$ at $500 \mathrm{rcf}$ and resuspended in a dextran solution (500 $\mathrm{kDa}$, Farmacosmos) (10 wt/wt \% dextran in CM), to achieve uniform cell seeding, at a density of 200,000 cells per $35 \mu \mathrm{l} .^{38}$ The cell suspension $(35 \mu \mathrm{l})$ was placed on top of each scaffold as a droplet, which was drawn into the scaffolds' pores and retained inside due to the hydrophobicity of the wellplate. Seeded scaffolds were incubated for 4 hours at $37^{\circ} \mathrm{C}$ / $5 \% \mathrm{CO}_{2}$ to allow cells to attach. After this time, scaffolds were transferred to new wells containing $1.5 \mathrm{ml}$ of basic media (BM) (CM supplemented with $200 \mu \mathrm{M}$ L-Ascorbic acid 2phosphate). BM was replaced after $24 \mathrm{~h}$ and every two or three days from then on. To evaluate hMSCs osteogenic differentiation, after 7 days in BM, scaffolds were cultured in mineralization media (MM) consisting of BM supplemented with dexamethasone (10 nM) (Sigma-Aldrich) and $\beta$-glycerophosphate (10 mm) (Sigma-Aldrich) for another 28 days or until mineral nodules were observed (49 days in the case of MgAl-CFX_s, or 35 days in the case of ZrP-GTM_s). 


\section{Imaging of cell viability in 3D scaffolds}

After $1 \mathrm{~h}$ seeding and after 7 days of culture, dead cells were stained for 20 minutes prior to fixation using the Live/Dead ${ }^{\mathrm{TM}}$ fixable far-red dead cell stain kit (Thermo Fisher Scientific) at a concentration of $0.5 \mu \mathrm{l}$ stain in $500 \mu \mathrm{l}$ Hank's Balanced Salt Solution per scaffold. Subsequently, samples were washed, fixed with $4 \%$ paraformaldehyde for $30 \mathrm{~min}$ and permeabilized using $0.1 \%$ Triton X-100 for $30 \mathrm{~min}$. Samples were incubated with phalloidin (Alexa Fluor 488, 1:75 in PBS) for $1 \mathrm{~h}$ at RT as counterstain. Finally, samples were washed 3 times with PBS. Scaffolds cross sections were imaged with a fluorescent microscope (Eclipse, Ti2-e, NIKON). Background subtraction and brightness adjustments were performed on the images using the software Image $\mathrm{J}$, in order to clarify their visualization. No image quantification was performed on the processed images.

\section{Biochemical assays}

Alkaline phosphatase (ALP) assay

ALP activity was evaluated on MgAl-CFX_s or ZrP-GTM_s after 47 days and 35 days of culture in MM, respectively (timepoints day 56 and day 42, respectively). 3D scaffolds were collected at every timepoint and washed with PBS, stored at $-80^{\circ} \mathrm{C}$ and freeze-thawed 3 times, to improve lysis efficiency. Samples were incubated for $1 \mathrm{~h}$ at $\mathrm{RT}$ in a cell lysis buffer composed of $\mathrm{KH}_{2} \mathrm{PO}(0.1 \mathrm{M}), \mathrm{K}_{2} \mathrm{HPO}_{4}(0.1 \mathrm{~m})$, and Triton X-100 (0.1 v\%), at pH 7.8. The chemiluminescent substrate for ALP CDP star ${ }^{\circledR}$ ready to use reagent (Roche) was added to the cell lysate at a 1:4 ratio. Luminescence was measured using a spectrophotometer. Remaining cell lysates were kept for DNA quantification. ALP values were reported normalized to DNA content.

\section{DNA assay}

DNA assay was performed on cells cultured on all 3D scaffolds after 1 and 7 days in BM (timepoints day 1 and day 7). Additionally it was performed on PEOT/PBT_s, 10\% MgAI_s and 10\% ZrP_s after 28 days in MM (timepoint day 35), on MgAI-CFX_s after 28 and 49 days in MM (timepoints day 35 and day 56), and on ZrP-GTM_s after 28 and 35 days in MM (timepoint day 35 and day 42). CyQUANT cell proliferation assay kit (Thermo Fisher Scientific) was used. Lysed samples from ALP assay or frozen samples after collection, were incubated overnight at $56{ }^{\circ} \mathrm{C}$ in Proteinase $\mathrm{K}$ solution $(1 \mathrm{mg} / \mathrm{ml}$ Proteinase $\mathrm{K}$ (SigmaAldrich) in Tris/EDTA buffer) for matrix degradation and cell lysis. Subsequently, samples 
were freeze-thawed three times and incubated $1 \mathrm{~h}$ at RT with a 20X diluted lysis buffer from the kit containing RNase A (1:500) to degrade cellular RNA. Lysed samples were incubated with the fluorescent dye provided by the kit (1:1) for $15 \mathrm{~min}$ and fluorescence was measured (emission/excitation $=520 / 480 \mathrm{~nm}$ ) with a spectrophotometer. DNA concentrations were calculated from a DNA standard curve.

\section{Alizarin red $S$ (ARS) staining and quantification}

Calcium deposition was qualitatively determined by ARS staining on MgAl-CFX_s or ZrPGTM_s after 28 and 49 days (timepoints day 35 and day 56, respectively), or 28 and 35 days (timepoints day 35 and day 42, respectively) of culture in $M M$, respectively. PEOT/PBT_s, 10\% MgAl_s and 10\% ZrP_s scaffolds were stained after 28 days of culture in MM (timepoint day 35). Briefly, 3D scaffolds were fixed with 4\% paraformaldehyde and washed with distilled water. Subsequently, scaffolds were stained with ARS (60 mm, pH 4.1 - 4.3) for $20 \mathrm{~min}$ at RT, and washed with distilled water. Images of scaffolds' cross sections were taken using a stereomicroscope (Nikon SMZ25). After imaging, stained samples (whole scaffold) were incubated for $1 \mathrm{~h}$ at RT while shaking, followed by $10 \mathrm{~min}$ incubation at $85{ }^{\circ} \mathrm{C}$, with $30 \mathrm{v} \%$ acetic acid. Afterwards, scaffolds were removed and solutions were centrifuged at 20,000 rcf for $10 \mathrm{~min}$. An appropriate volume of ammonium hydroxide ( $5 \mathrm{~m}$ ) was added to the supernatants to bring the $\mathrm{pH}$ to 4.2 . The absorbance was measured at $405 \mathrm{~nm}$ using a spectrophotometer. Concentration of ARS was calculated from an ARS standard curve and the values were normalized to DNA content.

\section{Immunostaining}

Cells were fixed in 4\% paraformaldehyde after 35 days (PEOT/PBT_s and ZrP-GTM_s) or 47 days (MgAl-CFX_s) of culture in MM (timepoints day 42 and day 56, respectively). Triton-X 100 (0.1 v\%) was added and incubated for $30 \mathrm{~min}$. Samples were washed 3 times with PBS and then blocked for $1 \mathrm{~h}$ with blocking buffer (3\% BSA + 0.01\% Triton-X 100). Afterwards, primary antibodies were added (1:200 Collagen I (COL I) polyclonal antibody rabbit-derived (ab34710, Abcam), or 1:200 Osteopontin (OPN) polyclonal antibody rabbitderived (ab8448, Abcam), in washing buffer (10x diluted blocking buffer)) and incubated overnight. Samples were washed 3 times with washing buffer prior to secondary antibody incubation (1:200 Alexa Fluor 488 goat derived anti rabbit antibody (Thermo Fisher Scientific), for PEOT/PBT and MgAl-CFX scaffolds, or 1:200 Alexa Fluor 647 goat derived anti rabbit (Thermo Fisher Scientific), for ZrP-GTM based scaffolds, in washing buffer). 
After $1 \mathrm{~h}$ incubation, scaffolds were washed as before and stained for F-actin (647 Alexa Fluor Phalloidin (Thermo Fisher Scientific) for PEOT/PBT_s and MgAI-CFX_s, and 568 Alexa Fluor Phallodin (Thermo Fisher Scientific) for ZrP-GTM_s (1:75 in PBS)), incubated for $1 \mathrm{~h}$ and washed 3 times with PBS. Confocal laser scanning microscopy was performed with a tandem confocal system (Leica TCS SP8 STED), equipped with a white light laser (WLL). Samples were excited with the dye specific wavelengths using the WLL. Emission was detected with $\mathrm{HyD}$ detectors (F-actin, OPN, COL I). For optimal visualization in the reported images, F-actin was colored in green, COL I in red and OPN in blue for all sample types.

\section{Statistical analysis}

All data is shown as average with error bars indicating the standard deviation of at least three replicates. Analysis of statistics was conducted with GraphPad Prism (version 8.0.1). A one-way or two-way analysis of variance (ANOVA) was performed followed by a Tukey's post-hoc multiple comparison test to evaluate statistical significance and correct for multiple comparisons.

\section{RESULTS AND DISCUSSION}

\section{Scaffolds fabrication and characterization}

Although the sustained release of drugs from MgAl ${ }^{39-40}$ and $\mathrm{ZrP}{ }^{41-43}$ has been previously reported, the combination of antibiotic-loaded fillers with polymers has not been carefully examined in terms of release kinetics, antimicrobial functionality or assurance of no negative effect on the bone regeneration response. Most importantly, no previous studies have reported their processing into 3D AM scaffolds, but only as implant coatings ${ }^{44}$ or incorporated on electrospun scaffolds. ${ }^{45}$ Here, 3D polymer composite scaffolds with fillerantibiotic concentrations up to 20 wt\% (MgAl-CFX_s or ZrP-GTM_s, Table 1) were successfully fabricated for the first time via ME-AM of previously prepared melt-blended composite pellets (MgAl-CFX_p or ZrP-GTM_p) (Figure 1 and Figure S1). The biodegradable polyether-ester multiblock copolymer PEOT/PBT was selected as model thermoplastic polymer to produce the scaffolds due to its processability at high temperatures $\left(\geq 190^{\circ} \mathrm{C}\right)$, and its proven in vitro and in vivo applicability in the orthopedic field. ${ }^{46-50}$ All composite scaffolds exhibited interconnected macropores, and experimental 
filament diameters, as well as $X-Y$ and $Z$ pores sizes consistently matched the theoretical values (Figure S2). Scaffolds presenting pores of $>300 \mu \mathrm{m}$ and porosity values in the range of $\sim 50 \%$ (Figure S2B) suggest that these constructs will support bone regeneration. ${ }^{51}$

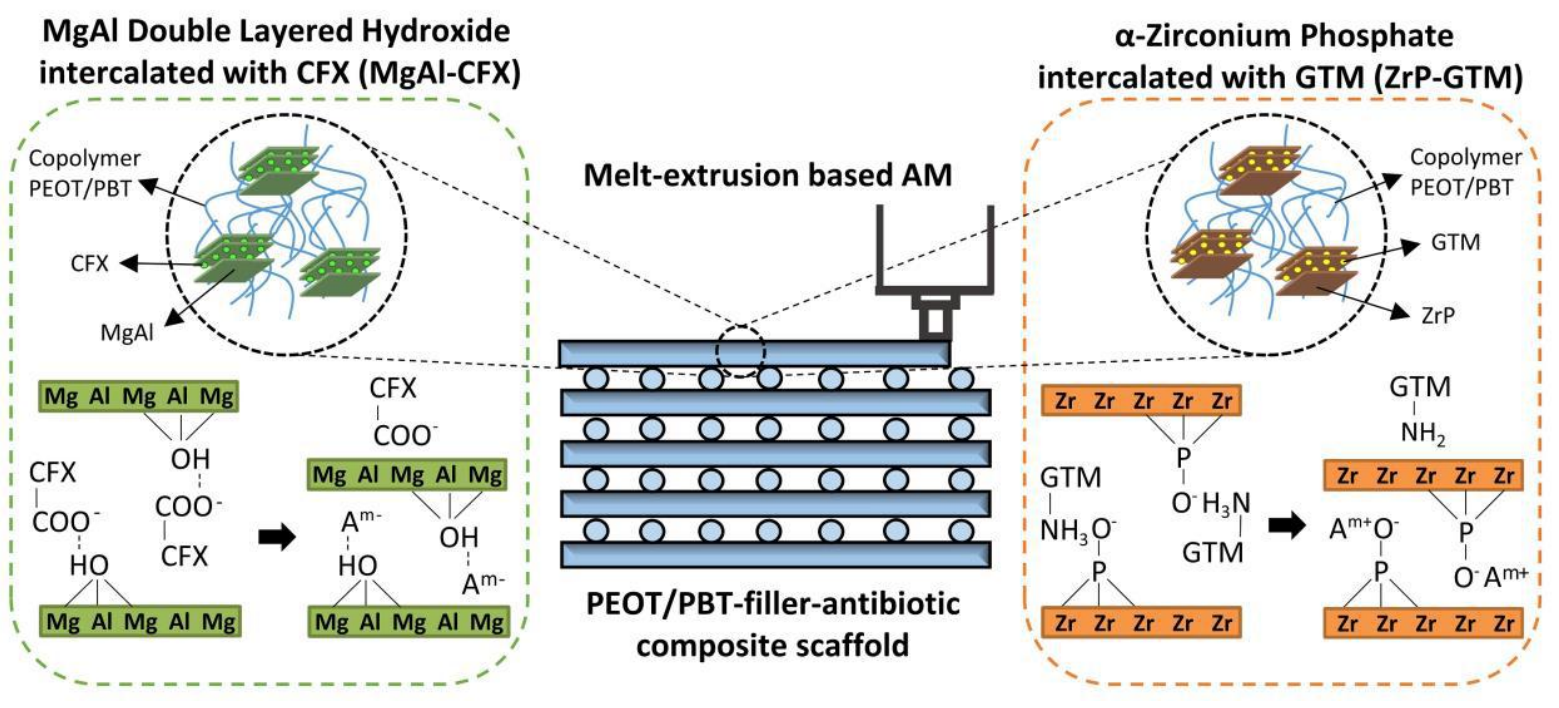

Figure 1. Schematic representation of the antibacterial composite scaffolds (PEOT/PBT-fillerantibiotic) fabricated by melt-extrusion AM. These polymeric composite scaffolds contain either MgAl intercalated with CFX (MgAl-CFX), or ZrP intercalated with GTM (ZrP-GTM). When submerged in an eluent solution, anions can exchange with CFX, which diffuses out of the filler and polymer matrix into the eluent. On the other hand, cations can exchange with GTM in the ZrP-GTM based system, allowing the diffusion of GTM out of the filler and polymer matrix. The release of antibiotics confer antimicrobial activity to the scaffolds.

As it is observed in Figure 2, BSEM images confirm an increasing amount of filler (visible as brighter points) with increasing filler-antibiotic concentrations on the surface and cross section of the scaffolds filaments. Using EDS the fillers were discriminated as MgAl and $\mathrm{ZrP}$ containing particles and the distinctive atomic elements of the CFX ( $F$ and N) and GTM ( $\mathrm{S}$ and $\mathrm{N}$ ) molecules were found to co-localize with their respective lamellar particles (Figure 2C-D). This strongly indicates that the filler and antibiotic were not separated in the filaments, but showed a preserved filler structure after the compounding and ME-AM process. Moreover, particle size was maintained for both types of fillers within the aftersynthesis values during the processing steps. A slight size reduction was observed during the ME-AM process, likely due to the higher shear forces in the print-head screw (Figure S3). Despite the presence of some filler aggregates close to the filaments' surface, the composite scaffolds surface roughness was not significantly altered in the microscale, presenting a smooth surface comparable to the PEOT/PBT scaffolds (Figure 2 and Figure 
S4). The sub-surface aggregates do form topographical projections, but given their low numbers and large separations (on the order of $100 \mu \mathrm{m}$ ), the cells are not likely to interact with multiple projections simultaneously.

A

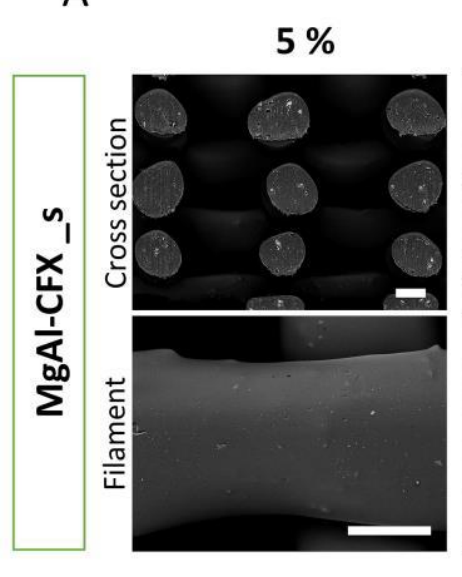

B

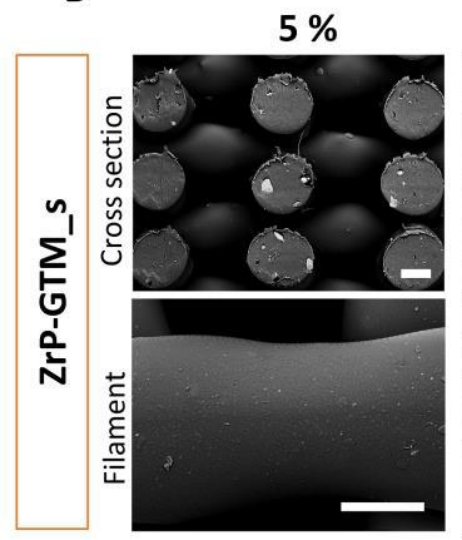

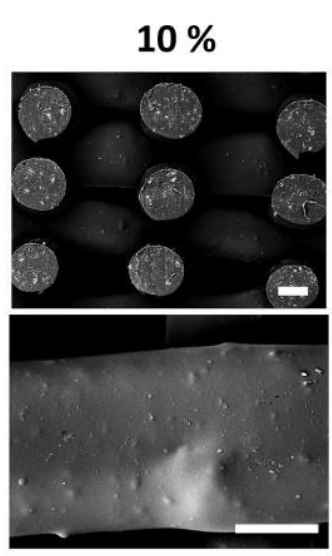

$10 \%$

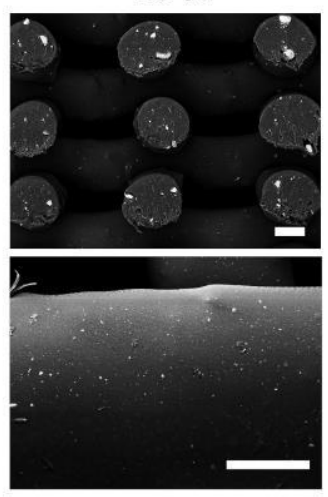

$\mathrm{C}$
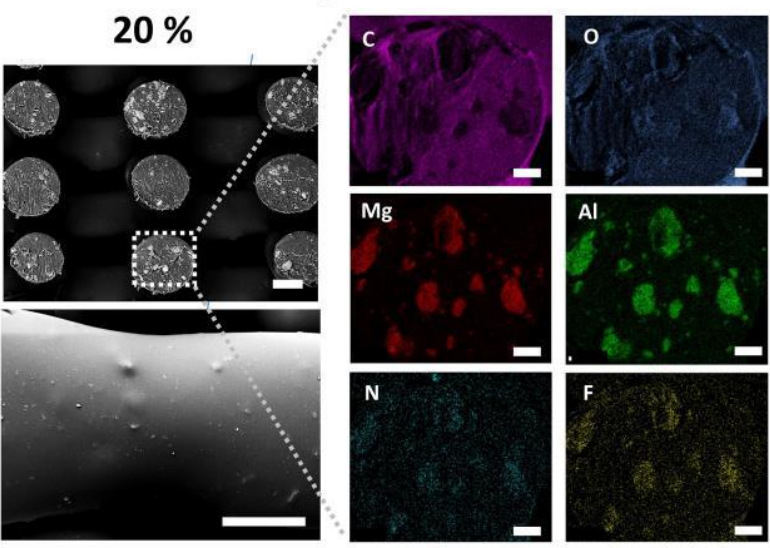

D

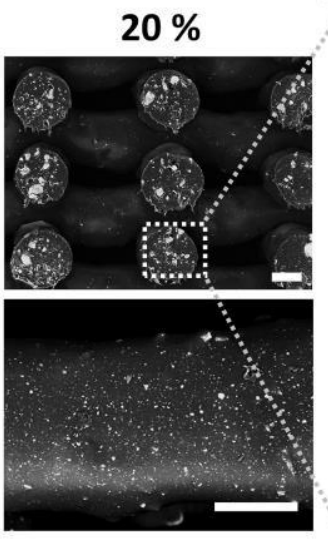

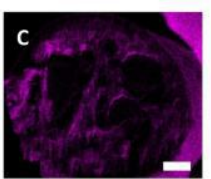

$\mathrm{Zr}$
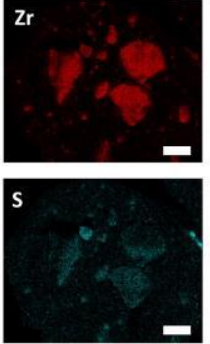
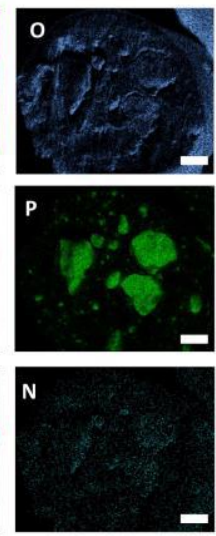

Figure 2. Filler distribution and antibiotics localization on composite scaffolds. BSEM micrographs of scaffolds cross sections and filaments surface depicting filler distribution (as white points) on (A) MgAl-CFX_s and (B) ZrP-GTM_s at different filler-antibiotic concentrations. Scale bars $250 \mu \mathrm{m}$. EDAX elemental mapping of representative fiber cross section of (C) $20 \% \mathrm{MgAl}-\mathrm{CFX}$ _s and (D) $20 \%$ ZrP-GTM_s. Scale bars $50 \mu \mathrm{m}$.

\section{In vitro antibiotic release}

In order to evaluate the kinetics of antibiotic release from MgAl-CFX_s and ZrP-GTM_s, composite scaffolds were incubated in APBS, and CFX and GTM released amounts were monitored over time. As demonstrated in Figure $3 \mathbf{A}$, by increasing the MgAl-CFX concentration within MgAl-CFX_s, larger amounts of CFX were released over time. A similar trend was also observed in the release profile of GTM from ZrP-GTM_s (Figure 3B). This tendency demonstrates the possibility of designing a customized scaffold, which releases the desired amount of antibiotic over time, by tuning the filler-antibiotic 
concentration mixed with the polymer matrix. Although both systems demonstrated a fillerantibiotic concentration-dependent release profile, significantly different release kinetics were observed on MgAl-CFX_s and ZrP-GTM_s. While the release of CFX from MgAlCFX_s was sustained over the course of the evaluated time (1 month), there was a GTM burst release from ZrP-GTM_s during the first $24 \mathrm{~h}$ followed by a slower release up to the completion of the study (Figure 3A-B). When active molecules are fixed into the inorganic lamellae of layered double hydroxides (LDH), such as MgAl, their de-intercalation mechanism occurs mainly by anion exchange, a process in which the intercalated active molecule and free anions in the elution solution can be exchanged via a concentration gradient. 52 On the other hand, the release of active molecules from metal hydrogen phosphates, such as ZrP, can occur not only by ion exchange (in this case, of cations), but also via the disruption of acid-base interactions between the active molecule and the filler, which rely on a $\mathrm{pH}$ switch in the environment about the acid dissociation constant (pKa) of the intercalated molecule (Figure 1). ${ }^{52} \mathrm{~A}$ series of factors are involved in the release kinetics of antibiotics when the lamellar particles are dispersed within a polymeric matrix: i) the permeation and diffusion of water molecules carrying counter ions through the polymer matrix; ii) the intra-particle diffusion of water, ions and active molecules, which depends on guest-host interactions and determines the rate of de-intercalation, and iii) the diffusion rate of active molecules (antibiotics in this study) from the polymer matrix. ${ }^{53} \mathrm{In}$ order to determine the release rate-limiting step, the release profiles of the $20 \mathrm{wt} \%$ fillerantibiotic scaffolds, taken as a representative cases, were fitted to various release kinetic models. 7 relevant models were used and the corresponding correlation coefficients are reported in Figure S5. Bhaskar model was found to best describe the release profile of CFX from 20\% MgAl-CFX_s. This model has previously been used to explain the release of active molecules from LDH systems and indicates that the release of CFX is an intraparticle diffusion controlled process, and therefore, ion exchange rate is the limiting step. 54-57 Accordingly, the decreased release rate of CFX over time (Figure $\mathbf{3 A}$ ) is likely due to the formation of a phase boundary between internal zones of the lamellar filler containing intercalated CFX and the external zones, in which anions from dPBS have already replaced the antibiotic, which progressively declines the antibiotic release process. ${ }^{58}$

To gain more insight into the release mechanism of 20\% ZrP-GTM_s, the total progress was separated into two stages: a rapid release stage I $(<24 \mathrm{~h})$ followed by a slow release stage II (>24h). The modified Freundlich kinetic model, which describes heterogeneous diffusion from flat surfaces of clays via ion exchange, was found to explain the release in 
Stage I (Figure S5). This implies that GTM diffuses into the medium solution via anion exchange during the first $24 \mathrm{~h}$, at a fast speed due to the antibiotic high solubility in waterbased neutral $\mathrm{pH}$ eluents. ${ }^{59-61}$ Since after the studied period the GTM released did not reach 100\% (Figure S5A), it is hypothesized that GTM released in stage I came from the ZrP particles located on the surface of scaffold filaments, after which the release of GTM from internal ZrP particles was hindered by the polymer network, resulting in the plateaulike profile observed in Stage II (Figure 3B). This type of behavior was already reported and is likely due to the low diffusion rate of water molecules through the polymer network, given by the poor water uptake/swellability capability of PEOT/PBT (4\% water uptake) at the specific PEO molecular weight (300 kDa) and PEOT:PBT segments ratio (55:45). 48, 53 In this case, the GTM still incorporated into the system could be released upon polymer degradation, a process that could be potentially speeded up by increasing the molecular weight of the PEO segments of the copolymer or by using a different and faster degrading material. Following the same reasoning, the fact that a plateau was not reached in the CFX release curve from MgAl-CFX_s, and that only $\sim 15-20 \%$ of the total CFX was released (Figure S5A) in the analyzed time, suggests that the CFX released during the evaluated period was also originated from the MgAl particles localized on the surface of the filaments. It is plausible that due to the low solubility of CFX at neutral $\mathrm{pH}$, and possibly the stronger filler-antibiotic interactions, the fast release seen in Stage I in 20\% ZrP-GTM_s did not happen in $20 \%$ MgAl-CFX_s.

\section{Effect of filler, $\mathrm{pH}$ and ionic concentration}

As mentioned above, the de-intercalation of molecules from fillers has been shown to be driven by ion exchange and $\mathrm{pH}$ changes in the eluent media. To further investigate these effects on our composite systems, easy to manufacture films were incubated in solutions with different $\mathrm{pH}$ values and ionic concentrations, and the release profiles were compared relative to each other without considering the absolute release amounts. In addition, the effect of filler was explored by preparing composite films (20\% MgAl-CFX_f) and films made of the same amount of CFX directly dispersed in the copolymer matrix (10\% CFX f $)$ was compared (Figure $3 \mathrm{C}$ ). The presence of the filler enabled the maintenance of a lower antibiotic release rate over time. The effect of filler on the sustained release of CFX was further confirmed upon incubation of $20 \% \mathrm{MgAl}-\mathrm{CFX} \mathrm{f}$ in acidic $\mathrm{pH}(\mathrm{pH}=4)$ (Figure $3 \mathrm{C}$ ). Here, the acidic attack dissolved the layered MgAl, allowing for a faster release of CFX, whose release was not governed by ion exchange anymore and became comparable to 


\section{Controlled release}

\section{Antibiotic release}

A
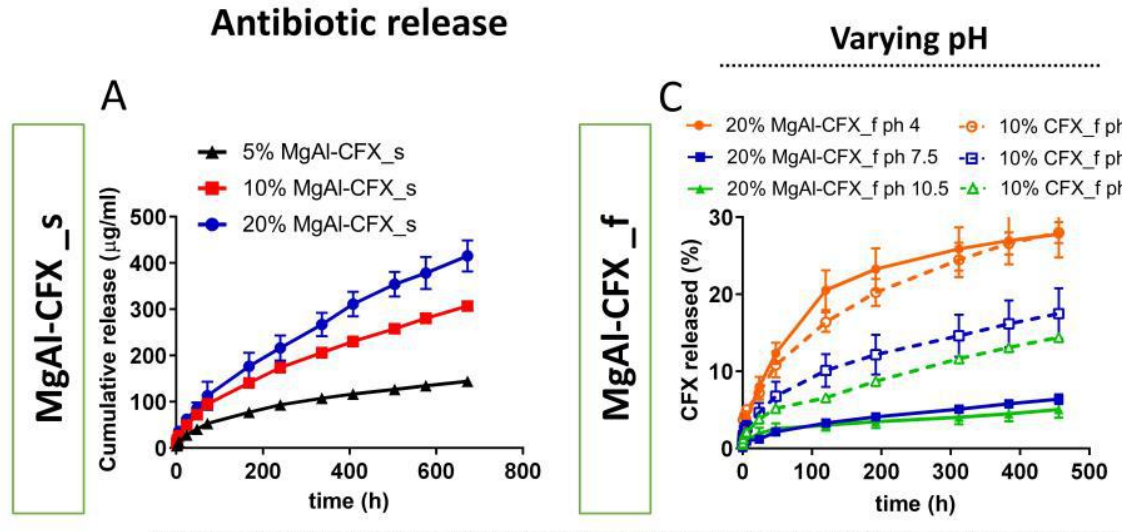

Varying ionic concentration
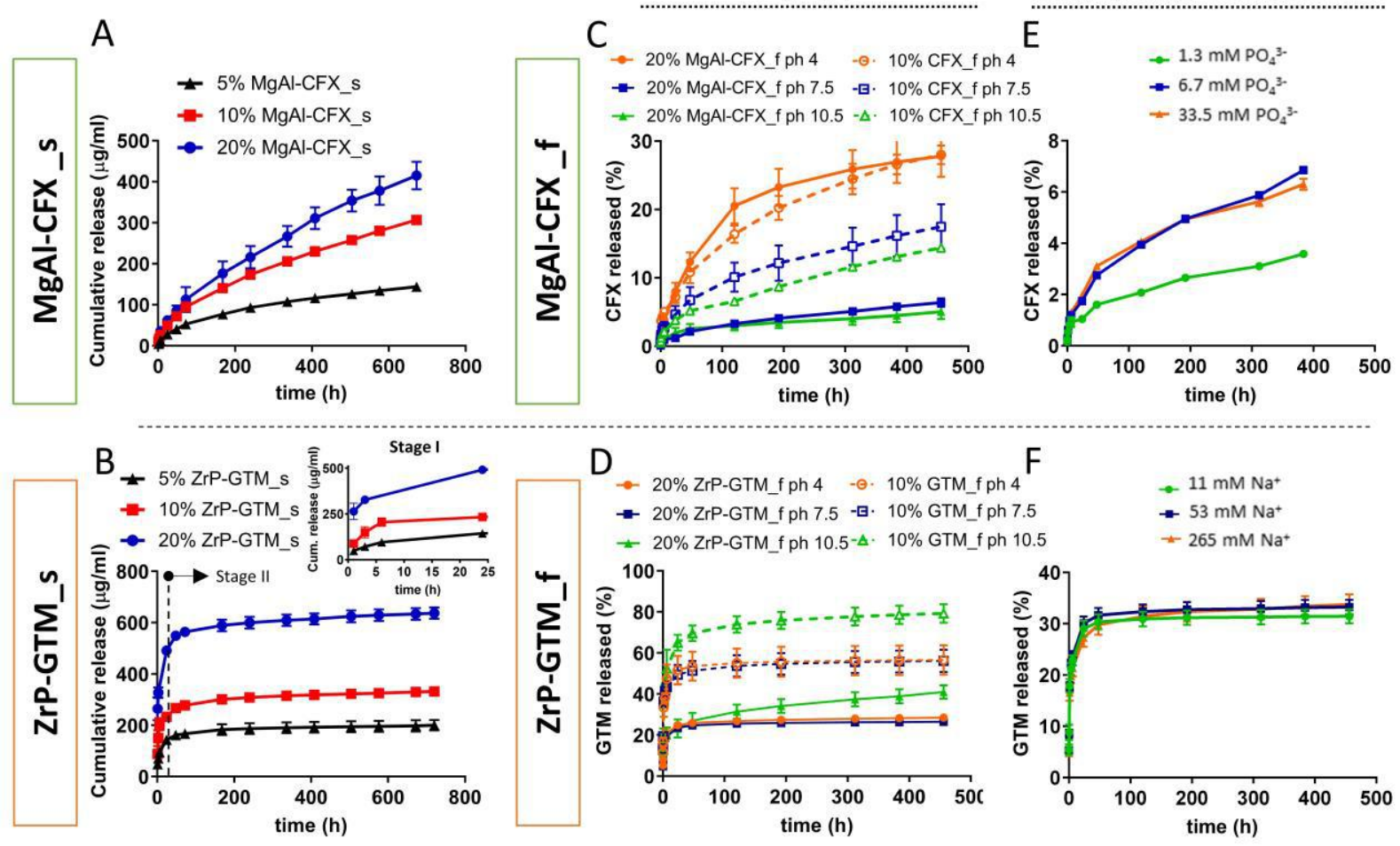

Figure 3. Antibiotic release profiles from MgAl-CFX and ZrP-GTM copolymer composites under different eluent conditions. Cumulative release of (A) CFX from MgAl-CFX_s and (B) GTM from ZrP-GTM_s immersed in dPBS during 4 weeks at $37^{\circ} \mathrm{C}$, proving the filler-antibiotic concentration dependent release. Influence of filler presence and $\mathrm{pH}$ of the eluent solution on the cumulative release percentage of (C) CFX and (D) GTM from filler-antibiotic composite films (20\% MgAl-CFX_f and $20 \%$ ZrP-GTM_f, respectively) and films composed of comparable amounts of antibiotic directly dispersed in the copolymer matrix (10\% CFX_f and 10\% GTM_f), immersed in buffers at acidic, neutral and basic $\mathrm{pH}$ during 3 weeks at $37^{\circ} \mathrm{C}$. Influence of the ionic concentration of the eluent solution on the cumulative release percentage of (E) CFX and (F) GTM from $20 \% \mathrm{MgAl}-$ CFX_f and $20 \%$ ZrP-GTM_f, respectively, during 3 weeks a $37^{\circ} \mathrm{C}$.

the release of CFX from 10\% CFX_f at $\mathrm{pH}=4 .{ }^{56,62}$ Interestingly, at $\mathrm{pH} \geq 7,10 \% \mathrm{CFX} f$ release profile remained unchanged and lower release rates than at $\mathrm{pH}=4$ were observed, due to lower CFX solubility at this $\mathrm{pH}$ range. Similarly, 20\% MgAl-CFX_f release profile did not vary at $\mathrm{pH} \geq 7$, as $\mathrm{MgAl}$ remained stable and intact (Figure $3 \mathrm{C}$ ). The role of $\mathrm{ZrP}$ on maintaining a more sustained release, especially in the stage I, was also demonstrated for 20\% ZrP-GTM_f, when comparing to 10\% GTM_f (Figure 3D). However, while MgAl in MgAl-CFX_f was affected by acidic pH, the release of GTM from 20\% ZrP-GTM_f was affected by alkaline conditions $(\mathrm{pH}=10.5)$, due to the different nature of the system (Figure 3D). Considering the isoelectric point of GTM $(\mathrm{pKa}=8),{ }^{63}$ the hydroxyl groups of GTM were deprotonated at $\mathrm{pH}=10.5$, disrupting the hydrogen bonds with the co- 
intercalated water molecules as well as producing a negatively charged GTM, which was repelled from the ZrP filler due to the disrupted acid-base electrostatic interactions between the GTM amine groups and the phosphate groups of the ZrP layers. ${ }^{41}$ It is hypothesized that oxidative degradation of PEOT/PBT, happening at alkaline conditions, 48, 64 allowed for further increase in the diffusion of GTM from the polymer matrix, which is visible as a slight increase in the release rate of GTM in Stage II on both 20\% ZrP-GTM_f and 10\% GTM_f release curves at $\mathrm{pH}=10$ (Figure 3D).

The aforementioned results indicate that the GTM release from ZrP-GTM_f in stage II can be controlled by varying the $\mathrm{pH}$ (acid base-interactions), without filler degradation. This mechanism of release was found to be more effective than ion exchange. As depicted in Figure 3F, the release of GTM does not vary upon increasing or decreasing the concentration of cations (sodium, $\mathrm{Na}^{+}$) in the eluent, within the evaluated concentration range. On the contrary, CFX release is clearly affected by the concentration of anions (phosphate, $\mathrm{PO}_{4}{ }^{3-}$ ) in the elution buffer, as depicted in Figure 3E, further demonstrating the ion-exchange related release of the system. Compared to other inorganic anions present in dPBS, phosphate ions present the highest affinity with LDH fillers, due to their higher charge. ${ }^{40}$ By a 5 -fold decrease in the phosphate concentration, CFX release decreased by $52 \%$ after 3 weeks. Interestingly, a 5 -fold increase over the dPBS phosphate base concentration did not affect the system, suggesting the existence of a concentration threshold.

Overall, by varying the filler and antibiotic type incorporated within the scaffold, two antibiotic delivery systems were fabricated, whose sustained release can be controlled not only upon changing the filler-antibiotic concentration, but also by varying both the eluent ionic concentration or $\mathrm{pH}$ (in the case of MgAl-CFX), or pH (in the case of ZrP-GTM). The ionic concentration (i.e. phosphate, calcium, sodium, potassium) is a parameter that cannot be predicted during the bone regeneration process. In this regard, in vivo release experiments would be necessary to understand the in situ antibiotic release from the scaffolds. On the other hand, the local $\mathrm{pH}$ in a fracture during bone healing is known to be maintained in the range of $\mathrm{pH} 6$ to $\mathrm{pH} 7.5$ and, in principle, our system is not tunable in this range. ${ }^{65-66}$ However, the property of some biocompatible and biodegradable polymers, such as the aliphatic polyesters PLA, PGA or PLGA, to dramatically decrease the local pH upon degradation (down to $\mathrm{pH}=4$ ), ${ }^{67}$ could be use advantageously to further customize the in vivo antibiotic release from filler-antibiotic composite scaffolds. 
The polymer degradation profile of the scaffold and its effect on the antibiotic release kinetics would also be a matter of evaluation in future studies. Previous literature suggests the slow degradation of the PEOT/PBT used in this study (PEO molecular weight $=300$ $\mathrm{kDa}$, PEOT:PBT weight ratio $=55: 45$ ), which is mainly due to the low molecular weight of the PEO hydrophilic segment preventing the swelling of the material and lowering the possibility of hydrolysis. Accordingly, the intrinsic viscosity and mechanical properties of PEOT/PBT with similar characteristics has been shown to remain constant after 24 weeks immersion in PBS at $37^{\circ} \mathrm{C}$. ${ }^{68}$ Similar results were obtained after 24 weeks implantation in vivo, where the mass loss was only up to $3 \%$ and minimum cracks were observed in the surface by SEM. ${ }^{69}$ It is plausible that in our case, the fillers increased the degradation time of the polymer, as previously shown on PEOT/PBT - nanosilicate scaffolds. ${ }^{70}$ Yet, long term in vitro and in vivo studies are required to further evaluate this.

\section{Thermal stability of antibiotics and scaffolds antimicrobial activity}

One of the main concerns involved in the AM of 3D scaffolds via high-temperature ME of polymers containing bioactive molecules is the preservation of their bioactivity after the process' thermal stresses. It has been previously demonstrated that the incorporation of some bioactive molecules into lamellar inorganic fillers increases their thermal stability. ${ }^{56}$, 71-72 However, CFX decomposes already at high temperatures due to its stable aromatic structure, what might have neutralized the potential shielding effect of the MgAl filler in our system, which in turn maintained CFX stability, as demonstrated by TGA (Figure S6A), as well as in previously studies. ${ }^{72}$ While in our TGA analysis CFX does not show any degradation bellow $255^{\circ} \mathrm{C}$, according to literature, MgAl-CFX weight loss bellow $255^{\circ} \mathrm{C}$ is attributed to the loss of MgAl interlamellar water, and CFX decarboxylation occurs at $\sim 146$ ${ }^{\circ} \mathrm{C}$. ${ }^{73-74}$ This inconsistency might be due to experimental conditions differences. Yet, HPLC confirmed molecular decomposition of CFX after the melt-blending process at around 150 ${ }^{\circ} \mathrm{C}(\sim 73 \%$ degradation), potentially attributed to the aforementioned decarboxylation of CFX at $146{ }^{\circ} \mathrm{C}$ (Figure S6B, C). ${ }^{74}$ Further decomposition occurred during the scaffolds production via ME at $190-195^{\circ} \mathrm{C}(\sim 45 \%$ degradation of the remaining GTM after melt blending, equivalent to $\sim 12.5 \%$ degradation of the total initial GTM loading).

Similarly, GTM decomposition starts at $220^{\circ} \mathrm{C}$ following some water loss, and the ZrP filler did not show to increase nor decrease its decomposition temperature, as shown in Figure S7A. ${ }^{63}$ The loss of interlamellar water of ZrP-GTM occurs in the range $20-220^{\circ} \mathrm{C}$, while 
the step above $220{ }^{\circ} \mathrm{C}$ is attributed to the condensation of the $\mathrm{PO}_{4}^{-3}$ groups and the decomposition of GTM. ${ }^{35}$ Accordingly, HPLC-MS analysis of GTM probed the stability of the molecule after each of the processing steps, with $\sim 1 \%$ and $~ 8 \%$ degradation after melt-blending and ME-AM, respectively (Figure S7B, D). Interestingly, this analysis also proved the preferential intercalation of the C1a GTM isoform during the ZrP-GTM synthesis process, due to its less steric bulky form, with respect to the other isoforms (Figure S7C).

Preliminary experiments demonstrated that the antimicrobial activity of the antibiotics was preserved after their intercalation within the filler, as well as in MgAl-CFX_f. Similarly, ZrPGTM and ZrP-GTM_f presented antibiotic activity (Table S1 and Table S2). Most importantly, the antimicrobial activity of both MgAl-CFX_s and ZrP-GTM_s against $S$. epidermidis and $P$. aeruginosa, was preserved on ME-AM scaffold (Figure 4), despite the aforementioned degradation. The antimicrobial activity of CFX consists of inhibiting the action of two essential enzymes that are involved in the modulation of the chromosomal supercoiling required for bacterial DNA synthesis, transcription and cell division. ${ }^{75}$ Despite its degradation, important active sites for CFX interaction with the target enzymes remained intact at the processing temperatures during melt-blending and posterior ME into scaffolds, thus preserving its antimicrobial activity. ${ }^{75}$ Importantly, the antimicrobial activity of CFX contained in MgAl-CFX_s was preserved at the same levels as unprocessed CFX, observed when comparing the experimental ZOI and the expected ZOI (i.e. ZOI given by corresponding amounts of pure unprocessed CFX) (Table S3 and Table S4). On the contrary, previous studies demonstrate a decrease in CFX efficiency against certain bacterial strains upon exposure to high temperatures $\left(120^{\circ} \mathrm{C}\right.$ for sterilization). ${ }^{30}$ However, these tests were performed under oxygen atmosphere, unlike the nitrogen conditions in which the ME-AM takes place, which in combination with MgAl might have acted as a protective shield in our system. In accordance with the thermal stability results of GTM and supported by previous GTM heat stability studies, ${ }^{30}$ the efficiency of this antibiotic released from ZrP-GTM_s was also preserved at the same levels as unprocessed GTM (Table S3 and Table S5). 


\section{S. Epidermidis}
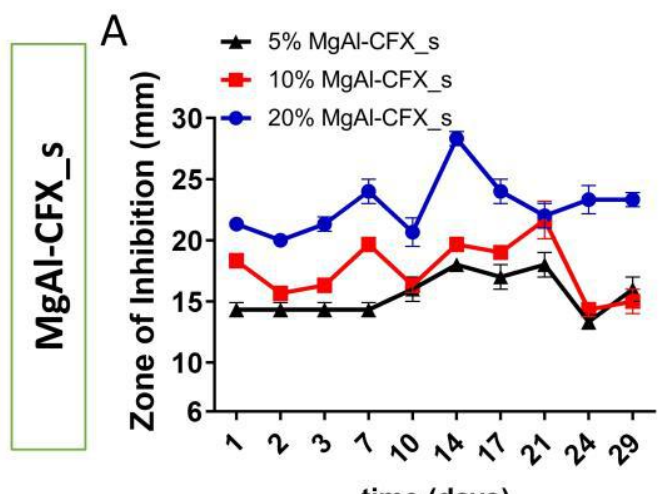

$\mathrm{C}$
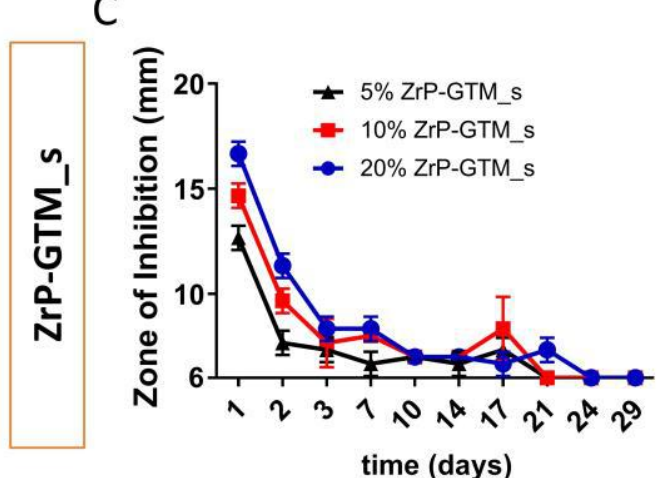
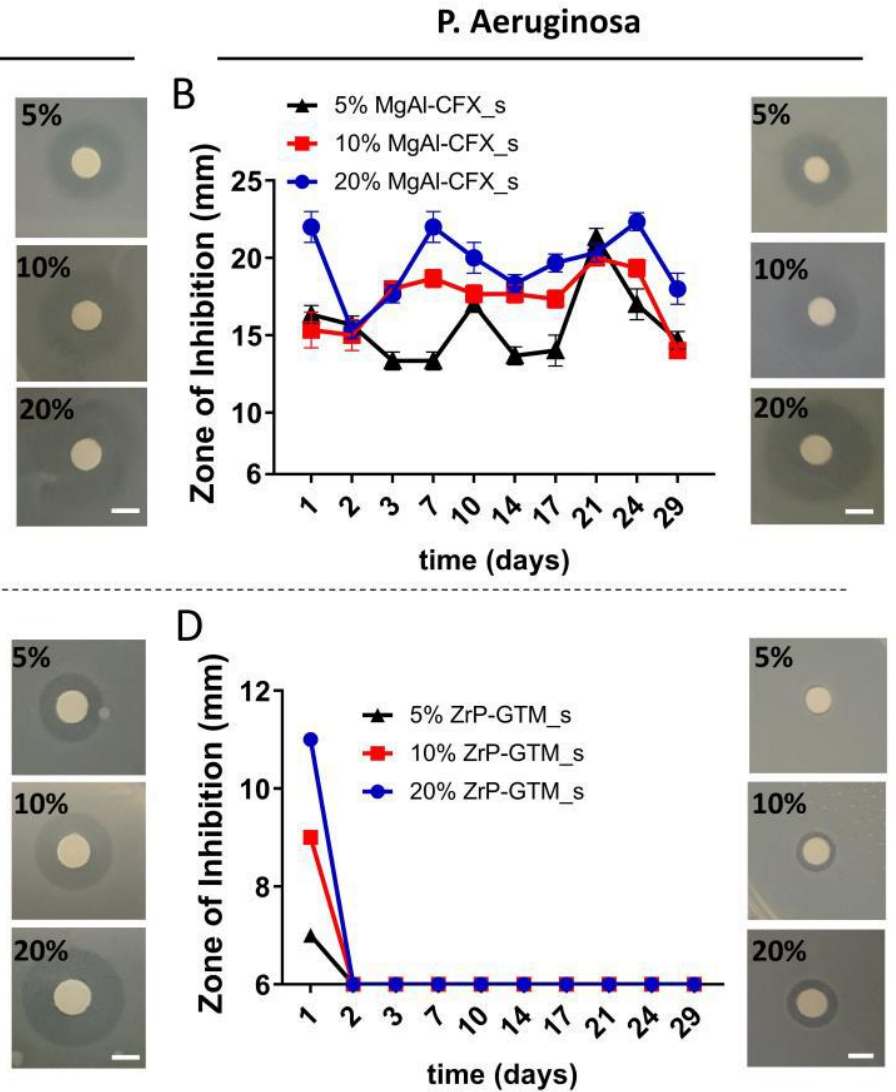

Figure 4. Antimicrobial activity of polymeric composite 3D AM scaffolds with different filler-antibiotic concentrations over 4 weeks. Graphs depicting ZOI of (A, B) MgAl-CFX_s and (C, D) ZrP-GTM_s in dPBS at $37^{\circ} \mathrm{C}$ against $S$. epidermidis and $P$. aeruginosa. Images of representative disk diffusion test plates depicting ZOIs around a disk impregnated with an aliquot of the scaffold release supernatant after the initial $24 \mathrm{~h}$ of release. Scale bars $5 \mathrm{~mm}$.

CFX MIC against both $S$. epidermidis and $P$. aeruginosa and is $1 \mu \mathrm{g} / \mathrm{ml}$ and $0.5 \mu \mathrm{g} / \mathrm{ml}$, respectively. ${ }^{76}$ Since the non-cumulative release of CFX from MgAl-CFX_s per timepoint was significantly above the MIC thresholds at all MgAl-CFX concentrations (Table S4 and Figure S8), the antimicrobial activity was preserved over the period evaluated (30 days) showing an increasing activity with increasing MgAl-CFX concentration (Figure 4A, B). On the other hand, the required GTM concentration to inhibit bacterial growth is higher (MIC against $S$. epidermidis: $1 \mathrm{ug} / \mathrm{ml}$; MIC against $P$. aeruginosa $4 \mathrm{ug} / \mathrm{ml}$ ). ${ }^{76}$ Therefore, in accordance with the amount of GTM released per timepoint (Table S5 and Figure S8), the antibacterial activity of ZrP-GTM_s against S. epidermidis lasted for 3 weeks, with a gradual decrease after a peak of activity in the first 48h (Figure 4C), while only $24 \mathrm{~h}$ against $P$. aeruginosa (Figure 4D). The concentration dependent activity was also observed in the case of ZrP-GTM_s s as it is shown in Figure 4C, D. 
While in vivo experiments would be necessary to fully understand the in situ antimicrobial function of the scaffolds, which is linked to the aforementioned in vivo conditions for antibiotic release (ionic concentration and $\mathrm{pH}$ ), this results already suggest the antimicrobial potential of the presented systems.

\section{Cell viability}

To further assess the cytotoxicity of the antibacterial systems, hMSCs viability was evaluated on MgAl-CFX_s and ZrP-GTM_s at the three different filler-antibiotic concentrations. Preliminary experiments demonstrated a significant reduction in cell number after 7 days of culture in 20\% MgAI-CFX_s and 20\% ZrP-GTM_s scaffolds (Figure S9A, B). Despite their well-known biocompatibility, 43,77 some reports describe the dose, time and size dependent cytotoxic effects of layered lamellar compounds, such as MgAl and $\mathrm{ZrP}$ when uptaken by cells. ${ }^{78-79}$ While this is not likely to occur during our in vitro experiments period as the fillers remain trapped within the copolymer matrix, fillers could potentially play a role in cell toxicity upon polymer degradation. Yet, we hypothesize that the sustained release of fillers into the surroundings, due to the slow polymer degradation process, would not lead to toxic amount of fillers as these are always under the toxicity limits previously reported in literature $(\sim 200-500 \mu \mathrm{l} / \mathrm{ml}) .43,80-82$ Thus, it is plausible that the high amount of antibiotic released during the $4 \mathrm{~h}$ seeding time concentrated in a small volume, negatively affected hMSCs attachment and viability. HMSCs cultured in tissue culture polystyrene showed a dose-dependence sensitivity to CFX (Figure S10A), which has shown to influence hMSCs proliferation upon continuous exposure. ${ }^{83}$ Similarly, although GTM has an impact on hMSCs viability only at high concentrations (>1000 $\mu \mathrm{g} / \mathrm{ml})$ (Figure S10B), short exposure (20 min) to high levels of GTM was found to influence osteoblasts proliferation rate up to 3 days post-exposure. ${ }^{84}$

To prevent a decrease in cell viability, surface extraction by pre-incubating the scaffolds in cell culture media prior to seeding (for $1.5 \mathrm{~h}$ ) was considered, as previously described. ${ }^{85}$ This method was used to reduce the initial drug burst release prior to cell contact and showed to significantly improve cell viability within our scaffolds (Figure 5D, E). Accordingly, no meaningful differences in cell number were found among filler-antibiotic concentrations and comparable levels of viability were maintained for 7 days. Representative live/dead images of the scaffolds cross sections further confirmed high hMSCs viability regardless of the filler-antibiotic concentration over 7 days for both MgAl- 
CFX_s and ZrP-GTM_s (Figure 5A-C). Interestingly, cells in the composite scaffolds showed a spread morphology comparable to the control samples (i.e. polymer-only scaffolds) after 1 day of culture, which changed to the characteristic elongated morphology of migrating cells after 7 days.

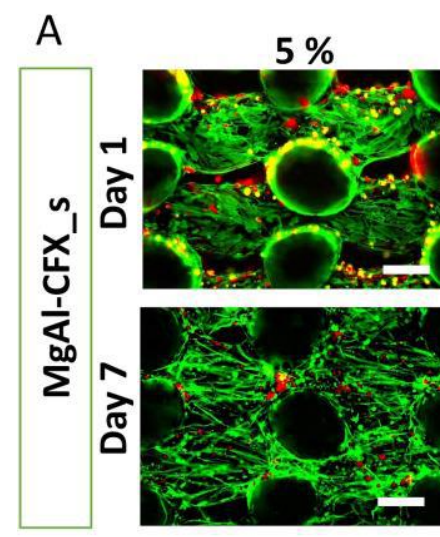

B

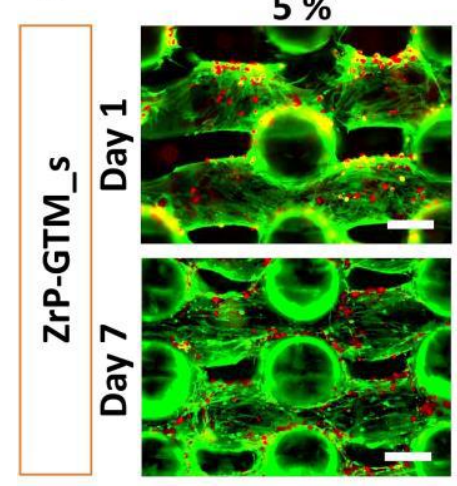

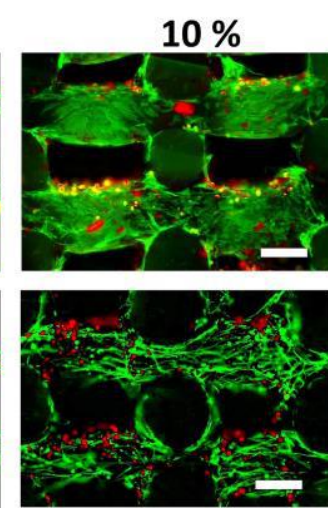
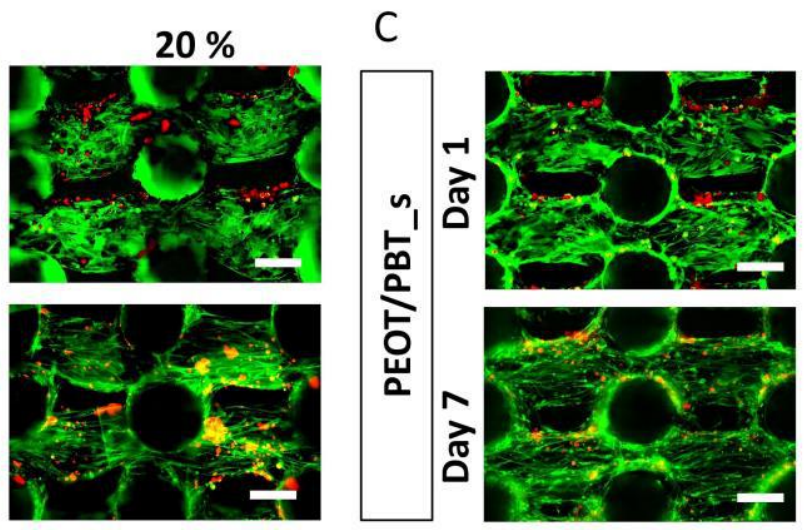

D
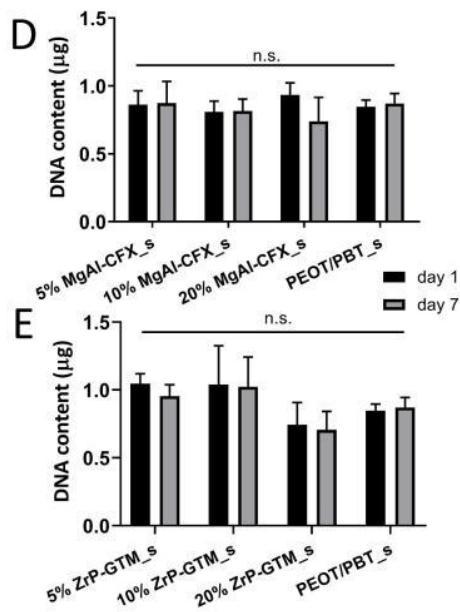

Figure 5. HMSCs viability is preserved after seeding MgAl-CFX_s and ZrP-CFX_s pre-incubated in media for $1.5 \mathrm{~h}$. Representative fluorescent images depicting cell viability ( $\mathrm{F}$-actin, green; dead cells, red) 1 day and 7 days post-seeding (a) MgAl-CFX_s, (b) ZrP-GTM_s and (c) PEOT/PBT_s. Quantification of DNA content 1 day and 7 days post seeding (d) MgAl-CFX_s and (e) ZrP-GTM_s, compared to PEOT/PBT_s control. Scale bars $250 \mu \mathrm{m}$. Statistical significance performed using two-way ANOVA with Tukey's multiple comparison test (n.s. $p>0.05$ ).

\section{Osteogenic differentiation}

In order to gain more insight into the potential of MgAl-CFX_s and ZrP-GTM_s to promote new bone formation, the osteogenic differentiation capacity of hMSCs seeded on the different scaffolds was analyzed. Preliminary results suggested that the osteogenic potential of hMSCs was influenced by the presence of the filler-antibiotic complexes. As 
depicted in Figure S11, after 28 days in mineralization media (MM), no mineral deposition was visualized or quantified on MgAl-CFX_s for all concentrations, nor on $20 \% \mathrm{ZrP}$ GTM_s, while a 10-fold reduction in calcium deposition was observed in 5 and $10 \%$ ZrPGTM_s with respect to the control samples (Figure S11). Yet, calcium deposits were observed in the polymer- and filler-only control scaffolds after 28 days in MM at levels reported in previous studies. ${ }^{38}$ This indicates that the fillers were not affecting hMSCs osteogenic behavior. This is further supported by a recently published report demonstrating the significant upregulation of several osteogenic genes including ALP, bone sialoprotein, Runt-related transcription factor 2 (RUNX2), OPN, and osteocalcin in osteoblasts when adding MgAl particles to the cell culture media. ${ }^{80} \mathrm{In}$ order to investigate if the antibiotics were impairing or just delaying the progression of hMSCs differentiation towards the creation of a mineralized matrix, scaffolds were cultured in MM for longer periods. After a total of 35 days in MM, all ZrP-GTM_s showed calcium deposition, which were inversely proportional to the ZrP-GTM concentration (Figure 6B). This suggests a GTM dose-dependent effect and its role in delaying mineralization. The amount of calcium in 5, 10, and $20 \%$ ZrP-GTM_s increased 3, 2 and 25-fold with respect to the previous timepoint, respectively. After a total of 49 days in MM, both 5 and $10 \%$ MgAl-CFX_s showed matrix mineralization, which was not observed at earlier timepoints, yet at levels lower than the control conditions.

Interestingly, 20\% MgAl-CFX_s did not mineralize even after 49 days in MM, suggesting that the continuous release of higher CFX amounts had a starker effect on the hMSCs differentiation process. This result was further supported by the lower DNA and higher values of ALP activity observed on 20\% MgAl-CFX_s compared to lower MgAl-CFX concentrations and the control (Figure S12), as the ALP activity is known to decrease during the mineralization process under optimum conditions. ${ }^{86} \mathrm{~A}$ strong inhibition of mineralization has been reported when confluent cultures of osteoblasts were treated with CFX. The effect was apparent with $2.5-5 \mu \mathrm{g} / \mathrm{ml} \mathrm{CFX}$ and progressively increased to more than a $90 \%$ decline in the calcium/protein ratio at $20-40 \mu \mathrm{g} / \mathrm{ml} \mathrm{CFX.}{ }^{87}$ These values are in the range of the release of CFX from 5-10\% MgAl-CFX_s and $20 \%$ MgAl-CFX_s, respectively, therefore supporting our results. While in vitro experiments agree in the dose dependent interference of CFX in the bone healing process, controversial results have been reported for in vivo studies. Abnormalities in cartilage morphology of fracture calluses were observed after treatment in rat models with ciprofloxacin $\left(2.4 \mathrm{\mu g} / \mathrm{ml}\right.$ in serum), ${ }^{88}$ while higher CFX release $(5 \mu \mathrm{g} / \mathrm{ml}$ per day) from a bone defect filler in rabbits allowed bone 
formation within 3 months in another study. ${ }^{89}$ This suggests that in vivo studies are necessary to gather a better insight over the effect of our scaffolds on bone formation. Compared to CFX, consistent in vitro and in vivo results have been previously published on the dose-dependency effect of GTM. For instance, mineralization in osteoblast and hMSCs cultures was not impaired after short exposure of up to $16 \mu \mathrm{g} / \mathrm{ml}$ GTM, ${ }^{84}$ or continuous exposure of $200 \mu \mathrm{g} / \mathrm{ml}$. ${ }^{90}$ Similarly, in vivo GTM serum concentration of 4.5 $\mu \mathrm{g} / \mathrm{mL},{ }^{91}$ or a continuous release from a bone filler with a peak of $58 \mu \mathrm{g}$ in the first $4 \mathrm{~h},{ }^{92}$ did not show reduced healing effect in rat models. However, a burst release from a bone cement (10 mg GTM released in 3 days) resulted in significantly less mineralized tissue than the control in a sheep model. ${ }^{93}$

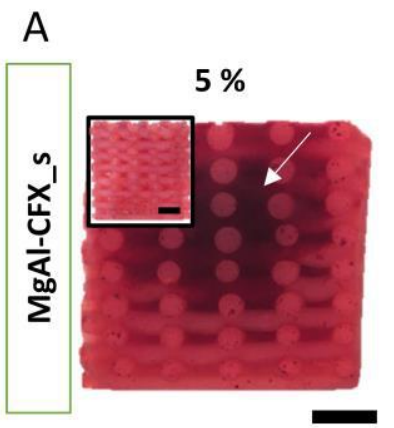

B

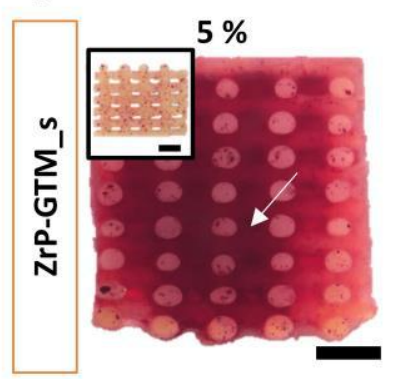

$10 \%$

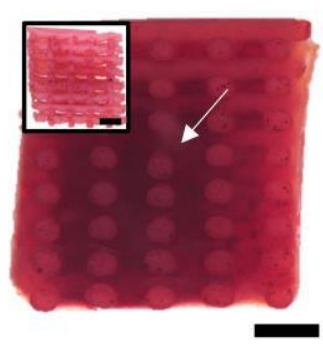

$10 \%$

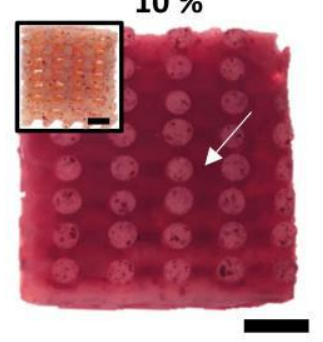

$20 \%$

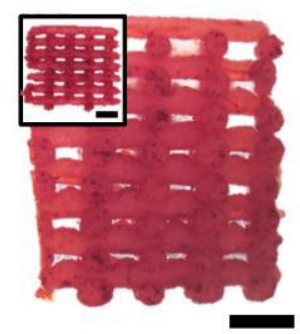

$20 \%$

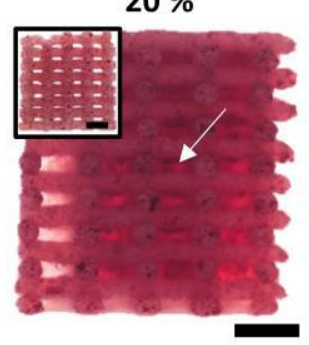

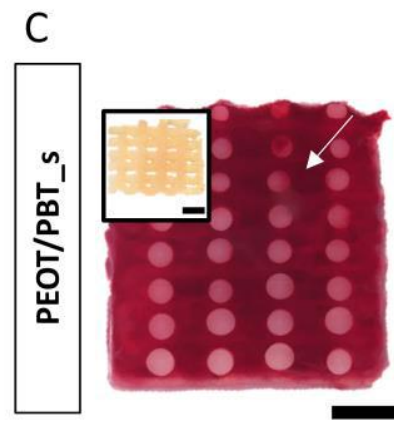

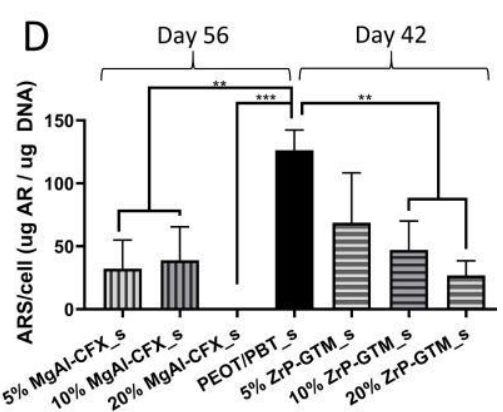

Figure 6. Influence of filler-antibiotic concentration on matrix mineralization. Representative stereomicroscopy images of scaffolds cross sections stained with ARS: (a) MgAl-CFX_s after 56 days of culture (49 days in MM) and (b) ZrP-GTM_s and (c) PEOT/PBT_s after 42 days of culture (35 days in MM). Inserts represent the corresponding control scaffolds without cells incubated in MM and stained with ARS. Scale bars $1 \mathrm{~mm}$. (d) Quantification of the ARS staining extracted from scaffolds normalized to cell number. Statistical significance performed using one-way ANOVA with Tukey's multiple comparison test $\left({ }^{* *} p<0.01 ;{ }^{* * *} p<0.001\right)$.

Along with the study in matrix mineralization, the progression in the osteogenic differentiation of hMSCs was further evaluated by immunostaining of two relevant osteogenic makers, COL I and OPN. COL I constitutes nearly $90 \%$ of bone organic ECM and serves as minerals nucleation sites. ${ }^{94}$ On the other hand, OPN is one of the most 
abundant non-collagenous proteins in bone. It reaches its peak during the mineralization period supporting its progression and the prevention of premature precipitation of calcium phosphate is suggested as one of its key functions. ${ }^{86,95}$ Interestingly, a dense layer of COL I fibrils was formed by hMSCs on top of scaffolds filaments regardless of the scaffold composition and no significant differences were observed compared to the PEOT/PBT control (Figure 7A and Figure S13). This implies that COL I fibers formation was not affected by antibiotics. However, OPN expression was reduced in MgAl-CFX_s when compared to PEOT/PBT_s and ZrP-GTM_s, qualitatively observed when comparing among scaffold types the ratio of OPN-stained hMSCs over the total cell number (Figure 7B and Figure S13). Intriguingly, this result lays in agreement with the delay in matrix mineralization observed on these scaffolds. Particularly, in $20 \%$ MgAl-CFX_s, where OPN expression is more clearly downregulated, the pronounced delay in the osteogenic differentiation of hMSCs cultured on these scaffolds is confirmed once more. However, the trend of the current results indicates that longer culture periods might lead to comparable osteogenesis and matrix mineralization results among different scaffolds types.

A
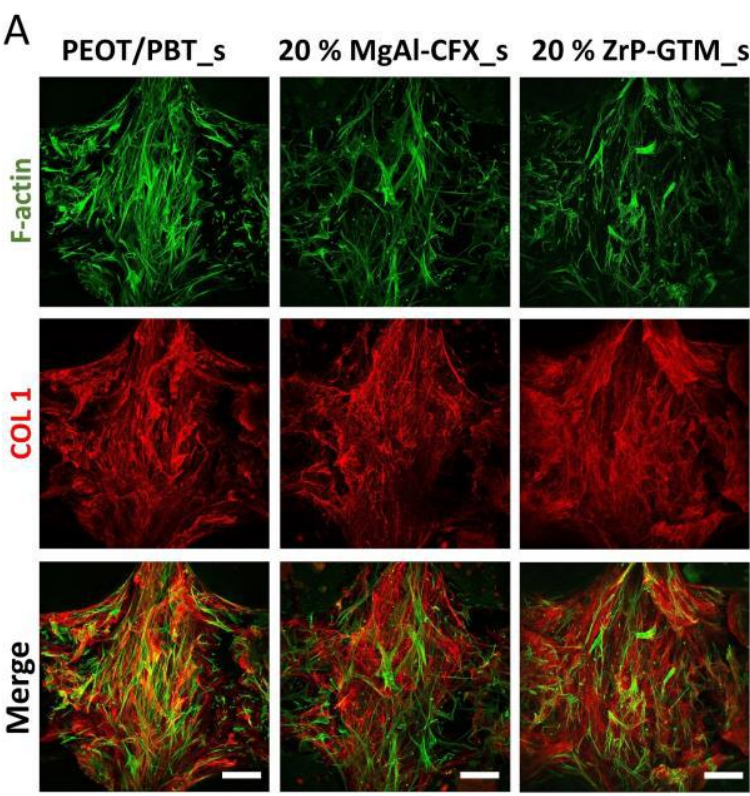

B
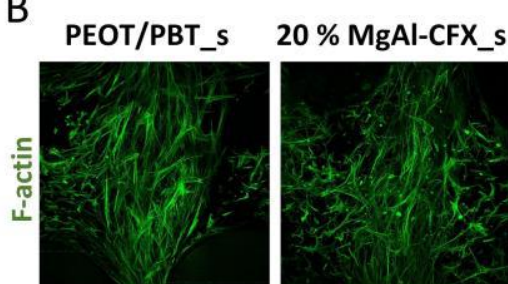

$20 \%$ ZrP-GTM
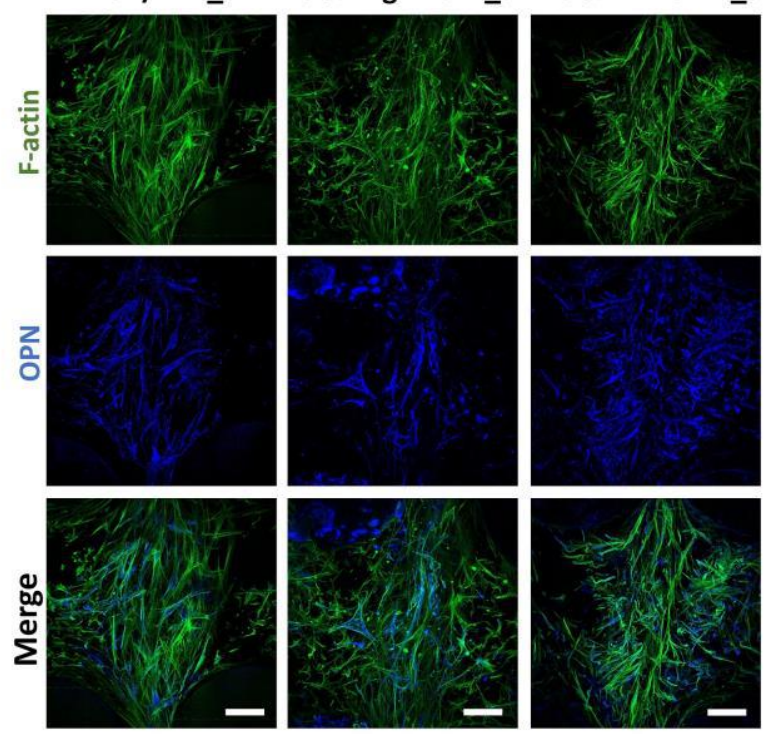

Figure 7. Influence of filler-antibiotic concentration on the osteogenic differentiation of hMSCs. Representative confocal microscopy images of hMSCs (F-actin, green) on top of the filaments of $20 \%$ MgAl-CFX_s after 54 days of culture (47 days in MM) and $20 \%$ ZrP-GTM_s and PEOT/PBT_s after 42 days of culture (35 days in $\mathrm{MM}$ ) and stained for the relevant osteogenic markers COL I (red) and OPN (blue). Scale bars $100 \mu \mathrm{m}$. 
Overall, the aforementioned results suggest that bone formation is supported in vitro on the filler-antibiotic based scaffolds presented in this study. Further studies should aim at evaluating the bone bonding and heterotopic bone formation capabilities of the scaffolds by in vitro biomineralization experiments. Yet, conclusions should be drawn carefully after these, since it has been previously shown that the formation of an apatite layer on the material surface in vitro is not always correlated with bone formation in vivo. ${ }^{96}$ Ultimately, in vivo experiments will be necessary to fully characterize the scaffolds bone regeneration performance.

\section{CONCLUSION}

Local delivery of antibiotics is desired to prevent infections after bone fracture of implantrelated surgery. The aim of this study was to develop antibiotic loaded 3D scaffolds fabricated via high temperature ME-AM. In order to protect antibiotics from the fabrication process thermal stresses and obtain a sustained release, the antibiotics CFX and GTM were intercalated within the lamellar structure of the inorganic layered fillers $\mathrm{MgAl}$ and $\mathrm{ZrP}$, respectively, prior to their dispersion within the polymeric matrix. Compared to no-filler systems, a more sustained release was obtained in the filler-based scaffolds. The antibiotic was released via diffusion through the polymer matrix and by a mechanism governed by ion exchange or $\mathrm{pH}$ changes. The functionality of the antibiotics after high temperature AM was confirmed against Gram + and Gram - bacterial strains demonstrating same antibacterial levels as unprocessed antibiotics. Importantly, the incorporation of fillers and antibiotics into the scaffolds neither affected hMSCs viability nor prevented their osteogenic differentiation, as verified by matrix mineralization and COL I and OPN expression. These newly proposed method enables the fabrication of dual functional scaffolds via direct incorporation of antibiotics in scaffolds fabricated at high temperatures, and has the potential to improve infection management while allowing bone regeneration. Moreover, this approach opens the door to future research on the use of other relevant antibiotics and biodegradable thermoplastic polymers to further optimize the system towards clinical applications. 


\section{Acknowledgement}

We are grateful to the FAST project funded under the H2020-NMP-PILOTS-2015 scheme (GA n. 685825) for financial support. Some of the materials used in this work were provided by the Texas A\&M Health Science Center College of Medicine Institute for Regenerative Medicine at Scott \& White through a grant from NCRR of the NIH (Grant \#P40RR017447). 


\section{References}

1. Darouiche, R. O., Treatment of Infections Associated with Surgical Implants. N Engl J Med 2004, 350 (14), 1422-1429.

2. Okike, K.; Bhattacharyya, T., Trends in the Management of Open Fractures. A Critical Analysis. J Bone Joint Surg Am 2006, 88 (12), 2739-2748.

3. Lew, D. P.; Waldvogel, F. A., Osteomyelitis. The Lancet 2004, 364 (9431), 369-379.

4. Garzoni, C.; Kelley, W. L., Staphylococcus Aureus: New Evidence for Intracellular Persistence. Trends Microbiol 2009, 17 (2), 59-65.

5. Arciola, C. R.; Campoccia, D.; Montanaro, L., Implant Infections: Adhesion, Biofilm Formation and Immune Evasion. Nature Reviews Microbiology 2018, 16 (7), 397-409.

6. Haas, D. W.; McAndrew, M. P., Bacterial Osteomyelitis in Adults: Evolving Considerations in Diagnosis and Treatment. Am J Med 1996, 101 (5), 550-561.

7. Trampuz, A.; Renz, N., Pocket Guide to Diagnosis and Treatment of Implant-Associated Infections after Fracture Fixation. 2 ed.; Pro-Implant Foundation: 2017

8. Holtom, P. D.; Pavkovic, S. A.; Bravos, P. D.; Patzakis, M. J.; Shepherd, L. E.; Frenkel, B., Inhibitory Effects of the Quinolone Antibiotics Trovafloxacin, Ciprofloxacin, and Levofloxacin on Osteoblastic Cells in Vitro. J Orthop Res 2000, 18 (5), 721-727.

9. Buchholz, H. W.; Engelbrecht, H., [Depot Effects of Various Antibiotics Mixed with Palacos Resins]. Der Chirurg; Zeitschrift fur alle Gebiete der operativen Medizen 1970, 41 (11), 511-515.

10. van Vugt, T. A. G.; Arts, J. J.; Geurts, J. A. P., Antibiotic-Loaded Polymethylmethacrylate Beads and Spacers in Treatment of Orthopedic Infections and the Role of Biofilm Formation. Front Microbiol 2019, 10, 1626-1626.

11. Kuehn, K.-D.; Ege, W.; Gopp, U., Acrylic Bone Cements: Composition and Properties. Orthopedic Clinics of North America 2005, 36 (1), 17-28.

12. Webb, J. C.; Spencer, R. F., The Role of Polymethylmethacrylate Bone Cement in Modern Orthopaedic Surgery. The Journal of bone and joint surgery. British volume 2007, 89 (7), 851-857.

13. Hanssen, A. D., Local Antibiotic Delivery Vehicles in the Treatment of Musculoskeletal Infection. Clinical Orthopaedics and Related Research ${ }^{\circledR} 2005$, 437, 91-96.

14. M., E.-H.; S., P.; J., M. R.; S., H. F., Biodegradable Antibiotic Delivery Systems. The Journal of bone and joint surgery. British volume 2011, 93-B (2), 151-157.

15. Mota, C.; Puppi, D.; Chiellini, F.; Chiellini, E., Additive Manufacturing Techniques for the Production of Tissue Engineering Constructs. J Tissue Eng Regen Med 2015, 9 (3), 174-190.

16. Chen, L.; Shao, L.; Wang, F.; Huang, Y.; Gao, F., Enhancement in Sustained Release of Antimicrobial Peptide and Bmp-2 from Degradable Three Dimensional-Printed Plga Scaffold for Bone Regeneration. RSC Advances 2019, 9 (19), 10494-10507.

17. Liu, P.; Fu, K.; Zeng, X.; Chen, N.; Wen, X., Fabrication and Characterization of Composite Meshes Loaded with Antimicrobial Peptides. ACS Applied Materials \& Interfaces 2019, 11 (27), 24609-24617.

18. Bakhshandeh, S.; Gorgin Karaji, Z.; Lietaert, K.; Fluit, A. C.; Boel, C. H. E.; Vogely, H. C.; Vermonden, T.; Hennink, W. E.; Weinans, H.; Zadpoor, A. A.; Amin Yavari, S., Simultaneous Delivery of Multiple Antibacterial Agents from Additively Manufactured Porous Biomaterials to Fully Eradicate Planktonic and Adherent Staphylococcus Aureus. ACS Appl Mater Interfaces 2017, 9 (31), 25691-25699.

19. Martins, C. H. G.; Carvalho, T. C.; Souza, M. G. M.; Ravagnani, C.; Peitl, O.; Zanotto, E. D.; Panzeri, H.; Casemiro, L. A., Assessment of Antimicrobial Effect of Biosilicate ${ }^{\circledR}$ against Anaerobic, Microaerophilic and Facultative Anaerobic Microorganisms. Journal of Materials Science: Materials in Medicine 2011, 22 (6), 1439-1446.

20. Yang, Y.; Yang, S.; Wang, Y.; Yu, Z.; Ao, H.; Zhang, H.; Qin, L.; Guillaume, O.; Eglin, D.; Richards, R. G.; Tang, T., Anti-Infective Efficacy, Cytocompatibility and Biocompatibility of a 3d-Printed Osteoconductive Composite Scaffold Functionalized with Quaternized Chitosan. Acta Biomater 2016, 46, 112-128.

21. Yang, Y.; Chu, L.; Yang, S.; Zhang, H.; Qin, L.; Guillaume, O.; Eglin, D.; Richards, R. G.; Tang, T., DualFunctional 3d-Printed Composite Scaffold for Inhibiting Bacterial Infection and Promoting Bone Regeneration in Infected Bone Defect Models. Acta Biomaterialia 2018, 79, 265-275. 
22. Martin, V.; Ribeiro, I. A.; Alves, M. M.; Gonçalves, L.; Claudio, R. A.; Grenho, L.; Fernandes, M. H.; Gomes, P.; Santos, C. F.; Bettencourt, A. F., Engineering a Multifunctional 3d-Printed Pla-Collagen-MinocyclineNanohydroxyapatite Scaffold with Combined Antimicrobial and Osteogenic Effects for Bone Regeneration. Materials Science and Engineering: C 2019, 101, 15-26.

23. Zhou, Z.; Yao, Q.; Li, L.; Zhang, X.; Wei, B.; Yuan, L.; Wang, L., Antimicrobial Activity of 3d-Printed Poly(Epsilon-Caprolactone) (Pcl) Composite Scaffolds Presenting Vancomycin-Loaded Polylactic AcidGlycolic Acid (Plga) Microspheres. Medical science monitor : international medical journal of experimental and clinical research 2018, 24, 6934-6945.

24. Visscher, L. E.; Dang, H. P.; Knackstedt, M. A.; Hutmacher, D. W.; Tran, P. A., 3d Printed Polycaprolactone Scaffolds with Dual Macro-Microporosity for Applications in Local Delivery of Antibiotics. Materials Science and Engineering: C 2018, 87, 78-89.

25. García-Alvarez, R.; Izquierdo-Barba, I.; Vallet-Regí, M., 3d Scaffold with Effective Multidrug Sequential Release against Bacteria Biofilm. Acta biomaterialia 2017, 49, 113-126.

26. Teo, E. Y.; Ong, S. Y.; Chong, M. S.; Zhang, Z.; Lu, J.; Moochhala, S.; Ho, B.; Teoh, S. H., Polycaprolactone-Based Fused Deposition Modeled Mesh for Delivery of Antibacterial Agents to Infected Wounds. Biomaterials 2011, 32 (1), 279-287.

27. Shim, J.-H.; Kim, M.-J.; Park, J. Y.; Pati, R. G.; Yun, Y.-P.; Kim, S. E.; Song, H.-R.; Cho, D.-W., ThreeDimensional Printing of Antibiotics-Loaded Poly-E-Caprolactone/Poly(Lactic-Co-Glycolic Acid) Scaffolds for Treatment of Chronic Osteomyelitis. Tissue Engineering and Regenerative Medicine 2015, 12 (5), 283293.

28. Camarero-Espinosa, S.; Calore, A.; Wilbers, A.; Harings, J.; Moroni, L., Additive Manufacturing of an Elastic Poly(Ester)Urethane for Cartilage Tissue Engineering. Acta Biomaterialia 2020, 102, 192-204.

29. Jaidev, L. R.; Chatterjee, K., Surface Functionalization of 3d Printed Polymer Scaffolds to Augment Stem Cell Response. Materials \& Design 2019, 161, 44-54.

30. Traub, W. H.; Leonhard, B., Heat Stability of the Antimicrobial Activity of Sixty-Two Antibacterial Agents. Journal of Antimicrobial Chemotherapy 1995, 35 (1), 149-154.

31. Campoccia, D.; Montanaro, L.; Speziale, P.; Arciola, C. R., Antibiotic-Loaded Biomaterials and the Risks for the Spread of Antibiotic Resistance Following Their Prophylactic and Therapeutic Clinical Use. Biomaterials 2010, 31 (25), 6363-6377.

32. Zilberman, M.; Elsner, J. J., Antibiotic-Eluting Medical Devices for Various Applications. J Control Release 2008, 130 (3), 202-215.

33. Arciola, C. R.; An, Y.; Campoccia, D.; Donati, M.; Montanaro, L., Etiology of Implant Orthopedic Infections: A Survey on 1027 Clinical Isolates. The International journal of artificial organs 2005, 28 (11), 1091-1100.

34. Costantino, U.; Marmottini, F.; Nocchetti, M.; Vivani, R., New Synthetic Routes to Hydrotalcite-Like Compounds- Characterisation and Properties of the Obtained Materials. European Journal of Inorganic Chemistry 1998, 1998 (10), 1439-1446.

35. Bastianini, M.; Scatto, M.; Sisani, M.; Scopece, P.; Patelli, A.; Petracci, A., Innovative Composites Based on Organic Modified Zirconium Phosphate and Peot/Pbt Copolymer. Journal of Composites Science 2018, $2(2), 31$.

36. Landers, R.; Pfister, A.; Hübner, U.; John, H.; Schmelzeisen, R.; Mülhaupt, R., Fabrication of Soft Tissue Engineering Scaffolds by Means of Rapid Prototyping Techniques. Journal of Materials Science 2002, 37 (15), 3107-3116.

37. ZHANG, X.; WYSS, U. P.; PICHORA, D.; GOOSEN, M. F., Biodegradable Controlled Antibiotic Release Devices for Osteomyelitis: Optimization of Release Properties. Journal of pharmacy and pharmacology 1994, 46 (9), 718-724.

38. Cámara-Torres, M.; Sinha, R.; Mota, C.; Moroni, L., Improving Cell Distribution on 3d Additive Manufactured Scaffolds through Engineered Seeding Media Density and Viscosity. Acta Biomaterialia 2020, 101, 183-195.

39. Rives, V.; del Arco, M.; Martín, C., Intercalation of Drugs in Layered Double Hydroxides and Their Controlled Release: A Review. Applied Clay Science 2014, 88-89, 239-269.

40. Saha, S.; Ray, S.; Acharya, R.; Chatterjee, T. K.; Chakraborty, J., Magnesium, Zinc and Calcium Aluminium Layered Double Hydroxide-Drug Nanohybrids: A Comprehensive Study. Applied Clay Science 2017, 135, 493-509. 
41. Díaz, A.; David, A.; Pérez, R.; González, M. L.; Báez, A.; Wark, S. E.; Zhang, P.; Clearfield, A.; Colón, J. L., Nanoencapsulation of Insulin into Zirconium Phosphate for Oral Delivery Applications. Biomacromolecules 2010, 11 (9), 2465-2470.

42. Kumar, C. V.; Chaudhari, A., Proteins Immobilized at the Galleries of Layered A-Zirconium Phosphate: Structure and Activity Studies. Journal of the American Chemical Society 2000, 122 (5), 830-837.

43. Saxena, V.; Diaz, A.; Clearfield, A.; Batteas, J. D.; Hussain, M. D., Zirconium Phosphate Nanoplatelets: A Biocompatible Nanomaterial for Drug Delivery to Cancer. Nanoscale 2013, 5 (6), 2328-2336.

44. Badar, M.; Rahim, M. I.; Kieke, M.; Ebel, T.; Rohde, M.; Hauser, H.; Behrens, P.; Mueller, P. P., Controlled Drug Release from Antibiotic-Loaded Layered Double Hydroxide Coatings on Porous Titanium Implants in a Mouse Model. Journal of Biomedical Materials Research Part A 2015, 103 (6), 2141-2149.

45. Valarezo, E.; Tammaro, L.; González, S.; Malagón, O.; Vittoria, V., Fabrication and Sustained Release Properties of Poly (E-Caprolactone) Electrospun Fibers Loaded with Layered Double Hydroxide Nanoparticles Intercalated with Amoxicillin. Applied Clay Science 2013, 72, 104-109.

46. Van Blitterswijk, C.; Leenders, H.; Baaker, D., The Effect of Peo Ratio on Degradation, Calcification and Bone Bonding of Peo/Pbt Copolymer (Polyactive). Cells and Materials 1993, 3 (1), 2.

47. Kuijer, R.; Bouwmeester, S. M.; Drees, M. E.; Surtel, D. M.; Terwindt-Rouwenhorst, E. W.; Van Der Linden, A.; Van Blitterswijk, C.; Bulstra, S., The Polymer Polyactivetm as a Bone-Filling Substance: An Experimental Study in Rabbits. Journal of Materials Science: Materials in Medicine 1998, 9 (8), 449-455.

48. Deschamps, A. A.; Claase, M. B.; Sleijster, W. J.; de Bruijn, J. D.; Grijpma, D. W.; Feijen, J., Design of Segmented Poly(Ether Ester) Materials and Structures for the Tissue Engineering of Bone. Journal of Controlled Release 2002, 78 (1), 175-186.

49. Jansen, E. J. P.; Pieper, J.; Gijbels, M. J. J.; Guldemond, N. A.; Riesle, J.; Van Rhijn, L. W.; Bulstra, S. K.; Kuijer, R., Peot/Pbt Based Scaffolds with Low Mechanical Properties Improve Cartilage Repair Tissue Formation in Osteochondral Defects. Journal of Biomedical Materials Research Part A 2009, 89A (2), 444452.

50. Di Luca, A.; Ostrowska, B.; Lorenzo-Moldero, I.; Lepedda, A.; Swieszkowski, W.; Van Blitterswijk, C.; Moroni, L., Gradients in Pore Size Enhance the Osteogenic Differentiation of Human Mesenchymal Stromal Cells in Three-Dimensional Scaffolds. Scientific Reports 2016, 6 (1), 22898.

51. Karageorgiou, V.; Kaplan, D., Porosity of 3d Biomaterial Scaffolds and Osteogenesis. Biomaterials 2005, 26 (27), 5474-5491.

52. Bringley, J. F.; Liebert, N. B., Controlled Chemical and Drug Delivery Via the Internal and External Surfaces of Layered Compounds. Journal of dispersion science and technology 2003, 24 (3-4), 589-605.

53. Bugatti, V.; Gorrasi, G.; Montanari, F.; Nocchetti, M.; Tammaro, L.; Vittoria, V., Modified Layered Double Hydroxides in Polycaprolactone as a Tunable Delivery System: In Vitro Release of Antimicrobial Benzoate Derivatives. Applied Clay Science 2011, 52 (1), 34-40.

54. Zhang, H.; Pan, D.; Zou, K.; He, J.; Duan, X., A Novel Core-Shell Structured Magnetic Organic-Inorganic Nanohybrid Involving Drug-Intercalated Layered Double Hydroxides Coated on a Magnesium Ferrite Core for Magnetically Controlled Drug Release. Journal of Materials Chemistry 2009, 19 (19), 3069-3077.

55. Perioli, L.; Posati, T.; Nocchetti, M.; Bellezza, F.; Costantino, U.; Cipiciani, A., Intercalation and Release of Antiinflammatory Drug Diclofenac into Nanosized Znal Hydrotalcite-Like Compound. Applied Clay Science 2011, 53 (3), 374-378.

56. Zhang, H.; Zou, K.; Guo, S.; Duan, X., Nanostructural Drug-Inorganic Clay Composites: Structure, Thermal Property and in Vitro Release of Captopril-Intercalated Mg-Al-Layered Double Hydroxides. Journal of Solid State Chemistry 2006, 179 (6), 1792-1801.

57. Bhaskar, R.; Murthy, R. S. R.; Miglani, B. D.; Viswanathan, K., Novel Method to Evaluate Diffusion Controlled Release of Drug from Resinate. International Journal of Pharmaceutics 1986, 28 (1), 59-66.

58. Ambrogi, V.; Fardella, G.; Grandolini, G.; Perioli, L., Intercalation Compounds of Hydrotalcite-Like Anionic Clays with Antiinflammatory Agents - I. Intercalation and in Vitro Release of Ibuprofen. International Journal of Pharmaceutics 2001, 220 (1), 23-32.

59. Gu, Z.; Thomas, A. C.; Xu, Z. P.; Campbell, J. H.; Lu, G. Q., In Vitro Sustained Release of Lmwh from Mgal-Layered Double Hydroxide Nanohybrids. Chemistry of Materials 2008, 20 (11), 3715-3722.

60. Yang, J.-H.; Han, Y.-S.; Park, M.; Park, T.; Hwang, S.-J.; Choy, J.-H., New Inorganic-Based Drug Delivery System of Indole-3-Acetic Acid-Layered Metal Hydroxide Nanohybrids with Controlled Release Rate. Chemistry of Materials 2007, 19 (10), 2679-2685. 
61. Kong, X.; Jin, L.; Wei, M.; Duan, X., Antioxidant Drugs Intercalated into Layered Double Hydroxide: Structure and in Vitro Release. Applied Clay Science 2010, 49 (3), 324-329.

62. Rojas, R.; Palena, M. C.; Jimenez-Kairuz, A. F.; Manzo, R. H.; Giacomelli, C. E., Modeling Drug Release from a Layered Double Hydroxide-lbuprofen Complex. Applied Clay Science 2012, 62-63, 15-20.

63. Rosenkrantz, B. E.; Greco, J. R.; Hoogerheide, J. G.; Oden, E. M., Gentamicin Sulfate. In Analytical Profiles of Drug Substances, Florey, K., Ed. Academic Press: 1981; Vol. 9, pp 295-340.

64. Carrow, J. K.; Di Luca, A.; Dolatshahi-Pirouz, A.; Moroni, L.; Gaharwar, A. K., 3d-Printed Bioactive Scaffolds from Nanosilicates and Peot/Pbt for Bone Tissue Engineering. Regenerative Biomaterials 2018, $6(1), 29-37$.

65. Beck-Broichsitter, B. E.; Christofzik, D. W.; Daschner, F.; Knöchel, R.; Smeets, R.; Warnke, P.; Wiltfang, J.; Becker, S. T., Endocultivation: Metabolism During Heterotopic Osteoinduction in Vivo-Monitoring with Fiber Optic Detection Devices. Tissue Engineering Part C: Methods 2012, 18 (10), 740-746.

66. Beck-Broichsitter, B. E.; Daschner, F.; Christofzik, D. W.; Knöchel, R.; Wiltfang, J.; Becker, S. T., Using Eddy Currents for Noninvasive in Vivo Ph Monitoring for Bone Tissue Engineering. Oral and maxillofacial surgery 2015, 19 (1), 55-60.

67. Liu, H.; Slamovich, E. B.; Webster, T. J., Less Harmful Acidic Degradation of Poly(Lacticco-Glycolic Acid) Bone Tissue Engineering Scaffolds through Titania Nanoparticle Addition. Int J Nanomedicine 2006, 1 (4), 541-545.

68. Deschamps, A. A.; Grijpma, D. W.; Feijen, J., Poly (Ethylene Oxide)/Poly (Butylene Terephthalate) Segmented Block Copolymers: The Effect of Copolymer Composition on Physical Properties and Degradation Behavior. Polymer 2001, 42 (23), 9335-9345.

69. Deschamps, A.; van Apeldoorn, A. A.; Hayen, H.; de Bruijn, J. D.; Karst, U.; Grijpma, D. W.; Feijen, J., In Vivo and in Vitro Degradation of Poly (Ether Ester) Block Copolymers Based on Poly (Ethylene Glycol) and Poly (Butylene Terephthalate). Biomaterials 2004, 25 (2), 247-258.

70. Carrow, J. K.; Di Luca, A.; Dolatshahi-Pirouz, A.; Moroni, L.; Gaharwar, A. K., 3d-Printed Bioactive Scaffolds from Nanosilicates and Peot/Pbt for Bone Tissue Engineering. Regenerative biomaterials 2019, $6(1), 29-37$.

71. Xia, S.-J.; Ni, Z.-M.; Xu, Q.; Hu, B.-X.; Hu, J., Layered Double Hydroxides as Supports for Intercalation and Sustained Release of Antihypertensive Drugs. Journal of Solid State Chemistry 2008, 181 (10), 26102619.

72. Kutlu, B.; Schröttner, P.; Leuteritz, A.; Boldt, R.; Jacobs, E.; Heinrich, G., Preparation of Melt-Spun Antimicrobially Modified Ldh/Polyolefin Nanocomposite Fibers. Materials Science and Engineering: $C$ 2014, 41, 8-16.

73. Costa, F. R.; Leuteritz, A.; Wagenknecht, U.; Jehnichen, D.; Häußler, L.; Heinrich, G., Intercalation of MgAl Layered Double Hydroxide by Anionic Surfactants: Preparation and Characterization. Applied Clay Science 2008, 38 (3), 153-164.

74. El-Gamel, N. E. A.; Hawash, M. F.; Fahmey, M. A., Structure Characterization and Spectroscopic Investigation of Ciprofloxacin Drug. Journal of Thermal Analysis and Calorimetry 2011, 108 (1), 253-262.

75. Chu, D. T.; Fernandes, P. B., Structure-Activity Relationships of the Fluoroquinolones. Antimicrob Agents Chemother 1989, 33 (2), 131-135.

76. The European Committee on Antimicrobial Susceptibility Testing. Breakpoint Tables for Interpretation of Mics and Zone Diameters. http://www.eucast.org.

77. Cunha, V. R. R.; de Souza, R. B.; da Fonseca Martins, A. M. C. R. P.; Koh, I. H. J.; Constantino, V. R. L., Accessing the Biocompatibility of Layered Double Hydroxide by Intramuscular Implantation: Histological and Microcirculation Evaluation. Scientific Reports 2016, 6, 30547.

78. Choi, S.-J.; Choy, J.-H., Layered Double Hydroxide Nanoparticles as Target-Specific Delivery Carriers: Uptake Mechanism and Toxicity. Nanomedicine 2011, 6 (5), 803-814.

79. Senapati, S.; Shukla, R.; Tripathi, Y. B.; Mahanta, A. K.; Rana, D.; Maiti, P., Engineered Cellular Uptake and Controlled Drug Delivery Using Two Dimensional Nanoparticle and Polymer for Cancer Treatment. Molecular Pharmaceutics 2018, 15 (2), 679-694.

80. Kang, H. R.; da Costa Fernandes, C. J.; da Silva, R. A.; Constantino, V. R. L.; Koh, I. H. J.; Zambuzzi, W. $\mathrm{F}$., Mg-Al and Zn-Al Layered Double Hydroxides Promote Dynamic Expression of Marker Genes in Osteogenic Differentiation by Modulating Mitogen-Activated Protein Kinases. Advanced Healthcare Materials 2018, 7 (4), 1700693. 
81. Choi, S.-J.; Oh, J.-M.; Choy, J.-H., Toxicological Effects of Inorganic Nanoparticles on Human Lung Cancer A549 Cells. Journal of Inorganic Biochemistry 2009, 103 (3), 463-471.

82. Li, Y.; Liu, D.; Ai, H.; Chang, Q.; Liu, D.; Xia, Y.; Liu, S.; Peng, N.; Xi, Z.; Yang, X., Biological Evaluation of Layered Double Hydroxides as Efficient Drug Vehicles. Nanotechnology 2010, 21 (10), 105101.

83. Sisto, F.; Bonomi, A.; Cavicchini, L.; Coccè, V.; Scaltrito, M. M.; Bondiolotti, G.; Alessandri, G.; Parati, E.; Pessina, A., Human Mesenchymal Stromal Cells Can Uptake and Release Ciprofloxacin, Acquiring $<$ Em>in Vitro</Em> Anti-Bacterial Activity. Cytotherapy 2014, 16 (2), 181-190.

84. Philp, A. M.; Raja, S.; Philp, A.; Newton Ede, M. P.; Jones, S. W., The Effect of Vancomycin and Gentamicin Antibiotics on Human Osteoblast Proliferation, Metabolic Function, and Bone Mineralization. Spine 2017, 42 (3), 202-207.

85. Huang, X.; Brazel, C. S., On the Importance and Mechanisms of Burst Release in Matrix-Controlled Drug Delivery Systems. Journal of Controlled Release 2001, 73 (2), 121-136.

86. Owen, T. A.; Aronow, M.; Shalhoub, V.; Barone, L. M.; Wilming, L.; Tassinari, M. S.; Kennedy, M. B.; Pockwinse, S.; Lian, J. B.; Stein, G. S., Progressive Development of the Rat Osteoblast Phenotype in Vitro: Reciprocal Relationships in Expression of Genes Associated with Osteoblast Proliferation and Differentiation During Formation of the Bone Extracellular Matrix. Journal of cellular physiology 1990, 143 (3), 420-430.

87. Holtom, P. D.; Pavkovic, S. A.; Bravos, P. D.; Patzakis, M. J.; Shepherd, L. E.; Frenkel, B., Inhibitory Effects of the Quinolone Antibiotics Trovafloxacin, Ciprofloxacin, and Levofloxacin on Osteoblastic Cells in Vitro. Journal of Orthopaedic Research 2000, 18 (5), 721-727.

88. M Huddleston, P.; M Steckelberg, J.; Hanssen, A. D.; Rouse, M.; E Bolander, M.; Patel, R., Ciprofloxacin Inhibition of Experimental Fracture-Healing*. The Journal of bone and joint surgery. American volume 2000, 82, 161-173.

89. Makinen, T. J.; Veiranto, M.; Lankinen, P.; Moritz, N.; Jalava, J.; Tormala, P.; Aro, H. T., In Vitro and in Vivo Release of Ciprofloxacin from Osteoconductive Bone Defect Filler. The Journal of antimicrobial chemotherapy 2005, 56 (6), 1063-1068.

90. Chang, Y.; Goldberg, V. M.; Caplan, A. I., Toxic Effects of Gentamicin on Marrow-Derived Human Mesenchymal Stem Cells. Clinical orthopaedics and related research 2006, 452, 242-249.

91. Haleem, A. A.; Rouse, M. S.; Lewallen, D. G.; Hanssen, A. D.; Steckelberg, J. M.; Patel, R., Gentamicin and Vancomycin Do Not Impair Experimental Fracture Healing. Clinical orthopaedics and related research 2004, (427), 22-24.

92. Fassbender, M.; Minkwitz, S.; Kronbach, Z.; Strobel, C.; Kadow-Romacker, A.; Schmidmaier, G.; Wildemann, B., Local Gentamicin Application Does Not Interfere with Bone Healing in a Rat Model. Bone 2013, 55 (2), 298-304.

93. Beuttel, E.; Bormann, N.; Pobloth, A.-M.; Duda, G. N.; Wildemann, B., Impact of Gentamicin-Loaded Bone Graft on Defect Healing in a Sheep Model. Materials (Basel) 2019, 12 (7), 1116.

94. Alford, A. I.; Kozloff, K. M.; Hankenson, K. D., Extracellular Matrix Networks in Bone Remodeling. The International Journal of Biochemistry \& Cell Biology 2015, 65, 20-31.

95. Roach, H. I., Why Does Bone Matrix Contain Non-Collagenous Proteins? The Possible Roles of Osteocalcin, Osteonectin, Osteopontin and Bone Sialoprotein in Bone Mineralisation and Resorption. Cell Biology International 1994, 18 (6), 617-628.

96. Barbieri, D.; Yuan, H.; Luo, X.; Farè, S.; Grijpma, D. W.; de Bruijn, J. D., Influence of Polymer Molecular Weight in Osteoinductive Composites for Bone Tissue Regeneration. Acta biomaterialia 2013, 9 (12), 9401-9413. 


\section{SUPPLEMENTARY FIGURES}

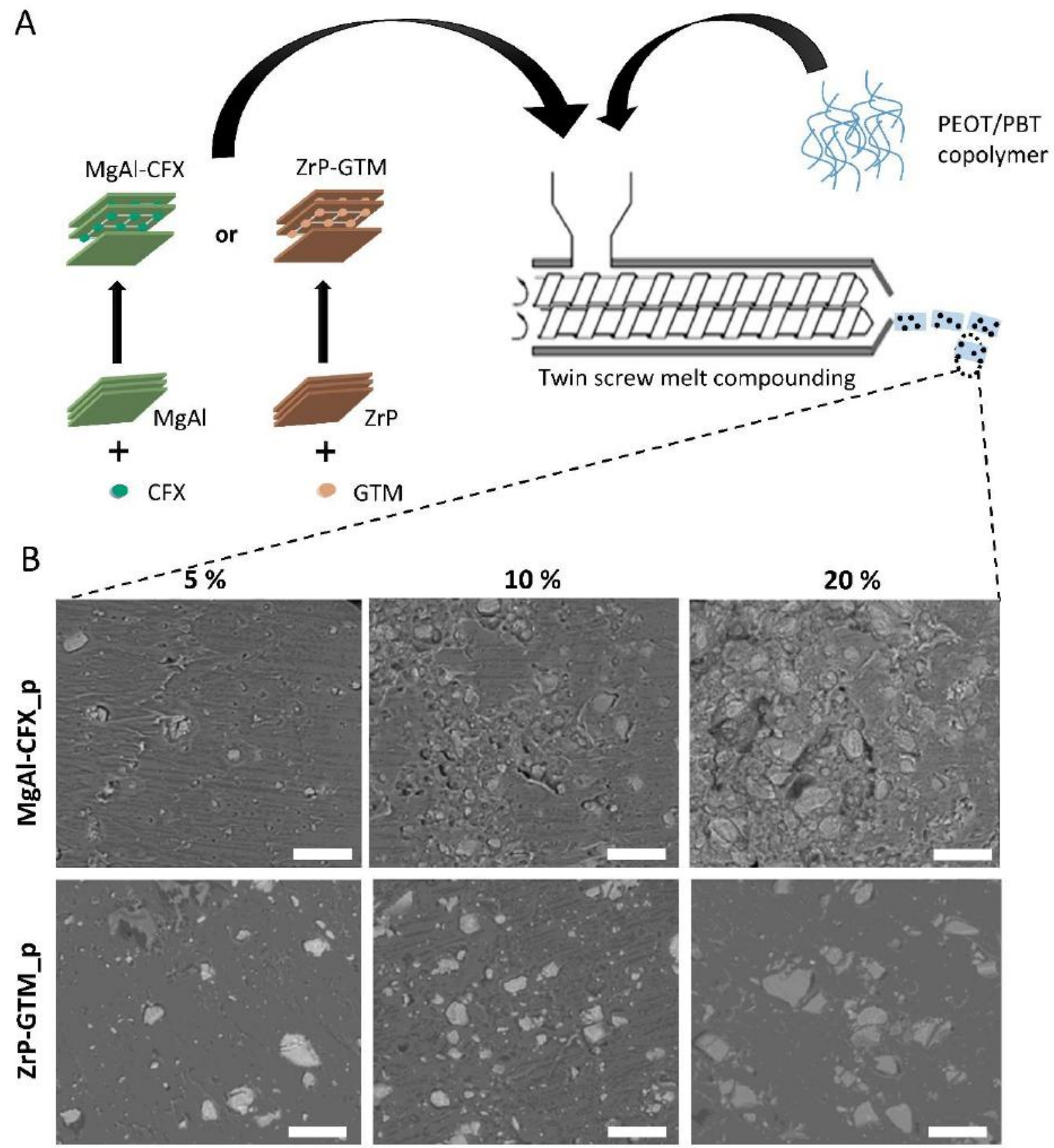

Figure S1. (A) Schematic representation of the melt-compounding process of the different copolymer-filler-antibiotic composites. Initially, CFX was intercalated within MgAl to obtain MgAlCFX, while GTM was intercalated within ZrP to obtain ZrP-GTM. Each of the two filler-antibiotic complexes separately were mixed at 5,10 and $20 \mathrm{wt} \%$ with PEOT/PBT using a twin screw extruder. Pellets of the 6 different materials (MgAl-CFX_p and ZrP-GTM_p) were obtained to be subsequently used for the fabrication of 3D scaffolds via ME-AM. (B) SEM micrographs of the cross section of MgAl-CFX_p and ZrP-GTM_p with different filler-antibiotic concentrations. Scale bars $200 \mu \mathrm{m}$. 
A

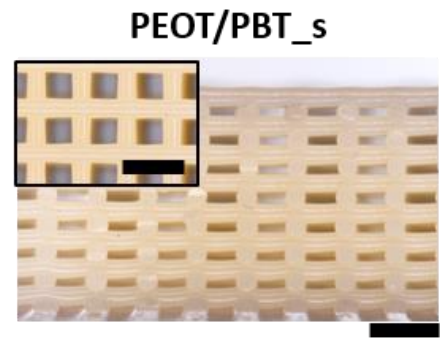

5\% MgAl-CFX_s

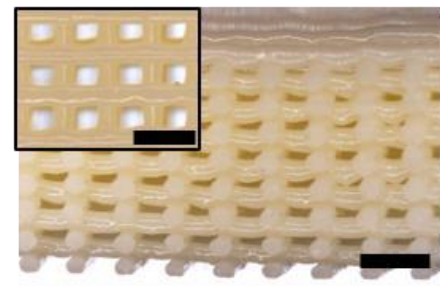

$10 \%$ MgAl-CFX_s
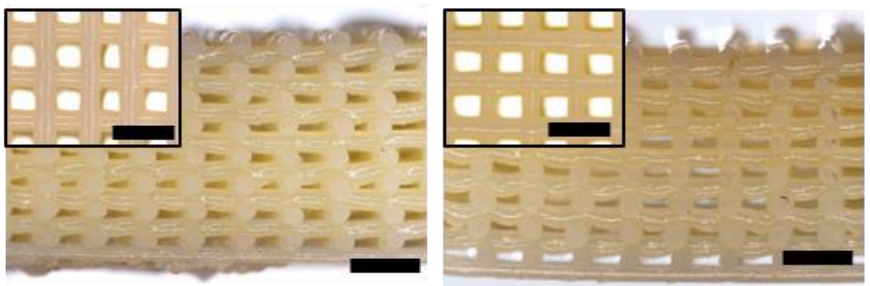

5\% ZrP-GTM_s

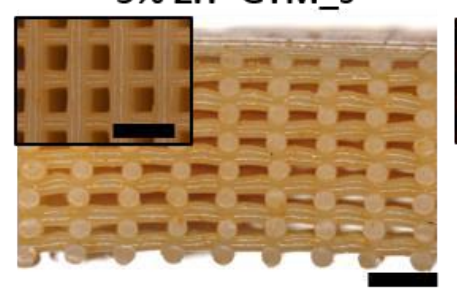

10\% ZrP-GTM_s

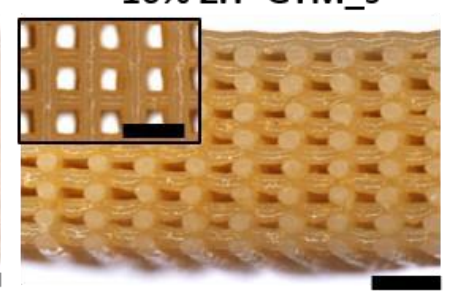

$20 \%$ ZrP-GTM_s

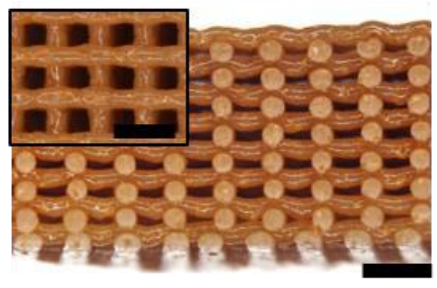

B

\begin{tabular}{ccccc}
\hline Scaffold & $\mathbf{d} \mathbf{1}(\boldsymbol{\mu m})$ & $\mathbf{d} \mathbf{(} \boldsymbol{\mu \mathrm { m } )}$ & $\mathbf{d} 3(\boldsymbol{\mu m})$ & Porosity $(\%)$ \\
\hline PEOT/PBT_s & $361 \pm 23$ & $810 \pm 23$ & $232 \pm 13$ & $48 \pm 3$ \\
\hline 5\% MgAl-CFX_s & $365 \pm 19$ & $805 \pm 11$ & $231 \pm 16$ & $48 \pm 2$ \\
\hline $10 \%$ MgAl-CFX_s & $358 \pm 23$ & $811 \pm 32$ & $225 \pm 24$ & $51 \pm 3$ \\
\hline $20 \%$ MgAl-CFX_s & $377 \pm 28$ & $804 \pm 26$ & $230 \pm 15$ & $51 \pm 1$ \\
\hline 5\% ZrP-GTM_s & $374 \pm 22$ & $814 \pm 40$ & $234 \pm 18$ & $46 \pm 9$ \\
\hline 10\% ZrP-GTM_s & $358 \pm 14$ & $808 \pm 21$ & $234 \pm 14$ & $47 \pm 3$ \\
\hline 20\%ZrP-GTM_s & $356 \pm 14$ & $812 \pm 27$ & $233 \pm 19$ & $46 \pm 2$ \\
\hline
\end{tabular}

Figure S2. (A) Stereomicroscopy images of PEOT/PBT_s and composite scaffolds cross section and top view (inserts) depicting the scaffolds' interconnected porosity in XYZ. Scale bars $1 \mathrm{~mm}$. (B) Experimental fiber diameter (d1), strand distance (d2), layer thickness (d3), and porosity calculated for each scaffold, 
A

\begin{tabular}{|c|c|}
\hline & Particle size D90 $(\mu \mathrm{m})$ \\
\hline MgAl-CFX & $1-20$ \\
\hline MgAl-CFX_p & $3-60$ \\
\hline MgAL-CFX_s & $3-20$ \\
\hline
\end{tabular}

B

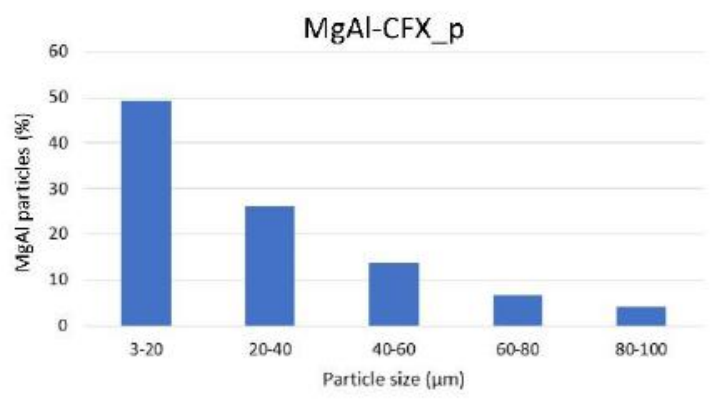

C

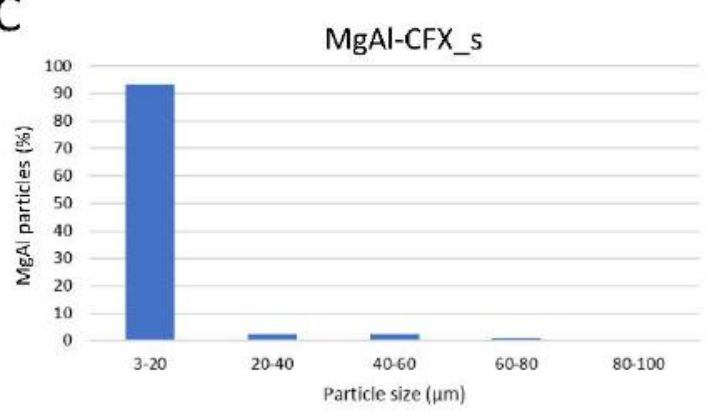

D

\begin{tabular}{|c|c|}
\hline & Particle size D90 $(\mu \mathrm{m})$ \\
\hline ZrP-GTM & $0.7-50$ \\
\hline ZrP-GTM_p & $5-40$ \\
\hline ZrP-GTM_S & $2-40$ \\
\hline
\end{tabular}

$\mathrm{E}$

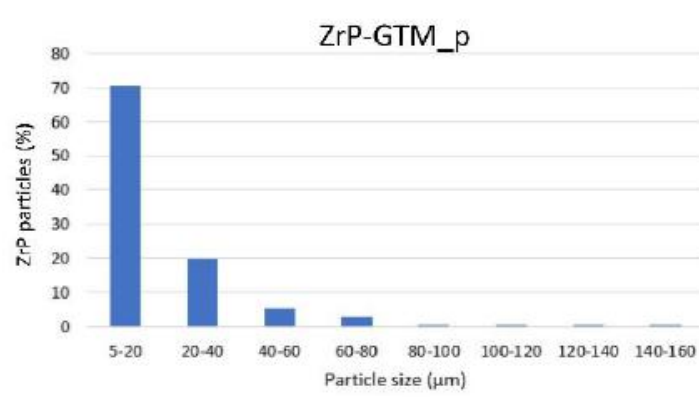

$\mathrm{F}$

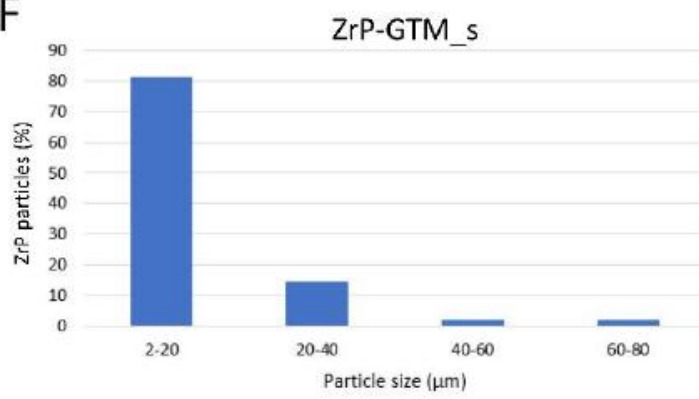

Figure S3. (A) MgAl-CFX particle size (D90) after synthesis, melt blending and ME-AM. (B) MgAlCFX size distribution on MgAl-CFX_p and (C) MgAl-CFX_s. (D) ZrP-GTM particle size (D90) after synthesis, melt-blending and ME-AM. (E) ZrP-GTM size distribution on ZrP-GTM_p and (F) ZrPGTM_s. 
5\% MgAl-CFX_s

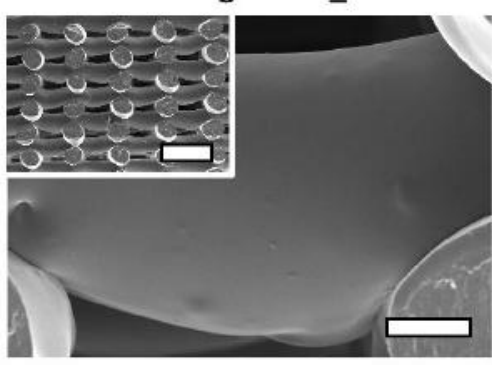

5\% ZrP-GTM_s

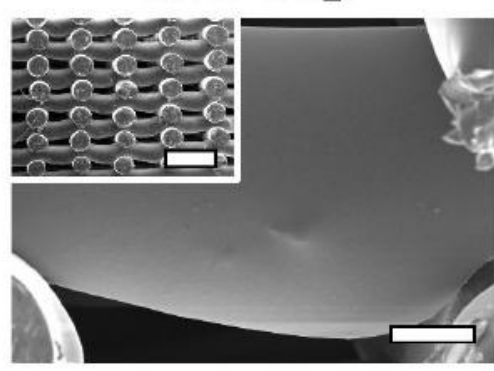

PEOT/PBT_s
10\% MgAl-CFX_s

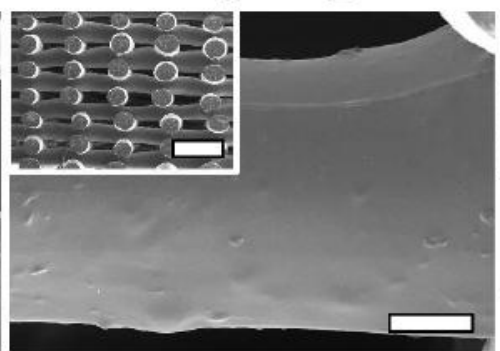

10\% ZrP-GTM_s

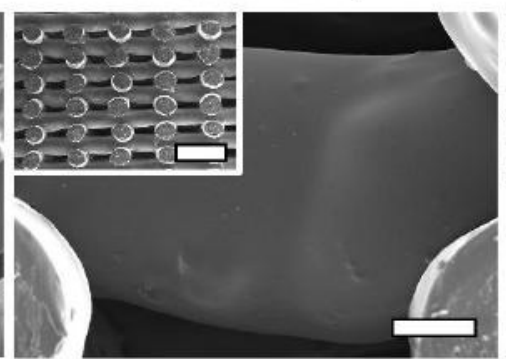

20\% MgAl-CFX_s

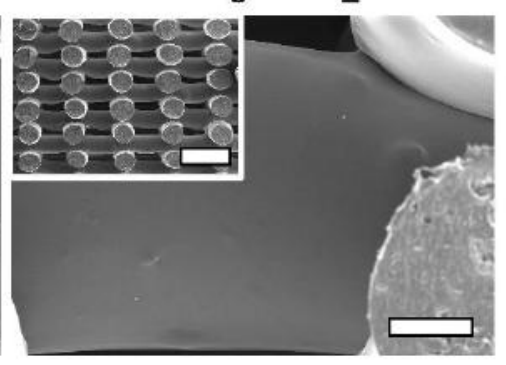

20\% ZrP-GTM_s

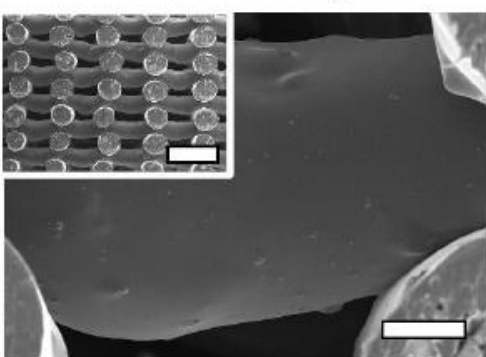

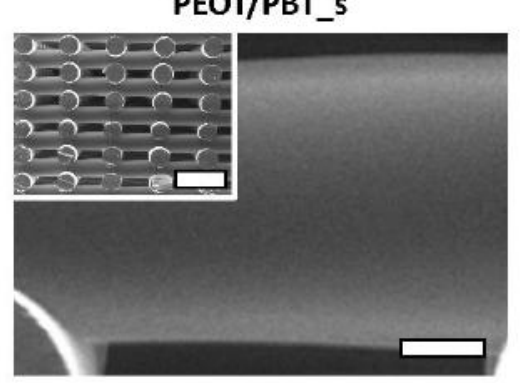

Figure S4. SEM micrographs of PEOT/PBT_s and composite scaffolds depicting the scaffolds' filaments surface roughness. Insert micrographs represent the scaffolds cross section. Scale bars $100 \mu \mathrm{m}$. insert corresponding to low magnification images. Sale bars $1 \mathrm{~mm}$. 
A
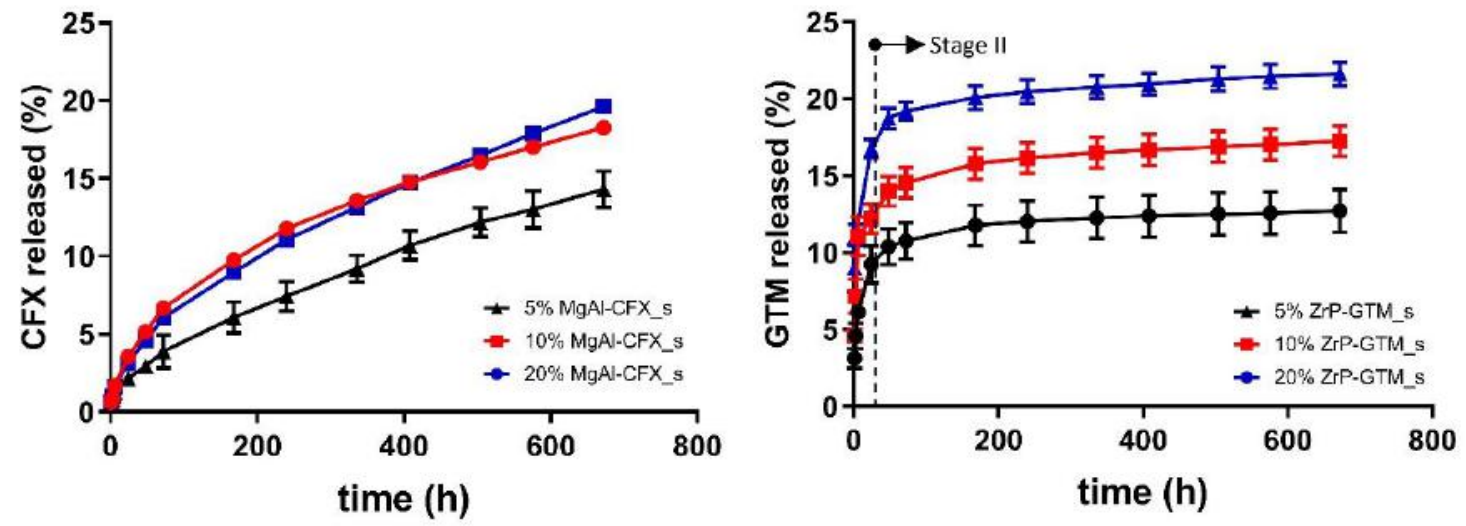

B

\begin{tabular}{|c|c|c|c|}
\hline \multirow[b]{2}{*}{ Model } & \multirow[b]{2}{*}{ Equation } & \multicolumn{2}{|c|}{$\mathbf{R}^{2}$} \\
\hline & & $\begin{array}{c}20 \% \text { MgAl- } \\
\text { CFX_s }\end{array}$ & $\begin{array}{c}20 \% \text { ZrP-GTM_s } \\
\text { (stage I) }\end{array}$ \\
\hline Zero order & $M_{t}-M_{0}=k t+a$ & 0.9757 & 0.7911 \\
\hline First order & $\log M_{t} / M_{0}=-k t+a$ & 0.9809 & 0.8004 \\
\hline Ritger-Peppas & $M_{t} / M_{0}=k t^{n}$ & 0.9949 & 0.9653 \\
\hline Higuchi & $M_{t} / M_{0}=k t^{1 / 2}$ & 0.9932 & 0.9027 \\
\hline Bhaskar & $\ln \left(1-M_{t} / M_{0}\right)=-k t^{0.65}$ & 0.9991 & 0.8758 \\
\hline $\begin{array}{l}\text { Modified } \\
\text { Freundlich }\end{array}$ & $1-M_{t} / M_{0}=k t^{a}$ & 0.8083 & 0.9766 \\
\hline $\begin{array}{l}\text { Parabolic } \\
\text { diffusion }\end{array}$ & $\left(1-M_{t} / M_{0}\right) / t=k t^{-0.5}+a$ & 0.9253 & 0.9614 \\
\hline
\end{tabular}

Figure S5. (A) Cumulative release (\%) of CFX and GTM from MgAl-CFX_s and ZrP-GTM_s, respectively. (B) Kinetic models used to fit the antibiotic release profiles from 20\% MgAl-CFX_s and 20\% ZrP-GTM_s (stage I only) and corresponding correlation coefficients $\left(R^{2}\right)$. $t$ refers to time, $M_{t}$ refers to amount of drug released in time $t, M_{0}$ refers to the total amount of drug loaded in the system, and $\mathrm{k}, \mathrm{a}$, and $\mathrm{n}$ are model-specific constants. 
A

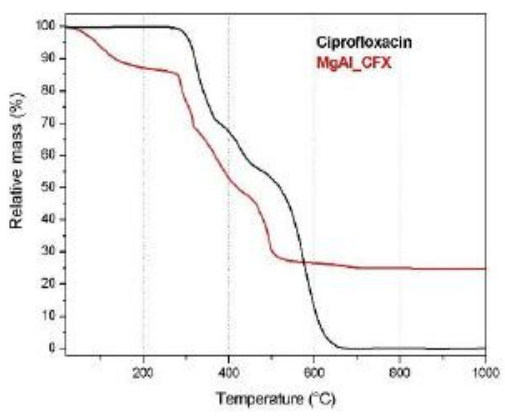

B

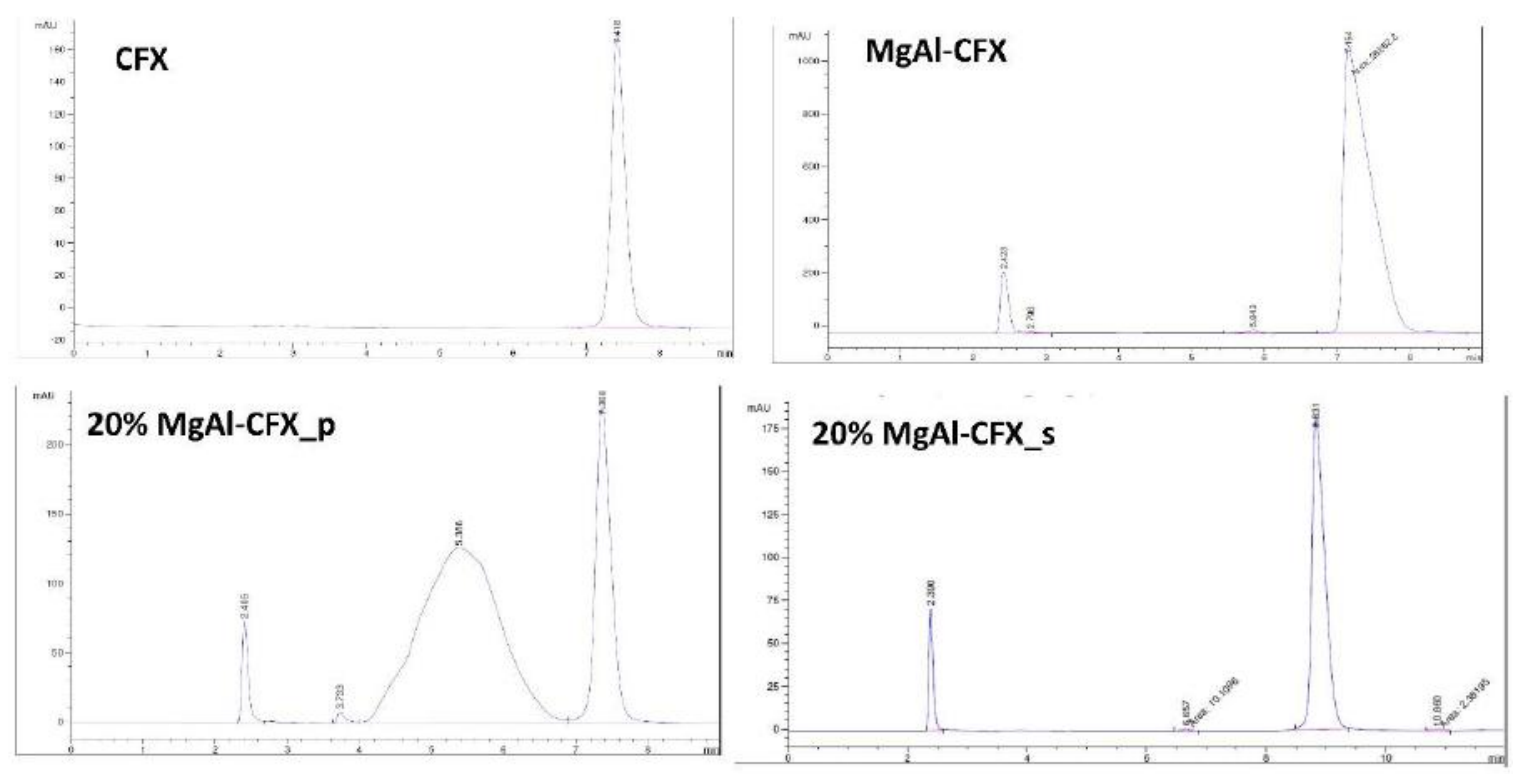

C

\begin{tabular}{c|c}
\hline & $\begin{array}{c}\text { Recovery complete } \\
\text { CFX molecule (\%) }\end{array}$ \\
\hline MgAl-CFX & 100 \\
20\% MgAl-CFX_p & 26,8 \\
20\% MgAl-CFX_s & $54.2 \pm 1.0$ \\
\hline
\end{tabular}

Figure S6. Thermal stability of CFX. (A) TGA curves of pure CFX and MgAl-CFX. (B) HPLC of pure CFX and CFX extracted from MgAI-CFX, 20\% MgAL-CFX_p and 20\% MgAl-CFX_s. (C) Percentage of complete CFX molecule recovery after each processing step. 
A

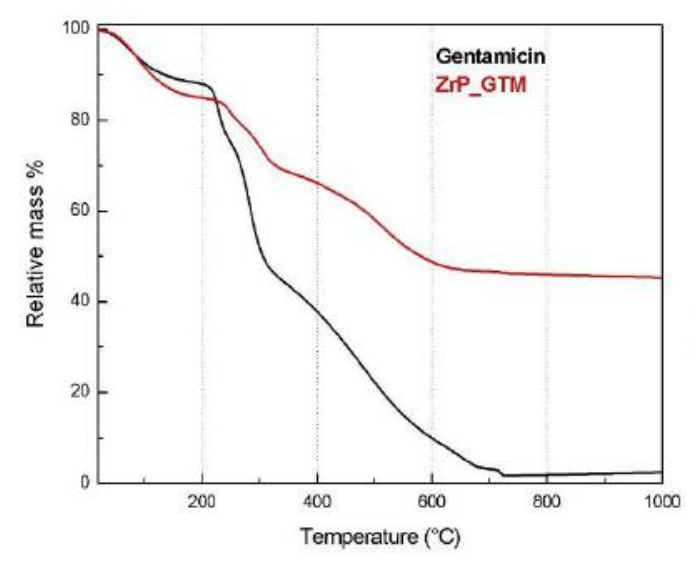

C

\begin{tabular}{|c|c|c|c|c|}
\hline & C1a (\%) & C2 (\%) & C2a (\%) & C1 (\%) \\
\hline GTM & 29.0 & 21.8 & 20.2 & 29.0 \\
\hline ZrP-GTM & 46.8 & 16.0 & 17.0 & 20.2 \\
\hline $20 \%$ ZrP_GTM_p & 44.4 & 16.9 & 17.2 & 21.4 \\
\hline $20 \% \mathrm{ZrP}$ GTM s & 43.7 & 16.8 & 17.7 & 21.8 \\
\hline
\end{tabular}

D

\begin{tabular}{c|c}
\hline & $\begin{array}{c}\text { Recovery complete } \\
\text { GTM molecule (\%) }\end{array}$ \\
\hline ZrP_GTM & $100.7 \pm 2.5$ \\
20\%ZrP_GTM_p & $99.3 \pm 2.1$ \\
20\%ZrP_GTM_S & $92.3 \pm 3.2$ \\
\hline
\end{tabular}

B
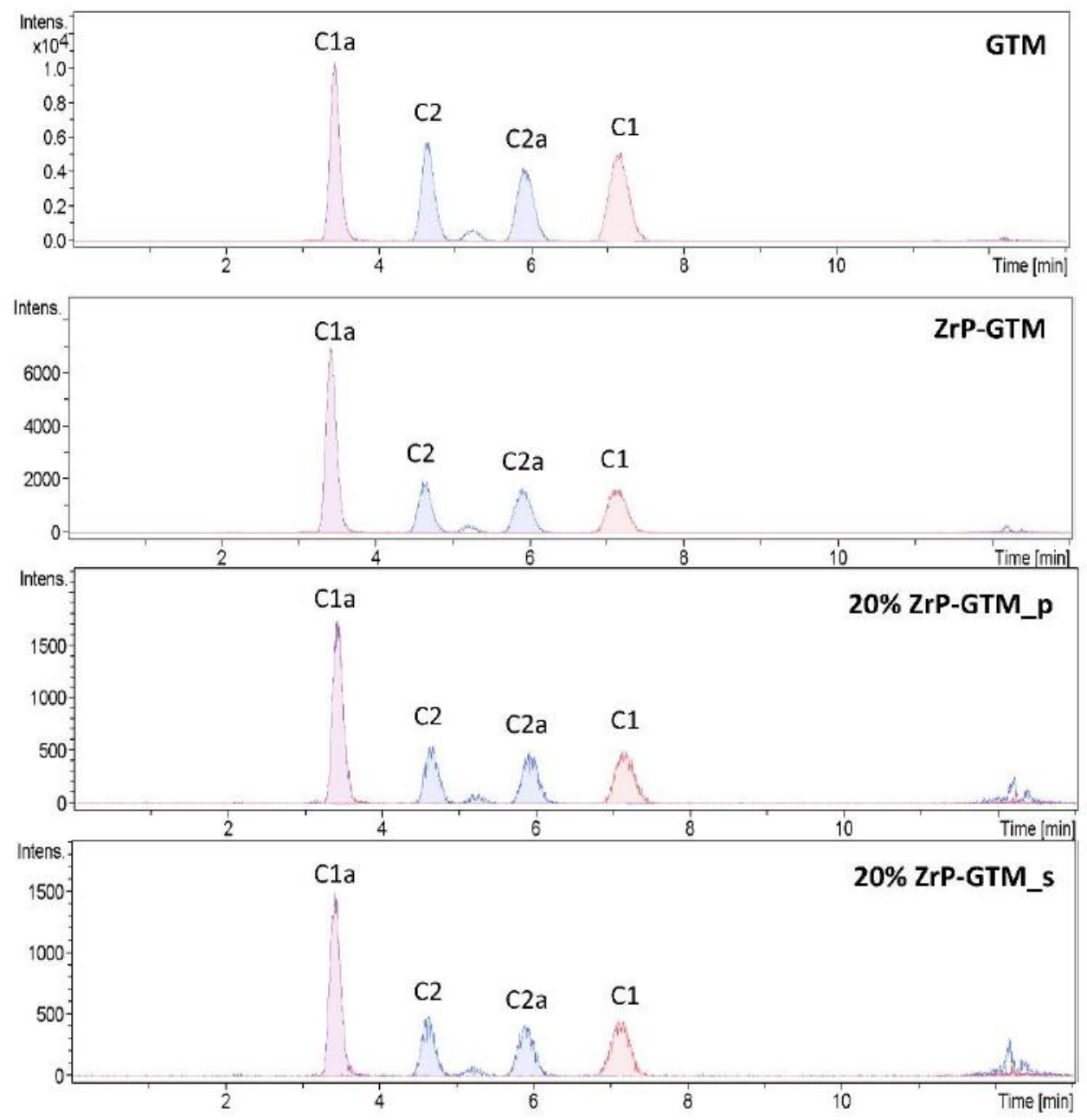

Figure S7. Thermal stability of GTM. (A) TGA curves of pure GTM and ZrP-GTM. (B) HPLC-MS of pure GTM and GTM extracted from ZrP-GTM, 20\% ZrP-GTM_p and 20\% ZrP-GTM_s. (C) Relative amount of each GTM isoform intercalated in ZrP after each processing step. (D) Percentage of complete GTM molecule recovery after each processing step. 
A

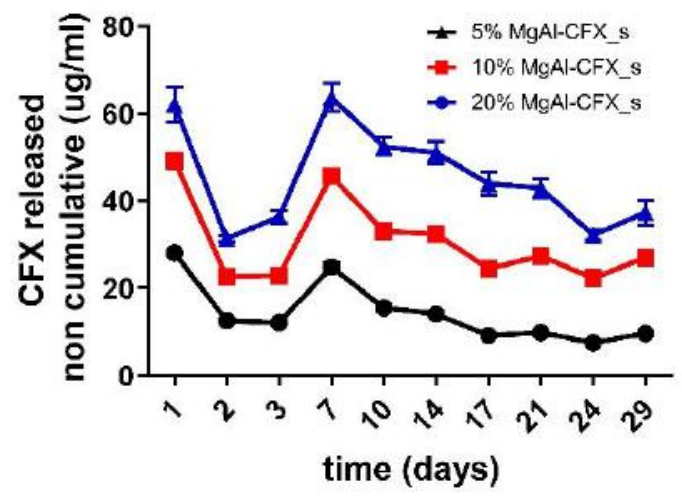

B

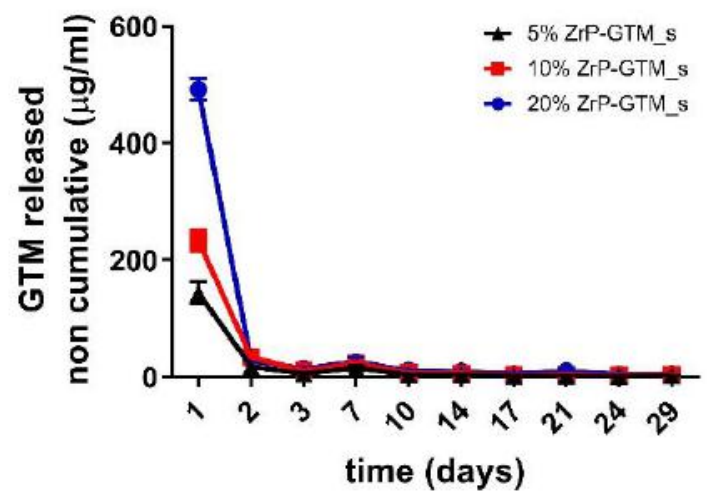

Figure S8. Non cumulative release of (A) CFX and (B) GTM over time from MgAl-CFX_s and ZrPGTM_s, respectively.

A

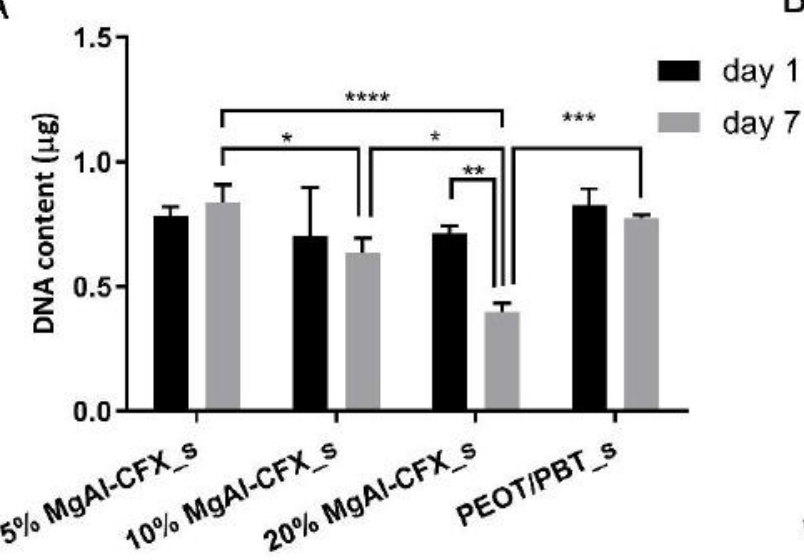

B

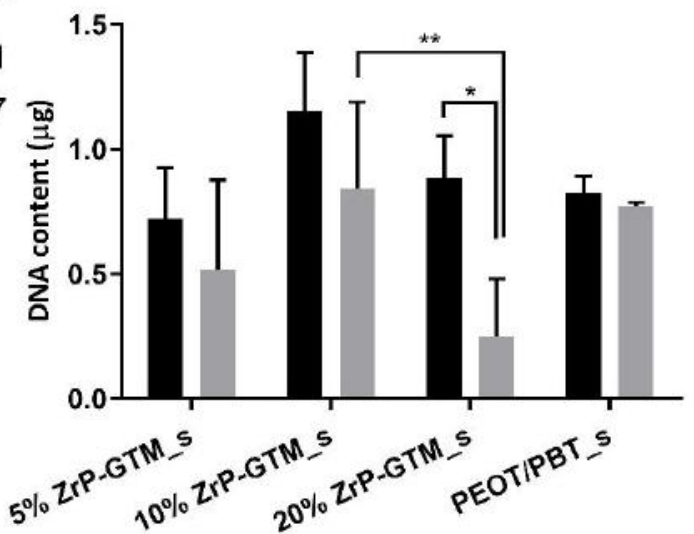

Figure S9. DNA content after 1 and 7 days of culture in BM in (A) MgAl-CFX_s and (B) ZrP-GTM_s, directly seeded without pre-incubation in media. Statistical significance performed using two-way ANOVA with Tukey's multiple comparison test $\left({ }^{*} p<0.05 ;{ }^{* *} p<0.01 ;{ }^{* * *} p<0.001 ;{ }^{* * *} p<0.0001\right)$. 
A

CFX toxicity

B

GTM toxicity
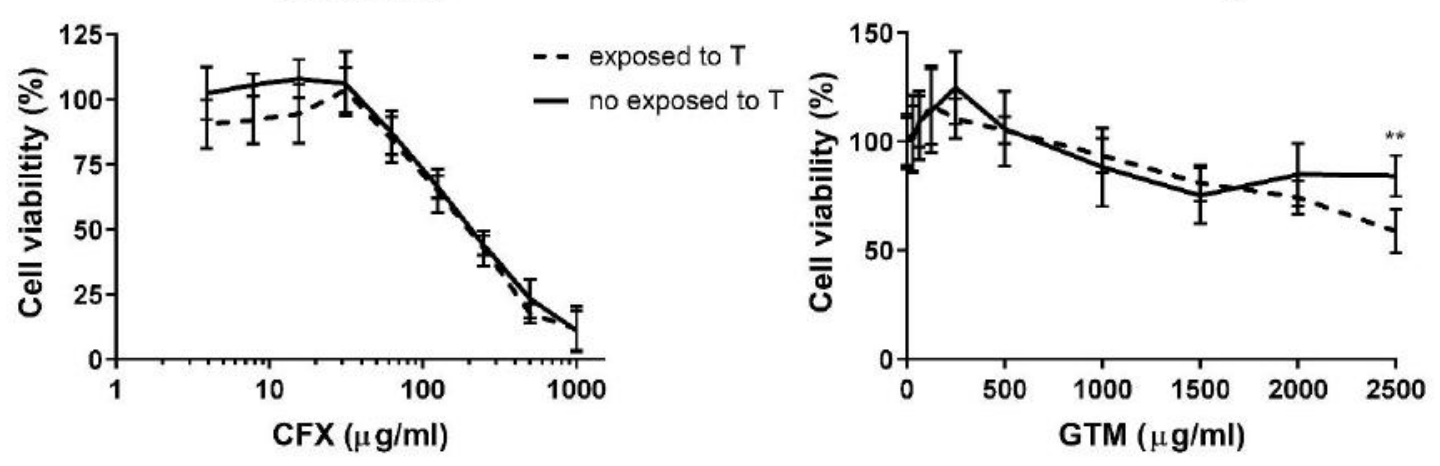

Figure S10. Dose dependence of toxicity of (A) CFX and (B) GTM towards hMSCs, in their pure form (not exposed to $\mathrm{T}$ ) or previously exposed to $200{ }^{\circ} \mathrm{C}$ for $30 \mathrm{~min}$ under a $\mathrm{N}_{2}$ atmosphere (exposed to T).

A

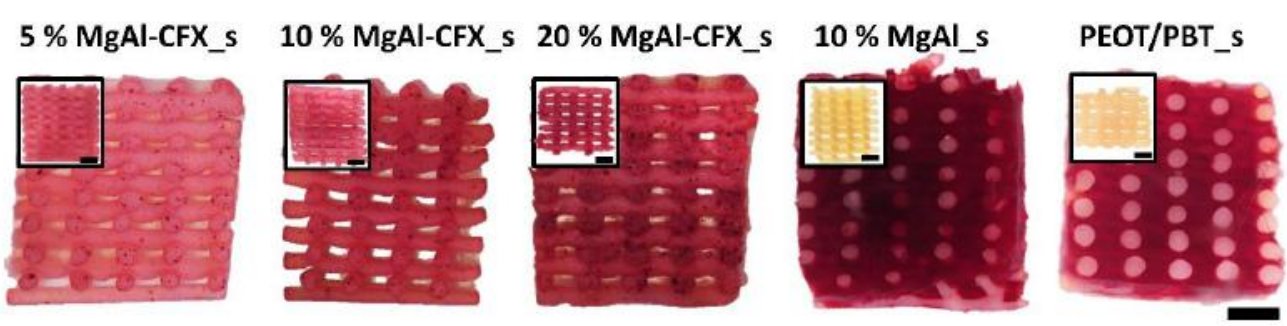

B
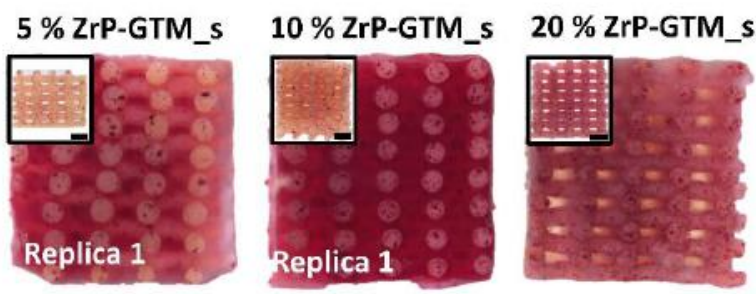

$10 \%$ ZrP_s
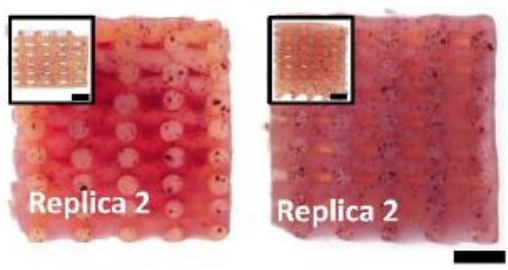

C
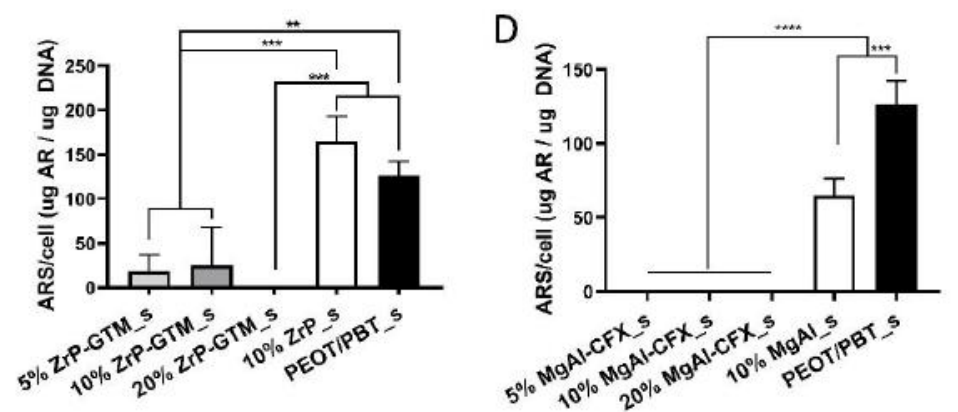

Figure S11. Stereomicroscopy images of (A) PEOT/PBT_s and MgAI-CFX_s and (B) ZrP-GTM_s cross sections stained with ARS after 35 days of culture (28 days in MM). Inserts represent the 
corresponding control scaffolds without cells incubated in MM and stained with ARS. Scale bars 1 $\mathrm{mm}$. (C, D) Quantification of the ARS extracted from scaffolds after 35 days of culture (28 days in $M M)$, normalized to total cell number. Statistical significance performed using one-way ANOVA with Tukey's multiple comparison test $\left({ }^{* *} \mathrm{p}<0.01 ;{ }^{* * *} \mathrm{p}<0.001 ;{ }^{* * *} \mathrm{p}<0.0001\right)$.

A

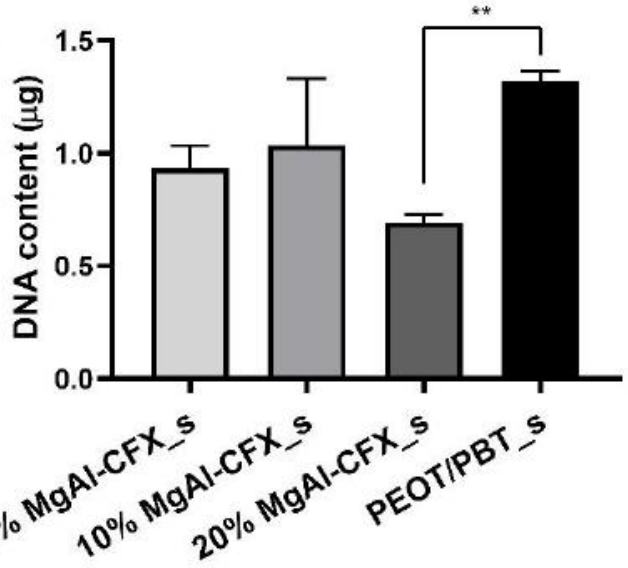

C

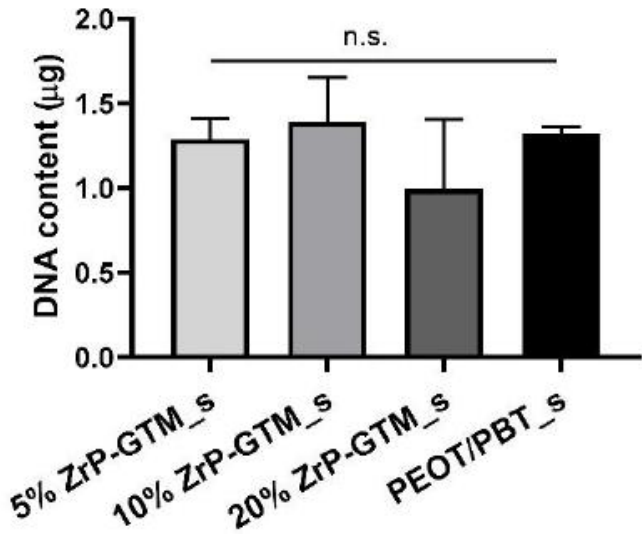

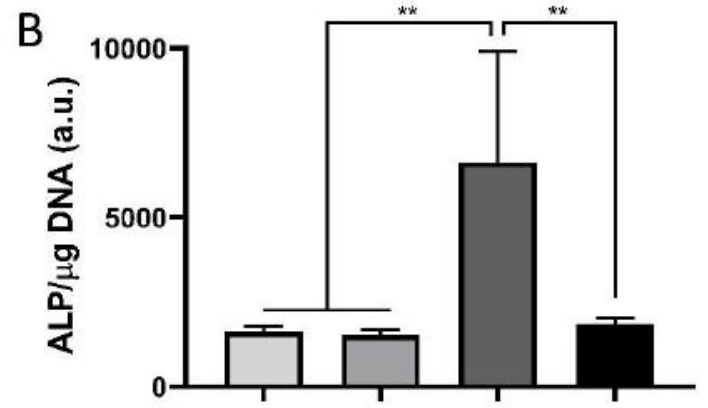

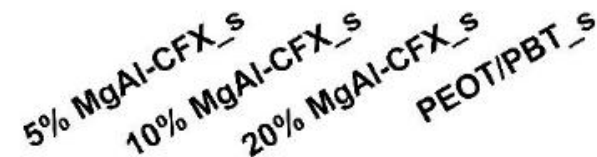

D

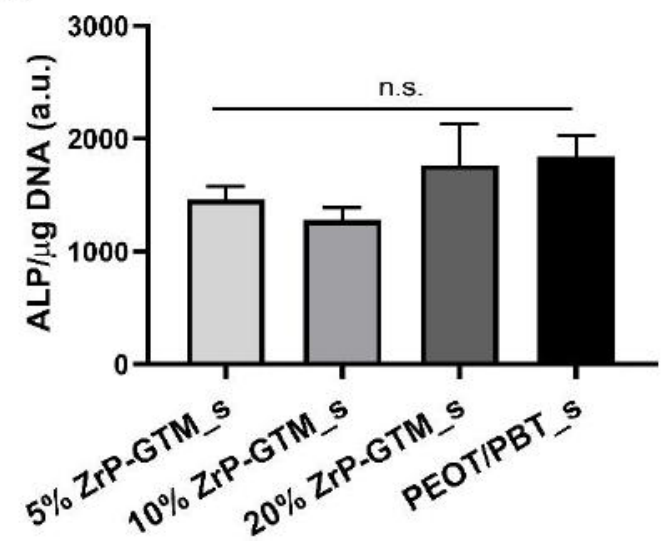

Figure S12. (A) DNA content and (B) ALP activity on MgAI-CFX_s after 56 days of culture (49 days in MM). (C) DNA content and (D) ALP activity on ZrP-GTM_s after 42 days of culture (35 days in MM). Statistical significance performed using one-way ANOVA with Tukey's multiple comparison test (n.s. $p>0.05,{ }^{* *} p<0.01$ ). 
A
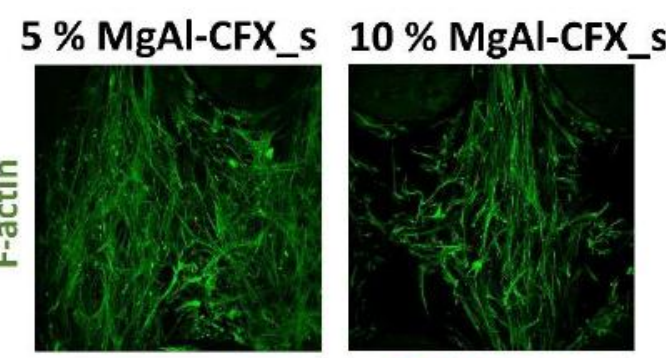

$5 \%$ ZrP-GTM_s $10 \%$ ZrP-GTM_s
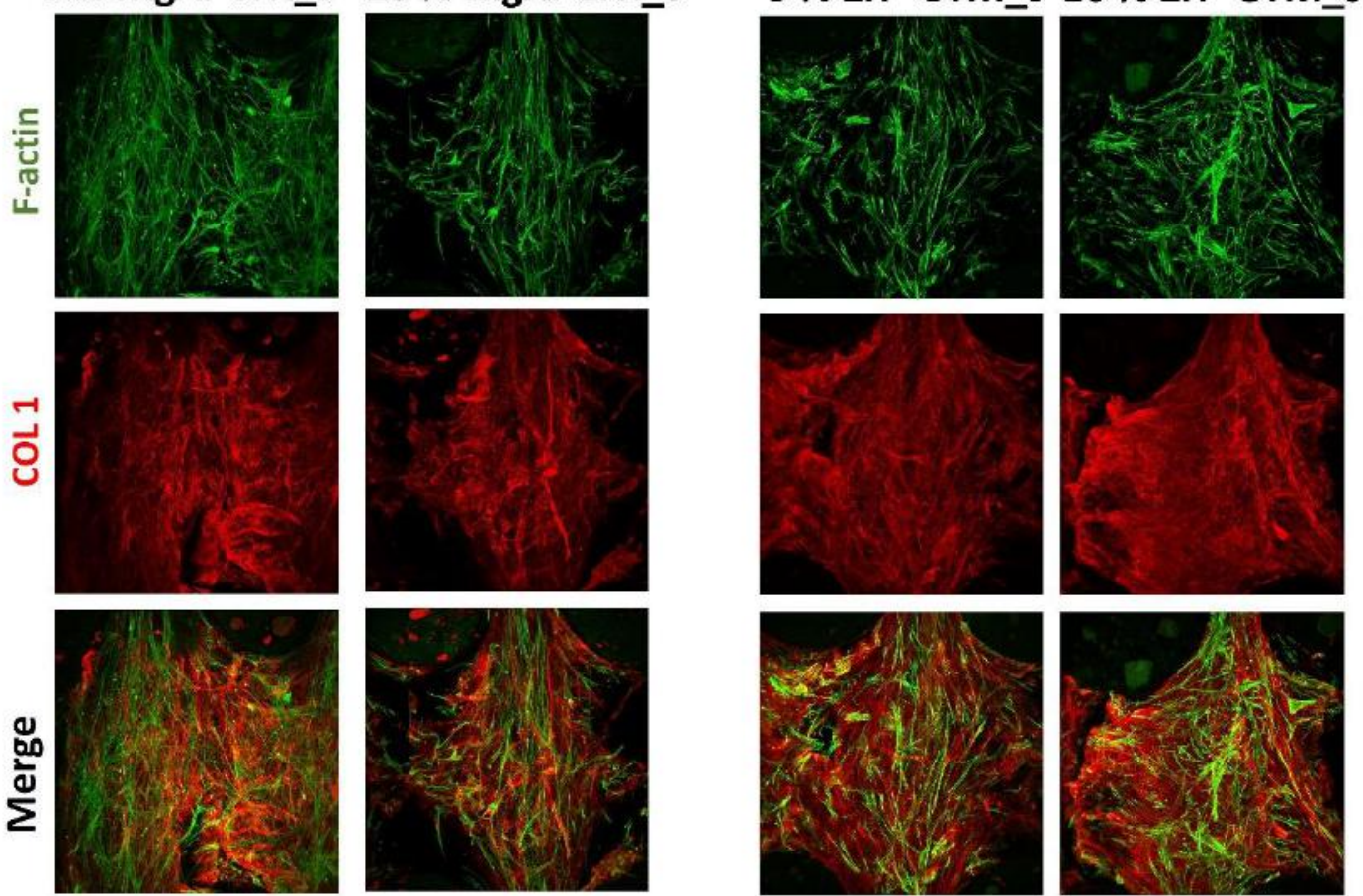

B

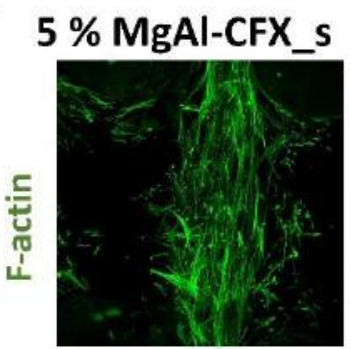

$10 \%$ MgAl-CFX_s

5 \% ZrP-GTM_s $10 \%$ ZrP-GTM_s
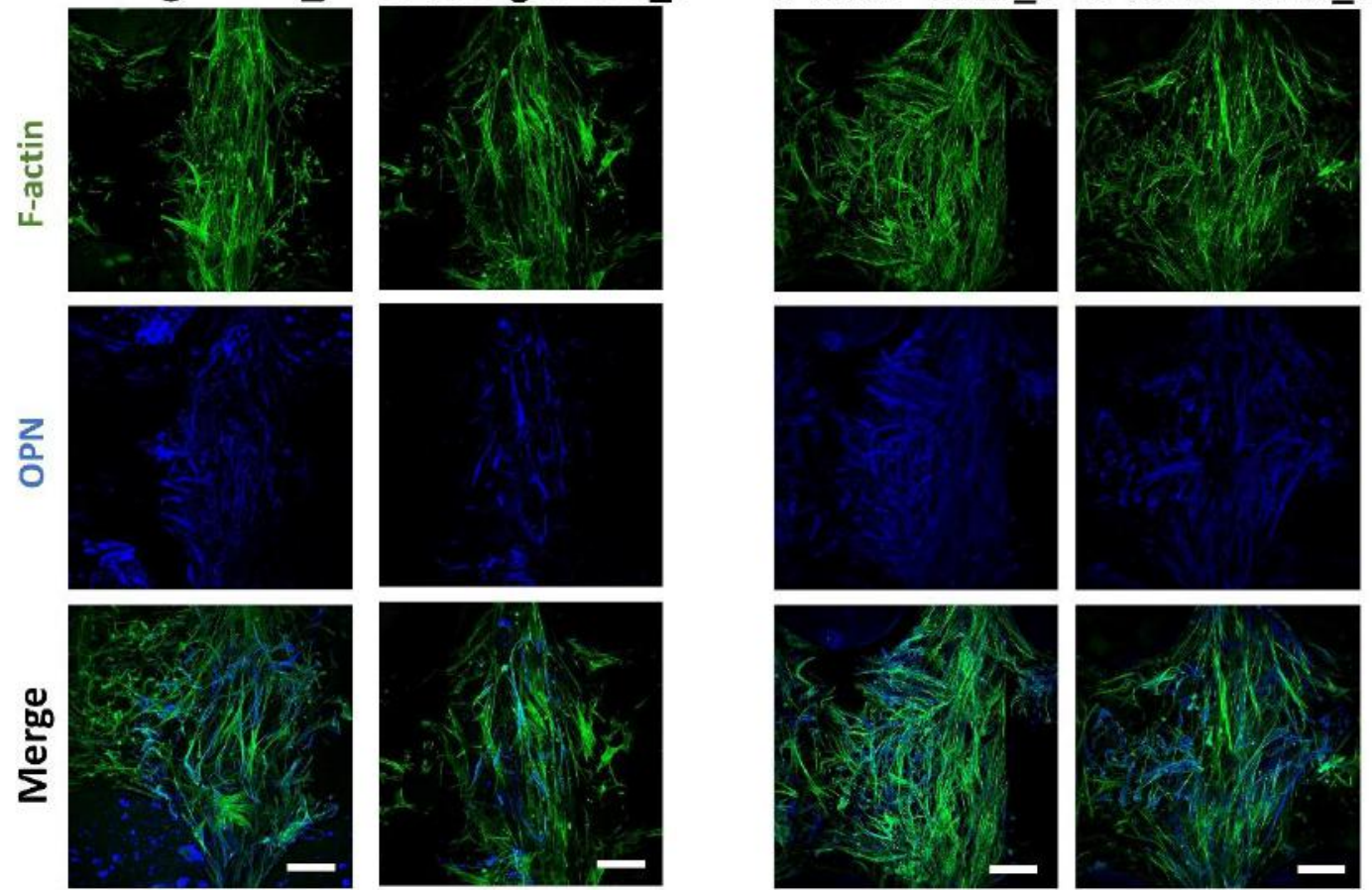

Figure S13. Representative confocal microscopy images of hMSCs (F-actin, green) on top of the filaments of (A) 5 and $10 \% \mathrm{MgAl}-\mathrm{CFX}$ s after 56 days of culture (49 days in MM) and (B) 5 and 10 $\%$ ZrP-GTM_s after 42 days of culture (35 days in MM) stained for relevant osteogenic markers COL1 (red) and OPN (blue). Scale bars $100 \mu \mathrm{m}$. 
Table S1. Antimicrobial activity of MgAl-CFX and ZrP-GTM at different concentrations against $S$. epidermidis and $P$. aeruginosa.

\begin{tabular}{|c|c|c|}
\hline $\begin{array}{l}\text { Samples } \\
(0.1 \mathrm{~g} / \mathrm{ml})\end{array}$ & $\begin{array}{c}\text { S. Epidermidis } \\
\text { R (log) }\end{array}$ & $\begin{array}{c}\text { P. Aeruginosa } \\
\text { R }(\log )\end{array}$ \\
\hline ZrP-GTM $5 \mathrm{mg} / \mathrm{ml}$ & $>4.7$ & $>6.7$ \\
\hline ZrP-GTM 10/ml & $>4.7$ & $>6.7$ \\
\hline ZrP-GTM 20m g/ml & $>4.7$ & $>6.7$ \\
\hline MgAl-CFX 5mg/ml & $>4.8$ & $>5.8$ \\
\hline $\begin{array}{l}\text { MgAl-CFX } 10 \\
\mathrm{mg} / \mathrm{ml}\end{array}$ & $>4.8$ & $>5.8$ \\
\hline $\begin{array}{c}\text { MgAl-CFX } 20 \\
\mathrm{mg} / \mathrm{ml}\end{array}$ & $>4.8$ & $>5.8$ \\
\hline
\end{tabular}

Table S2. Antimicrobial activity of MgAL-CFX_f and ZrP-GTM_f against $S$. epidermidis and $P$. aeruginosa.

\begin{tabular}{c|cc}
\hline $\begin{array}{c}\text { Samples } \\
\mathbf{( 0 . 1} \mathbf{g} / \mathbf{m l})\end{array}$ & $\begin{array}{c}\text { S. Epidermidis } \\
\mathbf{R}(\mathbf{l o g})\end{array}$ & $\begin{array}{c}\text { P. Aeruginosa } \\
\mathbf{R}(\mathbf{l o g})\end{array}$ \\
\hline $5 \%$ MgAl-CFX_f & 1.08 & $>6.85$ \\
\hline 10\% MgAl-CFX_f & 1.51 & $>6.85$ \\
$20 \%$ MgAl-CFX_f & 5.51 & $>6.85$ \\
5\% ZrP-GTM_f & $>4.94$ & 1.95 \\
10\% ZrP-GTM_f & $>4.94$ & 3.5 \\
20\% ZrP-GTM_f & $>4.94$ & 4.89 \\
\hline
\end{tabular}


Table S3. Susceptibility of $S$. epidermidis and $P$. aeruginosa and strains to pure GTM and CFX evaluated by the disk diffusion agar test.

\begin{tabular}{|c|c|c|c|c|c|c|c|c|c|}
\hline & \multicolumn{4}{|c|}{ S.Epidermidis } & \multicolumn{4}{|c|}{ P. Aeruginosa } \\
\hline & & \multicolumn{2}{|c|}{$\begin{array}{c}\text { GTM } \\
\text { zol (mm) }\end{array}$} & \multicolumn{2}{|c|}{$\begin{array}{c}\text { CFX } \\
\text { ZOI }(\mathrm{mm}) \\
\end{array}$} & \multicolumn{2}{|c|}{$\begin{array}{c}\text { GTM } \\
\text { ZOI (mm) }\end{array}$} & \multicolumn{2}{|c|}{$\begin{array}{c}\text { CFX } \\
\text { ZOI }(\mathrm{mm}) \\
\end{array}$} \\
\hline $\begin{array}{c}\text { Concentration } \\
(\mu \mathrm{g} / \mathrm{ml})\end{array}$ & $\begin{array}{c}\text { In disc } \\
(\mu \mathrm{g})\end{array}$ & Av. & s.d. & Av. & s.d. & Av. & s.d. & Av. & s.d. \\
\hline 500 & 10 & 16 & 1.2 & 33 & 2.0 & 9 & 0.0 & 25 & 0.6 \\
\hline 250 & 5 & 16 & 1.2 & 30 & 0.6 & 8 & 0.5 & 25 & 1.0 \\
\hline 100 & 2 & 12 & 0.0 & 25 & 0.6 & 6 & 0.0 & 23 & 1.0 \\
\hline 75 & 1.5 & 11 & 0.6 & 21 & 0.6 & 6 & 0.0 & 22 & 1.5 \\
\hline 50 & 1 & 10 & 0.6 & 18 & 0.0 & 6 & 0.0 & 18 & 0.6 \\
\hline 25 & 0.5 & 10 & 1.5 & 16 & 0.6 & 6 & 0.0 & 16 & 0.6 \\
\hline 15 & 0.3 & 7 & 1.2 & 12 & 0.6 & 6 & 0.0 & 14 & 0.6 \\
\hline 10 & 0.2 & 6 & 0.6 & 6 & 0.0 & 6 & 0.0 & 13 & 0.0 \\
\hline 5 & 0.1 & 6 & 0.0 & 6 & 0.0 & 6 & 0.0 & 6 & 0.0 \\
\hline 0 & 0 & 6 & 0.0 & 6 & 0.0 & 6 & 0.0 & 6 & 0.0 \\
\hline
\end{tabular}

Table S4. Non cumulative release of CFX over time from $20 \mathrm{wt} \% \mathrm{MgAl}-\mathrm{CFX} s \mathrm{~s}$ and the corresponding experimental and expected ZOI against $S$. epidermidis and $P$. aeruginosa. Expected ZOI were estimated from the susceptibility test in Table S3.

$20 \%$ MgAl-CFX_s

\begin{tabular}{|c|c|c|c|c|c|c|c|c|}
\hline \multirow{3}{*}{$\begin{array}{l}\text { time } \\
\text { (days) }\end{array}$} & & & \multicolumn{3}{|c|}{ S. Epidermidis } & \multicolumn{3}{|c|}{ P. Aeruginosa } \\
\hline & \multicolumn{2}{|c|}{ CFX release (ug) } & \multicolumn{2}{|c|}{$\begin{array}{c}\text { ZOI experimental } \\
(\mathrm{mm})\end{array}$} & \multirow{2}{*}{$\begin{array}{l}\text { ZOI expected } \\
(\mathrm{mm})\end{array}$} & \multicolumn{2}{|c|}{$\begin{array}{c}\text { ZOI experimental } \\
(\mathrm{mm})\end{array}$} & \multirow{2}{*}{$\begin{array}{c}\mathrm{ZOI} \text { expected } \\
(\mathrm{mm})\end{array}$} \\
\hline & Av. & s.d. & Av. & s.d. & & Av. & s.d. & \\
\hline 1 & 62.02 & 3.89 & 21.3 & 0.6 & $18-21$ & 22.0 & 1.0 & $18-22$ \\
\hline 2 & 31.37 & 0.75 & 20.0 & 0.0 & $16-18$ & 15.3 & 0.6 & $16-18$ \\
\hline 3 & 36.38 & 1.40 & 21.3 & 0.6 & $16-18$ & 17.7 & 0.6 & $16-18$ \\
\hline 7 & 63.67 & 3.23 & 24.0 & 1.0 & $18-22$ & 22.0 & 1.0 & $18-22$ \\
\hline 10 & 52.46 & 1.98 & 20.7 & 1.2 & 18 & 20.0 & 1.0 & 18 \\
\hline 14 & 51.06 & 2.50 & 28.3 & 0.6 & 18 & 18.3 & 0.6 & 18 \\
\hline 17 & 43.99 & 2.73 & 24.0 & 1.0 & $16-18$ & 19.7 & 0.6 & $16-18$ \\
\hline 21 & 42.93 & 2.27 & 22.0 & 1.0 & $16-18$ & 20.3 & 0.6 & $16-18$ \\
\hline 24 & 32.20 & 1.25 & 23.3 & 1.2 & $16-18$ & 22.3 & 0.6 & $16-18$ \\
\hline 29 & 37.34 & 2.86 & 23.3 & 0.6 & $16-18$ & 18.0 & 1.0 & $16-18$ \\
\hline
\end{tabular}


Table S5. Non cumulative release of GTM over time from 20 wt\% ZrP-GTM_s and the corresponding experimental and expected ZOI against $S$. epidermidis and $P$. aeruginosa. Expected ZOI were estimated from the susceptibility test in Table S3.

\begin{tabular}{|c|c|c|c|c|c|c|c|c|}
\hline \multicolumn{9}{|c|}{ 20\% ZrP-GTM_s } \\
\hline \multirow{3}{*}{$\begin{array}{l}\text { Time } \\
\text { (days) }\end{array}$} & \multirow{2}{*}{\multicolumn{2}{|c|}{ GTM release (ug) }} & \multicolumn{3}{|c|}{ S. Epidermidis } & \multicolumn{3}{|c|}{ P. Aeruginosa } \\
\hline & & & \multicolumn{2}{|c|}{$\begin{array}{c}\text { ZOI experimental } \\
(\mathrm{mm})\end{array}$} & \multirow{2}{*}{$\begin{array}{c}\text { zOI expected } \\
(\mathrm{mm})\end{array}$} & \multicolumn{2}{|c|}{$\begin{array}{c}\text { ZOI experimental } \\
(\mathrm{mm})\end{array}$} & \multirow{2}{*}{$\begin{array}{c}\text { ZOI expected } \\
(\mathrm{mm})\end{array}$} \\
\hline & Av. & s.d. & Av. & s.d. & & Av. & s.d. & \\
\hline 1 & 491.63 & 18.00 & 16.7 & 0.6 & 16 & 11.0 & 0.0 & 9 \\
\hline 2 & 28.50 & 1.18 & 11.3 & 0.6 & 10 & 6.0 & 0.0 & 6 \\
\hline 3 & 14.34 & 1.92 & 8.3 & 0.6 & 7 & 6.0 & 0.0 & 6 \\
\hline 7 & 25.50 & 7.93 & 8.3 & 0.6 & 10 & 6.0 & 0.0 & 6 \\
\hline 10 & 10.69 & 0.44 & 7.0 & 0.0 & 6 & 6.0 & 0.0 & 6 \\
\hline 14 & 9.50 & 1.03 & 7.0 & 0.0 & 6 & 6.0 & 0.0 & 6 \\
\hline 17 & 5.24 & 0.77 & 6.7 & 0.6 & 6 & 6.0 & 0.0 & 6 \\
\hline 21 & 9.93 & 5.64 & 7.3 & 0.6 & 6 & 6.0 & 0.0 & 6 \\
\hline 24 & 4.57 & 0.75 & 6.0 & 0.0 & 6 & 6.0 & 0.0 & 6 \\
\hline 29 & 5.04 & 1.19 & 6.0 & 0.0 & 6 & 6.0 & 0.0 & 6 \\
\hline
\end{tabular}

Table S6. Theoretical and experimental (determined by TGA) MgAl-CFX and ZrP-GTM loadings of MgAI-CFX_p and ZrP-GTM_p at different filler-antibiotic concentrations.

\begin{tabular}{|c|c|c|}
\hline & $\begin{array}{c}\text { Theoretical } \\
\text { loading (\%) }\end{array}$ & $\begin{array}{c}\text { Experimental } \\
\text { loading (\%) }\end{array}$ \\
\hline 5\% MgAl-CFX_p & 5 & 5.4 \\
\hline $10 \%$ MgAl-CFX_p & 10 & 7.1 \\
\hline $20 \%$ MgAl-CFX_p & 20 & 20.9 \\
\hline $5 \%$ ZrP-GTM_p & 5 & 8.0 \\
\hline $10 \%$ ZrP-GTM_p & 10 & 11.3 \\
\hline $20 \%$ ZrP-GTM_p & 20 & 18.6 \\
\hline
\end{tabular}





\section{Chapter 8}

General Discussion 

In this Chapter, the most important findings of the thesis will be discussed and contextualized within the overall aim of the thesis, which is the design and fabrication of bioactive melt extrusion additive manufactured (ME-AM) scaffolds for bone tissue regeneration. We highlight the reasons for material and cell source choice, the importance of cell confluency in osteogenic differentiation, and the unique functionality provided by the investigated scaffold modifications.

\section{Why PEOT/PBT?}

As noticed in the literature review within Chapter 2, poly( $\varepsilon$-caprolactone) (PCL) is the most used biodegradable thermoplastic polymer to fabricate ME-AM scaffolds for bone tissue engineering (BTE) applications. The main motivation for researchers to invest on this polymer is its approval by FDA or CE mark certification for drug delivery devices, tissue adhesion barriers, and 3D printed implants for bone regeneration, which consequently smoothens the clinical and marketing path of any subsequent newly designed PCL based scaffolds. However, most of the studies have been performed using research-grade PCL, which has been shown to differ from medical-grade PCL in terms of molecular weight, impurities content, physicochemical properties (mechanical properties, degradation) and processing parameters, often leading to results being unsuitable for translation. ${ }^{1-2}$ Other important reasons for PCL monopolizing ME-AM BTE research are its high compression strength; long degradation time; and its low printing temperature, which diminishes the printer hardware demands. ${ }^{2}$ Despite these appealing characteristics, PCL also possess some drawbacks, including its poor cell adhesion and limited cell spreading, ${ }^{3}$ which are non-optimal properties for applications in which strong cell adhesion and spreading are required, such as bone formation. ${ }^{4}$ For these reasons, the exploration of other synthetic polymers towards the design of an optimum scaffold for BTE is still desired. One of these polymers is the block copolymer poly(ethylene oxide terephthalate)/poly(butylene terephthalate) (PEOT/PBT), which is commercially available in medical-grade, as well as approved by regulatory authorities in the composition 1000PEOT70PBT30 (1000 kDa PEO segment and a PEOT:PBT ratio of 70:30) for its use as drug delivery system, cement restrictor and $3 \mathrm{D}$ printed scaffold for cartilage regeneration. PEOT/PBT copolymers are thermoplastic, biocompatible and biodegradable, and their mechanical, physicochemical and swelling properties can be tuned within a wide range by varying the PEO segment molecular weight, as well as the PEOT:PBT ratio, to meet the requirements of a specific regenerative application. ${ }^{5-8}$ Taking this into consideration, PEOT/PBT and scaffolds made 
from these materials have proven their in vitro and in vivo applicability in skeletal tissue regeneration, including bone ${ }^{9-16}$, cartilage ${ }^{17-20}$ and osteochondral tissue engineering, ${ }^{21-24}$ as well as in skin, ${ }^{25}$ and nerve regeneration, ${ }^{26}$ or islets of Langerhans delivery. ${ }^{27-29}$ Among other formulations, the PEOT/PBT used within this thesis, 300PEOT55PBT45 (300 kDa PEO segment and a PEOT:PBT ratio of 55:45), constitutes a valid alternative to PCL for the fabrication of ME-AM scaffolds for BTE. This is owed to its long degradation time (3\% mass loss after 24 weeks implantation in vivo), optimum mechanical properties (tensile modulus $\sim 50 \mathrm{MPa}$, compression modulus $\sim 120 \mathrm{MPa}$ ), and good cell attachment and spreading in vitro without further functionalization. ${ }^{5-8,10}$ Furthermore, it has a relatively low melting temperature $\left(\sim 160{ }^{\circ} \mathrm{C}\right)$ and low viscosity, which enables its easy processing through standard ME-AM platforms at $165-195^{\circ} \mathrm{C} .{ }^{30-31}$

\section{Why human mesenchymal stromal cells (hMSCs)?}

Because of their ability to differentiate into osteoblasts and their role in tissue regeneration, hMSCs are commonly used in tissue engineering. ${ }^{32}$ HMSCs have the ability to migrate chemotactically to injured tissues, where they differentiate into tissue-specific cells, and help in the regeneration process by the secretion of factors, which can modulate the inflammatory response, promote vascularization, and induce proliferation and differentiation of other endogenous cell types. ${ }^{33-34}$ In an attempt to support the endogenous homing of hMSCs, BTE approaches have considered the direct injection to the injured site of these cells or, most interestingly, the implantation of hMSC-laden scaffolds. ${ }^{35-36}$ When combined with hMSCs, the scaffold would not only mechanically support the fracture and promote a material-induced bone growth response, but also ideally boost the implanted hMSCs and, subsequently, other endogenous cell types, towards an accelerated bone regeneration response. HMSCs can be extracted and isolated from the patient's own bone marrow without leading to donor site morbidity, unlike other autografts, and can be expanded in culture to generate sufficient numbers while maintaining their multipotency. ${ }^{35}$

Prior to implantation, and regardless of the final scaffold application being cell-free or cellladen, the functionality of a newly designed scaffold is usually tested by seeding it with hMSCs and evaluating these cells ability to undergo osteogenic differentiation on the scaffold. ${ }^{37-38}$ Several reports have already dedicated initial efforts to evaluate this way the in vitro biological performance of PEOT/PBT (300PEOT55PBT45) ME-AM scaffolds for 
BTE. 13-16, 21-24 This common in vitro practice gives hints on the osteoinductive and osteoconductive properties of the scaffold, i.e. the scaffold's ability to recruit bone forming cells or simply support bone formation on scaffold surface, respectively. Accordingly, hMSCs were used in this thesis to test the developed ME-AM scaffold's ability to support or induce bone formation in vitro.

\section{Addressing the cell seeding problem on ME-AM scaffolds}

A challenge encountered when seeding hMSCs on PEOT/PBT ME-AM scaffolds was the poor cell attachment along the scaffold cross section, with the majority of cells sedimenting and forming a monolayer at the scaffold's bottom. This is a persistent issue associated with the macroporosity and synthetic nature of polymeric ME-AM scaffolds, as well as to gravity, 22, 39 which up to now has been managed by architectural or surface scaffold modifications, or by complicated seeding methods. Chapter 3 of this thesis has demonstrated that by simply altering the physical properties of the seeding media (viscosity or density), a significant enhancement on cell distribution along the scaffold cross section can be attained upon static seeding. This was possible by including the macromolecules (macroM) dextran or Ficoll to the seeding media at specific concentrations, which dramatically reduced hMSCs settling velocity, allowing cells to float longer in the suspension and giving them more time to attach to the scaffold filaments. This approach differs from previous efforts in terms of: i) its simplicity, as it does not require of additional equipment, such as a rocking bed for dynamic seeding, a vacuum chamber for plasma treatment, or complicated chemistry for a hydrogel carrier formation; ii) its universality, as it can be used for any scaffold type, regardless of its material and architecture; iii) its efficiency, as it avoids the use of large cell stocks; and iv) its temporary activity, as none of the supplements attach to the scaffold filaments and can be quickly expelled by cells when endocytosed.

Alternatively, enhanced cell attachment and distribution was achieved by fabricating PEOT/PBT scaffolds via atmospheric pressure plasma assisted AM in Chapter 4. In this case, cell attachment was induced by the deposition of hydrophilic and protein adhesive coatings on the scaffolds filaments, generated by plasma-polymerization, prior to conventional static seeding. Plasma had been previously used to treat 3D ME-AM scaffolds, ${ }^{40-42}$ but with our approach, we have tackled several issues that had been previously identified when this surface modification technique was used to treat scaffolds. 
First, the use of an atmospheric pressure plasma jet (APPJ) allowed to direct the plasma flame into the scaffold pores, thus avoiding the inhomogeneous and uncontrolled gradient functionalization along the scaffold volume resulting from conventional vacuum plasma. ${ }^{43}$ Furthermore, we investigated the use of plasma polymerization, which offers a more specific surface functionalization via the deposition of specific functional groups, in contrast to plasma activation with inert gases. Specifically, we employed organosilane precursors ((3-Aminopropyl) trimethoxysilane (APTMS) and a mixture of maleic anhydride and vinyltrimethoxysilane (MA-VTMOS)), which provide stable coatings resistant to delamination in aqueous conditions compared to other precursors. ${ }^{44-45}$ In this way, we were able to precisely analyze, for the first time, cell attachment to 3D ME-AM scaffolds as a function of specific surface charge and functional groups, in the presence or absence of serum. In particular, we observed that all plasma treatments increased the surface wettability of the scaffolds, enhanced cell-adhesive proteins adsorption to their surface, and allowed for homogeneous cell attachment along the scaffold cross section. Interestingly, APTMS treated scaffolds allowed cell attachment in the absence of serum, driven by electrostatic interactions between the cell and the charged scaffold surface, which possess relevant clinical applications.

\section{Homogeneous cell distribution and cell confluency in 3D ME-AM scaffolds are necessary for MSCs osteogenic differentiation and mineralization}

Although both macroM seeding and plasma treatment improved homogenous cell coverage on the PEOT/PBT scaffolds and led to high seeding efficiencies of $70-80 \%$, macroM seeding performed better at retaining cells along the scaffold cross section. While a large portion of the seeded cells adhered to the plasma treated scaffolds' bottom in form of a monolayer, reducing the cell number attached to the filaments throughout the scaffold's height, no monolayer was formed in the macroM seeded scaffolds. This was translated into a larger cell density and cell-cell contact points on the filaments of macroM seeded scaffolds, where cell agglomerates were already observed after seeding, and cells confluently covered the filaments after 1 day of culture. At the same timepoint, cell-cell contact was less abundant on the significantly less crowded plasma-treated scaffolds' filaments. Cell density and cell-cell contact in 2D cultures have been previously observed to greatly influence the osteogenic potential of hMSCs. According to the results in Chapter 3 and Chapter 4, we hypothesized that these parameters also played a key role in the differentiation of hMSCs on our ME-AM 3D scaffolds. Here, calcium deposition, a late 
marker for osteogenic differentiation, was observed only on scaffolds with high cell confluency on their filaments (macroM seeding), while osteogenic differentiation was delayed and calcium deposition was not observed on scaffolds with less cell density (plasma-assisted seeding), even after longer culture periods (54 days in MM). In 2D, studies have reported increased alkaline phosphatase (ALP) activity with increasing number of cell-cell contacts. ${ }^{46-47}$ Similarly, hMSCs plated at high density and cultured in osteogenic media have shown to lead to enhanced calcium deposition, compared to hMSCs plated at lower density. ${ }^{48-50}$ In particular, 100\% confluent hMSCs cultures have resulted into higher calcium deposition and osteocalcin protein expression, compared to lower confluency values, ${ }^{51}$ and over-crowding (> 100\%) has been shown to maintain the osteogenic differentiation potential of hMSCs. ${ }^{52-53}$ The upregulation of osteogenic markers through cell density variations in $2 \mathrm{D}$ has been explained by the activation of cell contactdependent signaling, such as gap junctions, ${ }^{46}$ and the activation of integrin ${ }^{50-51}$ and cadherin ${ }^{54}$ signaling pathways, which are all involved in the regulation of osteogenic differentiation. ${ }^{55-57}$

While cell studies in 2D have clearly demonstrated the positive correlation between cell confluency and enhanced osteogenic differentiation and calcium deposition, such effects have not been clearly observed so far in 3D. Some researchers have reported the detrimental effect of high seeding density on the ALP activity in osteoblasts or hMSCs, when cultured on 3D scaffolds, 58-59 while others have shown a positive impact of high cell seeding density on hMSC osteogenic differentiation. ${ }^{60-63}$ Overall, we hypothesize that these contradictory results in 3D are mainly owed to a lack of homogeneous cell distribution along the scaffold volume, which results in an inhomogeneous cell population and an erroneous averaged analysis. In this regard, we believe that the homogenous cell distribution attained with the macroM seeding method in Chapter 3 and with the plasmaassisted conventional seeding method in Chapter 4, allow us to draw more accurate conclusions on the effect of cell density, confluency and cell-cell contact on hMSCs osteogenic differentiation in 3D scaffolds, in particular in 3D ME-AM scaffolds. Taking this into account, it can be hypothesized that cell confluency is necessary for hMSCs osteogenic differentiation and calcium deposition on 3D AM-scaffolds, and this effect is potentially driven through the same mechanisms as in $2 \mathrm{D}$. 


\section{Cell confluency vs. other pro-osteogenic cues: in the race to osteogenic differentiation}

It cannot be dismissed that plasma-treated seeded scaffolds come along with an extra variable that has previously been shown to affect osteogenic differentiation on its own, i.e. surface chemistry. Functionalized 2D surfaces with polar functional groups have been shown to promote osteogenic differentiation, through an enhanced exposure of the integrin binding domains of adsorbed cell-adhesion proteins, which can lead to high levels of focal adhesion components and phosphorylated focal adhesion kinase ( $p-F A K)$ in the adhered cells, required for osteoblast differentiation. ${ }^{64-66}$ While in Chapter 4 we hypothesized that the lower protein adhesion to aged scaffolds (where osteogenic differentiation was evaluated) might have hampered MSC osteogenic stimulation and calcium deposition, it is also possible that the surface chemistry's pro-osteogenic effect is not as strong as the effect of cell confluency. Some studies in 2D have pointed out that substrates with increasing amount of polar functional groups or surface roughness give rise not only to enhanced calcium deposition, but also to increased cell attachment and density. ${ }^{67-68}$ This makes it difficult to identify the relevant osteogenic cues, among surface properties and cell confluency. Besides these, other cues that have been found to influence hMSCs osteogenic differentiation are, for example, substrate stiffness, substrate roughness, cell shape, and cell size. In some studies, where cells were exposed to competing prodifferentiation signals, cell-cell contact has been found to be a powerful cue, overruling the effect of other signals. For instance, through alterations in cytoskeletal tension, ALP and RUNX2 gene expression have been proven to be upregulated on cells with a large spread area, large perimeter, or with an aspect ratio around 2, compared to other shapes or sizes. 69-71 Yet, hMSCs cultured at high density also displayed the upregulation of osteogenic markers, together with calcium deposition, in spite of the physical constrains imposed by an increase density leading to "non-osteogenic morphologies" (i.e. small cell spread areas). ${ }^{70}$ Similarly, stiff substrates have been previously shown to direct hMSCs towards the osteogenic lineage, according to the upregulation of osteogenic markers. ${ }^{72}$ However, hMSCs cultured on stiff substrates were capable of depositing high amounts of calcium only when cultured at high density. ${ }^{49}$

The fact that cell confluency can override other pro-osteogenic factors in ME-AM scaffolds was further supported by the results observed in Chapter $\mathbf{5}$ and Chapter $\mathbf{6}$. In Chapter $\mathbf{5}$, hMSCs were seeded on PEOT/PBT-nanohydroxyapatite ( $\mathrm{nHA}$ ) composite scaffolds using 
the macroM seeding method and osteogenic differentiation was analyzed as a function of the HA content. Similarly, in Chapter 6 PEOT/PBT-reduced graphene oxide (rGO) composite scaffolds were seeded with hMSCs by the macroM seeding method, and osteogenic differentiation was assessed. While some previous studies had shown that the addition of HA and graphene based fillers to ME-AM composite scaffolds lead to the upregulation of osteogenic markers in hMSCs or other cell types in vitro, ${ }^{73-76}$ we did not observe significant differences in the osteogenic profile of these cells cultured on the composite scaffolds, compared to bare PEOT/PBT scaffolds. Indeed, hMSCs could undergo osteogenic differentiation and deposit a mineralized extracellular matrix (ECM) at the same levels on every scaffold type, regardless of the presence or absence of the fillers. One reason might be the polymer matrix being a barrier for fillers release in $\mathrm{nHA}$ containing scaffolds due to the poor exposure of the nHA particles to the surface, as suggested in Chapter 5 and by previous reports. ${ }^{74,77}$ This, together with the slow degradation time of $\mathrm{nHA}$, possibly led to suboptimal ion exchange dynamics between the scaffold and the medium and a calcium concentration in the medium, which was insufficient to affect calcium signaling pathways involved in osteogenesis. Nevertheless, calcium phosphate (CaP) re-precipitation after incubation in simulated body fluid was an indicator of the potential of these highly loaded HA scaffolds in vivo, in terms of tissue integration and osteoconduction. Another possible reason why differences were not observed in the osteogenic outcome between bare polymeric and filler-containing scaffolds could be correlated to the variable of seeding density being ruled out by seeding all the scaffold types with the macroM seeding method. In addition to accelerated osteogenic differentiation, previous studies have also observed enhanced cell attachment on such composite scaffolds, due to the fillers increasing the scaffold hydrophilicity and surface physical properties. ${ }^{78-80}$ Therefore, it is plausible that the observed accelerated cell differentiation on these composite scaffolds was induced by the higher cell density and confluency indirectly promoted by the fillers. Currently ongoing animal studies, in which such scaffolds were implanted in critical size ulnar defects in rabbits, will allow us to draw better conclusions on the bone regeneration performance of HA based composite scaffolds, compared to bare polymeric scaffolds.

These results raise an important question: if cell confluency is the main pro-osteogenic factor in ME-AM scaffolds, and it can be attained by simple strategies, such as macroM seeding on bare polymeric scaffolds, would it be necessary to invest on further scaffold modification strategies to regenerate bone, such as plasma treatments or the use of 
composite materials? Before answering this question, it is worth to mention that osteogenic differentiation was only attained in macroM seeded scaffolds when hMSCs were provided with osteogenic medium (Chapter 3 and Chapter 5), and it is not known whether endogenous factors would be sufficient to trigger bone formation in confluent scaffolds in an in vivo scenario. Previous in vivo studies evaluating the bone regeneration ability of cell-laden polymeric ME-AM scaffolds (seeded with hydrogel carriers or following protein coatings) have shown contradictory results, and it is not clear yet whether the presence of cells along with the scaffold has overall beneficial effects. ${ }^{81-84}$ Nevertheless, these studies have never assessed the state of cell confluency prior to scaffold implantation, and there is still no consensus on the optimum cell number, state of cell differentiation (the use of pre-differentiated or undifferentiated cells), and amount of viable cells after transplantation. 85 This highlights the necessity of future research on more controlled and systematic studies to investigate all these parameters. We believe that the macroM seeding method could serve as a reliable platform to study the bone regeneration capabilities of cell-laden scaffolds and to unravel the importance of cell density and confluency in vitro and in vivo. Given that the effect on in vivo bone regeneration of cell confluency in 3D scaffolds is still unknown, and that the use of the macroM seeding would be only applicable for cell-laden scaffolds strategies, the answer to the aforementioned question would be: yes, the investigation of other modification strategies such as plasma or the use of composites is still required.

\section{Unique functionalities of plasma-modified and composite ME-AM scaffolds}

\section{Plasma for better tissue integration}

As presented in Chapter 4, plasma activation and polymerization enhanced the surface energy and wettability of polymeric scaffolds, and led to protein adsorption and enhanced cell adhesion in vitro. In vivo, this could be translated into better scaffold integration with the existing bone defect ends and surrounding tissue (also called osseointegration), 86-88 which is critical for implant stability and is considered a prerequisite for implant loading and long-term clinical success. Moreover, these plasma modifications can promote endogenous cell adhesion, as a first step towards osteogenesis and bone formation. Notably, the aged scaffolds still showed to provide enhanced cell adhesion compared to untreated scaffolds. This experiment simulated the clinical scenario of scaffold production, where the storage after surface modification may occur for several days prior the 
implantation into the patient's defect. The automated production of scaffolds within an integrated ME-AM and APPJ platform further validates the translational potential of such plasma-modified scaffolds. Although not presented in this study, this integrated platform also allows to treat the scaffold layer by layer, to reach deep pores in large anatomical scaffolds, or zonally, to facilitate cell attachment in specific scaffold regions. ${ }^{89}$

\section{Composites for mechanical properties}

Among all the ME-AM scaffold modification strategies presented in Chapter 2, the incorporation of bioactive fillers within the scaffolds' polymer matrix towards composite scaffolds is the only method that enables increasing the scaffold mechanical properties without compromising its architecture and porosity. This is of great importance since, regardless of the polymer type used, polymeric ME-AM scaffolds still possess very low mechanical properties compared to bone, whereas scaffolds with high mechanical strength are fundamental for load bearing scenarios. In Chapter 5, we observed how the addition of $\mathrm{HA}$ led to a significant increase in the scaffold compression modulus and strength at yield, reaching the range of cortical bone mechanical properties. Although not discussed within this thesis, the addition of rGO in Chapter 6 and of lamellar inorganic fillers in Chapter 7 can also improve the mechanical properties of the PEOT/PBT scaffolds, in terms of compression modulus and yield compression strength. ${ }^{90}$

\section{Electrically conductive scaffolds}

In addition to improving their mechanical properties, the incorporation of fillers can provide the scaffold with other functional properties, such as electrical conductivity. In Chapter 6, polymeric scaffolds were rendered conductive with the addition of rGO fillers. Bone is a piezoelectric material and can generate electric potentials in response to mechanical stress, promoting bone resorption or formation. 91 Moreover, stem cell attachment, migration, proliferation, and differentiation have been shown to be influenced by electrical stimulation through the modification of the electrochemical environment, resulting in the activation of ion transporting channels, and membrane receptor complexes. ${ }^{92}$ For all these reasons, electrical stimulation has been considered as a tool in the clinics to stimulate bone healing, and its combination with electrically conductive scaffolds opened the door to a more localized therapy. ${ }^{93}$ During the last decades, scaffolds prepared through conventional fabrication methods and coated with conductive polymers, such as poly $(3,4-$ ethylenedioxythiophene) PEDOT, polypyrrole (PPy), and polyaniline (PANI) have been explored as conductive substrates for bone regeneration. 94 As a result of the 
establishment of AM as preferred scaffold fabrication technology, together with the rise of carbon-based materials as desired materials for biomedical applications, the field has recently been focused on the investigation of electrically conductive carbon based composite ME-AM scaffolds. ${ }^{95-96}$ The incorporation of the conductive material within the polymer matrix, rather than as a coating, provides mechanical reinforcement to the scaffold, enables to reduce its exposed amount ensuring higher biocompatibility, and avoids coating delamination due to weak interactions with the underlying substrate. Most recent studies have explored the in vivo bone formation capabilities of PCL/graphene and $\mathrm{PCL} /$ multiwall carbon nanotubes (MWCNT) ME-AM scaffolds combined with electrical stimulation. ${ }^{97-98}$ The presented results confirm the promising character of this line of research, to which we have contributed within this thesis mostly from a materials science point of view. Specifically, within Chapter 6 we evaluated how the densification of rGO into a more dosable and less inhalation-hazardous material affects the resulting composite scaffold printability, conductivity and physicochemical properties. Among different graphene based fillers, rGO is preferred due to its electrical conductivity, thermal stability, and availability at large scale through established synthesis methods. Our study reveals that while increasing the rGO bulk density leads to a more dosable material and to composites with a wider printability window, it also results in scaffolds with lower electrical conductivity, at a given wt\%. The solvent used for densification also showed to play a role in rGO compaction and subsequent dispersability within the composites. In this regard, for a given bulk density, densification in water resulted in a more compacted and aggregated rGO material than when densified in acetone, which reduced its dispersion within the polymer matrix and printability window. Overall, our study establishes relevant knowledge for potential future studies using rGO as a filler, and provides insight on the relevance of the rGO bulk density and volume occupancy for attaining ME-AM scaffolds with specific properties.

\section{Antimicrobial scaffolds for managing infections}

Beyond providing cues to promote bone formation, composite scaffolds can be used as drug carriers. In the clinics, up to $20 \%$ of open bone fractures, surgeries for fracture internal fixation, or implants revision surgeries develop into an infection. ${ }^{99}$ The gold standard clinical strategy to manage infections consists of implant removal, tissue debridement and defect filling with a bone cement (poly(methyl methacrylate) (PMMA)) impregnated with antibiotics, which allows the reduction of systemic antibiotic administration. ${ }^{100-101}$ With the 
advances in biodegradable calcium-based bone grafts in the last decade, some of these products have included antibiotics in their composition to treat osteomyelitis, while allowing bone regeneration in the defect for avoiding a revision surgery. ${ }^{101}$ Clinical studies have proven the ability of such systems (based on calcium sulfate, alone or combined with CaPs) in eradicating infections in $90-100 \%$ of the cases. Yet, bone healing and defect filling in non-unions was not always achieved, showing success rates ranging from 34 to $100 \%$. ${ }^{102-104}$ The fast degradation time of these bone void fillers (3-6 months), together with their low mechanical properties, have been identified as responsible parameters leading to failure in the bone regeneration process. For this reason, slower degrading and stiffer ME-AM scaffolds with antibiotic-eluting properties that can support the defect during the bone formation process are considered as more optimum alternatives. Generally, MEAM processes to obtain such scaffolds are limited to a few low melting temperature polymers, mainly PCL, in order to maintain the stability of the dispersed heat-sensitive drugs. As an alternative to developing new polymers with lower melting temperatures, or adding biocompatible plasticizers to the composites for tackling thermal stability issues, 105-106 we have investigated the use of lamellar protectors. In this regard, composite PEOT/PBT ME-AM scaffolds with lamellar inorganic fillers (magnesium aluminum layered double hydroxide (MgAl) and $\alpha$-Zirconium phosphate $(\mathrm{ZrP})$ ) intercalated with antibiotics (ciprofloxacin (CFX) and gentamicin (GTM), respectively) were presented in Chapter 7. These fillers had been previously shown to increase the thermal stability of sensitive drugs. 107-108 We also showed that the antibiotics maintained their antimicrobial activity after high temperature ME-AM, which was proven against some of the most common bacteria responsible for osteomyelitis ( $S$. epidermidis and $P$. aeuruginosa). In addition to this, we have shown that the fillers allowed for a more sustained antibiotics release, especially on scaffolds containing MgAl-CFX. The release profile was further tunable by adjusting the weight percentage of filler-antibiotic complexes within the scaffolds, as well as by the ionic concentration and $\mathrm{pH}$ changes in the surrounding environment. Such controlled antibiotic eluting systems are ideal, as they avoid potentially cytotoxic burst releases and prolong antimicrobial activity over time. Yet, such strategies require further development since standard antibiotic therapy for osteomyelitis lasts for 4-12 weeks, ${ }^{99}, 109$ a period that is much shorter than the time PEOT/PBT scaffolds' take to degrade, which is required for complete antibiotic release. In vivo, the PEOT/PBT used in this study (PEO molecular weight $=300 \mathrm{kDa}$, PEOT:PBT weight ratio= 55:45) showed only a $3 \%$ mass loss after 24 weeks of subcutaneous implantation in rats (film of thickness $400-600 \mu \mathrm{m}$ ). ${ }^{110}$ It is possible 
that our scaffolds porous structure, with increased surface exposed to surrounding body fluids, lower filament diameter (max $350 \mu \mathrm{m}$ ), and increased hydrophilicity given by the fillers, display shorter degradation times. In this regard, a careful evaluation of the scaffold degradation and antibiotic release in vivo is necessary, since a slow polymer degradation time can lead to a prolonged antibiotic treatment with local concentrations lower than the minimal inhibitory concentration (MIC), which can lead subsequently to the creation of antibiotic-resistant bacteria. ${ }^{111} \mathrm{~A}$ couple of approaches could be considered to accelerate the scaffold degradation time, and therefore the translation potential of such scaffolds. Inspired by other scaffold modification strategies, microporosity could be introduced in the composite scaffolds' filaments to enhance water permeability. Alternatively, a faster degrading thermoplastic material could be selected, such as PEOT/PBT with higher PEO molecular weight, or poly(lactic-co-glycolic) acid (PLGA) copolymers. ${ }^{110,112}$ Furthermore, the filler-antibiotic particles could be coated on the scaffold filaments, rather than being dispersed within the polymer matrix. Here, the fillers would still control the antibiotic release and prevent burst release, but the overall release would be completed faster due to the absence of the polymer matrix barrier. While the first suggested options would probably decrease the scaffold mechanical properties, the latter would not allow to control the amount of loaded antibiotic, which would mainly depend on the scaffold surface available area. Overall, it is evident that due to the control needed on multiple interdependent properties, tradeoffs are needed to be made to introduce antimicrobial properties to the scaffolds. Nevertheless, we encourage further investigations on the use of lamellar inorganic fillers for the incorporation of antibiotics within ME-AM scaffolds towards the development of an optimum system, as they are a reliable strategy to introduce bioactive molecules within high temperature melting polymers.

\section{In the future}

\section{Multifunctional scaffolds}

Looking to the future, a combination of the aforementioned scaffold modification strategies into a single scaffold is envisioned towards further enhancing their augmented bioactivity. For instance, a composite scaffold combining $\mathrm{nHA}$ and $\mathrm{rGO}$, would give rise to a multifunctional scaffold with electrical and antimicrobial properties (given by rGO), and mechanical and osteogenic properties, given by both $\mathrm{nHA}$ and $\mathrm{rGO}$. The synergistic effect of $\mathrm{nHA}$ and $\mathrm{rGO}$ added to the cell culture media in osteogenic differentiation in $2 \mathrm{D}$ has 
been previously shown, ${ }^{113}$ and such a binary combination has been already presented in 3D solvent based AM scaffolds. ${ }^{114}$ The combination of antimicrobial agents with nHA has also been investigated in solvent based AM scaffolds. In this regard, PLGA/HA scaffolds coated with quaternized chitosan proved their bone regeneration capabilities and infection management in an infected in vivo bone model. 115-116 This suggests the potential of combining our filler-antibiotic and HA into one composite scaffold for osteogenic and antimicrobial properties. Such binary composite scaffolds could be easily prepared by loading the single-filler composites used within this thesis into the double reservoir printhead developed by our group. ${ }^{89}$ This print-head would allow the in situ mixing of two materials at desired ratios towards a binary scaffold, simple or graded, avoiding the preprinting fabrication of binary composites my compounding. Ultimately, plasma treatment could further enhance the bioactivity of any of the suggested single or binary composite scaffolds.

\section{Other CaP fillers}

Due to the slow degradation time of $\mathrm{nHA}$ compared to other $\mathrm{CaP}$ fillers, such as tricalcium phosphate (TCP) or biphasic calcium phosphate (BCP), HA-based ME-AM scaffolds might maintain over a longer period of time their initial structural and mechanical properties, which are beneficial to support the fracture site while the new bone formation volume is still minimal. However, results in Chapter $\mathbf{5}$ have also suggested that the slow dissolution of $\mathrm{HA}$ might be hampering the osteoinductive potential of HA-based ME-AM scaffolds. Very few studies have compared side by side the osteogenic differentiation or bone regeneration capacity of ME-AM polymeric scaffolds containing different $\mathrm{CaP}$ fillers, and no definitive conclusions can be obtained from here. ${ }^{117}$ However, reports comparing the biological performance of different porous CaP scaffolds have shown that BCP or TCP scaffolds, with more optimum calcium exchange dynamics, outperform HA scaffolds in their in vitro and in vivo bone formation capacity. ${ }^{118-120}$ While the use of TCP as filler for ME-AM scaffolds has been studied in various reports, BCP, which is a combination of TCP and HA has not been so far a common filler of choice. In future studies, an exhaustive in vitro biological evaluation of ME-AM scaffolds containing BCP with different TCP/HA ratios would be of interest. By adjusting the TCP/HA ratio, a balance in mechanical properties, scaffold degradation time and ion exchange dynamics could be struck, towards an optimum ME-AM scaffold for BTE. The supplementation of the CaP filler with bioinorganics, such as strontium, silicon or magnesium, which are found in trace 
amounts in bone ECM and have shown to improve the bone regeneration capacity of CaPs, ${ }^{121}$ has also not been investigated in ME-AM scaffolds and could also be considered in the future.

\section{Other relevant antibiotics}

Future studies could be dedicated to investigate the loading of other antibiotic types within ME-AM scaffolds following the strategy presented in Chapter 7, which is based on the prior intercalation of antibiotics within lamellar inorganic fillers. Apart from the already tested CFX and GTM, MgAl and ZrP fillers can intercalate many other positively or negatively charged molecules, respectively, within their lamellar structure. This could enable the loading of a wide variety of antibiotics within ME-AM scaffolds, matching or widening the spectra of already existing local antibiotic delivery cements. Some of the antibiotics that could be considered are tobramycin, vancomycin, clindamycin, erythromycin or colistin. Although the first commercially available PMMA bone cements contained GTM, ${ }^{122}$ subsequent generations have broaden the spectra and included the aforementioned antibiotics, alone or combined, to cover a wider variety of pathogens involved in orthopedic infections. ${ }^{101,109}$ Similarly, vancomycin or tobramycin have also been included in the formulation of commercial degradable cements based on calcium sulfates, due to their broad antimicrobial coverage. 101, 123-124 Rifampin administration, combined with levofloxacin or ciprofloxacin, is a common systemic antibiotic therapy against Staphylococci, which is the most common bacterial strain in implant associated infections. ${ }^{99}$ While this antibiotic has not been included so far within commercialized gold standard cements, due to its interference with the polymerization mechanism of PMMA, 125 our polymer- intercalated filler approach could offer a suitable platform for its investigation within local delivery systems.

Despite the versatility of this system, the antibiotic size could be a limiting factor hampering its incorporation within the lamellar structure of the fillers. The access of a drug to the filler interlayer spaces can be restricted by its large molecular weight, or hydrodynamic radius, leading to adsorption to only the external filler surface. Among the aforementioned antibiotics, tobramycin, clindamycin, erythromycin and rifampin possess a molecular weight similar to that of CFX and GTM (300-800 Da), which have been already proven to fit within the interlayer spaces of lamellar fillers. ${ }^{126}$ On the other hand, vancomycin has a molecular weight of $\sim 1,500 \mathrm{Da}$, and previous studies have already demonstrated their uptake only on the outer surface of such fillers. ${ }^{127}$ This is an aspect to take into account, 
as it could influence the release kinetics, as well as imped the molecule heat protection. For this reason, a thorough investigation of each antibiotic loading efficiency and mode, release kinetics, and antimicrobial activity maintenance after scaffold processing is encouraged.

\section{Revisiting the polymer and cell choice}

As previously mentioned, PEOT/PBT was initially chosen as the base material within the composite scaffolds, since previous studies had reported its potential for BTE applications, in terms of optimal mechanical, physicochemical (degradation time, melting temperature), and biological properties (biocompatibility, cell adhesion). While our results demonstrate that PEOT/PBT did not interfere in the functional properties of PEOT/PBT-rGO composite scaffolds (electrical conductivity, antimicrobial properties) and PEOT/PBT plasma functionalized scaffolds (cell adhesion), it proved to be one of the players diminishing the overall functionality of PEOT/PBT-nHA and PEOT/PBT-filler-antibiotic composite scaffolds. Here, the interaction of the fillers with the surrounding environment was necessary to exert their bioactivity, and the slow degrading PEOT/PBT acted as a barrier, leading to suboptimal $\mathrm{CaP}$ exchange dynamics and antibiotic release durations, respectively, and challenging its choice as scaffold material. In these cases, we argued that the use of a polymer with faster degradation times and increased water uptake capabilities would had alleviated these issues, yet compromising other equally important properties, such as mechanical stability. We certainly believe that further research on biocompatible and printable polymers with optimum physicochemical properties is required to widen the polymer library and be able to strike a balance in these situations.

Re-evaluating the polymer choice also guides us to revisit other initial selections, such as the cell type chosen to assess the functionality of the newly developed scaffolds. Among different cell sources for osteoblastic cells, hMSCs are preferred by the scientific community for their use in BTE strategies over primary cells, such as osteoblasts derived from bone or periosteum. ${ }^{128}$ This is due to the possibility of isolating hMSCs from the bone marrow or adipose tissue in large numbers through minimal donor morbidity methods, in addition to their multi-lineage differentiation capabilities and participation in tissue regeneration processes. However, various reports have revealed that tissue source, isolation procedure, and donor characteristics (age, gender, and history) influence the phenotype, yield, growth rate, in vitro response to osteogenic signals, and in vivo bone formation capability of hMSCs. ${ }^{129-134}$ Such efficacy challenges are mostly overlooked in 
BTE research. In the present study, although ensuring hMSCs osteogenic potential in 2D conditions parallel to each experimental procedure in $3 \mathrm{D}$, such a variability was also not taken into consideration, and the extent of its effect on the reliability of the results and hypothesis presented within this thesis is not known. For this reason, the replication of the experiments with different hMSCs donors would be recommended to further proof all the hypothesis made.

Overall, we have proposed within this thesis significant scientific and technological advances applicable to the fabrication of more optimum ME-AM scaffolds for BTE. We have highlighted the advantages of each of them, as well as pointed out their limitations, while suggesting paths that future researchers could potentially take to further optimize our proposed systems. 


\section{Reference}

1. Hutmacher, D. W.; Lam, C. X. F., 13 - Scaffold and Implant Design: Considerations Relating to Characterization of Biodegradablity and Bioresorbability. In Degradation Rate of Bioresorbable Materials, Buchanan, F., Ed. Woodhead Publishing: 2008; pp 319-356.

2. Woodruff, M. A.; Hutmacher, D. W., The Return of a Forgotten Polymer-Polycaprolactone in the 21st Century. Progress in Polymer Science 2010, 35 (10), 1217-1256.

3. Yildirim, E. D.; Besunder, R.; Pappas, D.; Allen, F.; Guceri, S.; Sun, W., Accelerated Differentiation of Osteoblast Cells on Polycaprolactone Scaffolds Driven by a Combined Effect of Protein Coating and Plasma Modification. Biofabrication 2010, 2 (1), 014109.

4. Brunner, M.; Jurdic, P.; Tuckerman, J. P.; Block, M. R.; Bouvard, D., New Insights into Adhesion Signaling in Bone Formation. International review of cell and molecular biology 2013, 305, 1-68.

5. Deschamps, A. A.; Grijpma, D. W.; Feijen, J., Poly(Ethylene Oxide)/Poly(Butylene Terephthalate) Segmented Block Copolymers: The Effect of Copolymer Composition on Physical Properties and Degradation Behavior. Polymer 2001, 42 (23), 9335-9345.

6. Deschamps, A. A.; Claase, M. B.; Sleijster, W. J.; de Bruijn, J. D.; Grijpma, D. W.; Feijen, J., Design of Segmented Poly(Ether Ester) Materials and Structures for the Tissue Engineering of Bone. Journal of Controlled Release 2002, 78 (1), 175-186.

7. Deschamps, A.; Claase, M.; Sleijster, W.; de Bruijn, J.; Grijpma, D.; Feijen, J., Design of Segmented Poly(Ether Ester) Materials and Structures for Tissue Engineering of Bone. Journal of controlled release: official journal of the Controlled Release Society 2002, 78, 175-186.

8. Deschamps, A. A.; van Apeldoorn, A. A.; Hayen, H.; de Bruijn, J. D.; Karst, U.; Grijpma, D. W.; Feijen, J., In Vivo and in Vitro Degradation of Poly(Ether Ester) Block Copolymers Based on Poly(Ethylene Glycol) and Poly(Butylene Terephthalate). Biomaterials 2004, 25 (2), 247-258.

9. Van Blitterswijk, C.; Leenders, H.; Baaker, D., The Effect of Peo Ratio on Degradation, Calcification and Bone Bonding of Peo/Pbt Copolymer (Polyactive). Cells and Materials 1993, 3 (1), 2.

10. Claase, M. B.; Grijpma, D. W.; Mendes, S. C.; De Bruijn, J. D.; Feijen, J., Porous Peot/Pbt Scaffolds for Bone Tissue Engineering: Preparation, Characterization, and in Vitro Bone Marrow Cell Culturing. J Biomed Mater Res A 2003, 64 (2), 291-300.

11. Moroni, L.; de Wijn, J. R.; van Blitterswijk, C. A., Three-Dimensional Fiber-Deposited Peot/Pbt Copolymer Scaffolds for Tissue Engineering: Influence of Porosity, Molecular Network Mesh Size, and Swelling in Aqueous Media on Dynamic Mechanical Properties. J Biomed Mater Res A 2005, 75 (4), 957-965.

12. Claase, M. B.; de Bruijn, J. D.; Grijpma, D. W.; Feijen, J., Ectopic Bone Formation in Cell-Seeded Poly(Ethylene Oxide)/Poly(Butylene Terephthalate) Copolymer Scaffolds of Varying Porosity. Journal of materials science. Materials in medicine 2007, 18 (7), 1299-1307.

13. Nandakumar, A.; Barradas, A.; de Boer, J.; Moroni, L.; van Blitterswijk, C.; Habibovic, P., Combining Technologies to Create Bioactive Hybrid Scaffolds for Bone Tissue Engineering. Biomatter 2013, 3 (2).

14. Hendrikson, W. J.; Zeng, X.; Rouwkema, J.; van Blitterswijk, C. A.; van der Heide, E.; Moroni, L., Biological and Tribological Assessment of Poly(Ethylene Oxide Terephthalate)/Poly(Butylene Terephthalate), Polycaprolactone, and Poly (LIDI) Lactic Acid Plotted Scaffolds for Skeletal Tissue Regeneration. Adv Healthc Mater 2016, 5 (2), 232-243.

15. Di Luca, A.; Longoni, A.; Criscenti, G.; Mota, C.; van Blitterswijk, C.; Moroni, L., Toward Mimicking the Bone Structure: Design of Novel Hierarchical Scaffolds with a Tailored Radial Porosity Gradient. Biofabrication 2016, 8 (4), 045007.

16. Carrow, J. K.; Di Luca, A.; Dolatshahi-Pirouz, A.; Moroni, L.; Gaharwar, A. K., 3d-Printed Bioactive Scaffolds from Nanosilicates and Peot/Pbt for Bone Tissue Engineering. Regen Biomater 2019, 6 (1), 29 37.

17. Moroni, L.; Hendriks, J. A.; Schotel, R.; de Wijn, J. R.; van Blitterswijk, C. A., Design of Biphasic Polymeric 3-Dimensional Fiber Deposited Scaffolds for Cartilage Tissue Engineering Applications. Tissue engineering 2007, 13 (2), 361-371. 
18. Jansen, E. J.; Pieper, J.; Gijbels, M. J.; Guldemond, N. A.; Riesle, J.; Van Rhijn, L. W.; Bulstra, S. K.; Kuijer, R., Peot/Pbt Based Scaffolds with Low Mechanical Properties Improve Cartilage Repair Tissue Formation in Osteochondral Defects. J Biomed Mater Res A 2009, 89 (2), 444-452.

19. Rey-Rico, A.; Venkatesan, J. K.; Sohier, J.; Moroni, L.; Cucchiarini, M.; Madry, H., Adapted Chondrogenic Differentiation of Human Mesenchymal Stem Cells Via Controlled Release of Tgf-B1 from Poly(Ethylene Oxide)-Terephtalate/Poly(Butylene Terepthalate) Multiblock Scaffolds. J Biomed Mater Res A 2015, 103 (1), 371-383.

20. Barron, V.; Merghani, K.; Shaw, G.; Coleman, C. M.; Hayes, J. S.; Ansboro, S.; Manian, A.; O'Malley, G.; Connolly, E.; Nandakumar, A.; van Blitterswijk, C. A.; Habibovic, P.; Moroni, L.; Shannon, F.; Murphy, J. M.; Barry, F., Evaluation of Cartilage Repair by Mesenchymal Stem Cells Seeded on a Peot/Pbt Scaffold in an Osteochondral Defect. Ann Biomed Eng 2015, 43 (9), 2069-2082.

21. Bartha, L.; Hamann, D.; Pieper, J.; Péters, F.; Riesle, J.; Vajda, A.; Novak, P. K.; Hangody, L. R.; Vasarhelyi, G.; Bodó, L.; van Blitterswijk, C.; de Wijn, J.; Kenyeres, A.; Modis, L.; Balo, E.; Hangody, L., A Clinical Feasibility Study to Evaluate the Safety and Efficacy of Peot/Pbt Implants for Human Donor Site Filling During Mosaicplasty. European journal of orthopaedic surgery \& traumatology : orthopedie traumatologie 2013, 23 (1), 81-91.

22. Di Luca, A.; Longoni, A.; Criscenti, G.; Lorenzo-Moldero, I.; Klein-Gunnewiek, M.; Vancso, J.; van Blitterswijk, C.; Mota, C.; Moroni, L., Surface Energy and Stiffness Discrete Gradients in Additive Manufactured Scaffolds for Osteochondral Regeneration. Biofabrication 2016, 8 (1), 015014.

23. Di Luca, A.; Ostrowska, B.; Lorenzo-Moldero, I.; Lepedda, A.; Swieszkowski, W.; Van Blitterswijk, C.; Moroni, L., Gradients in Pore Size Enhance the Osteogenic Differentiation of Human Mesenchymal Stromal Cells in Three-Dimensional Scaffolds. Scientific reports 2016, 6.

24. Di Luca, A.; Lorenzo-Moldero, I.; Mota, C.; Lepedda, A.; Auhl, D.; Van Blitterswijk, C.; Moroni, L., Tuning Cell Differentiation into a 3d Scaffold Presenting a Pore Shape Gradient for Osteochondral Regeneration. Advanced Healthcare Materials 2016, 5 (14), 1753-1763.

25. Wang, H.; Pieper, J.; Peters, F.; van Blitterswijk, C. A.; Lamme, E. N., Synthetic Scaffold Morphology Controls Human Dermal Connective Tissue Formation. Journal of Biomedical Materials Research Part A: An Official Journal of The Society for Biomaterials, The Japanese Society for Biomaterials, and The Australian Society for Biomaterials and the Korean Society for Biomaterials 2005, 74 (4), 523-532.

26. Santos, D.; Wieringa, P.; Moroni, L.; Navarro, X.; Valle, J. D., Peot/Pbt Guides Enhance Nerve Regeneration in Long Gap Defects. Adv Healthc Mater 2017, 6 (3).

27. Buitinga, M.; Truckenmüller, R.; Engelse, M. A.; Moroni, L.; Ten Hoopen, H. W.; van Blitterswijk, C. A.; de Koning, E. J.; van Apeldoorn, A. A.; Karperien, M., Microwell Scaffolds for the Extrahepatic Transplantation of Islets of Langerhans. PloS one 2013, 8 (5), e64772.

28. Smink, A. M.; de Haan, B. J.; Paredes-Juarez, G. A.; Wolters, A. H.; Kuipers, J.; Giepmans, B. N.; Schwab, L.; Engelse, M. A.; van Apeldoorn, A. A.; de Koning, E.; Faas, M. M.; de Vos, P., Selection of Polymers for Application in Scaffolds Applicable for Human Pancreatic Islet Transplantation. Biomedical materials (Bristol, England) 2016, 11 (3), 035006.

29. Smink, A. M.; Hertsig, D. T.; Schwab, L.; van Apeldoorn, A. A.; de Koning, E.; Faas, M. M.; de Haan, B. J.; de Vos, P., A Retrievable, Efficacious Polymeric Scaffold for Subcutaneous Transplantation of Rat Pancreatic Islets. Annals of surgery 2017, 266 (1), 149-157.

30. Calore, A. R.; Sinha, R.; Harings, J.; Bernaerts, K. V.; Mota, C.; Moroni, L., Additive Manufacturing Using Melt Extruded Thermoplastics for Tissue Engineering. Methods in molecular biology (Clifton, N.J.) 2021, 2147, 75-99.

31. Vanzanella, V.; Scatto, M.; Zant, E.; Sisani, M.; Bastianini, M.; Grizzuti, N., The Rheology of Peot/Pbt Block Copolymers in the Melt State and in the Thermally-Induced Sol/Gel Transition Implications on the 3dPrinting Bio-Scaffold Process. Materials 2019, 12 (2), 226.

32. Caplan, A. I., Adult Mesenchymal Stem Cells for Tissue Engineering Versus Regenerative Medicine. Journal of cellular physiology 2007, 213 (2), 341-347.

33. da Silva Meirelles, L.; Fontes, A. M.; Covas, D. T.; Caplan, A. I., Mechanisms Involved in the Therapeutic Properties of Mesenchymal Stem Cells. Cytokine \& Growth Factor Reviews 2009, 20 (5), 419-427.

34. Schmitt, A.; van Griensven, M.; Imhoff, A. B.; Buchmann, S., Application of Stem Cells in Orthopedics. Stem Cells International 2012, 2012, 394962. 
35. Moroni, L.; Fornasari, P. M., Human Mesenchymal Stem Cells: A Bank Perspective on the Isolation, Characterization and Potential of Alternative Sources for the Regeneration of Musculoskeletal Tissues. Journal of cellular physiology 2013, 228 (4), 680-687.

36. Marolt Presen, D.; Traweger, A.; Gimona, M.; Redl, H., Mesenchymal Stromal Cell-Based Bone Regeneration Therapies: From Cell Transplantation and Tissue Engineering to Therapeutic Secretomes and Extracellular Vesicles. Frontiers in Bioengineering and Biotechnology 2019, 7 (352)

37. Barradas, A.; Yuan, H.; van Blitterswijk, C. A.; Habibovic, P., Osteoinductive Biomaterials: Current Knowledge of Properties, Experimental Models and Biological Mechanisms. Eur Cell Mater 2011, 21 (407), 29.

38. Ciapetti, G.; Granchi, D.; Baldini, N., The Combined Use of Mesenchymal Stromal Cells and Scaffolds for Bone Repair. Curr Pharm Des 2012, 18 (13), 1796-1820.

39. Leferink, A. M.; Hendrikson, W.; Rouwkema, J.; Karperien, M.; van Blitterswijk, C.; Moroni, L., Increased Cell Seeding Efficiency in Bioplotted Three-Dimensional Peot/Pbt Scaffolds. Journal of tissue engineering and regenerative medicine 2016, 10 (8), 679-689.

40. Jeon, H.; Lee, H.; Kim, G., A Surface-Modified Poly(E-Caprolactone) Scaffold Comprising Variable Nanosized Surface-Roughness Using a Plasma Treatment. Tissue Engineering Part C: Methods 2014, 20 (12), 951-963.

41. Lerman, M. J.; Smith, B. T.; Gerald, A. G.; Santoro, M.; Fookes, J. A.; Mikos, A. G.; Fisher, J. P., Aminated 3d Printed Polystyrene Maintains Stem Cell Proliferation and Osteogenic Differentiation. Tissue Engineering Part C: Methods 2019, 26 (2), 118-131.

42. Domingos, M.; Intranuovo, F.; Gloria, A.; Gristina, R.; Ambrosio, L.; Bártolo, P. J.; Favia, P., Improved Osteoblast Cell Affinity on Plasma-Modified 3-D Extruded Pcl Scaffolds. Acta Biomaterialia 2013, 9 (4), 5997-6005.

43. Cools, P.; Sainz-García, E.; Geyter, N. D.; Nikiforov, A.; Blajan, M.; Shimizu, K.; Alba-Elías, F.; Leys, C.; Morent, R., Influence of Dbd Inlet Geometry on the Homogeneity of Plasma-Polymerized Acrylic Acid Films: The Use of a Microplasma-Electrode Inlet Configuration. Plasma Processes and Polymers 2015, $12(10), 1153-1163$.

44. Bitar, R.; Cools, P.; De Geyter, N.; Morent, R., Acrylic Acid Plasma Polymerization for Biomedical Use. Applied Surface Science 2018, 448, 168-185.

45. Bulou, S.; Lecoq, E.; Loyer, F.; Frache, G.; Fouquet, T.; Gueye, M.; Belmonte, T.; Choquet, P., Study of a Pulsed Post-Discharge Plasma Deposition Process of Aptes: Synthesis of Highly Organic Pp-Aptes Thin Films with Nh2 Functionalized Polysilsesquioxane Evidences. Plasma Processes and Polymers 2019, 16 (4), 1800177.

46. Tang, J.; Peng, R.; Ding, J., The Regulation of Stem Cell Differentiation by Cell-Cell Contact on Micropatterned Material Surfaces. Biomaterials 2010, 31 (9), 2470-2476.

47. Wang, X.; Song, W.; Kawazoe, N.; Chen, G., The Osteogenic Differentiation of Mesenchymal Stem Cells by Controlled Cell-Cell Interaction on Micropatterned Surfaces. Journal of Biomedical Materials Research Part A 2013, 101 (12), 3388-3395.

48. Kim, K.; Dean, D.; Mikos, A. G.; Fisher, J. P., Effect of Initial Cell Seeding Density on Early Osteogenic Signal Expression of Rat Bone Marrow Stromal Cells Cultured on Cross-Linked Poly(Propylene Fumarate) Disks. Biomacromolecules 2009, 10 (7), 1810-1817.

49. Mao, A. S.; Shin, J.-W.; Mooney, D. J., Effects of Substrate Stiffness and Cell-Cell Contact on Mesenchymal Stem Cell Differentiation. Biomaterials 2016, 98, 184-191.

50. Noda, S.; Kawashima, N.; Yamamoto, M.; Hashimoto, K.; Nara, K.; Sekiya, I.; Okiji, T., Effect of Cell Culture Density on Dental Pulp-Derived Mesenchymal Stem Cells with Reference to Osteogenic Differentiation. Scientific Reports 2019, 9 (1), 5430.

51. Abo-Aziza, F. A. M.; A A, Z., The Impact of Confluence on Bone Marrow Mesenchymal Stem (Bmmsc) Proliferation and Osteogenic Differentiation. Int J Hematol Oncol Stem Cell Res 2017, 11 (2), 121-132.

52. Sotiropoulou, P. A.; Perez, S. A.; Salagianni, M.; Baxevanis, C. N.; Papamichail, M., Characterization of the Optimal Culture Conditions for Clinical Scale Production of Human Mesenchymal Stem Cells. Stem cells 2006, 24 (2), 462-471.

53. Haack-Sørensen, M.; Hansen, S. K.; Hansen, L.; Gaster, M.; Hyttel, P.; Ekblond, A.; Kastrup, J., Mesenchymal Stromal Cell Phenotype Is Not Influenced by Confluence During Culture Expansion. Stem Cell Reviews and Reports 2013, 9 (1), 44-58. 
54. Passanha, F. R.; Geuens, T.; Konig, S.; van Blitterswijk, C. A.; LaPointe, V. L. S., Cell Culture Dimensionality Influences Mesenchymal Stem Cell Fate through Cadherin-2 and Cadherin-11. Biomaterials 2020, 254, 120127.

55. Jaiswal, R. K.; Jaiswal, N.; Bruder, S. P.; Mbalaviele, G.; Marshak, D. R.; Pittenger, M. F., Adult Human Mesenchymal Stem Cell Differentiation to the Osteogenic or Adipogenic Lineage Is Regulated by MitogenActivated Protein Kinase. Journal of Biological Chemistry 2000, 275 (13), 9645-9652.

56. Ferrari, S. L.; Traianedes, K.; Thorne, M.; Lafage-Proust, M.-H.; Genever, P.; Cecchini, M. G.; Behar, V.; Bisello, A.; Chorev, M.; Rosenblatt, M.; Suva, L. J., A Role for N-Cadherin in the Development of the Differentiated Osteoblastic Phenotype. Journal of Bone and Mineral Research 2000, 15 (2), 198-208.

57. Salasznyk, R. M.; Klees, R. F.; Williams, W. A.; Boskey, A.; Plopper, G. E., Focal Adhesion Kinase Signaling Pathways Regulate the Osteogenic Differentiation of Human Mesenchymal Stem Cells. Experimental cell research 2007, 313 (1), 22-37.

58. Zhou, Y. F.; Sae-Lim, V.; Chou, A. M.; Hutmacher, D. W.; Lim, T. M., Does Seeding Density Affect in Vitro Mineral Nodules Formation in Novel Composite Scaffolds? J Biomed Mater Res A 2006, 78 (1), 183-193.

59. Lode, A.; Bernhardt, A.; Gelinsky, M., Cultivation of Human Bone Marrow Stromal Cells on ThreeDimensional Scaffolds of Mineralized Collagen: Influence of Seeding Density on Colonization, Proliferation and Osteogenic Differentiation. Journal of tissue engineering and regenerative medicine 2008, 2 (7), 400 407.

60. Holy, C. E.; Shoichet, M. S.; Davies, J. E., Engineering Three-Dimensional Bone Tissue in Vitro Using Biodegradable Scaffolds: Investigating Initial Cell-Seeding Density and Culture Period. Journal of Biomedical Materials Research: An Official Journal of The Society for Biomaterials, The Japanese Society for Biomaterials, and The Australian Society for Biomaterials and the Korean Society for Biomaterials 2000, 51 (3), 376-382.

61. Luo, F.; Hou, T.-Y.; Zhang, Z.-H.; Xie, Z.; Wu, X.-H.; Xu, J.-Z., Effects of Initial Cell Density and Hydrodynamic Culture on Osteogenic Activity of Tissue-Engineered Bone Grafts. PloS one 2013, 8 (1), e53697.

62. Wu, H.; Kang, N.; Wang, Q.; Dong, P.; Lv, X.; Cao, Y.; Xiao, R., The Dose-Effect Relationship between the Seeding Quantity of Human Marrow Mesenchymal Stem Cells and in Vivo Tissue-Engineered Bone Yield. Cell transplantation 2015, 24 (10), 1957-1968.

63. Yassin, M. A.; Leknes, K. N.; Pedersen, T. O.; Xing, Z.; Sun, Y.; Lie, S. A.; Finne-Wistrand, A.; Mustafa, K., Cell Seeding Density Is a Critical Determinant for Copolymer Scaffolds-Induced Bone Regeneration. $J$ Biomed Mater Res A 2015, 103 (11), 3649-3658.

64. Moursi, A. M.; Globus, R. K.; Damsky, C. H., Interactions between Integrin Receptors and Fibronectin Are Required for Calvarial Osteoblast Differentiation in Vitro. Journal of cell science 1997, 110 ( Pt 18), 21872196.

65. Keselowsky, B. G.; Collard, D. M.; García, A. J., Surface Chemistry Modulates Focal Adhesion Composition and Signaling through Changes in Integrin Binding. Biomaterials 2004, 25 (28), 5947-5954.

66. Griffin, M. F.; Ibrahim, A.; Seifalian, A. M.; Butler, P. E. M.; Kalaskar, D. M.; Ferretti, P., Chemical GroupDependent Plasma Polymerisation Preferentially Directs Adipose Stem Cell Differentiation Towards Osteogenic or Chondrogenic Lineages. Acta Biomater 2017, 50, 450-461.

67. Wang, P. Y.; Clements, L. R.; Thissen, H.; Jane, A.; Tsai, W. B.; Voelcker, N. H., Screening Mesenchymal Stem Cell Attachment and Differentiation on Porous Silicon Gradients. Advanced Functional Materials 2012, 22 (16), 3414-3423.

68. Wang, P.-Y.; Clements, L. R.; Thissen, H.; Tsai, W.-B.; Voelcker, N. H., Screening Rat Mesenchymal Stem Cell Attachment and Differentiation on Surface Chemistries Using Plasma Polymer Gradients. Acta biomaterialia 2015, 11, 58-67.

69. Peng, R.; Yao, X.; Ding, J., Effect of Cell Anisotropy on Differentiation of Stem Cells on Micropatterned Surfaces through the Controlled Single Cell Adhesion. Biomaterials 2011, 32 (32), 8048-8057.

70. Eyckmans, J.; Lin, G. L.; Chen, C. S., Adhesive and Mechanical Regulation of Mesenchymal Stem Cell Differentiation in Human Bone Marrow and Periosteum-Derived Progenitor Cells. Biol Open 2012, 1 (11), 1058-1068.

71. Yao, X.; Peng, R.; Ding, J., Effects of Aspect Ratios of Stem Cells on Lineage Commitments with and without Induction Media. Biomaterials 2013, 34 (4), 930-939. 
72. Engler, A. J.; Sen, S.; Sweeney, H. L.; Discher, D. E., Matrix Elasticity Directs Stem Cell Lineage Specification. Cell 2006, 126 (4), 677-689.

73. Yu, J.; Xu, Y.; Li, S.; Seifert, G. V.; Becker, M. L., Three-Dimensional Printing of Nano Hydroxyapatite/Poly(Ester Urea) Composite Scaffolds with Enhanced Bioactivity. Biomacromolecules 2017, 18 (12), 4171-4183.

74. Cho, Y. S.; Quan, M.; Lee, S.-H.; Hong, M. W.; Kim, Y. Y.; Cho, Y.-S., Assessment of Osteogenesis for 3d-Printed Polycaprolactone/Hydroxyapatite Composite Scaffold with Enhanced Exposure of Hydroxyapatite Using Rat Calvarial Defect Model. Composites Science and Technology 2019, 184, 107844.

75. Caetano, G. F.; Wang, W.; Chiang, W.-H.; Cooper, G.; Diver, C.; Blaker, J. J.; Frade, M. A.; Bártolo, P., 3d-Printed Poly( $\varepsilon$-Caprolactone)/Graphene Scaffolds Activated with P1-Latex Protein for Bone Regeneration. 3D printing and additive manufacturing 2018, 5 (2), 127-137.

76. Unagolla, J. M.; Jayasuriya, A. C., Enhanced Cell Functions on Graphene Oxide Incorporated 3d Printed Polycaprolactone Scaffolds. Materials Science and Engineering: C 2019, 102, 1-11.

77. Cho, Y. S.; Choi, S.; Lee, S.-H.; Kim, K. K.; Cho, Y.-S., Assessments of Polycaprolactone/Hydroxyapatite Composite Scaffold with Enhanced Biomimetic Mineralization by Exposure to Hydroxyapatite Via a 3dPrinting System and Alkaline Erosion. European Polymer Journal 2019, 113, 340-348.

78. Jiang, W.; Shi, J.; Li, W.; Sun, K., Morphology, Wettability, and Mechanical Properties of Polycaprolactone/Hydroxyapatite Composite Scaffolds with Interconnected Pore Structures Fabricated by a Mini-Deposition System. Polymer Engineering \& Science 2012, 52 (11), 2396-2402.

79. Jiang, W.; Shi, J.; Li, W.; Sun, K., Three Dimensional Melt-Deposition of Polycaprolactone/Bio-Derived Hydroxyapatite Composite into Scaffold for Bone Repair. Journal of Biomaterials Science, Polymer Edition 2013, 24 (5), 539-550.

80. Moncal, K. K.; Heo, D. N.; Godzik, K. P.; Sosnoski, D. M.; Mrowczynski, O. D.; Rizk, E.; Ozbolat, V.; Tucker, S. M.; Gerhard, E. M.; Dey, M., 3d Printing of Poly (E-Caprolactone)/Poly (D, L-Lactide-CoGlycolide)/Hydroxyapatite Composite Constructs for Bone Tissue Engineering. Journal of Materials Research 2018, 33 (14), 1972-1986.

81. Wojtowicz, A. M.; Templeman, K. L.; Hutmacher, D. W.; Guldberg, R. E.; García, A. J., Runx2 Overexpression in Bone Marrow Stromal Cells Accelerates Bone Formation in Critical-Sized Femoral Defects. Tissue engineering. Part A 2010, 16 (9), 2795-2808.

82. Rai, B.; Lin, J. L.; Lim, Z. X. H.; Guldberg, R. E.; Hutmacher, D. W.; Cool, S. M., Differences between in Vitro Viability and Differentiation and in Vivo Bone-Forming Efficacy of Human Mesenchymal Stem Cells Cultured on Pcl-Tcp Scaffolds. Biomaterials 2010, 31 (31), 7960-7970.

83. Reichert, J. C.; Cipitria, A.; Epari, D. R.; Saifzadeh, S.; Krishnakanth, P.; Berner, A.; Woodruff, M. A.; Schell, H.; Mehta, M.; Schuetz, M. A.; Duda, G. N.; Hutmacher, D. W., A Tissue Engineering Solution for Segmental Defect Regeneration in Load-Bearing Long Bones. Science Translational Medicine 2012, 4 (141), 141 ra193.

84. Kwon, D. Y.; Park, J. H.; Jang, S. H.; Park, J. Y.; Jang, J. W.; Min, B. H.; Kim, W.-D.; Lee, H. B.; Lee, J.; Kim, M. S., Bone Regeneration by Means of a Three-Dimensional Printed Scaffold in a Rat Cranial Defect. Journal of Tissue Engineering and Regenerative Medicine 2018, 12 (2), 516-528.

85. Janicki, P.; Boeuf, S.; Steck, E.; Egermann, M.; Kasten, P.; Richter, W., Prediction of in Vivo Bone Forming Potency of Bone Marrow-Derived Human Mesenchymal Stem Cells. Eur Cell Mater 2011, 21, 488-507.

86. Coelho, P. G.; Giro, G.; Teixeira, H. S.; Marin, C.; Witek, L.; Thompson, V. P.; Tovar, N.; Silva, N. R., Argon-Based Atmospheric Pressure Plasma Enhances Early Bone Response to Rough Titanium Surfaces. Journal of Biomedical Materials Research Part A 2012, 100 (7), 1901-1906.

87. Guastaldi, F. P. S.; Yoo, D.; Marin, C.; Jimbo, R.; Tovar, N.; Zanetta-Barbosa, D.; Coelho, P. G., Plasma Treatment Maintains Surface Energy of the Implant Surface and Enhances Osseointegration. International Journal of Biomaterials 2013, 2013, 354125.

88. Chou, W.-C.; Wang, R. C. C.; Huang, C.-L.; Lee, T.-M., The Effect of Plasma Treatment on the Osseointegration of Rough Titanium Implant: A Histo-Morphometric Study in Rabbits. Journal of Dental Sciences 2018, $13(3), 267-273$.

89. Sinha, R.; Cámara-Torres, M.; Scopece, P.; Verga Falzacappa, E.; Patelli, A.; Moroni, L.; Mota, C., A Hybrid Additive Manufacturing Platform to Create Bulk and Surface Composition Gradients on Scaffolds for Tissue Regeneration. Nature Communications 2021, 12 (1), 500. 
90. Sinha, R.; Sanchez, A.; Camara-Torres, M.; Uriszar-Aldaca, I. C.; Calore, A. R.; Harings, J.; Gambardella, A.; Ciccarelli, L.; Vanzanella, V.; Sisani, M.; Scatto, M.; Wendelbo, R.; Perez, S.; Villanueva, S.; Matanza, A.; Patelli, A.; Grizzuti, N.; Mota, C.; Moroni, L., Additive Manufactured Scaffolds for Bone Tissue Engineering: Physical Characterization of Thermoplastic Composites with Functional Fillers. bioRxiv 2021, 2021.2003.2023.436548.

91. Fukada, E.; Yasuda, I., On the Piezoelectric Effect of Bone. Journal of the Physical Society of Japan 1957, 12, 1158.

92. Thrivikraman, G.; Boda, S. K.; Basu, B., Unraveling the Mechanistic Effects of Electric Field Stimulation Towards Directing Stem Cell Fate and Function: A Tissue Engineering Perspective. Biomaterials 2018, 150, 60-86.

93. Griffin, M.; Bayat, A., Electrical Stimulation in Bone Healing: Critical Analysis by Evaluating Levels of Evidence. Eplasty 2011, 11.

94. Alegret, N.; Dominguez-Alfaro, A.; Mecerreyes, D., 3d Scaffolds Based on Conductive Polymers for Biomedical Applications. Biomacromolecules 2018, 20 (1), 73-89.

95. Vijayavenkataraman, S.; Kannan, S.; Cao, T.; Fuh, J. Y. H.; Sriram, G.; Lu, W. F., 3d-Printed Pcl/Ppy Conductive Scaffolds as Three-Dimensional Porous Nerve Guide Conduits (Ngcs) for Peripheral Nerve Injury Repair. Front Bioeng Biotechnol 2019, 7, 266.

96. Wibowo, A.; Vyas, C.; Cooper, G.; Qulub, F.; Suratman, R.; Mahyuddin, A. I.; Dirgantara, T.; Bartolo, P., 3d Printing of Polycaprolactone-Polyaniline Electroactive Scaffolds for Bone Tissue Engineering. Materials (Basel, Switzerland) 2020, 13 (3), 512.

97. Wang, W.; Junior, J. R. P.; Nalesso, P. R. L.; Musson, D.; Cornish, J.; Mendonça, F.; Caetano, G. F.; Bártolo, P., Engineered 3d Printed Poly( $\varepsilon$-Caprolactone)/Graphene Scaffolds for Bone Tissue Engineering. Materials Science and Engineering: C 2019, 100, 759-770.

98. e Silva, E. P.; Huang, B.; Helaehil, J. V.; Nalesso, P. R. L.; Bagne, L.; de Oliveira, M. A.; Albiazetti, G. C. C.; Aldalbahi, A.; El-Newehy, M.; Santamaria-Jr, M.; Mendonça, F. A. S.; Bártolo, P.; Caetano, G. F., In Vivo Study of Conductive 3d Printed Pcl/Mwcnts Scaffolds with Electrical Stimulation for Bone Tissue Engineering. Bio-Design and Manufacturing 2021.

99. Trampuz, A.; Widmer, A. F., Infections Associated with Orthopedic Implants. Current Opinion in Infectious Diseases 2006, 19 (4).

100. van Vugt, T. A. G.; Arts, J. J.; Geurts, J. A. P., Antibiotic-Loaded Polymethylmethacrylate Beads and Spacers in Treatment of Orthopedic Infections and the Role of Biofilm Formation. Front Microbiol 2019, 10, 1626-1626.

101. Trampuz, A.; Renz, N., Pocket Guide to Diagnosis and Treatment of Implant-Associated Infections after Fracture Fixation. 2 ed.; Pro-Implant Foundation: 2017

102. Ferguson, J. Y.; Dudareva, M.; Riley, N. D.; Stubbs, D.; Atkins, B. L.; McNally, M. A., The Use of a Biodegradable Antibiotic-Loaded Calcium Sulphate Carrier Containing Tobramycin for the Treatment of Chronic Osteomyelitis: A Series of 195 Cases. The bone \& joint journal 2014, 96-b (6), 829-836.

103. McNally, M. A.; Ferguson, J. Y.; Lau, A. C.; Diefenbeck, M.; Scarborough, M.; Ramsden, A. J.; Atkins, B. L., Single-Stage Treatment of Chronic Osteomyelitis with a New Absorbable, Gentamicin-Loaded, Calcium Sulphate/Hydroxyapatite Biocomposite: A Prospective Series of 100 Cases. The bone \& joint journal 2016, 98-b (9), 1289-1296.

104. Masrouha, K. Z.; Raad, M. E.; Saghieh, S. S., A Novel Treatment Approach to Infected Nonunion of Long Bones without Systemic Antibiotics. Strategies in trauma and limb reconstruction 2018, 13 (1), 13-18.

105. Pereira, G. G.; Figueiredo, S.; Fernandes, A. I.; Pinto, J. F., Polymer Selection for Hot-Melt Extrusion Coupled to Fused Deposition Modelling in Pharmaceutics. Pharmaceutics 2020, 12 (9), 795.

106. Shaqour, B.; Samaro, A.; Verleije, B.; Beyers, K.; Vervaet, C.; Cos, P., Production of Drug Delivery Systems Using Fused Filament Fabrication: A Systematic Review. Pharmaceutics 2020, 12 (6), 517.

107. Bugatti, V.; Gorrasi, G.; Montanari, F.; Nocchetti, M.; Tammaro, L.; Vittoria, V., Modified Layered Double Hydroxides in Polycaprolactone as a Tunable Delivery System: In Vitro Release of Antimicrobial Benzoate Derivatives. Applied Clay Science 2011, 52 (1), 34-40.

108. Kutlu, B.; Schröttner, P.; Leuteritz, A.; Boldt, R.; Jacobs, E.; Heinrich, G., Preparation of Melt-Spun Antimicrobially Modified Ldh/Polyolefin Nanocomposite Fibers. Materials Science and Engineering: $C$ 2014, 41, 8-16. 
109. Li, C.; Renz, N.; Trampuz, A.; Ojeda-Thies, C., Twenty Common Errors in the Diagnosis and Treatment of Periprosthetic Joint Infection. International orthopaedics 2020, 44 (1), 3-14.

110. Deschamps, A.; van Apeldoorn, A. A.; Hayen, H.; de Bruijn, J. D.; Karst, U.; Grijpma, D. W.; Feijen, J., In Vivo and in Vitro Degradation of Poly (Ether Ester) Block Copolymers Based on Poly (Ethylene Glycol) and Poly (Butylene Terephthalate). Biomaterials 2004, 25 (2), 247-258.

111. Li, B.; Webster, T. J., Bacteria Antibiotic Resistance: New Challenges and Opportunities for ImplantAssociated Orthopedic Infections. Journal of Orthopaedic Research® 2018, 36 (1), 22-32.

112. Park, T. G., Degradation of Poly (Lactic-Co-Glycolic Acid) Microspheres: Effect of Copolymer Composition. Biomaterials 1995, 16 (15), 1123-1130.

113. Lee, J. H.; Shin, Y. C.; Lee, S.-M.; Jin, O. S.; Kang, S. H.; Hong, S. W.; Jeong, C.-M.; Huh, J. B.; Han, D.W., Enhanced Osteogenesis by Reduced Graphene Oxide/Hydroxyapatite Nanocomposites. Scientific Reports 2015, 5, 18833.

114. Jakus, A. E.; Shah, R. N., Multi and Mixed 3d-Printing of Graphene-Hydroxyapatite Hybrid Materials for Complex Tissue Engineering. J Biomed Mater Res A 2017, 105 (1), 274-283.

115. Yang, Y.; Yang, S.; Wang, Y.; Yu, Z.; Ao, H.; Zhang, H.; Qin, L.; Guillaume, O.; Eglin, D.; Richards, R. G.; Tang, T., Anti-Infective Efficacy, Cytocompatibility and Biocompatibility of a 3d-Printed Osteoconductive Composite Scaffold Functionalized with Quaternized Chitosan. Acta Biomaterialia 2016, 46, 112-128.

116. Yang, Y.; Chu, L.; Yang, S.; Zhang, H.; Qin, L.; Guillaume, O.; Eglin, D.; Richards, R. G.; Tang, T., DualFunctional 3d-Printed Composite Scaffold for Inhibiting Bacterial Infection and Promoting Bone Regeneration in Infected Bone Defect Models. Acta Biomaterialia 2018, 79, 265-275.

117. Nyberg, E.; Rindone, A.; Dorafshar, A.; Grayson, W. L., Comparison of 3d-Printed Poly- $\varepsilon$-Caprolactone Scaffolds Functionalized with Tricalcium Phosphate, Hydroxyapatite, Bio-Oss, or Decellularized Bone Matrix. Tissue Engineering Part A 2016, 23 (11-12), 503-514.

118. Yuan, H.; Fernandes, H.; Habibovic, P.; de Boer, J.; Barradas, A. M. C.; de Ruiter, A.; Walsh, W. R.; van Blitterswijk, C. A.; de Bruijn, J. D., Osteoinductive Ceramics as a Synthetic Alternative to Autologous Bone Grafting. Proc Natl Acad Sci U S A 2010, 107 (31), 13614-13619.

119. Habibovic, P.; Yuan, H.; van der Valk, C. M.; Meijer, G.; van Blitterswijk, C. A.; de Groot, K., 3d Microenvironment as Essential Element for Osteoinduction by Biomaterials. Biomaterials 2005, 26 (17), 3565-3575.

120. Barradas, A. M.; Monticone, V.; Hulsman, M.; Danoux, C.; Fernandes, H.; Tahmasebi Birgani, Z.; Barrerede Groot, F.; Yuan, H.; Reinders, M.; Habibovic, P.; van Blitterswijk, C.; de Boer, J., Molecular Mechanisms of Biomaterial-Driven Osteogenic Differentiation in Human Mesenchymal Stromal Cells. Integrative biology : quantitative biosciences from nano to macro 2013, 5 (7), 920-931.

121. Lodoso-Torrecilla, I.; Klein Gunnewiek, R.; Grosfeld, E.-C.; de Vries, R. B. M.; Habibović, P.; Jansen, J. A.; van den Beucken, J. J. J. P., Bioinorganic Supplementation of Calcium Phosphate-Based Bone Substitutes to Improve in Vivo Performance: A Systematic Review and Meta-Analysis of Animal Studies. Biomaterials Science 2020, 8 (17), 4792-4809.

122. Neut, D.; Kluin, O.; Thompson, J.; van der Mei, H.; Busscher, H., Gentamicin Release from CommerciallyAvailable Gentamicin-Loaded Pmma Bone Cements in a Prosthesis-Related Interfacial Gap Model and Their Antibacterial Efficacy. BMC musculoskeletal disorders 2010, 11, 258.

123. Scott, C. P.; Higham, P. A.; Dumbleton, J. H., Effectiveness of Bone Cement Containing Tobramycin. An in Vitro Susceptibility Study of 99 Organisms Found in Infected Joint Arthroplasty. The Journal of bone and joint surgery. British volume 1999, 81 (3), 440-443.

124. Yusuf, E.; Croughs, P., Vancomycin Prophylaxis in Prosthetic Joint Surgery? Clinical Microbiology and Infection 2020, 26 (1), 3-5.

125. Funk, G. A.; Menuey, E. M.; Cole, K. A.; Schuman, T. P.; Kilway, K. V.; Mclff, T. E., Radical Scavenging of Poly(Methyl Methacrylate) Bone Cement by Rifampin and Clinically Relevant Properties of the RifampinLoaded Cement. Bone \& Joint Research 2019, 8 (2), 81-89.

126. Bastianini, M.; Scatto, M.; Sisani, M.; Scopece, P.; Patelli, A.; Petracci, A., Innovative Composites Based on Organic Modified Zirconium Phosphate and Peot/Pbt Copolymer. Journal of Composites Science 2018, $2(2), 31$.

127. Lopez, N. A.; Luengo, C. V.; Avena, M. J., Uptake/Release of Vancomycin on/from Mg-Al Layered Double Hydroxides. Adsorption 2019, 25 (7), 1349-1360. 
128. Mastrolia, I.; Foppiani, E. M.; Murgia, A.; Candini, O.; Samarelli, A. V.; Grisendi, G.; Veronesi, E.; Horwitz, E. M.; Dominici, M., Challenges in Clinical Development of Mesenchymal Stromal/Stem Cells: Concise Review. STEM CELLS Translational Medicine 2019, 8 (11), 1135-1148.

129. Mastrolia, I.; Foppiani, E. M.; Murgia, A.; Candini, O.; Samarelli, A. V.; Grisendi, G.; Veronesi, E.; Horwitz, E. M.; Dominici, M., Challenges in Clinical Development of Mesenchymal Stromal/Stem Cells: Concise Review. STEM CELLS Translational Medicine 2019, 8 (11), 1135-1148.

130. Payr, S.; Schuseil, T.; Unger, M.; Seeliger, C.; Tiefenboeck, T.; Balmayor, E. R.; van Griensven, M., Effect of Donor Age and 3d-Cultivation on Osteogenic Differentiation Capacity of Adipose-Derived Mesenchymal Stem Cells. Scientific Reports 2020, 10 (1), 10408.

131. Phinney, D. G.; Kopen, G.; Righter, W.; Webster, S.; Tremain, N.; Prockop, D. J., Donor Variation in the Growth Properties and Osteogenic Potential of Human Marrow Stromal Cells. Journal of cellular biochemistry 1999, 75 (3), 424-436.

132. Siddappa, R.; Licht, R.; van Blitterswijk, C.; de Boer, J., Donor Variation and Loss of Multipotency During in Vitro Expansion of Human Mesenchymal Stem Cells for Bone Tissue Engineering. Journal of orthopaedic research 2007, 25 (8), 1029-1041.

133. Siegel, G.; Kluba, T.; Hermanutz-Klein, U.; Bieback, K.; Northoff, H.; Schäfer, R., Phenotype, Donor Age and Gender Affect Function of Human Bone Marrow-Derived Mesenchymal Stromal Cells. BMC Medicine 2013, $11(1), 146$.

134. Yang, H. J.; Kim, K. J.; Kim, M. K.; Lee, S. J.; Ryu, Y. H.; Seo, B. F.; Oh, D. Y.; Ahn, S. T.; Lee, H. Y.; Rhie, J. W., The Stem Cell Potential and Multipotency of Human Adipose Tissue-Derived Stem Cells Vary by Cell Donor and Are Different from Those of Other Types of Stem Cells. Cells, tissues, organs 2014, $199(5-6), 373-383$. 


\section{Chapter 9}

Impact 



\section{Scientific impact}

Although bone has been studied since the birth of the tissue engineering (TE) field, scientists are still searching for the optimal strategy for its regeneration (Figure 1A). Bone is a complex tissue, in terms of mechanical properties, structure, and composition, and many molecular factors and cell types are involved in its formation and regeneration. For this reason, clinical problems, such as non-union fractures or craniofacial reconstructions, are also complex to approach, and not easy to resolve. Among the three components of the classical tissue engineering paradigm (cells, scaffolds and growth factors), scaffolds have been the main focus of study in the bone tissue engineering (BTE) field. Besides providing a bioactive environment for new tissue formation, scaffolds in bone regeneration have the additional significant role of providing mechanical support, mimicking the high strength of the native extracellular matrix. Aiming towards size and shape personalized structures with high mechanical properties, but at the same time porous for cell ingrowth, additive manufacturing (AM) has stood out within the last 15 years as one of the preferred biofabrication technologies for the fabrication of scaffolds for BTE applications. Over the years, large technological advancements have been made around AM to facilitate an efficient scaffold design, i.e. multiprinthead melt extrusion AM (ME-AM) platforms have been developed for the fabrication of multimaterial scaffolds, sophisticated light-based or bioprinting AM platforms have emerged, new printable polymer materials or bioinks have been designed. Yet, an AM scaffold with the optimal set of properties for efficient bone regeneration has not been fully attained, suggesting that further investigations and advancements are still required.

Within this thesis, courageous choices were made to generate large scientific and technological strides providing solutions to existing issues within the field. We believe that the diverse strategies presented, consisting on optimized or new composite materials, new surface modification techniques, and novel cell seeding methodologies, contribute to the better understanding of AM scaffolds and their use for bone regeneration applications and provides a stepping stone for future studies on the application of functional composites in BTE. We also consider that this research has a scientific impact beyond the BTE field and can inspire future research, in the biomedical, biomaterial and overall tissue engineering scientific community. 


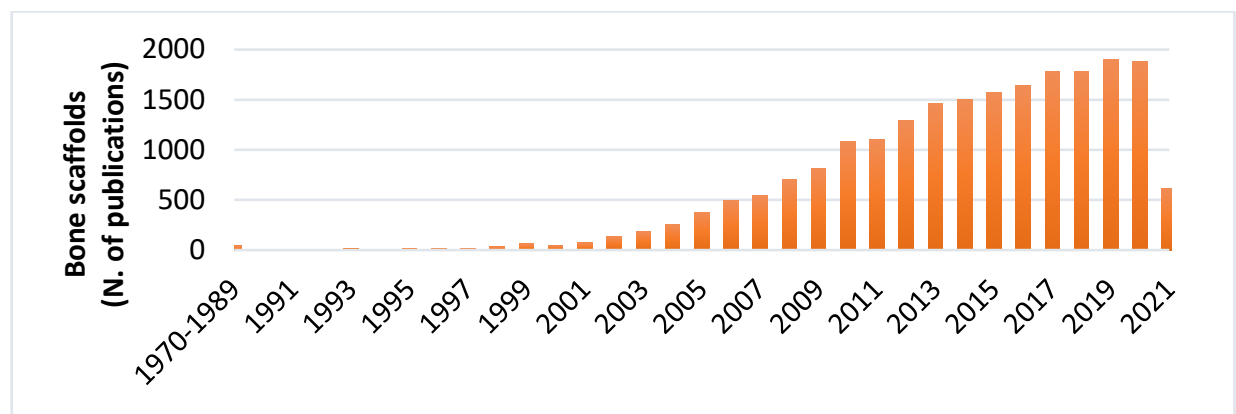

Figure 1. Scientific publications on new bone scaffolds since 1970. Pubmed search, April 2021.

Within the first chapters, we found extremely relevant to revisit the recurrent cell seeding efficiency problem on macroporous AM scaffolds. On one hand, in Chapter 3 we proposed a simple, reproducible and universal cell seeding approach that could be easily implemented by other research groups working with 3D AM for different applications. We are certain of the high scientific impact of this method, since it helps minimizing the required cell stock for in vitro experimentation and maximize cell seeding efficiency on scaffolds fabricated with any existing or newly developed synthetic material. It encourages researchers to redirect their efforts towards investigating more relevant issues, once the basic cell seeding problem is tackled. In Chapter 3, we went one step further and, while addressing the cell seeding issue, we explored a portion of the possibilities that a hybrid platform consisting of a melt extrusion printhead and plasma jet could bring to the design of surface-functionalized scaffolds. Our results open the door to future research on the effect of different plasma treatments on cell behavior on 3D ME-AM scaffolds. Furthermore, since this technique allows for a single-step workflow of scaffold production and surface homogeneous treatment, we envision its implementation within the scaffold fabrication pipeline of many laboratories in the future, as well as its impact in a smoother translation of ME-AM scaffolds into the clinic.

In subsequent chapters, several optimized or novel composite materials were investigated and complex methodologies from several scientific fields, transiting from material engineering, chemistry, and biology were used to analyze their suitability for BTE applications and their impact in the field. In particular, Chapter 4 showed that blending high concentrations of nanohydroxyapatite within a polymer, as a common focus of the community trying to mimic the bone composition, does not necessarily result in a highly osteoinductive material. Thus, here we raised important questions to the scientific community related to the appropriate calcium phosphate material and their incorporation 
method in ME-AM composite scaffolds for the stimulation of bone regeneration. On the other hand, the incorporation of graphene based materials into ME-AM scaffolds is not yet a well-stablished line of research in the field. For this reason, we believe that our study in Chapter 5 about the effect of rGO density and concentration on composite scaffolds production and cell-material interaction constitutes a stepping stone for scientists entering this field in the future. Finally, we believe that the approach presented in Chapter 6 to fabricate antibiotic-releasing AM scaffolds could open the door to future research on the use of other relevant inorganic nanofillers, antibiotics and biodegradable polymers to further optimize the system towards clinical applications.

\section{Social impact}

Bone is one of the most commonly injured organs in our body. Bone fractures can result from trauma, such as car accidents, or sport injuries, as well as from osteoporosis, a disease that weakens the bones and increases the risk of fragility fractures. Inappropriate initial treatments and co-morbidities, such as diabetes, genetic factors and poor life style, can increase the risk of delayed bone healing or non-unions. Resection of bone tumors, infections or prosthetic revisions in long bones can also result in non-unions. Around 4 million fractures are estimated to occur in Europe annually, from which approximately half a million require hospitalization and bone repair intervention. ${ }^{1}$ Non-unions, which occur in $5-10 \%$ of the cases, are the most difficult to treat and have a high financial impact, with a total cost of $€ 8,000$ to $€ 91,000$ per case. ${ }^{2-3}$ In European healthcare systems, around 82.8-93.3\% of this cost has been estimated to derive from indirect costs, such as productivity losses, due to long healing times leading to patients being unable to resume daily life activities and work within a short time. ${ }^{4}$ The remaining expenditures derive from care and intervention costs.

Current available clinical solutions for bone repair are based on natural and synthetic bone grafts. The natural grafts are divided into autografts and allografts, the latter ones including demineralized bone matrices (DBMs). The synthetic materials are based on ceramic, collagen-ceramic composites, polymers and bone morphogenetic proteins. In 2020, the global bone graft market size, based on allografts and synthetic substitutes, was estimated at $€ 2.36$ billion (Grand View Research, market analysis report 2021, ID: GVR-1-68038154-2). Due to the aging population and the increased incidence of osteoporosis and bone fractures, this market is expected to grow at an annual rate of $5.8 \%$ to reach $€ 3.7$ billion 
by 2028. Although the market is currently dominated by allograft materials ( 60\% share in 2020, mainly DBMs), the synthetic segment is anticipated to show significant growth over the years, due to the investment in product development, arise of new technologies and acceptance growth among patients. Bone grafts and substitutes are mostly used for spinal fusion, dental, and joint cemented prosthesis applications, while only $5 \%$ of the products are dedicated to long bone repair. This is partly because current marketed solutions, such as DBMs, or resorbable calcium phosphate cements have been shown to be inefficient in managing non-union scenarios in long bones, mainly due to their poor mechanical properties, unable to withstand the load required in these locations. Similar limitations are offered by collagen sponges infused with bone morphogenetic proteins (BMPs), which additionally have shown to induce undesired uncontrolled ectopic bone formation in clinical trials.

Here, AM scaffolds could bring many benefits for non-union defects. Such scaffolds can be fabricated to fit the specific patient complex defect using CT scan images of the patient's anatomy. This avoids the need of manipulation or modification of the scaffold intraoperatively, reducing the surgery time and ensuring good implant fixation within the defect. Moreover, AM scaffolds can be fabricated with specific structural parameters and mechanical properties from polymeric biodegradable materials or composites, to allow new bone formation and avoid a revision surgery. Despite their advantages, the implementation of biodegradable AM scaffolds in the clinics is still slow, as it is a rather new field and new medical devices require long regulatory paths, which subsequently leads to the current absence of companies marketing such products. Accordingly, many key players in the business of bone grafts, such as DePuy Synthes, Medtronic, Wright Medical, AlloSource, Stryker, Johnson and Johnson and Zimmer Biomet, only offer products based on DBM, ceramics or BMPs, and so far do not possess 3D AM scaffolds within their product portfolio. On the other hand, emerging small companies, such as Mimetis and Cerhum, have entered the sector by jumping in the 3D printing wave, yet developing AM ceramic scaffolds based on tricalcium phosphate (TCP) and hydroxyapatite (HA), whose performance in load bearing scenarios is arguable and still to be proven. Up to now, only one company (Osteopore International) has been able to commercialize 3D ME-AM polymeric scaffolds, for craniomaxilofacial defects, and currently is performing first-inhuman trials for their future application in long bone non-unions. Osteopore's poly(caprolactone) (PCL) scaffold was recently implanted in a patient with a non-union tibial defect at Maastricht University Hospital. The success of this clinical case, and many 
others to follow, will certainly open in the future the clinics' door to other novel ME-AM scaffolds. We believe that the research presented within this thesis will have a direct impact in shaping the future of such novel products, as we propose several strategies to improving their functionality, which could further accelerate bone healing times and reduce the socioeconomic burden associated with non-unions. Joining the success of the aforementioned basic polymeric ME-AM scaffolds with the extensive road of ceramics in the clinics, the clinical success of the PEOT/PBT-nHA scaffolds presented in Chapter 5 can be seen as a natural next step to come. Such scaffolds would combine i) the bioactivity and osteoconductivity provided by the ceramic component, ii) the mechanical support brought by the polymer, the reinforcing ceramic, and the scaffold structure itself, and iii) the concept of personalized medicine. Overall, a blend of properties that current commercial products lack. Although not directly investigated within this thesis, the further addition to these scaffolds of the antibiotic loaded lamellar fillers presented in Chapter $\mathbf{7}$, would certainly further boost their functionality and transform them into a very unique product. As mentioned in previous chapters, further optimization would be required before obtaining a "ready to use" scaffold, but we believe that we are on a good track, which will surely guide us to implement a novel, more reliable, probably cheaper and, importantly, functional therapy for bone non-union regeneration, compared to current gold standards. 


\section{References}

1. Hernlund, E.; Svedbom, A.; Ivergård, M.; Compston, J.; Cooper, C.; Stenmark, J.; McCloskey, E. V.; Jönsson, B.; Kanis, J. A., Osteoporosis in the European Union: Medical Management, Epidemiology and Economic Burden. A Report Prepared in Collaboration with the International Osteoporosis Foundation (Iof) and the European Federation of Pharmaceutical Industry Associations (Efpia). Arch Osteoporos 2013, 8 (1-2), 136.

2. Nandra, R.; Grover, L.; Porter, K., Fracture Non-Union Epidemiology and Treatment. Trauma 2016, 18(1), 3-11.

3. Mills, L. A.; Aitken, S. A.; Simpson, A., The Risk of Non-Union Per Fracture: Current Myths and Revised Figures from a Population of over 4 Million Adults. Acta orthopaedica 2017, 88 (4), 434-439.

4. Hak, D. J.; Fitzpatrick, D.; Bishop, J. A.; Marsh, J. L.; Tilp, S.; Schnettler, R.; Simpson, H.; Alt, V., Delayed Union and Nonunions: Epidemiology, Clinical Issues, and Financial Aspects. Injury 20 
Appendix 



\section{Summary}

Current clinical strategies to treat critical bone size defects, mainly based on autografts, are inefficient and have limitations. For this reason, the orthopedic field is seeking innovative and effective solutions to regenerate bone in such scenarios, with the help of tissue engineering. Melt extrusion additive manufacturing (ME-AM) has stood out as an ideal biofabrication technique to generate patient personalized, highly porous, biodegradable and mechanically robust scaffolds that can provide support for new bone tissue formation in non-unions. However, these scaffolds are made out of synthetic polymers lacking bioactivity, which generally require further functionalization to improve their biological performance. In this regard, several strategies have been investigated over the last decades to augment the bioactivity of ME-AM scaffolds, which are carefully reviewed in Chapter 2 of this thesis. Strategies such as architectural modifications, surface physical and chemical modifications, the incorporation of secondary fibrous or hydrogel networks within the scaffolds pores, and the use of filler-polymer composites are reviewed. As the search for optimal scaffolds that can boost bone regeneration continues, the aim of this thesis is to further contribute to the field by investigating different material-driven approaches to design and fabricate bioactive ME-AM scaffolds for bone tissue regeneration. To start with, Chapter $\mathbf{3}$ and Chapter $\mathbf{4}$ provide two elegant solutions to the recurrent cell seeding efficiency issue associated to macroporous 3D scaffolds. In Chapter 3, a simple and reliable static seeding method was developed to improve the cell seeding efficiency and distribution in ME-AM scaffolds. This method consisted of the supplementation of Dextran or Ficoll to the cell seeding media, which modified the medium viscosity and density, respectively, and reduced the settling velocity of cells, allowing for better attachment and distribution during the seeding process, compared to conventional static seeding. The macromolecules were completely removed after the seeding period and cell distribution along the scaffold cross section was dramatically enhanced. In addition, osteogenic differentiation of human mesenchymal stromal cells (hMSCs) was accelerated, in terms of enhanced mineralization and bone matrix maturation, compared to conventional seeding. In Chapter $\mathbf{4}$, the cell seeding issue was tackled from another perspective. In this case, ME-AM scaffolds were fabricated using a hybrid AM technique that combined ME-AM and an atmospheric pressure plasma jet, which was used to plasma-functionalize their surface. While argon activation resulted in an unspecific treatment, (3-Aminopropyl) trimethoxysilane (APTMS) and maleic anhydride- 
Vinyltrimethoxysilane (MA-VTMOS) plasma polymerization deposited positively and negatively charged coatings, respectively. This enabled to investigate the cell attachment mechanism to the scaffolds as a function of surface charge in the presence or absence of serum. Furthermore, the coatings increased the surface wettability of the scaffolds, enhanced cell-adhesive proteins adsorption, and allowed for homogeneous cell attachment along the scaffold cross section. Cell proliferation was enhanced even on aged scaffolds, and hMSCs were able to express osteogenic genes in all scaffold types.

After ensuring an efficient cell attachment, composite scaffolds comprising a polymer matrix mixed with bioactive fillers possessing distinct properties were fabricated in Chapters 5, 6 and 7. In Chapter 5, highly loaded PEOT/PBT-nanohydroxyapatite (nHA) composite scaffolds with up to $45 \mathrm{wt} \% \mathrm{nHA}$ were fabricated, mimicking the organicinorganic bone composition. Here, the effect of $\mathrm{nHA}$ content on the osteogenic differentiation of hMSCs was evaluated in depth, and results suggested that in spite of no observed differences among scaffold types at the gene and protein level, increased matrix mineralization was observed on $45 \mathrm{wt} \% \mathrm{nHA}$ scaffolds when cultured in mineralization and basic media. Moreover, a denser calcium phosphate layer reprecipitated on these scaffolds upon immersion in simulated body fluid. These results in combination with the enhanced compression modulus measured on $45 \mathrm{wt} \% \mathrm{nHA}$ scaffolds, could be predicting their enhanced performance in vivo. In Chapter 6, ME-AM composite scaffolds containing reduced graphene oxide ( $\mathrm{rGO}$ ) were fabricated to provide electrical conductivity, protein adhesion and osteogenic properties. Initially, the low-density rGO was densified by solvent evaporation, in order to ease handling and ensure safety during melt compounding. It was observed that high density led to more compacted rGO which facilitated the printing of these composites up to $15 \mathrm{wt} \%$ rGO formulations. On the other hand, composites prepared with medium density rGO possessed a lower printability window, but presented higher electrical conductivity. In addition, increasing rGO content led to increasing material hydrophilicity, protein adsorption, and antibacterial properties, without affecting hMSCs viability. Furthermore, 3\% rGO scaffolds were able to support hMSCs osteogenic differentiation. In Chapter 7, antibiotic-eluting scaffolds were fabricated via high temperature ME-AM. The antibiotics ciprofloxacin and gentamicin were intercalated within the lamellar inorganic fillers magnesium alluminium layer double hydroxide (MgAl) and zirconium phosphate $(\mathrm{ZrP})$, respectively, prior to their dispersion within the polymeric matrix, in order to protect them from the fabrication process thermal stresses and obtain a sustained release. Compared to no-filler systems, a more sustained release was obtained 
in the filler-based scaffolds, which was governed by diffusion through the polymer matrix and by ion exchange or $\mathrm{pH}$ changes. After printing, scaffolds demonstrated antimicrobial activity against Gram + and Gram - bacterial strains, at the same levels as unprocessed antibiotics. In addition, fillers and antibiotics did not affect hMSCs viability and osteogenic differentiation as shown by matrix mineralization and osteogenic proteins expression.

All the aforementioned results are discussed and placed into the state of the art context, in Chapter 8, while providing future perspectives. To finalize, a reflection on the scientific and societal impact of the research carried out in this thesis is presented in Chapter 9.

\section{Samenvatting}

De huidige klinische strategieën om kritieke botafwijkingen te behandelen, voornamelijk gebaseerd op autografts, zijn inefficiënt en hebben beperkingen. Om deze reden is de orthopedische sector op zoek naar innovatieve en effectieve oplossingen om bot te regenereren in dergelijke scenario's, met behulp van tissue engineering. Melt extrussion additive manufacturing (ME-AM) heeft zich onderscheiden als een ideale biofabricagetechniek om op de patiënt afgestemde, zeer poreuze, biologisch afbreekbare en mechanisch robuuste scaffolds te genereren die ondersteuning kunnen bieden voor de vorming van nieuw botweefsel in niet-unionen. Deze steigers zijn echter gemaakt van synthetische polymeren met een gebrek aan bioactiviteit, die over het algemeen verder gefunctionaliseerd moeten worden om hun biologische prestaties te verbeteren. In dit opzicht zijn er de laatste decennia verschillende strategieën onderzocht om de bioactiviteit van ME-AM steigers te vergroten, die in hoofdstuk 2 van dit proefschrift zorgvuldig worden besproken. Strategieën zoals architectonische modificaties, oppervlakte fysische en chemische modificaties, de incorporatie van secundaire vezelachtige of hydrogel netwerken binnen de scaffolds poriën, en het gebruik van vulstof-polymeer composieten worden besproken.Aangezien de zoektocht naar optimale steigers die botregeneratie kunnen stimuleren doorgaat, is het doel van dit proefschrift om verder bij te dragen aan het veld door verschillende materiaal-gedreven benaderingen te onderzoeken om bioactieve ME-AM scaffolds te ontwerpen en te fabriceren voor botweefsel engineering. Om te beginnen, hoofdstuk 3 en hoofdstuk 4 bieden twee elegante oplossingen voor de terugkerende cel seeding efficiency probleem geassocieerd met macroporeuze 3D scaffolds. In hoofdstuk 3, werd een eenvoudige en betrouwbare statische zaaien 
methode ontwikkeld om de cel zaaien efficiëntie en distributie in ME-AM scaffolds te verbeteren. Deze methode bestond uit de toevoeging van Dextran of Ficoll aan de cel zaaien media, die het medium viscositeit en dichtheid, respectievelijk gewijzigd, en verminderde de afwikkeling snelheid van de cellen, waardoor voor een betere hechting en distributie tijdens het zaaien proces, in vergelijking met conventionele statische zaaien. De macromoleculen werden volledig verwijderd na de zaaiperiode en de celdistributie langs de scaffoldsdoorsnede werd drastisch verbeterd. Bovendien werd de osteogene differentiatie van hMSCs versneld, in termen van verbeterde mineralisatie en botmatrix maturatie, vergeleken met conventionele seeding. In hoofdstuk 4 werd het probleem van de celzaaiing vanuit een ander perspectief benaderd. In dit geval werden ME-AM scaffolds vervaardigd met behulp van een hybride AM techniek die ME-AM combineerde met een atmosferische druk plasmastraal, die werd gebruikt om het oppervlak te plasmafunctionaliseren. Terwijl argon activering resulteerde in een niet-specifieke behandeling, (3-Aminopropyl) trimethoxysilaan (APTMS) en maleïnezuuranhydrideVinyltrimethoxysilaan (MA-VTMOS) plasma polymerisatie afgezet positief en negatief geladen coatings, respectievelijk. Dit maakte het mogelijk het aanhechtingsmechanisme van de cellen aan de scaffolds te onderzoeken als functie van de oppervlaktelading in aanwezigheid of afwezigheid van serum. Bovendien verhoogden de coatings de bevochtigbaarheid van het oppervlak van de scaffolds, verbeterden ze de adsorptie van celadhesieve eiwitten en maakten ze een homogene celhechting langs de doorsnede van de scaffolds mogelijk. De celproliferatie was verbeterd, zelfs op verouderde scaffolds, en hMSCs waren in staat om osteogene genen tot expressie te brengen in alle scaffold types. $\mathrm{Na}$ het verzekeren van een efficiënte celaanhechting, werden composiet scaffolds bestaande uit een polymeermatrix gemengd met bioactieve vulstoffen met verschillende eigenschappen gefabriceerd in de hoofdstukken 5, 6 en 7. In hoofdstuk 5 werden hoogbelaste PEOT/PBT-nHA composiet scaffolds met tot 45 wt $\%$ nHA gemaakt, die de organisch-inorganische botsamenstelling nabootsten. Hier werd het effect van HA gehalte op de osteogene differentiatie van hMSCs diepgaand geëvalueerd, en de resultaten gaven aan dat ondanks geen waargenomen verschillen tussen scafoldstypes op gen- en eiwitniveau, verhoogde matrixmineralisatie werd waargenomen op $45 \mathrm{wt} \% \mathrm{nHA}$ scaffolds wanneer gekweekt in MM en BM. Bovendien reprecipiteerde een dichtere CaP-laag op deze steigers na onderdompeling in SBF. Deze resultaten in combinatie met de verhoogde compressie modulus gemeten op $45 \mathrm{wt} \% \mathrm{nHA}$ scaffolds, zouden hun verbeterde prestaties in vivo kunnen voorspellen. In hoofdstuk 6, werden ME-AM composiet scaffolds met rGO 
gemaakt om elektrische geleiding, eiwit adhesie en osteogene eigenschappen te bieden. In eerste instantie werd de lage dichtheid rGO verdicht door oplosmiddel verdamping, om het hanteren te vergemakkelijken en de veiligheid te garanderen tijdens het smelten. $\mathrm{Er}$ werd vastgesteld dat een hoge dichtheid leidde tot meer verdichte rGO, wat het drukken van deze composieten tot $15 \mathrm{wt} \%$ rGO formuleringen vergemakkelijkte. Anderzijds hadden composieten bereid met rGO met een gemiddelde dichtheid een lagere bedrukbaarheid, maar een hoger elektrisch geleidingsvermogen. Ook leidde het verhogen van het rGOgehalte tot een verhoging van de materiaalhydrofiliciteit, eiwitadsorptie en antibacteriële eigenschappen, zonder de levensvatbaarheid van hMSC's aan te tasten. Bovendien waren $3 \%$ rGO scaffolds in staat om hMSCs osteogene differentiatie te ondersteunen. In hoofdstuk 7, werden antibiotica-eluting scaffolds gefabriceerd via hoge temperatuur MEAM. De antibiotica CFX en GTM werden geïntercaleerd in de lamellaire anorganische vulstoffen $\mathrm{MgAl}$ en $\mathrm{ZrP}$, respectievelijk, voordat ze werden gedispergeerd in de polymere matrix, om ze te beschermen tegen de thermische spanningen in het fabricageproces en om een duurzame afgifte te verkrijgen. Vergeleken met systemen zonder vulstof werd een duurzamere afgifte verkregen in de op vulstof gebaseerde scaffolds, die werd bepaald door diffusie door de polymere matrix en door ionenuitwisseling of $\mathrm{pH}$-veranderingen. $\mathrm{Na}$ het printen toonden de steigers antimicrobiële activiteit tegen Gram + en Gram bacteriestammen, op dezelfde niveaus als onbewerkte antibiotica. Bovendien hadden vulstoffen en antibiotica geen invloed op de levensvatbaarheid en osteogene differentiatie van hMSC's, zoals aangetoond door matrixmineralisatie en expressie van osteogene eiwitten.

Alle bovengenoemde resultaten worden in hoofdstuk 8 besproken en in de context van de huidige stand van de wetenschap geplaatst, waarbij ook toekomstperspectieven worden gegeven. Tot slot wordt in hoofdstuk 9 een beschouwing gegeven over de wetenschappelijke en maatschappelijke impact van het onderzoek dat in dit proefschrift is uitgevoerd. 



\section{List of Publications}

\section{Publications related to this thesis}

M. Cámara-Torres, R. Sinha, C. Mota, L. Moroni, Improving cell distribution on 3D additive manufactured scaffolds through engineered seeding media density and viscosity, Acta Biomaterialia 2020, 101, 183-195, DOI: 10.1016/j.actbio.2019.11.020.

M. Cámara-Torres, R. Sinha, P. Scopece, T. Neubert, K. Lachmann, A. Patelli, C. Mota, L. Moroni, Tuning Cell Behavior on 3D Scaffolds Fabricated by Atmospheric PlasmaAssisted Additive Manufacturing, ACS Applied Materials \& Interfaces 2021, 13 (3), 36313644, DOI: 10.1021/acsami.0c19687.

M. Cámara-Torres, S. Duarte, R. Sinha, A. Egizabal, N. Álvarez, M. Bastianini, M. Sisani, P. Scopece, M. Scatto, A. Bonetto, A. Marcomini, A. Sanchez, A. Patelli, C. Mota, L. Moroni, 3D additive manufactured composite scaffolds with antibiotic-loaded lamellar fillers for bone infection prevention and tissue regeneration, Bioactive Materials, Bioactive Materials 2021, 6 (4), 1073-10821073-1082, DOI:10.1016/j.bioactmat.2020.09.031.

M. Cámara-Torres, R.Sinha, S. Eqtesadi, R. Wendelbo, M. Scatto, P. Scopece, A. Sanchez, S. Villanueva, A. Egizabal, N. Álvarez, A. Patelli, C. Mota, L. Moroni, Effect of reduced graphene oxide (rGO) compaction degree and concentration on rGO-polymer composites printability and cell interactions. (Accepted in Nanoscale, 2021).

M. Cámara-Torres, R. Sinha, A. Sanchez, P. Habibovic, A. Patelli, C. Mota, L. Moroni, Effect of highly loaded nano hydroxyapatite composite scaffolds prepared via melt extrusion additive manufacturing on the osteogenic differentiation of human mesenchymal stromal cells. (Submitted).

M. Cámara-Torres, R. Sinha, C. Mota, L. Moroni, Boosting bone regeneration using augmented additive manufactured scaffolds. (In preparation)

\section{Other publications}

R. Sinha, M. Cámara-Torres, P. Scopece, E. Verga Falzacappa, A. Patelli, L. Moroni, C. Mota, A Hybrid Additive Manufacturing Platform to Create Bulk and Surface Composition Gradients on Scaffolds for Tissue Regeneration. Nature Communications 2021, 12 (1), 500, DOI: 10.1038/s41467-020-20865-y. 
R. Sinha, A. Sanchez, M. Camara-Torres, I. Calderon Uriszar-Aldaca, A.R. Calore, J. Harings, A. Gambardella, L. Ciccarelli, V. Vanzanella, M. Sisani, M. Scatto, R. Wendelbo, S. Perez, S. Villanueva, A. Matanza, A. Patelli, N. Grizzuti, C. Mota, L. Moroni, Additive manufactured scaffolds for bone tissue engineering: physical characterization of thermoplastic composites with functional fillers. ACS Applied Polymer Materials 2021.

\section{Scientific communications}

Oral presentations:

3D additive manufactured composite scaffolds mimicking the bone composition and the bone-to-soft tissue interface: effect of high loadings of hydroxyapatite on the osteogenic differentiation of hMSCs, $11^{\text {th }}$ World Biomaterials Congress, Online event, 2020.

High loading Reduced Graphene Oxide 3D Scaffolds for Bone Tissue Engineering, 11 th World Biomaterials Congress, Online event, 2020.

Boosting bone regeneration: Additive manufacturing of continuous gradient scaffolds using functional composite materials. 3D Medical Printing Conference \& Expo, Maastricht, The Netherlands, 2020.

3D additive manufactured scaffolds with antimicrobial activity for bone regeneration. eCM XIX: Orthopaedic Infection, Davos, Switzerland, 2019.

Continuous gradient hydroxyapatite-based 3D additive manufactured scaffolds for bone interface tissue engineering, TERMIS European Chapter Meeting, Rhodes, Greece, 2019. $3 \mathrm{D}$ additive manufactured scaffolds with antimicrobial activity for bone regeneration, $3^{\text {rd }}$ MERLN PhD Symposium, Maastricht University, The Netherlands, 2018.

3D additive manufactured scaffolds with antimicrobial activity for bone regeneration, 29 th Annual Conference of the European Society of Biomaterials, Maastricht, The Netherlands, 2018.

Poster presentations:

3D additive manufactured composite scaffolds for bone and bone-to-soft tissue interface: Effect of high loadings of hydroxyapatite on the osteogenic differentiation of hMSCs. $4^{\text {th }}$ MERLN PhD Symposium, Maastricht, The Netherlands, 2019. 
Atmospheric plasma assisted additive manufacturing of 3D scaffolds for tissue regeneration applications, 29 $9^{\text {th }}$ Annual Conference of the European Society of Biomaterials, Maastricht, The Netherlands, 2018.

Continuously graded reduced graphene oxide $3 \mathrm{~d}$ scaffolds for bone regeneration, TERMIS World Congress, Kyoto, Japan, 2018.

Novel static cell seeding method to improve cell distribution in 3D additive manufactured scaffolds, TERMIS World Congress, Kyoto, Japan, 2018.

Novel Static Cell Seeding Method for Improving Cell Distribution on 3D Additive Manufactured Scaffolds, 2 ${ }^{\text {nd }}$ MERLN PhD Symposium, Maastricht, The Netherlands, 2017. 



\section{Acknowledgement}

This long journey has finally come to an end, and I would like to acknowledge all the people that contributed by making it a bit easier and more enyojable.

First of all, I want to thank my parents, who everyday look forward to the day that I come back to their nest. They have always supported every decision I have made in my life, and will always continue doing so. They have taught me to be constant and persistent to achieve my goals. Also, thanks to my sister, who was brave enough to follow a similar path and become a PhD student herself. It seems I did not stress to her enough what a rollercoaster a $\mathrm{PhD}$ is. Well, we both always like amusement parks.

Next, I want to thank Omid. Simply, you were always there. From the first to the last day, you have encouraged me to be the best version of myself. You have been my life partner and my life coach. If science and business do not work for you, I ensure you a profitable career as a motivational speaker.

Special thanks also go to Lorenzo, for spotting something on me and hiring me as part of his team. I could not have thought of a better supervisor. You were always supportive, encouraging, and available to help with problems. I specially appreciated your nice words towards my work and working attitude, which certainly boosted my self-confidence in moments when I needed it the most.

Thank you also to Carlos, who was there on a daily basis, and became much more than a supervisor. I could always openly talk to you about my struggles, and you were there to help me whenever I needed it. You also allowed me to annoy you with plenty of transcendental questions, and even you rewarded me with the "Why?" title, which still hangs on my living room wall. You were the FAST team's GPS, and without your guidance the project would not have been as successful (and Ravi and I would still be lost in some street of Perugia).

Ravi, this PhD journey would not have been the same without you. I cannot remember a day in which I did not pay a visit to you in the in the lab or in your desk, to talk about our day, our problems, funny facts, and gossips. It felt like my daily therapy session. I really enjoyed our friendship, our FAST-meeting trips, and working with you. We made a good team combining your practical working attitude and my detective skills. It seems that when reviewing my papers, we exchanged roles and you became the picky one, but thank you 
for that also. As heritage, you left me some memorable quotes (i.e. "When you get a good result, take a picture and run"), for which I am very grateful and I applied to finish my PhD effectively.

Clarissa, you were one of my favorite persons in the lab, thank you for being my paranymph. Talking with you was always fun and exciting, gossip queen. Apart from that, I always felt I could talk to you about my struggles with my project, which did not look so dramatic beside yours. I really enjoyed sharing the printing bench with you on many occasions, it made working more attractive. Thank you for taking care of my cells in my absence and for thinking of me while in your trips and bringing me treats.

Filipa, you joined our FAST team at the end, but it felt like if you had been with us from the beginning. Thank you for the fresh air and positivity you brought to us. I really enjoyed working together on the in vivos, it was probably the most exciting time of the PhD. Special thank you for letting me hammer some glass vials, it was a stress relieve.

Sandra, I believe I will ever meet such a complete scientist as you are. I always admired your scientific knowledge, your productivity in the lab, and the high quality of your research. When I grow up, I want to be like you. I really appreciated our scientific discussions, your practical help in the lab, the times that you took care of my cells and, mostly, our friendship. It was always nice spending time with you, speak in Spanish, and share some gossip to spice up the day.

Special thanks to Tianyu, Huey Wen, Khadija, Rong, Urnaa and Rabeil. We were all at MERLN during the first half of my PhD, and I really enjoyed your company, our lunch times, coffee breaks, dinner times, badminton, and other plans we did together. I am happy we got to know each other, and I believe we will keep this friendship for many years more. Special thanks to Tianyu, who was there until the end. We supported each other during our whole PhD journey. I always enjoyed having lunch together, talking about our stuff, and going to Zumba. Thank you for letting me be your paranymph. I hope that we will see each other soon, in China.

Thank you to Stacy for being my master student, contributing to one of the publications and helping me to develop my patience and my mentoring abilities. To Shahzad, for being always friendly and keeping me company some evenings in the lab. To David G, Jip, Victor, Daniel and Danielle, for welcoming me when I arrived to MERLN. To Kenny and Andrea, for the time shared in the biofabrication lab. To Jiaping, David K, Vahid, Afonso, Adrian, 
Paul, Matt, Gabrielle, Shivesh, Tristan, Amit, Mirco, for being my colleagues in the biofabrication group, sharing some nice talks and scientific discussions, and helping me when I needed it. Thanks also to Eva, Marloes, Timo and Denis, for your help.

Last but not least, I would like to thank my collaborators within FAST: Prof. Alessandro Patelli, Ainhoa Egizabal, Noelia Álvarez, Dr. Alberto Sanchez, Dr. Sara Villanueva, Marco Scatto, Dr. Paolo Scopece, Dr. Rune Wendelbo, Dr. Siamaq Eqtesadi, Dr. Maria Bastianini, Dr. Michelle Sisani, Dr. Thomas Neubert, Dr. Kristina Lachmann, who helped me to make this thesis great, and with who I shared nice off-work times during our FAST meetings. 



\section{Biography}

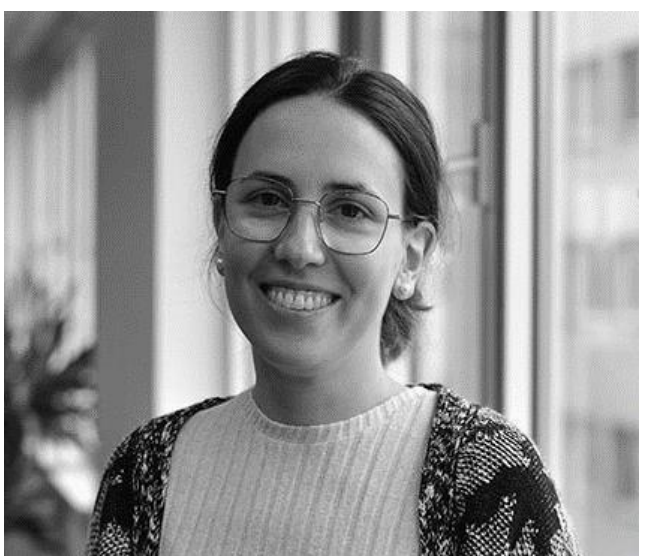

María was born on the $16^{\text {th }}$ August 1991 in Madrid, Spain. Pursuing her interest for engineering and biomedical sciences, she completed her BSc. in Biomedical Engineering at TECNUN School of Engineering- University of Navarra, Spain, in 2013. Aiming to gain international experience, she enrolled in a MSc. in Biomedical Engineering at FHAachen University of Applied Science, Germany. She performed her master thesis at the DWI-Leibniz Institute for Interactive Materials in Aachen, Germany, where she developed injectable magnetic microgels and macroporous hydrogels to obtain tissue regeneration structures in situ. With this experience, her passion for biomaterials and tissue engineering grew, and in 2016, she joined MERLN institute for Technology-inspired Regenerative Medicine, Maastricht University, as a PhD candidate. There, she worked on a multinational and interdisciplinary European project entitled FAST, which aimed at developing novel 3D-additive manufactured composite scaffolds for bone regeneration in critical-size defects. In her research, she explored the effect of newly developed functional composites and surface modifications on the differentiation of stem cells towards bone cells. Keeping in mind the ultimate goal of tissue engineering and regenerative medicine, in the future she aims at bringing new biomedical products to patients. 
